\title{
Micro-Systems for Time-Resolved Fluorescence Analysis Using CMOS Single-Photon Avalanche Diodes and Micro-LEDs
}

\author{
Bruce R. Rae
}

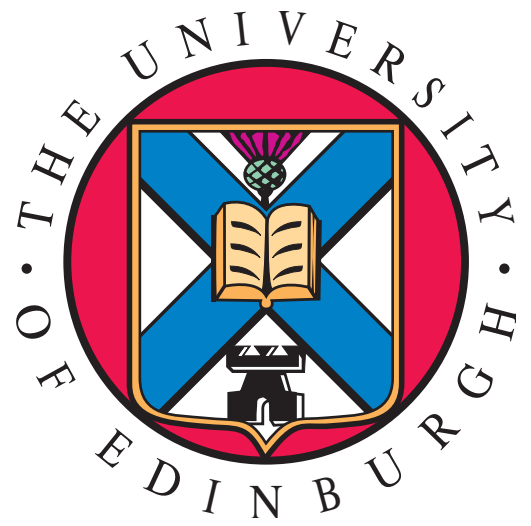




\section{Abstract}

Fluorescence based analysis is a fundamental research technique used in the life sciences. However, conventional fluorescence intensity measurements are prone to misinterpretation due to illumination and fluorophore concentration non-uniformities. Thus, there is a growing interest in time-resolved fluorescence detection, whereby the characteristic fluorescence decay time-constant (or lifetime) in response to an impulse excitation source is measured. The sensitivity of a sample's lifetime properties to the micro-environment provides an extremely powerful analysis tool. However, current fluorescence lifetime analysis equipment tends to be bulky, delicate and expensive, thereby restricting its use to research laboratories. Progress in miniaturisation of biological and chemical analysis instrumentation is creating low-cost, robust and portable diagnostic tools capable of high-throughput, with reduced reagent quantities and analysis times. Such devices will enable point-of-care or in-the-field diagnostics. It was the ultimate aim of this project to produce an integrated fluorescence lifetime analysis system capable of sub-nano second precision with an instrument measuring less than $1 \mathrm{~cm}^{3}$, something hitherto impossible with existing approaches. To accomplish this, advances in the development of AlInGaN micro-LEDs and high sensitivity CMOS detectors have been exploited. CMOS allows electronic circuitry to be integrated alongside the photodetectors and LED drivers to produce a highly integrated system capable of processing detector data directly without the need for additional external hardware.

In this work, a 16x4 array of single-photon avalanche diodes (SPADs) integrated in a $0.35 \mu \mathrm{m}$ high-voltage CMOS technology has been implemented which incorporates two 9-bit, in-pixel time-gated counter circuits, with a resolution of 400ps and on-chip timing generation, in order to directly process fluorescence decay data. The SPAD detector can accurately capture fluorescence lifetime data for samples with concentrations down to $10 \mathrm{nM}$, demonstrated using colloidal quantum dot and conventional fluorophores. The lifetimes captured using the on-chip time gated counters are shown to be equivalent to those processed using commercially available external time-correlated single-photon counting (TCSPC) hardware.

A compact excitation source, capable of producing sub-nano second optical pulses, was designed using AlInGaN micro-LEDs bump-bonded to a CMOS driver backplane. A series of driver array designs are presented which are electrically contacted to an equivalent array 
of micro-LEDs emitting at a wavelength of $370 \mathrm{~nm}$. The final micro-LED driver design is capable of producing optical pulses of 300ps in width (full width half maximum, FWHM) and a maximum DC optical output power of $550 \mu \mathrm{W}$, this is, to the best of our knowledge, the shortest reported optical pulse from a CMOS driven micro-LED device.

By integrating an array of CMOS SPAD detectors and an array of CMOS driven AlInGaN micro-LEDs, a complete micro-system for time-resolved fluorescence analysis has been realised. Two different system configurations are evaluated and the ability of both topologies to accurately capture lifetime data is demonstrated. By making use of standard CMOS foundry technologies, this work opens up the possibility of a low-cost, portable chemical/bio-diagnostic device. These first-generation prototypes described herein demonstrate the first time-resolved fluorescence lifetime analysis using an integrated micro-system approach. A number of possible design improvements have been identified which could significantly enhance future device performance resulting in increased detector and micro-LED array density, improved time-gate resolution, shorter excitation pulse widths with increased optical output power and improved excitation light filtering. The integration of sample handling elements has also been proposed, allowing the sample of interest to be accurately manipulated within the micro-environment during investigation. 


\section{Declaration of Originality}

I hereby declare that:

(a) the thesis has been composed entirely by myself;

(b) the work is my own, except where clearly indicated, and originated in the School of Engineering at The University of Edinburgh;

(c) the work has not been submitted for any other degree or professional qualification

Bruce Rothnie Rae 


\section{Acknowledgements}

This project was funded by the Engineering and Physical Sciences Research Council of the U.K.

I would like to thank Dr Robert Henderson for his dedicated supervision, encouragement, advice and guidance throughout my project. Many thanks to Dr David Renshaw for providing timely guidance, ensuring that I stuck to my deadlines and writing Matlab code that greatly advanced this project. Many thanks to Professor Edoardo Charbon of EPFL, Switzerland now at T.U. Delft in The Netherlands, for generously providing me with the SPAD structure which allowed this project to progress so rapidly. My thanks go to Professor Martin Dawson, Professor John Girkin, Dr Erdan Gu, Dr Chris Griffin and Jonathan McKendry at the Institute of Photonics for developing the micro-LED devices and working so closely with me during their integration with CMOS and their characterisation. Special thanks to the other members of Robert's research group Justin Richardson, Keith Muir, Richard Walker and Day-Uei Li for all the technical and not so technical discussions which made my $\mathrm{PhD}$ time so interesting and enjoyable. I would also like to thank Dr Katherine Cameron, who assisted me greatly when I first started my project, answering my many questions. I would like to acknowledge the important contributions made by Andrew Garrie of COSMIC at the University of Edinburgh for manufacturing the filter and slide holder for the micro-system and Martin Turner at ST Microelectronics for providing the original test PCB which provided me with an excellent platform to test my first chip designs. Thanks to Patricia Richardson and Robin Andrews from the University of Edinburgh Chemistry department and Jochen Arlt from COSMIC, the University of Edinburgh, for providing advice and access to equipment and fluorophores. I would also like to thank Bill Ashby at Optocap, Livingston for his assistance with the micro-LED bump-bonding process.

Finally, I would like to thank my partner Sabrina whose support, help, love and patience helped me keep perspective during my project. Her guidance on punctuation and grammar has also been an invaluable contribution to this thesis. My friends both here in Edinburgh and in Aberdeen who have supported me over the last few years. Last but by no means least, I would like to thank my family. My Mum, Dad and Sister for their love and support through my time at university and for encouraging me to pursue this goal. 


\section{Abbreviations}

ADC = Analogue-to-Digital Converter

AlInGaN = Aluminium Indium Gallium Nitride

CCD = Charge-Coupled Device

CFD $=$ Constant Fraction Discriminator

CMOS = Complimentary Metal Oxide Semiconductor

DBR = Distributed Bragg Reflector

DCR = Dark Count Rate

DNA = Deoxyribonucleic Acid

FF = Flip-Flop

FLIM = Fluorescence Lifetime Imaging Microscopy

FPGA $=$ Field-Programmable Gate Array

FRET $=$ Fluorescence Resonance Energy Transfer

FWHM = Full Width Half Maximum

GaAs = Gallium Arsenide

GaN = Gallium Nitride

GUI $=$ Graphical User Interface

IEM = Integration for Lifetime Extraction Method

InGaN = Indium Gallium Nitride

$\mathbf{I} / \mathbf{O}=$ Input/Output

IRF = Instrument Response Function

JTAG $=$ Joint Test Action Group

LED = Light-Emitting Diode

MCP = Micro-Channel Plate

MRI = Magnetic Resonance Imaging

PCB = Printed Circuit Board 
PCI $=$ Peripheral Component Interconnect

PDP $=$ Photon Detection Probability

PET $=$ Positron Emission Tomography

PMT = Photo-Multiplier Tube

RLD = Rapid Lifetime Determination

RMS = Root Mean Square

SPAD $=$ Single Photon Avalanche Diode

TCSPC $=$ Time-Correlated Single Photon Counting

$\mathbf{T i} / \mathbf{A u}=$ Titanium $/$ Gold

TRF $=$ Time Resolved Fluorescence

TTL $=$ Transistor-Transistor Logic

USB $=$ Universal Serial Bus

$\mathbf{U V}=$ Ultra-Violet

VCO $=$ Voltage Controlled Oscillator

VCSEL = Vertical Cavity Surface Emitting Laser

XML $=$ Extensible Markup Language

ZIF = Zero Insertion Force 


\section{Contents}

Abstract ........................ . . . ii

Declaration of Originality ................. iv

Acknowledgements .................... . . v

Abbreviations ........................ vi

List of figures . . . . . . . . . . . . . . . . . . . xi

List of tables ....................... . . . . . . . . . . . . . . .

1 Introduction $\quad 1$

1.1 Miniaturisation of a Fluorescence Lifetime

Measurement System .................... 1

1.2 Aims ......................... 3

1.3 Thesis ......................... 3

1.4 Contribution to Knowledge . . . . . . . . . . . . . . . . 4

1.5 Thesis Outline . . . . . . . . . . . . . . . . 5

2 Fluorescence and Sample Analysis Techniques $\quad 7$

2.1 Fluorescence . . . . . . . . . . . . . . . . . . 7

2.1 Fluorescence Lifetime . . . . . . . . . . . . . . . 9

2.2 Excitation Sources . . . . . . . . . . . . . . . . 15

2.3 Detectors ........................ . . 17

2.4 Micro-Analytical Systems . . . . . . . . . . . . . . . . . 22

2.5 Non-Fluorescence Based Sensing Techniques . . . . . . . . . . . . . . . 27

2.6 Key Technologies . . . . . . . . . . . . . . . . . . . . . . . . . . . . . . . . . . .

2.6.1 The SPAD Detector .................. 28

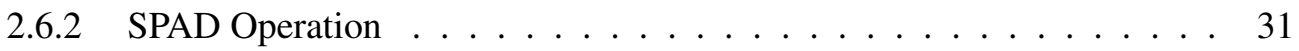

2.6.3 AlInGaN Micro-LEDs . . . . . . . . . . . . . . 38

2.6.4 Micro-LED Operation ................. . . 39

2.6.5 Micro-LED Performance . . . . . . . . . . . . . . . . 39

2.7 Summary ......................... . . . 40

3 Materials and Methods $\quad 43$

3.1 Hardware ........................ . . 43

3.1.1 Optical Bench . . . . . . . . . . . . . . . . 43

3.1.2 PicoQuant Laser Diode and Driver . . . . . . . . . . . . . . 45

3.1.3 Nikon TE2000-U Microscope . . . . . . . . . . . . . 45

3.1 .4 Filter Sets ...................... . . 48

3.1.5 Bench Top Optical Setup . . . . . . . . . . . . . . 50

3.1.6 Becker and Hickl TCSPC Module . . . . . . . . . . . . . . . . 52

3.1.7 Becker and Hickl Photo Multiplier Tube Detector Head . . . . . . . . . 53

3.2 PCB Test Boards . . . . . . . . . . . . . . . . . . . . . . 53

3.2.1 ST Microelectronics 908 Test Board . . . . . . . . . . . . . 53

3.2.2 The University of Edinburgh/Opal Kelly Test Board . . . . . . . . 55 
3.2 .3 Daughter Cards . . . . . . . . . . . . . . . . . . . . . . 56

3.3 Software ........................ . . 58

3.3 .1 Xilinx ISE and Altera Quartus . . . . . . . . . . . . . . . . 58

3.3 .2 Front Panel . . . . . . . . . . . . . . . . 58

3.3 .3 v2wreg ......................... 60

3.3 .4 vpciview ......................... 62

3.3 .5 Matlab ........................ 63

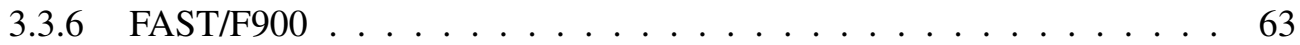

3.3 .7 PCB Fabrication . . . . . . . . . . . . . . . . . . . . . 64

3.3 .8 Cadence . . . . . . . . . . . . . . . . . . . . . . . . . . . . . . . . . 64

3.4 Experimental Procedure . . . . . . . . . . . . . . . . . . . . . . . . 64

3.4.1 Quantum Dots . . . . . . . . . . . . . . . . . . . . . . . . . . 64

3.4 .2 Other Fluorophores . . . . . . . . . . . . . . . . . . . . . . 65

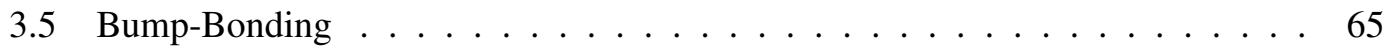

4 SPAD Detector and Circuits for Lifetime Estimation

4.1 SPAD Performance . . . . . . . . . . . . . . . . . . . 72

4.2 SPADs with Time-Gated Counters . . . . . . . . . . . . . . . . 73

4.2.1 Counter Circuit . . . . . . . . . . . . . . . . . . . . . . . . . . 75

4.2 .2 Timing Generator . . . . . . . . . . . . . . . . . . . 76

4.2 .3 Time-Gating . . . . . . . . . . . . . . . . . . 81

4.3 Time-Resolved Fluorescence Captured with SPADs . . . . . . . . . . . . . . 83

4.3.1 SPAD Sensitivity and Measurement Accuracy . . . . . . . . . . . . . 83

4.3.2 Fluorescein and Rhodamine B Evaluation . . . . . . . . . . . . . . 88

4.3.3 Lifetime Extraction from Decay Curves . . . . . . . . . . . . . . . . 89

4.4 Time-Resolved Fluorescence Captured using SPADs and On-Chip Counters . . 90

4.4.1 Lifetime Estimation Algorithms _. . . . . . . . . . . . . . . . 90

4.4.2 On-Chip Lifetime Measurement Sensitivity and Accuracy . . . . . . . 92

4.4.3 On-Chip Detection of Fluorescein, Rhodamine B and Coumarin 6 . . . 94

4.4.4 Lifetime Measurement Comparison . . . . . . . . . . . . . . . . 95

4.5 Discussion . . . . . . . . . . . . . . . . . . . . . . . . . . . . . 97

5 Micro-LED Devices and Drivers 100

$5.116 \times 4$ Current Controlled Micro-LED Driver Array . . . . . . . . . . . . . . 101

5.1 .1 Driver Circuitry . . . . . . . . . . . . . . . . . . . . . . 104

5.1.2 16x4 Driver Array Performance . . . . . . . . . . . . . . . . 106

$5.216 \times 16$ Voltage Controlled Micro-LED Driver Array . . . . . . . . . . . . . . 113

5.2 .1 Driver Circuitry . . . . . . . . . . . . . . . . . . . . . 115

5.2.2 Voltage Controlled Oscillator (VCO) and Linear Feedback Shift Register (LFSR) Circuits . . . . . . . . . . . . . . . . . 122

5.2 .3 Modes of Operation . . . . . . . . . . . . . . . . . . . . 123

5.2.4 Device Failure Due to Bump-Bonding Process . . . . . . . . . . . . . 124

$5.38 \times 8$ Voltage Controlled Micro-LED Driver Array . . . . . . . . . . . . . . . . 125

5.3.1 8x8 Driver Array Performance . . . . . . . . . . . . . . . . . . 128

5.4 Discussion . . . . . . . . . . . . . . . . . . . . . . . . . . 140

6 Micro-Systems for Time-Resolved Fluorescence Lifetime Detection 142 
6.1 Fluorescence System Transmission Model . . . . . . . . . . . . . . . . . . . 143

6.2 Single-Chip Micro-System for Time-Resolved Fluorescence Lifetime Detection 146 6.2 .1 System Setup . . . . . . . . . . . . . . . . . . . . . . . . . . . 146

6.2 .2 Excitation Light Filtering . . . . . . . . . . . . . . . . . . . . . 148

6.2.3 Fluorescence Lifetime Data Captured with Single Chip System . . . . . 150

6.2.4 Single-Chip Micro-System: Summary . . . . . . . . . . . . . . . . . . 151

6.3 Two-Chip Micro-System for Time-Resolved Fluorescence Lifetime Detection . 153

6.3.1 Fluorescence Lifetime Data Captured with Two-Chip System . . . . . 158

6.3.2 Two-Chip Micro-System: Summary . . . . . . . . . . . . . . . . . . . 160

6.4 Discussion . . . . . . . . . . . . . . . . . . . . . . . . . . . . 160

7 Future Work and Conclusions 163

7.1 Summary . . . . . . . . . . . . . . . . . . . . . 163

7.2 Critical Discussion . . . . . . . . . . . . . . . . . 165

7.3 Future Work . . . . . . . . . . . . . . . . . . . . . . . . . 167

7.4 Conclusions . . . . . . . . . . . . . . . . . . . . . . . . . 174

A Appendices $\quad \mathbf{1 7 6}$

A.1 16x4 SPAD Chip and 16x4 Micro-LED Driver Pin List . . . . . . . . . . 176

A.2 Single In-Pixel Counter Bit . . . . . . . . . . . . . . . . . . . . . . . . . . 179

A.3 8x8 Micro-LED Driver Pin List . . . . . . . . . . . . . . . . . . . . . . . . . . 180

A.4 VCO CIRCUIT . . . . . . . . . . . . . . . . . . . . . . . . . . 182

A.5 LFSR Circuit . . . . . . . . . . . . . . . . . . . . . . . . . . . 183

A.6 Verilog Used to Drive the 16x4 SPAD and Micro-LED Driver Chip . . . . . . . 184

A.7 Verilog Used to Drive the $16 \times 16$ and 8x8 Micro-LED Driver Chips . . . . . . 186

$\begin{array}{lll}\text { B Publications } & 187\end{array}$

$\begin{array}{ll}\text { References } & 254\end{array}$ 


\section{List of figures}

1.1 Commercially available fluorescence lifetime analysis systems; (a) MicroTime 100 by PicoQuant, an upright time-resolved fluorescence microscope. (b) OB920 fluorescence lifetime spectrometer by Edinburgh Instruments.

2.1 Jablonski Diagram. Promotion of an electron to a higher energy state due to excitation light and its subsequent relaxation to the ground state, coupled with the emission of a lower energy photon. . . . . . . . . . . . . . . .

2.2 In the frequency domain, modulation factor and phase difference can be used to estimate fluorescence lifetime. . . . . . . . . . . . . . . . . . . . 11

2.3 In the time domain, fluorescence intensity decay is measured directly as a function of time. . . . . . . . . . . . . . . . . . . . . 12

2.4 Time-gated fluorescence lifetime detection using an integrating photodiode with variable reset times. Presented by Patounakis et al. 2006. . . . . . . . . 18

2.5 Photodiode and VCSEL arrangement implemented by Thrush et al. 2004. . . . 24

2.6 Raw SPAD output pulses from a SPAD under varying intensity. . . . . . . . . . 28

2.7 Schematic cross-section and layout view of the circular SPAD employed in this project. This SPAD was implemented in a $0.35 \mu \mathrm{m}$ high-voltage CMOS technology. . . . . . . . . . . . . . . . . . . . . . . 29

2.8 Energy band diagrams in the Z-axis of the p+/deep n-well junction of a SPAD device. . . . . . . . . . . . . . . . . 30

2.9 The avalanche breakdown process. . . . . . . . . . . . . 31

2.10 Biasing of the SPAD detector to ensure TTL compatible output pulses upon detection of a photon. . . . . . . . . . . . . . . 32

2.11 Examples of dark count rate measurements as a function of the reverse bias voltage, VOP, for two different SPADs in the array. . . . . . . . . . . . . . . 34

2.12 In-pixel active quench circuit designed at FBK, Trento, Italy by David Stoppa et al. presented at the 2007 IEEE International Image Sensor Workshop . . . . 37

2.13 Cross-section diagrams after 4 AlInGaN fabrication steps. . . . . . . . . . . 38

2.14 IV curve of the UV (370nm) device. Measurement taken by Dr. Chris Griffin, Institute of Photonics, University of Strathclyde. . . . . . . . . . . . . . . 40

2.15 Wavelength spectra of a CMOS driven UV micro-LED device. . . . . . . . . . 40

3.1 Optical bench setup. . . . . . . . . . . . . . . . . . . . . . . . 44

3.2 Nikon TE2000-U Microscope used for SPAD chip characterisation. . . . . . . . 46

3.3 C-mount adaptor which housed a $1^{\prime \prime}$ diameter, $1^{\prime \prime}$ focal length lens used to re-size the image presented at the output port of the microscope. Image courtesy of Andrew Garrie, COSMIC at The University of Edinburgh. . . . . . . . . . . 47

3.4 Epi-fluorescence filter configuration. Image adapted from original presented in www.fluorescence.com/tutorial/fm-optic.htm, C\&L Instruments, Inc. 2009. . 49

3.5 Optical setup used to analyse samples held in cuvettes. . . . . . . . . . . . 50

3.6 Bench top analysis of a Fluorescein sample in a cuvette. . . . . . . . . . . . 51 
3.7 PCB test board used to characterise the $16 \times 4$ SPAD and counter array and $16 \times 4$ micro-LED array chip. . . . . . . . . . . . . . . . . . 54

3.8 PCB test board used to characterise the $8 \times 8$ micro-LED array. . . . . . . . . . 55

3.9 Daughter card used to re-map signals of the 908 Test Board from ST Microelectronics to allow compatibility with the 16x4 SPAD and Micro-LED array test chip. . . . . . . . . . . . . . . . . . . 56

3.10 Daughter card used to re-map signals of the University of Edinburgh/Opal Kelly test board to allow compatibility with the $16 \times 16$ and $8 \times 8$ Micro-LED array test chip. . . . . . . . . . . . . . . . . . . 57

3.11 Example of GUI created using XML code used to control digital I/O on chips being tested with Edinburgh University/Opal Kelly test board. . . . . . . . .

3.12 Screen shot of v2wreg GUI. A register map has been loaded which is used to control specific registers within the FPGA. This register map is intended to control the 16x4 SPADs with time-gated counters system. . . . . . . . . . . 61

3.13 The vpciview software was used to create images from time-gated counter data. 62

3.14 (a) Palomar 8000 automatic wire bonder from Palomer Technologies, Inc. and (b) a close up of the bonding tip. . . . . . . . . . . . . . . . . 66

3.15 Finetech Fineplacer flip-chip bonder from Finetech GmbH. . . . . . . . . . . 67

3.16 Stages of the bump-bonding process. (a) Gold ball-bonds are deposited on the CMOS driver array; (b) the micro-LED array is then flip-chip bonded to the CMOS die.

4.1 Illustration of a fluorescence lifetime decay captured using a time-gated counter circuit. . . . . . . . . . . . . . . . . 70

4.2 SPAD chip micrograph. . . . . . . . . . . . . . . . . . . . 71

4.3 PDP plots of the SPAD under different bias conditions and compared to a SPAD in $0.8 \mu \mathrm{m}$ technology. Comparison is also made to a SPAD without the die passivation layer removed. . . . . . . . . . . . . . . . . 72

4.4 System block diagram. . . . . . . . . . . . . . . . . . . . . . 74

4.5 SPAD circuit with passive quench transistor and two associated 9-bit ripple counters. . . . . . . . . . . . . . . . . . . . 75

4.6 On-chip 120-stage tapped delay line used to define counter time-gate and LED pulse widths. . . . . . . . . . . . . . . . . 78

4.7 An individual element of the 120-stage tapped delay line . . . . . . . . . . . 79

4.8 Circuit and timing diagram for the in-pixel time gate generator. . . . . . . . . . 80

4.9 Relationship between the on-chip generated time gate signal and the SPAD pulse in the counter. . . . . . . . . . . . . . . . . . . . . . . . 80

4.10 (a) Fixed and (b) variable width time-gate schemes. TG1 and TG2 represent the time-gate input signal to the first and second in-pixel counter circuits, respectively. 81

4.11 vpciview image and plotted horizontal pixel value of pulsed laser source, captured using a time-gate of fixed width and scanned position. . . . . . . . . 82

4.12 vpciview image and plotted horizontal pixel value of pulsed laser source, captured using a time-gate of variable width and fixed start position. . . . . . . 82 
4.13 Principle of TCSPC. (a) the sample of interest is excited with a short excitation pulse, resulting in a decaying fluorescence signal. (b) A single photon from this decay causes the detector to trigger. The time between the detector pulse and the subsequent excitation pulse is measured. (c) This measurement is used to place this photon count in a histogram time bin. As this process is repeated, a histogram representing the fluorescence decay is obtained. . . . . . . . . 83

4.14 Time resolved decay curves of quantum dot sample, evaluated at different

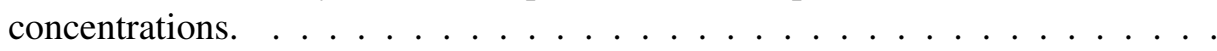

4.15 Time resolved decay curves of a $0.1 \mu \mathrm{M}$ quantum dot sample gathered for a fixed experimental time of 5 minutes and until 5000 counts were achieved in the peak channel (experimental time unrestricted). . . . . . . . . . . . . .

4.16 Time resolved decay curves of $1 \mu \mathrm{M}$ Fluorescein and Rhodamine B samples in Ethanol and $\mathrm{H}_{2} \mathrm{O}$, respectively. . . . . . . . . . . . . . . . . . . . 88

4.17 Fluorescence lifetime measurements obtained from quantum dot samples using SPAD detector and on-chip circuitry. . . . . . . . . . . . . . . . . 92

4.18 Fluorescein decay captured using 8 time-gates, each $2.4 n$ s wide. . . . . . . . . 94

4.19 Extracted lifetimes of quantum dot samples of varying concentration. Captured using different on-chip time-gated methods and external TCSPC hardware and processed using different extraction algorithms. . . . . . . . . . . . . 96

5.1 Floor plan of the original micro-LED driver test chip, part of the same test chip which featured the SPADs with counters array (Chapter 4). . . . . . . . . . 102

5.2 Micrograph of the individual top metal layer plates upon which each micro-LED in the array is bump-bonded. The SPAD detectors are situated in the circular gaps in the metal. . . . . . . . . . . . . . . . . . . . . 103

5.3 (a) Micrograph of 16x4 driver array with bump-bonded micro-LED array; (b) close-up of array highlighting array dimensions. . . . . . . . . . . . . 103

5.4 Current controlled micro-LED driver, situated under each electrode in the array. 104

5.5 Layout of a single micro-LED driver pixel within the $16 \times 4$ array. Situated within each driver is a single SPAD detector. . . . . . . . . . . . . . . 105

5.623 micro-LED devices operating simultaneously. . . . . . . . . . . . . 106

5.7 Uniformity of a CMOS driven UV (370nm) micro-LED array. . . . . . . . . 107

5.8 Input bias current mirror, with additional source resistance due to track length and bump-bonding. . . . . . . . . . . . . . . . . . . 108

5.9 Optical output power of a UV (370nm) device versus bias current for different elements in the array being driven by a DC signal. . . . . . . . . . . . . . . 109

5.10 Comparison of the average optical output power of a DC driven UV device and a square-wave $(50 \%$ duty cycle) driven device. . . . . . . . . . . . . . 110

5.11 The shortest and longest pulse widths achieved. . . . . . . . . . . . 111

5.12 Average optical pulse power versus bias current. . . . . . . . . . . . . 111

5.13 Average optical pulse power versus pulse width. . . . . . . . . . . . . . . 112

5.14 16x16 micro-LED driver array top level layout. . . . . . . . . . . . . . . . . 114

5.15 Driver circuit implemented in 16x16 micro-LED driver array. . . . . . . . . . 116

5.16 Layout of a single micro-LED driver pixel within the $16 \times 16$ array. . . . . . . 117

5.17 In-pixel level shifter circuit. . . . . . . . . . . . . . . . . . . . 118 
5.18 In-pixel pulse generator. Pulse width is equal to the inverter delay which is defined by VBMC2. When Mode Control is asserted the delay inverter is bi-passed and the inverter output tracks the circuit input. . . . . . . . .

5.19 The four main modes of driver operation (other than DC): (a) A square wave input signal derived from the on-chip VCO, (b) square wave signal derived from the on-chip LFSR circuit, (c) variable width pulsed-mode with repetition rate defined by VCO, (d) variable width pulsed-mode with repetition rate defined by LFSR. . . . . . . . . . . . . . . . . . . . . . . . . . . 123

5.20 Cross-section of an Austria Microsystems CMOS wafer. Image adapted from original presented in Austria Microsystems $0.35 \mu \mathrm{m}$ CMOS Process Parameters document. . . . . . . . . . . . . . . . . . . . . . . . . . . . 124

$5.218 \times 8$ micro-LED driver array top level layout. . . . . . . . . . . . . . . . . . 126

5.22 Layout view of a single pixel. In this revision a full metal stack has been dedicated to the bond site with the driver circuit located elsewhere in the pixel.

5.23 Uniformity of voltage driven UV $(370 \mathrm{~nm})$ micro-LED array (LED_VDD $=5 \mathrm{~V}$, LED_GND $=$ gnd!) . . . . . . . . . . . . . . . . . . . . . . . . . . 129

5.24 Current versus voltage characteristics of UV micro-LED device. . . . . . . . 130

5.25 Optical power versus current characteristics of UV micro-LED device. . . . . . 131

5.26 Optical pulse width versus the in-pixel current starving NMOS transistor gate bias voltage. . . . . . . . . . . . . . . . . . . 132

5.27 Shortest and longest optical pulses produced by $8 \times 8$ driver circuit. . . . . . . 133

5.28 Optical pulse width versus average optical power. . . . . . . . . . . . . . 134

5.29 LED_GND bias voltage versus average optical output power. . . . . . . . . . . 135

5.30 LED_GND bias voltage versus optical pulse width. . . . . . . . . . . . . 136

5.31 LED_GND is a global signal. When an LED driver is in the off state, the voltage across the LED is equal to the negative bias on LED ground. If this voltage is sufficient, current is sourced via M2 and the LED turns on. . . . . . . . . . . 137

5.32 A single micro-LED is addressed. As the negative bias on LED_GND increases, other array elements begin to turn on. . . . . . . . . . . . . . 137

5.33 Optical pulse width of PicoQuant diode laser. . . . . . . . . . . . . . . . . 139

5.34 Optical pulse width of CMOS driven micro-LED device. . . . . . . . . . . . 139

5.35 Light Emission from a point source at a distance from a photodetector. . . . . . 141

6.1 Signal losses from excitation source to signal detection in a fluorescence-based detection system. . . . . . . . . . . . . . . . . . . . 145

6.2 Cross-section of single-chip micro-system. . . . . . . . . . . . . . . . . . . 147

6.3 Micro-LED excitation of a quantum dot sample on a micro-cavity slide. . . . 147

6.4 Micro-LED emission spectra, SPAD PDP and quantum dot absorption (Adirondack Green) and emission spectra versus wavelength. This clearly highlights the spectral separation of the excitation wavelength and the detector sensitivity range. . . . . . . . . . . . . . . . . . . . . 149

6.5 IRF and fluorescent decay curves for quantum dot samples at three different emission wavelengths. . . . . . . . . . . . . . . 150

6.6 Cross-section of the two-chip micro-system. The sample of interest is introduced into the system in a micro-cavity slide sealed by a cover-slip. An optical filter prevents excitation light reaching the detector array. . . . . . . . 153 
6.7 (a) Top side of two-chip system daughter card used to interface with the CMOS driven micro-LED device, (b) the underside of the PCB is where the chip is situated, in this configuration the micro-LED chip faces the SPAD chip which is located on the master PCB . . . . . . . . . . . . . . . . . . . 154

6.8 Filter and sample holder situated between the micro-LED excitation source and the SPAD detector. Images courtesy of Andrew Garrie, COSMIC Laboratory, The University of Edinburgh. . . . . . . . . . . . . . . . . . . . . 155

6.9 Complete two-chip micro-system. Power and user control signals are provided via USB connection. The PCB daughter card is physically supported by the filter and sample holder and stacked header pins. . . . . . . . . . . . . 156

6.10 Two-chip micro-system for time-resolved fluorescence detection. Electrical connection between the FPGA test-board and the daughter card was made using stacked header pins. . . . . . . . . . . . . . . . . . . . . 157

6.11 Fluorescence lifetime decay curves from rhodamine 6G, fluorescein and quantum dot samples captured using the two-chip micro-system. . . . . . . . . 159

7.1 Alternative inverted SPAD bias configuration . . . . . . . . . . . . . . . . . 169

7.2 Metal bond stacks of varying dimensions. . . . . . . . . . . . . . 171

7.3 (a) Layout of $100 \mu \mathrm{m}$ pitch micro-LED driver test structure array. (b) Each pixel contained a $50 \mu \mathrm{m} \times 50 \mu \mathrm{m}$ bond-pad and driver circuit. . . . . . . . . . . 172

A.1 Single-bit of in-pixel counter used to count avalanche events. . . . . . . . . . 179

A.2 On-chip VCO implemented on 8x8 micro-LED driver array chip. . . . . . . . 182

A.3 One of seven on-chip LFSR circuits implemented on 8x8 micro-LED driver array chip. . . . . . . . . . . . . . . . . . . . . 183

A.4 Example of case statement used to define output location of on-chip tapped delay line. . . . . . . . . . . . . . . . . . . . . . . . . . 184 


\section{List of tables}

2.1 Summary of selected integrated systems for fluorescence detection . . . . . . 26

3.1 Summary of quantum dot samples used to evaluate micro-systems. . . . . . . . 64

3.2 Summary of non-quantum dot based fluorophores used. . . . . . . . . . . . . 65

4.1 Summary of SPAD device performance. . . . . . . . . . . . . . . . . 72

4.2 Summary of on-chip timing generator circuit performance. . . . . . . . . . 76

4.3 An overview of the experimental setup used in limiting dilution tests. . . . . . . 84

4.4 Count rate achieved, number of counts achieved in peak channel and experimental time versus concentration. ${ }^{*}$ extrapolated from time to achieve 5,000 and 500 counts, respectively. . . . . . . . . . . . . . . . 86

4.5 Lifetime estimations based on SPAD data processed by Becker and Hickl TCSPC module. . . . . . . . . . . . . . . . . . . . . . . . . . . . 89

4.6 Lifetime estimations based on SPAD data collected using on-chip time gating circuitry and processed using three different lifetime extraction algorithms. . . 93

4.7 $1 \mu \mathrm{M}$ coumarin- 6 and rhodamine-B lifetimes estimated using the 2-RLD algorithm. . . . . . . . . . . . . . . . . . . 95

4.8 Average extracted lifetimes of quantum dot samples of different concentration and the standard deviation across the different capture and extraction methods. . 96

5.1 Summary of $16 \times 16$ driver array features. . . . . . . . . . . . . . . . . . . 121

5.2 Peak optical pulse power versus pulse width and LED ground voltage. . . . . . 133

6.1 Summary of typical system transmission values. . . . . . . . . . . . . . . . 144

6.2 Summary of quantum dot samples used to evaluate system. . . . . . . . . . . 148

6.3 Extracted lifetimes from decay curves captured using single chip system . . . . 151 


\section{Chapter 1 \\ Introduction}

\subsection{Miniaturisation of a Fluorescence Lifetime Measurement System}

The use of fluorescence based methodologies is at the core of many modern instrumentation technologies, especially in the life sciences [1]. Originally, the interest was in the specific labelling of biological samples for imaging applications, but more recently this has expanded rapidly with the advent of deoxyribonucleic acid (DNA) sequencing and micro-array applications. In principle, the equipment needed for such spectroscopic instrumentation has not changed over several decades, in that a narrow wavelength source is required to excite the fluorophore of interest and the resulting fluorescence must pass through an optical device to separate the excitation light from the fluorescence emission, before being detected by a light sensitive instrument and the level of fluorescence determined. However, using modern fabrication and manufacturing techniques such instrumentation can now be made in complete integrated systems, with the potential for volume production. The range of methods for interpreting the fluorescence data has also expanded with the growth of fast electronics, originally destined for the telecommunications and computer markets, now being applied to fluorescence instrumentation.

Time-resolved fluorescence analysis is the measurement of the temporal properties of a fluorophore sample. Fluorescence lifetime detection provides a method of differentiating between spectrally overlapping samples which exhibit different lifetime properties [2]. The sensitivity of a sample's lifetime properties to the micro-environment provides an extremely powerful analysis tool. Lifetime analysis is predominantly performed in either the time or frequency domain. Due to limitations in light source technologies, lifetime analysis was originally performed in the frequency domain. With the advent of sub-nano second pulsed light sources the popularity of time domain analysis has increased. An expensive and complex array of equipment is required to perform conventional fluorescence lifetime analysis; such as a pico-second pulsed or modulated light source (often a laser source) and a sensitive detector 
such as a micro-channel plate photo-multiplier tube (MCP-PMT). Analysis is often performed using a microscope which contains a variety of optical lenses and filters. Commercially available fluorescence lifetime systems (Figure 1.1, [3], [4]) often contain more than one excitation source and a wide range of filters, adding further to system costs and complexity, thereby restricting its use to research laboratories. It is hoped that miniaturisation will result in a lower cost, more portable device with reduced complexity. Complementary metal oxide semiconductor (CMOS) technologies offer the ability to integrate many elements of the system while having the potential to be mass manufactured. Furthermore, by including much of the signal processing on-chip there is a great reduction in the amount of data that has to be broadcast off-chip for processing by external hardware. It was therefore the ultimate aim of this project to produce an integrated fluorescence lifetime analysis system capable of sub-nano second precision with an instrument measuring less than $1 \mathrm{~cm}^{3}$, something hitherto impossible with existing approaches. To accomplish this, advances in the development of aluminium indium gallium nitride (AlInGaN) micro-light emitting diodes (LEDs) and high sensitivity CMOS detectors have been exploited. CMOS allows electronic circuitry to be integrated alongside the photodetectors and LED drivers to produce a highly integrated system capable of processing detector data directly without the need for additional external hardware.

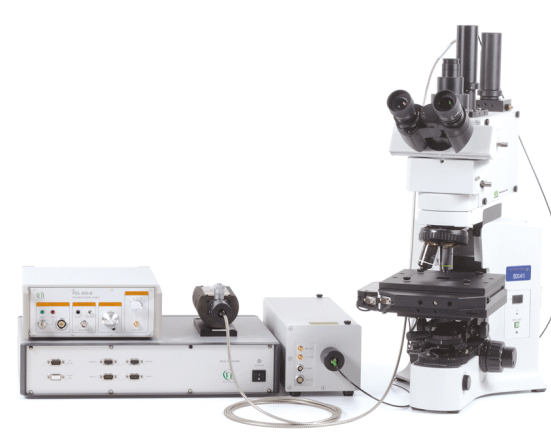

(a)

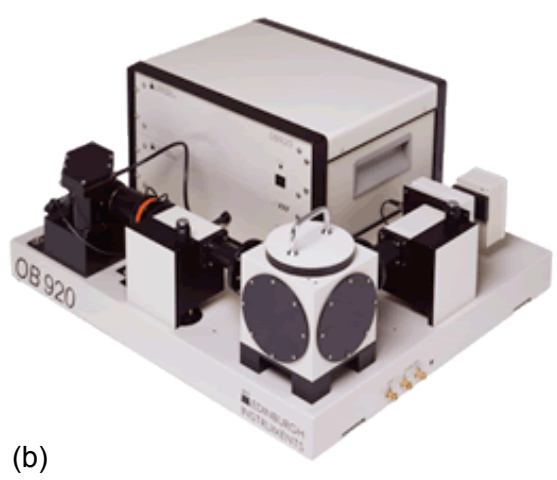

(b)

Figure 1.1: Commercially available fluorescence lifetime analysis systems; (a) MicroTime 100 by PicoQuant, an upright time-resolved fluorescence microscope. (b) OB920 fluorescence lifetime spectrometer by Edinburgh Instruments. 


\subsection{Aims}

The aim of this project was to develop a low-cost, robust micro-system for time-resolved fluorescence analysis. It was necessary to investigate the individual components of a time-resolved fluorescence system in order to develop elements with suitable performance and compatible micro-system dimensions. To achieve this, devices based on CMOS technology were designed which utilise the technology's ability to produce highly integrated, dense electronic circuits with dimensions in the order of millimeters. The performance of these devices had to be verified in a practical laboratory environment and their ability to stimulate and accurately capture fluorescence decay data demonstrated.

The specific aims of the project were:

- The development of on-chip circuitry capable of processing output data from an array of CMOS-based detectors with single-photon sensitivity.

- Design a CMOS circuit capable of driving an array of AlInGaN micro-LEDs producing sub-nanosecond optical pulses.

- Develop Verilog code for a field-programmable gate array chip to provide stimulus for both devices.

- Develop a graphical user interface (GUI) to provide user defined device inputs.

- Establish the performance limits of the excitation and detection elements in an experimental environment.

- Bring excitation and detection elements together on a single platform capable of driving both devices simultaneously.

- Demonstrate time-resolved fluorescence lifetime detection using a micro-system device.

\subsection{Thesis}

This thesis aims to demonstrate how existing optical and electronic elements can be integrated to form a micro-system for time-resolved fluorescence lifetime analysis.

A compact fluorescence sensing system is achieved by bringing together CMOS compatible detectors, with single-photon sensitivity, and electronic circuitry capable of processing detector 
data. Experimental results demonstrate how this detection system is capable of accurately capturing fluorescence lifetime data from samples with a wide range of concentration and that these lifetimes, captured using on-chip circuitry, are equivalent to those processed using commercially available external time-correlated single-photon counting hardware.

To successfully achieve a complete miniaturised time-resolved fluorescence micro-system, a compact excitation source, capable of producing sub-nano second optical pulses is also required. This thesis describes how AlInGaN micro-LEDs can be bump-bonded to a CMOS driver backplane to achieve an excitation source suitable for time-resolved analysis.

The ultimate aim of this thesis is to provide evidence of how these excitation and detection elements can be successfully amalgamated to form a micro-system for time-resolved analysis capable of accurately capturing fluorescence lifetime data.

\subsection{Contribution to Knowledge}

The ability to integrate SPAD detectors in a standard CMOS process allows signal processing circuitry to be included on the same substrate as the detection element. This work is, to the best of our knowledge, the first demonstration of a single-photon sensitive detection system with in-pixel time-gated counters and on-chip timing generation, in order to directly process fluorescence decay data. The limit of detection of the SPAD detector and in-pixel circuitry was found to be less than $10 \mathrm{nM}$ and lifetimes could be captured with a resolution of 408ps (minimum time-gate). Lifetime values extracted from decay data captured on-chip are shown to be comparable to those processed using commercially available photon counting hardware. Furthermore, this work demonstrates that AlInGaN micro-LEDs can be successfully bump-bonded to a CMOS backplane. The final micro-LED driver design is capable of producing optical pulses of 300ps in width (FWHM) and a maximum DC optical output power of $550 \mu \mathrm{W}$. Again to the best of our knowledge, this is the shortest reported optical pulse from a CMOS driven micro-LED device. By integrating an array of CMOS SPAD detectors and an array of CMOS driven AlInGaN micro-LEDs, a complete micro-system for time-resolved fluorescence analysis has been realised. Two different system configurations are evaluated and the ability of both topologies to accurately capture lifetime data is demonstrated. By making use of standard CMOS foundry technologies, this work opens up the possibility of a low-cost, portable chemical/bio-diagnostic device. The first-generation prototypes described 
herein demonstrate the first time-resolved fluorescence lifetime analysis using an integrated micro-system approach.

\subsection{Thesis Outline}

Chapter 2 introduces fluorescence and fluorescence lifetime and provides an overview of previous attempts at miniaturising different elements of the time-resolved fluorescence analysis system. The SPAD detector and AlInGaN micro-LED elements used in this project are also introduced and their features reviewed.

The Materials and Methods (Chapter 3) introduce various different pieces of test equipment and experimental procedures used to design and verify the different components of the micro-system. An overview of all critical pieces of design software is provided along with a description of laboratory equipment used during the evaluation of devices. Details of the fluorophores used are provided in addition to the bump-bonding process used to interface the AlInGaN micro-LEDs with the CMOS backplane.

Chapter 4 describes the design and test of an array of SPAD detectors with in-pixel signal processing circuitry, implemented in a high-voltage CMOS process. The sensitivity of the SPAD detector in an experimental environment is presented along with a demonstration of the in-pixel circuitries ability to accurately capture fluorescence lifetime decay data. Different lifetime extraction algorithms are also compared.

For a truly integrated system, an excitation light source is required. In Chapter 5 two CMOS circuits are described which are capable of driving arrays of AlInGaN micro-LEDs. The performance of these devices in DC, square-wave and pulsed mode is presented. For time-domain fluorescence lifetime analysis the pulse performance of the excitation source is critical. In this chapter, CMOS driven micro-LEDs producing sub-nano second optical pulses are demonstrated.

Having described miniaturised CMOS detection and excitation devices in Chapters 4 and 5 , respectively, Chapter 6 describes how these elements were brought together to create a micro-system for time-resolved fluorescence lifetime analysis. Two different micro-system topologies are presented. These systems integrate an AlInGaN micro-LED array with an array of single-photon avalanche diodes with integrated signal processing circuitry. A demonstration 
of each system's ability to excite a fluorescence sample and capture the ensuing lifetime decay curve is provided. The relative merits and drawbacks of each topology is discussed.

Chapter 7 concludes the thesis with a summary of the work and proposes a number of different areas of research that could be investigated in future to advance this work further.

A copy of all journal and conference publications arising from this project are provided in Appendix B. 


\section{Chapter 2 Fluorescence and Sample Analysis Techniques}

\subsection{Fluorescence}

Fluorescence detection for biological applications is a widely used and well-established field, as fluorescence sensing offers excellent sensitivity (the lowest level signal that can be accurately sensed or detected by the sensor [5]) and specificity (the detection method is specific to the agent of interest and avoids interference from other molecules). Fluorescence detection of biological samples is widely used in laboratories throughout the world due to the non-invasive and non-destructive nature of this sensing technique. Most molecules do not naturally fluoresce; however, by tagging a molecule or sample with a fluorescently labeled probe of high chemical specificity, it is possible to observe the molecular composition of the sample [6]. For a tagged sample to fluoresce it must first be stimulated by an external source. This normally takes the form of a light source, focussed on the sample of interest. The absorption of incident radiation excites the fluorophore to a higher vibrational energy level $\left(S_{2}-S_{n}\right.$ in Figure 2.1). The fluorophore then relaxes through a number of vibrational energy levels, until it is at the lowest vibrational energy level $\left(S_{1}\right)$. This process is known as internal conversion. After a given time, the electron will return to the ground state. It is this transition that is usually accompanied by a release of energy which can take the form of a photon (other relaxation mechanisms include phosphorescence and non-radiative recombination, see [7]). Due to the energy lost during the internal conversion process the emitted fluorescence photon is usually lower in energy (longer wavelength) than the absorbed photon, a phenomena known as the Stokes shift. A photon's wavelength is related to its energy via the following relationship:

$$
\lambda=\frac{h c}{E_{p h}}
$$

Where $\lambda$ is the wavelength of the photon, $h$ is Planck's Constant $\left(6.6261 \times 10^{-34} \mathrm{Js}\right), c$ is the velocity of light in a vacuum $\left(2.998 \times 10^{8} \mathrm{~ms}^{-1}\right)$ and $E_{p h}$ is the photon energy. 
This shift in wavelength from the absorbed photon to the emitted photon allows the excitation light to be separated from the resulting fluorescence emission using optical filters.

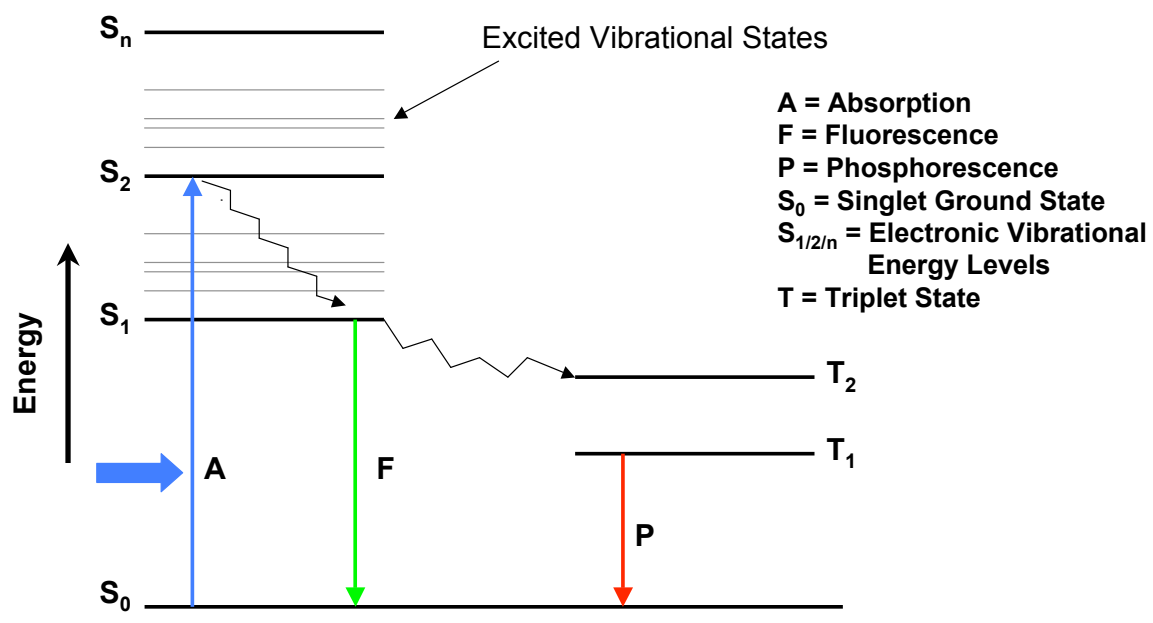

Figure 2.1: Jablonski Diagram. Promotion of an electron to a higher energy state due to excitation light and its subsequent relaxation to the ground state, coupled with the emission of a lower energy photon.

The intensity of fluorescence emission, relative to the intensity of the excitation source, is defined by the sample's quantum yield. Quantum yield is the ratio of the number of photons emitted and the number of photons absorbed. Alternatively, this can be expressed as the emission rate divided by the emission rate plus the non-radiative decay rate. The higher a sample's quantum yield, the brighter the emission. Due to energy losses during the internal conversion process, which results in the Stokes shift, the quantum efficiency of a sample is always less than unity.

In standard fluorescence intensity imaging a sensitive detector, such as a cooled charge-coupled device (CCD) or PMT, captures emissions from the fluorophore. The problem with measuring fluorescence intensity is that the intensity of the emitted fluorescent light is a function of the intensity and wavelength of the excitation light and also of the brightness and concentration of the fluorophore [8]. Thus, interpretation and qualification is hampered by non-homogenous probe concentration and non-uniform illumination. Furthermore, at very high fluorophore concentrations the excitation occurs only at or near the surface of the sample, and the fluorescence intensity becomes a non-linear function of the fluorophore concentration in the sample. 


\subsubsection{Fluorescence Lifetime}

Fluorophores have associated with them an exponential fluorescent decay transient after the removal of the excitation source, which defines their characteristic lifetime [9]. Due to the random nature of fluorescence emission, a fluorphore's associated lifetime is the average lifetime a sample spends in the excited state before photon emission occurs.

A sample's fluorescence lifetime, $\tau$, is determined by the rate at which the sample leaves the excited state (Equation 2.2). The transition can occur via two mechanisms, either by fluorescence emission (at rate $\Gamma$ ) or by competing non-radiative processes (represented collectively as $\mathbf{K}_{n t}$ ).

$$
\tau=\frac{1}{\Gamma+\Sigma K_{n t}}
$$

As discussed in Section 2.1 a fluorophore's quantum yield $(\Theta)$ is the ratio of absorbed photons to the number of emitted photons. This can be represented by Equation 2.3.

$$
\Theta=\frac{\Gamma}{\Gamma+\Sigma K_{n t}}
$$

For a given excitation light intensity, a fluorophore's brightness can be calculated if the molecular absorption coefficient $(\epsilon)$ is known, Equation 2.4.

$$
\text { MolecularBrightness }=q=\epsilon \Theta
$$

The absorption coefficient of a fluorophore is usually constant; therefore, changes in a fluorophore's brightness can usually be attributed to changes in the sample's quantum yield. Therefore, from equations 2.2 and 2.3, if the molecular brightness changes this will usually result in a change in sample lifetime. Due to the fact fluorescence intensity is a composite property of a sample, dependent on sample quantity and concentration as well as instrument set-up, it is very sensitive to sample variation and is subject to interference from scattered light. This makes the observation of small intensity changes very difficult. Conversely, fluorescence lifetime is an intrinsic fluorophore property, independent of sample volume and concentration. Lifetime analysis is also less sensitive to instrument setup. Fluorescence lifetime is therefore a 
more robust analysis method compared to intensity measurement, capable of observing subtle changes in sample conditions [10].

The rate of non-radiative recombination is dictated by the fluorophore's electron structure and its interaction with the environment. Non-radiative decay mechanisms include [7]:

- Inter-system crossing

- Collisional or static quenching

- Solvent effects

- Resonance energy transfer.

Fluorescence intensity is related to lifetime according to Equation 2.5 (for a mono-exponentially decaying sample). The equation assumes that the sample has been excited by an infinitely sharp ( $\delta$-function) light pulse. The time-dependent intensity at time $\mathrm{t}, \mathrm{I}(\mathrm{t})$, is given by:

$$
I(t)=I_{0} \exp \left(\frac{-t}{\tau}\right)
$$

Where $I_{0}$ is the intensity at time 0 and $\tau$ is the sample lifetime.

Fluorescence lifetime is independent of fluorophore concentration but dependent on the sample's local environment. Thus, lifetime detection allows precise quantitative data about both fluorophore distribution and local environment to be obtained, while avoiding the problems related to fluorescence intensity imaging such as photo-bleaching [11]. Fluorescence lifetime detection can also be used to differentiate between fluorophores with overlapping spectra, but exhibiting different decay characteristics. Typical fluorescence decay times of organic compounds fall between a few hundreds of picoseconds and several nanoseconds [2]. There are a number of different imaging experiments for which time-resolved detection can be used; these include, multiple fluorophore labeling [12], quantitative detection of ion concentrations and oxygen and energy transfer characteristics using fluorescence resonance energy transfer (FRET) [13]. The independence of time-resolved fluorescence from sample volume makes it ideally suited to miniaturisation [10]. 
There are two predominantly used techniques for measuring the fluorescence lifetime of a sample: the frequency-domain and time-domain methods.

\subsubsection{Frequency Domain}

In the frequency domain a sample is excited by an intensity modulated light source. This results in the fluorescence emission being modulated at the same frequency, but with a phase shift due to the intensity decay law (lifetime, Equation 2.5) of the sample [7], [14] and a reduction in the modulation depth (Figure 2.2).

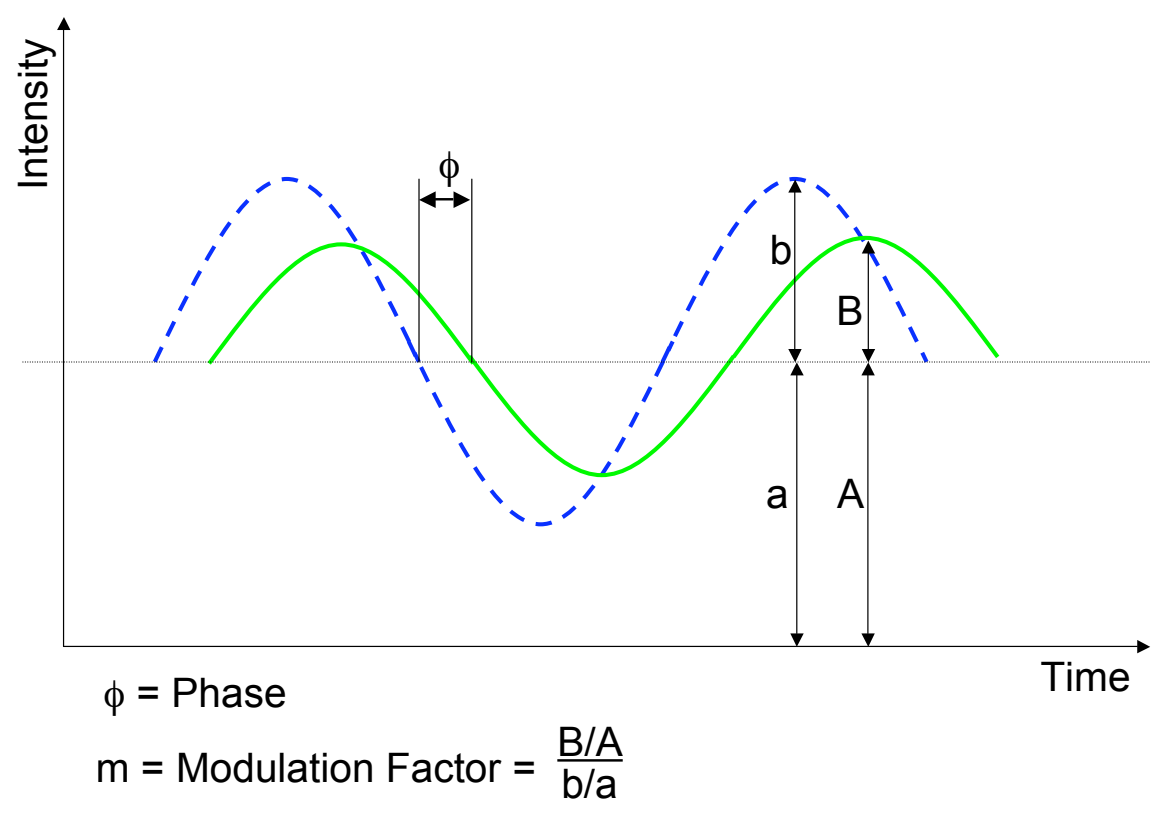

Figure 2.2: In the frequency domain, modulation factor and phase difference can be used to estimate fluorescence lifetime.

The modulation factor (Equation 2.7) and phase difference (Equation 2.6) are related to the fluorophore lifetime by:

$$
\begin{gathered}
\tan \phi=\omega \tau \\
m=\left(1+\omega^{2} \tau^{2}\right)^{-\frac{1}{2}}
\end{gathered}
$$


Where $\omega$ is equal to the angular frequency ( $2 \pi \mathrm{x}$ frequency). For samples which exhibit single exponential decay characteristics, the lifetime estimated by the phase and modulation methods will be the same. For multi-exponential decay curves, measurements must be taken over a number of frequencies and the results fit to a set of dispersion relationships [15].

\subsubsection{Time Domain}

In the time domain the intensity decay of a fluorescent sample is directly measured as a function of time, following absorption of a short excitation pulse (Figure 2.3). The fluorescence decay is captured using a time-gated detector. There are a number of different time gated detection methods; including, time-correlated single-photon counting (TCSPC), multi-gate photon counting, streak cameras and gated image intensifiers [16], [17], [18], [19].

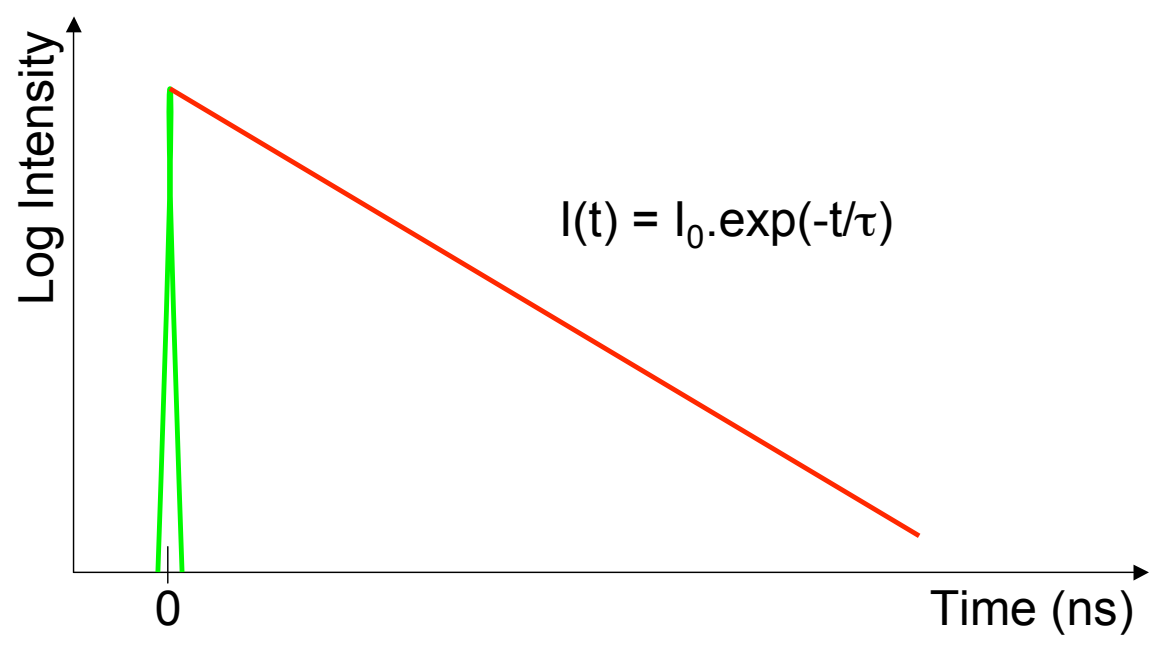

Figure 2.3: In the time domain, fuorescence intensity decay is measured directly as a function of time.

Until recently, the need for an extremely short pulsed laser source $(<1 \mathrm{~ns})$ has meant that time-domain fluorescence lifetime analysis has been restricted to research laboratories. With the advent of pico-second diode lasers and pulsed LED sources, however, time-domain fluorescence analysis has become more widespread as equipment costs and complexity are reduced [20]. The use of a pulsed light source means that the sample of interest is exposed to less excitation light, compared to the frequency-domain method, which can result in less sample damage and photo-bleaching effects. 


\subsubsection{Alternative Fluorescence Lifetime Analysis Methods}

TCSPC, time-gated detection and frequency domain techniques are the dominant methods of obtaining fluorescence lifetime data. There are however, a number of alternative methods of conducting lifetime measurements. These include the use of streak cameras, up-conversion methods and stroboscopic excitation.

Using a streak camera, very high timing resolution can be obtained (in the order of pico-seconds) [18]. Traditionally, streak cameras operate by sweeping incoming photons across the detection plane of the image sensor using high-voltage deflection plates. The resulting image contains temporal information in the axis across which the input photons were swept. From this, lifetime data can be recovered as the system also controls the speed and distance over which the photons are swept. Due to the requirement for high-voltage deflection plates, streak camera based lifetime analysis methods are not suitable for miniaturisation. However, recent work has seen the development of solid-state streak cameras, capable of high-frame rate operation [21]. In this work, temporal information is obtained by sampling the output of conventional integrating CMOS photodiodes onto on-chip capacitors. At present, this method can achieve a temporal resolution of 10ns. While this technique may allow solid-state streak cameras to be used in future fluorescence lifetime analysis systems, at present it does not provide sufficient resolution to be considered for inclusion in a micro-system solution.

Fluorescence lifetime analysis using up-conversion methods offers the ultimate time-resolution performance, defined by the pulse width of a laser source (femto-seconds for a Ti:Sapphire laser). The excitation light source is used as the input to an up-conversion crystal which only produces an up-converted version of the fluorescence emission during these laser input pulse. Through the use of a high-pass filter on the crystal output, only very short periods of the fluorescence decay can reach the detector. The time at which this short gate occurs can be altered through the use of an optical delay line. The need for two laser sources, an up-conversion crystal and an optical delay line (that provides just $1 \mathrm{~ns}$ of delay for every $30 \mathrm{~cm}$ of length) means that up-conversion is not an appropriate method for implementation in a micro-system for time-resolved fluorescence analysis.

A technique that has been reported as appropriate for implementation in a micro-system, is the use of stroboscopic excitation [22]. This technique greatly simplifies the design of the detection system, using the excitation source as the key system element for lifetime measurements. 
Using a pulsed excitation source with a variable repetition rate, the sample is excited and the resulting emission captured using a standard D.C. detector. When the repetition period of the excitation source is longer than the fluorescence lifetime of the sample under investigation, then the emission will fully decay between excitation pulses. As the repetition of the excitation source is increased, the time between pulses approaches that of the sample decay time and the fluorophore no longer fully relaxes to the ground state between excitation pulses. This results in a non-linear detector output which is dependent on the fluorescence lifetime of the sample [23]. In [24], stroboscopic time-resolved fluorescence measurements are demonstrated using a self-pulsing 650nm laser diode. These AlGaInP-based laser devices have been implemented on a micro scale ( $6 \mu \mathrm{m}$ width, $300 \mu \mathrm{m}$ length) and are capable of producing $380 \mathrm{ps}$ wide optical pulses. This, coupled with a greatly simplified detection system, makes this an ideal method for implementation in a micro-system. Unfortunately, accurate fluorescence lifetime determination is difficult to achieve using this method. This technique is more suited to observing lifetime changes during an assay or for distinguishing between samples of different lifetimes [23]. 


\subsection{Excitation Sources}

Traditionally, fluorescence excitation is achieved using laser sources or mercury or halogen lamps. These devices are physically large and expensive devices which require a filter to ensure a narrow-band excitation wavelength. For this reason, fluorescence analysis systems often contain several sources of different wavelength. Arc and incandescent lamps are commonly used excitation light sources due to their broadband continuous emission, but their size, low efficiency and low stability do not make them suitable for miniaturized portable analysis systems [25]. Gas discharge lamps have also been used for fluorescence excitation, these devices operate in a free-running mode and are difficult to control. Furthermore, the high supply voltage which they require $(>5 \mathrm{kV})$ does not make them compatible with CMOS electronics.

Currently, the standard excitation source for time-domain fluorescence lifetime analysis is the pulsed laser diode. Available over most of the visible wavelength spectrum these devices provide a low cost solution, relative to the femto-second Ti:Sapphire laser, to pulsed sample excitation. The specifications of a typical commercially available pulsed laser diode are summarised in Section 3.1.2. Once placed within a cooling heat sink the PicoQuant pulsed laser diode described in Section 3.1.2 measures $110 \times 75 \times 60 \mathrm{~mm}$, these devices are therefore significantly larger than devices based on CMOS technologies (which are in the order of a few millimeters squared).

The development of low-cost, miniaturised excitation sources for a full optical lab-on-a-chip is often neglected. Several groups have demonstrated fluorescence excitation using vertical cavity semiconductor devices [25], [26] and are discussed further in Section 2.4 where they have been integrated into micro-analytical devices. These devices, however, have not been integrated into a CMOS process and do not allow drive electronics and signal processing circuitry to be included on the same substrate.

In 2005, Davitt et al. [27] reported the use of a 32-element, linear array of light emitting diodes (LEDs) for fluorescence intensity measurements. Rectangular devices were fabricated measuring $200 \mu \mathrm{m}$ in length, $50 \mu \mathrm{m}$ high and at a pitch of $100 \mu \mathrm{m}$. Quantum well p-n heterostructure devices were fabricated with peak emission wavelengths at 290nm (AlGaN) and 340nm (AlGaInN). These devices were integrated into a small footprint $(20 \mathrm{x} 13 \mathrm{~cm})$ optical filter-based detection system used to identify aerosolized particles. A system incorporating a 32 element LED array, two photomultiplier tubes, three optical filters, one dichroic mirror and five 
optical lenses is presented and its ability to detected $140 \mu \mathrm{M}$ NADH fluorophore demonstrated. This paper presents an attempt at the miniaturisation of a fluorescence analysis tool using micro-LED devices. Despite the choice of a compact excitation device, the system still relies on a number of high-cost components and number of optical elements which require accurate alignment while further increasing device costs.

In 1995, Araki and Misawa [28] demonstrated the use of commercially available blue InGaN/AlGaN LEDs for fluorescence lifetime measurements. Driven by an external RLC (resistor, inductor, capacitor) circuit and controlled by an avalanche transistor, these devices generated $4 \mathrm{~ns}$ wide optical pulses with a $10 \mathrm{kHz}$ repetition rate and a peak optical power of $40 \mathrm{~mW}$. In order to operate, the avalanche transistor required a $300 \mathrm{~V}$ collector voltage, making the drive circuit incompatible with standard CMOS processes. In addition, the inclusion of an inductive component in the drive circuit makes its realisation in an integrated microelectronic circuit difficult. Using a high-gain photomultiplier tube and TCSPC hardware, accurate fluorescence lifetime measurements of Quinine-Sulfate are presented using these LED devices as an excitation source. This demonstrated how pulsed LEDs were suitable for consideration as a light source in time-domain fluorescence analysis.

Fluorescence lifetime analysis using micro-LED excitation was demonstrated in [29]. A 64x64 matrix-addressable LED array driven by external hardware with a pulse width of 2 ns was used to excite a sample of rhodamine-123, with the subsequent fluorescence decay being captured by a commercially available photomultiplier tube (PMT). These $\mathrm{InGaN} / \mathrm{GaN}$ devices measured $20 \mu \mathrm{m}$ in diameter and were capable of producing $40 \mathrm{nW}$ average optical power with a $4 \mathrm{~V}$ bias. Lifetime measurements of rhodamine-123 excited with a blue (460nm) micro-LED and capture using a fast photomultiplier are presented. Being matrix-addressable each array element requires a unique drive signal. As array sizes increases this creates a serious fan-out problem, and the ability to scale becomes limited by the ability to get drive signals to the edge of the LED array. This issue can be addressed by active address logic or by providing each element with a local driver circuit.

Although not designed for fluorescence analysis, Buß et al. 1995, demonstrated, to the best of our knowledge, the first example of planar vertical surface emitting LEDs bump-bonded to a CMOS back-plane comprising an array of driver circuits [30], [31]. An 8x8 array of GaAsP micro-LEDs on a GaP substrate is described. Each element measured 100x $100 \mu \mathrm{m}$ with a pitch of $200 \mu \mathrm{m}$. ZnNiAu is evaporated on the surface of each array element and provides 
local contact points. A GeNiAu contact situated on the back of the substrate provides the ground terminal for the whole array. Using a solder re-flow method, the micro-LED array is bump-bonded to an equivalent array of driver circuits realised in a CMOS technology (undefined process). Each element is bonded to a full bond pad and has a dedicated in-pixel driver circuit. Electrical characterisation of the CMOS driver array established that the circuit was capable of producing drive pulses with rise and fall times less than 250ns resulting in a cut-off frequency of greater than $1.45 \mathrm{MHz}$. This performance is a reflection of the CMOS process technologies available in 1995. This work established the design flow for the integration of mico-LED arrays with CMOS drive circuits.

\subsection{Detectors}

During fluorescence lifetime analysis, the number of photons emitted tends to be very low. Therefore, sensitive detection devices are required. For photon counting applications these devices require single-photon sensitivity, these include: micro-channel plate PMTs, high-speed amplified PMTs, discrete photodiodes and avalanche photodiodes. These devices tend to be discrete components, requiring separate power supplies and a communication interface. Furthermore, they tend to be physically large and delicate (especially PMT devices). PMT devices are also sensitive to magnetic fields making difficult their integration into medical devices such as magnetic resonance imagers (MRI).

A number of groups have demonstrated micro-scale fluorescence detection using a variety of different detectors. In [32], Patounakis et al. demonstrate CMOS detection of fluorescence lifetime decays using conventional CMOS photodiodes and on-chip signal processing circuitry. This devices relies on the integration of photodiode current to estimate photon intensity and does not display single-photon sensitivity. Each pixel measures 160x215 $\mu \mathrm{m}$ and includes a 100x $100 \mu \mathrm{m}$ n-well/p-sub photodiode, a collection capacitor and a trans-conductance circuit. Other on-chip circuitry includes $\Sigma \Delta$ analogue to digital converters for processing of the pixel data and static random access memory (SRAM) memory to store pixel values. Two key features of this design is the inclusion of an on-chip laser diode driver control circuit and the ability to release the photodiode from reset rapidly. By holding the photodiode in reset during sample excitation, the detector is insensitive to any impinging laser light. Using the inherent ability of CMOS to rapidly switch state, the photodiode can then be brought out of reset and allowed to capture the resulting fluorescence decay. By delaying the point at which the photodiode is 
brought out of reset (relative to the laser synchronisation signal), a time-gated histogram of the fluorescence lifetime can be obtained (Figure 2.4). This delay can accurately be controlled with an on-chip, 256-stage, programmable, variable delay line with a resolution of 230ps. This resolution is defined by the inverter delay time of the $0.25 \mu$ process in which this design was conceived. Despite presenting time-resolved detection of a laser turn-off edge, no fluorescence lifetime results are presented.

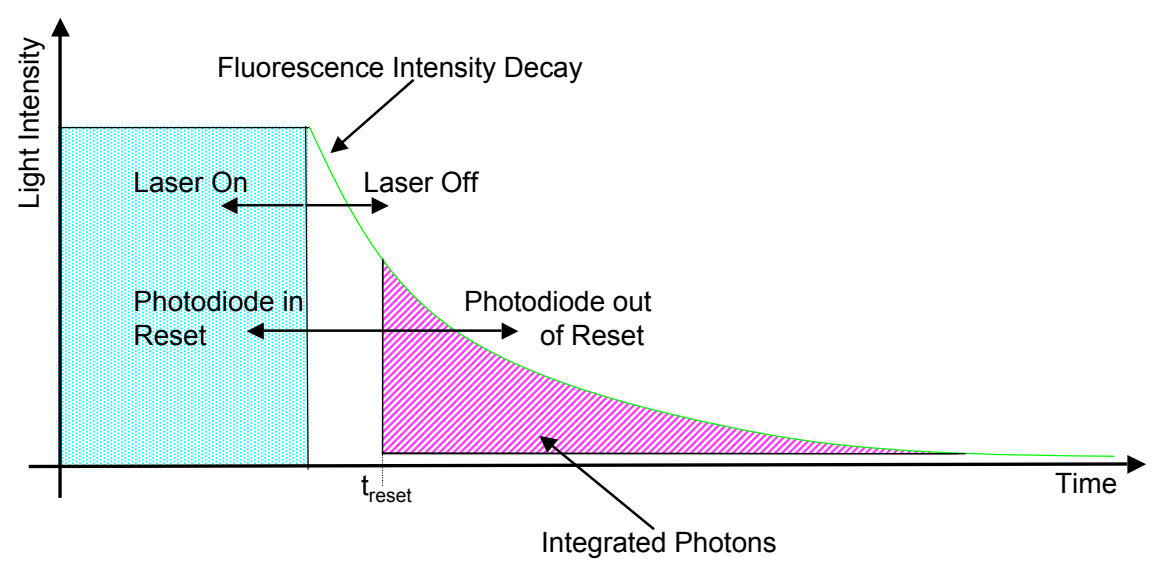

Figure 2.4: Time-gated fluorescence lifetime detection using an integrating photodiode with variable reset times. Presented by Patounakis et al. 2006.

There has been significant progress in recent years in the development of CMOS image sensors, mainly driven by the demand from the mobile telephone market. Originally developed for CCD image sensors, the pinned photodiode has now been utilised in CMOS image sensors, offering reduced dark current and transfer noise. In [33], a CMOS image sensor, aimed specifically at fluorescence lifetime imaging, with a 256x256 pinned photodiode array is implemented in a $0.18 \mu \mathrm{m}$ image sensor specific CMOS process. A novel two-stage charge transfer pixel structure allows excitation and background photons to be subtracted from the detected signal leaving only signal due to fluorescence emission. During sample illumination accumulated charge is transferred to a drain node in the pixel. During the resulting fluorescence emission transfer to the drain node is halted and charge is passed to an in-pixel storage diode. This process is repeated a number of times, allowing the amount of charge held in the storage diode to accumulate. Pixel read-out, via a transfer gate, is column based through a programmable gain amplifier. Fixed pattern readout noise is reduced using correlated double sampling. The subsequent amplifier output is processed by an on-chip analogue-to-digital converter before being broadcast off-chip. Similar to the work presented in [32], fluorescence decay data is 
calculated by varying the time at which the photodiode is switched from passing charge to the drain node to storing charge for readout. In this work, a timing resolution of 250ps has been achieved using an on-chip delay time controller. Each pixel measures $7.5 \times 7.5 \mu \mathrm{m}$ and includes a micro-lens array to improve fill factor. Using an external UV (375nm) laser diode as an excitation source, fluorescence lifetime measurement results are presented for fura- 2 fluorophore samples with varying concentration from 10-50nmol. From the results it is noted that the measured lifetime varies greatly with concentration $(4.75 \mathrm{~ns}$ for $10 \mathrm{nmol}$ down to $2.1 \mathrm{~ns}$ for 50nmol) suggesting that the systems ability to resolve fluorescence lifetimes may be skewed by low photon counts. In this paper the authors do not state which diluent was used however, the quoted lifetime of unbounded fura- 2 is $1.09 \mathrm{~ns}$ [34].

Recent developments in the design of CMOS compatible single-photon avalanche diodes [35] allow extremely sensitive detectors to be integrated alongside signal processing circuitry. In order to gather photon arrival time data, from which fluorescence lifetime can be extracted, a number of circuit techniques have been proposed. These include; on-chip time-to-digital converters [36] and in-pixel time-gated counters [37]. Single-photon avalanche diodes offer micro-scale single-photon detection capabilities and their ability to capture fluorescence data has been well-documented [38], [37]. They offer a number of other significant advantages; including being robust devices which are not destroyed by high light levels, insensitivity to magnetic fields and are relatively easy to manufacture [39].

The use of SPADs for fluorescence lifetime detection has been demonstrated in [38] and [40]. In [40] a 4x112 array of SPADs at a pitch of $25 \mu \mathrm{m}$, implemented in a $0.35 \mu \mathrm{m}$ CMOS process, is presented. This is thought to be the smallest reported pixel pitch in the literature. To achieve such small pixel pitch, very little circuitry is situated local to the diode. To minimise output data, an event driven readout structure has been implemented, requiring just 11 in-pixel transistors. Upon detection of a photon, this asynchronous readout mechanism outputs a timing pulse signalling an avalanche event along with the corresponding SPAD address, providing the array location of the event. This readout mechanism is achieved on a column basis and an avalanche event renders the whole column in-active. For lifetime measurements to be conducted, external hardware must be used. In [38], lifetime measurements of the fluorophore OGB-1 are presented and changes in the samples decay characteristics in response to varying calcium concentration demonstrated. This was achieved using a 4x4 SPAD array at a pitch of $32 \mu \mathrm{m}$, in a $0.35 \mu \mathrm{m}$ process. SPAD output pulses were broadcast off-chip and processed by 
external TCSPC hardware. Sample excitation was achieved using a femto-second Ti:Sapphire laser.

In [37], a $2 \times 7$ array of SPAD detectors with in-pixel counters and active quench circuitry was implemented in a $0.35 \mu \mathrm{m}$ high voltage technology. Each pixel measured $180 \mathrm{x} 150 \mu \mathrm{m}$ and a variety of different SPAD test structures were investigated. The most successful of these being a $20 \times 20 \mu \mathrm{m}$ detetector with a breakdown voltage of $28.3 \mathrm{~V}$, a dark count rate of $3 \mathrm{kHz}$ and an after-pulsing rate of $2 \mathrm{kHz}$ (with a dead time of 150ns). Fluorescence lifetime analysis is achieved using time-gated photon counting. A time-gate period is defined off-chip by a field-programmable gate array (FPGA), if an avalanche event occurs during this window then the counter will increment. Outwith this window, avalanche events are ignored. By shifting the time at which these count windows occur, a histogram of the fluorescence decay can be obtained. FPGA devices are usually implemented in the very latest CMOS technology. Therefore, by implementing the time-gate generation circuit on an FPGA the design can take advantage of the very short delay times offered by deep-submicron processes and high resolution performance can be achieved. Using this technique Mosconi et al. 2006 implement time-gates with a width of 500ps and a time resolution of 80ps. This work also demonstrates how active quench circuits can be implemented locally next to the SPAD detector (see Section 2.6.2.6). Results presented in this paper do not demonstrate fluorescence lifetime detection, instead the detection of a $10 \mathrm{~ns}$ laser pulse is presented. This work forms the basis of work presented in [41] where the detection of quantum dot samples down to concentrations of $0.327 \mathrm{nM}$ is demonstrated using SPAD detector array, connected to a single on-chip time gated counter and off-chip timing generator circuitry. Sample excitation is performed by an external laser diode.

A different signal processing approach is taken in [36]. A CMOS-based detection system intended for fluorescence lifetime imaging is reported which includes a 64x64 SPAD array and an on-chip time-to-digital converter circuit, implemented in a $0.35 \mu \mathrm{m}$ CMOS process. The active area of each SPAD is $15 \mu \mathrm{m}$ in diameter and the pixel pitch is $40 \mu \mathrm{m}$. Like [40], readout is event driven and is limited to one result per column per measurement cycle. This greatly limits the efficiency of the system, effectively creating a limit of 64 detected photon events per measurement cycle despite having 4096 pixels in the array. This limitation is eased slightly by the low photon detection probability associated with fluorescence lifetime measurements. To avoid avalanche events caused by the excitation source this design features the ability to 
gate the detector, rendering it inactive during sample excitation. The chip can also measure lifetime decays using two methods, time-gated detection and time-correlated single-photon counting. In time-gated mode, photon events are counted by a single on-chip counter and timing generation is performed off-chip. In TCSPC mode, the arrival time of each detected photon (relative to the excitation pulse) is evaluated using an on-chip time-to-digital converter based on a delay-locked loop circuit. Using a ring-oscillator structure, photon arrival time is measured by counting the number of cycles the ring oscillator performs between the excitation signal (which starts oscillations) and an avalanche event (which stops oscillations). Higher timing resolution is achieved by also evaluating the phase of the ring oscillator when it is halted. Using this technique a resolution of 350ps is achieved. Due to the high chip activity caused by the ring oscillator circuit this method leads to a large current consumption of $350 \mathrm{~mA}$. In this design just one ring oscillator circuit is implemented for the full array. Despite the authors claims, device current consumption would have to be addressed before this technique could be scaled to one time-to-digital converter per pixel. Fluorescence lifetime measurements of long-lifetime ( $>30 \mathrm{~ns}$ ) quantum dot samples are presented, using an external 406nm external laser diode as an excitation source. This system demonstrates an alternative approached to time-gated photon counting for on-chip fluorescence lifetime analysis.

Despite growing interest in fully integrated CMOS based SPAD systems, SPAD detectors based on other semiconductor materials have also become more widespread. Despite the inability to integrate electronics on the same substrate as the detection element, these devices are often packaged alongside a second external quenching device [42], [43]. The advantage of non-CMOS based devices is that the wavelength sensitivity of the device is no longer constrained by the junction depth of the CMOS process and can be tailored to individual applications. This can lead to SPAD detectors capable of detection in the near infra-red [44], [45]. Unfortunately, these devices cannot take advantage of the large scale production capabilities and investment that has been made in silicon-based CMOS technology and do not offer a low cost solution to single-photon counting. 


\subsection{Micro-Analytical Systems}

The design and application of bio-chips and micro-devices that can perform analysis for biomedical applications rapidly and inexpensively in a miniaturized environment has been the focus of much research [25], [46]. A need for the development of simple, robust, cost-effective medical devices capable of rapidly screening for multiple diseases and to monitor pathogens has been identified as a key step in the fight against infectious diseases, especially in developing areas [47]. The miniaturisation of diagnostic devices has the potential to increase throughput and reduce the cost of a wide range of diagnostic tests [48]. Furthermore, micro-scale systems often require reduced reagent quantities, resulting in reduced operating costs. The aim of much research into device miniaturisation is to produce a point-of-care device, capable of performing sample analysis quickly and easily at a patient's bed-side or in a doctor's surgery [49], [50].

Drug discovery is an area of research that could benefit from high-throughput miniaturised devices [51]. There is also on-going research into the development of implantable in vivo analysis devices [52]. Micro-analytical systems have been developed for the analysis of a wide range of analytes including oxygen [53], glucose, chemical and biological agents [54] as well as fluorophores and biological samples such as DNA [55]. One of the key challenges in the development of such devices is the integration of the different technologies required to produce a functional device. In a fluorescence-based device this would include sample excitation and detection elements alongside a sample handling mechanism such as microfluidics [56]. Many research groups have offered circuit designs which image or excite a sample of interest. It is the goal of this project to combine these elements in a single unit which takes advantage of CMOS technology to provide a degree of on-chip processing [57]. A CMOS process can provide both detectors and electronics for an integrated optical sensing system with no post-processing required for sensor integration [5].

In [49], a micro-system integrating a GaN thin-film LED alongside a CdS distributed Bragg reflector (DBR) filter, a PDMS microfluidic channel and Si PIN photodetector is presented. As this system was intended for intensity analysis, LED operation is DC and is driven by external hardware. Despite having a silicon substrate, this system includes no signal processing or LED control circuitry. The use of a microfluidic channel allows the sample of interest to be easily introduced into the micro-system. This device employs a planar topology, with the excitation and detection elements located on the same substrate, allowing the micro-fluidic device to be easily placed on top of the system with just $2 \mathrm{~mm}$ of separation between the 
sample and the detector. The PIN photodiode used has a large active area of $4 \mathrm{~mm}$ in diameter and measurements demonstrate how this system is capable of detecting fluorescence samples down to concentrations of $120 \mathrm{nM}$. This device demonstrates how fluorescence analysis can be performed in a micro-system environment, however, was limited by the poor filter performance which allowed just $40 \%$ of the emission light to reach the detector. Furthermore, by not including any signal processing or LED drive circuitry, this system requires a number of external pieces of hardware in order to operate.

Similar work is presented in [26], whereby a VCSEL excitation source emitting at $773 \mathrm{~nm}$ has been integrated alongside emission filters and PIN photodetectors. As in [49], this device is intended for fluorescence intensity analysis and the VCSEL light source was not designed for short pulse excitation. The VCSEL excitation source was capable of producing a continuous wave output with an output power that was dependent on the diameter of the device. Fluorescence detection is demonstrated with a $20 \mu \mathrm{m}$ diameter VCSEL device, with a maximum output power of $4 \mathrm{~mW}$. Detection is performed using a circular PIN photodiode, with a diameter of $1000 \mu \mathrm{m}$, which encircles the VCSEL device (Figure 2.5). Presented results show how the close proximity of the VCSEL and photodiode resulted in significant coupling of excitation light to the detector. Performance was improved through the integration of a DBR filter on the surface of the detector. Based on III-V materials the inclusion of CMOS electronics in this system is not possible. Furthermore, the large pixel size does not make this device suitable for implementation in an array format.

Building on this work, Porta and Summers [58] demonstrated how a single element could act as both a fluorescence excitation source and detector. In this work a resonant-cavity LED was implemented capable of producing $0.5 \mathrm{~mW}$ of optical power when forward biased and photo detection when reverse biased. While fluorescence detection is not demonstrated, photodiode characterisation using an external light source estimates that when sufficiently reverse biased (into the avalanche region of operation), these photodiodes would be capable of detecting the presence of $2 \times 10^{9}$ molecules per $\mathrm{cm}^{3}$. Fluorescence detection of a $50 \mu \mathrm{M}$ Cy5 fluorophore, using the same device, is confirmed by Summers et al., 2005 [59]. As with the work of Thrush et al. and Chediak et al. this device does not allow electronic circuitry to be included on the same substrate as the detector and excitation source. This device does however allow both system elements to be integrated on the same substrate. Furthermore, by operating the device in avalanche mode the pixel includes inherent signal gain, reducing integration time. 


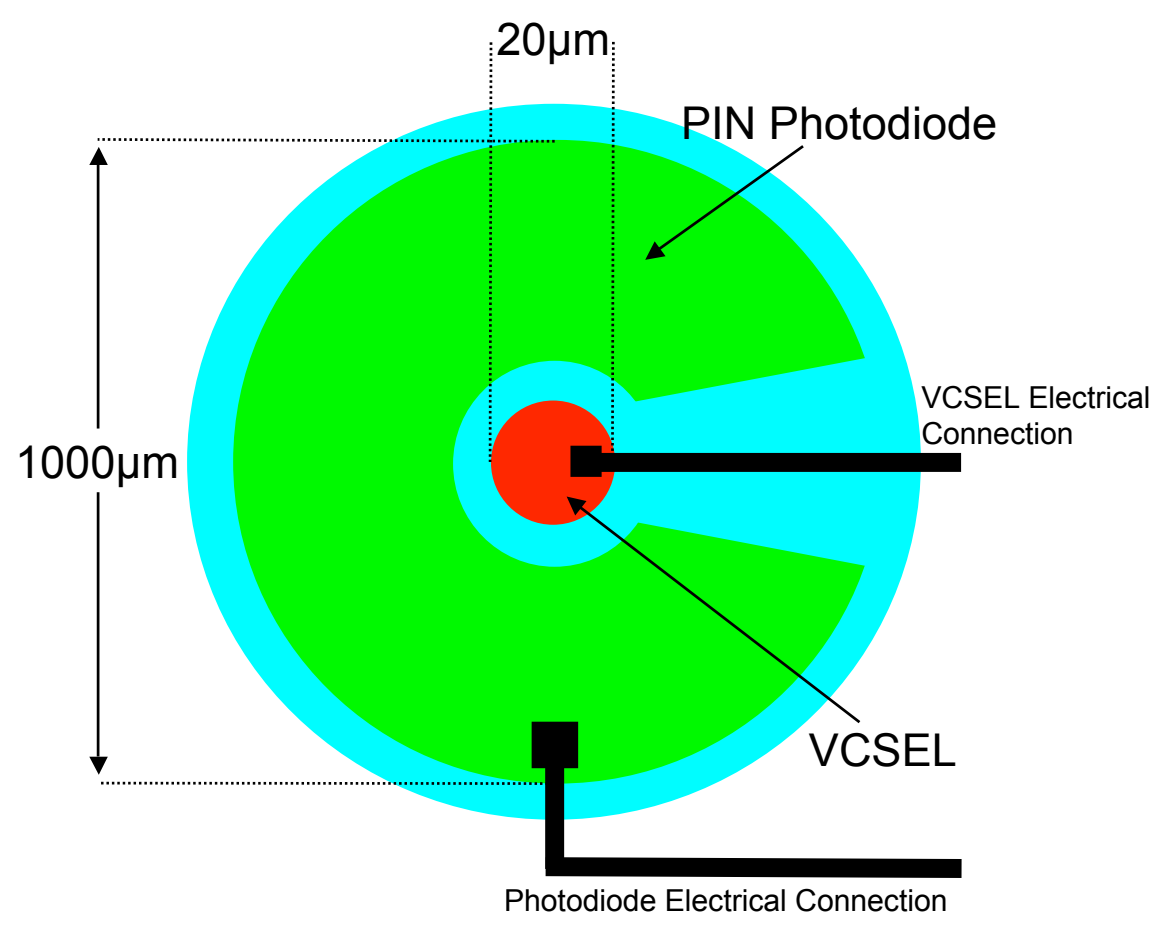

Figure 2.5: Photodiode and VCSEL arrangement implemented by Thrush et al. 2004 .

While many groups choose to introduce the sample of interest into the system via a micro-fluidic device, microscope slide or cuvette, Jang et al. [60] chose to interface molecular probes directly onto the transducer surface. Using a CMOS process, an array of n-well/p-substrate photodiodes were integrated alongside a fluorescence emission filter, readout circuitry and in-pixel 14-bit analogue-to-digital converters (ADC). An array of molecular capture probes were deposited onto the surface of a fibre-optic faceplate. This fibre-optic array is used to guide emission light to the photodiode array via a $\mathrm{ZnS} / \mathrm{Na}_{3} \mathrm{AIF}_{6}$ long pass filter. Each photodiode measures $50 \times 50 \mu \mathrm{m}$ with a pixel pitch of $250 \mu \mathrm{m}$. The sample of interest is washed over the surface of the device, hybridization occurs at sites whereby the target molecule in the sample interfaces with a complementary molecular capture probe located on the system surface. The sample of interest can be tagged with a fluorescence marker allowing sites where hybridization has occurred to be observed. While results of this technique are not presented, quantitative fluorescence intensity measurements of samples in solution demonstrate how the system is capable of sensing samples down to $0.125 \mathrm{nmol}$ in concentration. Based on a CMOS process, this system includes circuitry in-order to locally process detector data. However, the system does not include an integrated light source and uses 
an external low-power diode-pumped solid-state green laser $(532 \mathrm{~nm})$ for sample excitation. Furthermore, the CMOS device would require a significant number of post-processing steps in order to integrate the long pass filter and fiber-optic faceplate which are situated on the transducer array surface.

While the majority of micro-analysis devices are aimed at hand-held bio-diagnostic application, Bellis et al. 2006 [52] propose an in vivo miniature implantable fluorescence detector. A prototype device incorporates four discrete avalanche photodiode die attached to a narrow PCB board along with a single VCSEL chip with an emission wavelength of $650 \mathrm{~nm}$. This device is encased in a narrow glass tube. Located outside the tube, the prototype PCB widens to accommodate connections to external photon counting circuitry, avalanche quench circuits and a PC interface. No measured results are presented, however, this prototype demonstrates how the individual components required to perform fluorescence analysis in vivo can be integrated onto a single platform and packaged into a suitably sized device. The use of CMOS based avalanche photodiodes would allow photon counting and quench circuitry to be incorporated onto the same die as the detector, this would further increase system integration and minimise device size. With data communication being particularly challenging in an in vivo environment, on-chip signal processing circuitry could be designed to minimise the amount of data being broadcast off-chip, easing I/O requirements.

Many of the reported micro-systems focus on the detection of fluorescence intensity or lifetime for biomedical applications. In [53], a chemical sensing system is reported which integrates a gel-based thin-film sensor along with a CMOS photodetector array and an LED excitation element. The sensor is xerogel based and is a porous glass structure. This gel has been designed so that its fluorescence properties are dependent on the environments oxygen levels. The gel is spun onto the surface of a planar LED device. The LED excites the xerogel and the resulting fluorescence emission is monitored by a CMOS detector array. Measurement results show how the fluorescence intensity of the xerogel sensor changes with oxygen levels and how these changes can successfully be detected using a CMOS-based sensor. This work demonstrates how optical based micro-systems can be applied to a number of different sensing applications. By altering the chemical properties of the xerogel element, this system can be used to detect a wide variety of target analytes.

Table 2.1 provides an overview of selected systems presented in the literature which attempt to integrate a number of elements of a fluorescence analysis system. From the table it can 
be seen that research tends to focus on the integration of the detector and signal processing circuitry or the detector and excitation source and very little is presented on the integration of all three system elements. This results in systems which always require at least on high cost external device. For a low cost, miniaturised time resolved fluorescence system to be realised the integration of all three components must be addressed.

\begin{tabular}{|c|l|l|l|l|}
\hline \hline Reference & Application & Detector & Signal Processing & Excitation Source \\
\hline \hline$[32]$ & Fl. Lifetime & $\begin{array}{l}\text { CMOS } \\
\text { Photodetector }\end{array}$ & $\begin{array}{l}\text { Time-Gated } \\
\text { Pixel }\end{array}$ & $\begin{array}{l}\text { External } \\
\text { Laser Diode }\end{array}$ \\
\hline$[33]$ & Fl. Lifetime & $\begin{array}{l}\text { CMOS Pinned } \\
\text { Photodiode }\end{array}$ & $\begin{array}{l}\text { Time-Gate } \\
\text { Charge Transfer }\end{array}$ & $\begin{array}{l}\text { External } \\
\text { Laser Diode }\end{array}$ \\
\hline$[38]$ & Fl. Lifetime & $\begin{array}{l}\text { CMOS } \\
\text { SPAD }\end{array}$ & $\begin{array}{l}\text { External } \\
\text { TCSPC }\end{array}$ & $\begin{array}{l}\text { External } \\
\text { Ti:Sapphire Laser }\end{array}$ \\
\hline$[37]$ & Fl. Lifetime & CMOS & $\begin{array}{l}\text { In-Pixel Time } \\
\text { Gated Counters }\end{array}$ & $\begin{array}{l}\text { External } \\
\text { Laser Diode }\end{array}$ \\
\hline$[36]$ & Fl. Lifetime & $\begin{array}{l}\text { CMOS } \\
\text { SPAD }\end{array}$ & $\begin{array}{l}\text { On-Chip } \\
\text { TDC }\end{array}$ & $\begin{array}{l}\text { External } \\
\text { Laser Diode }\end{array}$ \\
\hline$[49]$ & Fl. Intensity & $\begin{array}{l}\text { Si PIN } \\
\text { Photodiode }\end{array}$ & None & $\begin{array}{l}\text { Integrated GaN } \\
\text { Thin Film LED }\end{array}$ \\
\hline$[26]$ & Fl. Intensity & $\begin{array}{l}\text { PIN } \\
\text { Photodiode }\end{array}$ & None & Integrated \\
& & VCSEL \\
\hline$[59]$ & Fl. Intensity & Reverse Bias & None & Forward Bias \\
& & Vertical Cavity & & Vertical Cavity \\
& & Device & Device \\
\hline$[60]$ & Fl. Intensity & $\begin{array}{l}\text { CMOS } \\
\text { Photodiode }\end{array}$ & $\begin{array}{l}\text { In-Pixel } \\
\text { ADCs }\end{array}$ & External \\
& & Laser Diode \\
\hline$[52]$ & In Vivo Fl. & $\begin{array}{l}\text { Discrete } \\
\text { APDs }\end{array}$ & External & Discrete \\
& Intensity & VCSEL Chip \\
\hline$[53]$ & Fl. Intensity \\
& Oxygen Sensor & CMOS & None & Discrete \\
& PED \\
\hline
\end{tabular}

Table 2.1: Summary of selected integrated systems for fluorescence detection 


\subsection{Non-Fluorescence Based Sensing Techniques}

There are a number of non-fluorescence based sensing techniques, these include:

- Mass Spectroscopy [61]

- Magnetic Bead Labeling [62]

- Electrochemical [63]

- Raman Spectroscopy [64]

- Interferometry [65]

- Surface Plasmon Resonance [66]

- Impedance Sensing [67]

- Gravimetric Sensing [68].

The main disadvantage of fluorescence based measurement techniques is the requirement to label the sample of interest with a fluorophore prior to analysis. This sample preparation adds to the experimental time and may result in an alteration in the behaviour of the sample under investigation. There are exceptions to this, where the autofluorescence characteristics of a sample are being observed [69]. It is for these reasons that there is much research into non-fluorescence based sensing techniques that do not require samples to be tagged with a marker. However, fluorescence based sample investigation remains the standard analysis technique used in medical and biological laboratories thanks to its excellent sensitivity and selectivity capabilities. Many companies have heavily invested in the development of fluorescence markers sensitive to a wide range of biological and chemical phenomena, making fluorescence sensing the most established and widely used of the analysis techniques. 


\subsection{Key Technologies}

The work described in this thesis was part of a collaborative, multi-disciplinary project which built on previous work carried out at a number of different institutions. Two key elements of the system were (i) the CMOS compatible single-photon avalanche diode (SPAD) detectors provided by Ecole Polytechnique Federale de Lausanne (EPFL) in Switzerland and (ii) the AlInGaN micro-LEDs developed by the Institute of Photonics at Strathclyde University, United Kingdom. An overview of these key technologies is provided in Sections 2.6.1 and 2.6.3, respectively.

\subsubsection{The SPAD Detector}

The concept of the avalanche photodiode was proposed a number of years ago by Haitz et al. [70]. In recent years much work has focussed on implementing single-photon avalanche diodes in a standard CMOS process [71], [41], [72]. SPADs realised in a foundry CMOS process were first demonstrated by Rochas et al. in 2002 [73]. If biased above breakdown, the optical gain of a SPAD becomes infinite, thereby ensuring Geiger mode operation. This process is described in Section 2.6.2.1. The SPAD is biased such that upon detection of a single-photon a transistor-transistor logic (TTL) compatible pulse is produced (Section 2.6.2.2). The amplitude of the SPAD output therefore provides no information on photon intensity. Intensity information is obtained by counting the pulses during a certain period of time or by measuring the mean time interval between successive pulses. This is clearly demonstrated in Figure 2.6.

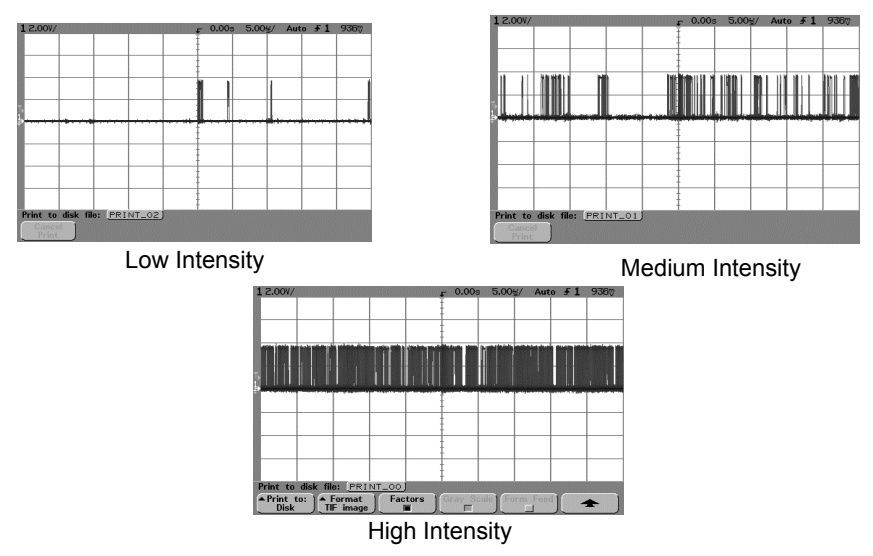

Figure 2.6: Raw SPAD output pulses from a SPAD under varying intensity. 
The design of the SPAD detector implemented in this project, was provided by Professor Edoardo Charbon at Ecole Polytechnique Federale de Lausanne (EPFL) and consists of a circular dual junction structure: $\mathrm{p}^{+}$anode/deep n-well/p-substrate (Figure 2.7), realised in a $0.35 \mu \mathrm{m}$ high-voltage crystalline silicon CMOS technology. The $\mathrm{p}^{+}$anode/deep n-well junction forms the avalanche multiplication region where the Geiger breakdown occurs. The $\mathrm{n}$-well/p-substrate junction allows the $\mathrm{p}^{+}$anode to be biased independently from the substrate and prevents electrical cross-talk. A p-well guard-ring surrounds the $\mathrm{p}^{+}$anode to prevent premature breakdown [74]. The device has a diameter of $6 \mu \mathrm{m}$ resulting in an active area of $28.27 \mu \mathrm{m}^{2}$.
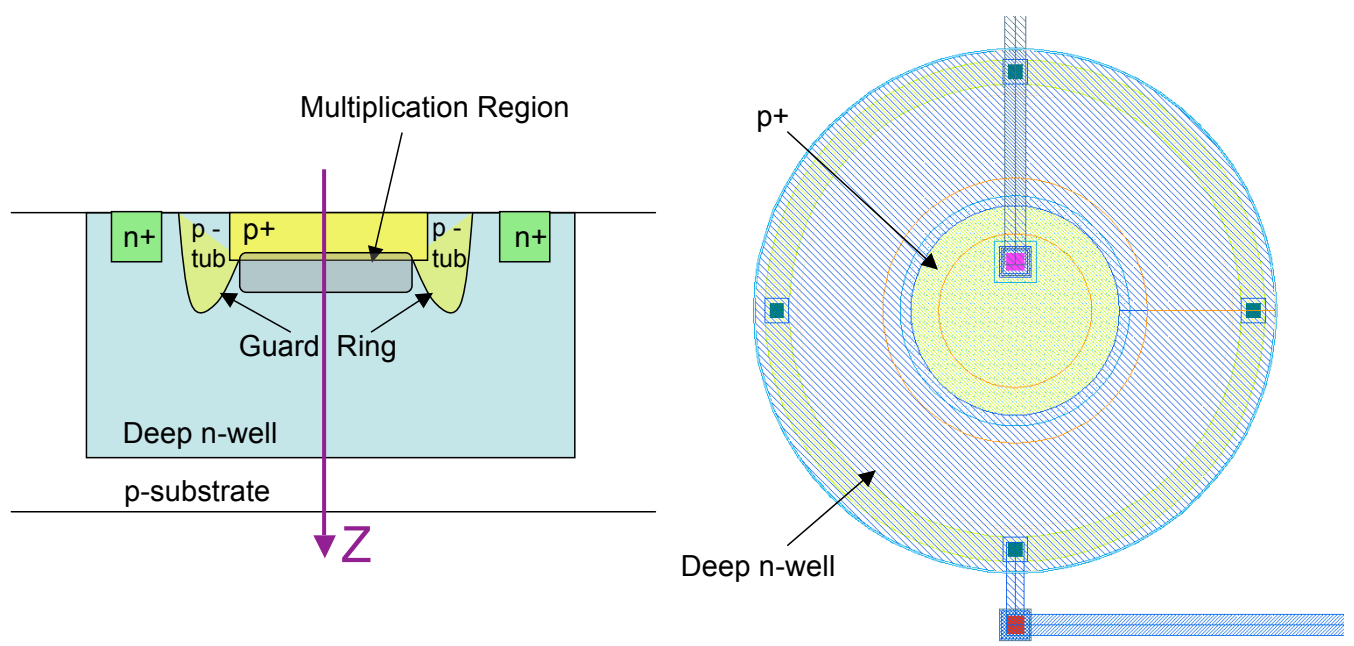

Figure 2.7: Schematic cross-section and layout view of the circular SPAD employed in this project. This SPAD was implemented in a $0.35 \mu \mathrm{m}$ high-voltage CMOS technology.

A diode biased beyond its reverse bias breakdown voltage will remain in a non-broken down state (zero current flowing) for a relatively long period of time (in the order of milliseconds). It is the occurrence of a primary free carrier within the high electric field p-n junction that triggers an avalanche breakdown event (Figure 2.8). It is always hoped that this primary free carrier is generated as a result of an incident photon. However, spurious breakdown events do occur due to thermal or tunnel generated carriers and trapped charges. These false breakdown events are indistinguishable from photon induced events in a Geiger mode avalanche photon diode and are therefore the primary cause of dark counts (Section 2.6.2.3). Trapped charge carriers result in a phenomenon known as after-pulsing (Section 2.6.2.4). 


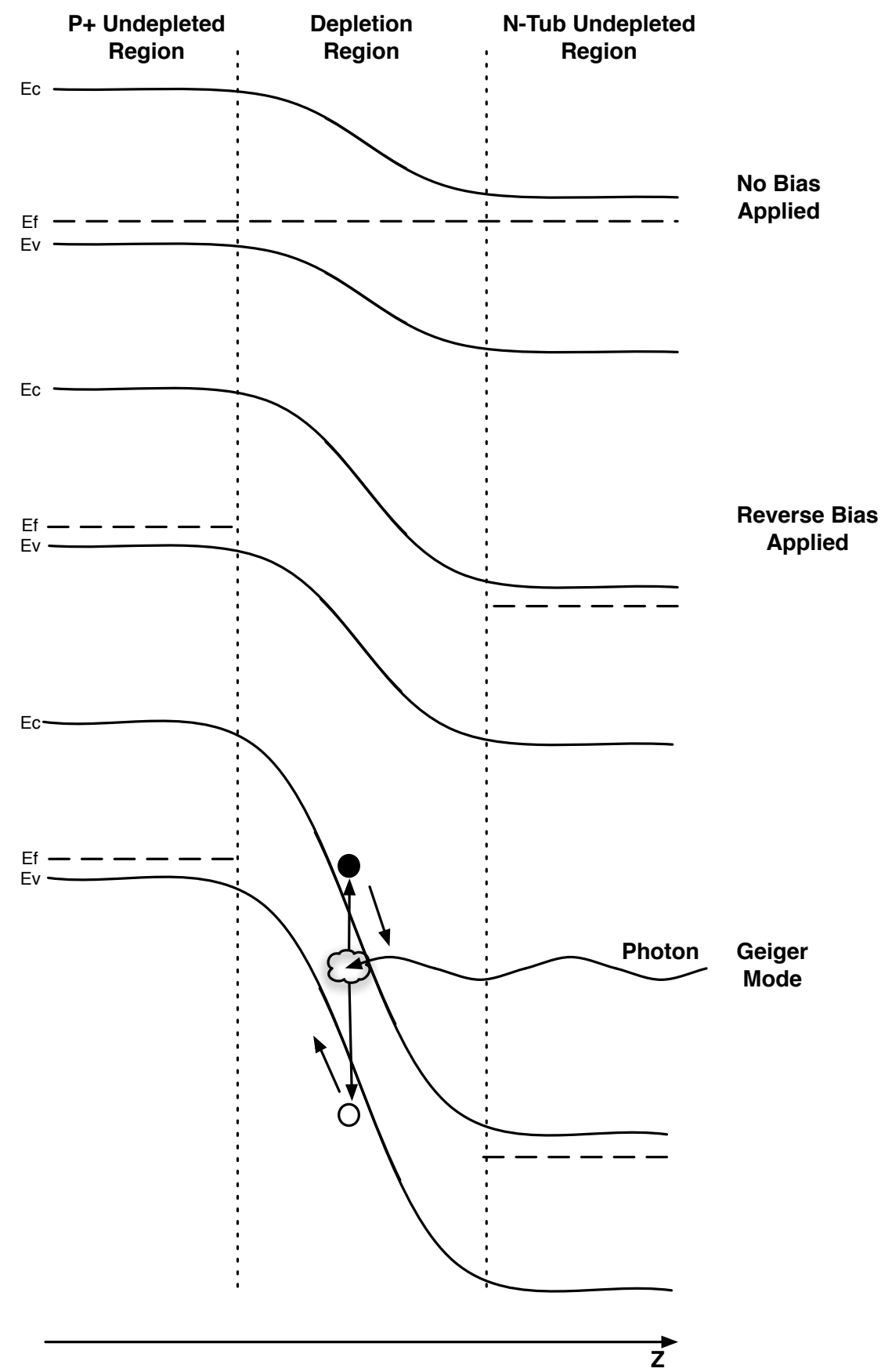

Figure 2.8: Energy band diagrams in the Z-axis of the p+/deep n-well junction of a SPAD device. 


\subsubsection{SPAD Operation}

\subsubsection{The Avalanche Process}

A photon of sufficient energy incident on the active area of the SPAD will generate a free electron-hole pair within the depletion region of the $\mathrm{p}-\mathrm{n}$ junction. Due to the high electric field within this region, caused by the large reverse bias voltage, the electrons and holes are accelerated towards the $\mathrm{p}$ and $\mathrm{n}$ regions, respectively. The accelerated free electron and hole collide with static electron-hole pairs in the junction, resulting in impact ionization. These newly created free electrons and holes are subsequently accelerated, resulting in further collisions and hence ionization events. As the number of free electrons increases, so does the current flowing through the SPAD device. The number of free electron-hole pairs, and hence current, continues to rise exponentially until quenching occurs (Section 2.6.2.6). The high electric field in the depletion region of a Geiger mode avalanche diode causes an exponential increase in the number of free electrons and holes due to impact ionization. This process is started as a result of the creation of a single free electron-hole pair caused by an incident photon.

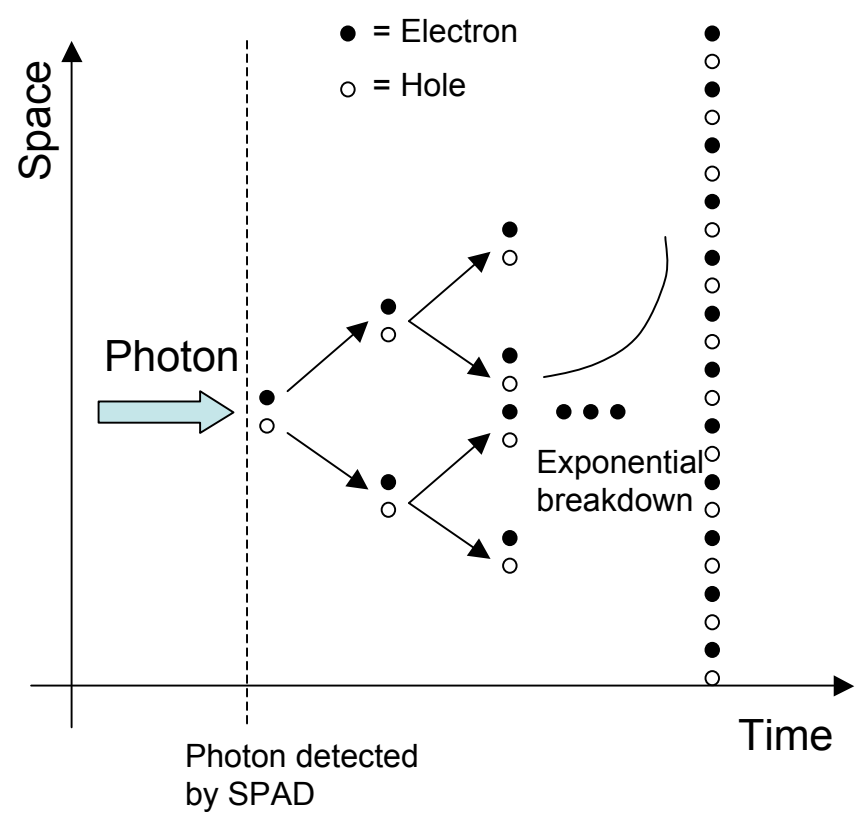

Figure 2.9: The avalanche breakdown process. 


\subsubsection{Biasing the SPAD}

In order to ensure the SPAD detectors compatibility with standard CMOS circuitry, the biasing of the SPAD must be carefully considered. The logic levels of the $0.35 \mu \mathrm{m}$ process used in this project were $0 \mathrm{~V}$ (logic 0 ) and $3 \mathrm{~V} 3$ (logic 1 , in electronics $3.3 \mathrm{~V}$ is denoted as $3 \mathrm{~V} 3$ ). Therefore, it had to be ensured that the output transition of the SPAD upon detection of a photon had a $3 \mathrm{~V} 3$ swing. This is achieved by setting up appropriate bias conditions (Figure 2.10). For the SPAD to operate in Geiger mode it must be biased above its breakdown voltage. The $\mathrm{p}^{+}$anode of the SPAD is therefore biased at a high negative voltage, VOP. The deep n-well cathode is connected to a positive power supply, SPAD_AVDD, via a quench resistor.

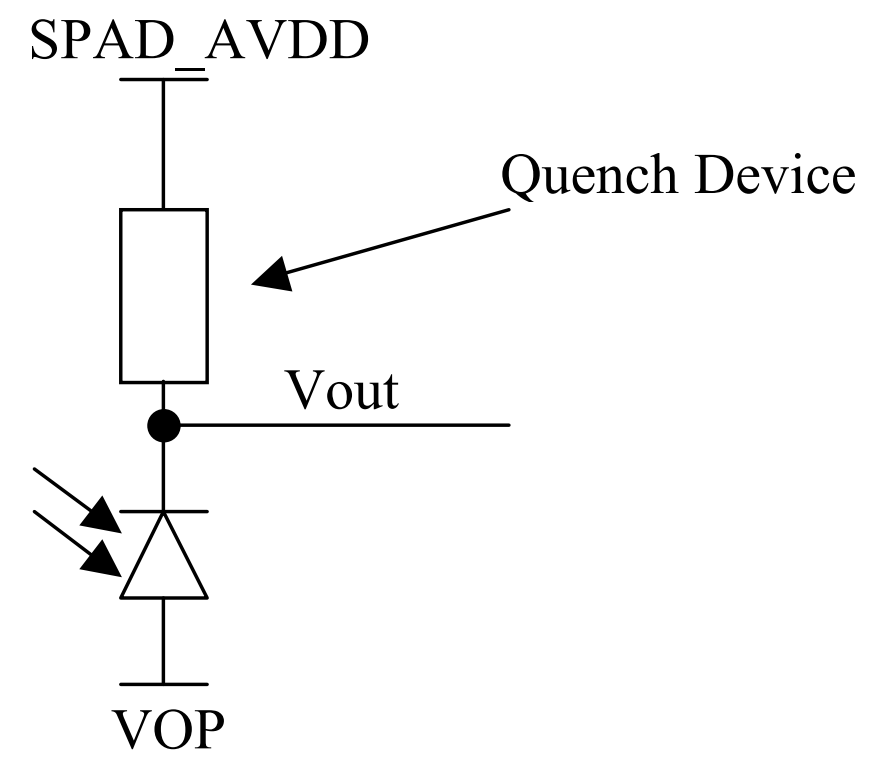

Figure 2.10: Biasing of the SPAD detector to ensure TTL compatible output pulses upon detection of a photon.

The key is to ensure that the excess bias voltage across the SPAD when in an armed state is equal to $3 \mathrm{~V} 3$ (for a $0.35 \mu \mathrm{m}$ process). The excess bias voltage is defined as:

$$
\text { ExcessBiasVoltage }(V e)=V O P+S P A D \_A V D D-V b d
$$

Where $V b d$ is the reverse bias breakdown voltage of the diode. As described in Section 2.6.2.6, when a SPAD breaks down the reverse bias voltage across the SPAD is lowered by the quenching circuit until this voltage is brought below $V b d$. According to Equation 2.8 the 
transition required to bring the SPAD out of avalanche breakdown is equal to Ve. This transition is seen at the Vout node in Figure 2.10. Being equal to 3V3, this transition voltage can then be detected and processed by standard CMOS logic circuitry.

If the reverse bias across the SPAD is insufficient the SPAD will never breakdown. Conversely, if the reverse bias voltage is too high the SPAD will be in permanent breakdown and will no longer be photon sensitive. For the SPAD used in this project the minimum reverse bias voltage was found to be approximately $20.8 \mathrm{~V}$. The SPAD went into permanent breakdown at voltages above approximately $24.05 \mathrm{~V}$.

\subsubsection{Dark Counts}

Dark counts are non-photon induced breakdown events and are a function of detector area and temperature. The primary causes of dark counts are thermal or tunnel generated carriers in the diode p-n junction [75]. As such, the dark count rate (DCR) of a SPAD is strongly temperature dependent. Dark counts place a limit on the minimum detectable number of photons and therefore defines the lower limit of the detectors dynamic range.

Dark count is also dependent on the reverse bias voltage placed across the SPAD. As this voltage is increased the SPADs sensitivity (PDP) increases, due to the higher electric field at the p-n junction increasing the likelihood of an avalanche breakdown event. However, this increased breakdown probability also increases the probability of a non-photon induced breakdown occurring. A measure of DCR versus the reverse bias voltage (VOP), for the SPADs implemented in this project, illustrates this in Figure 2.11. 


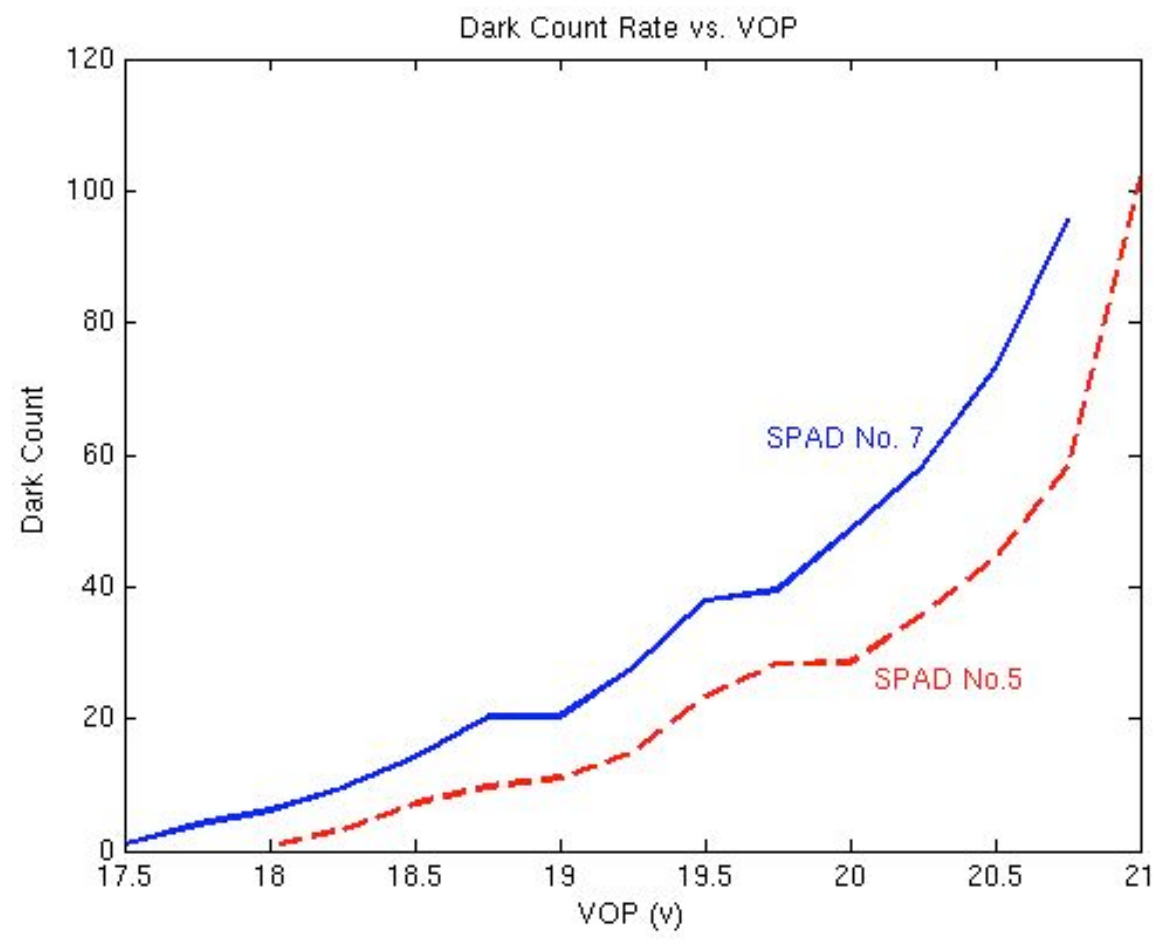

Figure 2.11: Examples of dark count rate measurements as a function of the reverse bias voltage, VOP, for two different SPADs in the array. 


\subsubsection{After-Pulsing}

After-pulsing is defined as spurious counts caused by carriers temporarily trapped in the depletion region during a breakdown event. After a short while these charges are released, causing a secondary Geiger pulse. The level of after-pulsing in a device is dependent on the quality of the silicon (which defines the trap concentration) and the number of carriers generated during a breakdown event [76]. The number of carriers generated is dependent on the diodes parasitic capacitance and the quench circuitry used. If the hold-off time of the quench circuit is not long enough, trapped charges do not dissipate and after-pulsing occurs. As quench time increases, the correlation between the initial pulse and after-pulses decreases. After-pulsing also shows an inverse temperature dependence, increasing as temperature decreases [77].

\subsubsection{Jitter}

Jitter or timing resolution is the statistical variation in the time-of-occurrence of a signal. In any timing critical electronic system signal jitter results in timing uncertainty. SPAD detector jitter results in variation in the time between a photon arriving at the sensor and the leading edge of the detector output pulse [78]. SPAD jitter can be attributed to variation in diffusion times of carriers generated outwith the depletion region, the transit times of carriers within the depletion region and fluctuations in the build up of avalanche current within the detector. Lateral diffusion of the avalanche process across the detector can also influence SPAD timing resolution. Therefore, the location of the detected photon within the active region of the detector can influence jitter performance [79]. In a time-resolved fluorescence lifetime analysis system SPAD jitter results in uncertainty in photon arrival times. This can result in a photon count being placed in an incorrect histogram time bin and hence, leads to skewing of the lifetime measurement.

\subsubsection{Quenching}

To halt the avalanche process, a quenching mechanism must be included as part of the SPAD pixel. The purpose of the quenching circuit is to halt the SPADs avalanche breakdown and to return the SPAD to an armed state, ready to detect another photon. There are two methods well documented in the literature that can be used to achieve this; namely passive quenching and active quenching. To stop the avalanche breakdown process the reverse bias voltage across 
the device must be reduced. The simplest and most compact way to achieve this is to place a resistive element in series with the SPAD device [80]. As current begins to flow in the diode, a voltage begins to develop across the resistor. This voltage drop increases as the current through the device increases. The SPAD and the resistor act as a voltage divider. As the voltage across the resistor increases, the reverse bias voltage across the SPAD decreases. This process continues until the SPAD is brought out of avalanche breakdown mode. As the free electrons and holes begin to recombine, the current flowing in the SPAD reduces. Thus, the voltage dropped across the resistive element decreases, the reverse bias across the SPAD increases and the SPAD is eventually brought back into Geiger mode of operation and is ready for another breakdown event. The resistance of the quenching device dictates the speed of quenching and the speed of diode recovery. This time is known as the dead time of the SPAD (the time in which the SPAD is insensitive to photons). This dead time defines the maximum count rate the SPAD is capable of and therefore places a limit on the maximum dynamic range of the detector. In this project a passive quenching mechanism was employed with a grounded PMOS transistor acting as the resistive element.

While passive quenching of an avalanche event in a SPAD can be achieved using a simple, compact circuit, it only offers limited user tunability in terms of setting the dead time of the detector and the recovery time. Using a passive circuit these values tend to be defined at the time of design, through the selection of the quench resistance. Passive quench resistance can be varied by the user if a transistor with an adjustable gate voltage is used as the resistive element. While active circuits increase circuit complexity and size they offer the user greater control over the detectors avalanche, quench and recovery dynamics. Active quench circuits are used to sense the occurrence of an avalanche event, hold the SPAD output node low for a period of time, before re-arming the detector by returning the SPAD to a Geiger mode bias state. A number of groups have implemented active quench circuits with SPADs, some have chosen to implement the quench circuit on a separate chip [81] while others located the quench circuit within the SPAD chip [41], [82]. An example of an in-pixel SPAD active quench circuit used to measure fluorescence lifetime data is presented in Figure 2.12 [41]. In this circuit the SPAD is reset by placing a 0 on the Precharge line, Precharge is then set high enabling the detector. Diode connected $\mathrm{M}_{p 2}$ acts to limit the current flow in the SPAD during breakdown. When an avalanche event occurs there is a voltage drop on the input to INV1 causing its output to rise, and thus $\mathrm{M}_{n 4}$ to conduct. This results in the IN node being pulled hard to ground via $\mathrm{M}_{n 4}$ and the Precharge NMOS transistor, $\mathrm{M}_{n 3}$. The SPAD can then be held in an off state for as long as is desired and 
is only re-armed when Precharge is set low. SPADoff can be used to hold the SPAD off at any time. By pulling the node IN hard to ground when an avalanche event is sensed and holding it in this state, the detection of after-pulse events can be minimised. The ability to disarm the SPAD detector via SPADoff can also be used to eliminate the detection of excitation light during a fluorescence lifetime experiment. The SPAD is disabled during sample excitation before being rapidly re-armed for fluorescence detection. Using this technique lifetime measurements can be performed without the need for an optical filter to remove excitation photons.

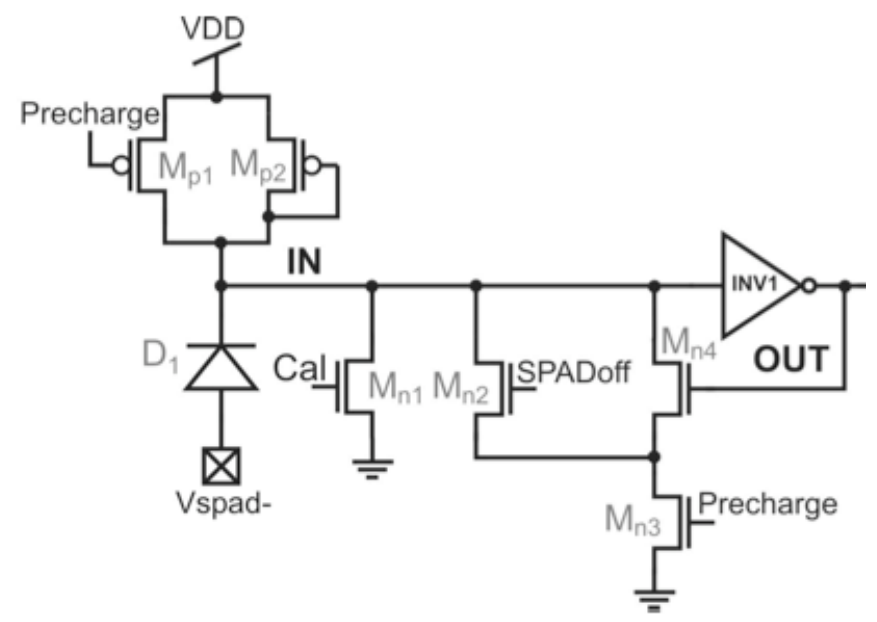

Figure 2.12: In-pixel active quench circuit designed at FBK, Trento, Italy by David Stoppa et al. presented at the 2007 IEEE International Image Sensor Workshop 


\subsubsection{AlInGaN Micro-LEDs}

Aluminium Indium Gallium Nitride (AlInGaN) micro-LEDs were developed at the Institute of Photonics at the University of Strathclyde [83] and were fabricated from "standard" InGaN/GaN quantum well ultra-violet (UV) LED wafers (planer n- and p- type GaN layers) grown on $c$-plane sapphire substrates by metal organic chemical vapour deposition [84]. An array of $64 \times 64$ spherical elements were fabricated with each pixel having a diameter of $72 \mu \mathrm{m}$ at a pitch of $100 \mu \mathrm{m}$. The emission wavelength of the LED devices is defined by the InGaN-GaN quantum-well width and composition [85]. These parameters can be controlled during manufacture and therefore, different emission wavelengths can be achieved. Contact to the $p$-area of the chip was achieved using Ni/Au evaporated contacts situated in each pixel. A global ground plane was achieved using Ti/Au deposition between the pixels, with four $n$-contact pads on each edge of the array. The AlInGaN micro-LED manufacturing process is summarised in Figure 2.13. The AlInGaN micro-LED fabrication steps are; (a) planer nand p-GaN layers grown on sapphire substrate; (b) the led structure is formed using standard photolithographic patterning and inductively coupled plasma etching, electrode contacts are formed. Contact to the n-type layer is done at the edge of the array; (c) The array is sealed in silicon dioxide; (d) chemical metal polishing is used to achieve a planar surface, with exposed contacts.

a)

\begin{tabular}{|c|}
\hline $\mathrm{p}-\mathrm{GaN}$ \\
\hline $\mathrm{n}-\mathrm{GaN}$ \\
\hline sapphire
\end{tabular}

c)

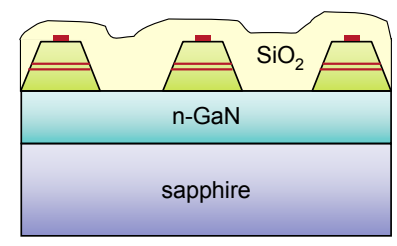

b)

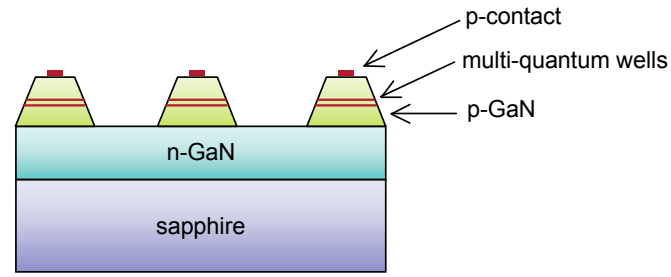

d)

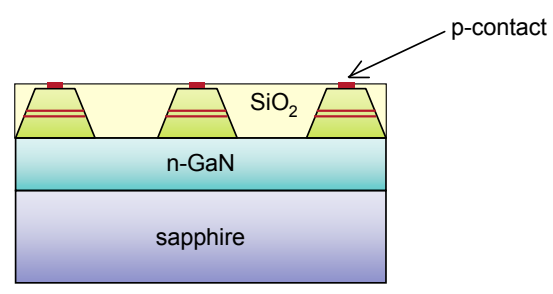

Figure 2.13: Cross-section diagrams after 4 AlInGaN fabrication steps. 


\subsubsection{Micro-LED Operation}

When a voltage is placed across a micro-LED device (forward-bias) current begins to flow, as voltage is dropped across the resistance of the device depletion region. This results in electrons and holes passing into the depletion region (p-n junction) of the diode. Upon crossing the depletion region electrons and holes will recombine with carriers of opposite conductivity type. As with all semiconductors, there are two competing recombination mechanisms in an LED: radiative and non-radiative. Non-radiative recombination results in vibrational energy in the lattice. The radiative recombination process results in the release of energy in the form of an emitted photon [86]. As the voltage across the device is increased the current flowing in the device increases resulting in increased photon emissions. The wavelength of the emitted photon is approximately equal to the band-gap energy of the semiconductor material. Band-gap energy and wavelength are related by Equation 2.9:

$$
E_{g} \approx h v
$$

Where $E_{g}$ is the semiconductor bandgap energy, $h$ is Planck's constant $6.6261 \times 10^{-34} \mathrm{Js}$ ) and $v$ is equal to the frequency of the photon.

Quantum well based devices have increased radiative efficiency and reduced recombination lifetime by confining the free carriers to the narrow well region. This results in increased optical output power (compared to p-n homo-junctions) and shorter rise and fall times. Furthermore, micro-LED structures have the potential for shorter rise and fall times (compared to conventional LED devices) thanks to the lower capacitance of each element in the array. As well as the capacitance of the micro-LED contacts there is also a contribution from the capacitance of the depletion region of the diode, which is device area dependent.

\subsubsection{Micro-LED Performance}

In this project, micro-LED arrays with an emission wavelength of $370 \mathrm{~nm}$ were investigated. The optical power of a micro-LED is defined by the current flowing in the device which in turn is defined by the voltage across the device, Figure 2.14 show the IV curve (current versus voltage) for the UV (370nm) device.

Figure 2.15 shows the wavelength spectra of a CMOS driven UV micro-LED device, measured 


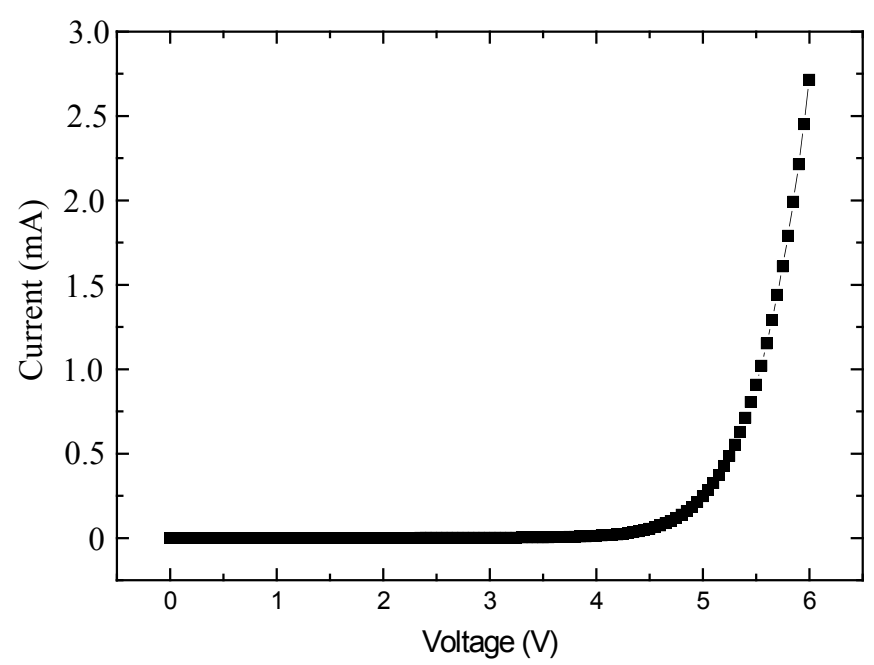

Figure 2.14: IV curve of the UV (370nm) device. Measurement taken by Dr. Chris Griffin, Institute of Photonics, University of Strathclyde.

using an Ocean Optics, USB2000+ fibre optic spectrometer. From these data it was found that the devices emission spectra peaks at a wavelength of $371 \mathrm{~nm}$.

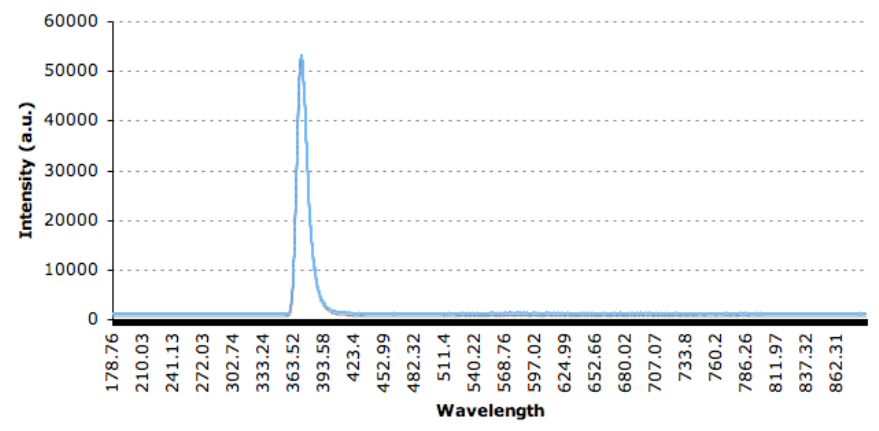

Figure 2.15: Wavelength spectra of a CMOS driven UV micro-LED device.

\subsection{Summary}

Measurement of fluorescence is a widely used analysis technique, with a wide range of application areas. Quantitative fluorescence intensity measurements are prone to misinterpretation due to illumination and fluorophore concentration non-uniformities. Thus, there is a growing interest in time-resolved fluorescence detection, whereby the characteristic fluorescence decay time-constant (or lifetime) in response to an impulse excitation source is 
measured. The sensitivity of a fluorophore's lifetime properties to the micro-environment additionally provides an extremely powerful analysis tool.

Conventional equipment required to perform fluorescence lifetime analysis is bulky and expensive. Typical set-ups involve a pico-second pulsed or modulated light source (often a laser source), a sensitive detector such as a micro-channel plate photo-multiplier tube (MCP-PMT), an optical system, frequently incorporating a microscope for low volume measurements, as well as a variety of optical lenses and filters. Optical lab-on-a-chip applications for point-of-care diagnostics equipment are now motivating research into the miniaturisation of fluorescence lifetime instrumentation.

Sections 2.2, 2.3 and 2.4 summarise previous attempts to miniaturise elements of fluorescence analysis systems. It has been identified that research has tended to focus on the miniaturisation of individual system components while the integration of all elements of the fluorescence system (excitation, detection and signal processing) has been neglected. Furthermore, those groups that have successfully integrated excitation and detection elements have targeted fluorescence intensity analysis. A review of previous work highlights how miniaturised light sources suitable for time-resolved fluorescence lifetime analysis have been developed. With the most promising of these appearing to be integrated VCSELs, resonant-cavity LEDs and AlInGaN micro-LEDs. These devices can be fabricated in an array format and have a small footprint. Furthermore, the integration of AlInGaN with CMOS drive circuitry has been demonstrated, offering the ability to precisely control individual elements. In addition, there has been a lot of progress in the integration of detection devices with signal processing circuitry, particularly using CMOS technology. CMOS compatible detectors offer the opportunity to produce highly integrated fluorescence detection systems capable of locally processing detector data. Recent developments in the design of CMOS compatible single-photon avalanche diodes allow extremely sensitive detectors to be integrated alongside signal processing circuitry. In order to gather photon arrival data, from which fluorescence lifetime can be extracted, a number of techniques have been proposed in the literature. These include; on-chip time-to-digital converters, in-pixel time-gated counters and pinned-photodiodes.

The feasibility of integrated detectors and signal processing circuitry and miniaturised light sources for fluorescence lifetime analysis has been demonstrated. The integration of these elements to form a low-cost, compact lifetime system has been identified as an area requiring an innovative solution. Such a device will have to incorporate the essential elements of a lifetime 
system in a device which is easy to set-up and align and which occupies a small footprint. For a lifetime system to operate successfully the synchronisation of the excitation and detection elements is a key factor. This is achievable in a micro-system whereby both the excitation and detection devices are based on the same underlying technology. CMOS offers a platform on which excitation device drivers, detection devices and signal processing circuitry can be realised. 


\section{Chapter 3 \\ Materials and Methods}

This multi-disciplinary project required a wide and diverse range of hardware and software tools to allow CMOS based optical systems to be designed and characterised. This included software used to design, simulate and verify CMOS chips before fabrication, and the optical hardware used to assess the functionality of prototype chips.

\subsection{Hardware}

\subsubsection{Optical Bench}

All optical work was conducted in a dark room within the Collaborative Optical, Spectroscopy, Micromanipulation and Imaging Centre (COSMIC) of the University of Edinburgh. The optics, microscope, excitation source and detector were mounted on a vibration isolation table (ThorLabs, Frame - PFA51504, Breadboard - PBI52525, Air Compressor - PTA511). The excitation light from laser diode was passed through two barrel lenses, in order to convert the rectangular beam profile of the solid-state light source into a circular one. The light then passed through a beam expander and the beam height was adjusted via a periscope. The expanded beam was focused into the rear port of the microscope (see Section 3.1.3) via a $150 \mathrm{~mm}$ focal length convex lens (see Figure 3.1). All optics mounts, posts, lenses and mirrors were manufactured by Thor Labs Inc. 


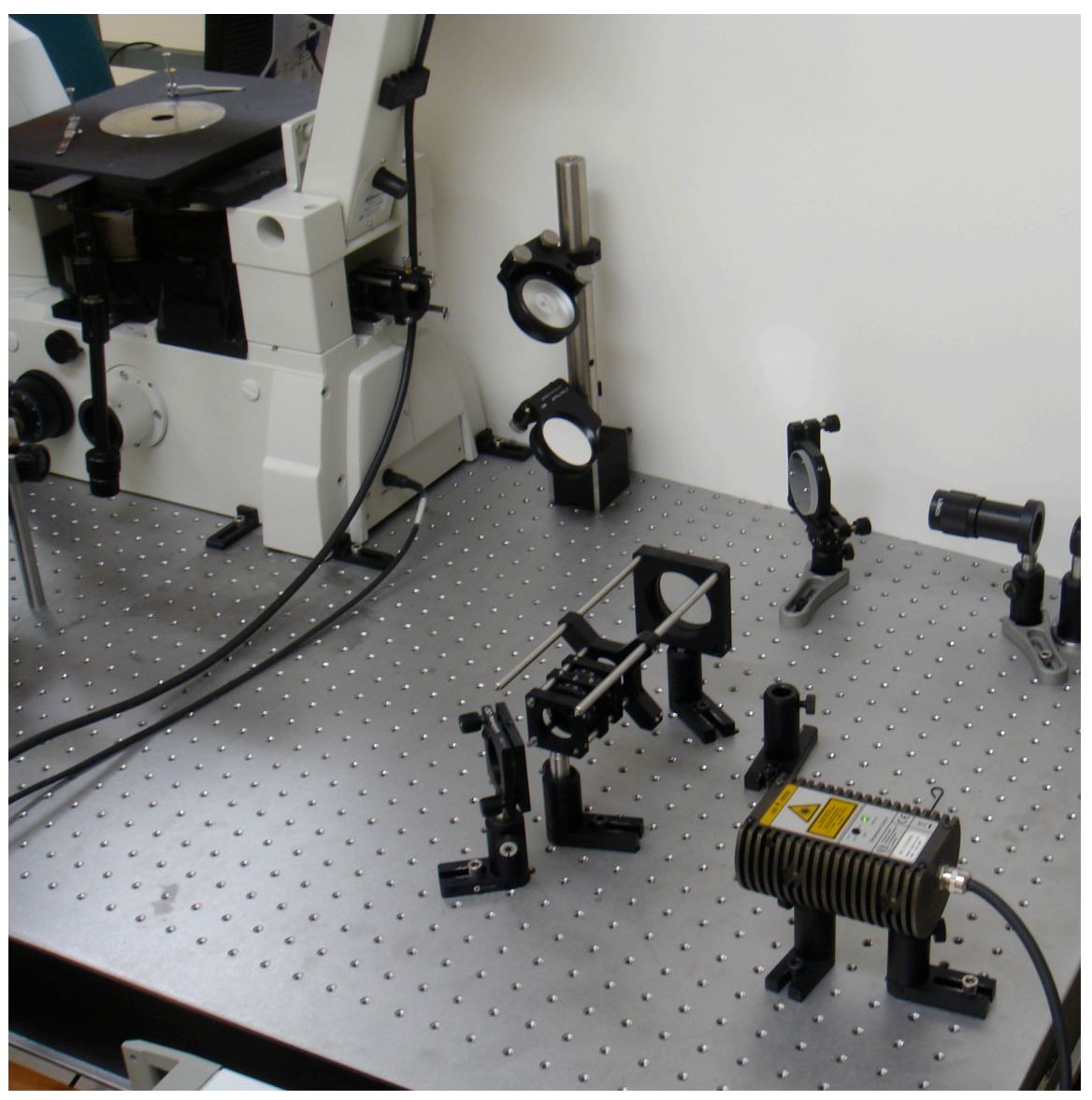

Figure 3.1: Optical bench setup. 


\subsubsection{PicoQuant Laser Diode and Driver}

Experiments requiring a pulsed laser source made use of a picosecond pulsed diode laser from PicoQuant GmbH (diode laser head model: LDH-P-C-470, pulsed diode laser driver model: PDL 800-B). The diode laser head had an emission wavelength that peaked at 467nm and had a minimum pulse width of $68 \mathrm{ps}$. The diode laser driver produced a clock signal with a core frequency of $40 \mathrm{MHz}$ that could be divided by a factor of $1,2,4,8$ or 16 thereby producing laser pulses with a user selectable frequency of $40,20,10,5$ or $2.5 \mathrm{MHz}$. The driver module could also receive an external trigger input, allowing the laser to be synchronised with other instruments. The delay time from the trigger input to the optical output from the laser was specified by the manufacturer to be $35 \pm 5 \mathrm{~ns}$ with a jitter of less than $40 \mathrm{ps}$. The PDL 800 -B laser driver was also capable of providing a synchronisation output, this was a $-800 \mathrm{mV}$, 6ns output pulse. The delay time from the falling edge of the synchronisation output pulse to the laser output pulse is specified by the manufacturer to be $12 \mathrm{~ns}$ with less than $20 \mathrm{ps}$ jitter. The diode laser head had a maximum average optical power at $40 \mathrm{MHz}$ of $1.2 \mathrm{~mW}$. When the repetition rate is reduced to $5 \mathrm{MHz}$ or $2.5 \mathrm{MHz}$, frequencies better suited to time-resolved fluorescence measurements, the maximum average optical power was $0.15 \mathrm{~mW}$ and $0.078 \mathrm{~mW}$, respectively. The diode laser was a Class IIIR laser; thus, the blink response of the eye provided suitable protection.

\subsubsection{Nikon TE2000-U Microscope}

A number of experiments were conducted with the detector array mounted to the output port of a Nikon TE2000-U inverted research microscope (Figure 3.2). The Nikon TE2000-U microscope is configured for epi-fluorescence microscopy. In this configuration the excitation light from the laser diode is coupled into the rear of the microscope and directed toward the objective (via an excitation filter) using a dichroic mirror (see Section 3.1.4). The objective is then used to focus the excitation light onto the sample of interest. The returning fluorescence emission is gathered by the same objective and separated from the excitation light by the dichroic mirror and emission filter. Therefore, there is no direct path between the excitation light and the collection objective, only reflected light, greatly improving the signal-to-noise ratio of the system. Any reflected light that is gathered is removed by the filter set.

The TE2000-U microscope has 4 output ports (eyepiece, front, left and right). The SPAD detector array and FPGA test board were mounted on the optical table to the right-hand port 
of the microscope. The test board was mounted onto X-and Y-translation stages to allow fine positioning of the die within the image plane. The side ports of the microscope are designed to be compatible with the c-mount standard. The image is focused $17.52 \mathrm{~mm}$ from the end of the port with a maximum image size of approximately $1^{\prime \prime}$ in diameter. Therefore, the image required resizing to match the size of the detector. This was achieved by placing a $1^{\prime \prime}$ diameter, $1^{\prime \prime}$ focal-length lens in the focal plane of the c-port. An adaptor was built which correctly located the lens at the c-port focal point and would position the detector at the focal point of this lens (see Figure 3.3). The aim of this was to maximise the number of photons reaching the detector rather than maintaining image quality. When image focus needs to be retained during image resizing, this requires the use of a macro-imaging lens placed at distance from the c-port whereby the ratio of the distance from the c-port and the distance to the detector is equal to the desired reduction in image size. The c-mount adaptor was designed and manufactured by Andrew Garrie a technician at COSMIC, The University of Edinburgh.

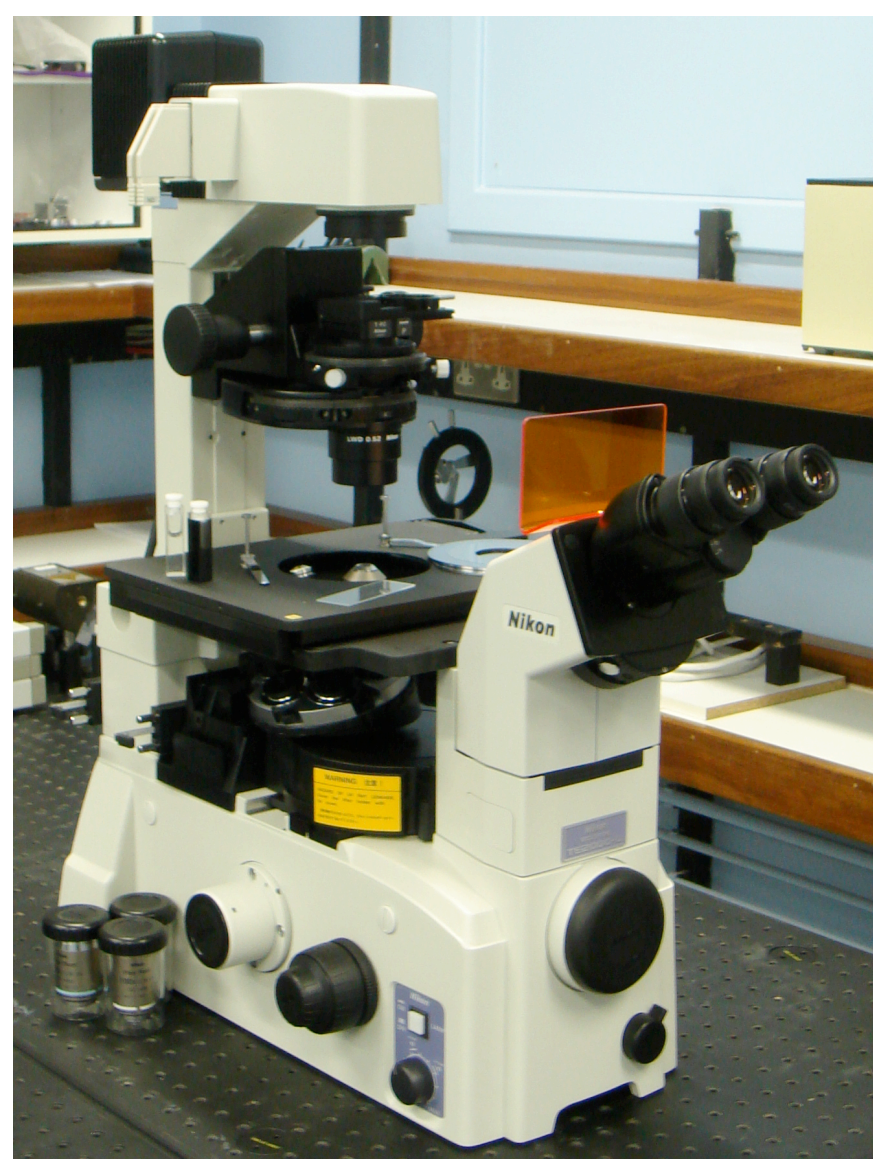

Figure 3.2: Nikon TE2000-U Microscope used for SPAD chip characterisation. 


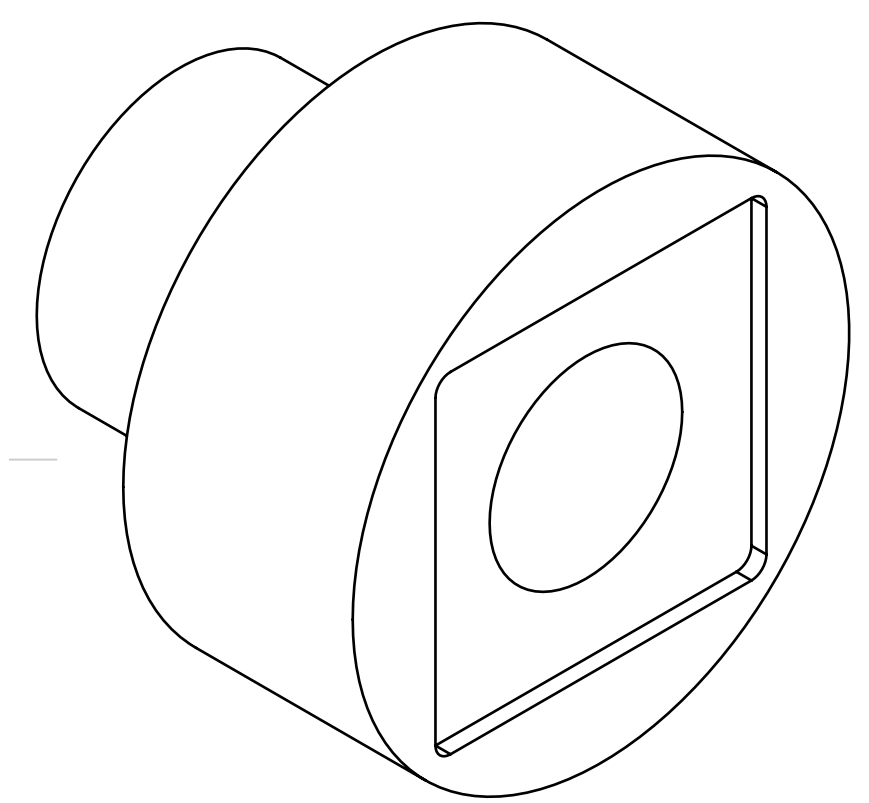

Figure 3.3: $C$-mount adaptor which housed a $1^{\prime \prime}$ diameter, $1^{\prime \prime}$ focal length lens used to re-size the image presented at the output port of the microscope. Image courtesy of Andrew Garrie, COSMIC at The University of Edinburgh. 


\subsubsection{Filter Sets}

Due to the Stoke's shift phenomenon (Section 2.1) it is possible to separate fluorescence excitation and emission light using a set of filters and a dichroic mirror. All experiments, which made use of the microscope, were conducted using the same $467 \mathrm{~nm}$ pulsed diode laser (Section 3.1.2), therefore a 470nm $( \pm 11 \mathrm{~nm})$ excitation filter (Semrock BrightLine Single-Band Filter, FF01-470/22) was used. The purpose of this filter is to minimise the amount of stray light, from sources other than the laser, entering the excitation path of the microscope. The excitation filter is housed in a filter cube along with a dichroic mirror and emission filter. The dichroic mirror is used to separate the sample emission light from laser excitation light in epi-fluoresence microscope configurations. The dichroic mirror used (Semrock BrightLine Single-Edge Dichroic Beam-Splitter, FF506-Di02) reflected all light below a wavelength of $506 \mathrm{~nm}$. Any photons of wavelength longer than $506 \mathrm{~nm}$ will pass through the mirror. The wavelength characteristics of the emission filter is dictated by the wavelength of emitted photons from the sample of interest. The purpose of the emission filter is to block any excitation light reaching the detector. For this project a 514nm long-pass filter (Semrock RazorEdge Long Wave Pass Raman Filter, LP02-514RU) was chosen. This allowed any sample with an emission wavelength above $514 \mathrm{~nm}$ to be analysed. The separation of excitation and emission light by a filter set in an epi-fluorescence configuration is depicted diagrammatically in Figure 3.4. 


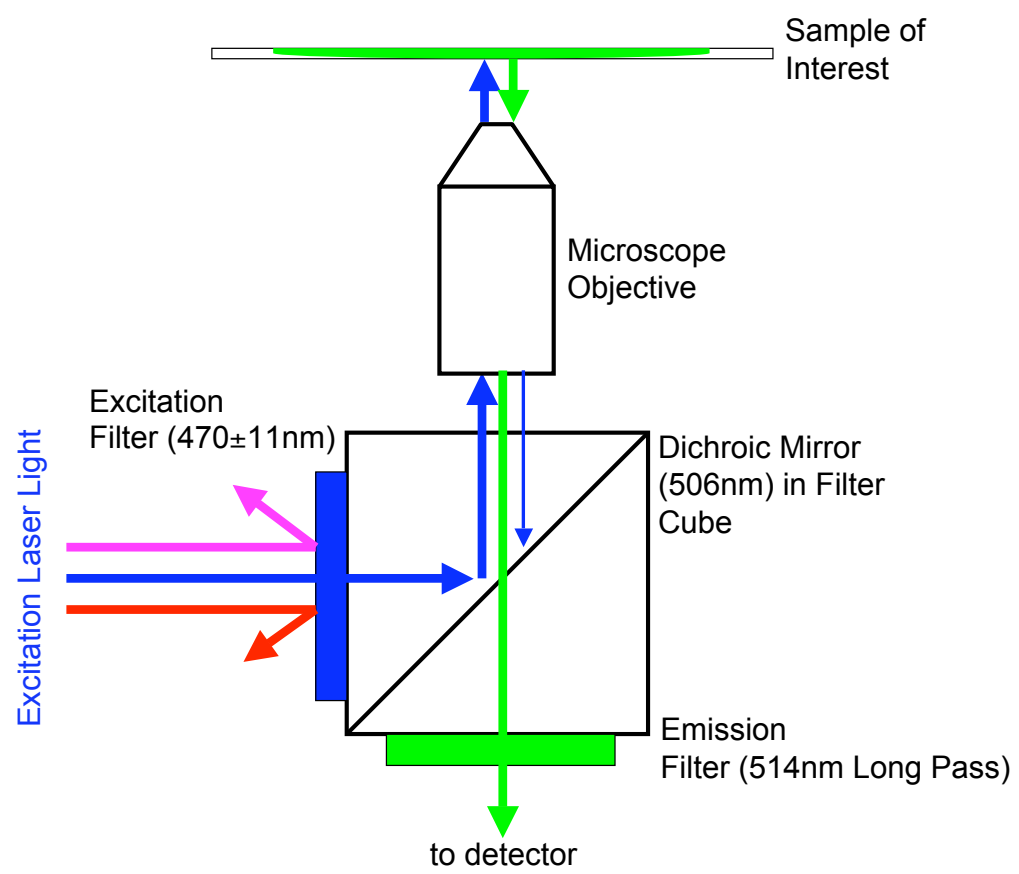

Figure 3.4: Epi-fluorescence filter configuration. Image adapted from original presented in www.fluorescence.com/tutorial/fm-optic.htm, C\&L Instruments, Inc. 2009. 


\subsubsection{Bench Top Optical Setup}

As an alternative to the optical setup offered by the microscope (Section 3.1.3) a set of bench top optics was constructed which would allow analysis of bulk samples held in a cuvette. Figure 3.5 is a schematic representation of the setup. Figure 3.6 shows a photograph of the optical setup.

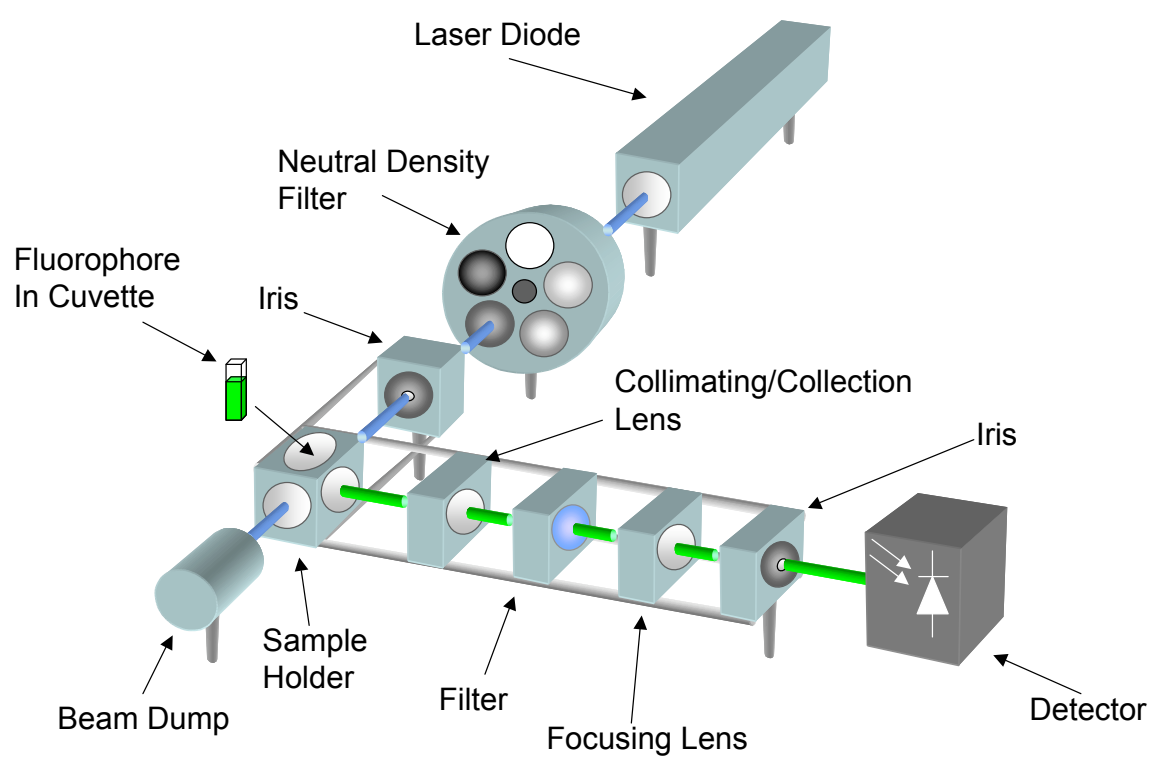

Figure 3.5: Optical setup used to analyse samples held in cuvettes.

The collection/collimating lens and the focussing lens are both $25 \mathrm{~mm}$ diameter, $25 \mathrm{~mm}$ focal length PCX lenses from Edmund Optics (A45-097). A 488nm Semrock RazorEdge Long Wave Pass Raman Filter (LP02-488RU-25) was used. All posts, post-holders, cage-plates, cage-cube and iris were obtained from ThorLabs inc. and mounted on a Melles Griot optical bread board. 


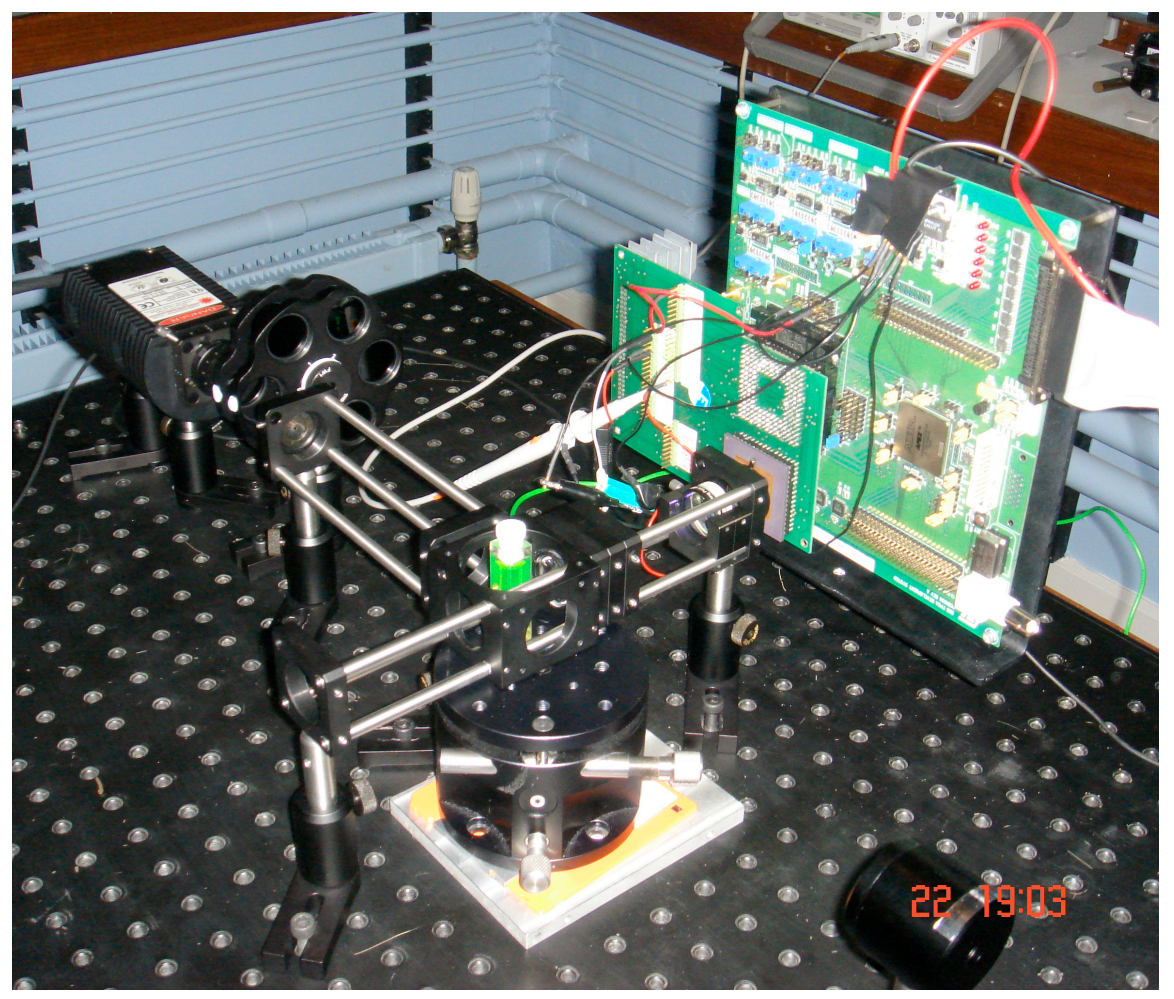

Figure 3.6: Bench top analysis of a Fluorescein sample in a cuvette. 


\subsubsection{Time-Correlated Single-Photon Counting (TCSPC)}

The operation of the Becker and Hickl card described in Section 3.1.6 is based on the principle of time-correlated single-photon counting. This technique counts the number of photons detected and places them into time bins which correspond to their arrival time. This time is measured relative to a second input signal which is synchronised to the excitation source. What is measured is the time difference between a photons arrival and the subsequent excitation pulse. Performing TCSPC in this way is known as reverse mode. By repeating this process many times a histogram can be built up which represents a distribution of the photon arrival times. For lifetime measurements this will represent the fluorescence decay curve [16].

\subsubsection{Becker and Hickl TCSPC Module}

The Becker and Hickl time correlated single-photon counting module (SPC-730) is a PCI based system capable of recording fluorescence lifetime decays with high accuracy. The system is based around a Time-to-Amplitude Converter (TAC) and a Analogue-to-Digital Converter (ADC) and has been designed to work in the reversed start-stop mode. The ADC has 4096 time channels (12-bit) per decay (with a minimum time per channel of $815 \mathrm{fs}$ ) with a maximum of 65536 counts per channel. The device has an electrical time resolution of 6ps FWHM. The device saturates at count rates beyond 6.5MHz. The Becker and Hickl TCSPC module was used during the characterisation of the micro-LED devices and the SPADs. It was also used as a commercially available time-resolved fluorescence system against which results obtained with the on-chip circuitry could be compared.

The Becker and Hickl card required a synchronisation input from the excitation source and an input from the detector. The detector input passes through a constant fraction discriminator (CFD) which could be set to trigger at a constant fraction of the pulse amplitude. This is used to eliminate events caused by after-pulsing or other sources of noise resulting in partial transitions of the detector output. It also reduces pulse-height variation induced timing jitter. The specifications on the input signals were that they had to conform to the NIM (Nuclear Instrumentation Methods) standard which states that the module would trigger on the falling edge of a 0 to $-800 \mathrm{mV}$ (logic levels 0 and 1, respectively) negative going input signal (both on the synchronisation and CFD inputs). This standard is used to ensure compatibility of all optical devices. Thus, the photomultiplier tube (see Section 3.1.7) and the Picoquant PLD-800b laser diode driver both produced compatible outputs. On the other hand the SPAD chip produces 
positive going 0 to $3.3 \mathrm{~V}$ output pulses. Similarly, when the micro-LED devices were being used as an excitation source they produced a synchronisation output which was a 0 to $3.3 \mathrm{~V}$ clock signal. In order to make these signals compatible with the Becker and Hickl TCSPC module it was necessary to invert and attenuate these signals. This was achieved using a Becker and Hickl A-PPI passive pulse inverter and by placing 50-ohm terminator stubs in parallel with the signals to reduce the pulse amplitude.

\subsubsection{Becker and Hickl Photo Multiplier Tube Detector Head}

Evaluation of the micro-LED device performance and the capture of time-resolved fluorescence data was also achieved using a Becker and Hickl high speed photo-multiplier tube (PMT) detector head (PMH-100-0). This device has been specifically designed for photon counting applications and contains a fast PMT and a high gain, high bandwidth amplifier capable of producing a $300 \mathrm{mV}$ (amplitude), 1.5ns (width) output pulse upon detection of a single photon. The wavelength response of the detector head is 300 to $650 \mathrm{~nm}$.

\subsection{PCB Test Boards}

\subsubsection{ST Microelectronics 908 Test Board}

The 908 PCB test board (Figure 3.7) was designed and built by ST Microelectronics and was originally intended for the in-house evaluation of CMOS camera modules. The board contained an Altera Apex (EP20K600EFC672-1X) FPGA chip as well as 14 analogue voltage sources and regulated power supplies. The board required an external $7.5 \mathrm{~V}$ power supply and communication with a desktop PC was achieved via a peripheral component interconnect (PCI) link. Programming of the FPGA was via a joint test action group (JTAG) link to the PC.

The board was modified to be compatible with the SPAD/micro-LED chip through the addition of a PCB daughter card (see Section 3.2.3). This card plugged into the 225-pin zero insertion force (ZIF) socket of the 908 test board and re-mapped the analogue, digital and power supply signals to the appropriate pins of a second socket which matched the pin-out map of the chip. 


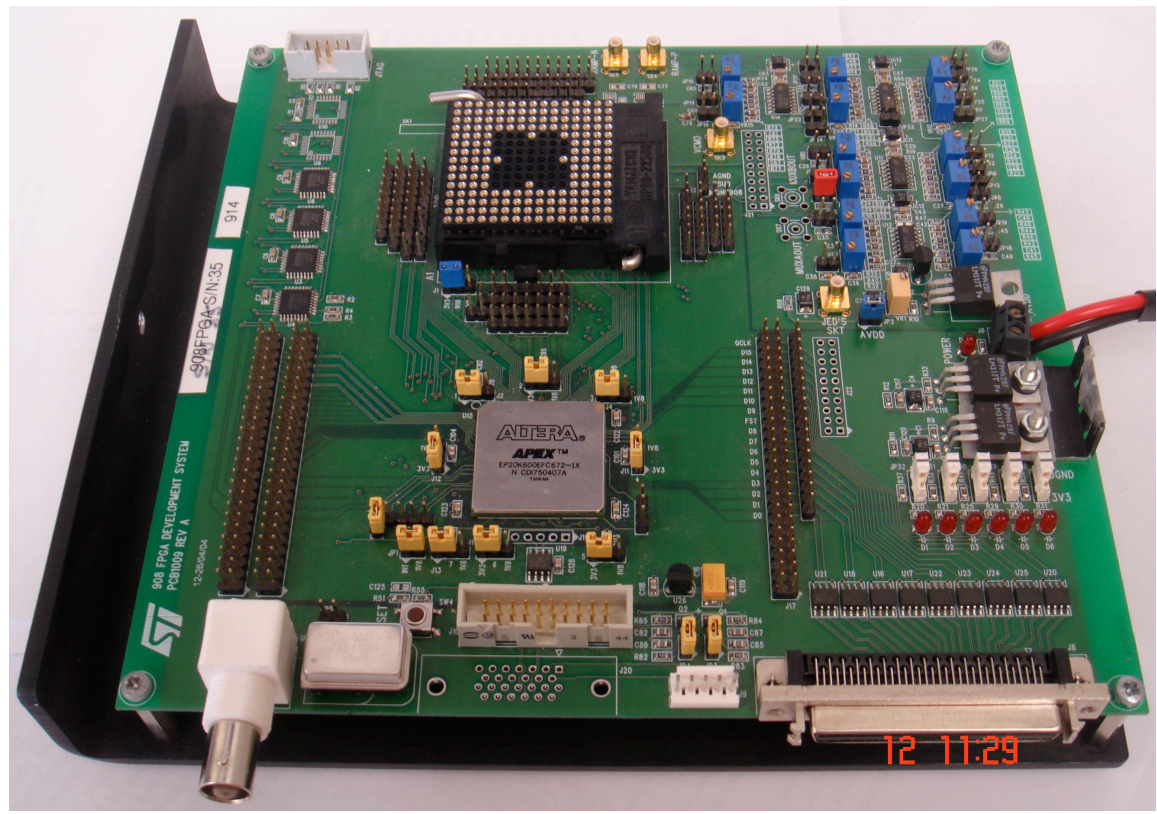

Figure 3.7: $P C B$ test board used to characterise the 16x4 SPAD and counter array and 16x4 micro-LED array chip. 


\subsubsection{The University of Edinburgh/Opal Kelly Test Board}

In order to reduce the overall size of the micro-analysis system a second FPGA test board was designed. This test board was intended as a generic board for use in a number of other department projects. The board was designed by Keith Muir (Institute for Integrated Micro and Nano Systems, Edinburgh University). Like the ST Microelectronics 908 test board (Section 3.2.1), this board incorporated an FPGA chip, 12 analogue voltage sources (each capable of delivering 100mA), 4 current sources, 4 current sinks and regulated power supplies $(2 \times 1.1 \mathrm{~A}$ and $1 \times 100 \mathrm{~mA}$ ). The regulated power supplies, volatge and current sources could be powered from an external power supply or the 5V USB supply. The FPGA chip chosen was a Xilinx Spartan-3 (XC3S1000-4FG320) and was situated within an Opal Kelly (XEM3010) USB 2.0 FPGA integration module (Opal Kelly, Inc.). This credit card sized PCB contained an FPGA device (with 3 on-board phase-locked loops, PLLs), dedicated power supply regulators (3.3V and $1.2 \mathrm{~V}$ switched-mode, capable of powering peripheral devices) and a USB 2.0 interface with access to 114 digital I/Os from the FPGA. The USB 2.0 interface was used to download verilog bit files to the FPGA, provide FPGA-PC communication and act as the power supply to the test board. This interfaced with the generic test board via two 80-Pin surface mount high-density headers. The generic test board included a $15 \times 15$ pin ZIF socket with connections mapped to the SPAD/micro-LED chip design. The test board setup with daughter card (to drive $8 \times 8$ micro-LED device) can be seen in Figure 3.8. All verilog that was originally used in conjunction with the ST 908 Test Board (Section 3.2.1) was ported over to this test board to allow all chips used during this project to be driven by the same FPGA test board.

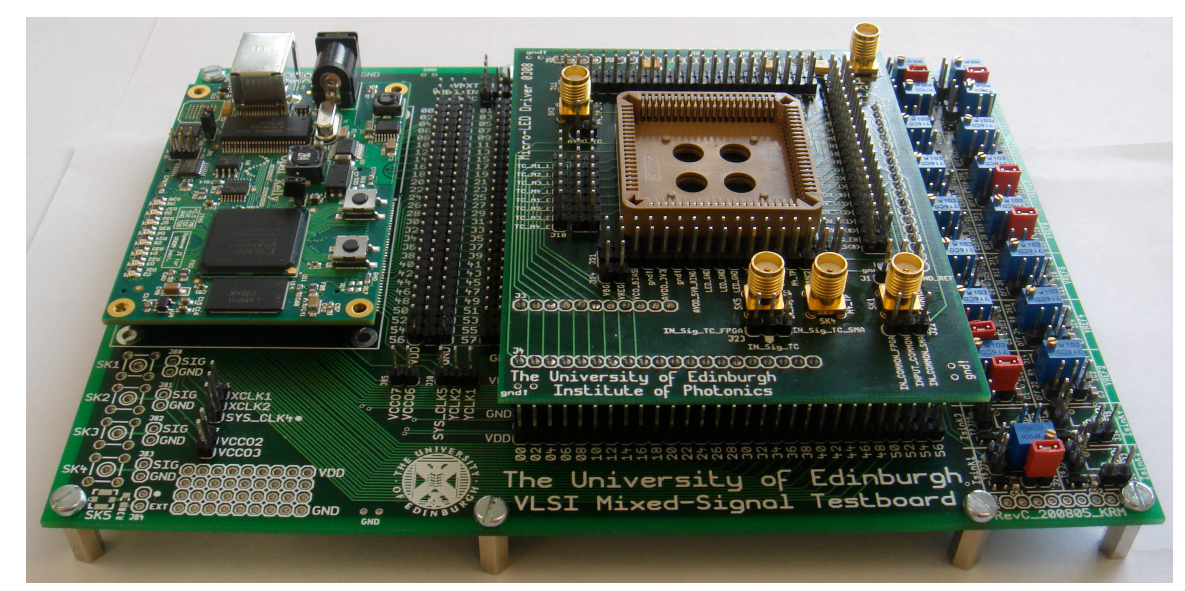

Figure 3.8: $P C B$ test board used to characterise the $8 \times 8$ micro-LED array. 


\subsubsection{Daughter Cards}

In order to make the two generic FPGA test boards used in this project (Sections 3.2.1 and 3.2.2) compatible with the pin-out of specific chip designs, it was necessary to design daughter PCBs. These boards were used re-map the signals of the generic test board to ensure that the correct signals (power, digital and analogue) went to the correct locations of the device socket. These boards are small and relatively inexpensive, making the generic test board compatible with virtually any chip package and pin-out (as long as the signal count of the chip does not exceed that of the underlying test board). Photographs of the daughter cards used during this project can be seen in Figures 3.9 and 3.10.

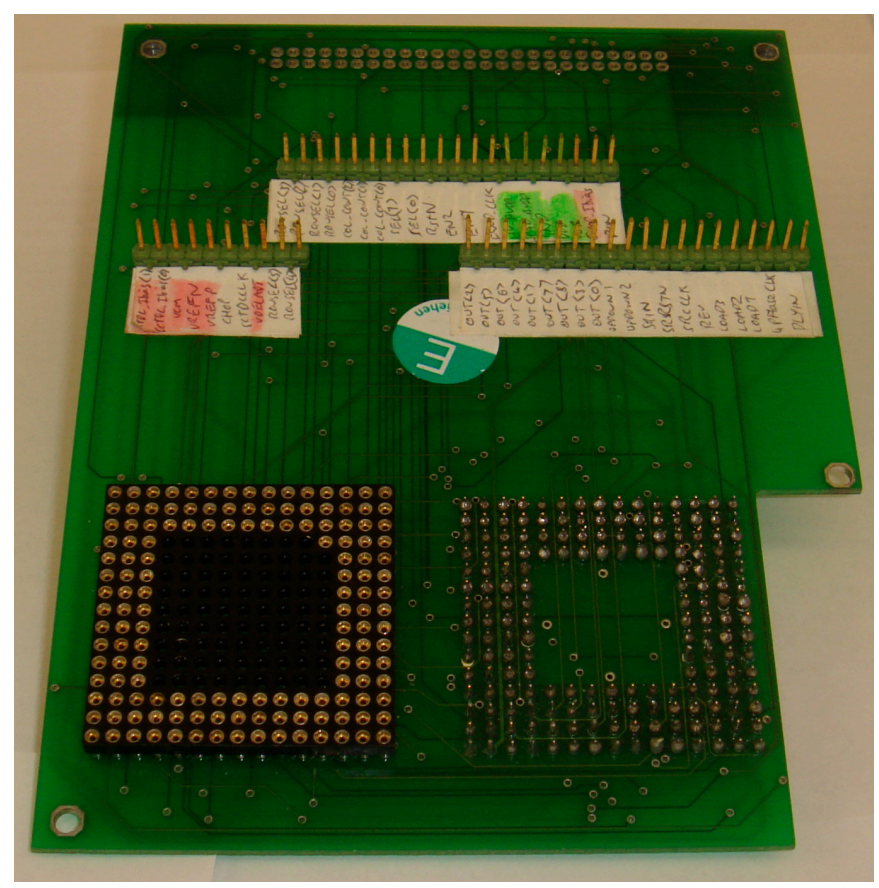

Figure 3.9: Daughter card used to re-map signals of the 908 Test Board from ST Microelectronics to allow compatibility with the 16x4 SPAD and Micro-LED array test chip. 


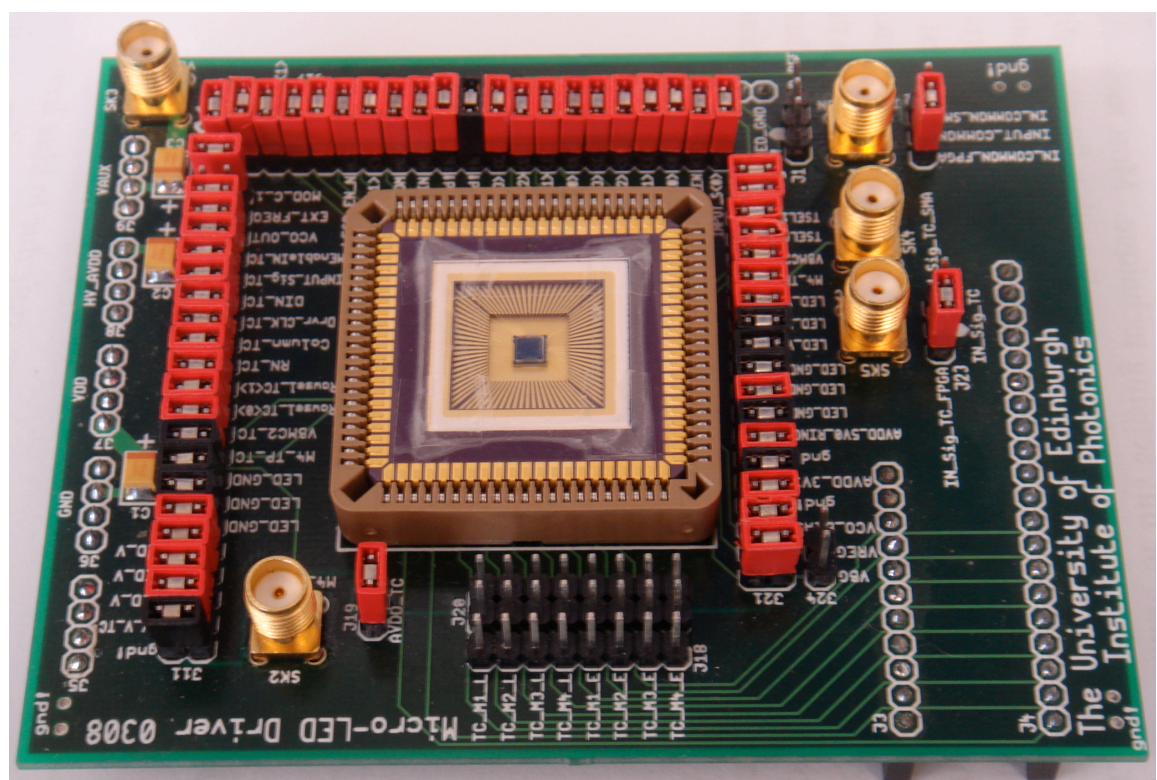

Figure 3.10: Daughter card used to re-map signals of the University of Edinburgh/Opal Kelly test board to allow compatibility with the $16 \times 16$ and $8 \times 8$ Micro-LED array test chip. 


\subsection{Software}

\subsubsection{Xilinx ISE and Altera Quartus}

Verilog code was written using Xilinx ISE, version 8.1.03i (for use with the Opal Kelly test board, Section 3.2.2 ) and Altera Quartus II, version 5.0 (for use with the ST 908 test board) software (see Appendices A.6 and A.7). These packages allowed Verilog code to be simulated, checked for syntax errors and processed into a series of files ready for download onto the FPGA.

\subsubsection{Front Panel}

Front Panel software (version 3.0.10) provided by Opal Kelly was used to act as a user interface to the Opal Kelly FPGA test board. This software package handled the downloading of verilog bit files to the test board as well as USB communication between the graphical user interface (GUI) and the FPGA. Front Panel allows the user to construct their own GUI using the XML programming language. An example of the GUI designed to provide user control of the $16 \times 4$ SPAD and counter chip can be seen in Figure 3.11. Using this interface the user could write values into registers within the FPGA. A full copy of the XML code used to construct the GUIs designed during this project can be found on the enclosed supplementary material CD. 


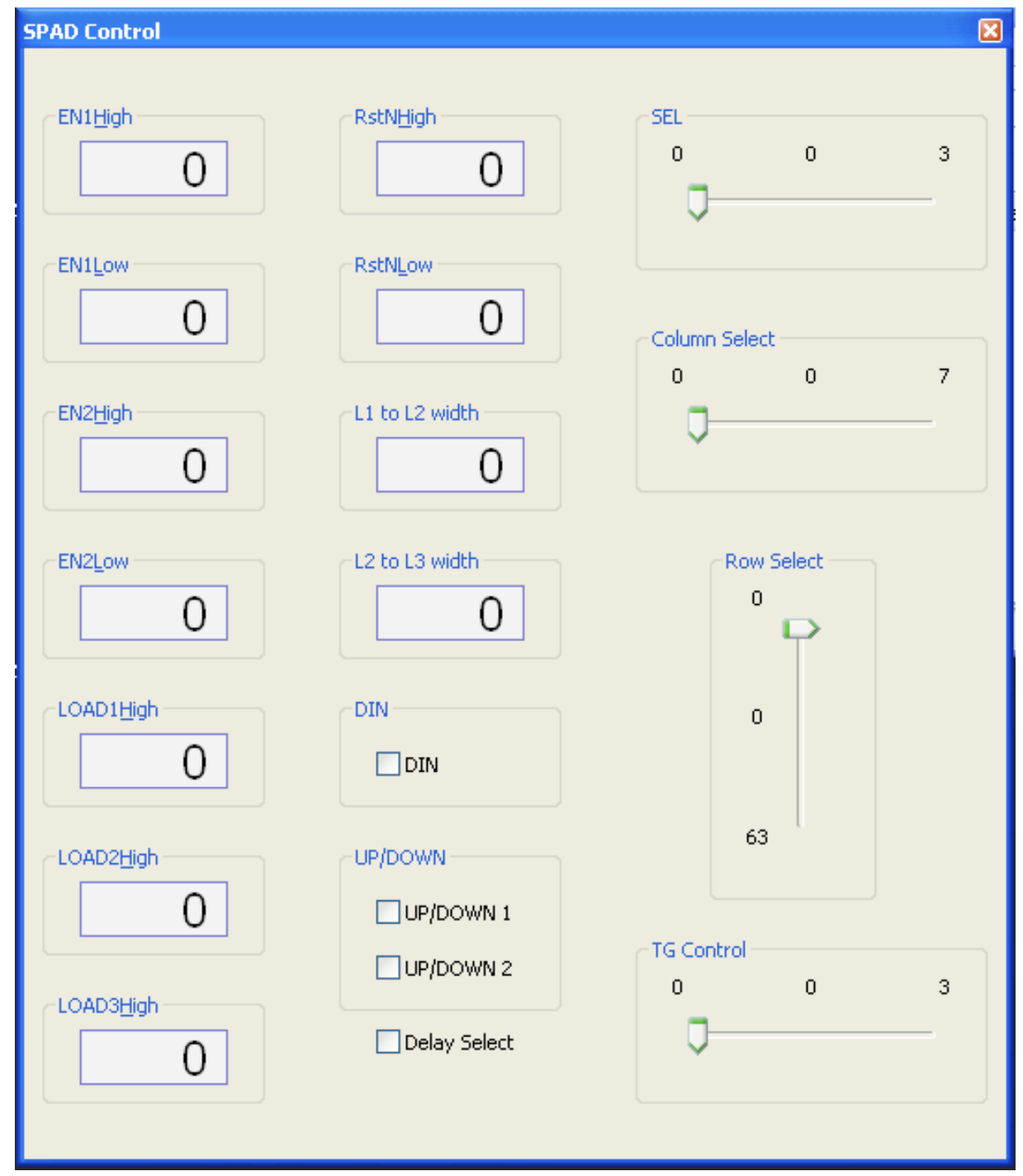

Figure 3.11: Example of GUI created using XML code used to control digital I/O on chips being tested with Edinburgh University/Opal Kelly test board. 


\subsection{3 v2wreg}

v2wreg was a software package written by STMicroelectronics and was used to set the value of registers within the FPGA of the ST 908 test board. This interface could be modified to be compatible with individual users FPGA code. Using this package a GUI was generated that allowed the user to control various aspects of the SPAD/micro-LED driver chip including which array element was being addressed and the length of delay generated by the on-chip delay circuit (Figure 3.12).

Using this package the width and position (in time) of each time gate could be selected. The user could also define the number of times a time gate is evaluated and the counter allowed to increment before the counter value is read out and the counter reset. Due to the low light levels associated with time-resolved fluorescence analysis, a photon is not expected in every time gate. Therefore, the number of times a time gate period can be evaluated before the counter is read out can be greater than 512 (the maximum number of counts for a 9-bit counter). Care must be taken by the user however, not to select a value which causes roll-over of the on-chip counter and corruption of lifetime data. 


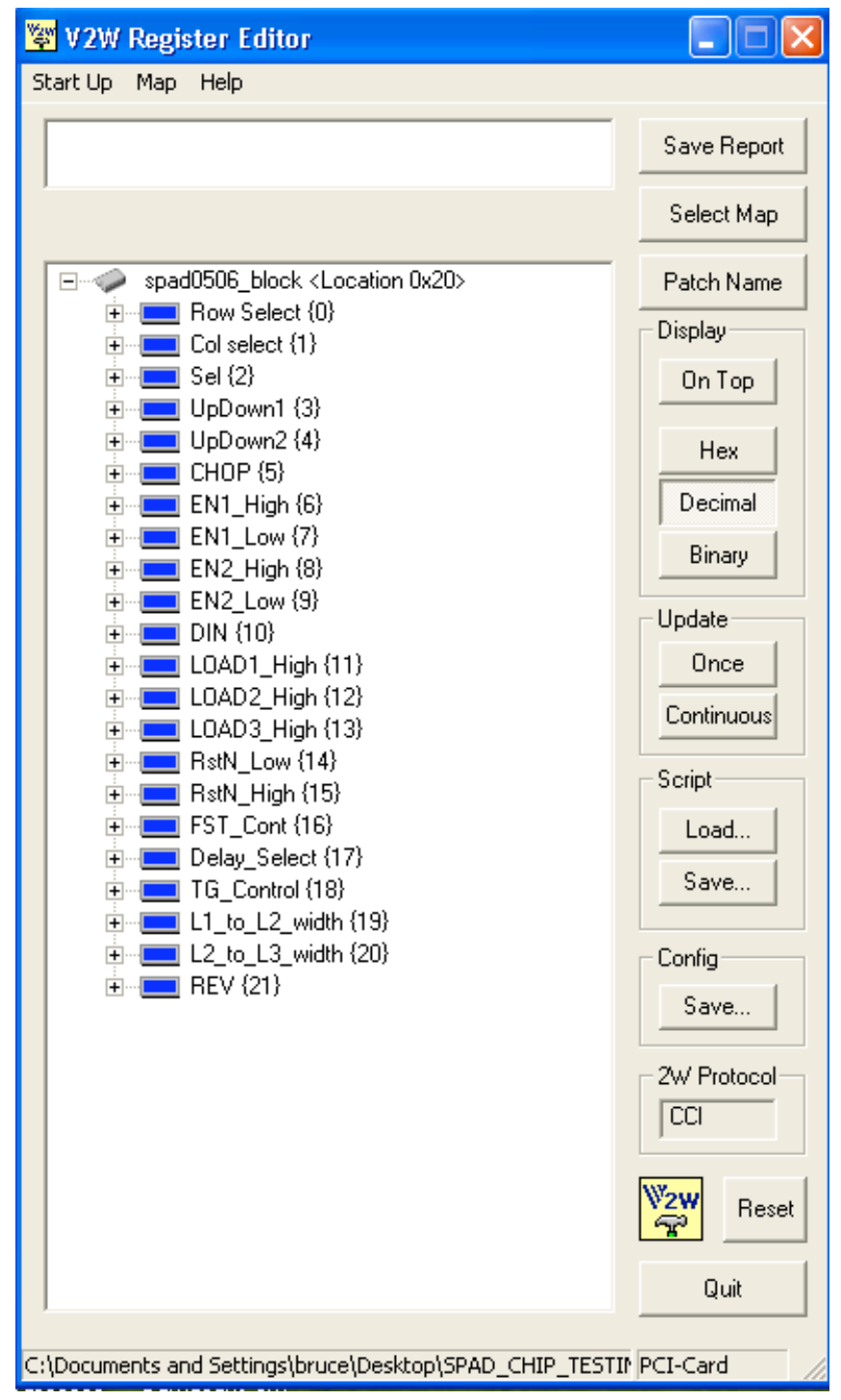

Figure 3.12: Screen shot of v2wreg GUI. A register map has been loaded which is used to control specific registers within the FPGA. This register map is intended to control the 16x4 SPADs with time-gated counters system. 


\subsection{4 vpciview}

vpciview was written by STMicroelectronics and was originally intended to capture and evaluate data from standard CMOS camera modules. The software was modified to allow it to interpret output data from an array of single-photon avalanche diodes connected to 9 bit counters. Using this software, grayscale images could be constructed whereby each image pixel was associated with a particular detector pixel and the grayscale value was a function of the counter output data (Black $=0$ counts, White $=512$ counts). The number of times a time-gate period is evaluated is user defined via the v2wreg interface and the pixel value is equal to the counter value after this period. Using the vpciview software the user can define the size of the image generated. Sequences of images can be dumped to file for post processing. Figure 3.13 is a screen shot of the vpciview interface.

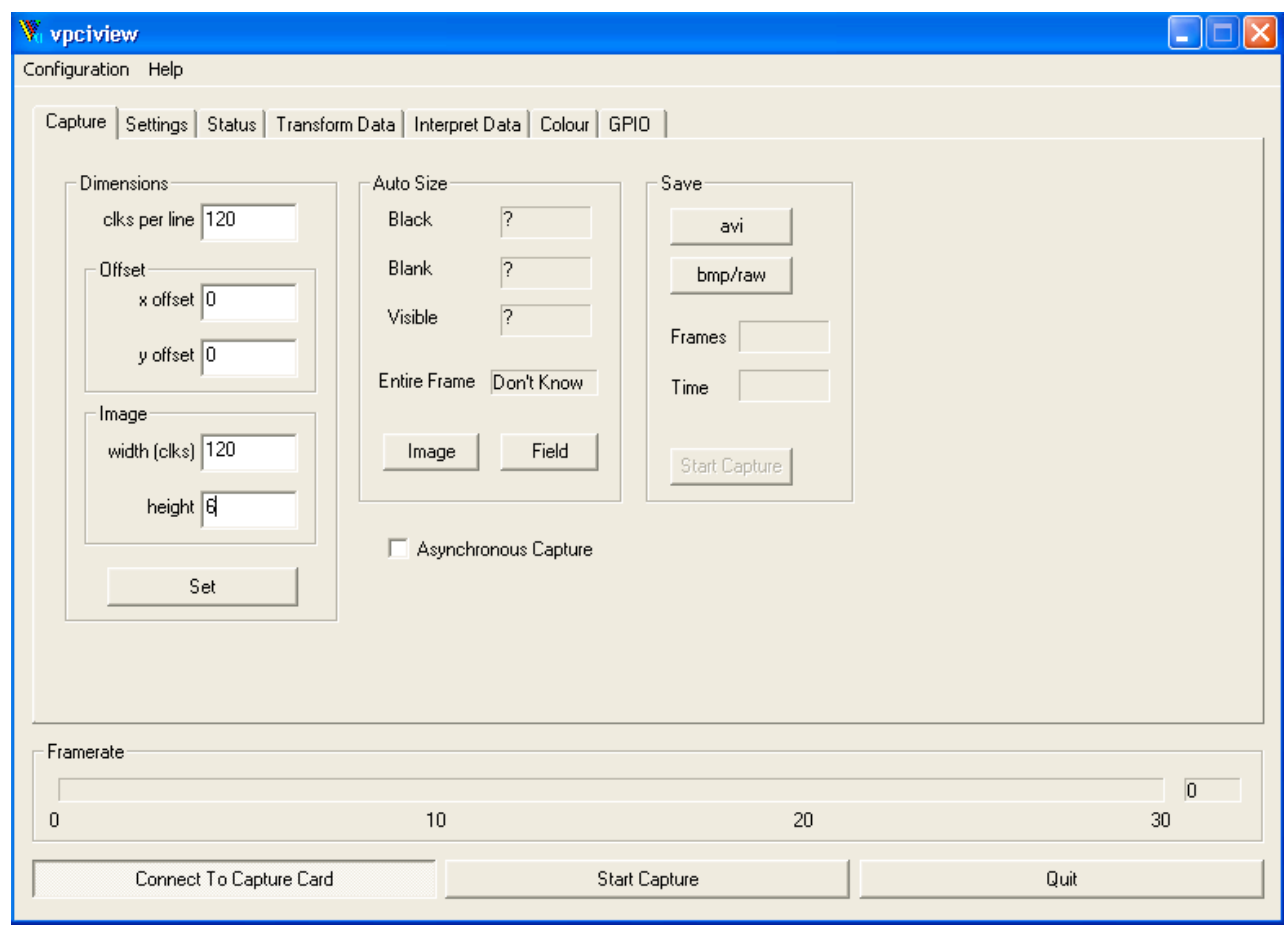

Figure 3.13: The vpciview software was used to create images from time-gated counter data. 


\subsubsection{Matlab}

The processing of on-chip counter data gathered via the vpciview interface (Section 3.3.4) was written by Dr David Renshaw and was performed using the mathematics software package Matlab (The Mathworks, Inc.). A series of images, dumped to file using the vpciview software, were read into Matlab. Code written in Matlab was used to assess the value of each pixel in each image. These values were summed in order to generate a single number equivalent to the total number of counts from a time gate period that had been evaluated many times.

\subsubsection{FAST/F900}

Fluorescence Analysis Software Technology (FAST) and F900 are both software packages by Edinburgh Instruments used to analyse fluorescence kinetics. These packages were used to analyse fluorescence lifetime decay curves obtained using the Becker and Hickl TCSPC module (Section 3.1.6) and to verify lifetime predictions made by the 2-gate and multi-gate RLD algorithms and the IEM algorithm (Section 4.4.1). Multi-exponential decay curves could be assessed using a number of different analysis tool, including global lifetime data analysis and lifetime distribution analysis. The fitting of exponential curves to the decay data in order to predict the lifetime of the sample can be achieved using two methods; tail fitting or reconvolution fitting. Once an exponential fit has been made to the raw data the software provides a number of methods for assessing the quality of the fit. Along with a prediction of the sample lifetime $(\tau)$ the software also returns values for the reduced chi-squared test, which assess goodness of fit and the weighted residuals which assesses the difference between the original data and the fitted curve. 


\subsubsection{PCB Fabrication}

The design and layout of all printed circuit boards (PCBs) during this project was achieved using Target 3001 CAD software (version 12) for PCB design (www.ibfriedrich.com). Using this package PCBs were designed before being submitted for manufacture. This process was coordinated by PCB-Pool, PCB manufacture was carried out by Beta Layout (details of both organisation can be found at www.pcb-pool.com).

\subsubsection{Cadence}

All schematic capture, circuit simulation and chip layout was performed using the Cadence design suite (version 5.10). Schematic capture was achieved using Cadence's Schematic Composer environment. Circuit simulation was performed using the Cadence Spectre simulator and results presented using the WaveScan tool. Circuit layout was carried in the Cadence Virtuoso layout environment. Two layout verification tools were used to perform layout versus schematic (LVS) and design rule check (DRC) tests, Assura by Cadence and Calibre by Mentor Graphics. Design rules and standard cells were provided by the foundry (Austria Microsystems, HIT-Kit Version 3.70).

\subsection{Experimental Procedure}

\subsubsection{Quantum Dots}

The measurement of fluorescence decay curves was demonstrated using Cadmium Selenide Zinc Sulphide (CdSe/ZnS) quantum dots from Evident Technologies Inc. in a toluene solution (EviDot Test Kit 490-620). Table 3.1 contains a summary of the quantum dot samples used.

\begin{tabular}{lllll}
\hline & $\begin{array}{l}\text { Peak } \\
\text { absorption } \\
(\mathrm{nm})\end{array}$ & $\begin{array}{l}\text { First } \\
\text { absorption } \\
\text { peak }(\mathrm{nm})\end{array}$ & $\begin{array}{l}\text { Emission } \\
\text { peak }(\mathrm{nm})\end{array}$ & $\begin{array}{l}\text { Supplied } \\
\text { Concentration } \\
\left(\mathrm{nmol} \mathrm{ml}^{-1}\right)\end{array}$ \\
\hline Sample & $<400$ & 510 & 526 & 77.03 \\
Adirondack Green & $<400$ & 525 & 543 & 68.77 \\
Hops Yellow & $<400$ & 531 & 555 & 57.17 \\
\hline
\end{tabular}

Table 3.1: Summary of quantum dot samples used to evaluate micro-systems.

Experiments were conducted with quantum dot samples at concentrations as supplied by the 
manufacturer and at diluted concentrations. Dilutions were prepared using high-performance liquid chromatography (HPLC) grade toluene. All samples were stored at $4{ }^{\circ} \mathrm{C}$.

The properties of quantum dots are highly tunable and dependent on the size of individual dots in the sample. Altering the size of the quantum dot particles changes the bandgap properties of the dot. Thus, the emission wavelength of a quantum dot sample is dictated by the physical size of the particles [87]. The fluorescence lifetime of the quantum dots used is quoted by the manufacturer as being between 15 to $20 \mathrm{~ns}$. The use of quantum dots in biomolecular analysis is discussed in [88].

\subsubsection{Other Fluorophores}

A number of other fluorophores were used throughout the project including; fluorescein, rhodamine B, coumarin 6 and rhodamine 6G (Sigma-Aldrich, Inc.), these were mixed in solution with ethanol, water, methanol and water, respectively. These samples offer significantly shorter lifetimes than the quantum dots. Typically fluorescein in water has a lifetime of $4.1 \mathrm{~ns}$, rhodamine B in water has a lifetime of $1.68 \mathrm{~ns}$, coumarin 6 in ethanol has a lifetime of $2.5 \mathrm{~ns}$ and rhodamine $6 \mathrm{G}$ in water has a lifetime of 4.08ns [34]. An overview of the fluorophores used can be found in Table 3.2.

\begin{tabular}{lllll}
\hline Fluorophore & Solvent & $\begin{array}{l}\text { Excitation } \\
\text { Wavelength }(\mathrm{nm})\end{array}$ & $\begin{array}{l}\text { Emission } \\
\text { Wavelength }(\mathrm{nm})\end{array}$ & $\begin{array}{l}\text { Typical (ns) } \\
\text { Lifetime (ns) }\end{array}$ \\
\hline Fluorescein & Ethanol & 495 & 517 & 4.0 \\
Coumarin 6 & Methanol & 460 & 505 & 2.5 \\
Rhodamine B & $\mathrm{H}_{2} \mathrm{O}$ & 562 & 583 & 1.68 \\
Rhodamine 6G & $\mathrm{H}_{2} \mathrm{O}$ & 525 & 555 & 4.08 \\
\hline
\end{tabular}

Table 3.2: Summary of non-quantum dot based fluorophores used.

\subsection{Bump-Bonding}

In recent years semi-conductor companies have been looking to chip-stacking as an alternative to the shrinking of transistor dimensions as a means of maintaining Moore's Law [89]. There are a number of techniques that have been developed to achieve this, including through-silicon vias [90], conductive glues and solder re-flow methods. Another method that is used to achieve vertical interconnects between stacked devices is bump-bonding. This method was chosen to 
provide connection between the CMOS driver array and the AlInGaN micro-LED array.

Bump-bonding was performed at OptoCap Ltd, Livingston UK (www.optocap.com, 2009). The process of bump-bonding involves two main steps: the deposition of gold ball bonds on the CMOS, followed by the bringing together of the CMOS array and the micro-LED array for bonding. The CMOS device was mounted in a Palomar 8000 automatic wire bonder (Palomar Technologies, Inc. see Figure 3.14 (a)) and a bonding tip (Figure 3.14 (b)) is fed a $20 \mu \mathrm{m}$ diameter gold wire via a capillary. A combination of thermal, electrical and ultrasonic energy is then applied to bond the gold wire to the die, forming a $60 \mu \mathrm{m}$ diameter ball. This process is automated and the bonding machine visits each electrode in the driver array, repeating this process until every element in the array (including the ground pads) is populated with a gold ball bond.
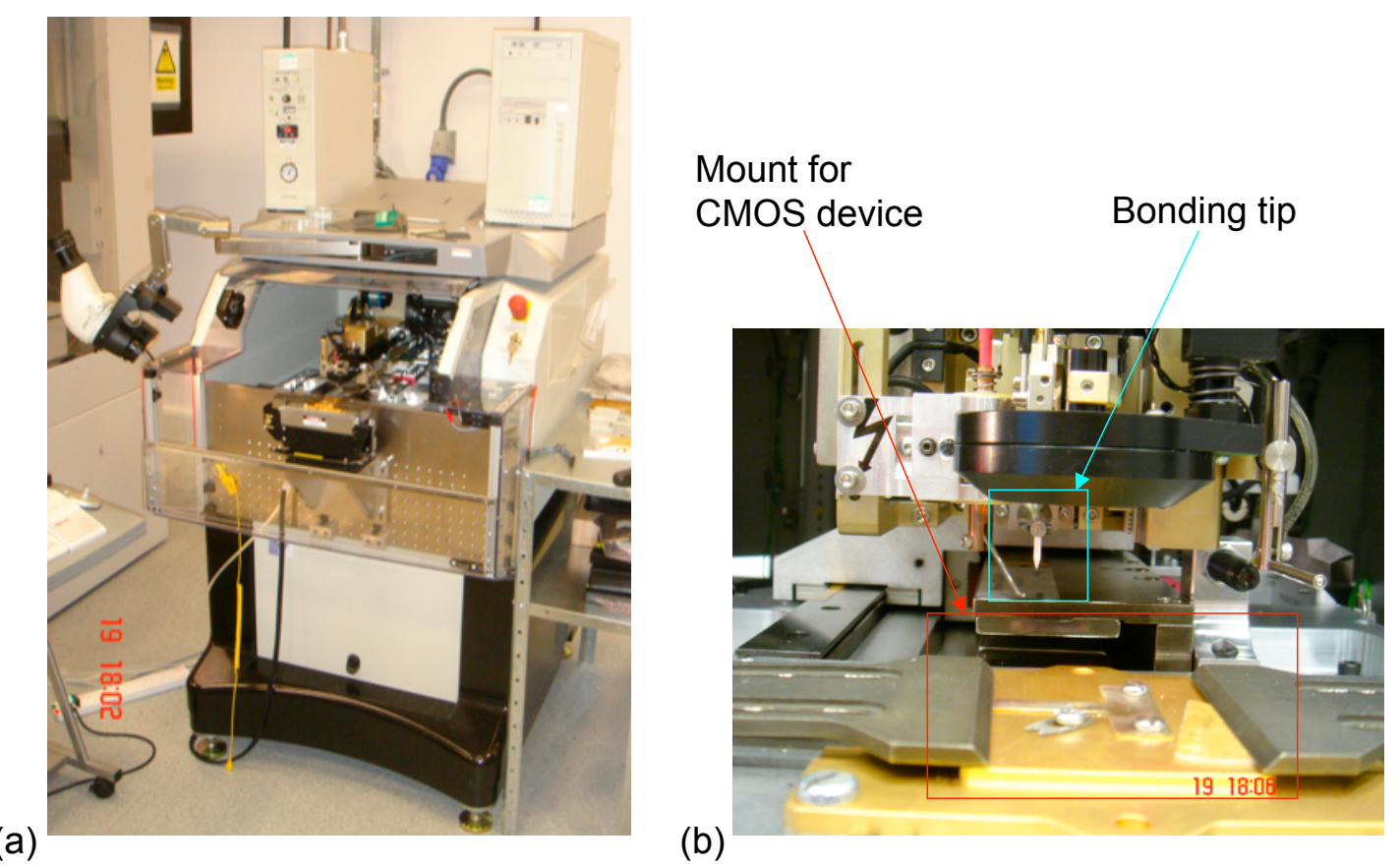

Figure 3.14: (a) Palomar 8000 automatic wire bonder from Palomer Technologies, Inc. and (b) a close up of the bonding tip.

The CMOS LED driver array was then transferred to a Finetech Fineplacer (Finetech GmbH and Co. KG) flip-chip bonder (Figure 3.15). The CMOS device was held on a mechanical arm using a vacuum head, while the LED array was placed on a heated vacuum plate. The two devices are carefully aligned before being brought together. Ultrasonic energy $(1500 \mathrm{~mW}$ for 
$800 \mathrm{~ms})$ and thermal energy $\left(80^{\circ} \mathrm{C}\right.$ rising to $200^{\circ} \mathrm{C}$ over 50 seconds) was then applied, melting the gold ball bonds situated between the two arrays and creating the bonds.

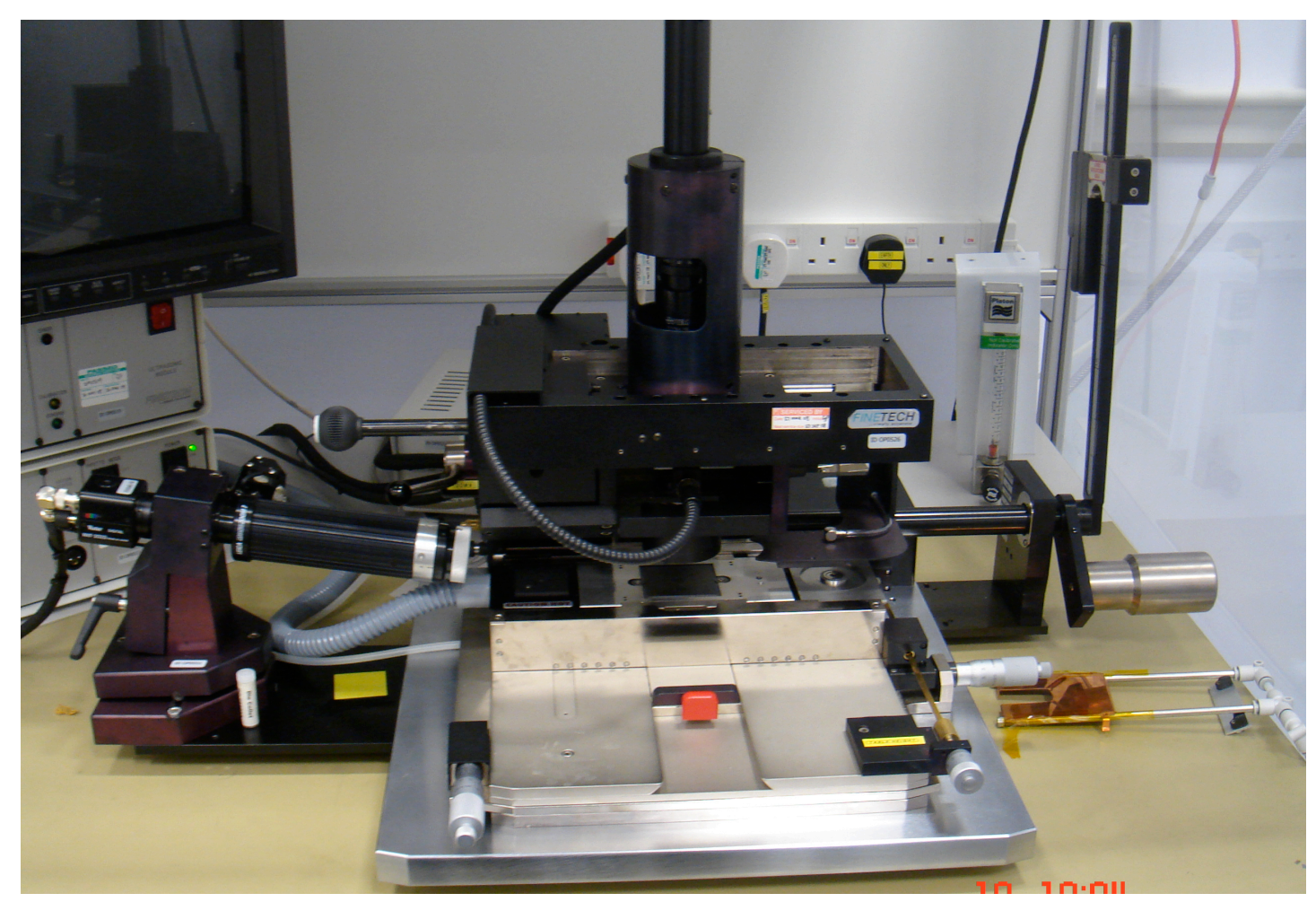

Figure 3.15: Finetech Fineplacer flip-chip bonder from Finetech GmbH.

Figure 3.16 shows micrograph images of a micro-LED driver chip during the bump-bonding process. In (a) the CMOS device has had an array of gold ball bonds placed on each of the electrode sites. In (b) the AlInGaN micro-LED array on a sapphire substrate has been flip-chip bonded to the CMOS device. 


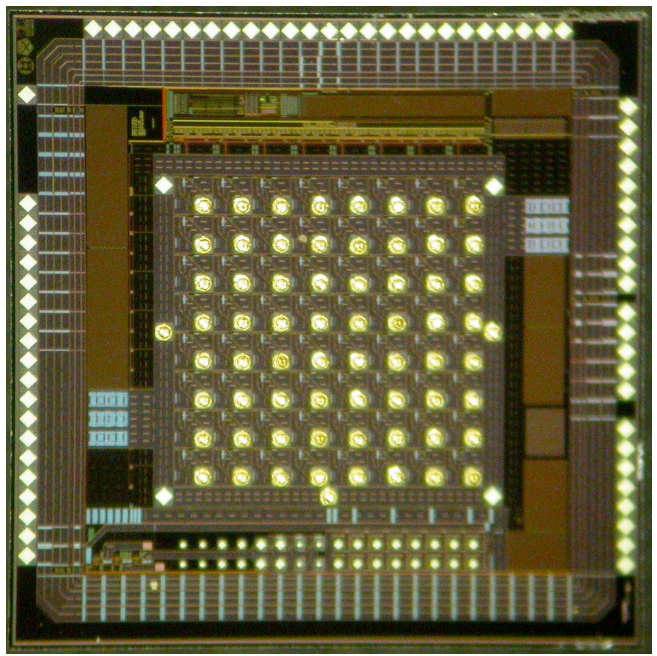

(a)

(b)

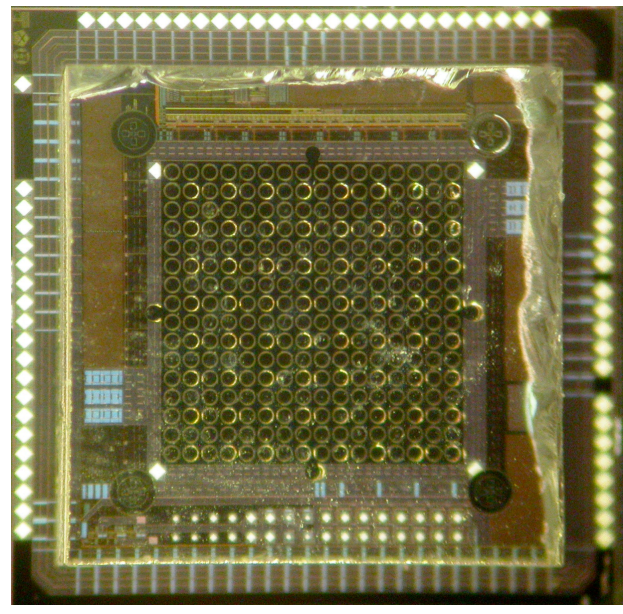

Figure 3.16: Stages of the bump-bonding process. (a) Gold ball-bonds are deposited on the CMOS driver array; (b) the micro-LED array is then fip-chip bonded to the CMOS die. 


\section{Chapter 4 SPAD Detector and Circuits for Lifetime Estimation}

The ability to implement SPAD detectors in a CMOS process allows the detector to be implemented on the same substrate as signal processing circuitry. Photon arrival data from the SPAD can be processed directly on-chip, removing the need to pass all SPAD avalanche events off-chip. This greatly reduces the bandwidth requirements of the device output. The aim of this work was to develop an array of SPADs with each detector having associated in-pixel signal processing circuitry capable of capturing fluorescence lifetime decay data. By processing the data on-chip the need for external photon counting hardware is removed. In this chapter the SPAD detector, in-pixel time-gated photon counting circuitry and on-chip timing circuitry are introduced. The sensitivity and accuracy of the detector array is evaluated through a series of fluorescence lifetime experiments, performed with samples of different concentration. This experiment has been performed using commercially available external photon counting hardware and in-pixel circuitry to process the SPAD output data.

Commercially available, single channel time-correlated single-photon counting PCI modules intended for time domain, time-resolved fluorescence measurements are designed to be used in conjunction with external single detectors, such as a PMT or a large-area avalanche photodiode. These discrete detectors are extremely sensitive and, in conjunction with the TCSPC module, have high timing accuracy; however, such systems are expensive. The detectors are also highly sensitive to environmental factors and when coupled with the TCSPC module (housed in a separate PC) this makes for a rather bulky system.

It was hypothesised that a more compact micro-system for time-resolved fluorescence could be achieved by making use of CMOS technology's ability to integrate signal processing circuitry on the same chip as a sensor array, thereby allowing detector data to be directly processed. This chapter describes how time domain, time-gated fluorescence lifetime analysis has been implemented on a CMOS chip. Using this method, the sample of interest is excited by a pulsed light source. The subsequent lifetime decay is captured within a series of two or more 
gated count windows. Using the count values obtained in each window a histogram of the fluorescence decay curve can be generated (Figure 4.1). A fluorescence lifetime is then obtained by applying a lifetime extraction algorithm to the histogram data.

A SPAD detector has been implemented which allows single photon detection through the action of avalanche breakdown in a $\mathrm{p}+$ /deep $\mathrm{n}$-tub photodiode, reverse biased above its breakdown voltage (Geiger mode). These are situated in a $16 \times 4$ array pitch-matched to the micro-LEDs, allowing histogram and lifetime analysis without the need for external photon counting hardware. Direct observation of SPAD output pulses is also possible from an array of addressable SPADs situated directly within the micro-LEDs for confirmation of the integrated lifetime analysis techniques.

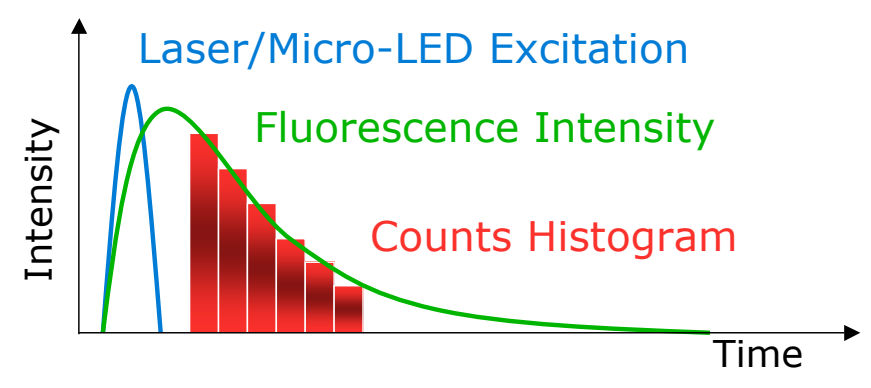

Figure 4.1: Illustration of a fluorescence lifetime decay captured using a time-gated counter circuit.

This chapter describes how single-photon avalanche diodes integrated into a CMOS process have been used to develop a multi-channel, multi-detector implemented on a single CMOS die along with on-chip circuitry capable of processing raw SPAD data in order to perform time-resolved fluorescence measurements within a low-cost, compact and robust micro-system. There are many advantages of solid state detectors over conventional imagers including; high quantum efficiency, magnetic field immunity, radiation hardness, robustness, long operating lifetime and potentially lower costs [77]. All these factors make CMOS-based imagers ideal for medical applications. A micrograph of the chip, fabricated in a $0.35 \mu \mathrm{m}$ high-voltage CMOS process from Austria Microsystems, can be seen in Figure 4.2. An overview of the performance of the implemented SPAD detector is provided followed by a description of the time-gated counter circuits that have been realised. Experimental data is provided which demonstrates that the SPADs are capable of accurately capturing fluorescence lifetimes using samples of concentrations down to $10 \mathrm{nM}$ and that these lifetimes can be successfully processed using both commercially available external TCSPC hardware and on-chip time-gated counters. 


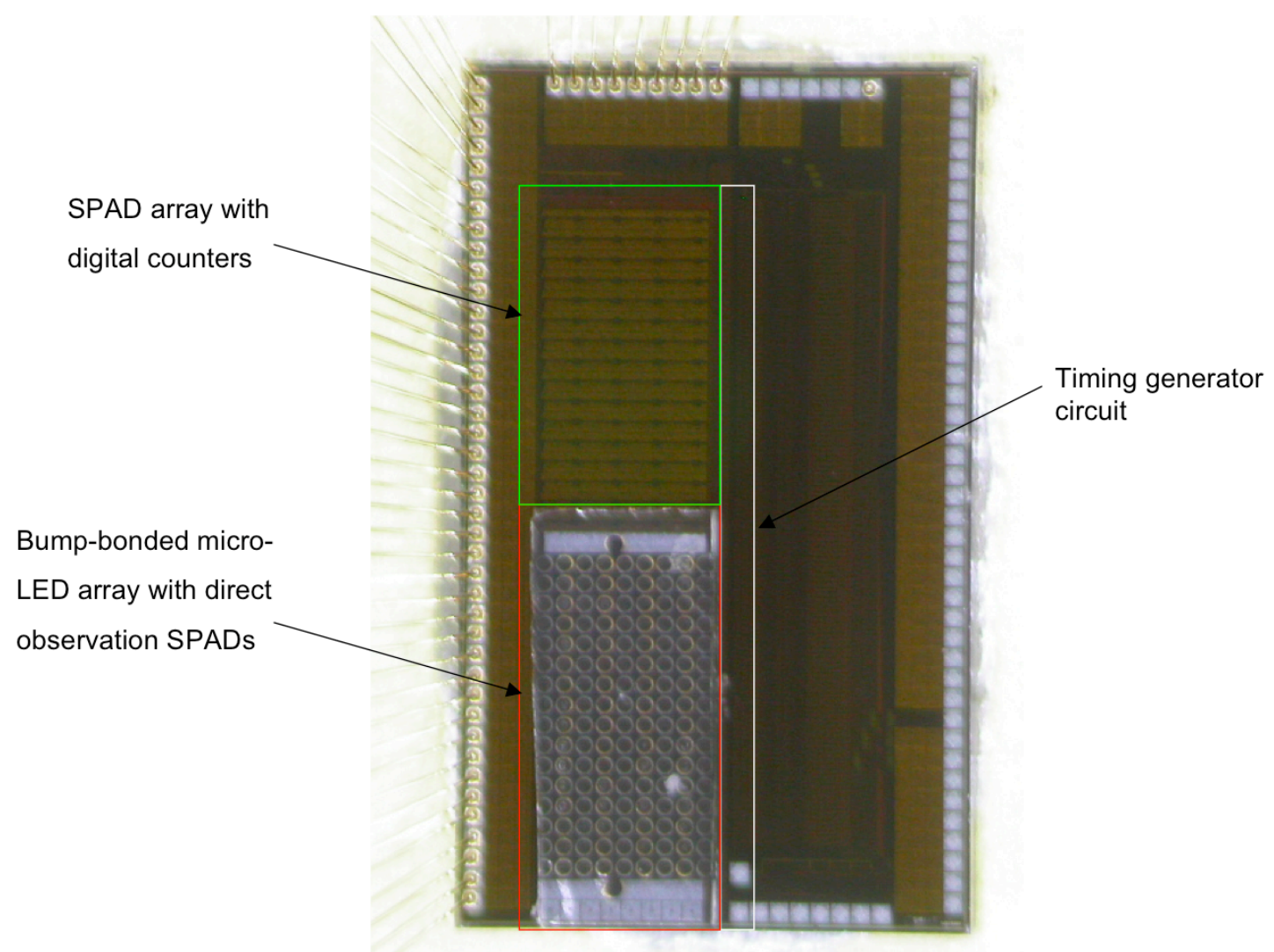

Figure 4.2: SPAD chip micrograph. 


\subsection{SPAD Performance}

Table 4.1 summarises the measured performance of the SPAD detector used in this project $\left({ }^{*}\right.$ Achieved after removal of the device passivation layer, ${ }^{\dagger}$ Measurements performed by Edoardo Charbon at EPFL, Switzerland).

\begin{tabular}{ll}
\hline Metric & Performance \\
\hline Average Dark Count Rate & $50 \mathrm{~Hz}$ \\
Dead Time & $40 \mathrm{~ns}$ \\
Saturation & $25 \mathrm{MHz}$ \\
Wavelength Range & $350-1000 \mathrm{~nm}^{\dagger}$ \\
Photon Detection Probability (PDP) & $0.1-40 \%^{\dagger}$ \\
PDP Peak & $440-466 \mathrm{~nm}^{\dagger}$ \\
Jitter & $114 \mathrm{ps}(\mathrm{FWHM})^{\dagger}$ \\
\hline
\end{tabular}

Table 4.1: Summary of SPAD device performance.

The performance of the SPAD was evaluated by collaborators at the EPFL, Lausanne, Switzerland [74]. The SPADs PDP measured across the full visible wavelength spectrum is shown in Figure 4.3.

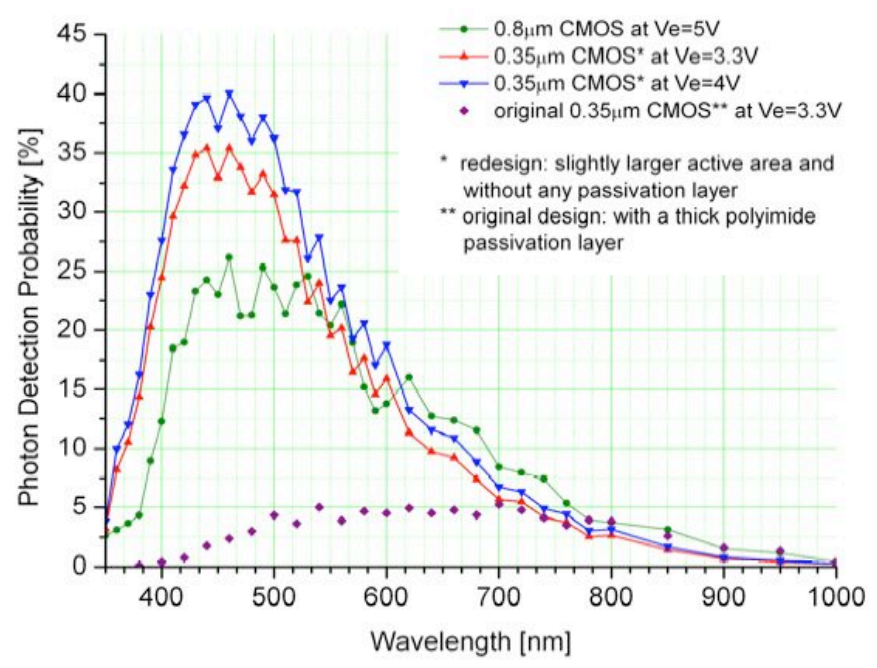

Figure 4.3: PDP plots of the SPAD under different bias conditions and compared to a SPAD in $0.8 \mu \mathrm{m}$ technology. Comparison is also made to a SPAD without the die passivation layer removed. 


\subsection{SPADs with Time-Gated Counters}

One of the advantages of being able to integrate a detector or detector array into a CMOS process is the ability to include signal processing and control circuitry on the same chip. As part of this project a 2-D CMOS time-resolved imaging system has been designed, consisting of a fully addressable array of 16x4 array of SPADs integrated with on-chip signal processing and timing circuits. Each pixel measured $100 \mu \mathrm{m}$ x $200 \mu \mathrm{m}$. The pixels incorporated two 9-bit ripple up-down counters with a novel time-gating mechanism allowing fully programmable scanning of time resolved events over a 48ns range with a 408ps resolution. The imager was controlled by a FPGA and photon count histograms were captured and displayed by a PC. Histograms could be displayed in real-time by a PC video image frame-grabber (vpciview, Section 3.3.4) allowing real-time monitoring of the profile and characteristics of fast time domain events such as would be encountered in fluorescence lifetime imaging. Figure 4.4 shows a system block diagram. Both the SPAD counter array and the micro-LED array (Chapter 5) were based on this architecture. By processing raw SPAD data locally within each pixel, the amount of data that would otherwise have to broadcast across the chip and potentially off-chip is minimised. A complete list and description of each pin of the 16x4 SPAD chip can be found in Appendix A.1. 


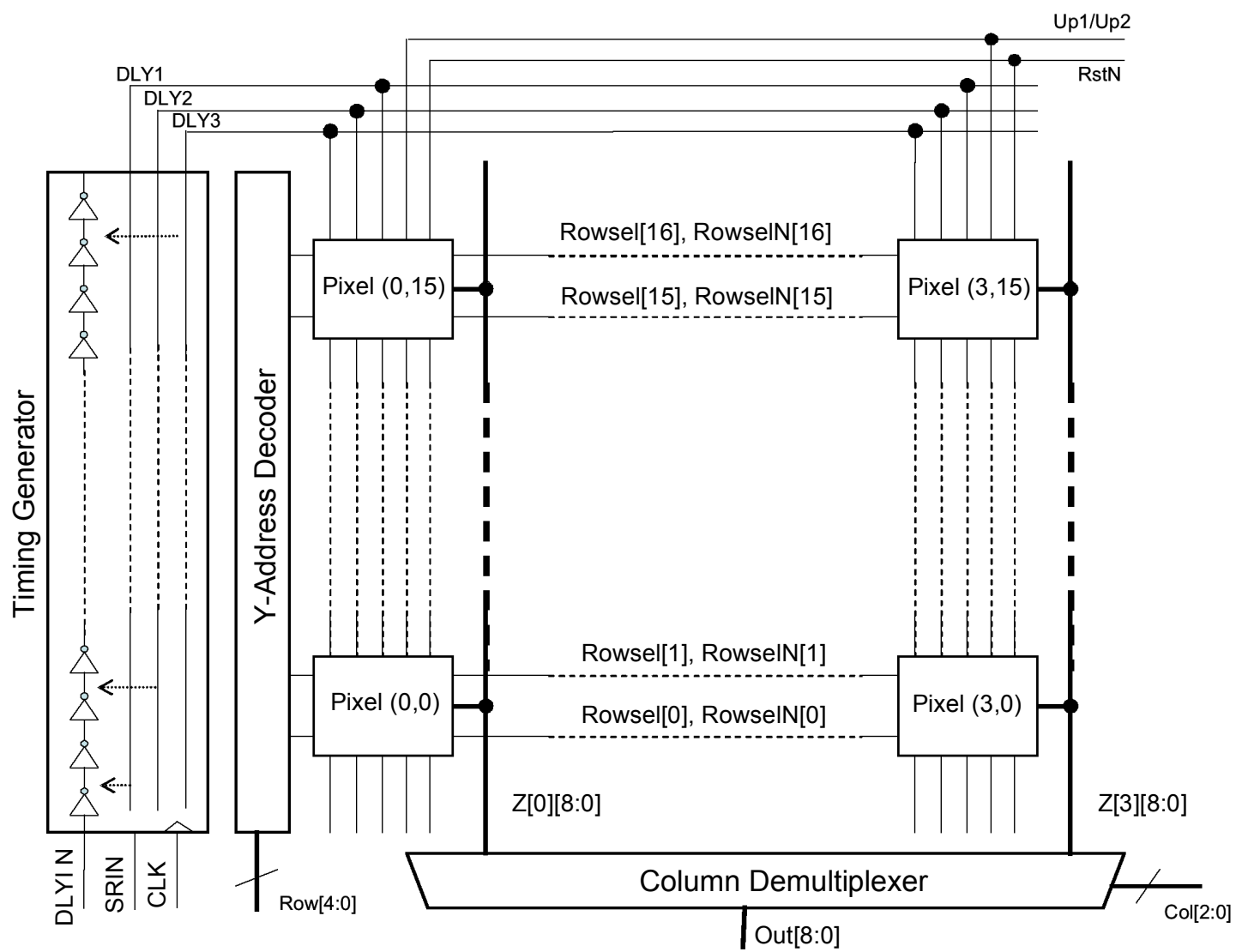

Figure 4.4: System block diagram. 


\subsubsection{Counter Circuit}

Within each pixel two 9-bit up/down ripple counter circuits were implemented, these use toggle (T-type) flip-flops (FF). The SPAD pulses (Chapter 2, Figure 2.6) provided the asynchronous clock to the first T-type FF in the counter (Figure 4.5). A ripple counter was chosen to minimise the clock loading, since no synchronous count behaviour is required. Time-gated operation is accomplished by providing the toggle input of the first T-type FF in the counter with short pulses, which are generated within the pixel from delayed versions of the $3.68 \mathrm{MHz}$ system clock broadcast to the array from the on-chip timing generator (Section 4.6). A schematic view of a single counter bit can be found in Appendix A.1. The 9-bit word-length of each counter circuit allows 512 counts to be gathered before it is necessary to read-out the counter data. Failure to do this results in a loss of information due to wrap-round of the counter (the next count after 512 returns the counter output to 0 ).

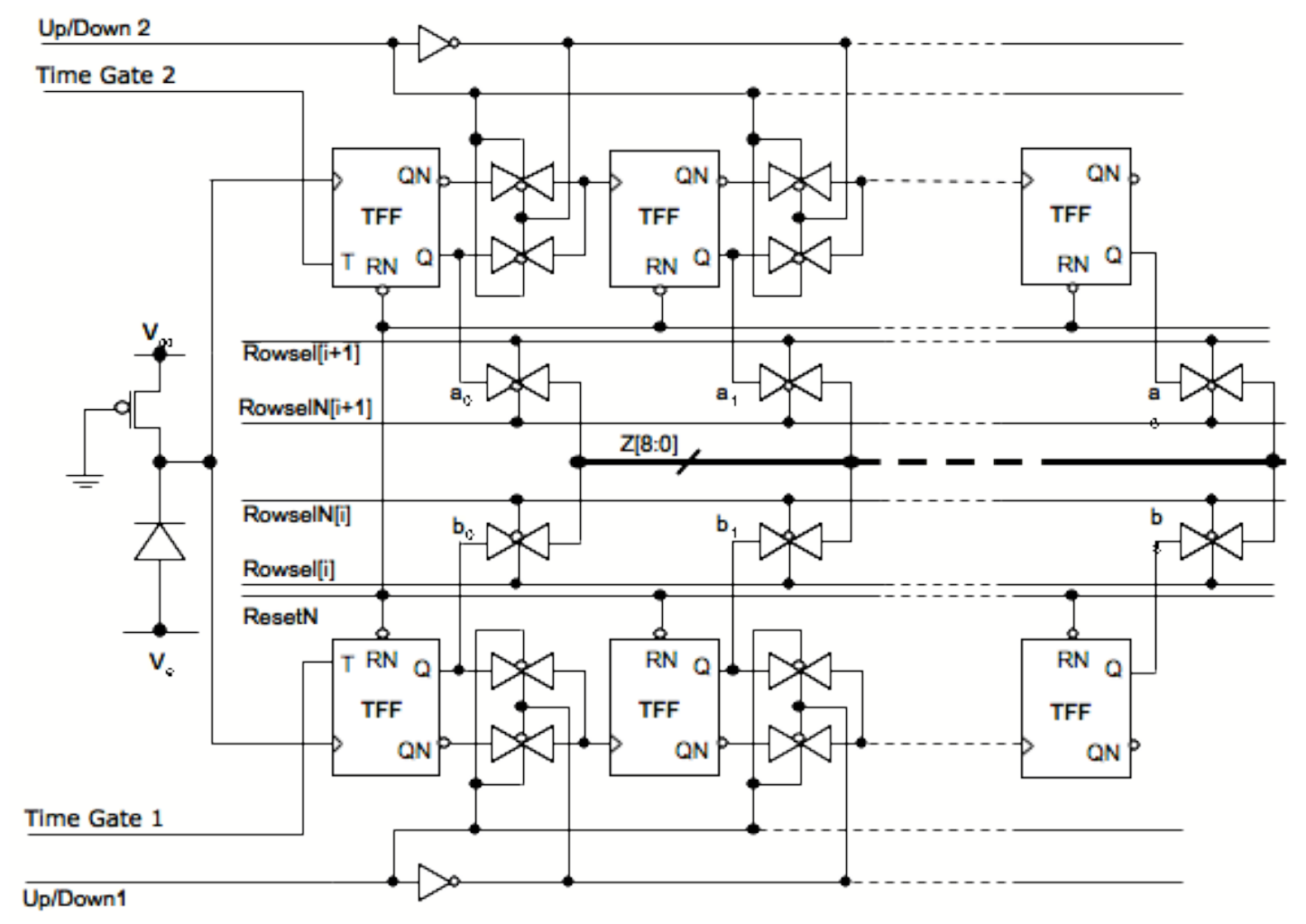

Figure 4.5: SPAD circuit with passive quench transistor and two associated 9-bit ripple counters. 


\subsubsection{Timing Generator}

The timing generator (Figure 4.6) consists of a 120-element tapped delay line composed of current limited buffers. The buffer unit delay is 408 ps with 44 ps RMS jitter at $3.3 \mathrm{~V}$ at room temperature. Three delayed versions of the $3.68 \mathrm{MHz}$ system clock are generated, each delayed output can be selected independently under the control of a latched shift register. Time-gate widths can be selected from 408ps to $48 \mathrm{~ns}$ with a resolution of 408ps. The performance of the on-chip timing generator is summarised in Table 4.2.

Each element of the delay line (Figure 4.7) consists of a two-inverter buffer with an in-line current starving transistor. The gate bias of the current starving transistor was passed off-chip, allowing the user to control the delay through each element in the delay chain. This allows the user to extend the maximum length of the delay generator at the expense of minimum time-gate width.

\begin{tabular}{ll}
\hline Metric & Performance \\
\hline Buffer Unit Delay & $408 \mathrm{ps}$ \\
RMS Jitter (3.3V at room temp.) & $44 \mathrm{ps}$ \\
System Clock & $3.68 \mathrm{MHz}$ \\
Max Time-gate Width & $48 \mathrm{~ns}$ \\
Min Time-gate Width & $408 \mathrm{ps}$ \\
Time-gate Resolution & $408 \mathrm{ps}$ \\
\hline
\end{tabular}

Table 4.2: Summary of on-chip timing generator circuit performance.

The delay line generates three delayed versions of the system clock (SQIN, SQIND and SQINDD). The time delay between these three signals is user definable, by selecting the element of the tapped delay line which outputs the delayed clock. These three delayed clock signals are then broadcast globally across the chip to each pixel in the array. Circuitry within each pixel then generates time gates (counter enable) of width equal to the time delay between the signals. A schematic of the circuitry used to achieve this and a timing diagram of the process is shown in Figure 4.8. By using the difference between two signals broadcast to each pixel via the same route, jitter in the enable signal is minimised, as is the bandwidth requirement of the clock bus drivers. The delay setup by the tapped delay line is user definable via PC control of the FPGA. In this way, the time gates can be easily modified to fit the sample of interest.

A criticism of the gated counting method is that it is relatively photon inefficient as it rejects all photons arriving outwith the narrow time-gate period. In this design each SPAD has two 
associated counters. Thus, two time-gate periods can be collected simultaneously, thereby improving photon efficiency. This feature lent itself particularly well to the 2-gate rapid lifetime determination algorithm (Section 4.4.1), minimising sample exposure to potentially damaging excitation light. As can be seen in Figure 4.8, the time-gate logic circuit within the pixel generates two time-gate pulses equal to the delay between the first and second clock delay signals (SQIN and SQIND, respectively) and the second and third clock delay signals (SQIND and SQINDD, respectively). The temporal relationship between the time-gate signal and a photon pulse from the SPAD is shown in Figure 4.9. 


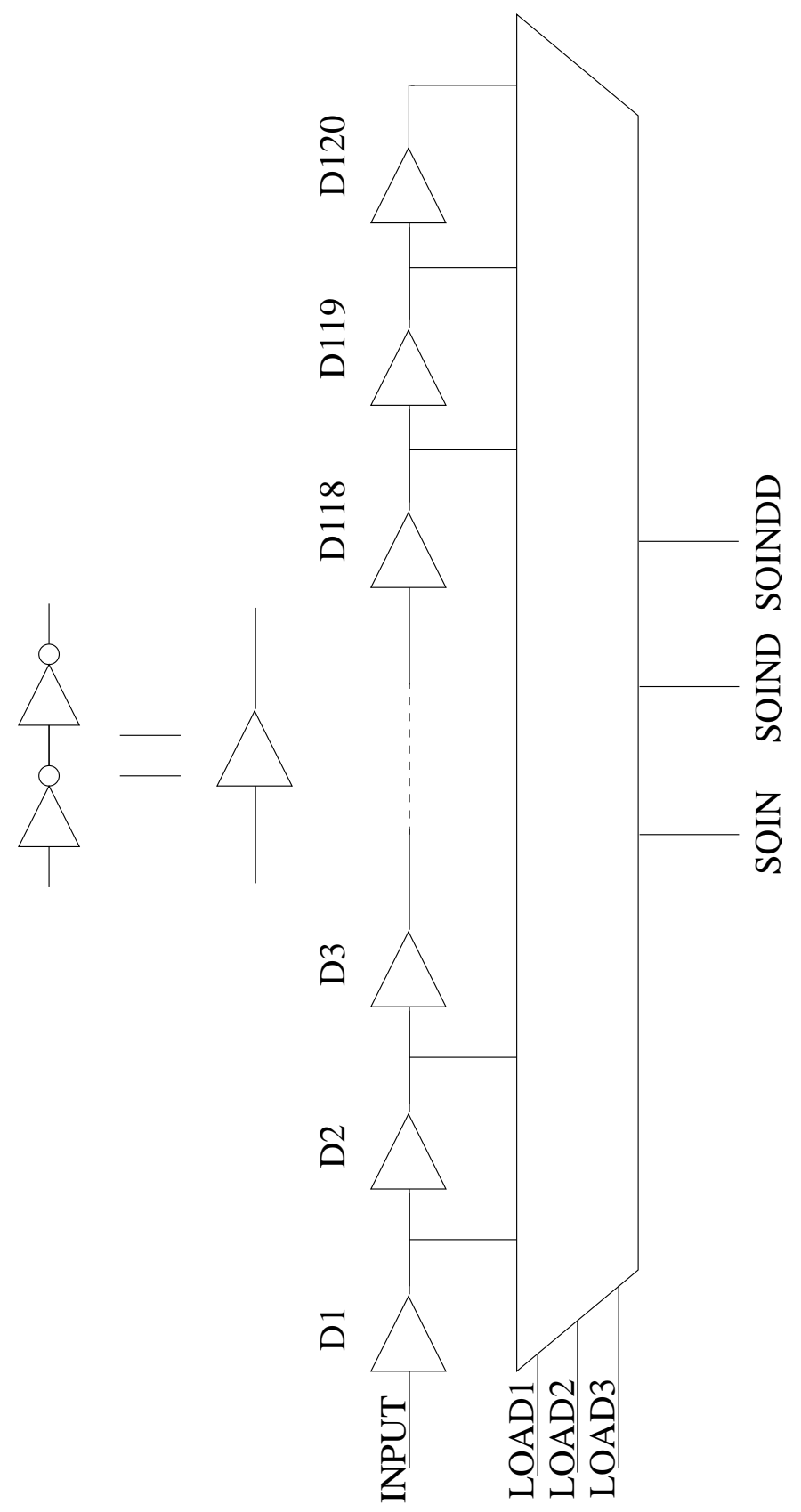

Figure 4.6: On-chip 120-stage tapped delay line used to define counter time-gate and LED pulse widths. 


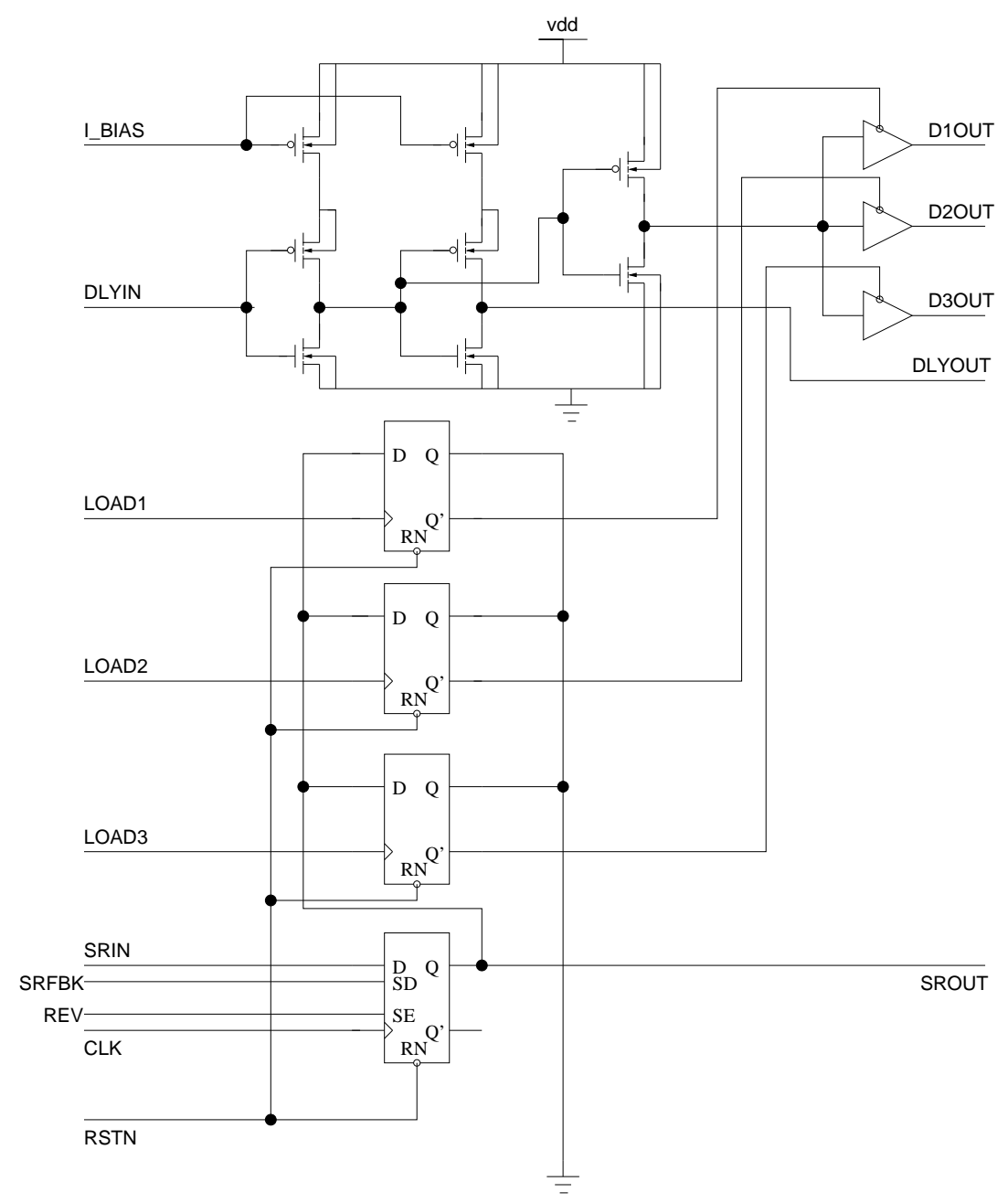

Figure 4.7: An individual element of the 120-stage tapped delay line 

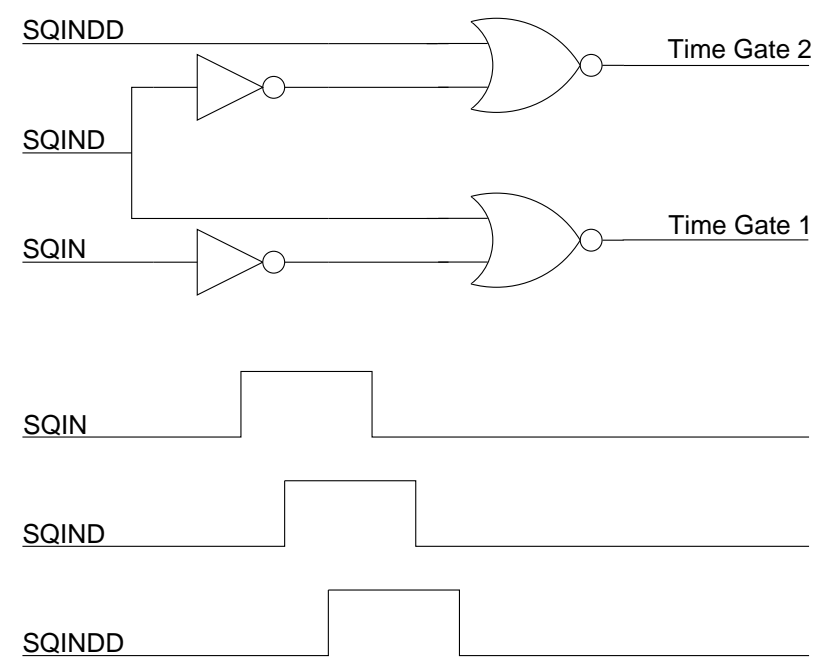

Time Gate 1

Time Gate 2

Figure 4.8: Circuit and timing diagram for the in-pixel time gate generator.

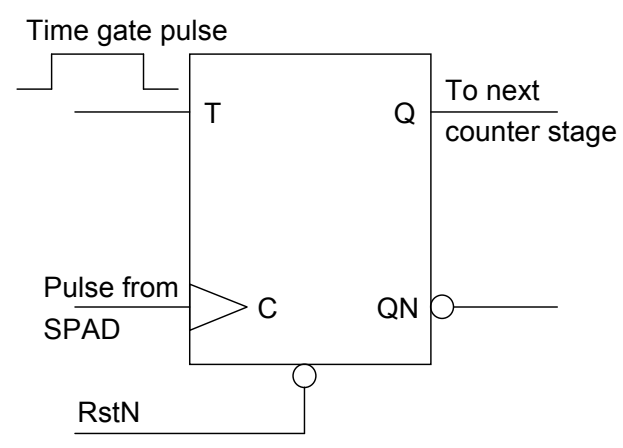

\begin{tabular}{|l|l|l|l|}
\hline Temporal Relation & Count & Temporal Relation & \multirow{2}{*}{ Count } \\
\cline { 1 - 1 } Gate & \multirow{2}{*}{ Yes } & \multicolumn{2}{|l|}{ Gate } \\
Photon
\end{tabular}

Figure 4.9: Relationship between the on-chip generated time gate signal and the SPAD pulse in the counter. 


\subsubsection{Time-Gating}

The on-chip timing generator circuit made two time gating methods possible. Time-gates of fixed width could be defined and the position of both the start and stop time changed (Figure 4.10 (a)). Alternatively, the start (or stop) time of the time gate could be fixed and the stop (or start) time could be altered, resulting in a time-gate of increasing (or decreasing) gate width (Figure 4.10 (b)).
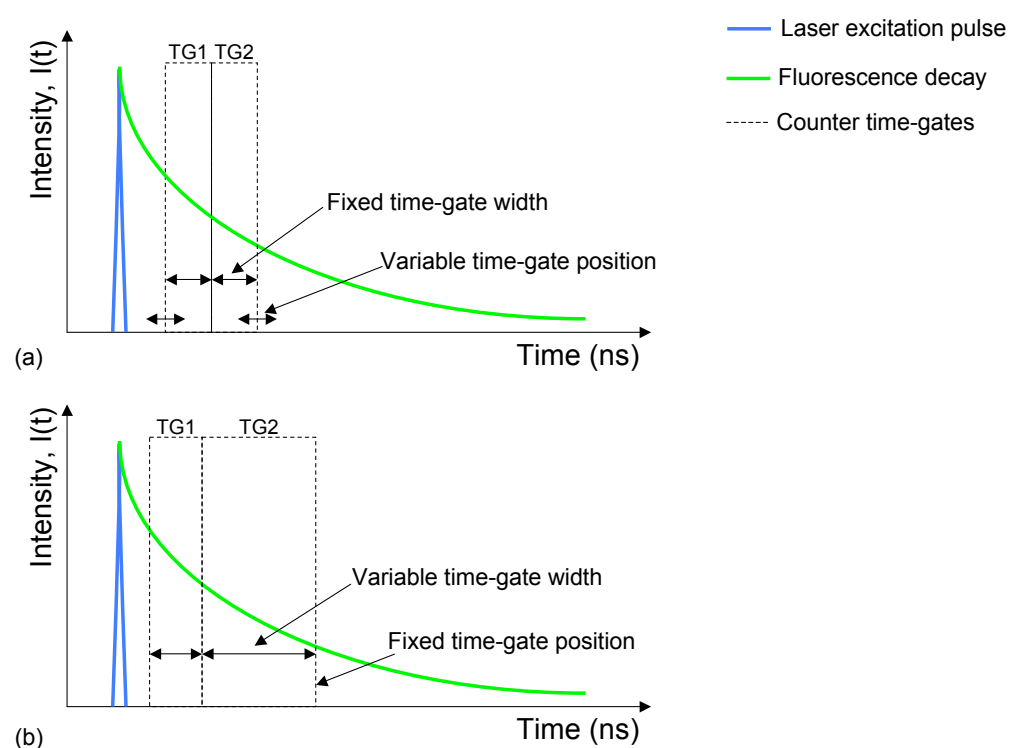

Figure 4.10: (a) Fixed and (b) variable width time-gate schemes. TG1 and TG2 represent the time-gate input signal to the first and second in-pixel counter circuits, respectively.

The operation of the two time-gating schemes was clearly demonstrated experimentally. By focussing a pulsed laser source (Section 3.1.2) with a repetition rate which was less than 48ns (the longest delay possible) and sweeping the position (fixed width scheme) or width (variable width scheme) of the time-gate, it was possible to locate the laser pulse (in time). During time-gate periods in which the laser pulse was exciting the SPAD detector, the counter would be active and the number of avalanche events detected was large. Conversely, during time-gate periods in which the laser was turned off the number of avalanche events detected was equal to the dark count rate of the detector. The difference in the two time-gating schemes can be seen from the results presented in Figures 4.11 and 4.12. 

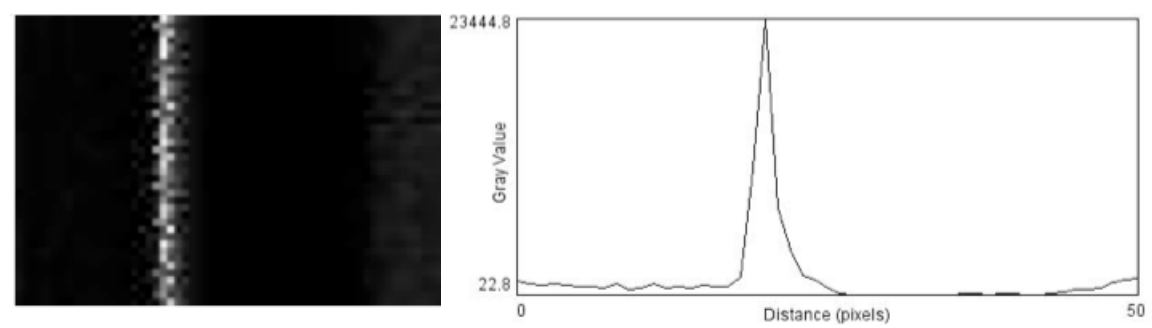

Figure 4.11: vpciview image and plotted horizontal pixel value of pulsed laser source, captured using a time-gate of fixed width and scanned position.
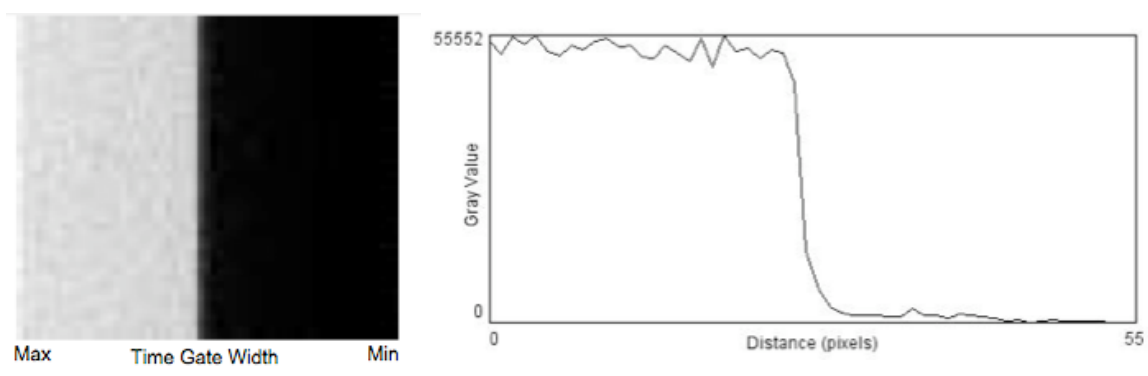

Figure 4.12: vpciview image and plotted horizontal pixel value of pulsed laser source, captured using a time-gate of variable width and fixed start position. 


\subsection{Time-Resolved Fluorescence Captured with SPADs}

Initial time-resolved fluorescence (TRF) lifetime experiments were conducted using the on-chip SPAD detectors and an external Becker and Hickl TCSPC module (Section 3.1.6). This allowed the performance of the detectors to be evaluated independent of the on-chip signal processing circuitry. The principle of TCSPC is shown in Figure 4.13. Using a variety of fluorophores of different lifetimes, concentration and intensity, results were gathered and lifetimes extracted using FAST/F900 software from Edinburgh Instruments (Section 3.3.6).

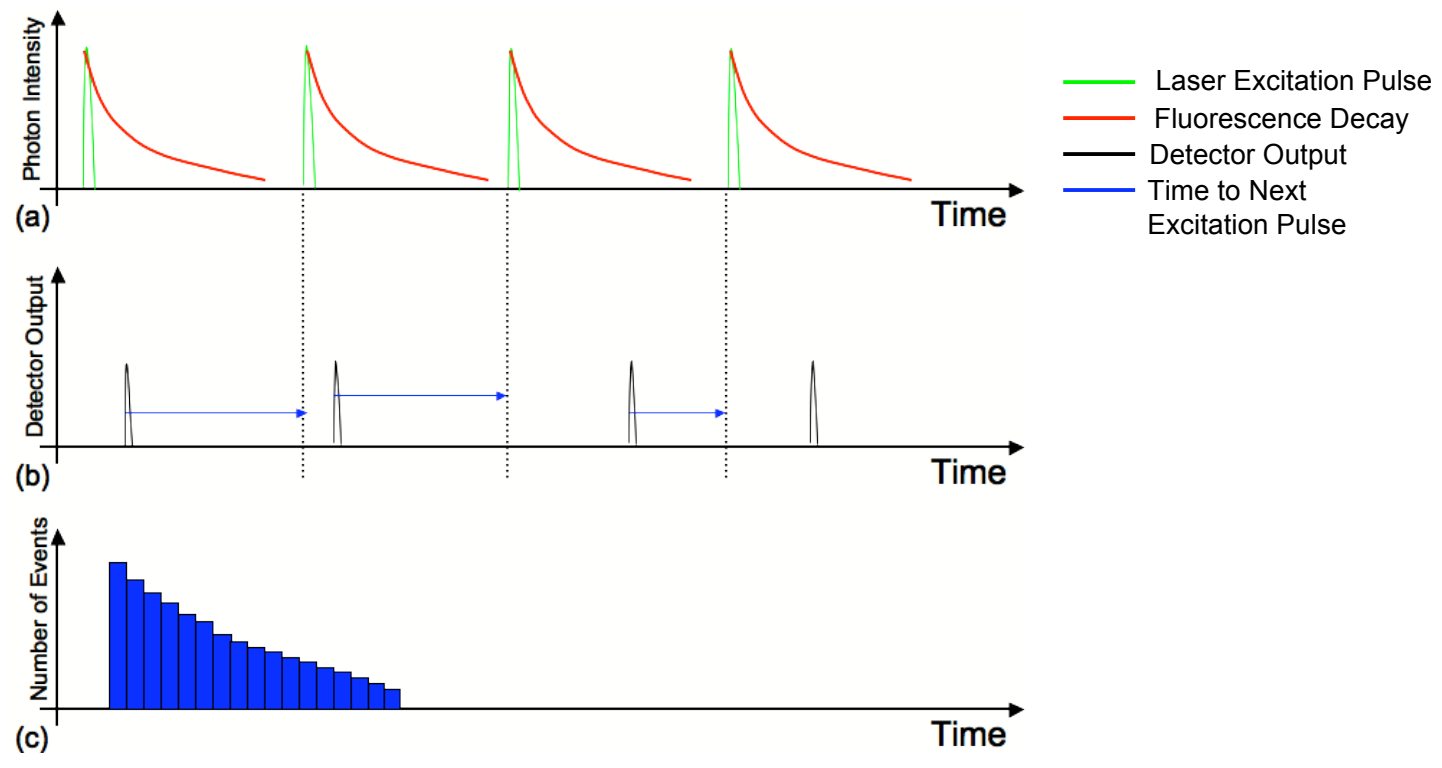

Figure 4.13: Principle of TCSPC. (a) the sample of interest is excited with a short excitation pulse, resulting in a decaying fluorescence signal. (b) A single photon from this decay causes the detector to trigger. The time between the detector pulse and the subsequent excitation pulse is measured. (c) This measurement is used to place this photon count in a histogram time bin. As this process is repeated, a histogram representing the fluorescence decay is obtained.

\subsubsection{SPAD Sensitivity and Measurement Accuracy}

To assess the sensitivity of the SPAD detector fluorescence lifetime analysis of a series of quantum dot samples (Section 3.4.1) of different concentrations was conducted. Quantum dot samples with an emission wavelength of 548nm were prepared at concentrations of 50, 25, 10, $1,0.1$ and $0.01 \mu \mathrm{M} .45 \mu \mathrm{L}$ of each sample was loaded into a single cavity (15mm diameter) glass microscope slide (Fisher Scientific, UK, MNK-140-010A) and sealed with a $0.12 \mathrm{~mm}$ thick 
borosilicate glass cover slip (Fisher Scientific, UK, MNJ-300-020T). As stated previously, the lifetime of a fluorophore is independent of sample concentration. By conducting a series of lifetime experiments for a fixed period of time with different fluorophore concentrations, a set of decay curves should be obtained whereby only the number of counts in the peak channel of each decay varies. The time constant of each curve should be identical.

A Nikon TE2000-U Microscope (Section 3.1.3) was used, with a x20 objective and the filter set described in Section 3.1.4 and the PicoQuant 467nm pulsed diode laser light source (Section 3.1.2). The SPAD detector was placed at a side output port of the microscope. The IRF was obtained using a sample of Ludox to scatter the excitation light. An overview of the experimental setup is provided in Table 4.3.

\begin{tabular}{ll}
\hline Setting & Value \\
\hline Laser Rep. Rate & $5 \mathrm{MHz}$ \\
Laser Average Power & $0.15 \mathrm{~mW}$ \\
Microscope Objective & X20 \\
SPAD VOP & $-19.5 \mathrm{~V}$ \\
SPAD_AVDD & $3.3 \mathrm{~V}$ \\
SPAD Address & Row 15, Column 2 \\
Sample & CdSe/ZnS Quantum Dots \\
Sample Volume & $45 \mu \mathrm{L}$ \\
Sample Emission Wavelength & $548 \mathrm{~nm}$ \\
\hline
\end{tabular}

Table 4.3: An overview of the experimental setup used in limiting dilution tests.

Figure 4.14 shows how the maximum number of counts in the peak channel of the decay curve increments appropriately according to the sample concentration and how the decay curves remain parallel as they all represent the same sample lifetime. It was found that the SPADs were sensitive to approximately $0.01 \mu \mathrm{M}$. A reduction in the concentration of the quantum dot sample correlated closely with a reduction in the number of photon counts per second. For the $0.01 \mu \mathrm{M}$ sample, the count rate per second was just $2.24 \times 10^{2}$. The nature of TCSPC experiments means that lifetime decay curves can still be produced if the user is willing to extend the time over which the experiment is conducted. The problem associated with this is that the noise floor of the experiment continues to rise as the experimental time is extended. As demonstrated in Figure 4.15, the time resolved fluorescence decay curves for a $0.1 \mu \mathrm{M}$ quantum dot sample (548nm emission wavelength) gathered for a fixed experimental time of 5 minutes and for an experiment which was allowed to run until 5,000 counts were gathered in the peak channel. It took 21 minutes 58 seconds to achieve 5,000 counts in the peak channel, at a count 


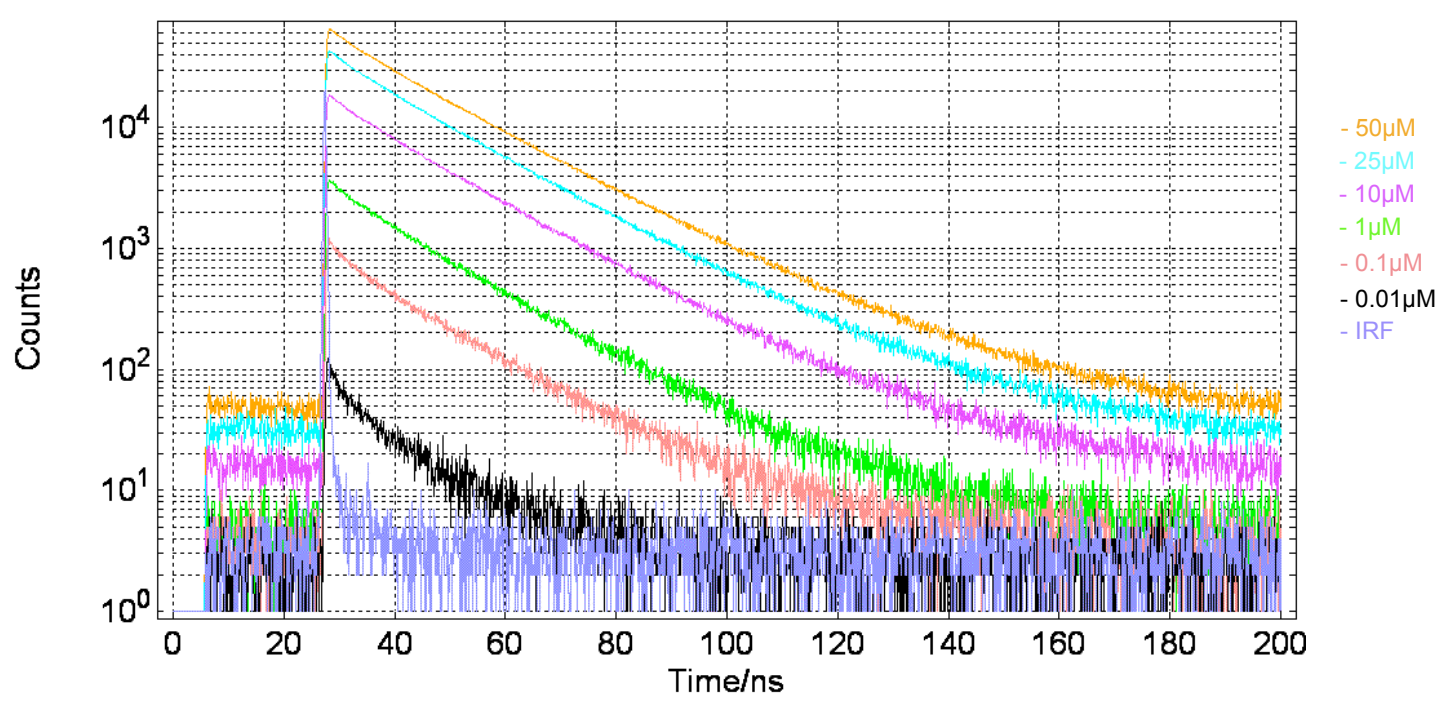

Figure 4.14: Time resolved decay curves of quantum dot sample, evaluated at different concentrations.

rate of $1.65 \times 10^{3}$. The noise floor of the decay curve rose with the increase in experimental time. It should be noted, however, that the signal-to-noise ratio improved since the peak due to fluorescence emission rose faster than the background dark counts.

The concentration of the sample of interest dictates the number of photons per second detected by the SPAD detector (counts per second) and thus the number of counts achieved in the peak channel (for an experiment of fixed time) or the experimental time. Another issue associated with extending the experimental time is the increased exposure of the sample to photon energy (from the excitation source), which can lead to photo-bleaching of the sample and/or sample damage. While lifetime measurements are immune to the effects of photo-bleaching, photo-bleaching results in a further decrease in the number of counts gathered per second. Table 4.4 summarises the counts per second that were achieved and the time taken to gather 10,000 counts in the peak channel for different quantum dot sample concentrations. These data were gathered using the same experimental setup described in Table 4.3.

Given that the SPAD is biased as a single-photon detector it may be expected that it would achieve a higher count rate than is reported. The low count rate observed could be attributed to the physical size of the SPAD detector, as the active area of the SPAD detector is only $6 \mu \mathrm{m}$ in diameter $\left(\right.$ Area $=28.27 \mathrm{pm}^{2}$ ). This results in a fill factor of just $0.14 \%$ for a $100 \times 200 \mu \mathrm{m}$ pixel. 


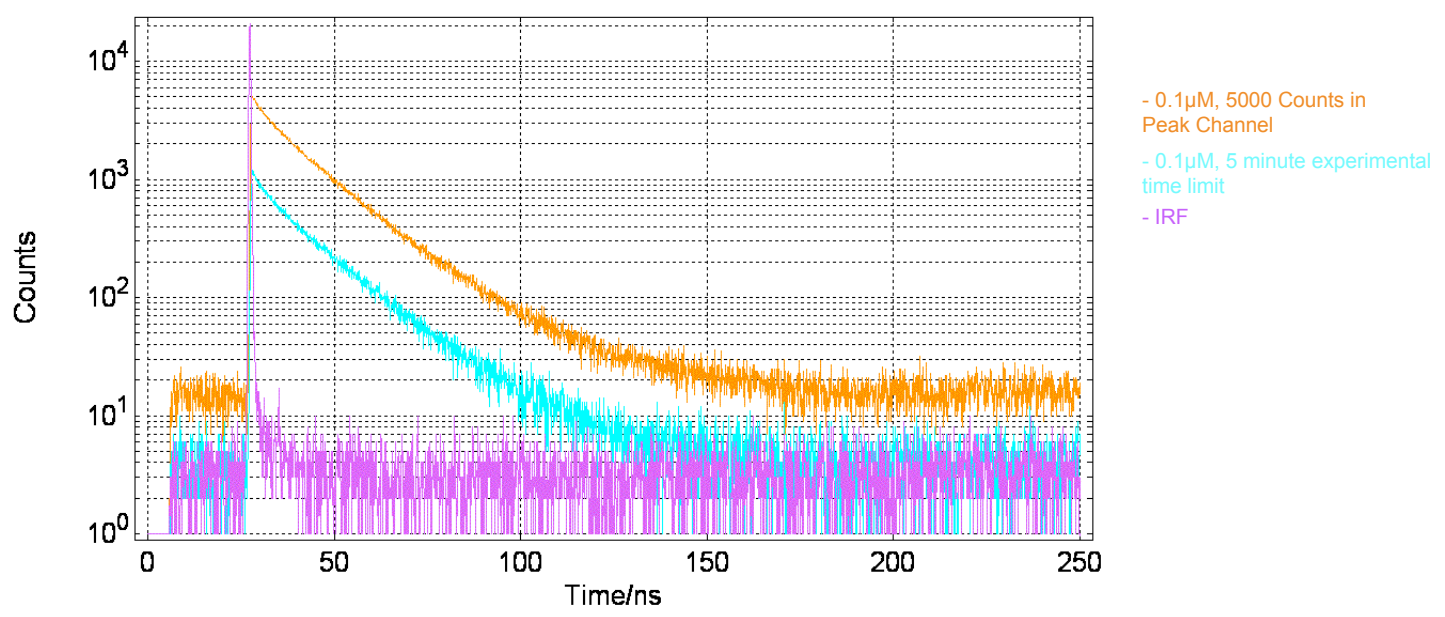

Figure 4.15: Time resolved decay curves of a $0.1 \mu \mathrm{M}$ quantum dot sample gathered for a fixed experimental time of 5 minutes and until 5000 counts were achieved in the peak channel (experimental time unrestricted).

The results were gathered using the output from a single SPAD in the array. For comparison, the diameter of the Becker and Hickl PMT described in Section 3.1.7 is $8 \mathrm{~mm}$ (Area $=0.5 \mu \mathrm{m}^{2}$ ), which is $1.7 \times 10^{6}$ times larger than the active area of the SPAD detector. For this reason a convex lens was placed at the output port of the microscope in an attempt to maximise the number of photons gathered by the detector. A number of companies produce single large area Geiger mode SPAD detectors [91], [92] with comparable sensor diameters to that of a PMT. SensL of Cork, Ireland produce single large area detectors which are created from an array of smaller detectors connected in parallel. Another way to circumvent the problem of small fill factor is to

\begin{tabular}{llll}
\hline Concentration & Counts per Second & $\begin{array}{l}\text { No. of Counts in } \\
\text { Peak Channel after } \\
5 \text { mins }\end{array}$ & $\begin{array}{l}\text { Time to Reach } 10000 \\
10000 \text { Counts in } \\
\text { Peak Channel }\end{array}$ \\
\hline $50 \mu \mathrm{M}$ & $1.15 \times 10^{5}$ & 65298 & 0 minutes 46 seconds \\
$25 \mu \mathrm{M}$ & $7.54 \times 10^{4}$ & 42646 & 1 minute 12 seconds \\
$10 \mu \mathrm{M}$ & $3.09 \times 10^{4}$ & 18486 & 2 minutes 37 seconds \\
$1 \mu \mathrm{M}$ & $5.73 \times 10^{3}$ & 3705 & 13 minutes 46 seconds \\
$0.1 \mu \mathrm{M}$ & $1.63 \times 10^{3}$ & 1203 & 43 minutes 56 seconds \\
$0.01 \mu \mathrm{M}$ & $2.24 \times 10^{2}$ & 125 & 425 minutes 20 seconds* \\
\hline
\end{tabular}

Table 4.4: Count rate achieved, number of counts achieved in peak channel and experimental time versus concentration. * extrapolated from time to achieve 5,000 and 500 counts, respectively. 
introduce optical concentrators [93] or micro-lenses [94], [95]. These elements aim to recover the detector fill factor by focusing incident light away from the non-active chip electronics and towards the active SPAD area. [93] present simulation results which demonstrate optical concentrator devices with concentration factor of up to 65 (ratio of concentrator collection diameter to output diameter).

\subsubsection{System Noise}

The chip had no way to disable individual detectors within the array. Despite only a single SPAD being addressed and read out, all elements in the array remained active. This results in a high level of on-chip activity when the array is bathed by photons, with SPADs breaking down across the array. The effect of these breakdown events is noise on the SPAD bias supplies, which are common to all elements in the array. It is possible that this noise contribution, which is linked to array activity and hence incident photon levels, is the reason for inconsistency in the noise floor of the decays featured in Figure 4.14. Decay curves associated with samples of higher concentration (and thus increased emission light levels) have the highest noise floor. There are a number of potential solutions to this problem; for instance, the CFD crossing level of the Becker and Hickl TCSPC card could be adjusted to reduce sensitivity to fluctuations on the detector input line. Alternatively, the SPAD bias supply lines could be decoupled further, this could take the form of additional off-chip discrete capacitors or, in the event of a chip redesign, on-chip capacitors local within each pixel. Finally, the SPAD circuit could be redesigned with the option of dis-arming unused SPADs in the array. Stoppa et al. [37] have demonstrated pixels with actively quenched SPADs which can be held off and then armed rapidly. In addition to minimising SPAD activity this system can also be used to gate the SPAD during sample excitation. By taking advantage of CMOS's ability to switch states at high speed (approximately 200ps in a $0.35 \mu \mathrm{m}$ process) it is possible to render the SPAD insensitive to photons during the excitation period. The SPAD can then be quickly re-armed and be active, ready to capture the resulting fluorescence decay. The process of disabling the photodetector element during the sample excitation phase is also demonstrated in [9], whereby a conventional integrating photodiode is held in reset until after a laser pulse. The photodiode is then taken out of reset and allowed to integrate during the fluorescence decay. This method of photodetector activation/deactivation removes the need for any discrete optical emission filters in the system, thereby reducing overall system size and costs. It is hoped that any future SPAD-based design to be incorporated into a micro-scale time resolved analysis device would feature this technique. 


\subsubsection{Fluorescein and Rhodamine B Evaluation}

The majority of device characterisation was performed using the Quantum Dot samples (Section 3.4.1) owing to their favourable excitation and emission wavelengths, high quantum yield and long lifetimes. It was considered important to assess the performance of the SPAD detector using conventional, bio-compatible fluorophores with shorter lifetimes. Thus, decay curves were compared for Fluorescein (diluted in Ethanol) and Rhodamine B (in $\mathrm{H}_{2} \mathrm{O}$ ), both at a concentration of $1 \mu \mathrm{M}$ using the SPAD detector and processed by the Becker and Hickl card (Figure 4.16). The IRF was obtained using a sample of Ludox to scatter the excitation light. These results were gathered using the microscope described in Section 3.1.3.

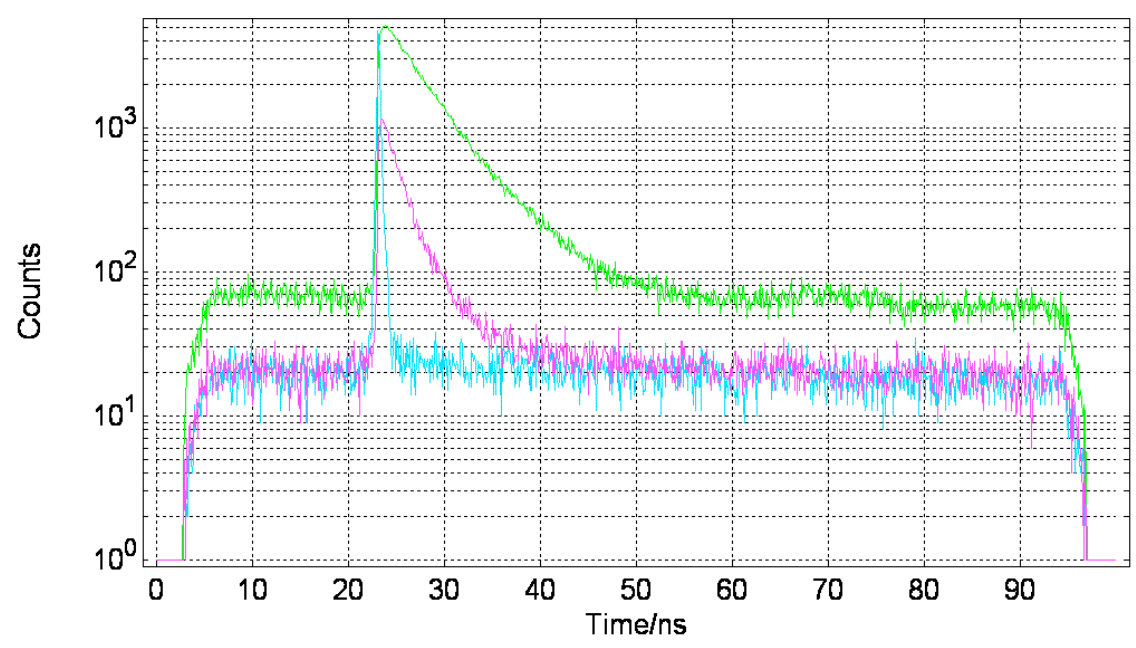

Figure 4.16: Time resolved decay curves of $1 \mu M$ Fluorescein and Rhodamine B samples in Ethanol and $\mathrm{H}_{2} \mathrm{O}$, respectively. 


\subsubsection{Lifetime Extraction from Decay Curves}

Using F900/FAST software from Edinburgh Instruments (Section 3.3.6) estimations of sample lifetimes could be obtained through the fitting of exponential curves to the data presented in Section 4.3.1 and 4.3.2. Table 4.5 summarises the extracted lifetimes based on the decay curves presented in Sections 4.3.1 and 4.3.2. Measurement error is based on 114ps RMS SPAD jitter plus 4ps RMS TCSPC module jitter. Curve fitting was performed using discrete components analysis of the data.

\begin{tabular}{llll}
\hline Sample & Solvent & Concentration & Estimated Lifetime (ns) \\
\hline 548nm Quantum Dots & Toluene & $50 \mu \mathrm{M}$ & $14.7( \pm 114 \mathrm{ps})$ \\
548nm Quantum Dots & Toluene & $25 \mu \mathrm{M}$ & $14.3( \pm 114 \mathrm{ps})$ \\
548nm Quantum Dots & Toluene & $10 \mu \mathrm{M}$ & $14.2( \pm 114 \mathrm{ps})$ \\
548nm Quantum Dots & Toluene & $1 \mu \mathrm{M}$ & $13.3( \pm 114 \mathrm{ps})$ \\
548nm Quantum Dots & Toluene & $0.1 \mu \mathrm{M}$ & $14.6( \pm 114 \mathrm{ps})$ \\
548nm Quantum Dots & Toluene & $0.01 \mu \mathrm{M}$ & $10.5( \pm 114 \mathrm{ps})$ \\
Fluorescein & Ethanol & $1 \mu \mathrm{M}$ & $4.2( \pm 114 \mathrm{ps})$ \\
Rhodamine B & $\mathrm{H}_{2} \mathrm{O}$ & $1 \mu \mathrm{M}$ & $1.7( \pm 114 \mathrm{ps})$ \\
\hline
\end{tabular}

Table 4.5: Lifetime estimations based on SPAD data processed by Becker and Hickl TCSPC module.

The results presented in Table 4.5 are in good agreement with lifetime values quoted by the quantum dot manufacturer (Evident Technologies, Inc.), with the exception of the lowest concentration $(0.01 \mu \mathrm{M})$ sample. At this low concentration only a small portion of the decay can be observed above the noise floor (Figure 4.14). This severely limits the fitting range that can be chosen for the lifetime extraction algorithm and can lead to skewed results. Another phenomenon that has been observed for low concentration quantum dot samples is that background fluorescence greatly affects the ability to make accurate lifetime estimations (Gerard Giraud, COSMIC, University of Edinburgh, personal communication, December 2008). At low sample concentrations (nM), fluorescence emission from other sources can affect results. Lifetime measurements of non-quantum dot based fluorophores are also in good agreement with values quoted in the literature. In [34] a list of typical fluorescence lifetimes for various fluorophores is provided, the quoted lifetime for fluorescein and rhodamine B is 4.0ns and $1.68 \mathrm{~ns}$, respectively. Comparing this to the measured lifetimes gives an error of 178ps and $57 \mathrm{ps}$ for fluorescein and rhodamine B. It should be noted that there are a large number of factors that can influence lifetime, including temperature, solvent quality and fluorophore quality. 


\subsection{Time-Resolved Fluorescence Captured using SPADs and On-Chip Counters}

Fluorescence lifetime estimations were made using the in-pixel time gated counters. A histogram of the decay curve can be generated by allowing the circuit to count the number of SPAD avalanche events within a fixed time period, changing the position of this time-gate period and repeating the process. The width and number of time-gates used to generate the histogram was user definable and results were compared for a number of different time-gate settings. Count values are read from the chip at the end of each count period and displayed using the vpciview software tool. The image generated is indicative of the number of counts gathered in each time period. These images were then processed using Matlab to produce a set of numbers equal to the total number of counts in each time gate period. Histograms of decay curves were produced from this data set and lifetime estimation algorithms could be applied.

\subsubsection{Lifetime Estimation Algorithms}

An extraction algorithm was employed to extract a fluorescence lifetime from the data obtained using the on-chip time-gating circuitry. Three algorithms were chosen: the multi-gate rapid-lifetime-determination algorithm (RLD-M) [96], the two-gate rapid-lifetime-determination algorithm (2-RLD) [97], [98] and the direct integration for lifetime extraction method (IEM) [99]. All three of these algorithms were capable of extracting a single exponential from a decay curve. The IEM algorithm used was proposed by $\mathrm{Dr}$ Day-Uei Li, a colleague at The University of Edinburgh. The advantage of the IEM algorithm is that it does not use any logarithms to calculate the lifetime and can therefore be easily implemented on-chip or alternatively within an FPGA. In [99], the IEM algorithm (Equation 4.1) is introduced (and compared to other lifetime extraction algorithms) and the integration of the algorithm into each pixel of a multi-detector system is proposed. This is further evidence of how the size of future micro-systems for time-resolved fluorescence lifetime analysis and the reliance on external lifetime extraction software can be reduced. During this project the algorithms were applied to the data away from the laboratory after data had been gathered. 


$$
\tau=h \cdot \frac{\left(N_{1} / 2\right)+N_{2}+\ldots+N_{N-1}+\left(N_{N} / 2\right)}{N_{1}-N_{k}}
$$

Where $\tau$ is the extracted lifetime, $h$ is the width of a time gate and $N_{k}$ is the number of counts in time gate $k$.

The 2-RLD algorithm (Equation 4.2) is the simplest algorithm used and requires data from just two time-gates to produce a lifetime estimation.

$$
\tau=\frac{h}{\ln \left(\frac{N_{1}}{N_{2}}\right)}
$$

Where $\tau$ is the extracted lifetime, $h$ is the width of a time-gate, $N_{1}$ is the number of counts gathered in the first (earliest) time-gate and $N_{2}$ is the number of counts gathered in the second (latest) time-gate.

The M-RLD algorithm (Equation 4.3) is an extension of the 2-RLD algorithm and can be applied to data gathered over any number of time gates.

$$
\tau=-\frac{M\left(\Sigma t_{k}^{2}\right)-\left(\Sigma t_{k}\right)^{2}}{M \Sigma\left(t_{k} \cdot \ln \left(N_{k}\right)\right)-\Sigma t_{k} \cdot \Sigma \ln \left(N_{k}\right)}
$$

Where $\tau$ is the extracted lifetime, $M$ is the number of time gates, $N_{k}$ is the number of counts in time-gate $k$ and $t_{k}$ is the start position (in time) of each time-gate. 


\subsubsection{On-Chip Lifetime Measurement Sensitivity and Accuracy}

The sensitivity of the SPADs (with the on-chip time-gating circuitry enabled) weas tested by determining the fluorescence lifetimes of a series of diluted quantum dot samples. The ST Microelectronics 908 test board, on which the SPAD chip was situated, was mounted against the output port of the microscope. Data was transferred to a PCB via PCI link for processing using the vpciview software and Matlab. Finally, lifetime extraction algorithms were applied to the data. To allow each of the lifetime algorithms to be used and evaluated, experiments were repeated using different numbers of time-gates to assess the effect that this and the time-gate width has on lifetime estimations. Florescence decays were captured using 23x800ps time-gates, 9x2ns time-gates and 2x8.8ns time-gates. Figure 4.17 demonstrates the system was capable of capturing decay curves over a wide range of sample concentrations, from $50 \mu \mathrm{M}$ down to $0.01 \mu \mathrm{M}$. The decay curves presented were all captured using a quantum dot sample with an emission wavelength of $548 \mathrm{~nm}$. The on-chip delay line has a maximum delay of $48 \mathrm{~ns}$. Due to the delay between the input trigger and the optical output from the laser driver of $29.2 \mathrm{~ns}$, this effectively cut the maximum observation window to $18.8 \mathrm{~ns}$. Given that the quoted lifetime of the quantum dots from the manufacturer was approximately $15 \mathrm{~ns}$ and that a decay curve lasts approximately five times the extracted lifetime, only a portion of the sample's decay curve can be captured using the on-chip method.

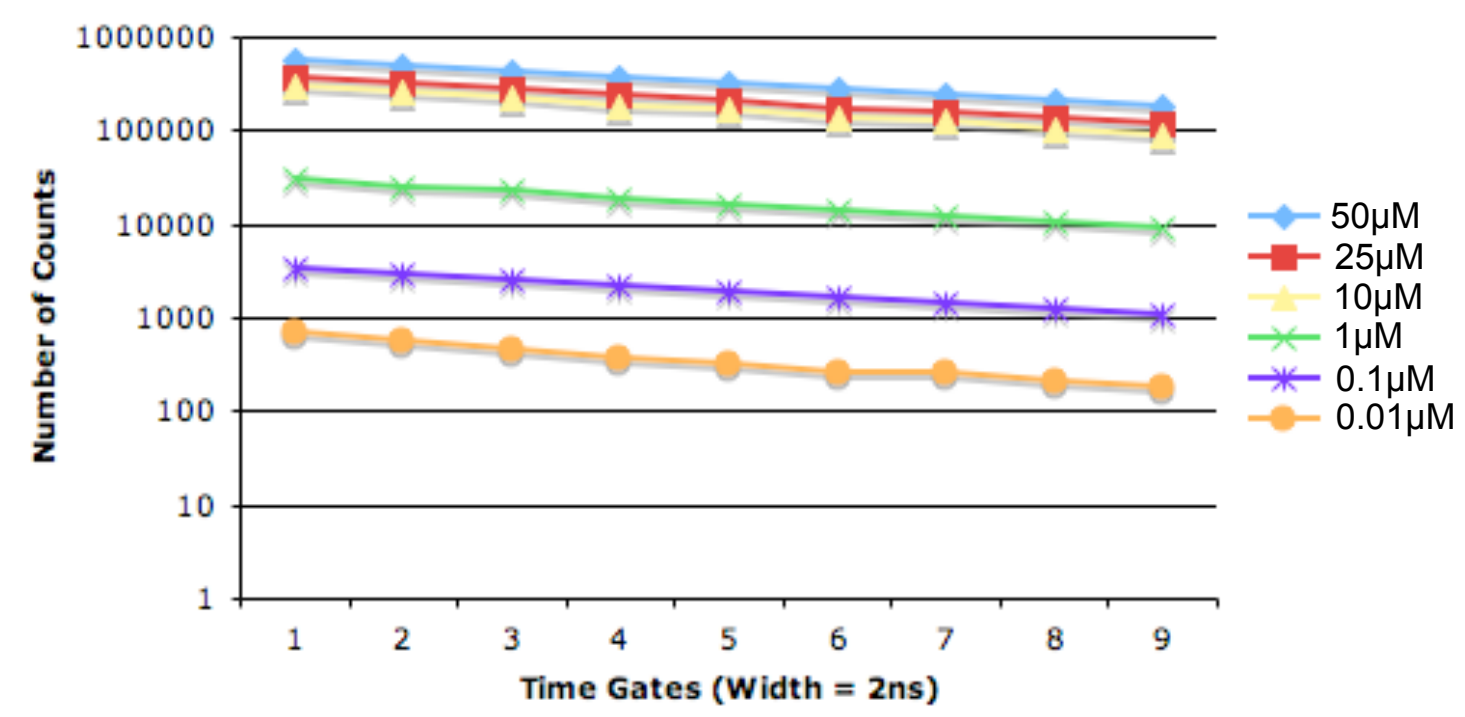

Figure 4.17: Fluorescence lifetime measurements obtained from quantum dot samples using SPAD detector and on-chip circuitry. 
Extracted lifetime values are presented (Table 4.6), using different numbers of time gates and different extraction algorithms. These measurements were obtained using the same $548 \mathrm{~nm}$ emission wavelength quantum dot sample as was used for the decay curves (Figure 4.17). Measurement error is based on 114ps RMS SPAD jitter plus 44ps on-chip time-gate jitter. These results are in good agreement with the lifetimes extracted from decay curve histograms produced using the Becker and Hickl TCSPC module (Table 4.5).

\begin{tabular}{l|ll|ll|ll|l}
\hline & \multicolumn{2}{|c|}{$23-G a t e s$} & \multicolumn{2}{c|}{ 9-Gates } & \multicolumn{2}{c|}{ 2-Gates } & Measurement \\
Concentration & M-RLD & IEM & M-RLD & IEM & 2-RLD & IEM & Error \\
\hline $50 \mu \mathrm{M}$ & $13.7 \mathrm{~ns}$ & $14.9 \mathrm{~ns}$ & $14.2 \mathrm{~ns}$ & $14.4 \mathrm{~ns}$ & $14.1 \mathrm{~ns}$ & $14.6 \mathrm{~ns}$ & $( \pm 122 \mathrm{ps})$ \\
$25 \mu \mathrm{M}$ & $13.7 \mathrm{~ns}$ & $13.1 \mathrm{~ns}$ & $14.0 \mathrm{~ns}$ & $13.7 \mathrm{~ns}$ & $13.7 \mathrm{~ns}$ & $14.1 \mathrm{~ns}$ & $( \pm 122 \mathrm{ps})$ \\
$10 \mu \mathrm{M}$ & $13.2 \mathrm{~ns}$ & $12.9 \mathrm{~ns}$ & $13.5 \mathrm{~ns}$ & $13.3 \mathrm{~ns}$ & $13.4 \mathrm{~ns}$ & $13.9 \mathrm{~ns}$ & $( \pm 122 \mathrm{ps})$ \\
$1 \mu \mathrm{M}$ & $12.8 \mathrm{~ns}$ & $13.1 \mathrm{~ns}$ & $13.8 \mathrm{~ns}$ & $13.8 \mathrm{~ns}$ & $13.0 \mathrm{~ns}$ & $13.5 \mathrm{~ns}$ & $( \pm 122 \mathrm{ps})$ \\
$0.1 \mu \mathrm{M}$ & $12.3 \mathrm{~ns}$ & $22.2 \mathrm{~ns}$ & $15.0 \mathrm{~ns}$ & $17.3 \mathrm{~ns}$ & $12.6 \mathrm{~ns}$ & $13.1 \mathrm{~ns}$ & $( \pm 122 \mathrm{ps})$ \\
$0.01 \mu \mathrm{M}$ & $11.6 \mathrm{~ns}$ & $8.7 \mathrm{~ns}$ & $10.6 \mathrm{~ns}$ & $11.9 \mathrm{~ns}$ & $13.4 \mathrm{~ns}$ & $13.8 \mathrm{~ns}$ & $( \pm 122 \mathrm{ps})$ \\
\hline
\end{tabular}

Table 4.6: Lifetime estimations based on SPAD data collected using on-chip time gating circuitry and processed using three different lifetime extraction algorithms.

At higher concentrations and hence higher photon counts per time gate, all three lifetime extraction algorithms performed well regardless of the number of time-gates used to capture the decay (Table 4.6). As sample concentration is reduced, error appears in the extracted lifetime. This was particularly evident for experiments which capture fluorescence decays using a greater number of shorter time-gates. Error was greatest for experiments using 23 time-gates to capture decay curves from samples of concentrations $0.1 \mu \mathrm{M}$ and $0.01 \mu \mathrm{M}$. Experiments conducted with longer time gates appeared to perform better at lower concentrations. This could be due to the greater number of photon counts that can be gathered per time-gate, since when wider time-gates were used the error per time-gate was less. Samples of lower concentration resulted in a lower number of emitted photons upon excitation, when a wider time-gate was used the chances of detecting a photon in that period increases. As the number of counts gathered increased, the accuracy of the algorithm improved. 


\subsubsection{On-Chip Detection of Fluorescein, Rhodamine B and Coumarin 6}

It was of interest to use on-chip circuitry to gather time-resolved fluorescence data for fluorophores other than quantum dots. The test setup described in Section 3.1.5 was used to capture the decay of a $1 \mu \mathrm{M}$ fluorescein sample (in ethanol) using eight time-gates of $2.4 \mathrm{~ns}$ (Figure 4.18).

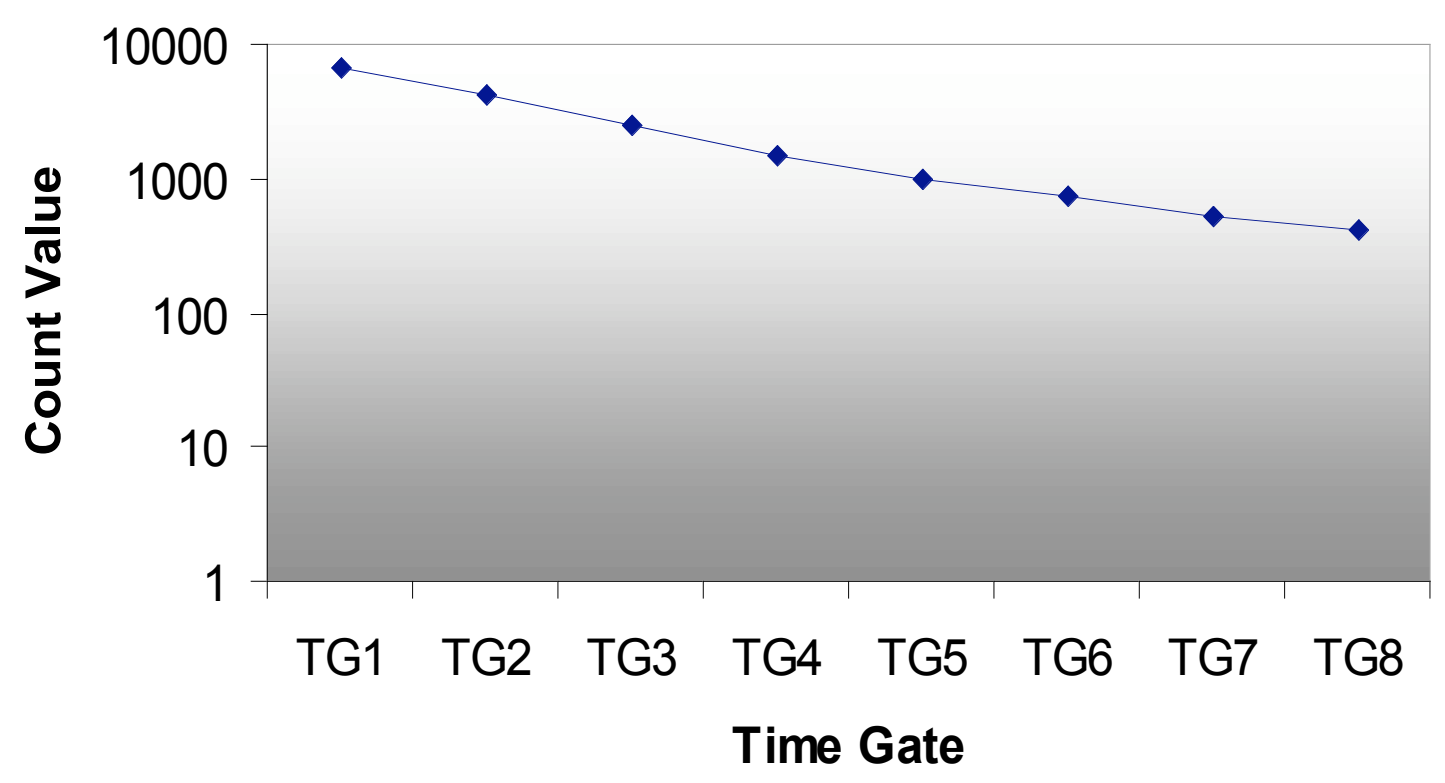

Figure 4.18: Fluorescein decay captured using 8 time-gates, each 2.4ns wide.

A lifetime of $5.4 \mathrm{~ns}( \pm 122 \mathrm{ps})$ was extracted from the decay curve in Figure 4.18 using the IEM algorithm. There is a $1.25 \mathrm{~ns}$ difference in the lifetime extracted here compared to the lifetime obtained using the Becker and Hickl card (Table 4.5). This could be due to a reduced collection time for the on-chip analysis and imperfection in the optical setup. The optical path of the bench top setup was not as well optimised as the microscope setup, leading to greater photon losses and an increased likelihood of noise and contamination.

The 2-RLD algorithm also performed well with several different fluorophores (Table 4.7). This could be further evidence of the greater robustness of wider time-gate schemes to system noise. Calibrated system measurement error is based on 180ps PMT jitter plus 4ps TCSPC module jitter.

When performing time-gating experiments it is necessary to select the width of time-gates used to capture the decay. This is dictated by a number of factors, including: the intended lifetime 


\begin{tabular}{|c|c|c|c|c|c|c|}
\hline Fluorophore & Solvent & $\begin{array}{l}\text { Time-gate } \\
\text { Width (ns) }\end{array}$ & $\begin{array}{l}\text { Counts } \\
\text { in TG1 }\end{array}$ & $\begin{array}{l}\text { Counts } \\
\text { in TG2 }\end{array}$ & $\begin{array}{l}\text { RLD } \\
\text { Lifetime } \\
\text { (ns) }\end{array}$ & $\begin{array}{l}\text { Lifetime from } \\
\text { Calibrated } \\
\text { System (ns) }\end{array}$ \\
\hline Coun & Methanol & 6.12 & 107411 & 12298 & $2.8( \pm 122 \mathrm{ps})$ & $2.4( \pm 180 \mathrm{ps})$ \\
\hline Rhodamine B & $\mathrm{H}_{2} \mathrm{O}$ & 4.08 & 1947 & 338 & $2.3( \pm 122 \mathrm{ps})$ & $2.2( \pm 180 \mathrm{ps})$ \\
\hline
\end{tabular}

Table 4.7: $1 \mu M$ coumarin-6 and rhodamine-B lifetimes estimated using the 2-RLD algorithm.

extraction algorithm, the desired histogram resolution and the predicted lifetime of the sample under investigation. For the 2-RLD algorithm it is stated in [17] that the optimum time-gate width should be 2.5 times the lifetime of the sample. It is for this reason, that the time-gates stated in Table 4.7 were chosen. Due to the longer lifetime of the quantum dot samples and the limited range of the on-chip time-gate generating circuit, the same rule could not be applied during experiments investigating the lifetimes of quantum dot samples.

\subsubsection{Lifetime Measurement Comparison}

Figure 4.19 plots the measured lifetime of quantum dot samples of concentrations ranging from $50 \mu \mathrm{M}$ down to $0.01 \mu \mathrm{M}$. These results were gathered using external TCSPC hardware (and extracted using discrete components analysis) and on-chip time-gated counting, captured using 23, 9 and 2 gates (and extracted using both the IEM and RLD algorithms). From this graph it can be seen that variation in extracted lifetimes is greatest for the samples of lowest concentration $(0.1$ and $0.01 \mu \mathrm{M})$. This can be attributed to the low number of counts that can be gathered, as demonstrated in Table 4.4. For concentrations of $1 \mu \mathrm{M}$ and above, lifetime predictions are much more consistent across the different capturing and extraction methods. The variation in estimated lifetime for each sample is summarised in Table 4.8. These results show how the standard deviation of the predicted lifetime is significantly greater for samples of low concentration. One observation that is made from Figure 4.19, is how variation in the estimated lifetime is lowest for the 2-gate photon counting method. As has been discussed, this can be attributed to the use of wider time gates, which improves photon efficiency and hence increases the number of gathered photons, reducing statistical variation. There will also be a level of variation in estimated lifetime between the different extraction algorithms due to the different mathematical methods employed. From Figure 4.19 and Table 4.8 it can be seen that for concentrations of $1 \mu \mathrm{M}$ and above this variation has a variation of less than $1.3 \mathrm{~ns}$. 


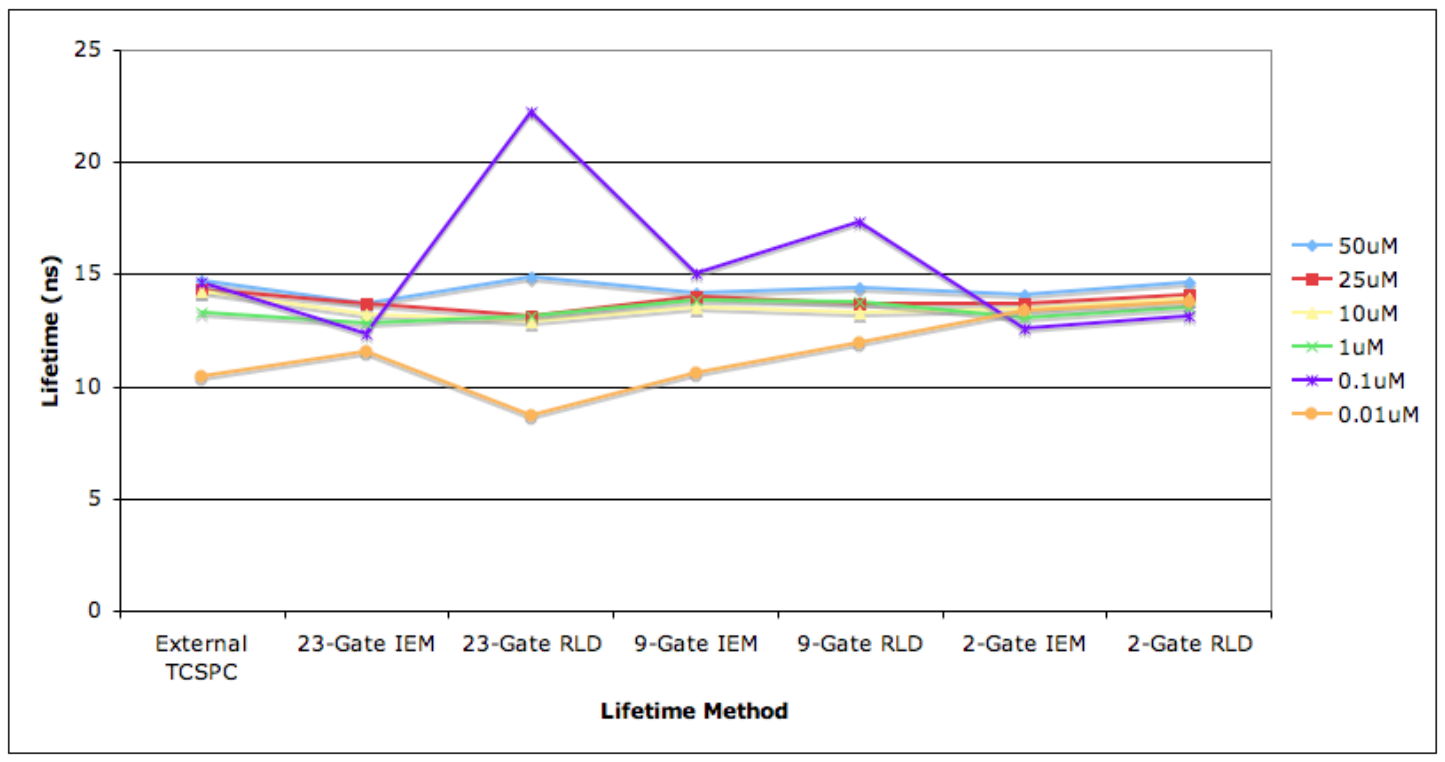

Figure 4.19: Extracted lifetimes of quantum dot samples of varying concentration. Captured using different on-chip time-gated methods and external TCSPC hardware and processed using different extraction algorithms.

\begin{tabular}{llc}
\hline Sample Concentration & Average Lifetime (ns) & Maximum/Minimum Estimated Lifetime (ns) \\
\hline $50 \mu \mathrm{M}$ & 14.367 & $14.9 / 13.7$ \\
$25 \mu \mathrm{M}$ & 13.79 & $14.3 / 13.1$ \\
$10 \mu \mathrm{M}$ & 13.492 & $14.2 / 12.9$ \\
$1 \mu \mathrm{M}$ & 13.335 & $13.8 / 12.8$ \\
$0.1 \mu \mathrm{M}$ & 15.305 & $22.2 / 12.3$ \\
$0.01 \mu \mathrm{M}$ & 11.491 & $13.8 / 8.7$ \\
\hline
\end{tabular}

Table 4.8: Average extracted lifetimes of quantum dot samples of different concentration and the standard deviation across the different capture and extraction methods. 


\subsection{Discussion}

The ability to capture accurate time-resolved fluorescence lifetime data using SPADs and signal processing circuitry integrated in the same CMOS die has been demonstrated. This provides a compact, low cost solution to the detection and processing of lifetime data within a time-resolved fluorescence micro-system. A number of lifetime extraction algorithms have been introduced and evaluated. All three algorithms (2-RLD, N-RLD and IEM) performed well with the counter data. The IEM algorithm holds much promise due to its ability to be implemented on-chip. This has clear advantages, allowing lifetime data to be processed in real-time and without the need for external hardware and software. This would further enhance system integration and lead to a reduction in the time between sample measurement and lifetime estimation. A system whereby the IEM algorithm has been implemented in FPGA firmware, which provides a clear route to an on-chip implementation, has been demonstrated by colleagues at The University of Edinburgh and has been shown to successfully display real-time fluorescence lifetime data from each SPAD in an array of 32x32 (to be published).

The high timing accuracy inherent in the operation of a SPAD detector is ideally suited to time-resolved photon detection and its ability to operate in low light level conditions was demonstrated with the detection of fluorescence emission from low concentration samples. The poor fill factor of the SPAD device is the main issue associated with such detectors. The integration of a detector with signal processing circuitry requires large pixel dimensions. This problem is exacerbated by the large clearance area required by the SPAD from the nearest on-chip circuitry due to the deep n-well used. A fill factor of just $0.14 \%$ results in low count rates and limits the SPAD's efficiency in an experimental environment. The comparison of the SPADs performance to commercially available detectors demonstrated that this is an issue that requires much improvement. In this project, the problem was crudely addressed by placing a convex focusing lens in front of the device. Future designs could include optical concentrators or micro-lenses to address this issue. By directing incident photons away from non-sensitive circuitry and towards the active area of the SPAD, the sensor's fill factor would greatly improve. This would improve the performance of all SPADs in the array, allowing conventional imaging setup to be realised. The lens placed in front of the array focusses light on a small portion of the array and results in a reduction in image quality. Micro-lens technology for CMOS imagers is a well established technology and is used routinely in commercial photodiode based image sensors [100] along with filter arrays. These techniques, if applied to a SPAD based device, 
would improve photon efficiency and hence count rate; thus, reducing experiment times and/or allowing even lower concentration samples to be analysed within a realistic time-scale. The addition of filters would allow future designers to tune the spectral sensitivity of the detector, resulting in improved background light rejection.

One of the key features of CMOS technology is the ability to rapidly switch signals high and low and, therefore circuitry on and off. The design described here has made use of this to define short time-gates that enable and disable the in-pixel counters for time-gated photon counting. While these time-gates define the state of the counting circuitry, they do not control the activity of the SPAD detector. While the counter at the output of the SPAD may be off, the detector itself remains active. This is true for every element in the array. It has been shown that the high level of SPAD activity across the array results in noise on the common SPAD bias supplies (VOP and SPAD_AVDD). It is thought that this results in modulation of both the SPADs PDP and dark count characteristics and could lead to increased detector noise in high light level conditions (Section 4.3.1.1). By taking advantage of the rapid switching properties of CMOS, it has been shown [101] that it is possible to de-activate the SPAD detector outwith the time-gate period, which would greatly reduce on-chip activity and hence improve noise performance. This technique could be used as part of a micro-system to render the SPAD insensitive to light during the excitation phase of a time-resolved fluorescence experiment before rapidly activating the SPAD to capture the fluorescence decay. This filtering in time technique would remove the need for an optical filter, further reducing system costs and size.

The 9-bit counters within each pixel allowed 512 counts to be accumulated in each time-gate period before requiring to be read out and reset. Failure to do this resulted in loss of data. The photon arrival statistics associated with low light level fluorescence lifetime experiments means that a photon will not be detected in every time-gate period. It was therefore possible to increase the number of times a time-gate period was evaluated before the readout process was done. The maximum number of counts achieved per read out could be increased by extending the number of bits in the counter [102]. This would lead to an increase in pixel size and thus a reduction in fill factor if implemented in the same $0.35 \mu \mathrm{m}$ technology. By implementing such a circuit in a smaller dimension technology, the pixel size could be retained or reduced; thus improving fill factor and potentially increasing the array density.

By increasing the pixel size or moving to a smaller technology it would also be possible to include additional counters within the pixel. This would allow a greater number of time-gate 
periods to be evaluated simultaneously. This feature has been demonstrated here using two counter circuits to capture two different time-gate periods simultaneously and efficiently implementing the 2-RLD lifetime extraction algorithm. This technique could be extended further to more efficiently implement multi-gate algorithms (M-RLD and IEM, Section 4.4.1), improving photon efficiency and reducing sample exposure to excitation light and experimental time. It is interesting to note how the 2-RLD algorithm is less prone to error at lower sample concentrations due to the use of wider time-gates and hence longer photon collection times. This increases the number of counts per time bin as less photons fall outwith the time-gate window. By collecting two time-gate periods simultaneously using the two in-pixel counters, photon efficiency and hence the number of photons counted for a given experimental time is further enhanced.

A direct comparison of the performance of the on-chip time-gated circuitry and a commercially available time-correlated single-photon counting module has been made. While the histogram resolution (bin width) that has been achieved in the on-chip solution is not as fine as the commercial system (400ps versus $813 \mathrm{fs}$ ), the accuracy of extracted lifetimes from the captured decay data is comparable. It should also be kept in mind that the resolution that can be achieved with a CMOS solution is defined by the minimum gate delay of the technology. In $0.35 \mu \mathrm{m}$ CMOS technology this gate delay is equal to approximately 200ps (hence a 400ps delay through a two-stage buffer). As CMOS technology dimensions scale the minimum gate delay also reduces. For example, in $0.13 \mu \mathrm{m}$ CMOS technology the minimum gate delay is approximately 20-30ps. It is therefore reasonable to predict that as SPAD technologies become available in smaller dimension technologies [72], the time-bin resolution of a time-gated system will improve. This improvement would be in addition to the advantages of greater signal processing circuit complexity and/or improved pixel pitch. It should be noted, however, that in order for SPADs to be successfully implemented in such technologies the issue of extremely high dark count $(90 \mathrm{kHz}$ at room temperature [72]) must be addressed [103]. 


\section{Chapter 5 Micro-LED Devices and Drivers}

One of the most expensive, delicate and bulky components of any time-resolved fluorescence system is the pulsed laser excitation source [104]. Such devices have a single, fixed emission wavelength; therefore, fluorescence imaging systems often contain multiple laser sources to span a greater excitation wavelength range. This greatly adds to the cost and size of the system. Many commonly used fluorophores are excited in the near ultraviolet (UV) and blue regions of the optical spectrum. The advent of Gallium Nitride $(\mathrm{GaN})$ and other blue emitting semiconductor sources, around 15 years ago [105], has helped stimulate interest in the use of these sources instead of lasers or mercury discharge lamps for excitation in fluorescence-based instrumentation. The advantages of these sources include low cost, reliability, compactness and good wavelength matching to many standard fluorophores. In addition, their ability to produce short (sub nano-second) pulses offers the opportunity for low cost lifetime measurements.

Previously, GaN micro-LED arrays developed at The Institute of Photonics at the University of Strathclyde, were matrix addressed and externally driven by a commercially available LED driver module (Electro-Optic Device, Inc., ETX-10A, single supply, 5-volt, pulsed laser diode driver with integrated high voltage power supply)[106], [85], [84]. Using this technique only a single element in the array could be active at any one time. There are also difficulties associated with scaling the number of elements in the array as each device required a unique row and column signal. This presented fan-out problems due to the number of address lines that were required. CMOS driven micro-LEDs present a solution to this problem thanks to the ability to multiplex addressing control signals. The use of latched driver inputs in the array also meant each element in the array could hold its state, allowing multiple devices to be activated simultaneously.

Throughout this project there were a number of iterations of the micro-LED driver array design. The aim was to produce short optical pulses of sufficient power to excite fluorescent dyes for time-resolved measurements. This chapter briefly describes the AlInGaN micro-LED devices developed at the Institute of Photonics before introducing the two different driver circuits that have been implemented here and detailing their performance. The bump-bonding process 
used to attach the driving CMOS die to the GaN LED array is described and a number of issues associated with this technique are discussed. A comparison of the performance of the CMOS-driven micro-LED array versus a commercially available diode laser is also made.

\subsection{6x4 Current Controlled Micro-LED Driver Array}

The original micro-LED driver array formed part of the same test chip as the SPADs with counter array (described in Chapter 4). In Figure 5.1 a floor plan of the chip shows how the two arrays shared the die area.

A 16x4 array of drivers has been implemented here. Each pixel measures $100 \mu \mathrm{m}$ by $200 \mu \mathrm{m}$ and contains a single micro-LED driver circuit, capable of switching up to $50 \mathrm{~V}$ onto a metal electrode formed from the top metal layer of the $0.35 \mu \mathrm{m}$ high voltage CMOS process. The electrode array was depassivated by pad opening at the foundry to reveal the aluminium top-plate electrodes for bump-bonding (Figure 5.2). Incorporated into each electrode is a SPAD detector.

Of the four metal layers available in the $0.35 \mu \mathrm{m}$ high voltage CMOS process from Austria Microsystems, the first two layers were used for signal routing, the third formed a shield to protect the underlying electronics from the high-voltage signals being driven onto the array and the fourth layer was used to pattern the electrodes. Each electrode occupied almost the full pixel space $(100 \mu \mathrm{m} \times 200 \mu \mathrm{m})$ with $19090 \mu \mathrm{m}^{2}$ of top metal exposed. Electrode selection was controlled using row and column addressing. Micrograph images of the bump-bonded 16x4 CMOS and LED arrays can be seen in Figure 5.3.

A SPAD was placed within each driver pixel and had the same structure as those described in Section 2.6.1. No signal processing circuitry was located within the pixel, with the detector output being fed directly off-chip for observation and analysis.

The number and position of the LED pixels that are active is user definable and any combination from a single LED up to all 64 elements of the array can be active at any one time. During analysis, all 64 SPADs in the array are active. However, the chosen address defines which SPADs are present on the output bus, thus the address of the detecting SPAD does not have to correspond to the address of the active micro-LED. 




Figure 5.1: Floor plan of the original micro-LED driver test chip, part of the same test chip which featured the SPADs with counters array (Chapter 4). 


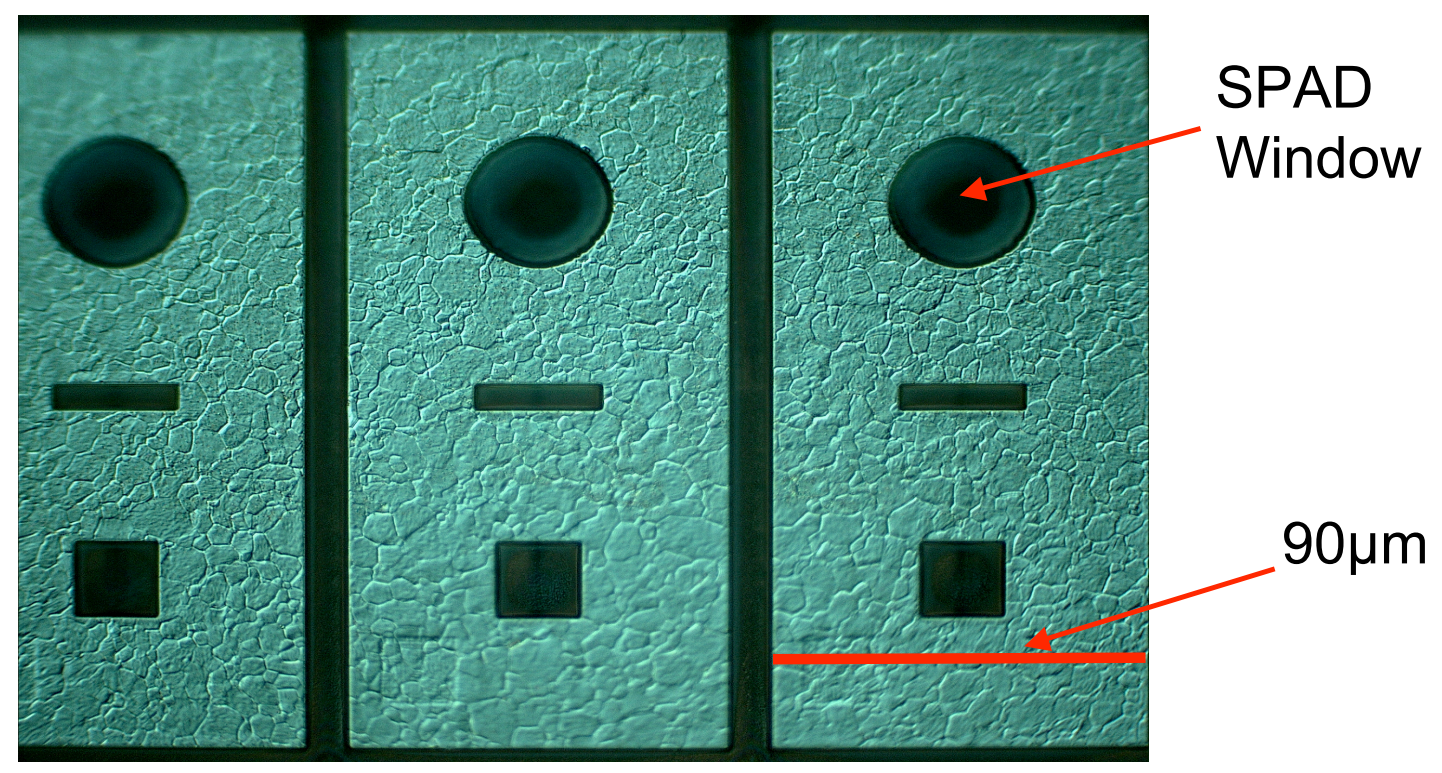

Figure 5.2: Micrograph of the individual top metal layer plates upon which each micro-LED in the array is bump-bonded. The SPAD detectors are situated in the circular gaps in the metal.
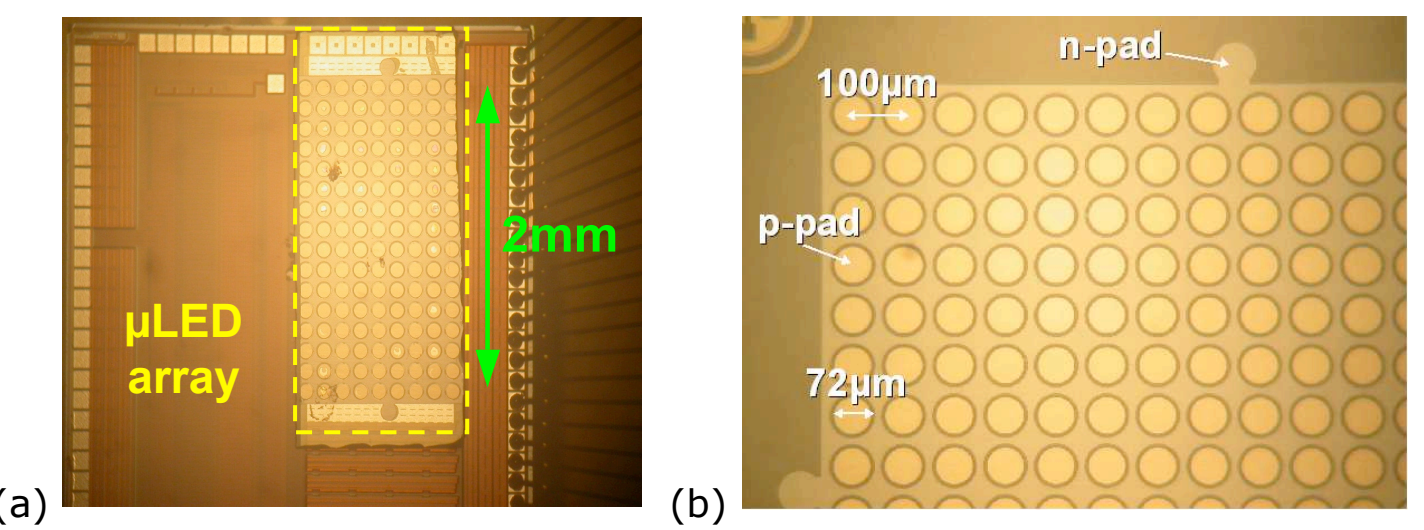

Figure 5.3: (a) Micrograph of 16x4 driver array with bump-bonded micro-LED array; (b) close-up of array highlighting array dimensions. 


\subsubsection{Driver Circuitry}

Each of the 64 elements in the array contained a dedicated driver circuit situated directly below the electrode. A schematic of the driver circuit implemented can be seen in Figure 5.4.

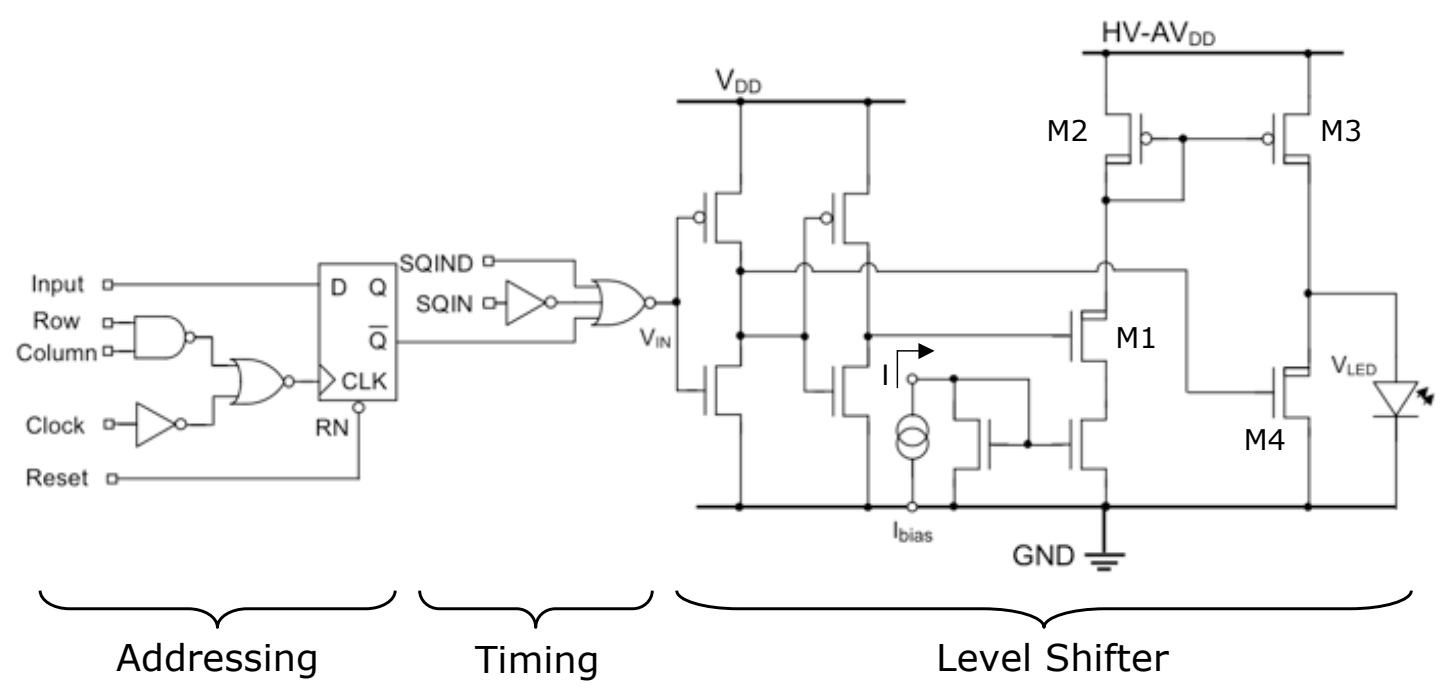

Figure 5.4: Current controlled micro-LED driver, situated under each electrode in the array.

The addressing and timing circuitry within the driver circuit used 3.3V logic, a level-shifter was then used to convert this to a user definable voltage $\left(\mathrm{HV}_{-} \mathrm{AV}_{D D}\right)$ that could be varied from $3.3 \mathrm{~V}$ up to $50 \mathrm{~V}$. On-chip $\mathrm{X}$ - and Y-decoders define the row and column signals and the reset signal was global. The D-type flip-flop allowed the driver state to be stored, allowing devices to remain on after address logic had been de-asserted. In order to assert a driver state, the desired logic is placed on the input line. On the following rising edge of the clock, this state is passed to the D-type flip-flop output. The clock signal can only be seen by the flip-flop if the correct addressing logic has been asserted. The timing signals, SQIN and SQIND, were generated by the same timing generator circuit used to define the time-gates for the SPAD counter circuit (Section 4.6). The difference in time between these two signals is used to generate an input pulse to the level-shifter in the circuit. Inputs SQIN and SQIND can also be disabled, allowing DC and square wave signals to be used as the input to the driver circuit. The input signal to the level shifter (Vin) defines how long transistor M1 is turned on. The current, Ibias, is mirrored via transistors M2 and M3 to the micro-LED device. Ibias is used to control the current flowing in the LED. The voltage seen by the LED is defined by HV_AV $D D$. Therefore, the current in the device is a trade-off between the IV curve of the device (Figure 2.14) and Ibias. When Vin 
goes low, M1 is turned off and M4 is turned on, discharging the micro-LED rapidly to ground. By making use of a level-shifter circuit just four high-voltage (thick-oxide) transistors were required. These are physically large and occupy the majority of the pixel space. A layout view of a single driver pixel can be seen in Figure 5.5.

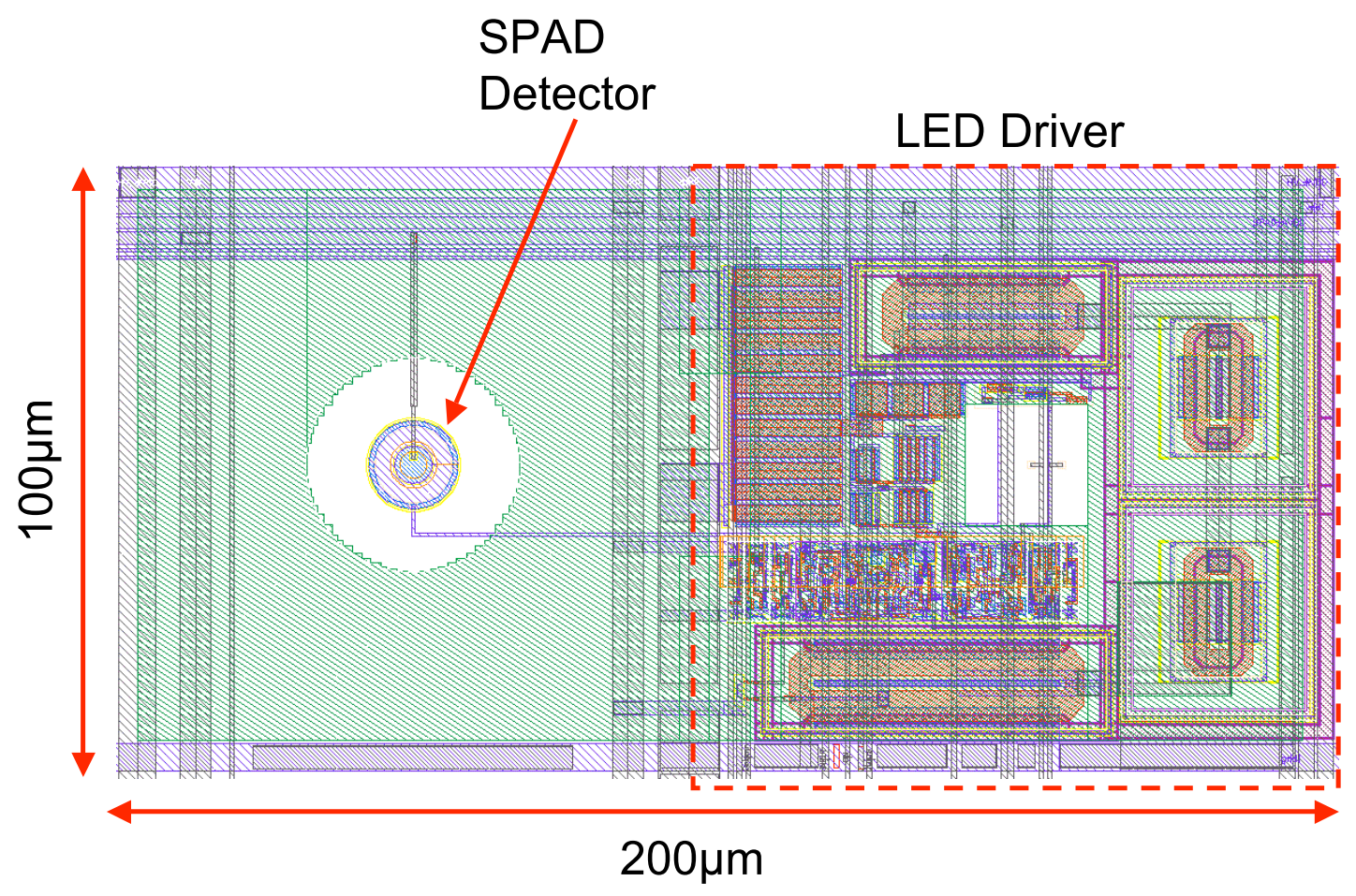

Figure 5.5: Layout of a single micro-LED driver pixel within the 16x4 array. Situated within each driver is a single SPAD detector. 


\subsubsection{6x4 Driver Array Performance}

A number of micro-LEDs can be driven simultaneously in a user definable pattern (Figure 5.6) In a micro-fluorescence system this feature could be used to stimulate multiple areas of the sample of interest (or multiple samples) simultaneously.

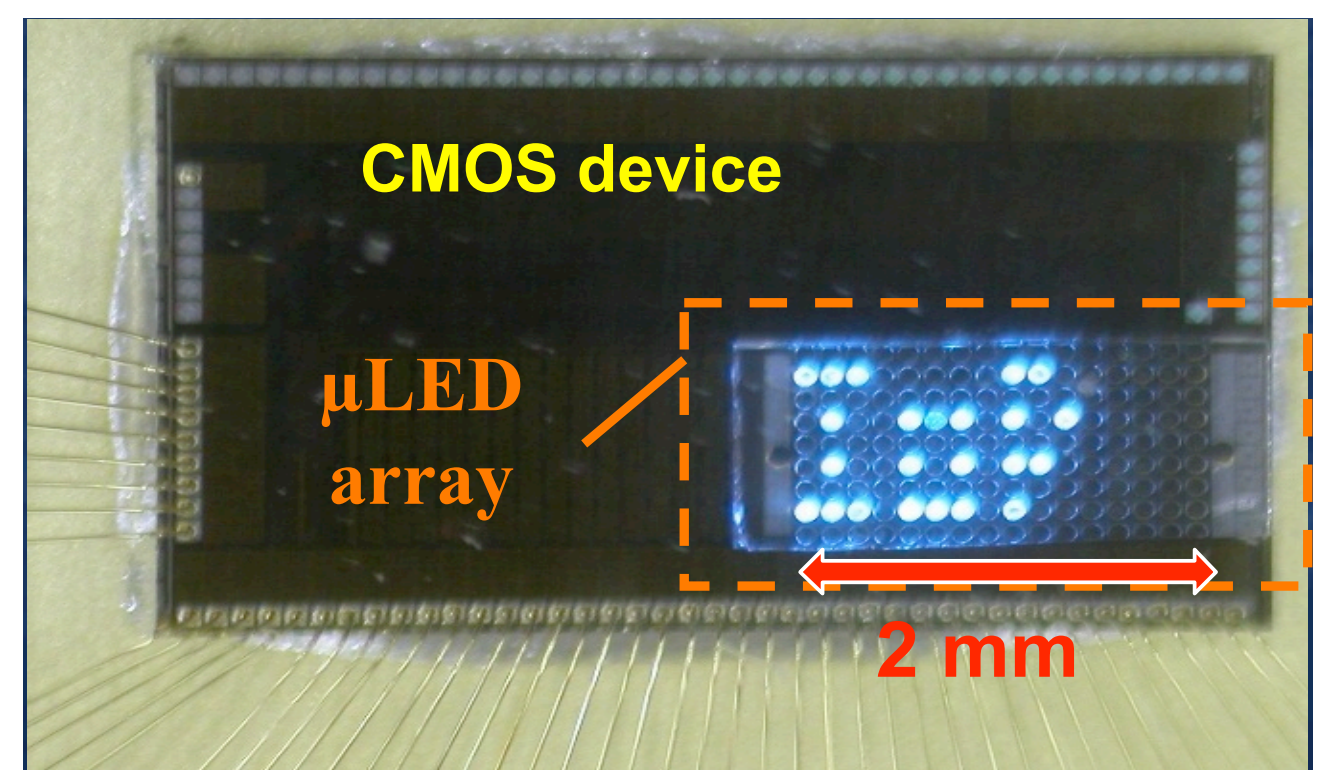

Figure 5.6: 23 micro-LED devices operating simultaneously. 


\subsubsection{Array Uniformity}

Good array uniformity can be achieved by controlling the current flowing in each LED. The exponential IV characteristics of the LED mean that small changes in LED voltage result in large changes in current flow in the device. The optical output power, with respect current characteristics, are approximately linear (until the device reaches saturation). A current controlled driver reduces the effects of variation in the drive strength of each element in the array.

The uniformity of a CMOS driven UV (370nm) micro-LED array is presented in Figure 5.7. The optical output power was measured for each element in the array biased at the same voltage $\left(\mathrm{HV}_{\text {AAvdd }}=15 \mathrm{~V}\right)$ and current $($ Ibias $=2 \mathrm{~mA})$. The average optical power across the array was $6.34 \mu \mathrm{W}$. However, not every element in the array was functional, this could be caused by failure in the bump-bonding process. When other LED/CMOS arrays were bump-bonded, it was also found that a number of elements were non-functional, but the addresses of these elements were not consistent between devices. This failure appeared much more dramatically in a subsequent chip design (see Section 5.2).

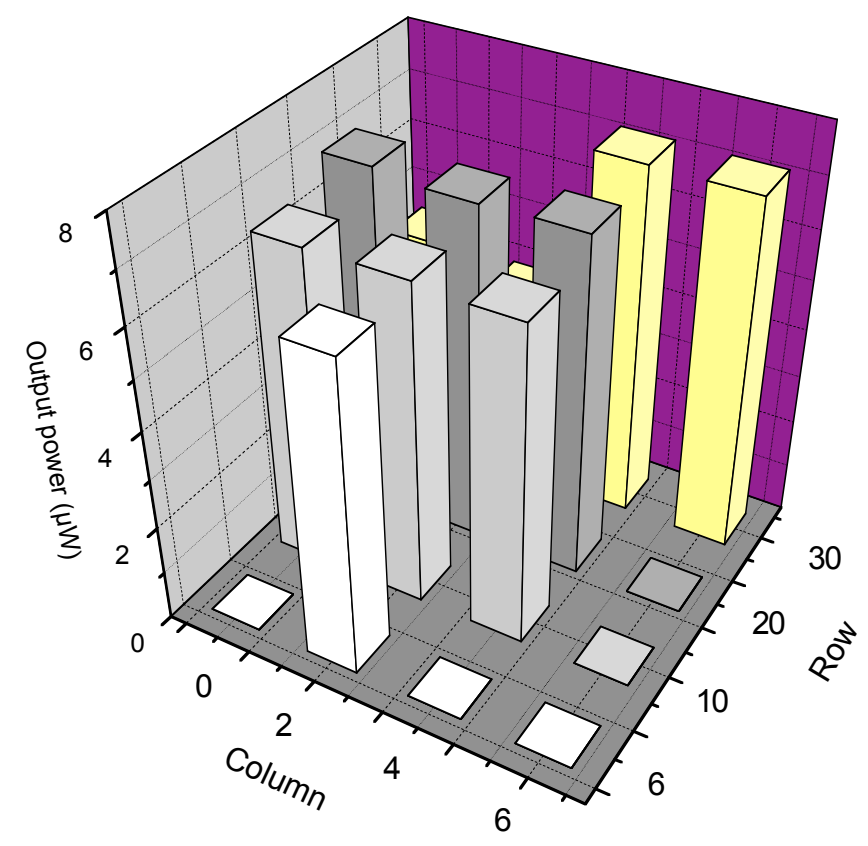

Figure 5.7: Uniformity of a CMOS driven UV (370nm) micro-LED array.

Variation in the bump-bonding process can also lead to non-uniformity in LED power across 
the array. In this design the bias current Ibias is mirrored from a single transistor, situated to the right of the driver array, to each of the array elements. Elements further away from this transistor will have different ground path characteristics. This problem is further exasperated by variation or failure in the n-type ground bump-bond contacts at the four edges of the array.

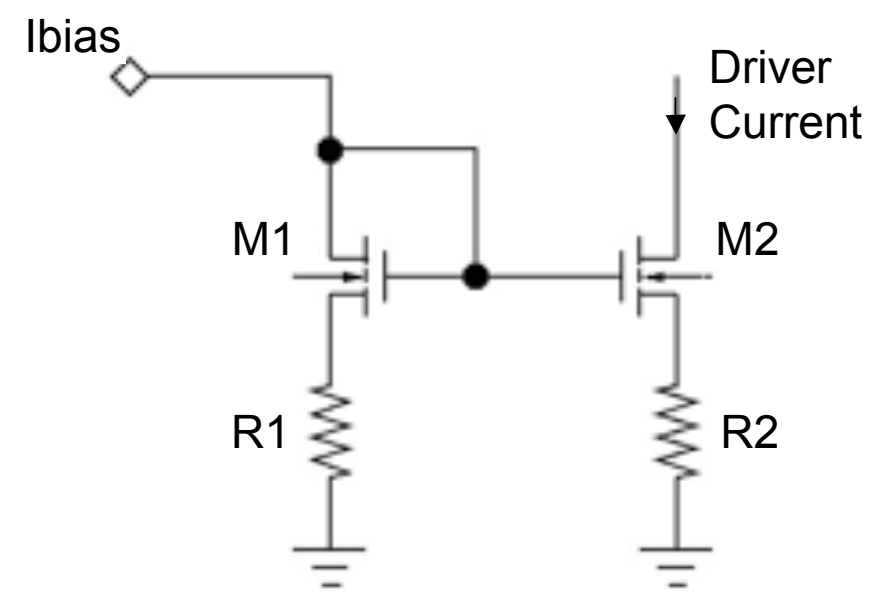

Figure 5.8: Input bias current mirror, with additional source resistance due to track length and bump-bonding.

As illustrated in Figure 5.8, if R1 is equal to $100 \Omega$, R2 is equal to $200 \Omega$ and Ibias is $5 \mathrm{~mA}$, then there is a $500 \mathrm{mV}$ drop across $\mathrm{R} 1$ and a $1 \mathrm{~V}$ drop across $\mathrm{R} 2$. This results in a different gate-source voltage for M1 and M2 and therefore a different current in both. The resistance, $\mathrm{R} 2$, could potentially be different in every element in the array. 


\subsubsection{Optical Power and Pulse Performance}

The optical output power of different elements in the array was assessed using a Coherent FieldMaxII-TO power meter. When the bias current is swept from 0 to $5 \mathrm{~mA}$ and the high voltage supply is fixed at $15 \mathrm{~V}$, a maximum optical output power of $10 \mu \mathrm{W}$ is attained (Figure 5.9). These output powers are in good agreement with those reported in [107], where power measurements were made on a matrix-driven micro-LED array flip-chip bonded to a printed circuit board.

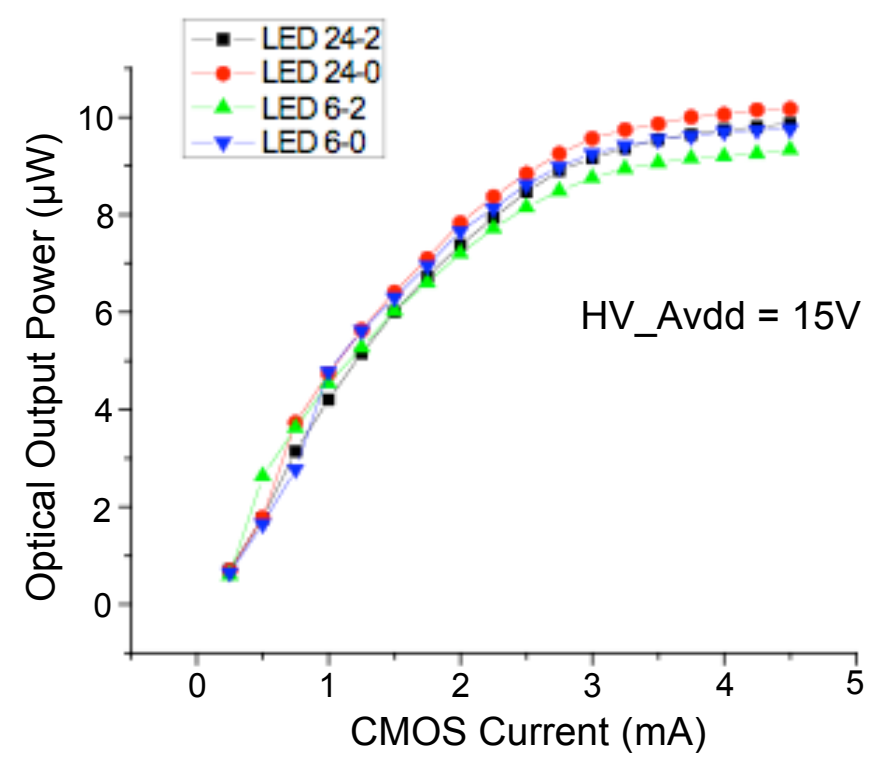

Figure 5.9: Optical output power of a UV (370nm) device versus bias current for different elements in the array being driven by a DC signal.

A square wave input signal can also be used as the driver input. For a 50\% duty ratio square wave the resultant optical output power is half that of a DC driven device (Figure 5.10).

Pulse performance would be critical for the successful implementation of the micro-LEDs in a time domain miniaturised fluorescence lifetime analysis device. Due to the increased capacitance on the output of the driver attributed to the presence of the bump-bonds and micro-LED the circuit was incapable of producing 400ps optical pulses, which is the shortest electrical pulse width that the on-chip delay circuit could achieve. The capacitance restricts the rise and fall time of the driver output and the peak voltage attained in the pulse time was insufficient to turn on an LED element. The pulse width is fully adjustable and user-selectable and is defined by the delay range of the on-chip timing generator circuit. Figure 5.11 shows 


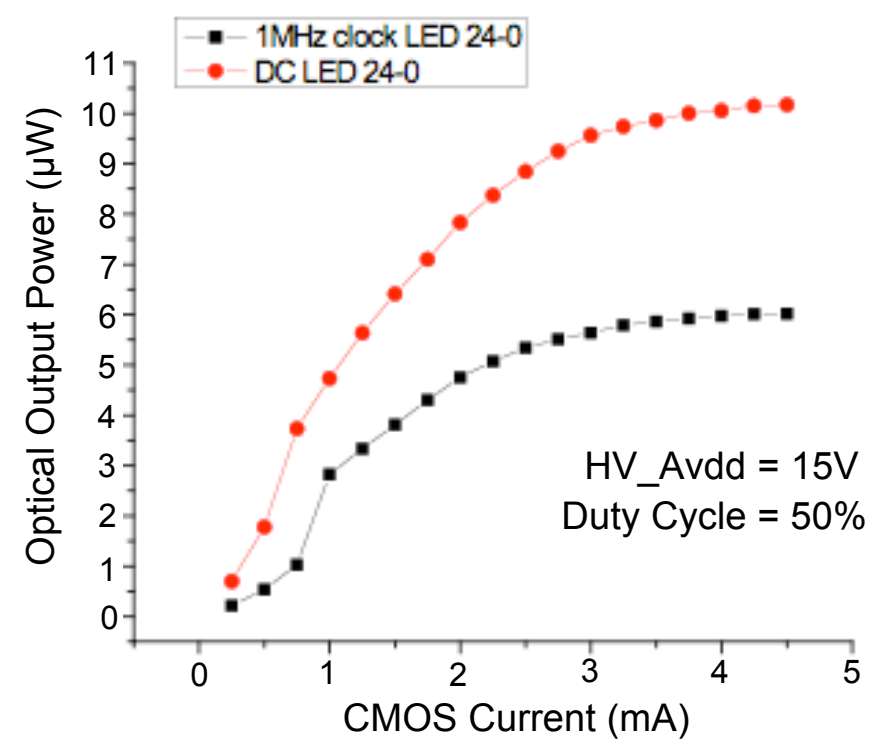

Figure 5.10: Comparison of the average optical output power of a DC driven UV device and a square-wave (50\% duty cycle) driven device.

the longest and shortest optical pulses that were achieved. The 400ps resolution of the timing generator also defines the resolution of achievable pulse widths. The optical pulse width can therefore be adjusted from $1.12 \mathrm{~ns}$ to $47.87 \mathrm{~ns}$ (full width half maximum, FWHM) in steps of 400ps.

The average optical output power of a micro-LED in pulsed mode is dependent on three variables, bias current, repetition rate and pulse width. Figure 5.12 demonstrates how the output power changes with varying bias current for a fixed pulse width of $20 \mathrm{~ns}$.

When the electrical pulse width from the driver is short, the rise and fall times of the pulse below the turn-on voltage of the LED begin to dominate. This resulted in lower than expected optical output power and limits the performance of the driver when short pulses are required (Figure 5.13). 


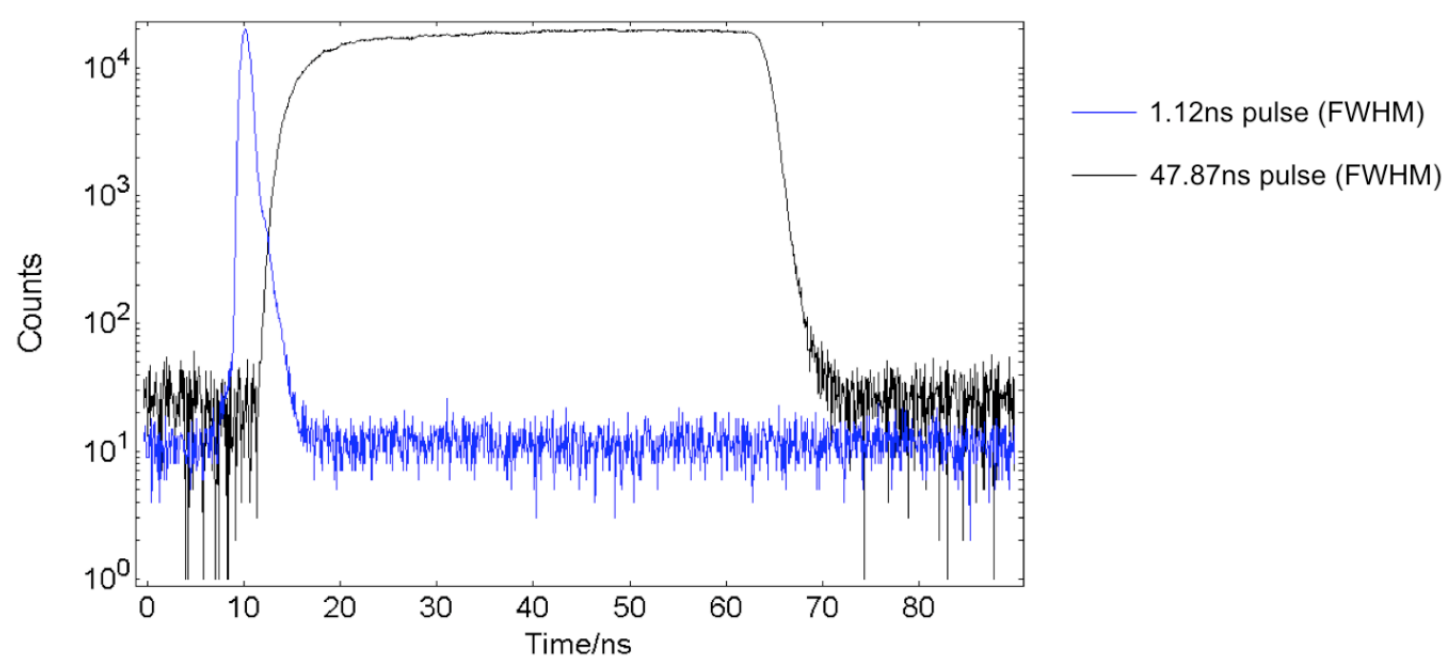

Figure 5.11: The shortest and longest pulse widths achieved.

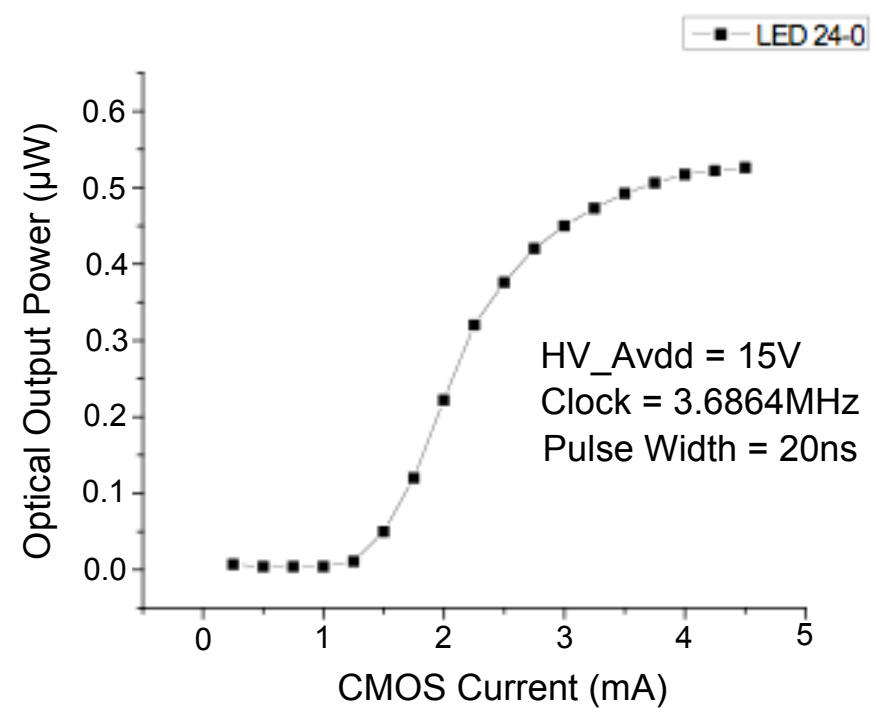

Figure 5.12: Average optical pulse power versus bias current. 


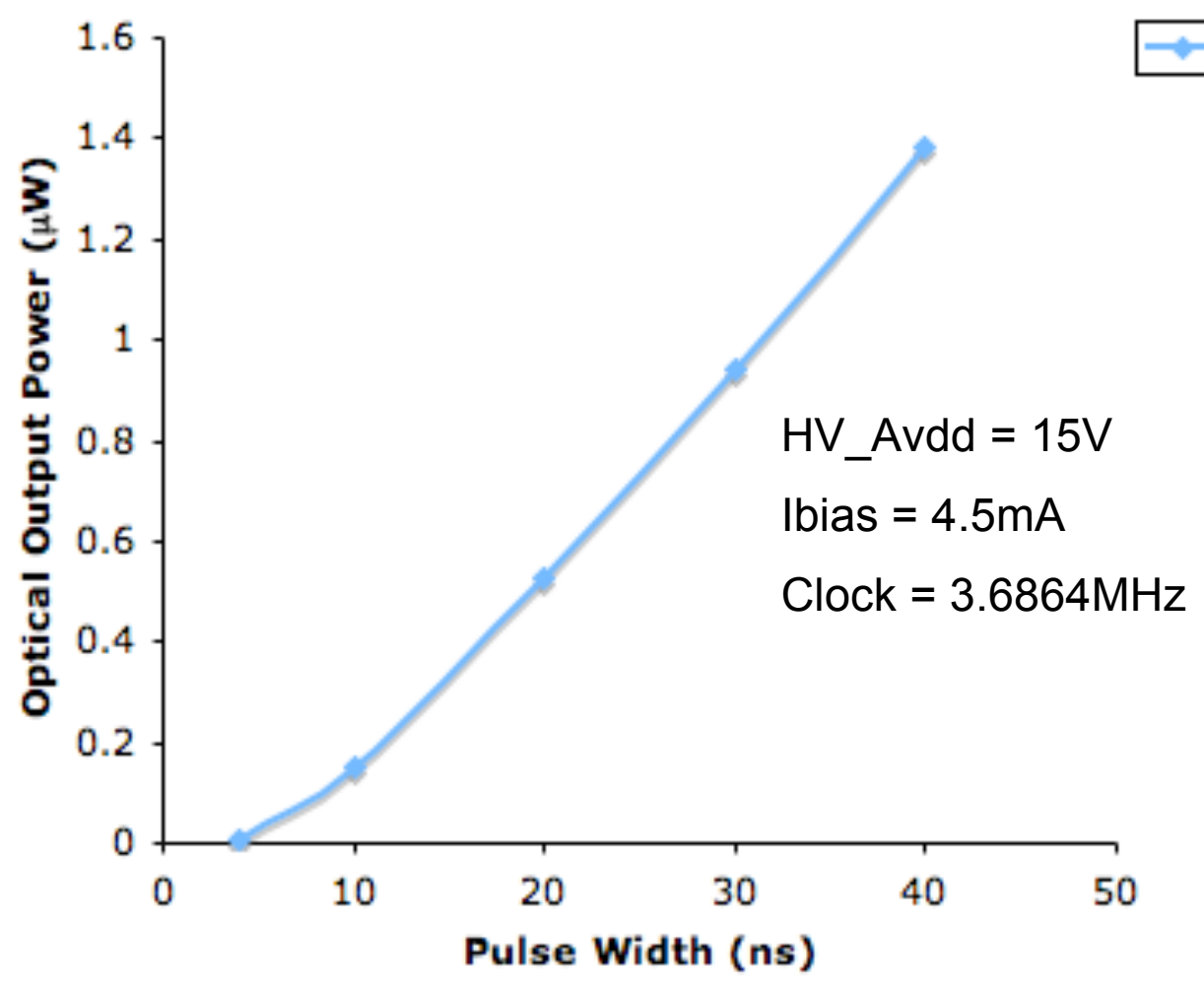

Figure 5.13: Average optical pulse power versus pulse width. 


\subsection{6x16 Voltage Controlled Micro-LED Driver Array}

The 16x16 driver array described herein was a dedicated micro-LED driver chip. The aim of this design was to increase the number of elements in the array, increase optical output power and to reduce the minimum optical pulse width, with respect to the driver design described in Section 5.1.

To achieve these goals, a voltage controlled (rather than current driven) driver was implemented, thereby sacrificing array uniformity in favour of optical power. The design was also implemented in a standard $0.35 \mu \mathrm{m}$ CMOS technology from Austria Microsystems rather than a high voltage process. Each pixel measured $100 \mu \mathrm{m}$ by $100 \mu \mathrm{m}$. The metal electrode upon which each LED is bump-bonded was formed from the top metal layer of the CMOS process and occupied the full area of each pixel. All drive circuitry was situated under each electrode (chip micrograph, Figure 5.14). In this CMOS process, two variants of transistor were available; standard 3.3V transistors and physically larger 5V transistors. The turn-on voltage of a UV LED is approximately $4.5 \mathrm{~V}$ (Figure 2.14), therefore it was necessary to implement the higher voltage $5 \mathrm{~V}$ transistors in the output stage of the driver circuit. To allow these devices to handle higher voltages, a thicker gate-oxide is used in the transistor design. This increases the minimum gate length (from $0.35 \mu \mathrm{m}$ to $0.5 \mu \mathrm{m}$ ) and the dimensions of the transistor. In an area limited design such as this, the additional area occupied by these high-voltage transistors posed a problem. Their use was therefore limited to the output stages of the device.

Additional features that were included in the design were; (i) a variable frequency voltage controlled oscillator (VCO) to allow clock signals to be produced on-chip; (ii) a series of linear feedback shift registers (LFSR's) producing pseudo-random bit patterns that could act as the driver input, mimicking data communications. 




Figure 5.14: $16 x 16$ micro-LED driver array top level layout. 


\subsubsection{Driver Circuitry}

A dedicated driver circuit (Figures 5.15 and 5.16) was situated below each electrode in the array.

The addressing logic used in this design was identical to that of the original $16 \times 4$ driver array (Section 5.1), allowing an element to remain in a particular state while other elements in the array are being addressed. All driver input signals were based on $3.3 \mathrm{~V}$ logic before being level-shifted to a higher user-definable voltage (LED_VDD) which could have a maximum value of $5 \mathrm{~V}$ (Figure 5.17). This allowed standard 3.3V logic to be used for the addressing and control logic in the pixel before the signal level is increased to LED_VDD which requires the use of physically larger transistors capable of handling $5 \mathrm{~V}$.

The driver had four different modes of operation: DC, square-wave, pulsed or externally driven. A square wave, DC or external signal on INPUT_SIG could be passed directly to the driver input if MODE_CONTROL_1 was asserted high. When MODE_CONTROL_1 was set low the driver was placed in pulsed mode. In pulsed mode, the square-wave input signal is passed through inverter I1, and the time delay through I1 defined the pulse width (Figure 5.18).

The inverter delay could be adjusted via the gate voltage (VBMC2) of the current starving transistor M1. The level-shifted DC, pulsed, or square wave signal is then passed to a chain of inverters designed using transistor capable of handling up to $5 \mathrm{~V}$. To minimise load capacitance on the input signal while maximising the drive strength of the circuit, the output buffer comprised a chain of inverters of ever-increasing transistor width/length ratios was implemented. The capacitive load seen by the driver input is defined by the width and length of the transistors of the first stage of the buffer chain. The drive strength of an inverter is also proportional to transistor width and length. Therefore relatively small transistor dimensions were chosen for the first stage of the output buffer (PMOS width $=2.3 \mu \mathrm{m}$, length $=0.5 \mu \mathrm{m}$, NMOS width $=1 \mu \mathrm{m}$, length $=0.5 \mu \mathrm{m}$ ). In order to increase the circuit drive strength, the output signal then passes through a series of transistors of increasing width (length is kept at a minimum $=0.5 \mu \mathrm{m}$ ), culminating in an output inverter with a $1080 \mu \mathrm{m}$ wide PMOS transistor and a $450 \mu \mathrm{m}$ wide NMOS transistor. Due to the difference in electron and hole mobility, a ratio of approximately 2.5 is maintained between the width of the PMOS and NMOS transistors in each inverter. This ensures that the rise and fall times of the inverter output are equal. 


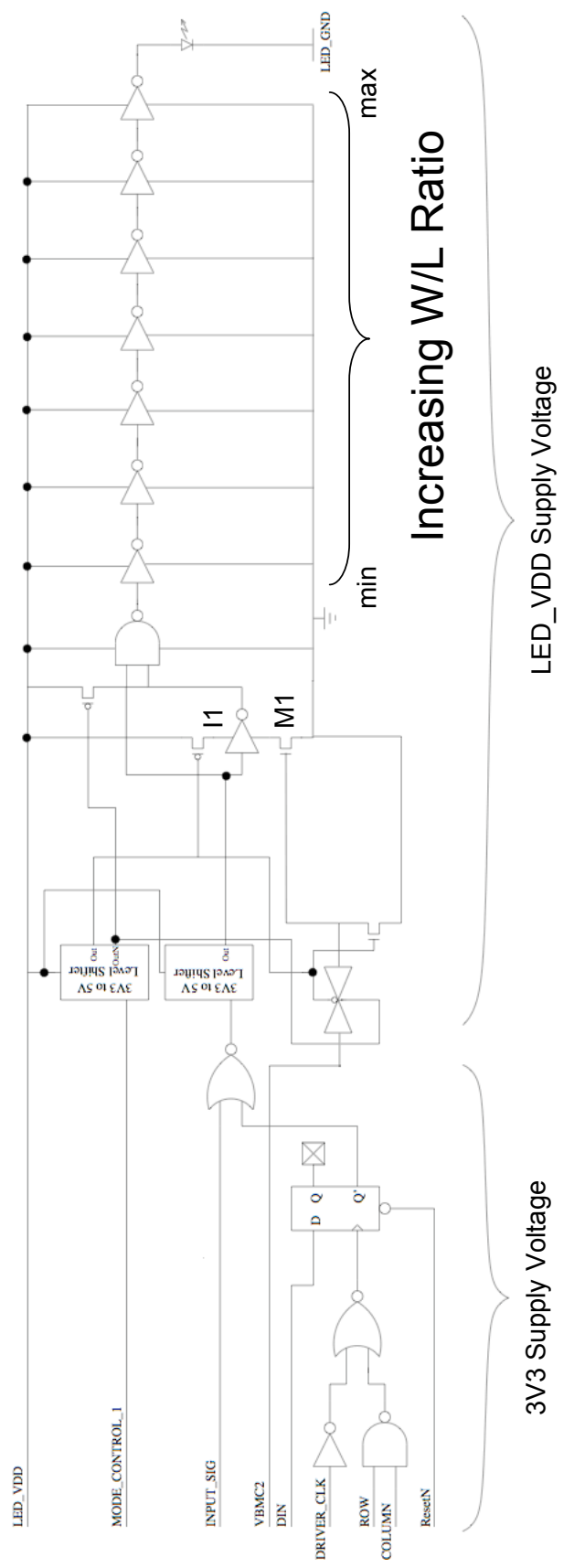

Figure 5.15: Driver circuit implemented in $16 \times 16$ micro-LED driver array. 


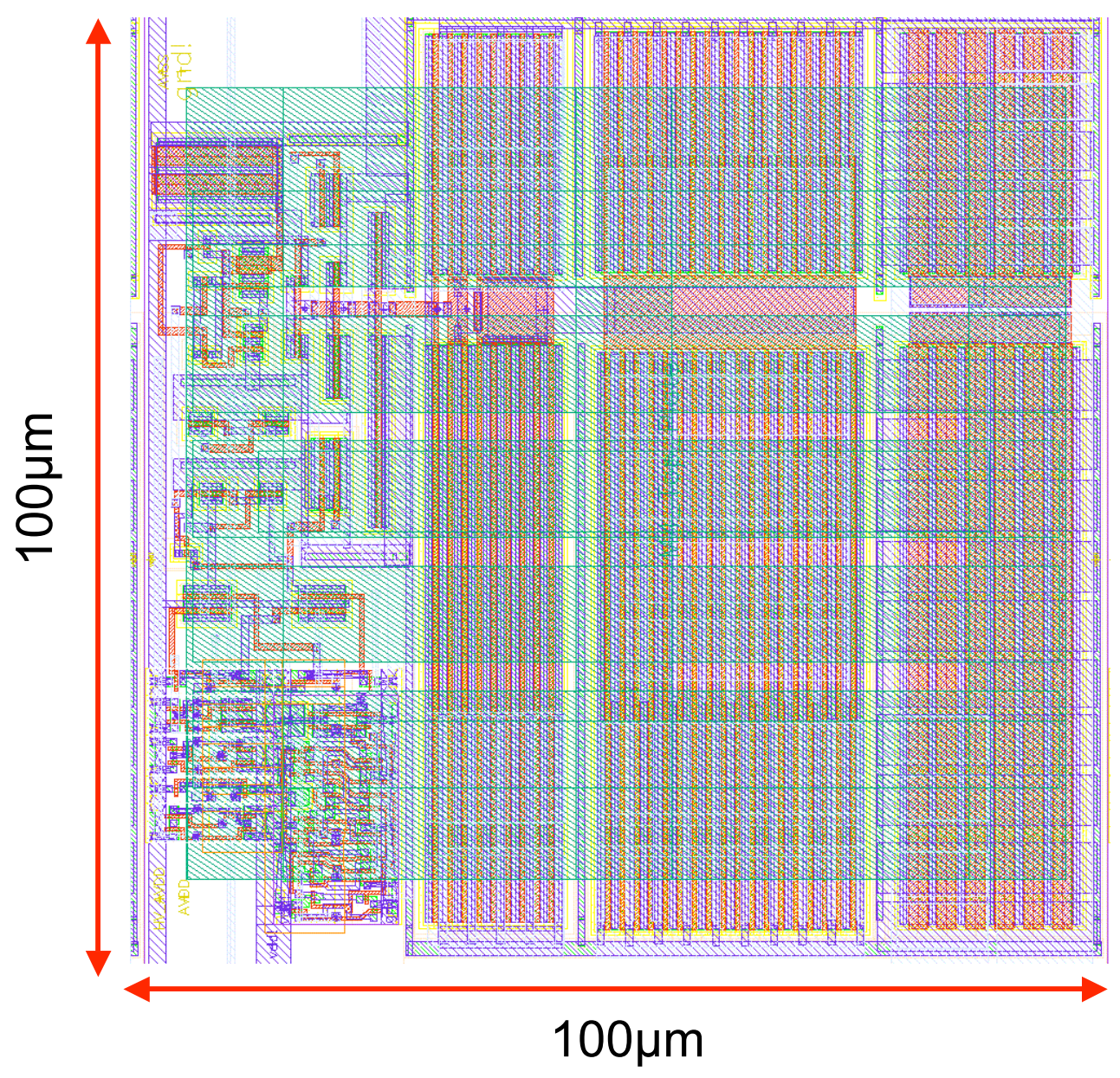

Figure 5.16: Layout of a single micro-LED driver pixel within the $16 x 16$ array. 


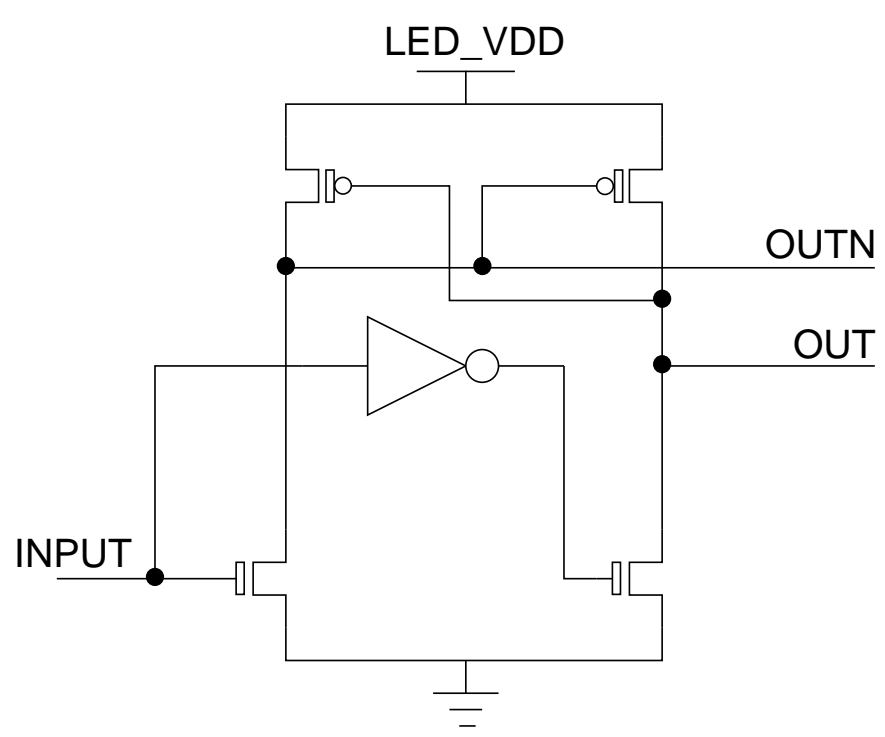

Figure 5.17: In-pixel level shifter circuit. 

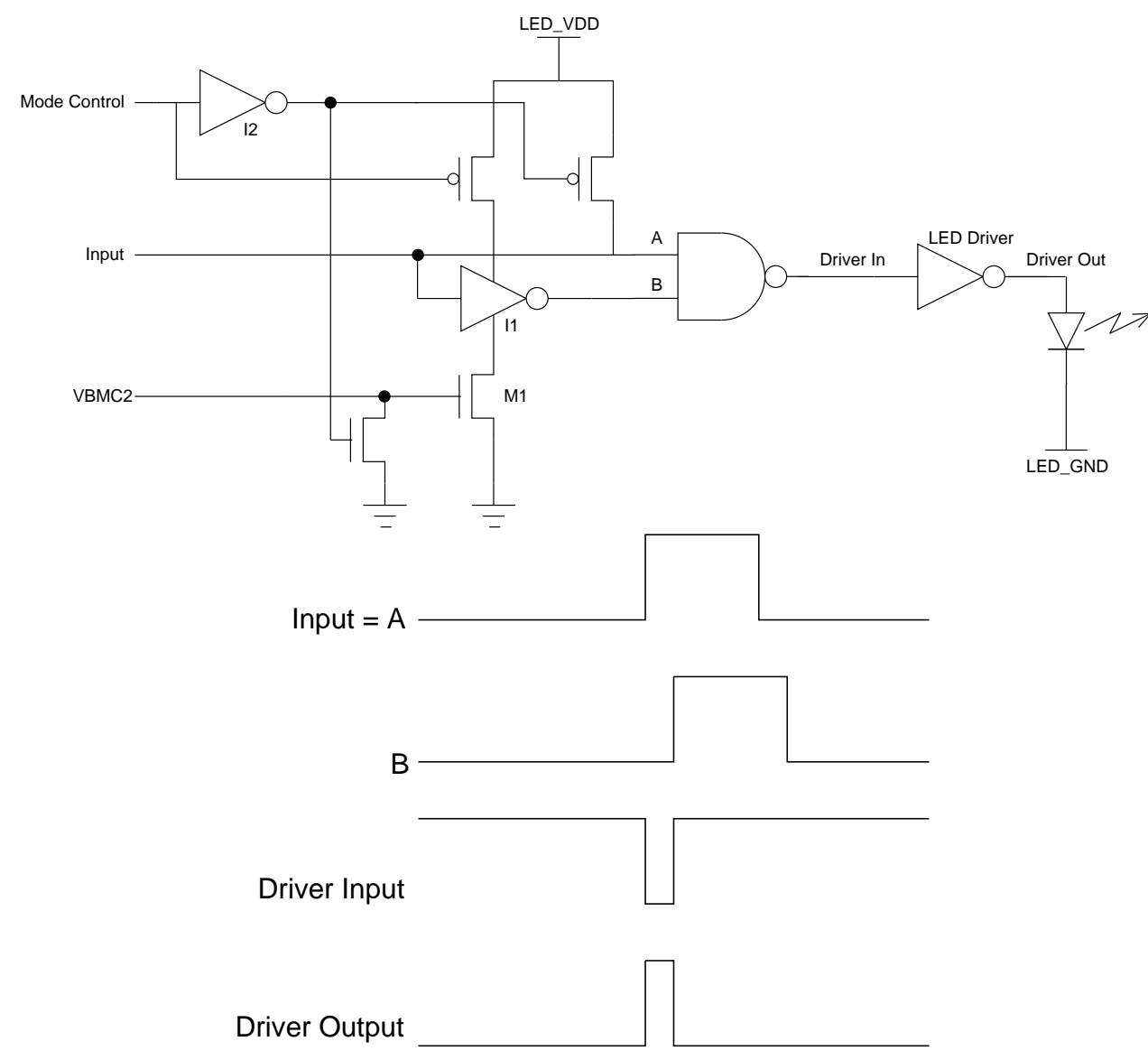

Figure 5.18: In-pixel pulse generator. Pulse width is equal to the inverter delay which is defined by VBMC2. When Mode Control is asserted the delay inverter is bi-passed and the inverter output tracks the circuit input. 
The current delivered by the driver was set by the IV curve of the micro-LED device and the user-definable output voltage (LED_VDD).

No voltage is dropped across the diode when the LED driver first begins to supply current to a micro-LED. Therefore, the drain-source voltage $\left(\mathrm{V}_{D S}\right)$ across the PMOS transistor of the driver output stage is equal to -LED_VDD (0V - LED_VDD). Since the gate-source voltage $\left(\mathrm{V}_{G S}\right)$ is also equal to -LED_VDD and the transistor threshold voltage $\left(\mathrm{V}_{T}\right)$ is equal to $-0.97 \mathrm{~V}$, this places the transistor in the saturation region of operation. When in saturation the transistor is capable of delivering greater than $500 \mathrm{~mA}$ per device. Equation 5.1 is a first order calculation of the current handling capabilities of the output PMOS transistor when in saturation. $K^{\prime}$ is the device-transconductance factor and is defined by the foundry (Austria Microsystems $0.35 \mu \mathrm{m}$ CMOS Process Parameters document).

\begin{tabular}{ll}
\hline Device Width $(W)$ & $1080 \mu \mathrm{m}$ \\
Device Length $(L)$ & $0.5 \mu \mathrm{m}$ \\
$K^{\prime}$ & $31 \mu \mathrm{A} / \mathrm{V}^{2}$ (typical) \\
$V_{T}$ & $-0.97 \mathrm{~V}$ \\
\hline
\end{tabular}

$$
\begin{gathered}
I_{D}=\frac{K^{\prime} W}{2 L}\left(V_{G S}-V_{T}\right)^{2} \\
I_{D}=\frac{31 \times 10^{-6} \times 1080 \times 10^{-6}}{2 \times 0.5 \times 10^{-6}}(-5+0.97)^{2} \\
I_{D}=543.7 \mathrm{~mA}
\end{gathered}
$$

As current begins to flow in the diode the voltage dropped across the device begins to increase, resulting in a reduction in the drain-source voltage of the PMOS output transistor. This causes the transistor to move from the saturation to the linear region of operation. Once in the linear region Equation 5.2 predicts the maximum current within the transistor (first order calculation). This calculation assumes $4 \mathrm{~V}$ have been dropped across the LED and uses the same $W, L, K^{\prime}$ and $V_{T}$ values as Equation 5.1. 


$$
\begin{gathered}
I_{D}=-\frac{K^{\prime} W}{L}\left(V_{G S}-V_{T}-\frac{V_{D S}}{2}\right) V_{D S} \\
I_{D}=-\frac{31 \times 10^{-6} \times 1080 \times 10^{-6}}{0.5 \times 10^{-6}}\left(-5+0.97+\frac{1}{2}\right) 1 \\
I_{D}=236.37 \mathrm{~mA}
\end{gathered}
$$

The initial large current that the PMOS transistor can deliver when in saturation could potentially improve transistor rise time when the driver is first switched on, or when in pulsed mode. As the transistor moves into the linear region of operation the current that can be delivered reduces. Therefore, the current that the driver output stage can deliver when in DC mode is limited to approximately $236.37 \mathrm{~mA}$

The current delivered by each driver should be limited to approximately $100 \mathrm{~mA}$ per device, due to the limited current handling capabilities of the on-chip power supply routing and package bond-wires. The performance of the driver is summarised in Table 5.1.

\begin{tabular}{l|l}
\hline Array Size & $16 \times 16$ \\
Driver Pitch & $100 \mu \mathrm{m}$ \\
Shortest Electrical Pulse & $244 \mathrm{ps}$ \\
Max. Voltage & $5 \mathrm{~V}$ \\
Pulse Repetition Frequency Range & $7 \mathrm{MHz}-800 \mathrm{MHz}$ \\
Max. Delivered Current per Driver & $100 \mathrm{~mA}$ \\
\hline
\end{tabular}

Table 5.1: Summary of 16x16 driver array features.

A notable feature of the new $16 \times 16$ driver array is that the ground terminal of the LED array (n-contacts) has been separated from the ground of the driver circuit. This technique allowed the LED to be biased above or below ground prior to an excitation signal being applied by the driver. By placing a negative bias on LED ground (LED_GND), a voltage greater than 5V (limit of the CMOS process) could be placed across each device and, hence, greater optical output powers could be achieved. 


\subsubsection{Voltage Controlled Oscillator (VCO) and Linear Feedback Shift Register (LFSR) Circuits}

A voltage controlled oscillator ( $\mathrm{VCO}$ ) was implemented alongside the 16x16 driver array. This circuit was capable of producing a square wave signal with a tunable frequency range from $7 \mathrm{MHz}$ to $800 \mathrm{MHz}$. The design featured fine and course adjustment of the VCO frequency. The core frequency of the VCO was defined by the number of elements in the ring oscillator and the delay through each of these elements. Current starving transistors were placed within the ring oscillator and the gate voltage of these transistors was defined off-chip, thus allowing fine adjustment of the core ring oscillator frequency. The output of the ring oscillator was then passed to a digital divider circuit capable of dividing the input signal by $0,4,16$ or 64 and hence producing a course selection of lower frequency signals.

The VCO output could be subsequently used as the input signal to the drivers of the main array, defining the repetition rate of a square wave or pulsed input signal. By producing a square wave input signal on-chip, the need for an off-chip clock (such as a crystal oscillator) has been removed, potentially reducing system size and cost.

A much mooted application for the CMOS driven micro-LED devices, other than time-resolved fluorescence lifetime devices, has been their use in optical communications systems. Their relatively small dimensions, tunable emission wavelength and low cost makes them well-suited for chip-to-chip, fibre optic and free-space communications applications. To investigate this potential application, linear feedback shift register (LFSR) circuits were included in the design.

VCO and LFSR circuit diagrams can be found in Appendices A.4 and A.5.

For a light source to be successfully used within a time-resolved fluorescence lifetime analysis system it is necessary to provide a synchronisation signal that can be used to derive the start (or stop) signal for a TCSPC module or time-gating circuitry. The VCO frequency seen by each driver in the array (after course division) was therefore buffered off-chip and could be observed on a SMA socket on the daughter card. This signal was intended to be used as the input signal of the timing generator circuit described in Section 4.6. The time-gate signal for the SPAD counter circuit could then be synchronised to the sample excitation. 


\subsubsection{Modes of Operation}

The four main modes of driver operation (other than DC) are demonstrated in Figure 5.19. In addition to these operating modes the driver input signal can be derived from an external source fed directly into the device.

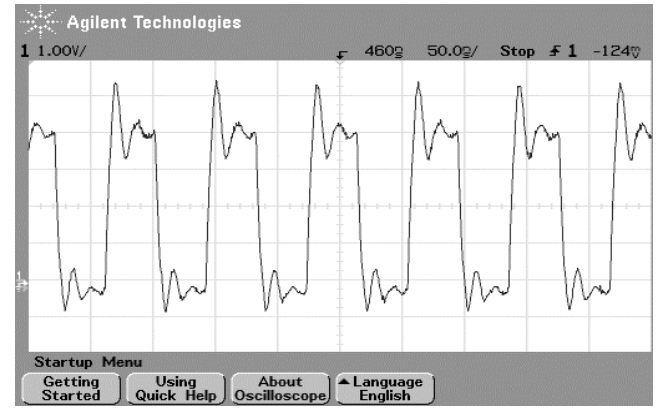

(a) VCO Square Wave

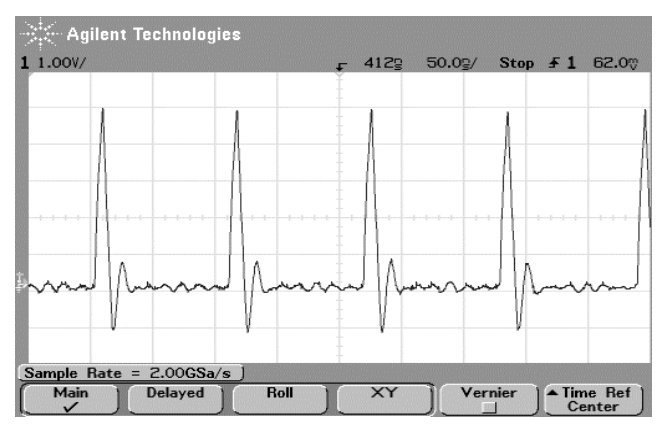

(c) VCO Pulsed

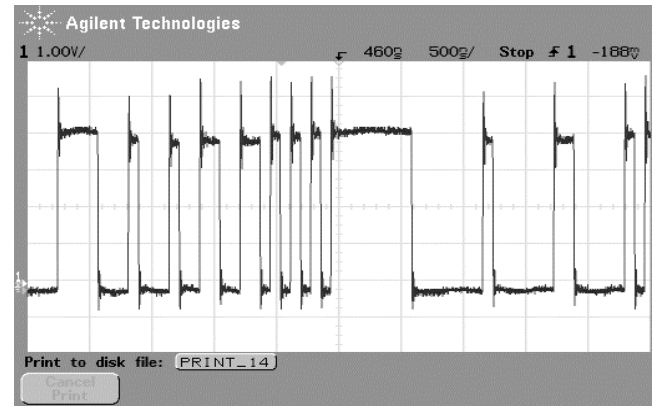

(b) LFSR Square Wave

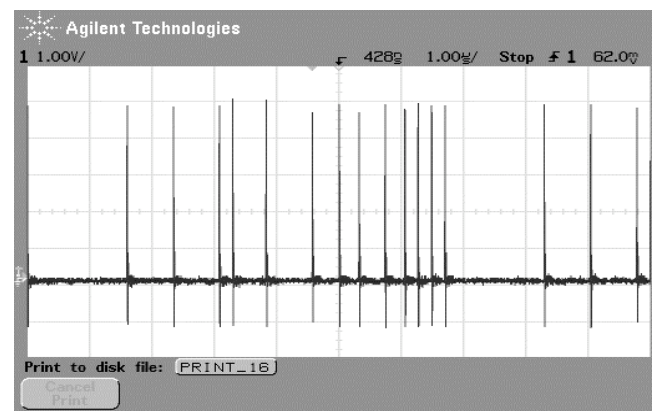

(d) LFSR Pulsed

Figure 5.19: The four main modes of driver operation (other than DC): (a) A square wave input signal derived from the on-chip VCO, (b) square wave signal derived from the on-chip LFSR circuit, (c) variable width pulsed-mode with repetition rate defined by VCO, (d) variable width pulsed-mode with repetition rate defined by LFSR. 


\subsubsection{Device Failure Due to Bump-Bonding Process}

A number of issues were encountered during the evaluation of bump-bonded chips. Unlike the $16 \times 4$ driver array which was implemented in a $0.35 \mu \mathrm{m}$ high-voltage process, the $16 \times 16$ driver array was fabricated in a standard $0.35 \mu \mathrm{m}$ process. In order to handle higher voltages, the high-voltage process included a thicker top metal layer $(2800 \mathrm{~nm})$ compared to the standard process $(925 \mathrm{~nm})$. It was found that this additional thickness provided extra mechanical strength for the top-metal electrodes to which the micro-LED devices were bump-bonded. The forces exerted on the chip during the bump-bonding process resulted in mechanical failure of the $16 \times 16$ array. This caused short-circuits between the metal electrodes and the underlying drive circuit beneath them, rendering the device non-functional. While short circuits were not observed in the 16x4 device, not all array elements were functional (Figure 5.7) this could be attributed to local mechanical failure of the top metal electrodes. This failure can be explained further with reference to Figure 5.20. The top metal electrode was formed from the Metal 4 layer, the thickness of which is dependent on the process used. The underlying driver circuitry was then implemented using the lower level metals (metal layers 1-3) and silicon. Damage to the metal 4 to metal 3 inter-metal dielectric (IMD3) was observed.

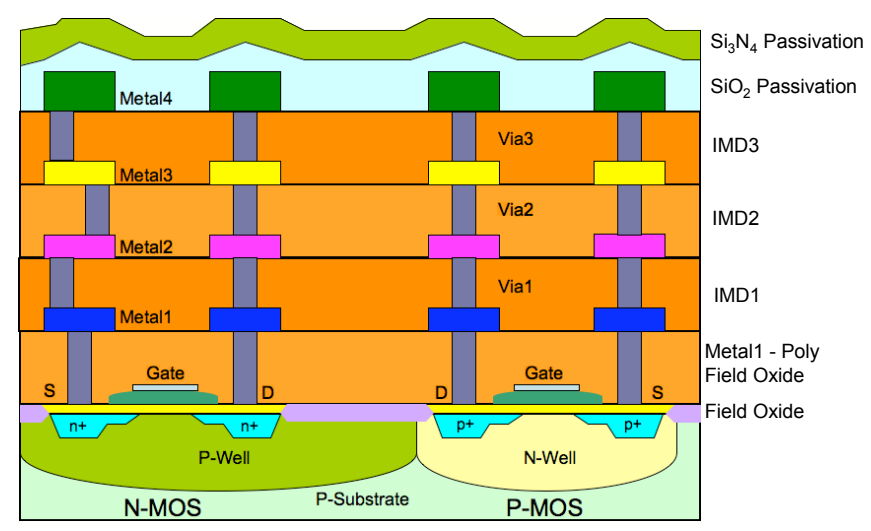

IMD = Inter-Metal Dielectric

Figure 5.20: Cross-section of an Austria Microsystems CMOS wafer. Image adapted from

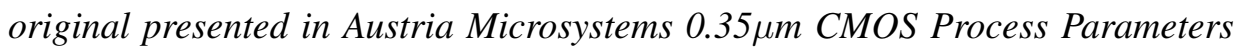
document.

Placing circuitry below bonding pads is a large research area known as CUPs (circuits under pads), [108]. It is clear that a rather naive approach was taken to the implementation of driver circuits under metal electrodes upon which a second device was to be bonded. In light of the mechanical failure of the $16 \times 16$ driver array device it was decided that a new driver chip should 
be implemented with a more robust design.

\subsection{8x8 Voltage Controlled Micro-LED Driver Array}

A new driver array design was implemented to provide extra mechanical strength to the metal electrode upon which the LEDs are bonded. Bond pads, which are usually part of the standard cell library provided by the foundry, are constructed using all four metal layers of the process with a dense array of vias between each layer. While this does not allow any circuitry to be placed beneath them, this technique does provide a robust platform that can be repeatedly bonded to. To allow a full metal bond stack to be included within each array element, the number of drivers was reduced to $64(8 \times 8)$ and the pixel pitch extended to $200 \mu \mathrm{m}$. This allowed a $100 \mu \times 100 \mu \mathrm{m}$ full metal bond stack to be implemented within each pixel alongside a dedicated driver circuit (Figure 5.21).

The driver circuit in the $8 \times 8$ driver array was identical to that described in Section 5.2.1, with the driver output being placed on the full metal bond-stack. A layout view of a single driver pixel is shown in Figure 5.22. A complete list and description of each pin of the 8x8 driver chip can be found in Appendix A.3. 


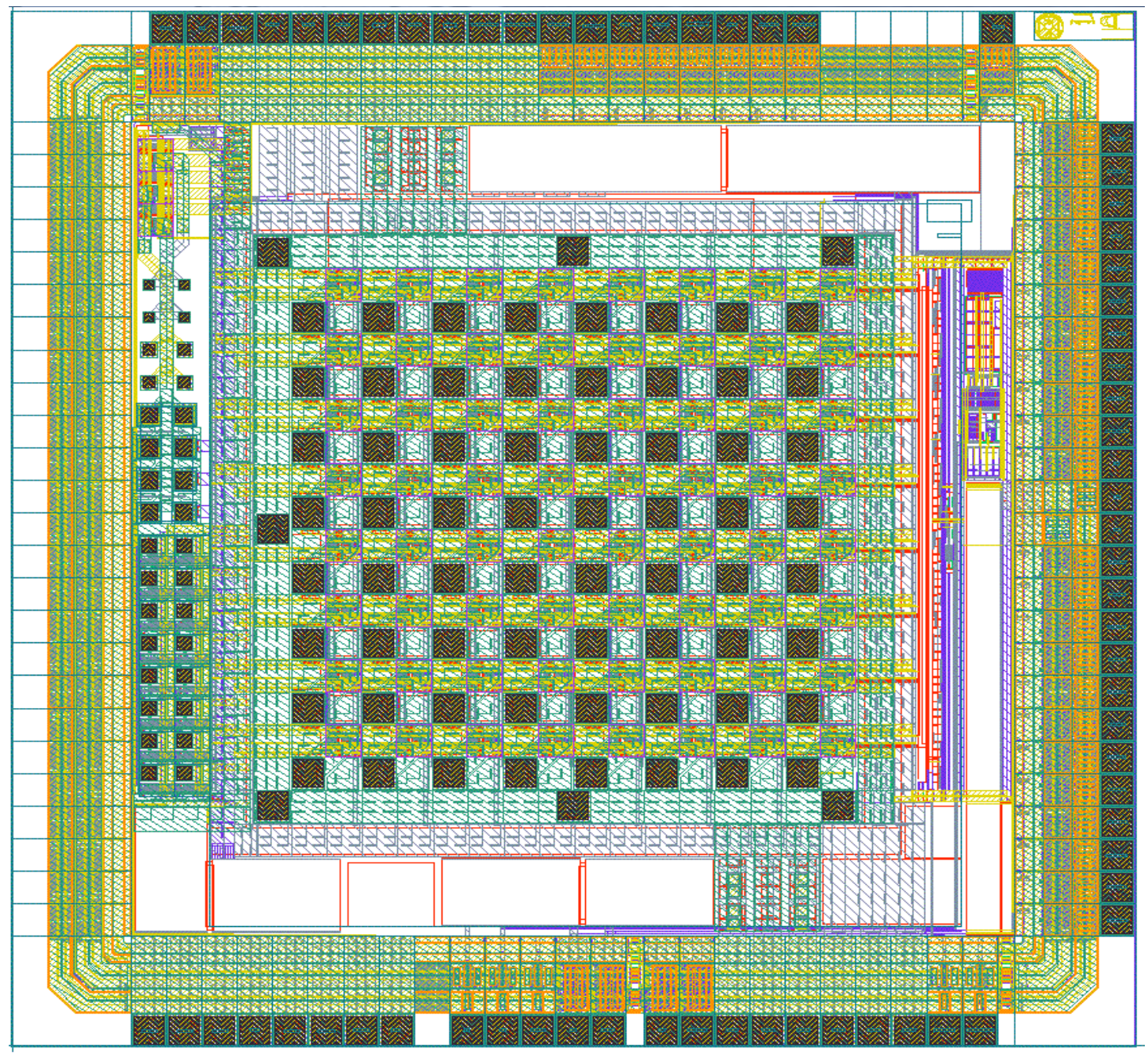

Figure 5.21: $8 x 8$ micro-LED driver array top level layout. 


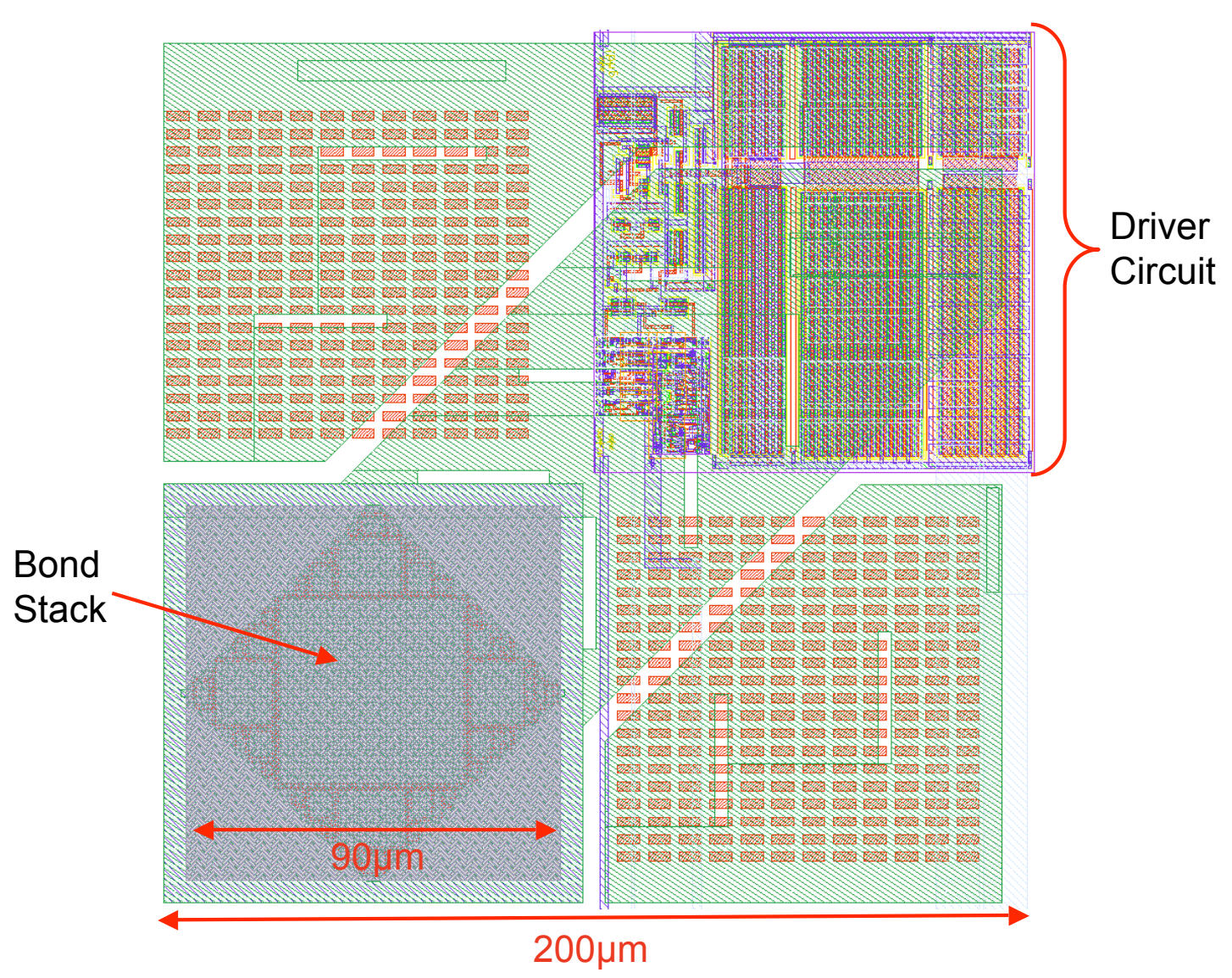

Figure 5.22: Layout view of a single pixel. In this revision a full metal stack has been dedicated to the bond site with the driver circuit located elsewhere in the pixel. 


\subsubsection{8x8 Driver Array Performance}

The 8x8 micro-LED driver also included on-chip VCO (which could be observed off-chip) and LFSR circuits. The driver performance reported herein was assessed using a UV (370nm) emission wavelength micro-LED array.

\subsubsection{Array Uniformity}

The new bond-stack provided a successful platform upon which the micro-LED array could be bump-bonded and it was found that all 64 devices in the array were functional. Though, in this voltage driven device, array uniformity was sacrificed in favour of optical output power. This is highlighted in Figure 5.23 which demonstrates the large variation in optical output power across the array for a fixed DC LED voltage driving a UV (370nm) micro-LED array. The average optical power across all elements in the array was $70.675 \mu \mathrm{W}$, representing a 10 -fold increase in optical power compared to the average power reported in Section 5.1.2.1. This was achieved with a lower LED voltage ( $4.88 \mathrm{~V}$ compared to $15 \mathrm{~V}$ ) but with an increased current consumption per element (4.93mA per element compared to the clamped 2mA in the $16 \times 4$ array). However, there are large variations in the optical output power produced by each element, ranging from $129.5 \mu \mathrm{W}$ to $28.2 \mu \mathrm{W}$. 


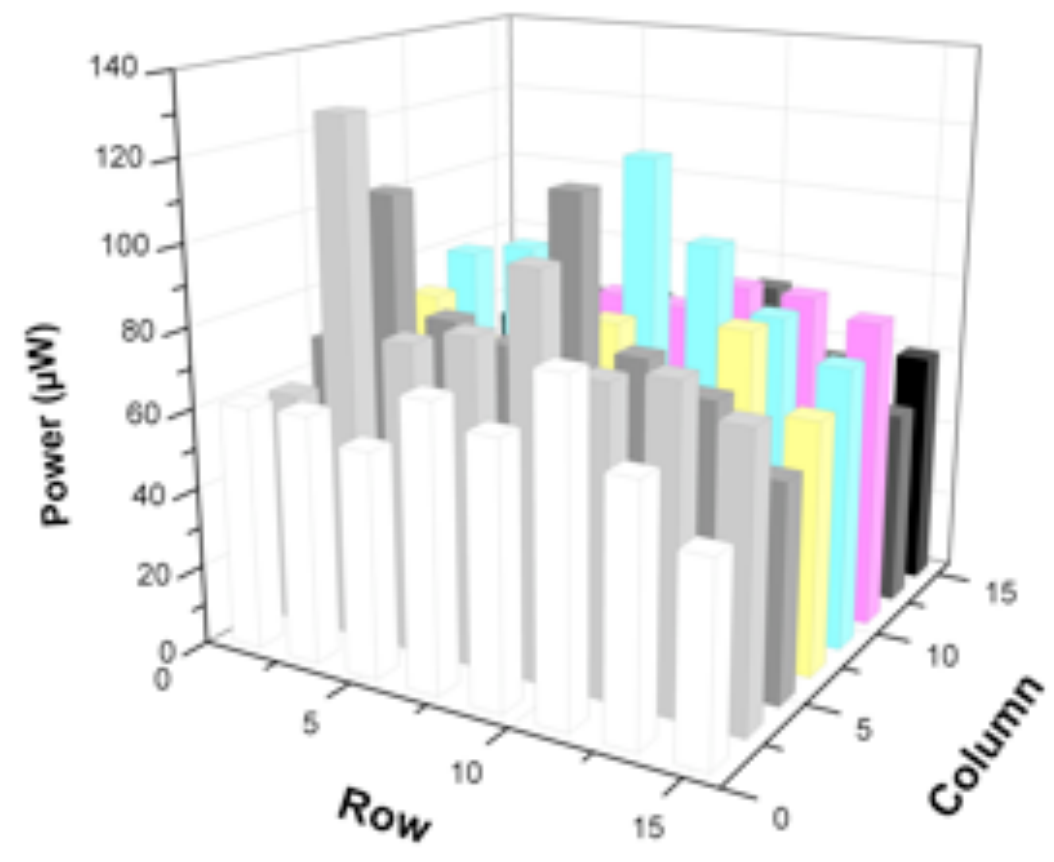

Figure 5.23: Uniformity of voltage driven $U V(370 \mathrm{~nm})$ micro-LED array $\left(L E D_{-} V D D=5 \mathrm{~V}\right.$, LED_GND = gnd!). 


\subsubsection{DC Performance}

While driving LEDs with voltage rather than current leads to poorer array uniformity it does allow for the maximum amount of current to be drawn by the LED for a given drive voltage. The current drawn is therefore dictated by the IV curve of the LED device. The current consumption of the driver array increases as the DC voltage placed onto a single device is increased (Figure 5.24). This increased current consumption correlates with increased optical output power (Figure 5.25). With a DC drive current of $80 \mathrm{~mA}$ being delivered to a single device the optical output power measured approximately $550 \mu \mathrm{W}$.

Output power saturation is caused by a saturation of the LED active region (the quantum wells) with carriers. Any further injection of carriers no longer results in increased optical power, instead these excess carriers lead to heating which can cause device damage or destruction [86].

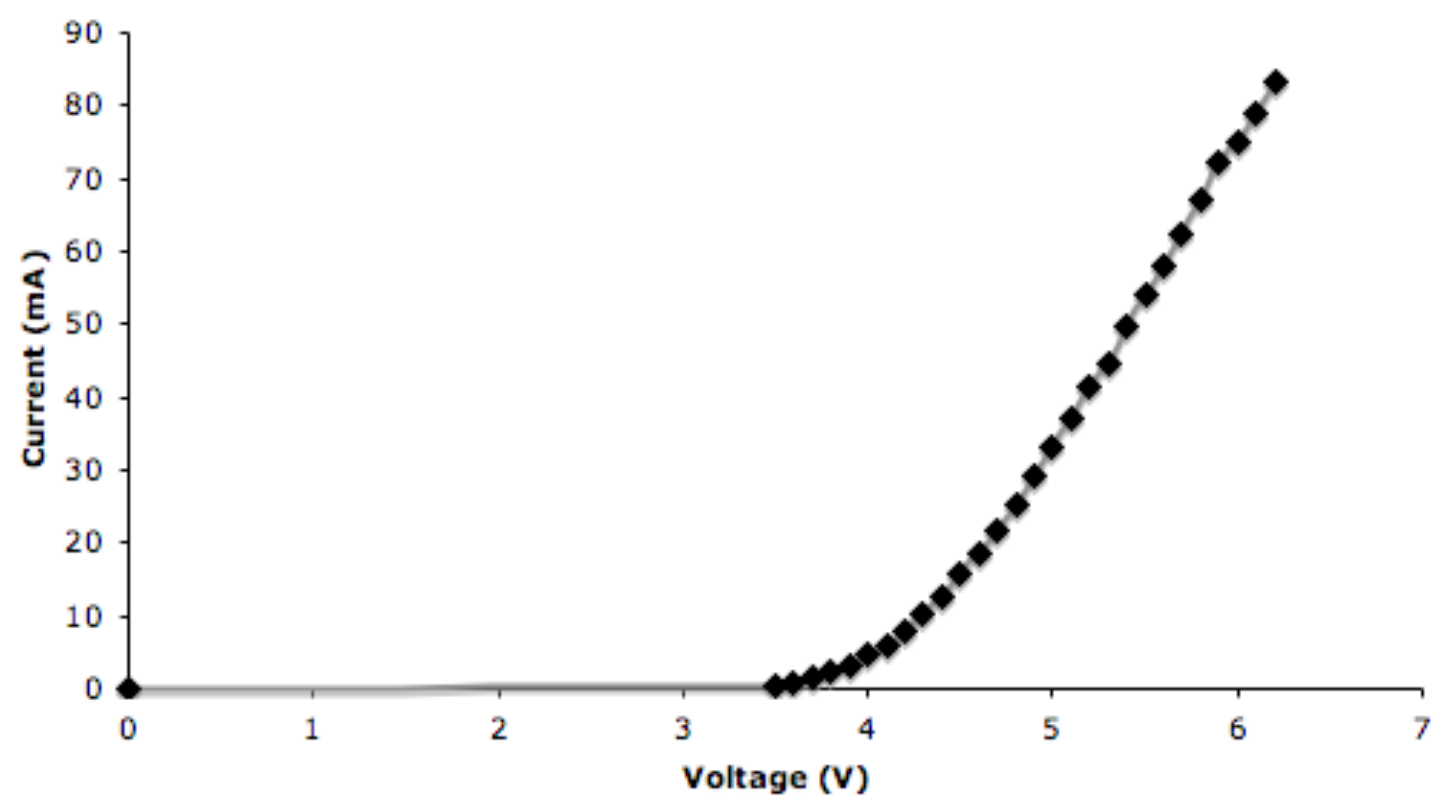

Figure 5.24: Current versus voltage characteristics of UV micro-LED device. 


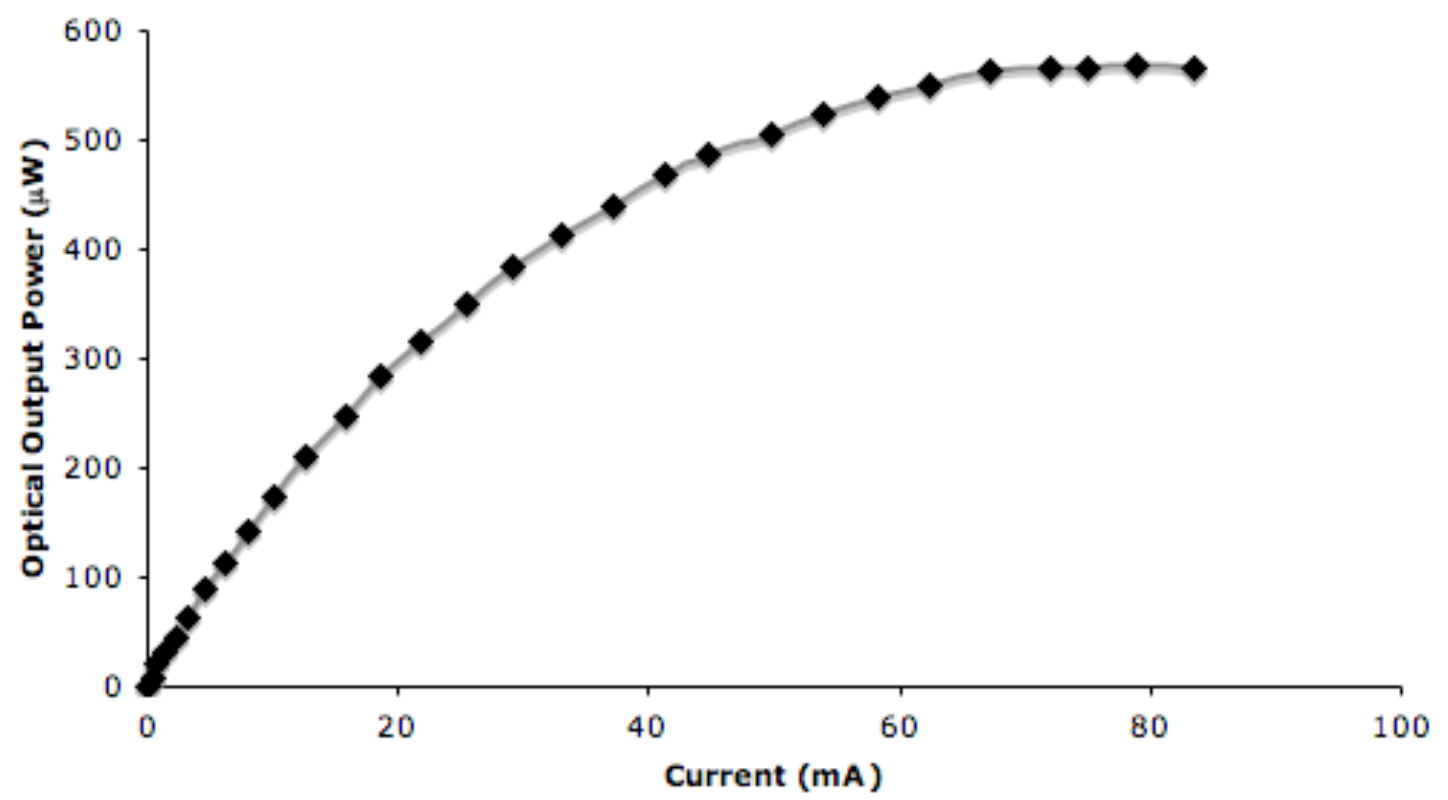

Figure 5.25: Optical power versus current characteristics of UV micro-LED device. 


\subsubsection{Pulsed Performance}

The width of the electrical pulse produced by the driver was defined by the delay through a single inverter within each pixel. This delay was controlled by a current starving NMOS transistor in the inverter circuit (see Section 5.2.1 for circuit details). The optical pulse width relates to the bias voltage on the gate of the NMOS current starving transistor (Figure 5.26).

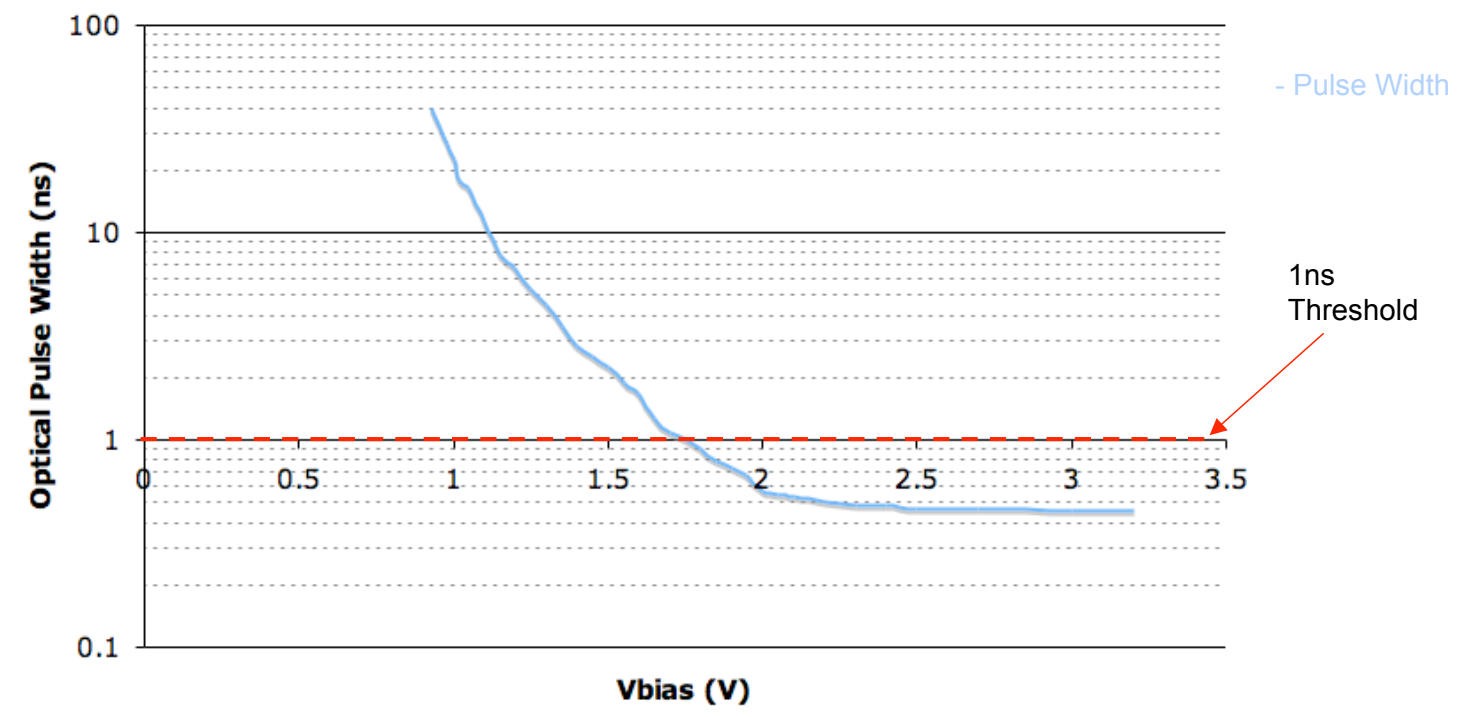

Figure 5.26: Optical pulse width versus the in-pixel current starving NMOS transistor gate bias voltage.

Using this technique, optical pulse widths from 300ps up to 39.89 ns could be produced. Figure 5.27 demonstrates the longest and shortest optical pulse widths achieved.

As the pulse width is reduced the average optical power is reduced linearly (Figure 5.28).

Peak pulse power is critical for time-resolved fluorescence applications requiring pulsed excitation light. This can be derived from the measured average optical power using Equation 5.3:

$$
\frac{P_{A v g}}{P_{o}}=\frac{\tau}{T}
$$

Where $P_{A v g}$ is the average optical power, $P_{o}$ is the peak pulse power, $\tau$ is the pulse width and $T$ is the pulse period. 


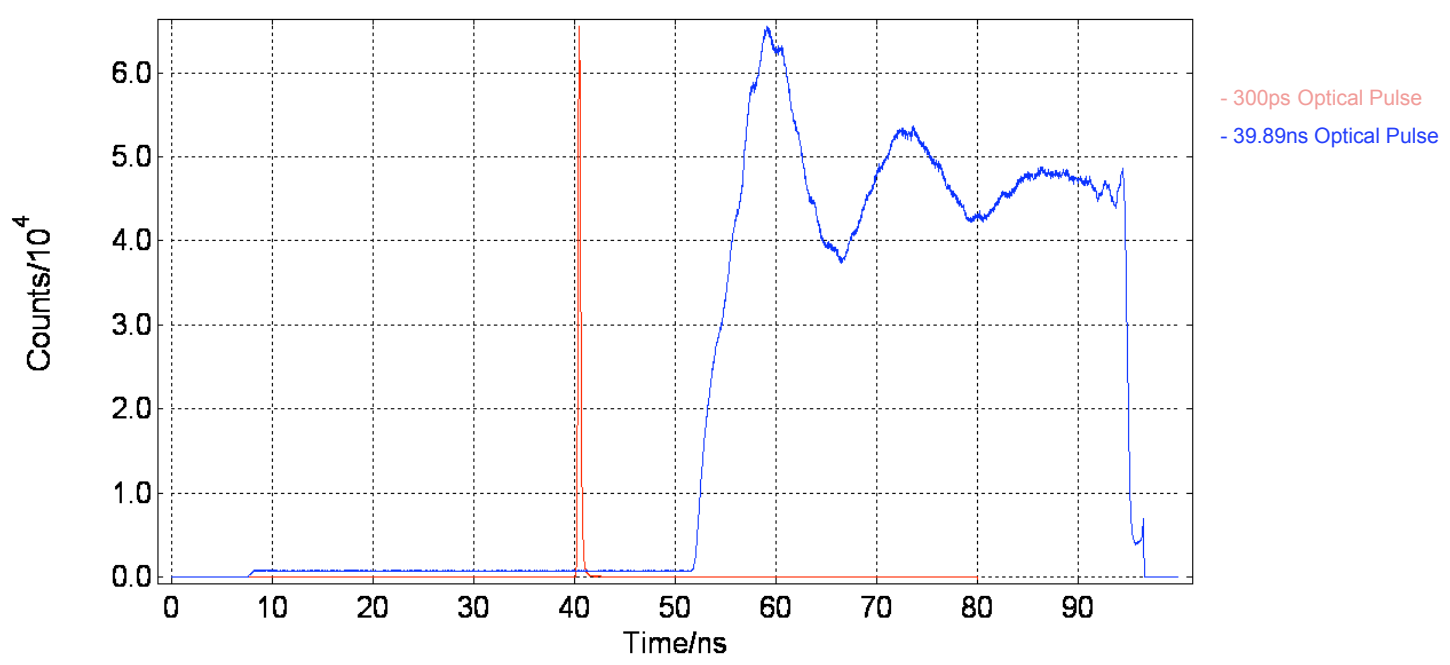

Figure 5.27: Shortest and longest optical pulses produced by $8 \times 8$ driver circuit.

Table 5.2 summarises peak optical pulse power and energy for various pulse widths and LED ground bias voltages. These results show how placing a negative bias voltage on the ground terminal of the micro-LED array leads to a significant increase in pulse power.

\begin{tabular}{lllllll}
\hline VBMC2 & $\begin{array}{l}\text { Pulse } \\
\text { Width }\end{array}$ & LED_GND & $\begin{array}{l}\text { Repetition } \\
\text { Rate }\end{array}$ & $\begin{array}{l}\text { Average } \\
\text { Power }\end{array}$ & $\begin{array}{l}\text { Peak } \\
\text { Power }\end{array}$ & $\begin{array}{l}\text { Pulse } \\
\text { Energy }\end{array}$ \\
\hline $2.7 \mathrm{~V}$ & $464 \mathrm{ps}$ & gnd! & $12 \mathrm{MHz}$ & $0.012 \mu \mathrm{W}$ & $2.16 \mu \mathrm{W}$ & $0.99 \times 10^{-15} \mathrm{~J}$ \\
$2.7 \mathrm{~V}$ & $464 \mathrm{ps}$ & $-1.5 \mathrm{~V}$ & $12 \mathrm{MHz}$ & $0.953 \mu \mathrm{W}$ & $171.16 \mu \mathrm{W}$ & $7.94 \times 10^{-14} \mathrm{~J}$ \\
$1.5 \mathrm{~V}$ & $2.22 \mathrm{~ns}$ & gnd! & $48.5 \mathrm{MHz}$ & $10.88 \mu \mathrm{W}$ & $100.96 \mu \mathrm{W}$ & $0.224 \times 10^{-12} \mathrm{~J}$ \\
$1.5 \mathrm{~V}$ & $2.22 \mathrm{~ns}$ & $-1.5 \mathrm{~V}$ & $48.5 \mathrm{MHz}$ & $67.2 \mu \mathrm{W}$ & $623.6 \mu \mathrm{W}$ & $1.384 \times 10^{-12} \mathrm{~J}$ \\
$0.97 \mathrm{~V}$ & $39.89 \mathrm{~ns}$ & gnd! & $12 \mathrm{MHz}$ & $174.3 \mu \mathrm{W}$ & $364.1 \mu \mathrm{W}$ & $14.52 \times 10^{-12} \mathrm{~J}$ \\
\hline
\end{tabular}

Table 5.2: Peak optical pulse power versus pulse width and LED ground voltage.

A measure of LED_GND versus average optical output power was conducted for a fixed pulse width and repetition rate (Figure 5.29). This shows how the optical output power of a 464ps pulse can be increased from less than $0.1 \mu \mathrm{W}$ to approximately $1 \mu \mathrm{W}$ by placing a $-1.5 \mathrm{~V}$ bias on the ground terminal of the LED. One caveat is that by negatively biasing the ground terminal of the LED the device is closer to its turn on voltage prior to an electrical pulse being applied by the CMOS driver. Thus, for very short pulses (sub nano-second) where pulse rise and fall times dominate, it may be the case that this bias condition extends the optical pulse width of the LED (Figure 5.30). Another factor which may place a limit on the minimum achievable pulse width 


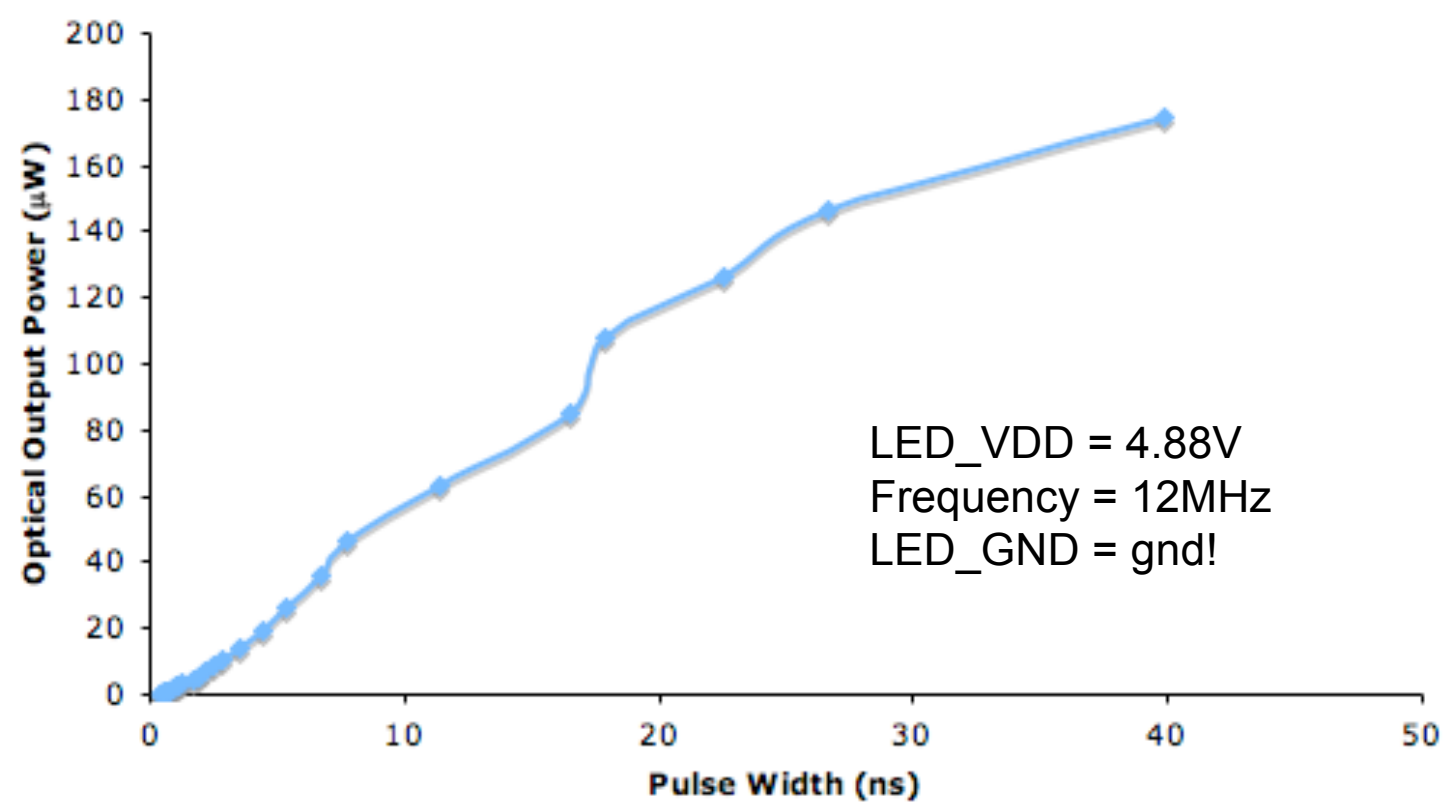

Figure 5.28: Optical pulse width versus average optical power.

is the spontaneous minority carrier recombination lifetime within the LED device, as the LED output intensity is directly dictated by the minority carrier concentration. Both the rise and fall time of the LED are influenced by carrier recombination lifetime. This can be addressed by ensuring there is a large difference between the on-voltage across the LED and the off-voltage. When the LED is turned off, this results in carrier sweep out, whereby the created electric field forces free carriers out of the LED active region. This sweep out time can influence the LED fall time and can be shortened further by placing a negative bias across the LED when in the off state, thereby increasing the electric field in the depletion region [86].

It should be noted that caution should taken when biasing the micro-LED devices via the ground terminal of the array (Figure 5.31). The signal LED_GND is a global signal, common to every element in the array regardless of which element is being addressed. Therefore, it is possible to turn on every element in the array by applying a sufficient negative bias voltage to the ground terminal. The $p$-contact of elements that are not being addressed is tied to gnd!. At LED_GND bias voltages of around -3V all elements in the array begin to emit light. This results in artificially high optical power measurements. In a miniaturised fluorescence lifetime reader application this would result in a decrease in signal-to-noise ratio and would skew lifetime estimations. As LED_GND is a global signal, when an LED driver is in the off state, the 


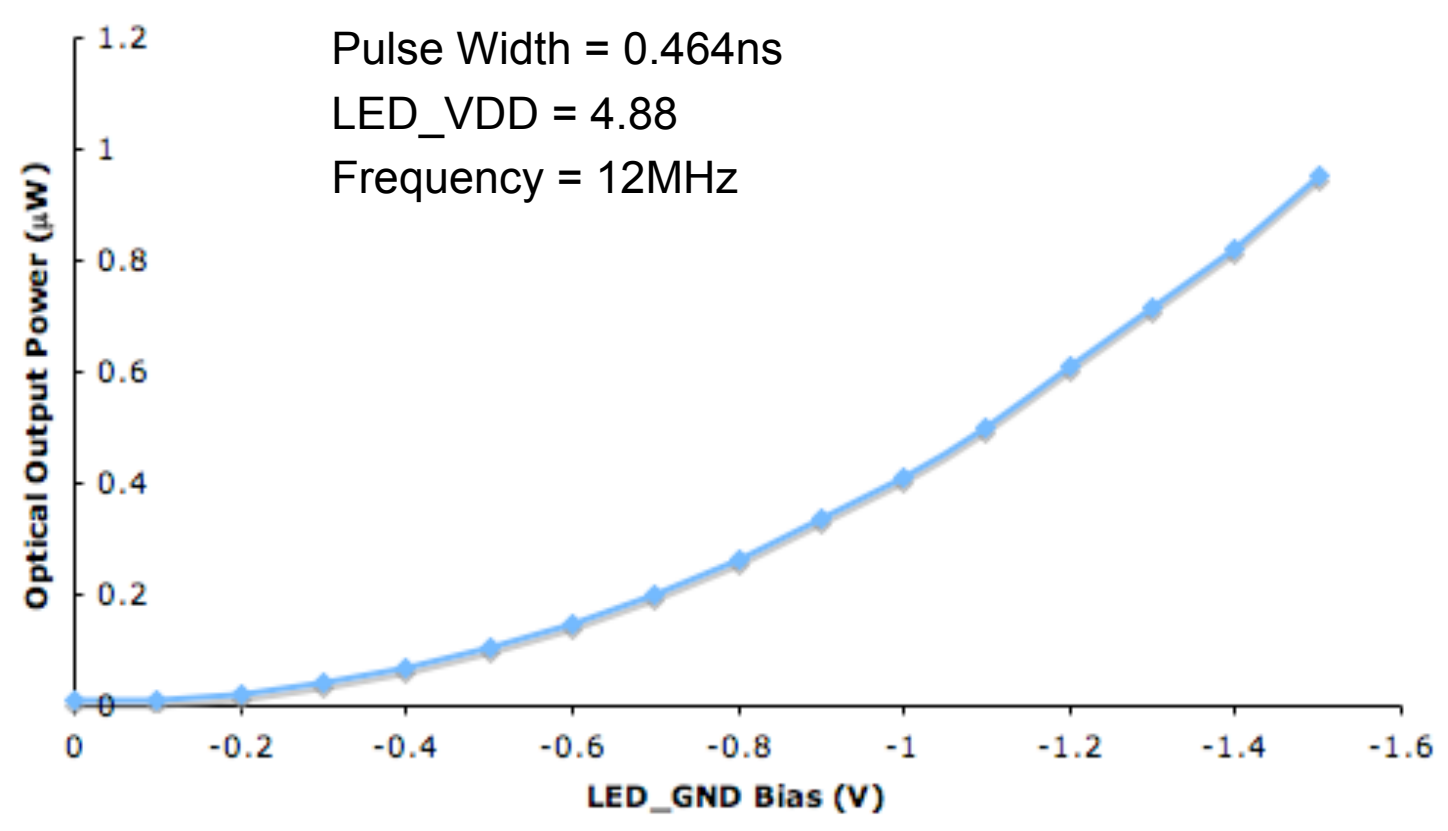

Figure 5.29: LED_GND bias voltage versus average optical output power.

voltage across the LED is equal to the negative bias on LED ground. If this voltage is sufficient, current is sourced via M2 and the LED turns on. This effect is clearly visible under microscope observation (Figure 5.32). As the negative bias applied to LED_GND increases the brighter non-addressed array elements become. 


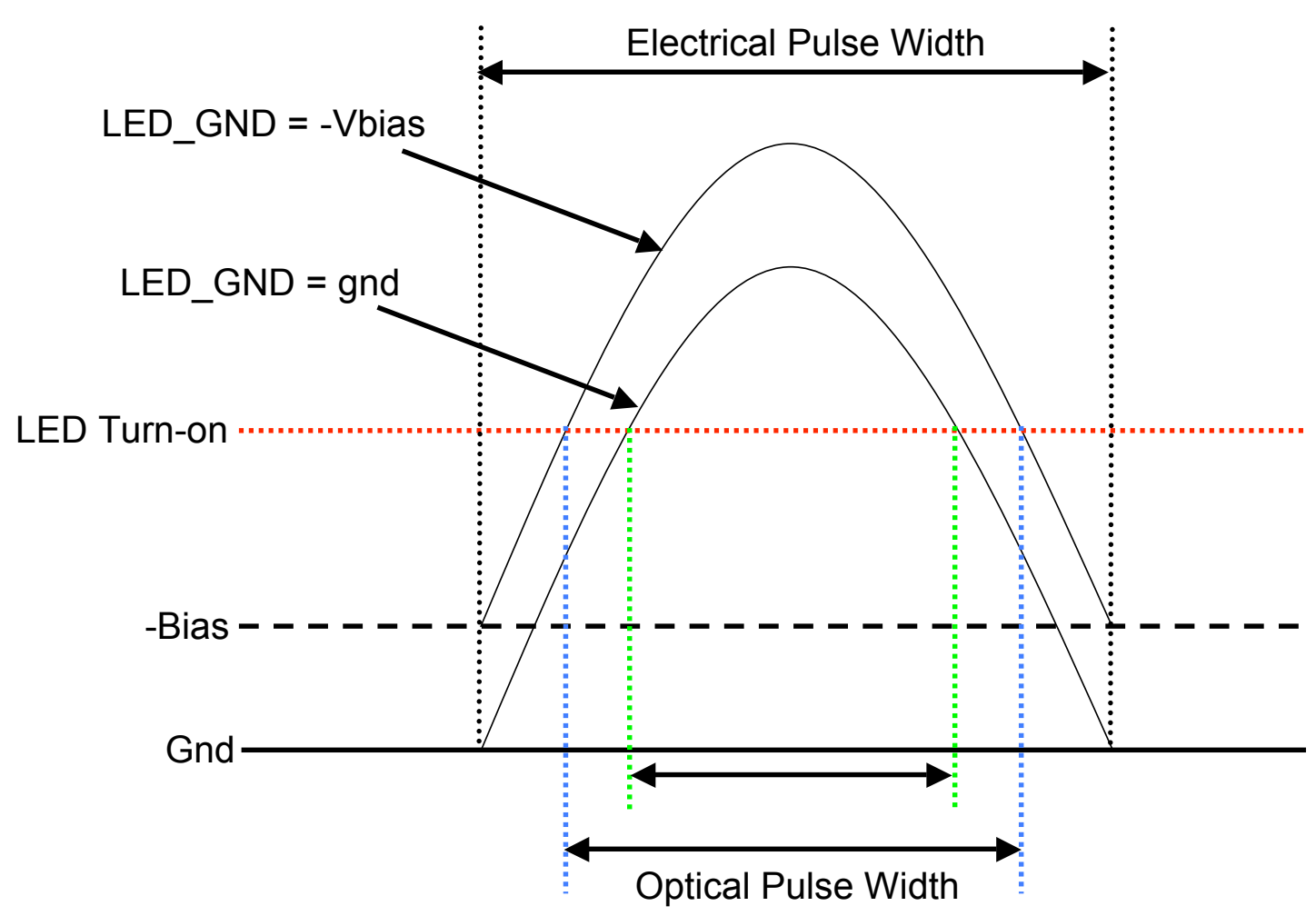

Figure 5.30: LED_GND bias voltage versus optical pulse width. 


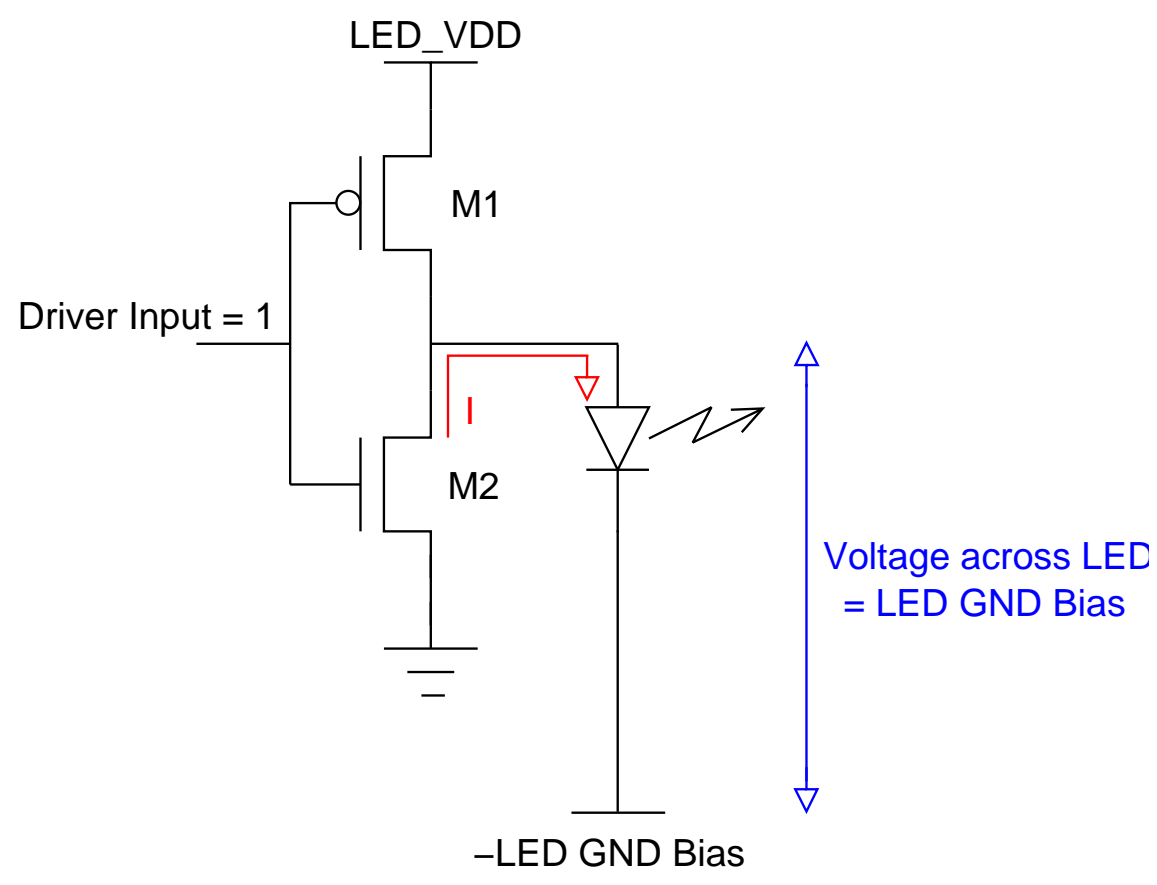

Figure 5.31: LED_GND is a global signal. When an LED driver is in the off state, the voltage across the LED is equal to the negative bias on LED ground. If this voltage is sufficient, current is sourced via M2 and the LED turns on.

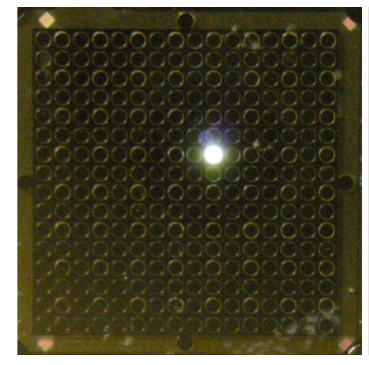

LED_GND $=-1 V$

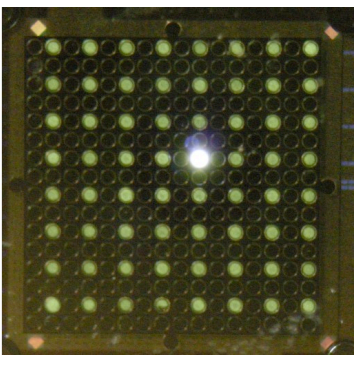

LED_GND $=-2 V$

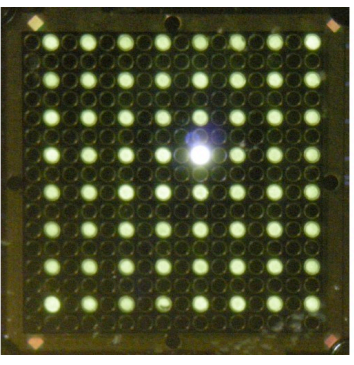

LED_GND $=-3 V$

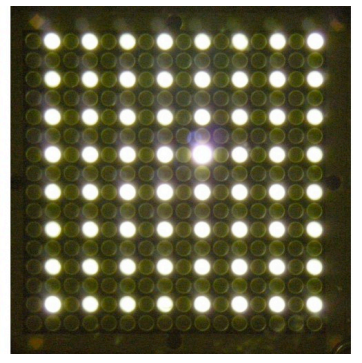

LED_GND $=-4 \mathrm{~V}$

Figure 5.32: A single micro-LED is addressed. As the negative bias on LED_GND increases, other array elements begin to turn on. 


\subsubsection{Device Performance Compared to Commercial Laser Diode}

With a shortest pulse of 300ps and adjustable repetition rate, pulse width and power; the micro-LEDs are ideally suited for time-resolved fluorescence applications. Comparing the micro-LED devices to the PicoQuant LDH-470 diode laser (Section 3.1.2), the diode laser is capable of producing shorter pulse widths (68ps minimum) at a higher peak optical pulse power $(458.8 \mathrm{~mW})$. However, the user cannot control the pulse width. Furthermore, in a micro-system environment the physical size of the device is critical. To ensure device output power stability the diode laser head is shrouded by a Peltier cooler. This results in a device which is $118 \mathrm{~mm}$ long, $74 \mathrm{~mm}$ wide and $58 \mathrm{~mm}$ high. Each micro-LED element measures just $72 \mu \mathrm{m}$ in diameter, $1.036 \mathrm{~mm}$ in height and requires no cooling.

With 64 individually addressable devices within the micro-LED array, the optical power can be varied by enabling a greater number of devices in the array. The ability to address different devices also allows for spatial control over areas of sample that are to be excited. With the CMOS backplane this can be achieved without the need for any moving parts. Varying focus position of the diode laser would require an adjustable stage. This has been exploited in [109] whereby confocal microscopy has been demonstrated using a matrix-addressable micro-LED array with no moving parts.

Pulse profile (in time) is critical when assessing the performance of a light source in a time-resolved fluorescence system. Figure 5.33 shows the pulse profile of the PicoQuant laser diode, emitting at $467 \mathrm{~nm}$ with a repetition rate of $40 \mathrm{MHz}$. This produces a $54 \mathrm{ps}$ FWHM pulse with an average power of $1.3 \mathrm{~mW}$ and a peak power of $257 \mathrm{~mW}$. There is a significant "knee" on the falling edge of the pulse. While this does not effect the FWHM pulse width, it significantly extends the length of time light is emitted from the laser (by approximately $0.75 \mathrm{~ns})$. In a fluorescence lifetime experiment this "knee" could potentially influence decay curves and hence the estimated lifetime.

When the 300ps micro-LED pulse is plotted on a logarithmic scale (Figure 5.27) a similar "knee" can be seen on the falling edge of the optical pulse. When plotted on a linear scale (Figure 5.34) it can be compared to the optical pulse profile of the PicoQuant diode laser. The "knee" of the micro-LED pulse is significantly less pronounced than that of the diode laser. Despite having a wider FWHM (300ps compared to 54ps) the pulse power after 300ps is significantly less in the micro-LED device. 


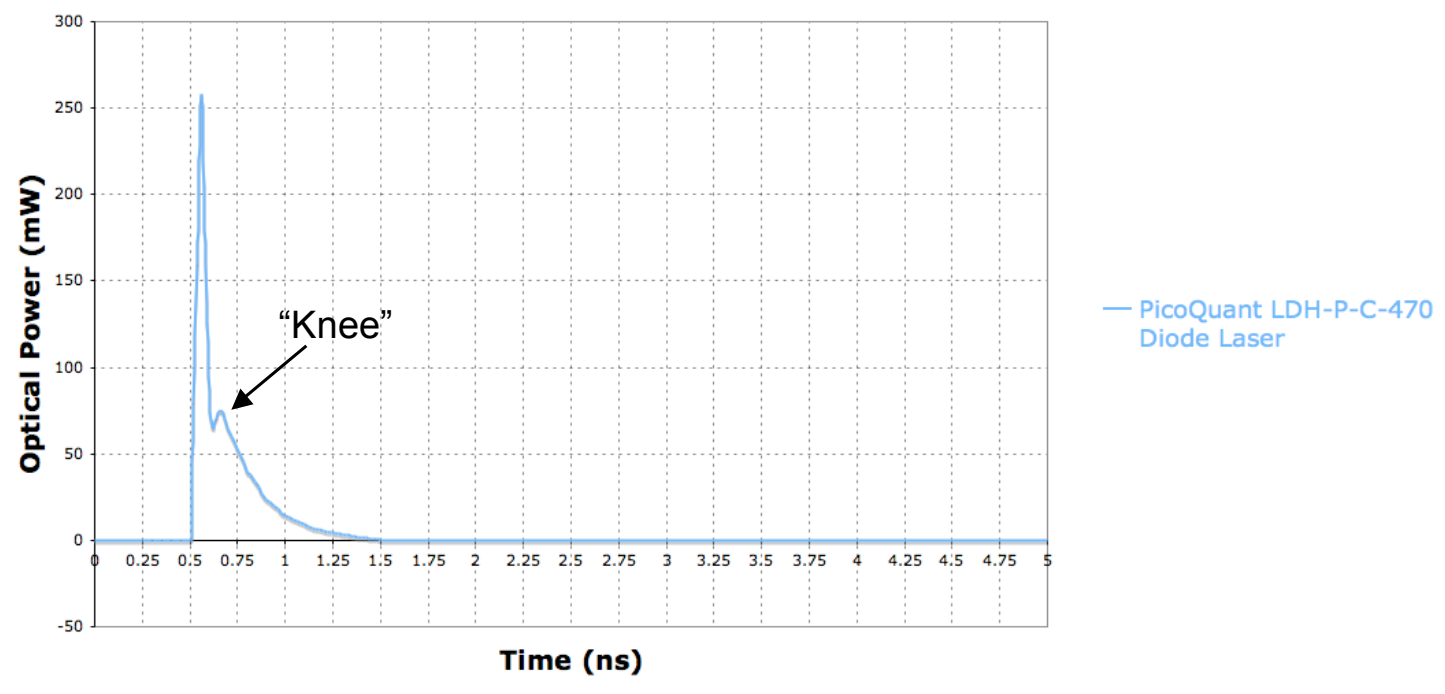

Figure 5.33: Optical pulse width of PicoQuant diode laser.

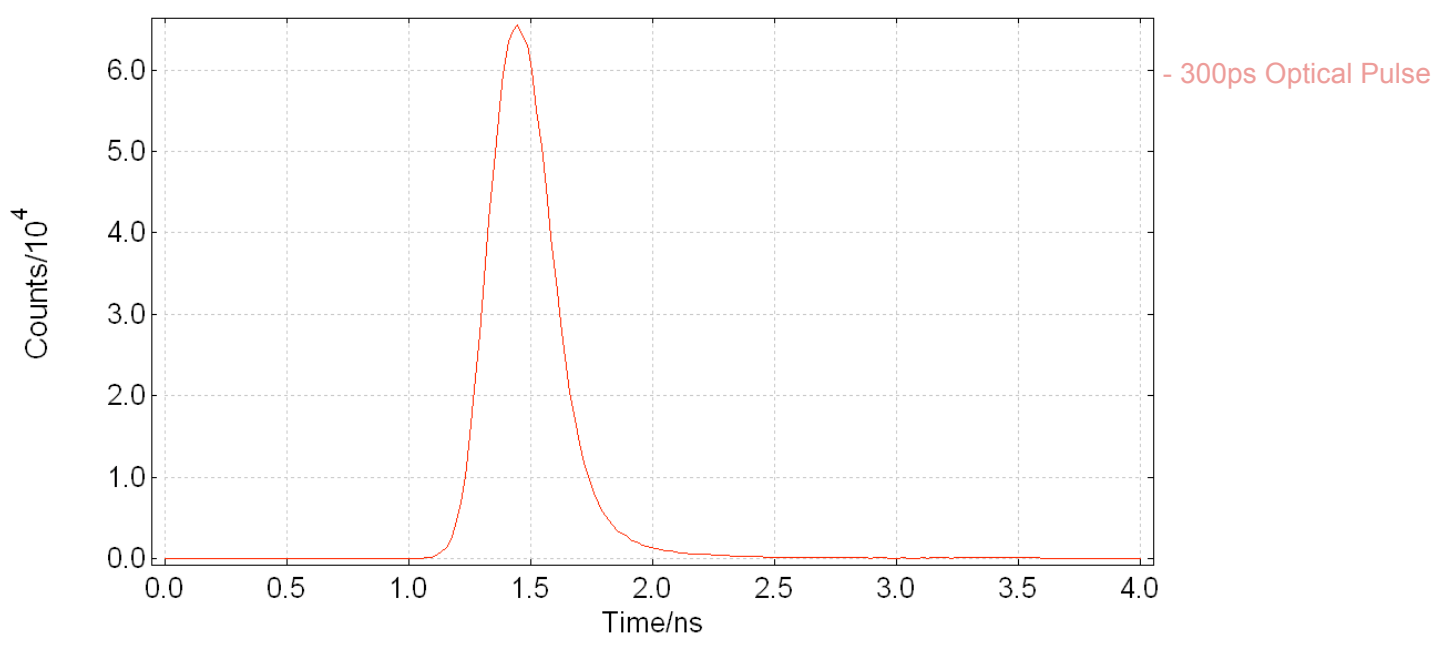

Figure 5.34: Optical pulse width of CMOS driven micro-LED device. 


\subsection{Discussion}

It has been shown that the CMOS driven micro-LED devices can be a suitable alternative to a pulsed laser as an excitation source in a miniaturised fluorescence lifetime system. The driver design went through a number of iterations in an attempt to minimise pulse width and maximise pulse power. A valuable lesson was also learnt from the 16x16 driver array device (Section 5.2) regarding the effects of the forces exerted upon the chip during the bump-bonding process. In light of this, a new pixel architecture was devised with a full metal bond-stack upon which each micro-LED device could be bump-bonded. This provided a more reliable and robust platform with initial devices showing $100 \%$ device functionality.

The 16x4 micro-LED driver array demonstrated how a CMOS circuit was capable of driving AlInGaN micro-LEDs. A UV (370nm) array was used in the first demonstration of a micro-system for time-resolved fluorescence detection (see Chapter 6). A second generation of voltage based driver arrays was developed, resulting in an $8 \times 8$ driver array capable of driving micro-LEDs with a comparable performance to a commercially available diode laser.

The divergent nature of LED light emission results in a reduction in optical power density as the distance from the device is increased. This effect is much less pronounced in a laser device which produces a collimated emission. It is for this reason that micro-LED devices are ideally suited to micro-system devices rather than a bench top application. As the distance between the micro-LED emitter and the sample of interest is decreased the optical power density incident on the sample increases. This can be demonstrated with reference to Figure 5.35 and Equation 5.4. For simplicity, Equation 5.4 makes the assumption that the photodetector is curved and matches the curve of the emitted light, as depicted in Figure 5.35. The equation shows how the amount of light detected by the photodetector increases with the square of its distance from a point light source. This argument also holds true for the distance between the sample of interest and the detection device.

$$
S=4 \pi R^{2}
$$

Where $S$ equals the surface area of a sphere (the exposed photodiode area) and $R$ the radius of sphere (the distance between the sample and photodiode or micro-LED and sample).

While the optical power of the excitation light from the commercially available diode laser 


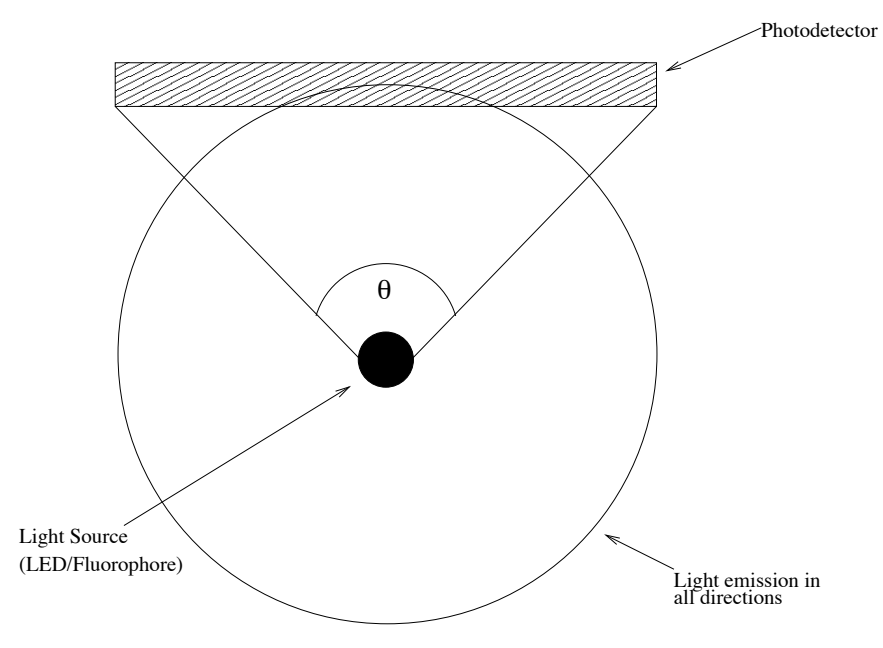

Figure 5.35: Light Emission from a point source at a distance from a photodetector.

has been shown to be significantly greater than that of the CMOS driven micro-LED devices, many significant advantages of the CMOS devices have been noted, with their physical size making them ideal for a micro-system application. The $8 \times 8$ driver array described in this chapter provided a stable platform to which the LED arrays could be bump-bonded. The power and pulse performance of this array also improved on the original 16x4 array. For the majority of fluorophores a 300ps excitation pulse is sufficiently short so as not to affect their decay characteristics. In addition, it has been shown that while minimum FWHM of a micro-LED device pulse is greater than the diode laser, the pulse profile exhibits less persistence with a cleaner falling edge, potentially resulting in less skewing of fluorescence decay curve characteristics. 


\section{Chapter 6}

\section{Micro-Systems for Time-Resolved Fluorescence Lifetime Detection}

Conventional equipment used for time-resolved fluorescence lifetime analysis tends to be physically large, delicate and expensive. A narrow wavelength excitation source is required and the resulting fluorescence must pass through an optical device to separate the excitation light from the fluorescence emission, before being detected by a light sensitive instrument. External hardware is then required to process data from the detector and determine the sample lifetime. However, using modern fabrication and manufacturing techniques, such instrumentation can now be made in complete integrated systems with the potential for volume production.

In Chapter 4 an array of CMOS single-photon avalanche diodes with on-chip signal-processing circuitry was introduced and the ability to accurately capture and estimate lifetime data was demonstrated. Chapter 5 provided details of several compact CMOS driven micro-LED arrays, capable of producing sub-nanosecond optical pulses, that were designed and fabricated during this project. In this chapter, micro-systems incorporating both of these devices are presented and their ability to capture time-resolved fluorescence lifetime data is demonstrated. Two different micro-system design topologies were investigated; (i) a single chip solution using the 16x4 array of CMOS driven micro-LEDs as the excitation source and the SPADs situated within the driver array as the detector. (ii) The second topology incorporated dedicated excitation and detector devices in a two chip sandwich structure. Excitation was performed using the $8 \mathrm{x} 8$ CMOS driven micro-LED array. Detection was performed by a set of SPADs situated within the original 16x4 SPAD array. Signal processing was performed using a commercially available TCSPC module. Prototypes of these integrated micro-systems were developed and this chapter demonstrates their ability to perform fluorescence excitation and detection of both quantum dot samples in colloidal suspension and other fluorophores. It is hoped that such a micro-system could lead to a low-cost, robust time-resolved fluorescence lifetime detection system, making this technique more accessible to a wider range of users. 


\subsection{Fluorescence System Transmission Model}

In any optical system there are a number of factors that can potentially contribute to signal degradation. These sources of error are summarised in Figure 6.1 which provides a flow diagram of an LED/SPAD based fluorescence detection system. The number of photons generated by an LED is directly proportional to the injected current (until saturation occurs) and the device's conversion efficiency. The divergent nature of LED photon emission means that not all photons are guaranteed to reach the sample of interest. The fluorescence sample will have a quantum yield which defines the number of fluorescence photons emitted for every absorbed excitation photon. Fluorescence emission is omni-directional and only a proportion of the emitted photons will reach the detector area. One of the current draw-backs of CMOS based SPAD detectors is their poor fill factor. Therefore, only a small proportion of the photons which reach the detector will fall on a photo-sensitive area. The detector has a quantum efficiency or photon-detection probability which dictates how many of these photons are detected and how much current is generated for every detected photon. The electrical output of the detector must then be sensed by read-out electronics; at this stage additional electrical noise can be introduced and the sensitivity and efficiency of such circuitry is also critical.

The external conversion efficiency $\left(\eta_{Q E}\right)$ of an LED can be calculated by dividing the optical output power by the electrical input power:

$$
\eta_{Q E}=\frac{P}{I V}
$$

Where $P$ is equal to the micro-LED optical output power, $V$ is the LED bias voltage and $I$ is the current in the LED device.

From the results presented in Section 5.3.1.2 the external conversion efficiency for a single UV micro-LED in DC mode and biased with 6V can be calculated as:

$$
\begin{array}{r}
\eta_{Q E}=\left(\frac{550 x 10^{-6}}{\left(75 \times 10^{-3} x 6 V\right)}\right) * 100 \\
\eta_{Q E}=0.122 \%
\end{array}
$$


Table 6.1 provides a summary of typical values for the efficiencies of each stage of Figure 6.1. Consideration must also be given to the collection angle of the fluorescence gathering optics or detector within the system (see Section 5.4), as the omni-directional nature of fluorescence emission prevents all photons from being gathered.

\begin{tabular}{ll}
\hline Property & Typical Value \\
\hline LED Conversion Efficiency & $0.122 \%$ \\
Fluorophore Quantum Yield & Fluorescein $=95 \%^{*}$ \\
& Rhodamine B $=31 \%^{*}$ \\
& Evidenttech Quantum Dots $=50 \%^{* *}$ \\
Photodetector Fill Factor & $0.14 \%$ \\
Photodetector Quantum Efficiency & $35 \%$ at 460nm \\
Readout Noise & N/A in SPAD system \\
Photodetector Dark Count & $50 \mathrm{~Hz}$ \\
\hline
\end{tabular}

Table 6.1: Summary of typical system transmission values.

${ }^{*}[7]$

${ }^{* *}$ www.evidenttech.com 


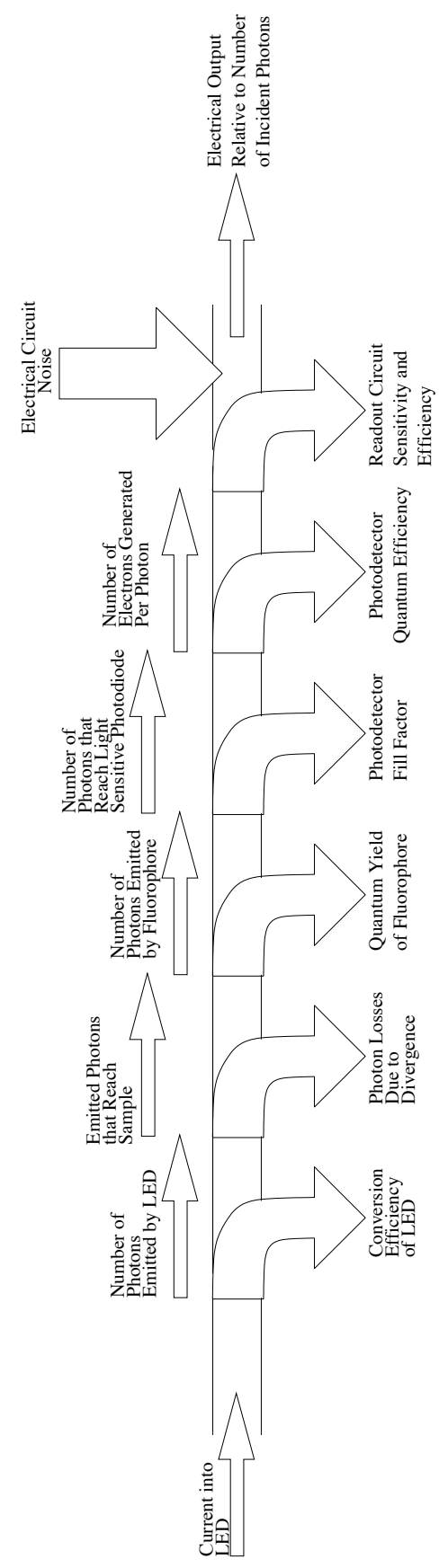

Figure 6.1: Signal losses from excitation source to signal detection in a fluorescence-based detection system. 


\subsection{Single-Chip Micro-System for Time-Resolved Fluorescence Lifetime Detection}

A 16x4 CMOS current-based micro-LED driver array was bump-bonded to an equivalent array of UV (370nm) micro-LEDs which acted as the excitation source. The design and performance of which is described in Section 5.1. SPAD detectors situated within the driver array acted as the detector (performance presented in Chapter 4). The sample of interest was presented to the system on a slide placed above the micro-LED array. The optically transparent sapphire substrate of the micro-LED array allowed returning fluorescence light to pass through the micro-LED array, reaching the SPAD array below.

\subsubsection{System Setup}

In order to successfully perform time resolved fluorescence lifetime analysis, the TCSPC module required an input pulse synchronised to the micro-LED excitation pulse. This was achieved using the system clock of the ST Microelectronics 908 Test Board (Section 3.2.1), which acted as the input signal to the on-chip delay generator from which the excitation pulse is derived. Each micro-LED pulse was synchronised to the rising edge of the system clock which was therefore used as the synchronisation pulse for the TCSPC module. To ensure compatibility of the $3.3 \mathrm{~V}$ clock signal and the TCSPC synchronisation input it was necessary to attenuate and invert the system clock as described in Section 3.1.6. The TCSPC card was operated in reverse mode and the pulse train from the SPAD detectors were passed, via a pulse inverter, to the constant fraction discriminator input of the module. The micro-LED pulse frequency was set by the system clock and was set to be $3.6864 \mathrm{MHz}$. The micro-LED excitation pulse width was chosen to be $8 \mathrm{~ns}$. The LED received a $4 \mathrm{~mA}$ bias current and the electrode was driven to $13 \mathrm{~V}$, producing short pulses with fast rise and fall times $(2.15 \mathrm{~ns}$ and $3.31 \mathrm{~ns}$ respectively, for a pulse width of 8ns). Samples were held in a micro-cavity slide (Agar Scientific Ltd, UK) and sealed by a $24 \mathrm{~mm} \times 24 \mathrm{~mm}$ cover slip to avoid evaporation. The slide was placed on top of the chip and excited by a pulse train from a single micro-LED. Figure 6.2 shows a schematic of the optical stack.

A photograph of the micro-LED array exciting a quantum dot sample mounted on a micro-cavity slide is shown in Figure 6.3. 


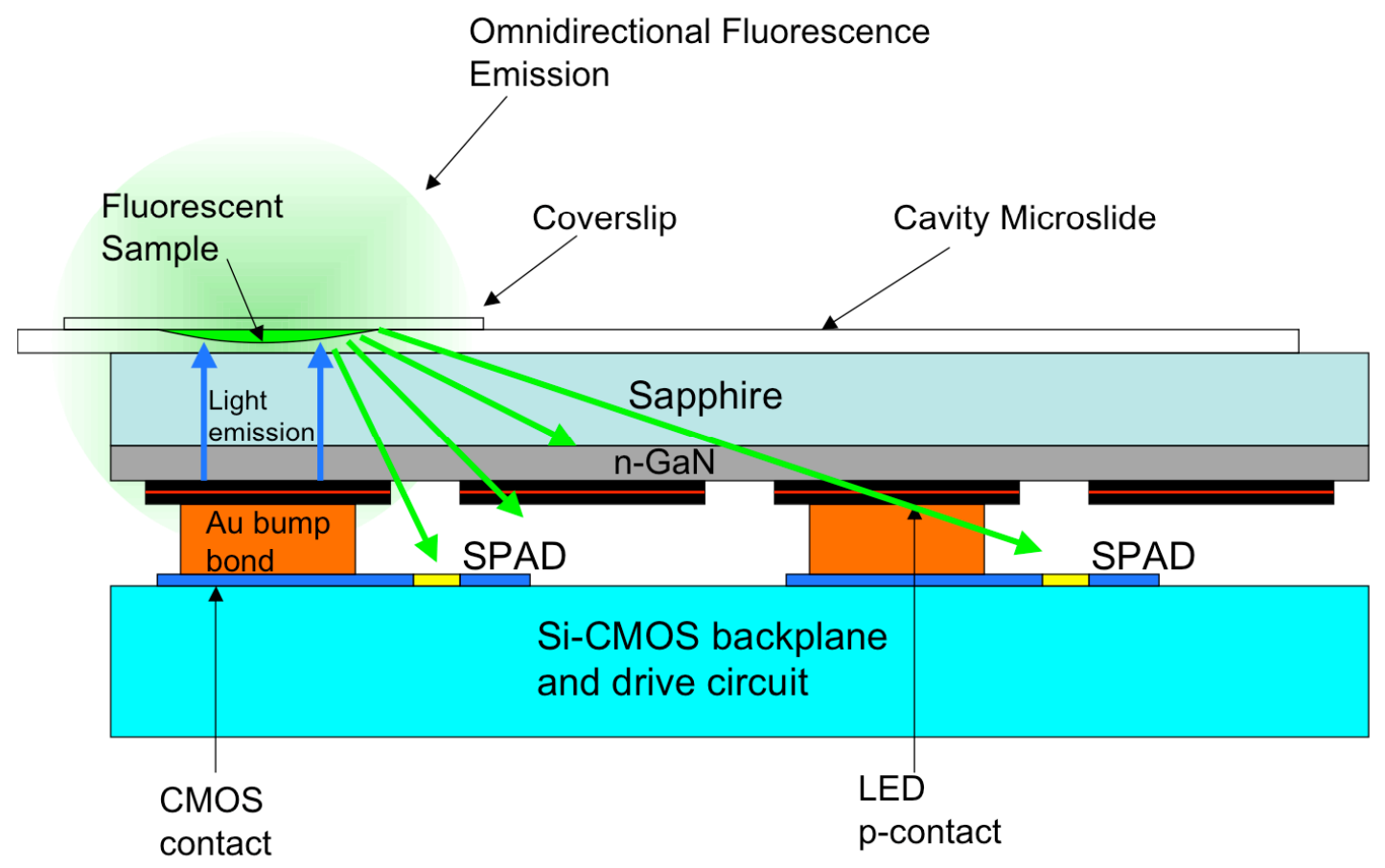

Figure 6.2: Cross-section of single-chip micro-system.

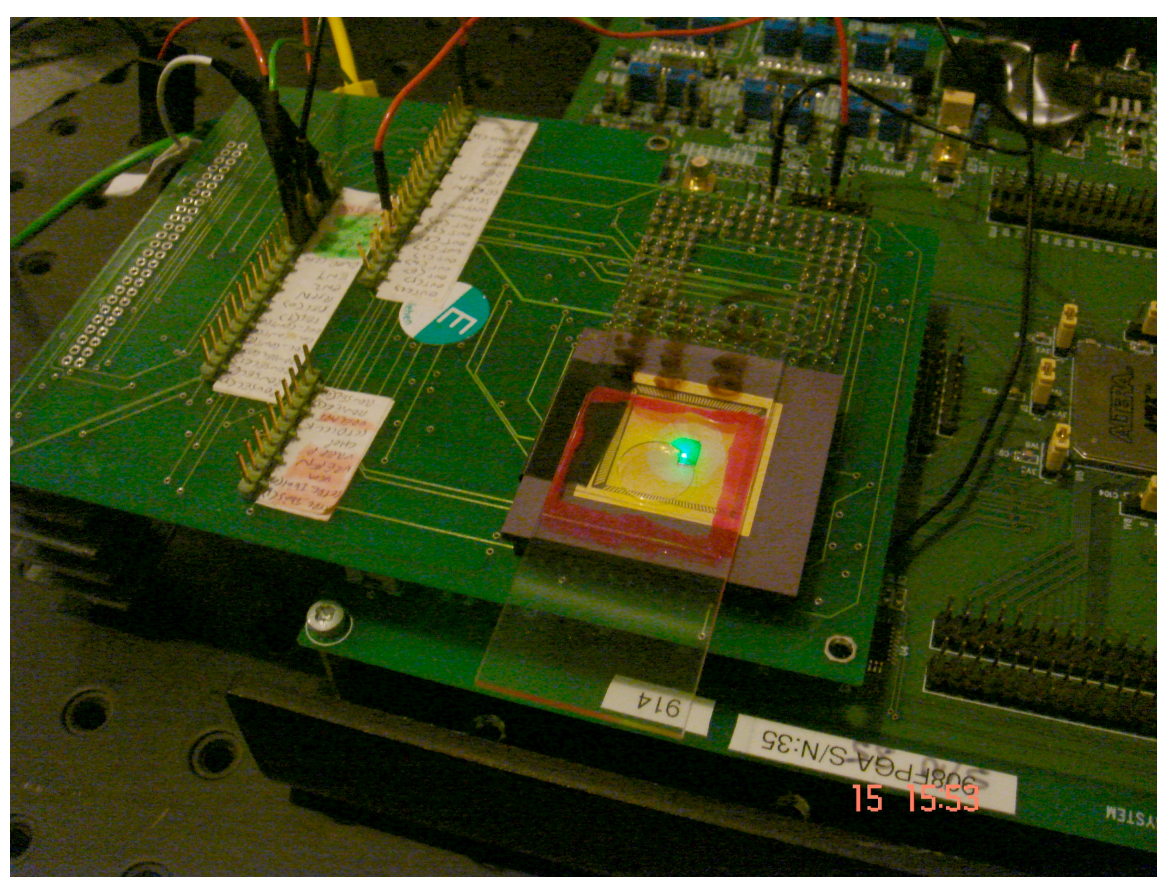

Figure 6.3: Micro-LED excitation of a quantum dot sample on a micro-cavity slide. 


\subsubsection{Excitation Light Filtering}

The number and position of the LED pixels that were active was user definable and any combination from a single LED up to all 64 elements of the array could be active at any one time. During analysis all 64 SPADs in the array are active. However, the address chosen by the user defines which SPADs are present on the output bus; thus, the address of the detecting SPAD does not have to correspond to the address of the exciting micro-LED. Due to the lack of optical filter to avoid direct coupling of excitation light from the micro-LED to the detector, the SPAD chosen was situated 8 rows away $(800 \mu \mathrm{m})$ from the emitting LED pixel. This offset the detector from the excitation source, so that the excitation source no longer directly faced the detector. It should be noted that this does not completely eliminate photon counts due to direct LED excitation of the SPAD, as can be seen from the results presented in Section 6.2.3. Photon counts due to directly coupled light from the LEDs were further minimised by choosing a 370nm UV excitation source. At 370nm, the photon detection probability (PDP) of the SPADs was $7 \%$. However, this wavelength was ideal for the excitation of the selected quantum dots, which were used to characterise the system and are summarised in Table 6.2.

\begin{tabular}{lllll}
\hline & $\begin{array}{l}\text { Peak } \\
\text { absorption } \\
(\mathrm{nm})\end{array}$ & $\begin{array}{l}\text { First } \\
\text { absorption } \\
\text { peak }(\mathrm{nm})\end{array}$ & $\begin{array}{l}\text { Emission } \\
\text { peak }(\mathrm{nm})\end{array}$ & $\begin{array}{l}\text { Concentration } \\
\left(\mathrm{nmol} \mathrm{ml}^{-1}\right)\end{array}$ \\
\hline Adirondack Green & $<400$ & 510 & 526 & 77.03 \\
Catskill Green & $<400$ & 525 & 543 & 68.77 \\
Hops Yellow & $<400$ & 531 & 555 & 57.17 \\
\hline
\end{tabular}

Table 6.2: Summary of quantum dot samples used to evaluate system.

At 526nm (the emission wavelength of the Adirondack Green quantum dots) the PDP of the SPADs increased to $25 \%$. This insensitivity to shorter wavelengths results in filtering of the excitation light. This process is summarised in Figure 6.4, which overlays the spectral properties of the LED, quantum dot sample and SPAD. The LED and sample emission spectra were measured using a Triax 550 spectrometer (Jobin Yvon, Inc.), with the sample being excited by a $15 \mathrm{~mW}, 375 \mathrm{~nm}$ UV laser (Power Technology, Inc.). For the absorption, $200 \mu 1$ of Adirondack Green quantum dots was mixed with $1800 \mu \mathrm{l}$ of toluene in a quartz cuvette and its absorption spectrum was measured with a Lambda 2 spectrophotometer (Perkin Elmer). 


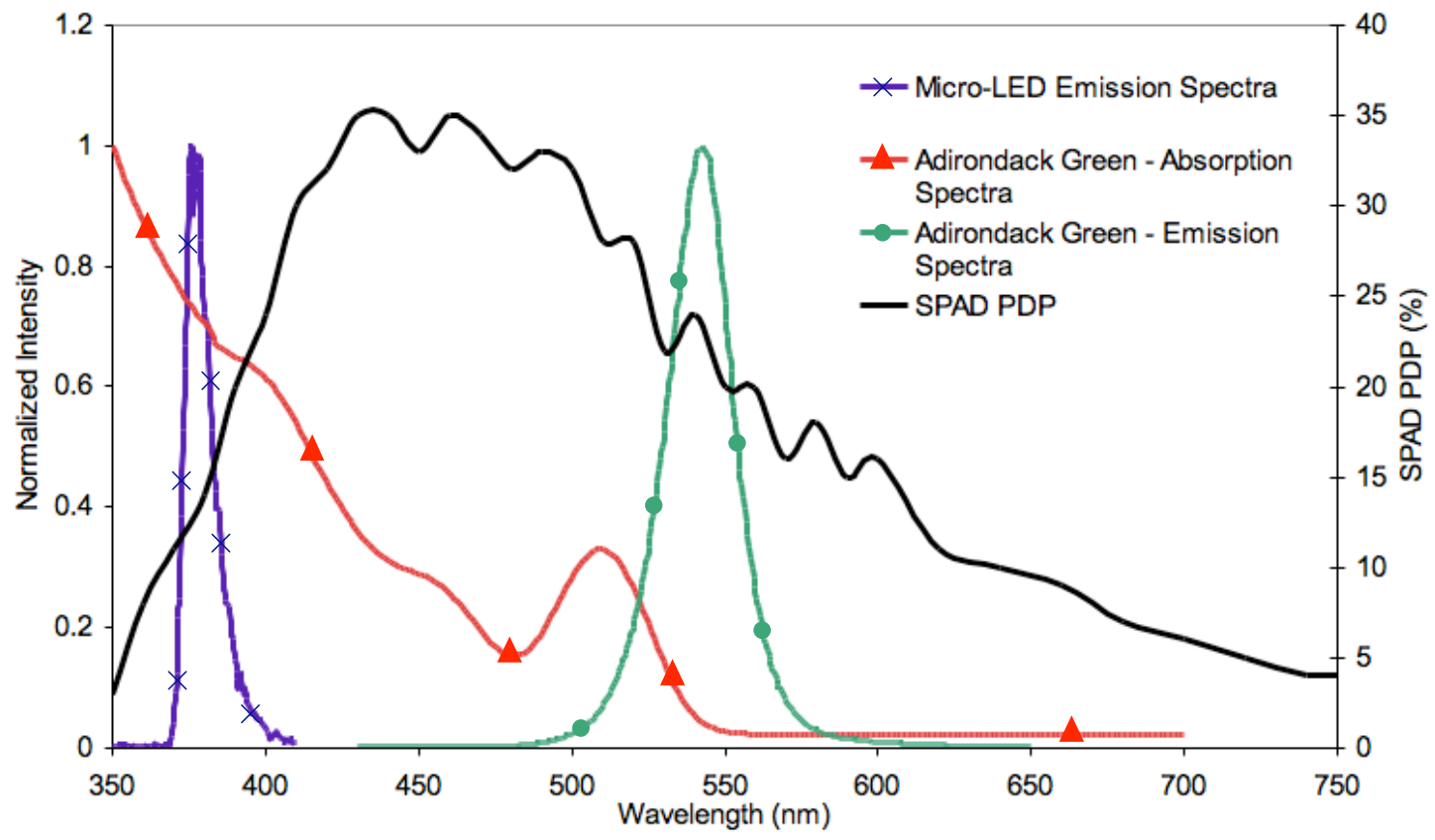

Figure 6.4: Micro-LED emission spectra, SPAD PDP and quantum dot absorption (Adirondack Green) and emission spectra versus wavelength. This clearly highlights the spectral separation of the excitation wavelength and the detector sensitivity range. 


\subsubsection{Fluorescence Lifetime Data Captured with Single Chip System}

The output of the SPADs was passed off-chip to a TCSPC module which created a histogram based on photon arrival times, relative to the excitation pulse. The fluorescence decay of three quantum dot samples captured in this way is presented in Figure 6.5. The concentrations of these samples was as supplied by the manufacturer, $77.03 \mathrm{nmol} \mathrm{ml}^{-1}, 68.77 \mathrm{nmol} \mathrm{ml}^{-1}$ and $57.17 \mathrm{nmol} \mathrm{ml}^{-1}$ for Adirondack Green, Catskill Green and Hops Yellow, respectively. The acquisition time for each decay curve was less than 10 minutes. Due to the absence of any optical spectral filtering in the system, the excitation pulse was also detected by the SPADs and can be observed in the resulting histogram as the large broad peak from which the fluorescence decay emerges. The ratio of detected excitation light to detected fluorescence emission was dependent on several factors. One such factor was the intensity of excitation light from the LED. This could be controlled by the user by adjusting the peak voltage, the bias current of the driver and the width of the excitation pulse. The intensity of fluorescence emission from the sample was also an important factor and is influenced by sample concentration. Finally, the relative distance from the excitation micro-LED and the relevant detection SPAD affected the level of directly coupled light from the LED reaching the detector.

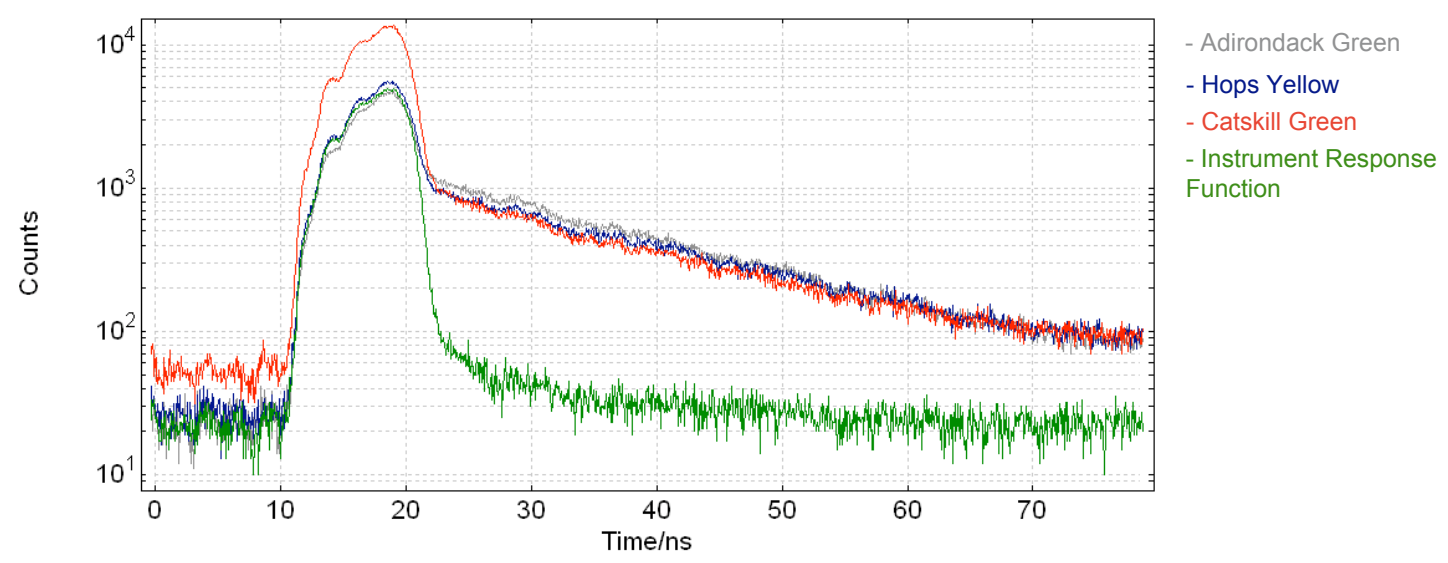

Figure 6.5: IRF and fluorescent decay curves for quantum dot samples at three different emission wavelengths.

As can be seen from the results in Figure 6.5, the fluorescence decay curves can clearly be distinguished from the instrument response function (IRF). The data gathered was exported and analysed using FAST lifetime extraction software by Edinburgh Instruments (Section 3.3.6) [110]. Lifetimes extracted by the discrete deconvolution of the IRF and the decay curves 
presented in Figure 6.5 are summarised in Table 6.3 (114ps estimated measurement error, based on 114ps RMS jitter from the SPAD detector plus 4ps RMS jitter of the TCSPC module) for Adirondack Green, Catskill Green and Hops Yellow, respectively. These extracted lifetime values are longer than those measured using the SPAD detector and microscope. This could be due to the use of a wider excitation pulse ( $8 \mathrm{~ns}$ compared to 68ps) which may interfere with decay dynamics.

\begin{tabular}{lll}
\hline Sample & Lifetime & Measurement Error \\
\hline Adirondack Green & $17.8 \mathrm{~ns}$ & $\pm 114 \mathrm{ps}$ \\
Catskill Green & $17.2 \mathrm{~ns}$ & $\pm 114 \mathrm{ps}$ \\
Hops Yellow & $19.1 \mathrm{~ns}$ & $\pm 114 \mathrm{ps}$ \\
\hline
\end{tabular}

Table 6.3: Extracted lifetimes from decay curves captured using single chip system

\subsubsection{Single-Chip Micro-System: Summary}

A compact fluorescence lifetime analysis system has been demonstrated, which incorporates sample excitation via a CMOS driven UV micro-LED array and emission detection using an array of SPAD detectors located on the same CMOS chip. By placing the sample directly on top of the excitation source and detector array, the dimensions of the system are minimised thereby minimising photon loss due to divergent light. As the excitation pulse width was decreased, the intensity of the micro-LED emission was reduced, which in turn reduced the fluorescence emission. This led to a lower signal-to-noise ratio and thus a less accurate lifetime measurement. To allow an $8 \mathrm{~ns}$ excitation pulse to be used, fluorophores with a relatively long lifetime were used. In addition to their compatible wavelength properties, as described previously, the lifetimes of the quantum dot samples were ideally matched for excitation with an 8 ns excitation pulse.

Results demonstrate the systems ability, in conjunction with external TCSPC hardware, to accurately measure fluorescence lifetime data of commercially available quantum dot samples. The system removed the need for complicated optical alignment, with the sample being introduced on a simple micro-cavity slide. The SPADs insensitivity to UV wavelengths also allowed the system to operate without the need for optical filters. It is clear that a future design will need to address the issue of detection of directly coupled excitation light, either through the inclusion of an optical filter or by electronically time-gating the SPAD detector to render 
it insensitive during the excitation period. It is this high level of coupled excitation light that limits the sensitivity of the single chip system and prevents lower concentration samples from being analysed. It is hoped that with the inclusion of an optical filter or an electronically gated detector, the minimum detection limit of the system can be improved. However, the ability of such a micro-system, based on a standard CMOS process, to conduct lifetime analysis without the need for expensive optics and filters should open the way to the development of a compact (potentially portable), robust and inexpensive solution to time resolved fluorescence measurements. 


\subsection{Two-Chip Micro-System for Time-Resolved Fluorescence Lifetime Detection}

Filtering of the sample excitation light was necessary in order to produce credible decay curves that were not dominated by a large excitation pulse. While the micro-system presented in Section 6.2 offered a single chip solution to micro-scale time resolved fluorescence detection, the structure of the device did not allow optical filters to be placed between the excitation and detection elements. Micro-fabrication steps could be performed to place micro-filters between the elements but this would be an expensive and complicated solution [111], [112]. To allow an optical filter to be placed between the excitation and detection elements and to make use of the higher power and shorter pulse widths that the 8x8 dedicated micro-LED driver array (Section 5.3) provided, a two chip "sandwich" structure was proposed (Figure 6.6).

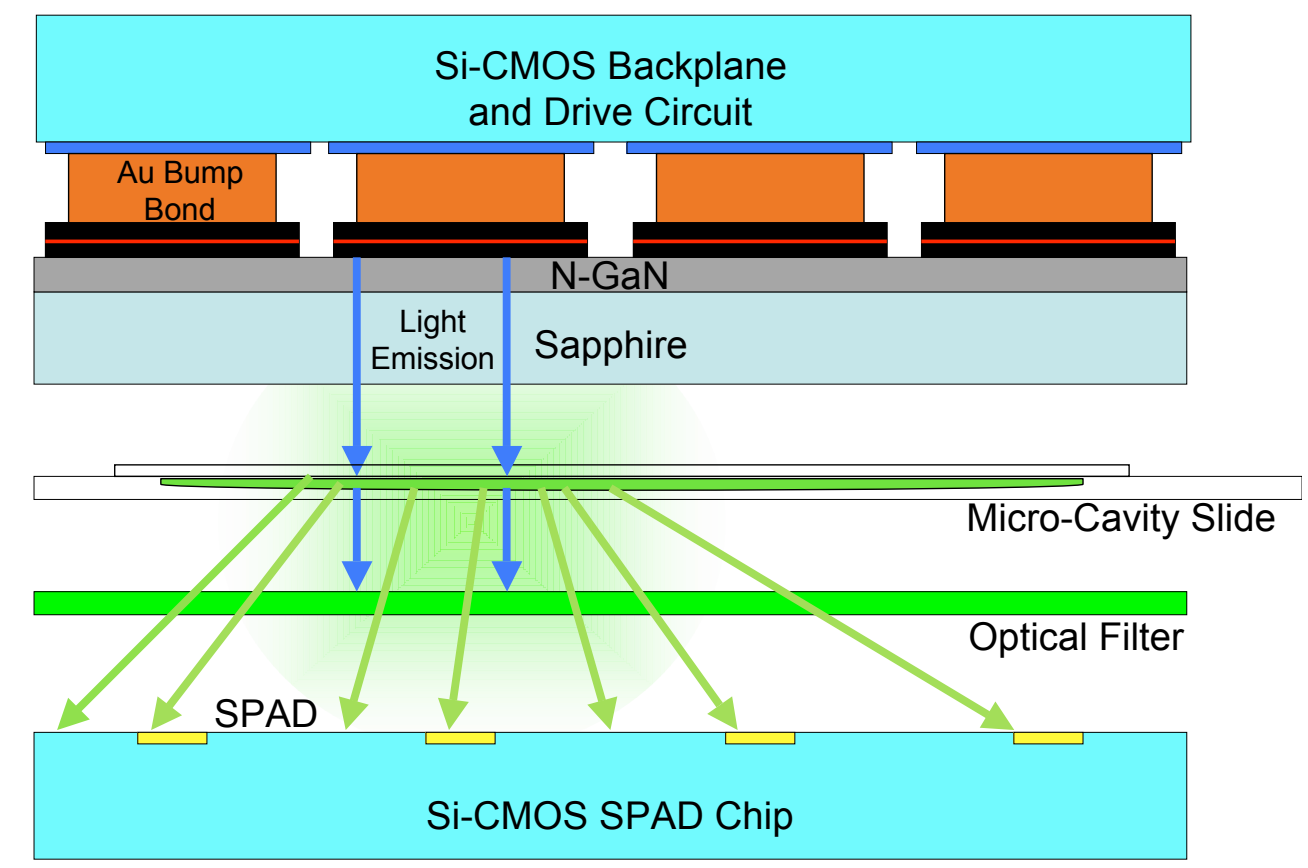

Figure 6.6: Cross-section of the two-chip micro-system. The sample of interest is introduced into the system in a micro-cavity slide sealed by a cover-slip. An optical filter prevents excitation light reaching the detector array.

A dedicated daughter card (Figure 6.7) was designed, with the micro-LED device situated on the under-side of the PCB, facing the SPAD detector chip located on the The University of Edinburgh $\backslash$ Opal Kelly Test Board (Section 3.2.2). Electrical connection to the daughter card was made via stacked header pins. This technique allowed the distance between the micro-LED 
device and the SPAD detector chip to be adjusted. At present the two devices are separated by a distance of approximately $1 \mathrm{~cm}$, although it is hoped that this distance can be reduced to as little as $3 \mathrm{~mm}$. Both devices shared the same core power supplies and ground connections. These supplies and all other bias supplies, apart from the negative supply of the SPAD detector, were generated on the test board PCB and derived from the 5V supply of the USB connection. The negative supply required by the SPAD detector was generated by an external power supply. The devices shared a single FPGA situated on the test board, which generated the digital input signals to both devices.

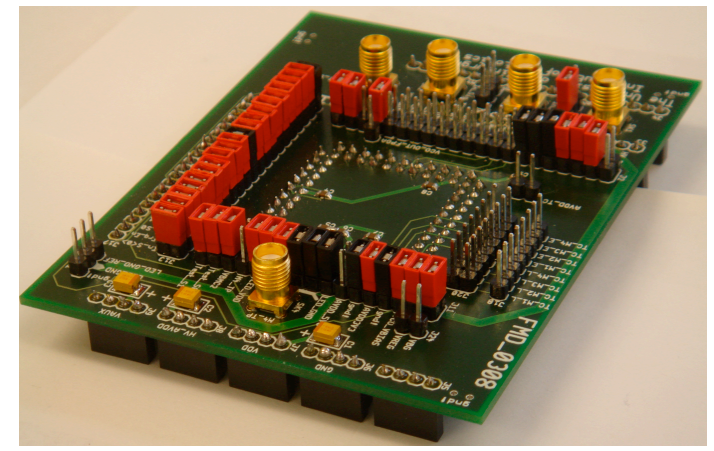

(a)

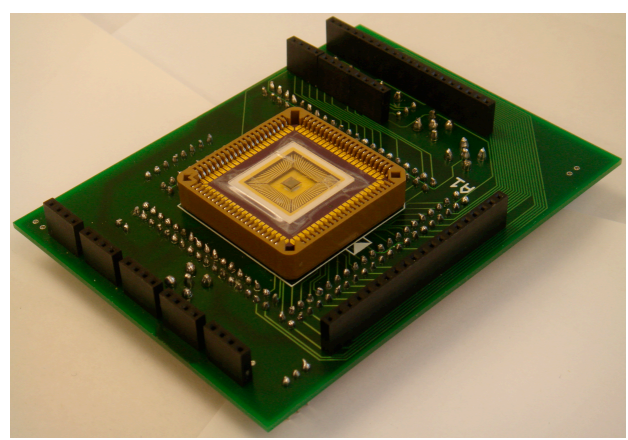

(b)

Figure 6.7: (a) Top side of two-chip system daughter card used to interface with the CMOS driven micro-LED device, (b) the underside of the PCB is where the chip is situated, in this configuration the micro-LED chip faces the SPAD chip which is located on the master $P C B$.

An optical filter and the sample of interest were placed between the devices. A plastic holder was designed to house these two elements (Figure 6.8). This holder provided a light tight enclosure for the packaged SPAD chip, an optical filter, a sample held in a micro-cavity slide and a packaged micro-LED device.

Due to the inability of the VPCIVIEW data capture software (Section 3.3.4) to communicate with the SPAD device via USB it was not possible to capture and process data from the in-pixel counter circuits. Instead, raw SPAD pulses were passed off-chip and processed by external TCSPC hardware (Becker and Hickl SPC-730, Section 3.1.6).

The output from the on-chip VCO, situated on the micro-LED driver, defined the repetition rate of the LED device. This signal was also passed off-chip and could be observed on a SMA connector on the daughter card. This signal was used as the synchronisation input to the TCSPC 

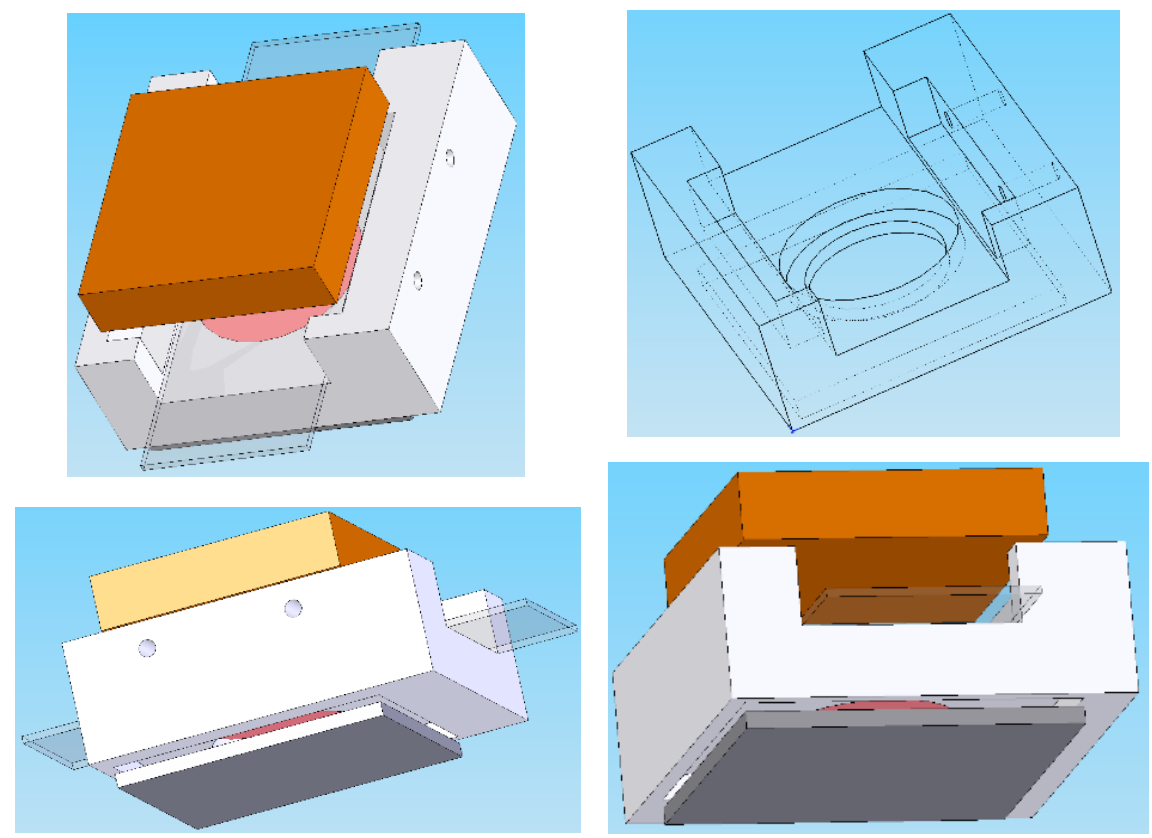

Figure 6.8: Filter and sample holder situated between the micro-LED excitation source and the SPAD detector. Images courtesy of Andrew Garrie, COSMIC Laboratory, The University of Edinburgh.

module. A schematic of the complete system can be seen in Figure 6.9.

The micro-LED excitation source emitted light at a wavelength of $370 \mathrm{~nm}$, a $514 \mathrm{~nm}$ long pass filter was therefore chosen to separate the excitation light from the fluorescence emission. This allowed a range of samples with emission spectra greater than $514 \mathrm{~nm}$ to be evaluated.

A photograph of the implemented two-chip micro-system is shown in Figure 6.10. 


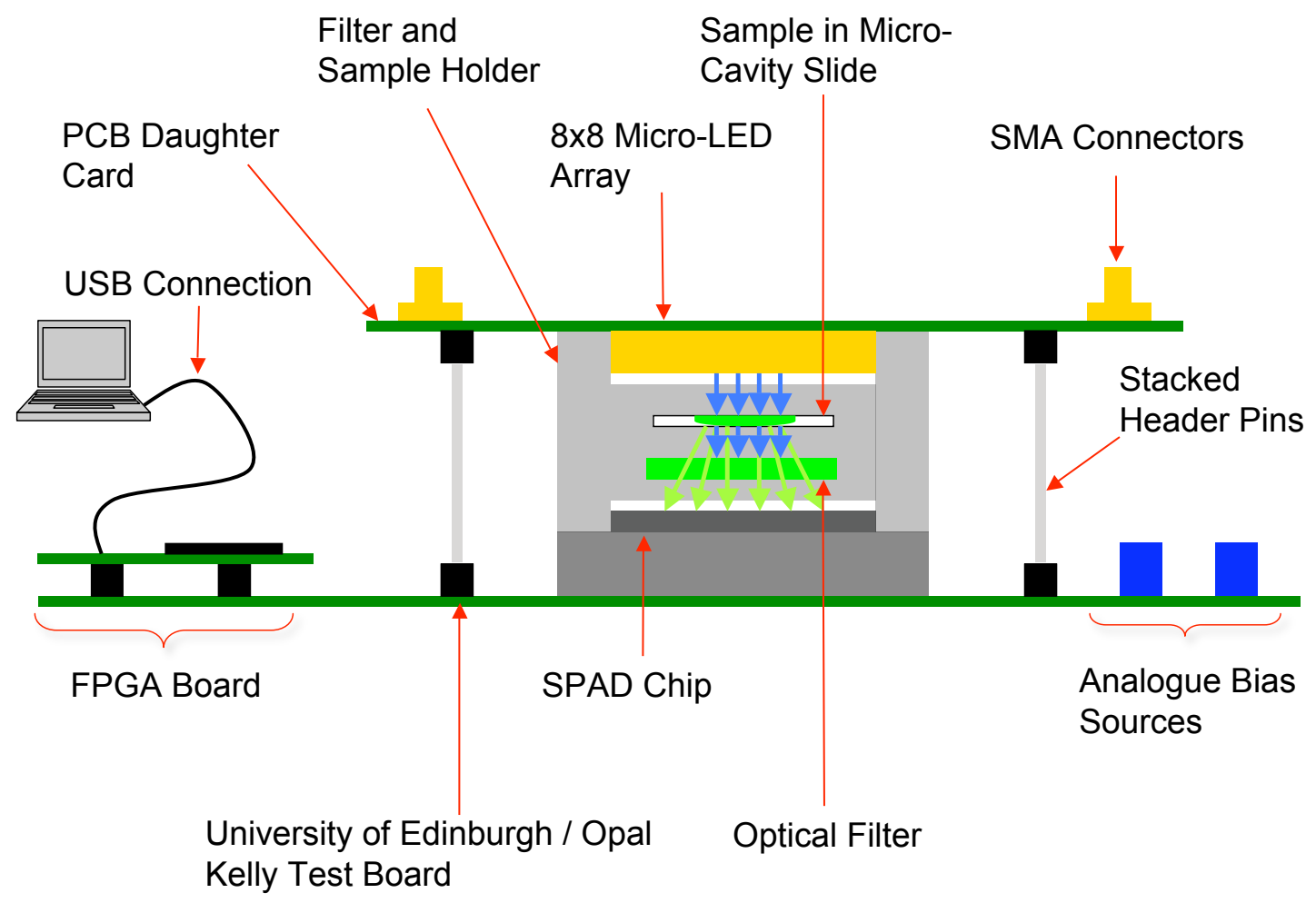

Figure 6.9: Complete two-chip micro-system. Power and user control signals are provided via USB connection. The PCB daughter card is physically supported by the filter and sample holder and stacked header pins. 


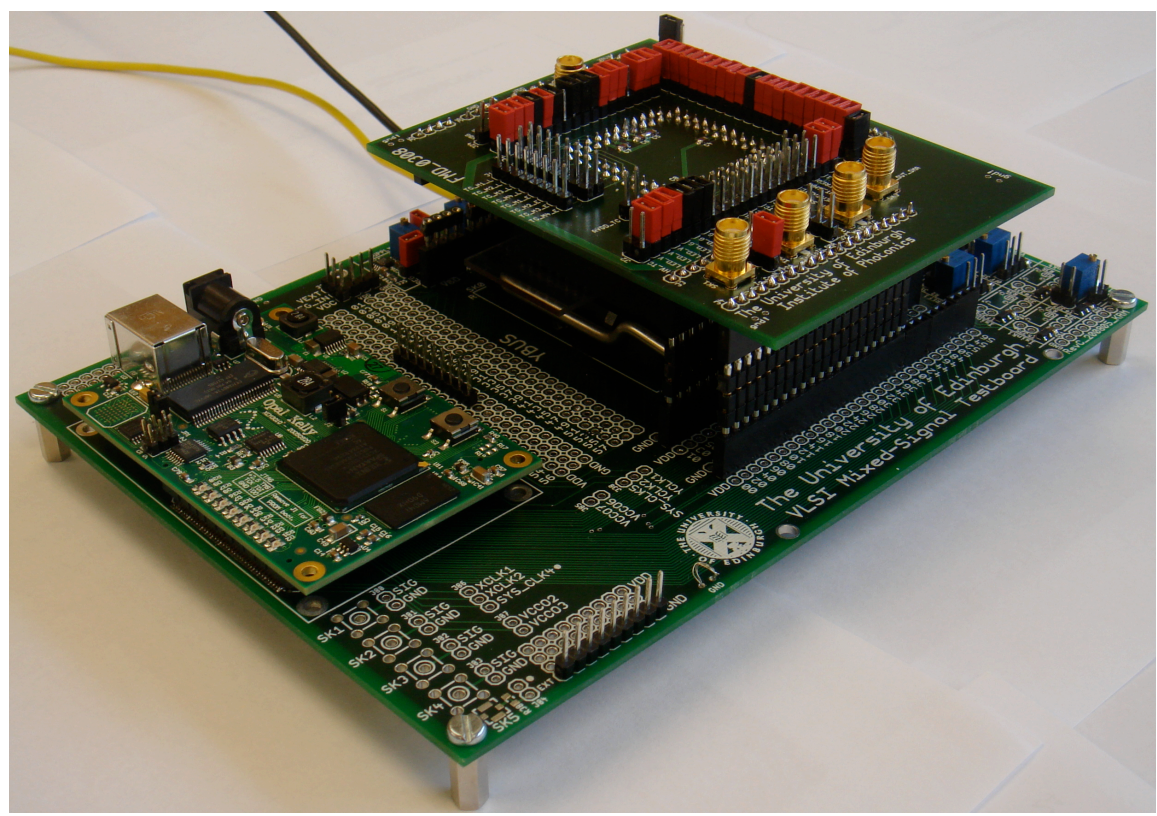

Figure 6.10: Two-chip micro-system for time-resolved fluorescence detection. Electrical connection between the FPGA test-board and the daughter card was made using stacked header pins. 


\subsubsection{Fluorescence Lifetime Data Captured with Two-Chip System}

It was hoped that the inclusion of an optical filter and shorter excitation pulses would allow shorter lifetime samples to be evaluated (with respect to the 15-20ns lifetimes of the quantum dot samples). In order to assess this, samples of rhodamine $6 \mathrm{G}$, fluorescein and quantum dots were analysed. Initial results revealed that while the inclusion of an optical filter in the system greatly attenuated the levels of excitation light reaching the detector, output data was still dominated by micro-LED light. This can be attributed to inefficiency in the filter at $370 \mathrm{~nm}$. Despite being a long pass filter specified with a roll-on at $514 \mathrm{~nm}$ and less than $1 \%$ transmission in the stop-band, it was found that at 370nm it had $30 \%$ transmission. This problem was further exasperated by the divergent nature of the LED light reaching the filter. Edge filters from Semrock, Inc. are specifically designed for normal incident light. Very few photons from the LED source would reach the filter at this angle of incidence. The efficiency of the filter was therefore further compromised. This problem was solved by stacking two optical filters, greatly improving the attenuation of excitation light at $370 \mathrm{~nm}$. While this solution does not provide an elegant, compact or cost-efficient solution, it did allow initial measurements of short lifetime fluorophores to be made. Figure 6.11 shows time-resolved fluorescence lifetime data for rhodamine $6 \mathrm{G}$, fluorescein and quantum dot samples (at $1 \mathrm{mM}, 1 \mathrm{mM}$ and $57 \mu \mathrm{M}$ concentration, respectively) along with an IRF curve of the 2.5ns micro-LED optical pulse that was used to excite the samples. The acquisition time for each decay curve was less than 10 minutes. When these decay curves are compared to the results presented in Section 6.2.3 for the single chip system, it is clear that the inclusion of an optical filter greatly benefits the quality of the decay data. Thus, the ability to effectively remove excitation photons from the resulting fluorescence photons through optical filtering has been demonstrated. The use of the $8 \times 8$ micro-LED driver chip also greatly improved the micro-system's ability to resolve fluorescence lifetime decay curves by providing a higher optical power and shorter pulse width excitation source. These two factors played a key role in improving the performance of the two-chip micro-system over that of the single chip system. From the decay curves presented, lifetimes of $5.98 \mathrm{~ns}, 4.49 \mathrm{~ns}$ and $13.27 \mathrm{~ns}$ were estimated for the rhodamine $6 \mathrm{G}$, fluorescein and quantum dot samples, respectively. The extracted lifetimes of the fluorescein and quantum dot samples agree well with the results presented in Section 4.3.3 and 4.4.2. However, the lifetime extracted for rhodamine 6G was longer than that reported in the literature [113] (3.99ns). This could be due to sample contamination or degradation due to age. It is unlikely that this discrepancy can be attributed to system offset as the lifetime estimations for fluorescein and quantum dot samples 
are in good agreement with previously reported results. However, further system evaluation is required to confirm this.

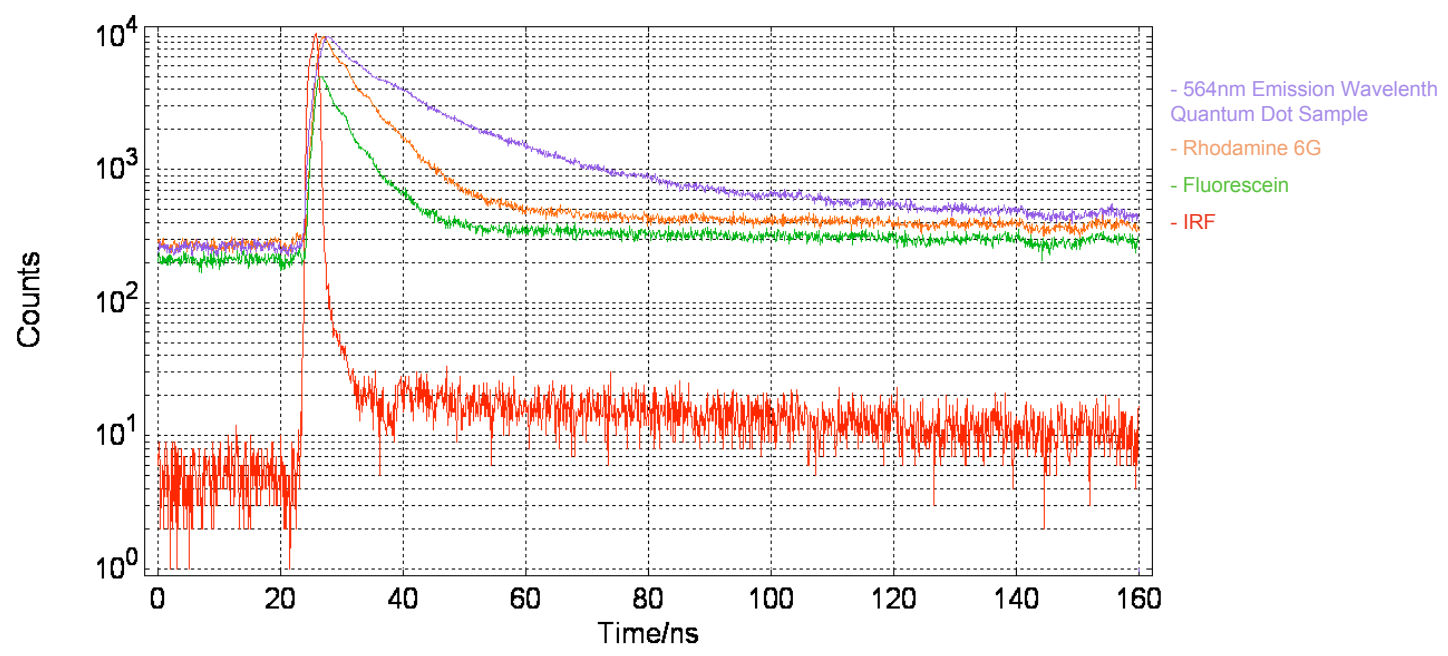

Figure 6.11: Fluorescence lifetime decay curves from rhodamine $6 G$, fluorescein and quantum dot samples captured using the two-chip micro-system.

The higher than expected noise floor could be attributed to the level of chip activity during the excitation and detection phase. As has been discussed in Section 4.3.1.1, the inability to suppress SPAD activity on the chip results in noise on the SPAD bias signal which is thought to lead to spurious counts. Furthermore, to obtain these decay curves the experiment was allowed to run for approximately 30 minutes. This extended experimental time resulted in a greater number of dark counts. It is thought that this high noise floor led to slight skewing of the lifetime data. [114] show the effect that a low signal-to-noise ratio has on the extracted lifetime data. An improved signal-to-noise ratio and lifetime estimation could be achieved by optimisation of the optical path through the inclusion of an optical filter which is better matched to the excitation source. A redesigned SPAD detector device would include the ability to disable non-addressed SPADs and to time-gate the detectors, which may remove the need for an optical filter entirely. 


\subsubsection{Two-Chip Micro-System: Summary}

The two-chip micro-system design allows the user to place an optical filter between the excitation source and the detector array. This key feature has been shown to allow the system to capture improved decay curve data. The results presented show how the excitation light was effectively filtered out. Unfortunately, two stacked optical filters were required. This was due to the short wavelength characteristics of the original long-pass filter and it is thought that with the inclusion of a better matched filter, these results could be reproduced with a single filter.

The two-chip system also allowed electrical signals to be shared between the two chips. Both devices shared a common ground and power supply, which was derived from the USB supply of the FPGA board using low drop-out voltage regulators situated on the PCB test board. This minimised the need for multiple external power supplies, greatly reducing the size and cost of the system. The ability to easily pass signals between the chips is particularly significant for time-resolved fluorescence lifetime applications where synchronisation of the excitation and detector devices is of great importance. In this design it was possible to use the on-chip VCO circuit situated within the micro-LED driver device to not only define the repetition rate of the pulsed micro-LEDs, but also to act as the input signal to the timing-generator used to define the time-gates of in-pixel counters within the SPAD device. Using this technique the position of the counter time-gates are inherently synchronised to the sample excitation pulse. The development of a software programme capable of interfacing with the SPAD counter chip via USB will allow this set up to be fully evaluated. By utilising the on-chip counter circuits, the need for an external TCSPC module would be eliminated. It should be noted that in Chapter 4 the capability of the time-gated SPAD counter circuit has been fully evaluated and their ability to accurately capture fluorescence lifetime data demonstrated. This, coupled with the inclusion of on-board (or on-chip) generation of the negative SPAD detector bias voltage, would result in a compact micro-system for time resolved fluorescence lifetime measurements. Data communication and device power would be entirely handled via USB connection to a computer, with data interpretation and lifetime extraction being performed in software.

\subsection{Discussion}

Two different micro-system topologies for time-resolved fluorescence lifetime measurements were implemented and evaluated. The first system was capable of performing fluorescence 
lifetime measurements using a single chip. This provided a low-cost solution to time resolved fluorescence detection. The system design meant that there were no issues with the alignment of the excitation and detection elements and samples could be conveniently introduced to the system by placing the slide on top of the packaged chip. Unfortunately, this configuration did not allow an optical filter to be placed between the excitation source and detector array, resulting in a significant amount of excitation light being coupled into the detector, which was observed in the resulting decay curve. Furthermore, the optical output power of the micro-LED device had to be traded off against pulse width. In order to provide sufficient optical power to obtain a usable SPAD count rate from the samples' fluorescence emission it was necessary to use an $8 \mathrm{~ns}$ excitation pulse. The lack of optical filter and the need to use relatively wide excitation pulses prevented short lifetime sample being evaluated.

In a bid to address the issue of excitation light filtering and the need for shorter excitation pulse widths, a two-chip micro-system was implemented. This system made use of a re-designed micro-LED driver array (Section 5.3), capable of producing significantly shorter and higher power optical pulses. A two-chip stacked topology also allowed optical filters to be placed between the excitation and detection elements. These two additions to the micro-system greatly improved device performance, resulting in a device capable of accurately detecting decay curves of shorter lifetime fluorophores. By removing the need for an excitation filter through the time gating of the SPAD detector, the distances between the excitation source, sample and detector could be reduced, minimising the divergence of the excitation light and resulting fluorescence and thereby improving system efficiency and performance.

It is clear that both system architectures would benefit from a number of design improvements. The introduction of a time-gated SPAD, removing the need for an optical filter, may allow the single chip solution to become a valid option. The two-chip design would also benefit from this change; however, it would also benefit from the inclusion of a filter which is better matched to the $370 \mathrm{~nm}$ excitation wavelength of the micro-LEDs. The two-chip device offers the opportunity to include on-chip signal processing, which would remove the need for an external TCSPC module, greatly reducing system costs and size. To achieve this a software program capable of communicating with the SPAD device via a USB interface must be designed.

A micro-system solution will enable the development of low cost, portable fluorescence lifetime readers for many optical lab-on-a-chip applications such as point-of-care diagnostics equipment and the synthesis and/or read out of DNA micro-arrays. With each element being 
separately addressable, there is the potential to excite many fluorescent samples in parallel [29]. By integrating the excitation source with a photodetector and on-chip driving electronics, our devices will contribute to the development of lab-on-a-chip (LoC) systems [115]. This includes work by Chodavarapu et al. [14] which aims to carry out fluorescence detection using a CMOS-based system which incorporates a detector with signal processing circuitry. Their system does not, however, include an integrated excitation source and uses a discrete LED. Cleary et al. [116] demonstrated TCSPC on a micro-scale using integrated optics and microfluidics, however, their system relies on a pulsed diode laser as an excitation source and a discrete SPAD detector. The system presented here offers a greater level of integration, placing the excitation source in the same micro-scale system as the sample and detector. 


\section{Future Work and Conclusions}

\subsection{Summary}

The data presented in this thesis has demonstrated two different designs for a micro-system which aims to provide a low-cost, compact and robust alternative to high-powered pulsed lasers and fragile, expensive photo-multiplier-tube based detection systems for time-resolved fluorescence detection. The designs utilise the ability to integrate photon detection elements on the same CMOS substrate as signal processing circuitry to form a compact detection system based on single-photon avalanche diodes. It has been shown that these SPAD devices are capable of accurately capturing and processing decay data. Sample excitation was achieved using an array of CMOS driven AlInGaN micro-LEDs, capable of producing sub-nanosecond optical pulses and which occupy a small footprint. The SPAD detector array and the initial micro-LED driver array were fabricated in a high-voltage $0.35 \mu \mathrm{m}$ technology. Two further iterations of the micro-LED driver array design were implemented in a low-voltage $0.35 \mu \mathrm{m}$ technology. The micro-system has been shown to operate successfully without the need for expensive optics.

By making use of standard CMOS foundry technologies, this work opens up the possibility of mass manufacturing a low-cost micro-system. This would greatly increase access to the technique of fluorescence lifetime analysis, since conventional systems are currently associated with prohibitively high costs.

Through a critical review of published work aimed at miniaturising elements of a fluorescence lifetime system (Chapter 2), it was identified that a fully integrated micro-system for fluorescence lifetime analysis incorporating excitation, detection and signal processing elements had not previously been realised. A time-domain lifetime system requires an excitation source capable of producing short optical pulses and the ability to accurately capture the arrival times of the resulting fluorescence photons.

Chapter 4 outlines the design and test of a SPAD detector array realised in a high-voltage $0.35 \mu \mathrm{m}$ CMOS technology. A 16x4 array of SPAD detectors with in-pixel time-gated counters 
and on-chip time-gated circuitry is described. The sensitivity of the SPAD detector was found to be less than $10 \mathrm{nM}$, as demonstrated using detector data processed by both a commercially available external TCSPC module and using in-pixel signal processing circuitry. An on-chip timing generator can produce time-gates with a resolution of 408ps and 44ps RMS jitter. Using this circuit, time-gates of user definable width from a minimum of $408 \mathrm{ps}$ to a maximum of $48 \mathrm{~ns}$ can be produced. By gating the operation of in-pixel counter circuits, histogram data of fluorescence decay curves can be captured. The extraction of lifetime values from counter data is presented using three different lifetime extraction algorithms. All three algorithms performed well; however, the ability to realise the IEM algorithm on-chip has been identified as a clear route to further system integration. The integration of the detection device with signal processing circuitry on the same CMOS chip removes the need for external photon counting hardware. The ability to capture accurate time-resolved fluorescence lifetime data using SPADs and signal processing circuitry integrated in the same CMOS die provides a compact, low-cost solution to the detection and processing of lifetime data suitable for integration within a time-resolved fluorescence micro-system. As CMOS technology dimensions are reduced, the minimum gate delay of the process is also reduced. This has a direct impact on the minimum time-gate width that can be achieved, improving device resolution and therefore allowing shorter lifetimes to be detected with greater precision. With the advent of SPAD detectors in deep sub-micron technologies, it is hoped that this can be matched by performance improvements in the signal processing circuitry.

CMOS driven arrays of AlInGaN micro-LEDs were evaluated independently (Chapter 5), before being integrated with the SPAD detector array in a micro-system. Initially, a current controlled driver was conceived. This device allowed the bump-bonding process to be established and was capable of producing optical pulses down to $1.12 \mathrm{~ns}$ in width. The inclusion of SPAD detectors within this array allowed a single chip micro-system for time resolved fluorescence detection to be investigated. It was decided that a redesigned driver should aim to reduce the optical pulse width to below $1 \mathrm{~ns}$ and increase the optical output power of the device. To achieve this, a voltage driven design was implemented, sacrificing array uniformity for power performance which was capable of delivering 300ps wide pulses. An initial 16x16 array of voltage based LED drivers needed to be redesigned due to mechanical failure of the metal electrodes during the bump-bonding process. This redesign sacrificed pixel pitch and hence array density to ensure there was a robust and reliable platform upon which to bump-bond the micro-LED array. This redesign resulted in an $8 \times 8$ driver array with 
$100 \%$ device functionality. With a pulse width of 300ps the LED array was suitable as an excitation source for the investigation of short lifetime fluorophores. Micro-LED performance results presented in this thesis were for a device with an emission spectra which peaked at $371 \mathrm{~nm}$. With minor modifications to the AlInGaN device structure it is possible to modify the emission spectra. It can be envisaged that a number of CMOS driven micro-LED devices could be designed to cover a wide wavelength range. Micro-LED devices have already been demonstrated from 290nm to 470nm [117], [118], [119], [120]. Devices could be selected on the basis of how well their emission spectra matches the absorption spectra of the sample of interest. As devices with different emission properties emerge it is hoped that new applications for CMOS driven micro-LED will emerge such as chip-to-chip communications, mask-free photolithography, 3-D imaging and ranging.

Having evaluated the performance of the SPAD detector with signal processing circuitry and the CMOS driven AlInGaN micro-LED array as separate devices, Chapter 6 describes how these elements were brought together to demonstrate two different designs for a time-resolved fluorescence lifetime analysis micro-system. The aim of this work was to produce a compact system capable of exciting a sample of interest which contains a fluorescence marker and to detect and process the resulting fluorescence emission photons. A single-chip and a two-chip stack approach were both demonstrated and results of fluorescence decays captured using these devices were presented. From these data it is clear that the inability to separate the excitation light from the fluorescence emission is a key hurdle which must be overcome for a viable system. In the present project, this was addressed by placing an optical filter between the SPAD and micro-LED devices in the two-chip stack configuration. This approach was not possible in the single chip solution due to the dimensions of the device. The lack of filter had a detrimental effect on the captured fluorescence lifetime decays. This problem can be overcome electronically, as discussed below. These systems are, to the best of our knowledge, the first demonstration of a complete micro-system for time-resolved fluorescence detection to incorporate both an array of excitation sources and an array of detectors with single-photon sensitivity.

\subsection{Critical Discussion}

This project has demonstrated how a compact, low-cost micro-system for time resolved fluorescence can be realised using CMOS-based excitation, detection and signal processing 
elements. System wide signal compatibility was achieved by basing all elements on the same $0.35 \mu \mathrm{m}$ CMOS process. This key feature allowed for a compact system in which all devices could be controlled by the same FPGA device and inter-device communication and synchronisation could be achieved easily. While device performance is not yet comparable to more established experimental setups using commercially available equipment, these prototype systems demonstrate the benefits and potential of an integrated system. This work addresses a gap that has been identified in previous research work in bringing together all the components of a fluorescence lifetime system to form a truly integrated system. This work provides a platform for future, to improve the performance of each element of the system that will, in turn, lead to performance improvements of the system as a whole.

Despite demonstrating the ability of the on-chip time-gated circuits to capture lifetime data (Chapter 4), a demonstration of this in a micro-system environment has not been achieved. This was due to a change in test platform towards the end of the project. Initial time-gated detection tests were performed using a PCI based PCB platform. The development of firmware and software to transfer counter data to a computer was a critical component of this project. In order to develop a more compact system compatible with modern computer communication methods a USB based platform was designed. This required the re-development of firmware and software to control the device. Unfortunately, time constraints prevented the development of software capable of interpreting and analysing time-gated counter data via the USB interface. The design of such software will be a key piece of future work in the development of the micro-system.

The ability to introduce an optical filter in the two-chip system saw dramatic improvements in system performance thanks to the reduction in coupled excitation light from the LED-array to the SPAD detectors. However, the separation of the two devices is still not on the same scale as the excitation and detection elements. With each micro-LED measuring $72 \mu \mathrm{m}$ in diameter, each SPAD having an active area of just $6 \mu \mathrm{m}$ in diameter and both arrayed at a pitch of $200 \mu \mathrm{m}$ it is felt that a separation of $1 \mathrm{~cm}$ between the devices is still too large. The divergent nature of the LED emission means that any reduction in distance between the device and the sample of interest will be met with a squared reduction in the beam size, increasing the optical power focussed on the sample. Conversely, should it be required that a greater sample area be excited then this distance can be increased at the expense of reduced optical power density. The same argument can be applied to the detection of fluorescence emission. The omni-directional nature 
of fluorescence emission means that as the detector is brought closer to the sample this should be met with a squared increase in the number of photons impinging on a unit area of the detector. This is a particularly relevant point for CMOS based SPAD detectors due to the small fill factor of such devices. It is hoped that a reduction in the stack height will lead to a greater number of photon counts per second and, thus, reduced measurement times. Furthermore, the inclusion of micro-lens has the potential to improve the performance of both the micro-LED and SPAD devices, reducing the divergence of excitation light and improving detector fill factor.

This system will greatly benefit from progress being reported in the literature. Especially advances in the field of detector design and their integration with ever-more sophisticated signal processing circuitry. As these designs are realised in ever smaller CMOS processes, the potential timing resolution of the system improves leading to more accurate, detailed results. As array dimensions increase the potential for a fully integrated lifetime imager becomes a possibility.

\subsection{Future Work}

This project has offered an indication of how relatively low cost devices can be brought together to create a compact micro-system for time resolved fluorescence analysis. The implementation and testing of these micro-systems revealed a number of design modifications that could significantly improve system performance.

The need to better discriminate between excitation light and fluorescence emission has been identified as design area which requires improvement. This issue is conventionally solved using optical filters. For a micro-system which aims to reduce the overall cost of the system, the inclusion of a suitable optical filter could prove expensive. This problem can be overcome electronically, by taking advantage of the ability to switch logic states in CMOS extremely quickly. This allows the SPAD to be dis-armed during the excitation phase of the process, rendering the detector insensitive to photons. After the excitation phase the SPAD can be rapidly armed to capture the ensuing fluorescence decay. This technique has been demonstrated by other research groups and can be incorporated into an active quench circuit. Furthermore, the ability to disable SPADs within the array could reduce system noise.

The ability to reject ambient light is an inherent feature of the SPAD counter circuitry. Owing to their ability to count both up and down. Ambient light rejection can therefore be achieved 
by exciting the fluorophore, setting a time-gate period during lifetime of the fluorescence decay and allowing the counter to count up. The time-gate position can then be moved to after the fluorescence decay, and the counter allowed to count down from the value it had previously reached. Any counts removed during this time period would be due to ambient light only. The residual counter value at the end of this process can therefore be attributed to the fluorescence from the sample of interest. To evaluate this, additional verilog code controlling the timing and mode of counter operation would be required.

As has been described in Section 7.2, the ability to capture counter data from the chip via USB was lost during the transition from a PCI based FPGA test board to a USB based PCB. Micro-system performance was therefore assessed using an external commercially available TCSPC module. The development of a suitable software program, capable of capturing chip counter data and presenting it to the user, would enable the micro-system to operate without the need for any external hardware. The system would simply require a computer with a USB link, via which power could be sourced, and could potentially supply the negative SPAD bias voltage.

The generation of the negative SPAD bias supply either on-chip or on the system PCB would allow the system size and cost to be further reduced by removing the need for an external voltage source. To achieve this, the bias voltage must be derived from the $5 \mathrm{~V}$ supply of the USB interface. On-chip, this can be achieved using a charge-pump circuit. This task is made easier as the SPAD bias supply does not source large currents (current consumption is zero except during a breakdown event). In a conventional two-well semi-conductor process, a charge-pump would be capable of producing a positive bias voltage. This would therefore require a different SPAD bias strategy to the one employed during this project. By biasing the n-well node of the SPAD detector with a high positive voltage and the p-anode tied to ground, the SPAD can operate in Geiger mode. The quench transistor is placed between the p-anode and ground (Figure 7.1). This biasing scheme has the added advantage of turning the p-anode of the SPAD into the moving node of the detector. This node is physically smaller and therefore has less capacitance, resulting in a potentially faster moving breakdown event in which less charge carriers flow (leading to improved noise performance). The SPAD bias voltage could also be produced using discrete components situated on the system PCB.

The need to provide a full metal bond stack within each pixel of the micro-LED driver array resulted in an increase in the pixel dimensions and pitch. On the periphery of the array a number 


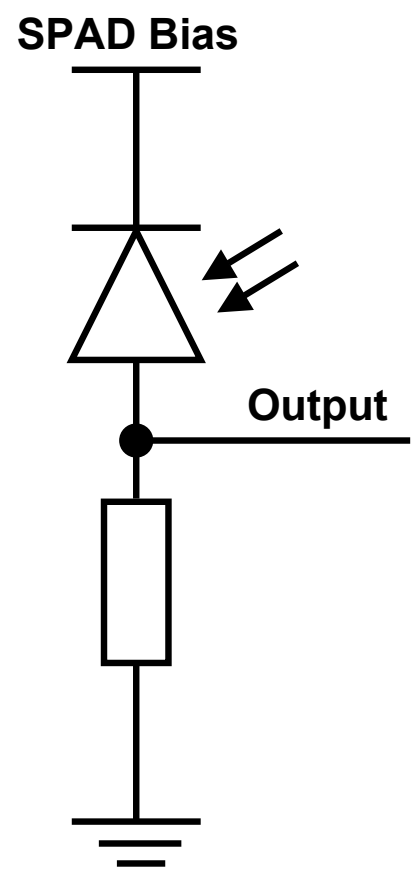

Figure 7.1: Alternative inverted SPAD bias configuration

of test structures were implemented in an attempt to improve the pixel pitch and therefore increase array density. In the design of the main $8 \times 8$ array a pragmatic approach was taken and a $100 \mu \mathrm{m} \times 100 \mu \mathrm{m}$ bond-stack was situated within each pixel. These are the same dimensions as the standard bond pads situated at the periphery of the chip. It is hoped that by reducing the size of the bond-stack, pixel dimensions can also be reduced. This presents a challenging bump-bonding problem, as the micro-LED must be able to reliably adhere to the CMOS device. To assess the capabilities of the bump-bonding process a series of metal bond stacks of varying dimensions have been laid out as test structures (Figure 7.2). The test structures have bond sites that vary in dimensions from $50 \mu \mathrm{m} \times 50 \mu \mathrm{m}$ down to $25 \mu \mathrm{m} \times 25 \mu \mathrm{m}$. Each of the three groups of test structures has a single LED driver to drive all four pads, allowing the bump-bonding process to be verified. Should bump-bonding to these smaller pad structures prove successful it is hoped that device performance will improve. A smaller metal-stack will have a lower capacitance, potentially leading to improved pulse performance. Furthermore, a reduced bond-pad size will allow denser arrays to be implemented. Alternatively, if pixel pitch remains constant a smaller pad structure will increase the amount of area that can be dedicated to the driver device. This will allow driver structures of greater complexity to be designed and would allow larger output driver devices to be implemented, increasing the drive strength of the device. 
In order to assess how device performance improves with reduced bond-stack dimensions and in a bid to return to the original $100 \mu \mathrm{m}$ pitch of the $16 \times 16$ driver array device, a second test structure block was included at the periphery of the $8 \times 8$ array. This structure included a $2 \times 8$ pixel array at a pitch of $100 \mu \mathrm{m}$. Each pixel included a $50 \mu \mathrm{m}$ bond stack and a driver circuit laid out in the remaining three quarters of the pixel. For a layout view of this test structure, see Figure 7.3. The functionality of these reduced dimension bond-pads has been verified electrically, however, their performance is yet to be assessed. 


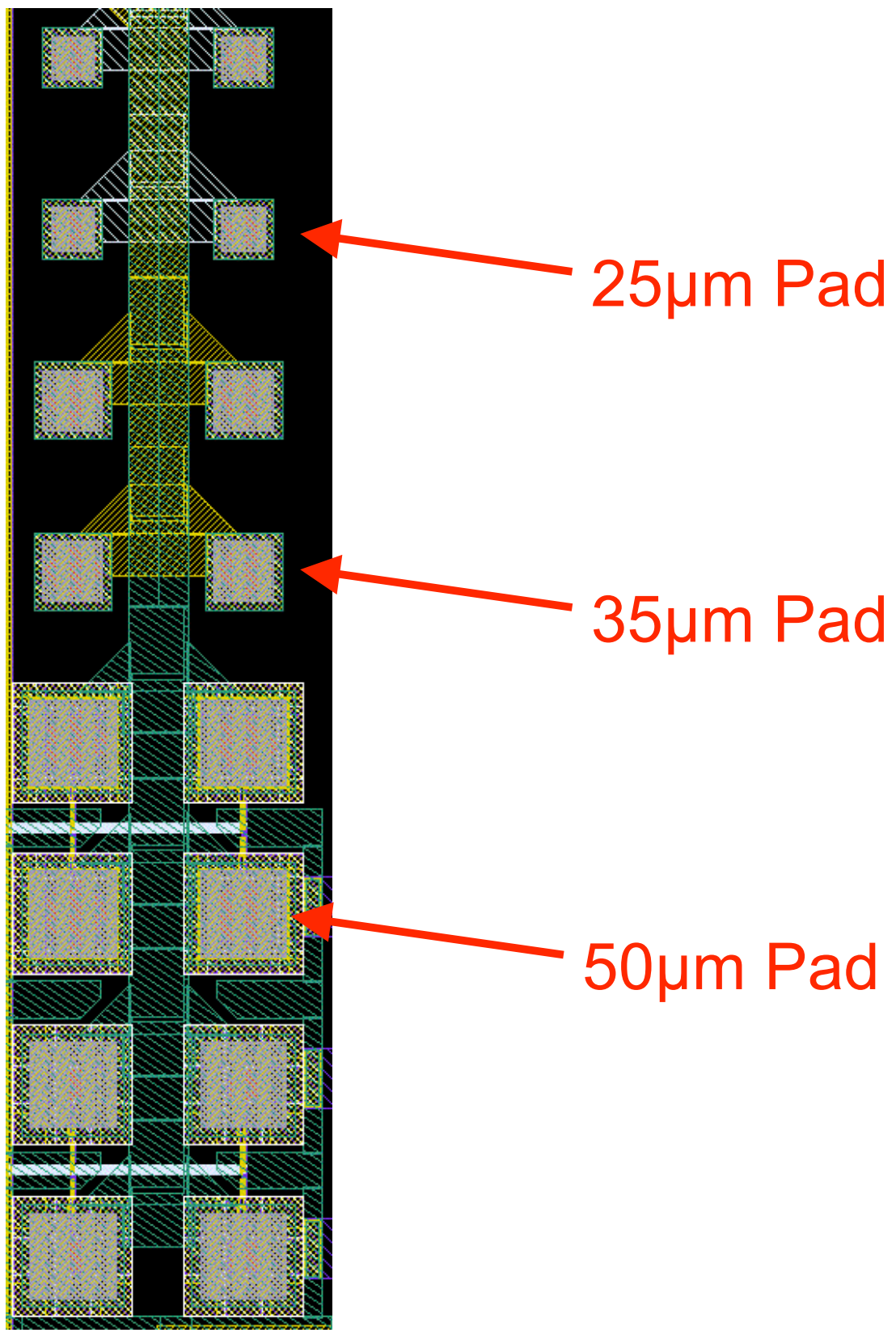

Figure 7.2: Metal bond stacks of varying dimensions. 


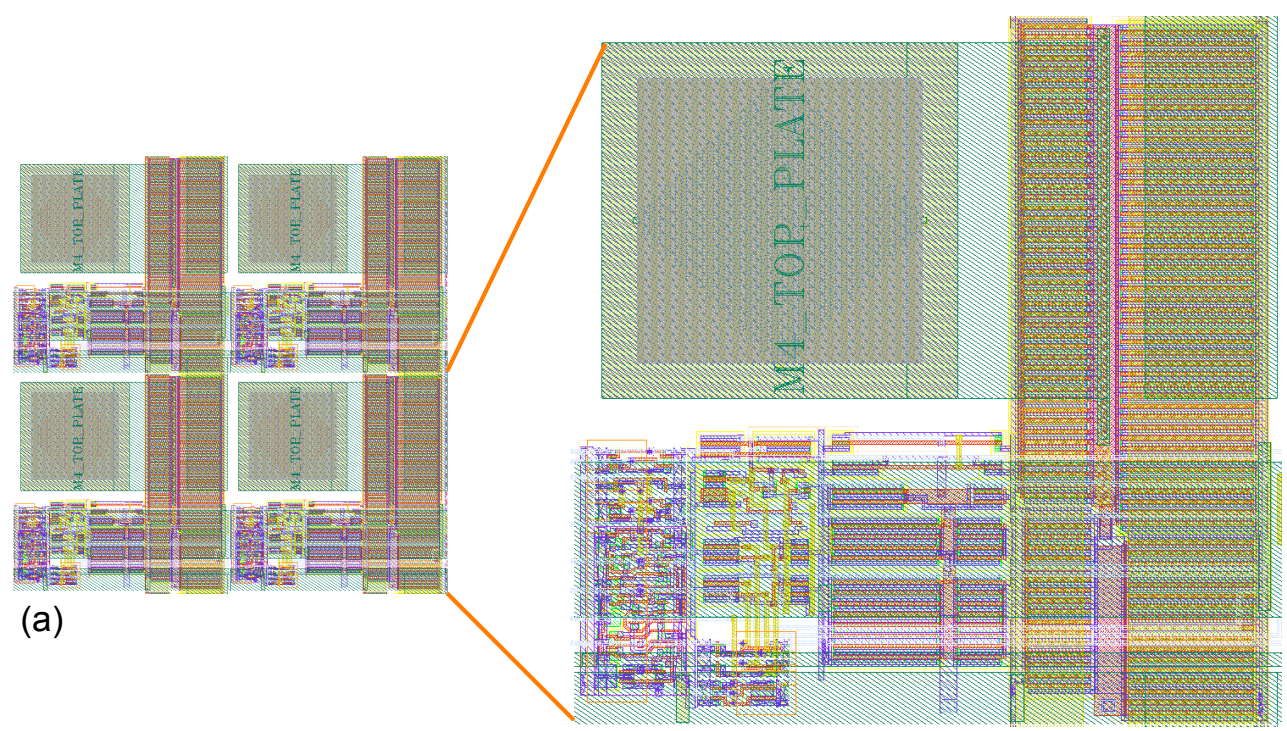

(b)

Figure 7.3: (a) Layout of 100 $\mu$ m pitch micro-LED driver test structure array. (b) Each pixel contained a $50 \mu \mathrm{m} \times 50 \mu \mathrm{m}$ bond-pad and driver circuit. 
As CMOS device dimensions shrink in newer process technologies, the number of metal layers made available for signal routing above the silicon tends to increase. For instance, the foundry United Microelectronics Corporation (UMC) provides 6 metal layers in their $0.18 \mu \mathrm{m}$ technology. In their $0.13 \mu \mathrm{m}$ process, 8 metal layers are available and this increases to 9 metal layers in the 90nm technology (www.umc.com and www.europractice-ic.com, both visited 2009). The exception to this rule is in technologies dedicated to the fabrication of image sensors whereby the metal stack height is kept small to improve optical performance. If a micro-LED driver array were to be implemented in a deep sub-micron technology, these metal layers would be available. It may therefore be possible to implement a metal bond stack using, for example, the top four metal layers. This may provide sufficient mechanical strength to withstand the bump-bonding process and would allow circuitry to be placed underneath the pads and signals to be routed using the remaining metal layers. This would allow pixel pitch, and therefore array density, to be improved. Furthermore, as CMOS process dimensions shrink the minimum gate length of a transistor in that process is reduced. This allows smaller transistors to be utilised and therefore the area of a circuit block can be reduced. In the case of a micro-LED driver circuit this would allow pixel dimensions to be reduced without a reduction in drive strength, in turn allowing for denser driver arrays. Conversely, it may be decided that the pixel pitch should remain the same. This would allow more complex circuits to be implemented in the given area. As transistor gate length is reduced, the minimum signal delay through a device is also reduced. Therefore, it is hoped that a micro-LED driver implemented in a smaller CMOS technology would be capable of producing shorter electrical stimulus pulses, resulting in shorter optical pulses.

Shorter gate delays through a transistor in smaller dimension technologies would also allow the minimum time-gate width, generated by the on-chip tapped delay line, of the SPAD chip presented in Chapter 4 to be reduced. This would allow lifetime decay curves to be captured with a finer resolution and allow faster decay events to be observed. Until recently, implementing SPAD detectors in deep sub-micron CMOS technologies has proven difficult, with detectors exhibiting large dark count rates. A number of recent publications [72], suggest that the problems associated with the implementation of SPADs in such technologies are beginning to be understood and overcome. This would allow circuits of greater complexity to be situated within the pixel (or at the periphery of the array) and denser detector arrays to be achieved. 
This thesis has presented performance results for CMOS driven UV micro-LED arrays. These devices emit at a wavelength of $370 \mathrm{~nm}$. In a micro-system environment these devices work well with the SPAD detectors as the SPAD device is naturally insensitive at 370nm (Section 6.2.2). Micro-LED devices at other wavelengths have been presented in the literature [84]. The inclusion of suitable filtering (optical or electronic) would allow such devices to be successfully implemented alongside SPAD detectors within a micro-system. This would allow fluorophores of different excitation spectra to be analysed. Furthermore, initial work carried out using micro-LED structures which emit at 450nm demonstrate a lower turn-on voltage and increased optical output power. This would allow such devices to excite a sample of interest with a greater intensity and may open-up new applications for micro-LED devices. Another emerging application area of high power micro-LEDs, capable of producing short optical pulses, is the optical pumping of organic polymer lasers [121], [122]. This would result in a hybrid organic semiconductor/gallium nitride/CMOS pixel that could potentially be useful for biomedical applications.

Sample handling is the focus of much work in the field of biomedical micro-systems and lab-on-chip devices. It can therefore be envisaged that the micro-systems presented here could incorporate a sample handling element such as a micro-fluidics device. This would allow a sample of interest to be easily presented for investigation and may also allow mixing and reaction dynamics to be evaluated. For a hand-held or point-of-care device this fluidics element could be disposable, therefore minimising the risk of cross-contamination. There are a number of sample handling techniques that have the potential to be incorporated into a micro-system for fluorescence lifetime analysis. A simple micro-fluidics channel pumped by a syringe would allow sample to enter and exit the system in a simple, controlled manner. Conversely, a significantly more complex system can also be envisaged by making use of electro-wetting [123], [124], [125] or dielectrophoretic based devices [126]. These would allow a sample to be accurately manipulated within the micro-environment. These techniques have the added advantage of allowing other sample interrogation techniques to be implemented alongside fluorescence, such as impedance or capacitance sensing.

\subsection{Conclusions}

This project has shown that it is possible to conduct fluorescence lifetime measurements in a micro-system environment using low cost components (relative to currently used fluorescence 
lifetime analysis equipment). This could pave the way to a compact (potentially portable), robust and inexpensive solution to time-resolved fluorescence measurements. The development of this device is on-going and system performance benefits will be achieved if improvements are made in any area of the system design. This could include shorter optical pulses or higher output power from the micro-LED device, increased detector sensitivity and more sophisticated signal processing circuitry. A number of these performance improvements could be achieved by migrating the current design to a more advanced CMOS technology. This system should benefit from on-going work across the fields of miniaturised light sources and detection systems. 


\section{Appendix A Appendices}

\section{A.1 16x4 SPAD Chip and 16x4 Micro-LED Driver Pin List}

\begin{tabular}{|c|c|c|}
\hline Pin Name & Pin Type & Description \\
\hline DLYIN & Digital Input & $\begin{array}{l}\text { Differential gating control circuit } \\
\text { (DGCC) input signal, which gets } \\
\text { delayed to generate the SQUOT } \\
\text { signals for the two array blocks. } \\
\text { Provides the first, undelayed, square } \\
\text { wave signal to the EWOD/ } \mu \mathrm{LED} \text { array }\end{array}$ \\
\hline 4Phase_CLK & Digital Input & $\begin{array}{l}\text { Clock signal for the four phase } \\
\text { enable/updown generator }\end{array}$ \\
\hline LOAD1 & Digital Input & $\begin{array}{l}\text { Defines the delay between SQOUTD1 } \\
\text { and DLYIN }\end{array}$ \\
\hline LOAD2 & Digital Input & $\begin{array}{l}\text { Defines the delay between SQOUTD2 } \\
\text { and DLYIN }\end{array}$ \\
\hline LOAD3 & Digital Input & $\begin{array}{l}\text { Defines the delay between SQOUTD3 } \\
\text { and DLYIN }\end{array}$ \\
\hline REV & Digital Input & $\begin{array}{l}\text { Reverses the direction of the DGCC to } \\
\text { reduce the delays between the SQOUT } \\
\text { signals and DLYIN }\end{array}$ \\
\hline SRCLK & Digital Input & $\begin{array}{l}\text { Provides the clocked input to the first } \\
\text { bit of each delay element in the DGCC }\end{array}$ \\
\hline SRRST & Digital Input & Reset signal for the DGCC \\
\hline SRIN & Digital Input & DGCC input signal \\
\hline UPDOWN2 & Digital Input & $\begin{array}{l}\text { Defines the direction of counting in the } \\
\text { second counter in the } 9 \text { bit counter array }\end{array}$ \\
\hline
\end{tabular}




\begin{tabular}{|c|c|c|}
\hline UPDOWN1 & Digital Input & $\begin{array}{l}\text { Defines the direction of counting in the } \\
\text { first counter in the } 9 \text { bit counter array } \\
\text { and the } 18 \text { bit counter }\end{array}$ \\
\hline $\mathrm{OUT}<8: 0>$ & Digital Output & $\begin{array}{l}\text { Output bus. When ROWSEL }<5: 0>= \\
0-32 \text { Output is from SPAD Counter } \\
\text { array. When ROWSEL }<5: 0>=0 \\
64: 2 \text { Output from EWOD } / \mu \text { LED array. } \\
\text { When ROWSEL }<5: 0>=34-46: 2 \\
\text { Output from TDCBTJ block. When } \\
\text { ROWSEL }<5: 0>=48 \text { Output from } \\
\text { Top Test Structures }\end{array}$ \\
\hline DIN & Digital Input & $\begin{array}{l}\text { Low voltage input for setting electrode } \\
\text { state in } \mathrm{EWOD} / \mu \mathrm{LED} \text { driver }\end{array}$ \\
\hline EWOD_Ibias & Analogue Input & $\begin{array}{l}\text { Bias current to provide stable input } \\
\text { current to ensure fast transition of the } \\
\mu \text { LED driver }\end{array}$ \\
\hline VOP & Power Supply & Negative (-20V) SPAD power supply \\
\hline vdd! & Power Supply & Positive, $3.3 \mathrm{~V}$, circuit power supply \\
\hline gnd! & Power Supply & Circuit ground \\
\hline SPAD_AVDD & Power Supply & $\begin{array}{l}\text { Separate } 3.3 \mathrm{~V} \text { power supply for local } \\
\text { SPAD circuitry }\end{array}$ \\
\hline HV_AVDD & Power Supply & $\begin{array}{l}\text { High Voltage }(50 \mathrm{~V}) \text { power supply for } \\
\text { EWOD/ } \mu \text { LED driver circuit }\end{array}$ \\
\hline EWOD_CLK & Digital Input & $\begin{array}{l}\text { Ensures only the addressed } \\
\text { EWOD/ } \mu \text { LED driver can change } \\
\text { state }\end{array}$ \\
\hline EN1 & Digital Input & $\begin{array}{l}\text { External enable signal for the first } \\
\text { counter of the 9bit counter array and } \\
\text { the } 18 \text { bit counter array }\end{array}$ \\
\hline EN2 & Digital Input & $\begin{array}{l}\text { External enable signal for the second } \\
\text { counter of the } 9 \text { bit counter array }\end{array}$ \\
\hline RstN & Digital Input & Global reset signal \\
\hline
\end{tabular}




\begin{tabular}{|c|c|c|}
\hline $\mathrm{SEL}<0>$ & Digital Input & $\begin{array}{l}\text { Multiplexer control, selects mode of } \\
\text { operation for both arrays }\end{array}$ \\
\hline $\mathrm{SEL}<1>$ & Digital Input & $\begin{array}{l}\text { Multiplexer control, selects mode of } \\
\text { operation for counter array }\end{array}$ \\
\hline COL_CONT $<2: 0>$ & Digital Input & $\begin{array}{l}\text { Multiplexer control line which selects } \\
\text { which array column is going to be read } \\
\text { out }\end{array}$ \\
\hline ROWSEL $<5: 0>$ & Digital Input & $\begin{array}{l}\text { Multiplexer control line which selects } \\
\text { which row/experiment is going to be } \\
\text { present on the output bus }\end{array}$ \\
\hline VDELADJ & Analogue Input & $\begin{array}{l}\text { Provides an analogue reference signal } \\
\text { for TDCBTJ block }\end{array}$ \\
\hline SCTDCCLK & Digital Input & Clock signal for TDCBTJ block \\
\hline $\mathrm{CHOP}$ & Digital Input & $\begin{array}{l}\text { Square wave input signal for Top Test } \\
\text { Structure and Direction control signal } \\
\text { for } \Sigma \Delta \text { block }\end{array}$ \\
\hline VREFP & Analogue Input & $\Sigma \Delta$ reference voltage \\
\hline VREFN & Analogue Input & $\Sigma \Delta$ reference voltage \\
\hline $\mathrm{VCM}$ & Analogue Input & $\Sigma \Delta$ control signal \\
\hline SCTDC_Ibias $<1>$ & Analogue Input & $10 \mu \mathrm{A}$ bias current for $\Sigma \Delta$ block \\
\hline SCTDC_Ibias $<0>$ & Analogue Input & $100 \mu \mathrm{A}$ bias current for $\Sigma \Delta$ block \\
\hline
\end{tabular}




\section{A.2 Single In-Pixel Counter Bit}

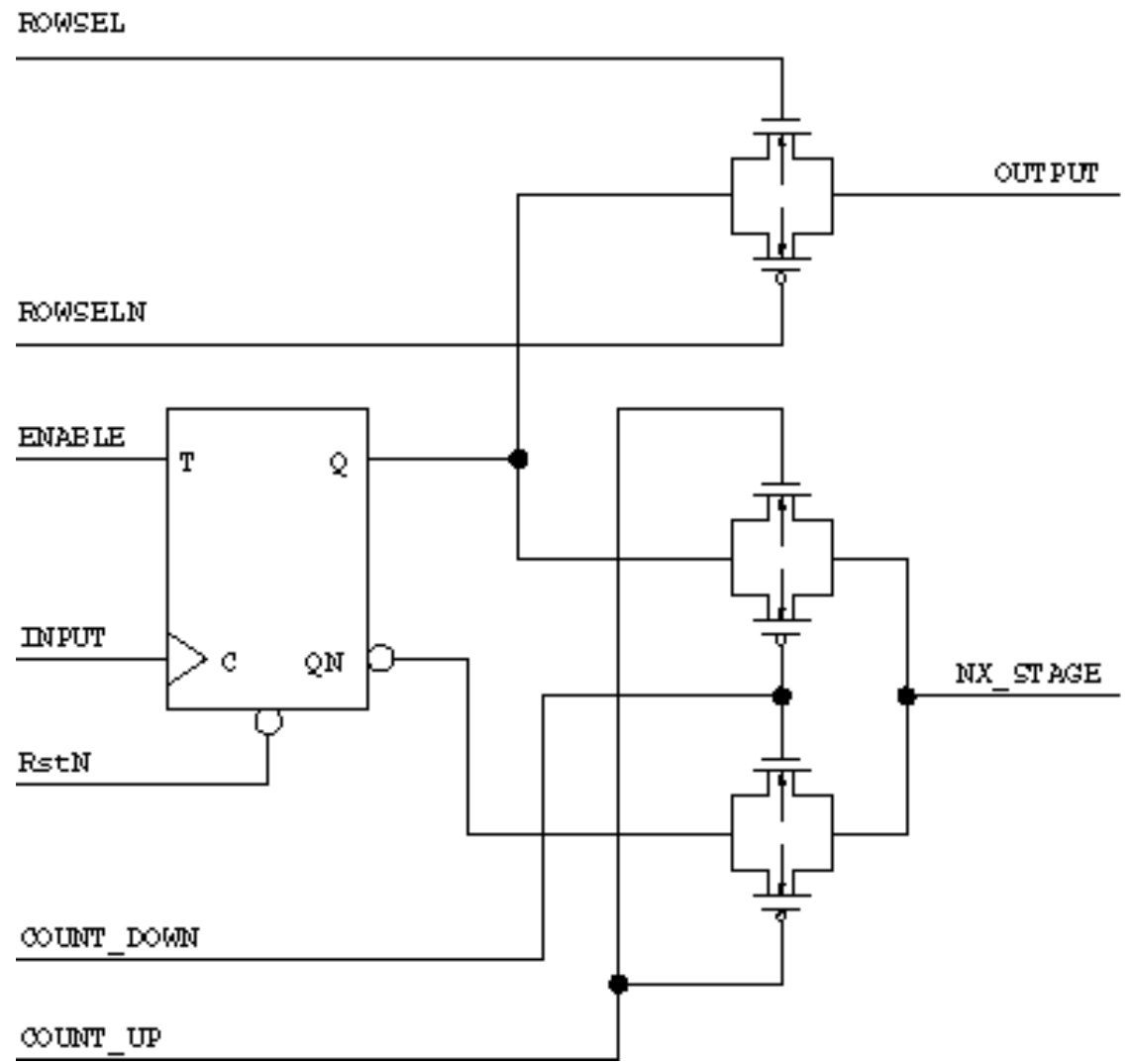

Figure A.1: Single-bit of in-pixel counter used to count avalanche events. 


\section{A.3 8x8 Micro-LED Driver Pin List}

\begin{tabular}{|c|c|c|}
\hline LED_VDD & Power Supply & LED power supply \\
\hline LED_GND & Power Supply & LED ground \\
\hline VCO_OUT & Digital Output & $\begin{array}{l}\text { Allows on-chip VCO output to be } \\
\text { observed }\end{array}$ \\
\hline EXT_FREQ & Digital Output & $\begin{array}{l}\text { Allows } 1024 \text { division of VCO output to } \\
\text { be observed }\end{array}$ \\
\hline MOD_CONT_1 & Digital Input & $\begin{array}{l}\text { Driver mode select, } 1=\text { pulsed mode, } 0 \\
=\mathrm{DC} \text { mode }\end{array}$ \\
\hline RESET_N & Digital Input & Driver reset \\
\hline VCO_EN & Digital Input & On-chip VCO enable \\
\hline CLK_SEL $<1: 0>$ & Digital Input & $\begin{array}{l}\text { Coarse control of on-chip VCO output } \\
\text { frequency, } 00=\text { no division, } 01=\text { core } \\
\text { frequency } \div 4,10=\text { core frequency } \div \\
16,11=\text { core frequency } \div 64\end{array}$ \\
\hline DRIVER_CLK & Digital Input & $\begin{array}{l}\text { Ensures only the addressed micro-LED } \\
\text { driver can change state }\end{array}$ \\
\hline LFSR_EN_N & Digital Input & Enable signal for on-chip LFSR circuit \\
\hline INPUT_SEL $<1: 0>$ & Digital Input & $\begin{array}{l}\text { Selects driver input source, } 00=\text { VCO, } \\
01=\text { INPUT_COMMON, } 1 \mathrm{X}=\mathrm{LFSR}\end{array}$ \\
\hline INPUT_COMMON & Digital Input & External driver input signal \\
\hline DIN & Digital Input & $\begin{array}{l}\text { Low voltage input for setting electrode } \\
\text { state of micro-LED driver }\end{array}$ \\
\hline COL_SEL $<3: 0>$ & Digital Input & $\begin{array}{l}\text { Multiplexer control line which selects } \\
\text { driver column address }\end{array}$ \\
\hline ROW_SEL $<3: 0>$ & Digital Input & $\begin{array}{l}\text { Multiplexer control line which selects } \\
\text { driver row address }\end{array}$ \\
\hline EXT_FREQ_EN & Digital Input & $\begin{array}{l}\text { Enables VCO output to be observed on } \\
\text { VCO_OUT pad }\end{array}$ \\
\hline VBIAS_MOD_CONT_2 & Analogue Input & Input bias, defines driver pulse width \\
\hline M4_TOP_PLATE & Analogue Output & $\begin{array}{l}\text { Allows observation of driver electrical } \\
\text { output }\end{array}$ \\
\hline AVDD5V0_RING & Power Supply & $5 \mathrm{~V}$ pad ring power supply \\
\hline
\end{tabular}




\begin{tabular}{|c|c|c|}
\hline AVDD3V3 & Power Supply & $3.3 \mathrm{~V}$ pad ring power supply \\
\hline VCO_BIAS & Analogue Input & $\begin{array}{l}\text { Analogue bias, allows fine control of } \\
\text { core VCO frequency }\end{array}$ \\
\hline AVDD_TC & Power Supply & Test cell $3.3 \mathrm{~V}$ power supply \\
\hline gnd! & Power Supply & Circuit ground \\
\hline HV_AVDD_TC & Power Supply & Test cell LED power supply \\
\hline M4_Top_Place_TC & Analogue Output & Test cell driver electrical output \\
\hline Vbias_ModeCont2_TC & Analogue Input & $\begin{array}{l}\text { Test cell input bias, defines driver pulse } \\
\text { width }\end{array}$ \\
\hline ROWSEL $<0: 1>$ & Digital Input & $\begin{array}{l}\text { Test cell multiplexer control line which } \\
\text { selects driver row address }\end{array}$ \\
\hline RN_TC & Digital Input & Test cell reset \\
\hline Column_TC & Digital Input & $\begin{array}{l}\text { Test cell multiplexer control line which } \\
\text { selects driver column address }\end{array}$ \\
\hline Driver_CLK_TC & Digital Input & $\begin{array}{l}\text { Test cell input, ensures only the } \\
\text { addressed micro-LED driver can } \\
\text { change state }\end{array}$ \\
\hline DIN_TC & Digital Input & $\begin{array}{l}\text { Test cell low voltage input for setting } \\
\text { electrode state of micro-LED driver }\end{array}$ \\
\hline INPUT_Sig_TC & Digital Input & Test cell driver input signal \\
\hline CMEnableIN_TC & Digital Input & $\begin{array}{l}\text { Test cell mode select, } 1=\text { pulsed mode, } \\
0=\text { DC mode }\end{array}$ \\
\hline
\end{tabular}




\section{A.4 VCO CIRCUIT}

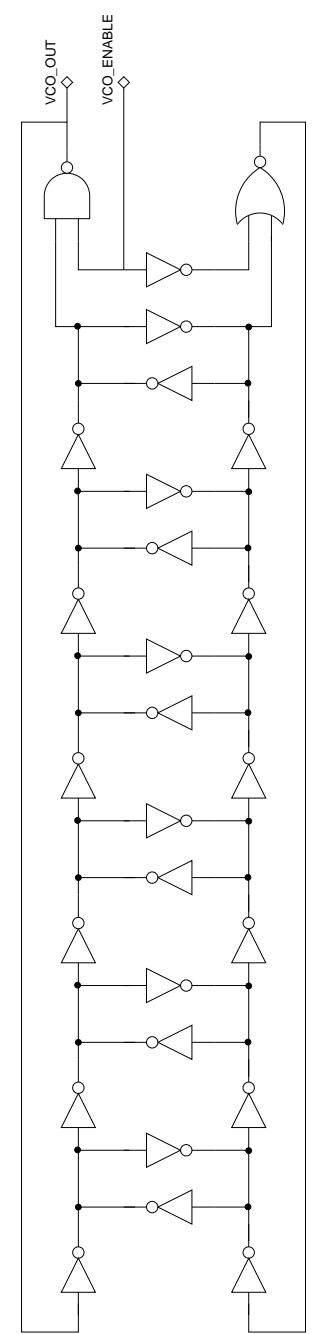

Figure A.2: On-chip VCO implemented on $8 x 8$ micro-LED driver array chip. 


\section{A.5 LFSR Circuit}

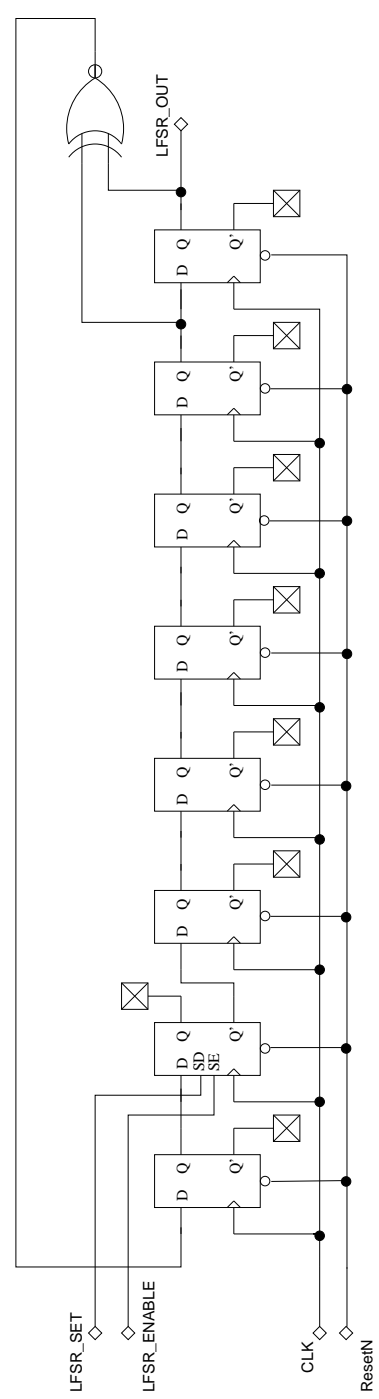

Figure A.3: One of seven on-chip LFSR circuits implemented on $8 x 8$ micro-LED driver array chip. 


\section{A.6 Verilog Used to Drive the 16x4 SPAD and Micro-LED Driver Chip}

The verilog used to drive the 16x4 SPAD and micro-LED driver chip was originally written using the Altera Quatus II software for use with the Altera Apex (EP20K600EFC672-1X) FPGA chip located on the ST Microelectronics 908 Test Board (Section 3.2.1). The majority of signals defined by the code are static DC logic signals used to define such parameters as device address, operating mode etc. However, a number of timed signals were required to allow the user to select which element of the tapped delay-line the counter time-gate signals were going to be output from. This in turn selects the width of the time-gate window for the counter circuit. Additional timed signals were required by the PCI interface card to allow data to be grabbed by the controlling PC at defined intervals, upon grabbing the data it was necessary for the in-pixel counters to then be reset. To achieve this, counters were included in the verilog code that could monitor the position of the delayed input signal within the timing generator. On every system clock edge, the output position of the tapped delay line could advance by one element. Using a counter that increments on every clock edge, case statements and a user selectable input parameter equal to between 1 and 120 (the number of elements in the tapped delay line), the time-gate width can be selected. An example of a case statement used to define the start of a time gate period (LOAD1) which will occur when the clock counter output (counter_out) is equal to the user definable signal LOAD1_High can be seen in Figure A.4.

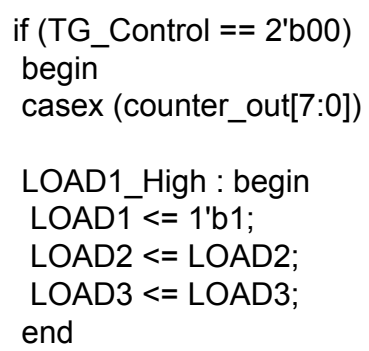

Figure A.4: Example of case statement used to define output location of on-chip tapped delay line.

A pseudo-code overview of the verilog used to define a time-gate period and for counter data to be passed to the computer via PCI interface is given below.

Verilog counter increments on every rising edge of the system clock

When counter output $=$ Load1_High $($ which is user definable via GUI) 
Set Load $1=1$ for one clock cycle (this defines the start of time gate 1 )

When counter output $=$ Load2_High (which is user definable via GUI)

Set Load $2=1$ for one clock cycle (this defines the end of time gate 1 and the start of time gate 2)

When counter output $=$ Load3_High (which is user definable via GUI)

Set Load $3=1$ for one clock cycle (this defines the end of time gate 2 )

Time gate periods are evaluated a user definable number of times

When 8 MSB's of counter out $=$ RstN_Low

Data in addressed counter is passed via PCI to computer, this defines the value of one pixel in the image

On-chip counters are reset

Verilog counters are reset

The above process is repeated until a value for every pixel in a line of the image is obtained Line width is defined by the user input signal FST_Cont

Count 2 increments every time an on-chip counter is reset and pixel data is passed to the PC when Count $2=$ FST_Cont

A frame start signal is sent via PCI to the computer and a new line of the image is taken

The code used to define where in the tapped delay line the time-gate control signals are output, and hence the time-gate width, is also used to define the electrical pulse width of the micro-LED drivers situated within the same chip.

The above code was adapted later in the project to be used within a Xilinx Spartan-3 (XC3S1000-4FG320) FPGA which was situated on the The University of Edinburgh $\backslash$ Opal Kelly Test Board (3.2.2) and could be controlled via a USB rather than PCI interface.

A full copy of the Verilog code used to control the 16x4 SPAD and Micro-LED Driver Chip can be found on the enclosed supplementary material CD. 


\section{A.7 Verilog Used to Drive the 16x16 and 8x8 Micro-LED Driver Chips}

The verilog required to drive the $16 \times 16$ and $8 \times 8$ micro-LED driver chips was significantly simpler with respect to the verilog required by the original 16x4 array (Section A.6). The electrical pulse that was output by each driver was generated within each pixel and was defined off-chip by a voltage bias which controlled the gate voltage of a current starving transistor within an inverter circuit. Furthermore, with no detection element on the device no data had to be transmit back to the controlling PC. Verilog, written specifically for the USB controlled Xilinx Spartan-3 (XC3S1000-4FG320) FPGA of The University of Edinburgh $\backslash$ Opal Kelly Test Board (3.2.2) was therefore designed. This verilog defined all DC static input signals required by the chip to operate. Each signal was assigned a USB address allowing the GUI to be designed so that the user could define the state of each signal.

A full copy of the Verilog code used to control the 16x16 and 8x8 Micro-LED Driver Chips can be found on the enclosed supplementary material CD. 


\section{Appendix B Publications}

B.R. Rae, K.R. Muir, D. Renshaw, R.K. Henderson, J. Girkin, Z. Gong, J. McKendry, E. Gu, M.D. Dawson, "A vertically integrated CMOS micro-system for time-resolved fluorescence analysis," Accepted for inclusion in IEEE BioCAS Conference, 2009.

D.-U. Li, R. Walker, J. Richardson, B. Rae, A. Buts, D. Renshaw and R. Henderson, "FPGA implementation of a video-rate fluorescence lifetime imaging system with a 32x32 CMOS single-photon avalanche diode array," IEEE International Symposium on Circuits and Systems, pp. 3082-3085, May 2009.

J. McKendry, B.R. Rae, Z. Gong, K.R. Muir, B. Guilhabert, D. Massoubre, E. Gu, D. Renshaw, M.D. Dawson and R.K. Henderson, "Individually-addressable AlInGaN micro-LED arrays with CMOS control and sub-nanosecond output pulses," IEEE Photonics Technology Letters, Issue 99, April 2009.

D.-U. Li, R. Walker, J. Richardson, B. Rae, A. Buts, D. Renshaw and R. Henderson, ”Hardware implementation and calibration of background noise for an integration-based fluorescence lifetime sensing algorithm," Journal of the Optical Society of America: A, vol. 26, no. 4, pp. 804-814, April 2009.

Z. Gong, D. Massoubre, J. McKendry, H.X. Zhang, C. Griffin, B. Guilhabert, E. Gu, J.M. Girkin, M.D. Dawson, B.R. Rae and R.K. Henderson, "Flip-chip, micro-pixellated InGaN light-emitting diode arrays: attractive sources for micro-displays, colour conversion, and fluorescence detection," Physica Status Solidi C, pp. 1-4, January 2009.

D.-U. Li, B. Rae, D. Renshaw, R. Henderson and E. Bonnist, "On-chip fluorescence lifetime extraction using synchronous gating - theoretical error analysis and practical implementation," Biosignals 2008 - International Conference on Bio-Inspired Systems and Signal Processing, January 2008.

Y. Li, W. Parkes, L.I. Haworth, A.A. Stokes, K.R. Muir, P. Li, A.J. Collin, N.G. Hutcheon, R. Henderson, B. Rae, A.J. Walton, ”Anoidic $\mathrm{Ta}_{2} \mathrm{O}_{5}$ for CMOS compatible low voltage 
electrowetting-on-dielectric device fabrication," Solid-State Electronics, vol. 52, pp. 1382-1387, 2008.

B.R. Rae, C. Griffin, J. McKendry, J.M. Girkin, H.X. Zhang, E. Gu, D. Renshaw, E. Charbon, M.D. Dawson and R.K. Henderson, "CMOS driven micro-pixel LEDs integrated with single photon avalanche diodes for time resolved fluorescence measurements," Journal of Physics D: Applied Physics, vol. 41, April 2008.

B.R. Rae, C. Griffin, K.R. Muir, J.M. Girkin, E. Gu, D. Renshaw, E. Charbon, M.D. Dawson and R.K Henderson, "A microsystem for time-resolved fluorescence analysis using CMOS single-photon avalanche diodes and micro-LEDs," IEEE International Solid-State Circuits Conference, pp. 166-603, February 2008.

C. Griffin, J. McKendry, H.X. Zhang, E. Gu, B.R. Rae, R. Henderson, D. Renshaw, J.M. Girkin and M.D. Dawson, "CMOS-integrated flip-chip, micro-pixel InGaN LED arrays for on-chip microfluorimetry," IEEE Lasers and Electro-Optics Society Conference, pp. 588-589, October 2007.

J. McKendry, C. Griffin, H.X. Zhang, Z.Gong, B. Guilhabert, D. Massoubre, E. Gu, M.D. Dawson, B.R. Rae, D. Renshaw and R. Henderson, "Micro-pixellated flip-chip InGaN light emitting diodes integrated with CMOS," 2nd European Optical Society Topical Meeting on Optical Microsystems, September 2007.

Y.A. Li, W. Parkes, L.I. Haworth, A.A. Stokes, K.R. Muir, P. Li, A.J. Collin, N.G. Hutcheon, R. Henderson, B. Rae and A.J. Walton, "Anodic $\mathrm{Ta}_{2} \mathrm{O}_{5}$ for CMOS compatible low voltage electrowetting-on-dielectric device fabrication," IEEE European Solid-State Device Research Conference, September 2007.

B.R. Rae, D. Renshaw, E. Charbon and R. Henderson, "A 2-D time resolved photon counting imager in $0.35 \mu \mathrm{m}$ technology," European Optical Society Conference on Frontiers in Electronic Imaging, pp. 50-51, June 2007.

T.D. Ross, R.K. Henderson, B. Rae and D. Renshaw, "A buried triple-junction self-reset pixel in a $0.35 \mu \mathrm{m}$ high voltage CMOS process," International Image Sensor Workshop, 2007.

R. Henderson, B. Rae, D. Renshaw and E. Charbon, "Oversampled time estimation techniques for precision photonic detectors," Invited Paper to The VLSI System on Chip Conference - 
Special Session on Precision Imaging, vol. 249, pp. 48-51, October 2006. 


\section{A Vertically Integrated CMOS Micro-System for Time- Resolved Fluorescence Analysis}

\author{
Bruce R. Rae, Keith R. Muir, David \\ Renshaw and Robert K. Henderson \\ Joint Research Institute for Integrated Systems \\ Institute for Integrated Micro and Nano \\ Systems \\ School of Engineering \\ The University of Edinburgh \\ The King's Buildings, Mayfield Road \\ Edinburgh, EH9 3JL, U.K. \\ Bruce.Rae@ed.ac.uk
}

\author{
John Girkin \\ Department of \\ Physics \\ Durham University \\ South Road \\ Durham, DH1 3LE,
}

\author{
Zheng Gong, Jonathan McKendry, \\ Erdan Gu and Martin D. Dawson \\ Institute of Photonics \\ University of Strathcylde \\ 106 Rottenrow \\ Glasgow, G4 0NW, U.K
}

Abstract-We describe a two-chip micro-scale time-resolved fluorescence analyzer integrating excitation, detection and filtering. A new 8x8 array of drivers integrated in standard lowvoltage $0.35 \mu \mathrm{m}$ CMOS is bump-bonded to AlInGaN blue micropixellated light-emitting diodes (micro-LED). The array is capable of producing sample excitation pulses with a width of 777ps (FWHM) enabling short lifetime fluorophores to be $777 \mathrm{ps}$ (FWHM) enabling short lifetime fluorophores to be
excited. The fluorescence emission is detected by a second, excited. The fluorescence emission is detected by a second,
vertically-opposed $16 \times 4$ array of single-photon avalanche diodes vertically-opposed 16x4 array of single-photon avalanche diodes
(SPADs) fabricated in $0.35 \mu \mathrm{m}$ high-voltage CMOS technology with in-pixel time-gated photon counting circuitry. This constitutes the smallest reported solid-state micro-system for fluorescence decay analysis, replacing lasers, photomultiplier tubes, bulk optics and discrete electronics. The system is demonstrated with measurements of fluorescent colloidal quantum dot and Rhodamine samples.

$$
\text { I. INTRODUCTION }
$$

Quantitative fluorescence intensity measurements are prone to misinterpretation due to illumination and fluorophore concentration non-uniformities. Thus, there is a growing interest in time-resolved fluorescence detection, whereby the characteristic fluorescence decay time-constant (or lifetime) in response to an impulse excitation source is measured. In addition, the sensitivity of a fluorophore's lifetime properties to the local microenvironment provides an extremely powerful analysis tool.

Conventional equipment required to perform fluorescence lifetime analysis is bulky and expensive. Typical set-ups include a pico-second pulsed or modulated light source (often a laser source), a sensitive detector such as a micro-channel plate photo-multiplier tube (MCP-PMT), an optical system, frequently incorporating a microscope for low volume measurements, as well as a variety of optical lenses and filters. Optical lab-on-a-chip (LoC) applications for point-of-care

This work was supported in part by UK Engineering and Physical diagnostics equipment are now motivating research into the miniaturisation of fluorescence lifetime instrumentation.

CMOS compatible detectors offer the opportunity to produce highly integrated fluorescence detection systems capable of monolithic detector data processing, at low cost. Recent developments in the design of CMOS compatible single-photon avalanche diodes [1] allow extremely sensitive detectors to be interated alongside signal processing circuitry. In order to gather photon arrival time data, from which fluorescence lifetimes can be extracted, a number of chip time-to-digital converters [2], in-pixel time-gated counters [3] and pinned-photodiodes [4].

A truly miniaturised optical LoC requires miniature and low-cost excitation sources, as well as detectors. Several fluorescence lifetime detector arrays have now been demonstrated in CMOS [2-4]. However, the aspect of miniaturised excitation sources has been largely neglected, relying on pulsed lasers with expensive and bulky drive electronics.

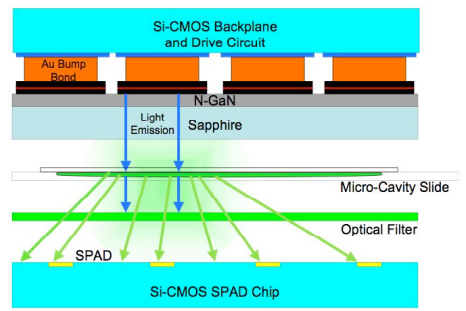

Figure 1: Cross-section of the two-chip micro-system. The sample of interest is introduced in a micro-cavity slide sealed by a cover-slip. An optical filter prevents excitation light reaching the detector array. 
Micro-LED devices have recently been demonstrated to produce sub-nanosecond pulses suitable for lifetime analysis [5], where excitation of a fluorescence sample was demonstrated using external LED drivers and a PMT detector.

In this paper, we combine a dedicated CMOS-driven micro-LED chip with a second CMOS SPAD array in a twochip "sandwich" structure (Fig. 1). Incorporating an excitation source with a photodetector, on-chip driving electronics and lifetime signal processing circuitry, our devices represent a highly integrated LoC system [6]. By including much of the signal processing and timing on-chip, the $\mathrm{I} / \mathrm{O}$ requirements and jitter sensitivity of the system is greatly reduced. The pixellation of detector and emitter arrays ease alignment constraints, reduce sample photobleaching and provide multiplexed analysis capability. To the best of our knowledge, multiplexed analysis capability. To the best of our knowledge,

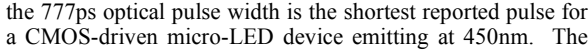
inclusion of an optical filter reduces measurement error caused by the detection of scattered excitation light. The accurate detection of commonly used, short lifetime fluorophores (Rhodamine) and longer lifetime colloidal semiconductor quantum dots is presented down to nanomolar concentration.

\section{DEVICE IMPLEMENTATION}

\section{A. Excitation Array}

Sample excitation is achieved using an $8 \times 8$ array of $72 \mu \mathrm{m}$ diameter, AlInGaN blue micro-pixellated light-emitting diodes (micro-LEDs) fabricated from "standard" InGaN/GaN quantum well blue LED wafers (planer n- and p- type GaN layers), grown on $c$-plane sapphire substrates by metal organic chemical vapor deposition [7]. This micro-LED array is bump-bonded to an equivalent array of LED driver circuits realised in a standard low-voltage $0.35 \mu \mathrm{m}$ CMOS technology (Fig. 2). Each array element (or pixel) is individually addressable, with a dedicated driver circuit per micro-LED. The wavelength spectra of the CMOS-driven blue micro-LED device peaks at a wavelength of $450 \mathrm{~nm}$.

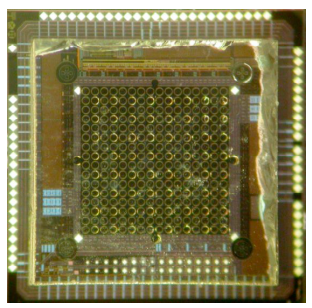

Figure 2: AlInGaN micro-LED array bump-bonded to an $8 \times 8 \mathrm{CMOS}$ driver array

The CMOS driver array pixels measure $200 \mu \mathrm{m} \times 200 \mu \mathrm{m}$, at $200 \mu \mathrm{m}$ pitch. Each pixel contains a dedicated driver circuit, driving a full metal bond-stack to which the micro-LED is bump-bonded (Figs. 3 and 4). All driver input signals were based on $3.3 \mathrm{~V}$ logic before being level-shifted to a higher user-definable voltage $\left(L E D_{-} V D D\right)$, to a maximum of $5 \mathrm{~V}$. This allows standard $3.3 \mathrm{~V}$ logic to be used for the addressing and control circuitry in the pixel before the signal level is increased to $L E D \_V D D$ (requiring the use of physically larger transistors able to withstand $5 \mathrm{~V}$ ).

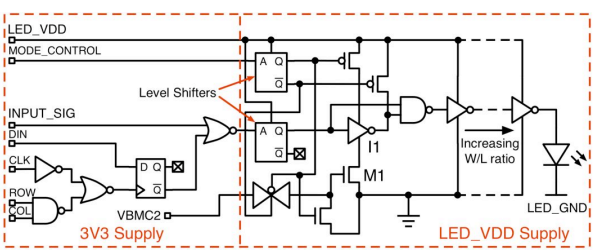

Figure 3: CMOS driver element, illustrating the output buffer and short pulse generation circuitry.

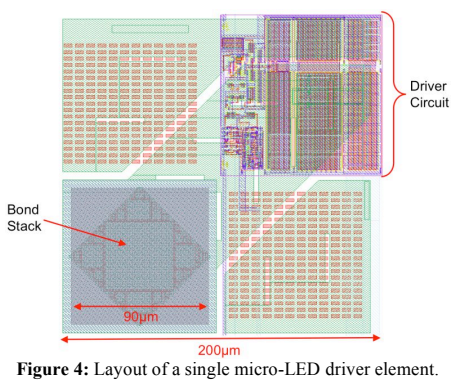

The driver circuit is capable of producing optical pulses of user-definable width variable from $47.48 \mathrm{~ns}$ down to $777 \mathrm{ps}$, FWHM $( \pm 180$ ps estimated measurement error, based on PMT RMS jitter), Fig. 5. By placing a square-wave signal on INPUT_SIG, the delay through inverter I1 defines the pulse width. The inverter delay can be adjusted via the gate voltage $(V B M C 2)$ of the current starving NMOS transistor M1. The level-shifted DC, pulsed, or square-wave signal is then passed through an output buffer designed using $5 \mathrm{~V}$ transistors. To minimise load capacitance on the input signal while maximising the drive strength of the circuit, an output buffer comprising a chain of inverters of increasing transistor width/length ratios has been implemented.

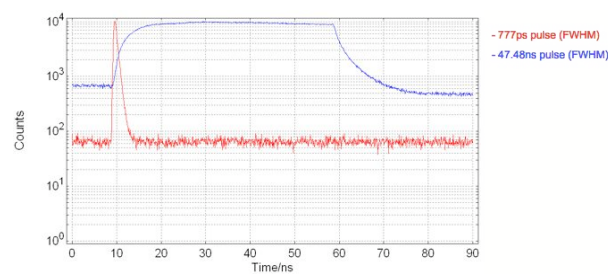

Figure 5: Shortest and longest micro-LED excitation pulses. 
An on-chip voltage controlled oscillator (VCO) has also been implemented within the $8 \times 8$ driver array. Comprising of a current starved ring oscillator, this circuit is capable of producing a square-wave signal with a tunable frequency range from $7 \mathrm{MHz}$ to $800 \mathrm{MHz}$. The VCO output can be used as the input signal to the drivers of the main array, defining the repetition rate of a square-wave or pulsed input signal. By producing a square-wave input signal on-chip, the need for an off-chip clock (such as a crystal oscillator) has been removed, potentially reducing system size and cost. The performance of the micro-LED excitation array is summarized in Table I.

TABLE I

\begin{tabular}{cc}
\multicolumn{2}{c}{ SUMMARY OF MICRO-LED DRIVER ARRAY } \\
\hline \hline Performance & \\
\hline Array Size & $8 \times 8$ \\
Driver Pitch & $200 \mu \mathrm{m}$ \\
Shortest Optical Pulse & $777 \mathrm{ps}$ \\
Excitation Wavelength & $450 \mathrm{~nm}$ \\
Max. Voltage & $5 \mathrm{~V}$ \\
Max. Driver Current & $236 \mathrm{~mA}(\mathrm{DC})$ \\
VCO Frequency Range & $7 \mathrm{MHz}-800 \mathrm{MHz}$ \\
Die Size & $3.18 \times 3.18 \mathrm{~mm}^{2}$ \\
\hline \hline
\end{tabular}

B. Detection Array

Our SPAD detector array is the same as that reported in [8] now combined with the separate emitter chip described in this paper. It consists of a $16 \times 4,200 \mu \mathrm{m}$ pitch array of SPAD detectors implemented in a $0.35 \mu \mathrm{m}$ high-voltage CMOS process. The pixels contain a $28.27 \mu \mathrm{m}^{2}$ active area SPAD structure as reported in [9] biased in Geiger mode at $-19.5 \mathrm{~V}$ via a passive quench PMOS transistor. SPAD output pulses can either be buffered off-chip via an inverter circuit located locally within the SPAD pixel for processing by external photon counting hardware, or processed locally within each pixel by time-gated ripple counters. These counters allow histogram and lifetime analysis without the need for external photon counting hardware and significantly reduce the quantity of data required to be broadcast off-chip.

Synchronisation of the time-gated detection circuit and the micro-LED excitation array is achieved by using the VCO circuit located on the LED driver chip as the input to the timing generator circuit situated on the SPAD chip. This circuit defines the positions of the time-gate. In this way, the time-gate is inherently synchronised with the excitation pulse, reducing errors due to uncorrelated timing jitter.

C. System Configuration

Fig. 6 shows the configuration of the two-chip system. A dedicated daughter card was designed, with the micro-LED device situated on the under-side of the $\mathrm{PCB}$, facing the SPAD detector chip located on the main PCB test board. Electrical connection to the daughter card is made via stacked header pins. This technique allows the distance between the micro-LED device and the SPAD detector chip to be adjusted. Both devices share the same core power supplies and ground connections. These supplies and all other bias supplies, apart from the negative SPAD bias, are generated on the test board PCB and derived from the 5V supply of the USB2.0 connection. The negative SPAD supply is generated by an external power source. The devices share a single FPGA situated on a Xilinx experimentation module PCB (Opal Kelly, XEM3010), which generates the digital input signals to both devices.

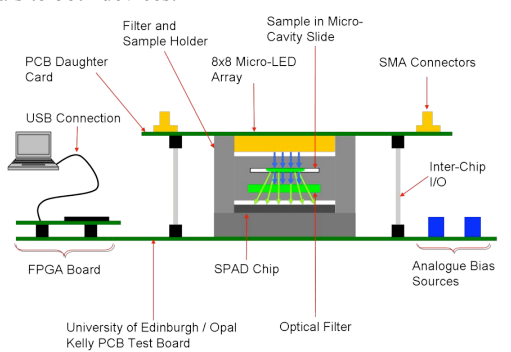

Figure 6: Complete two-chip micro-system. The PCB daughter card is physically supported by the filter and sample holder and stacked header pins. The excitation and detection arrays have a minimum separation of $3 \mathrm{~mm}$, between which an optical filter and the sample of interest are placed. A plastic holder was designed to house these two elements, providing a light tight enclosure for the packaged SPAD chip, an optical filter, a sample held in a micro-cavity slide and a packaged micro-LED device.

The output from the on-chip VCO, situated on the microLED driver, defines the repetition rate of the LED device. This signal is passed off-chip (to a SMA connector on the daughter card) and is used as the synchronisation input to either a time-correlated single photon counting (TCSPC) module (Becker and Hickl, SPC-130), or the timing generator circuit on-board the detector chip (Fig. 7). A $514 \mathrm{~nm}$ long pass filter (Semrock, LP02-514RU-25) was chosen to separate the excitation light from the fluorescence emission. This allows a range of fluorophores with emission spectra greater than $514 \mathrm{~nm}$ to be evaluated while maximising the rejection of excitation light.

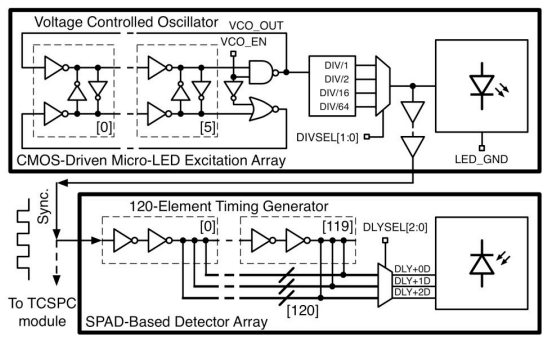

Figure 7: The on-chip VCO provides the square-wave input to the micro-LED driver array and the SPAD time-gate generator circuit.

SPAD output pulses can be passed off-chip to SMA connectors and used as the input to the constant fraction discriminator of the TCSPC module. Alternatively, the SPAD pulses can be processed locally using the in-pixel counters. In this mode, counter data is broadcast off-chip to the FPGA. 


\section{RESULTS}

In order to establish the sensitivity of the SPAD detector and on-chip time-gating circuitry, a dilution experiment was performed where different concentrations of $\mathrm{CdSe} / \mathrm{ZnS}$ quantum dot samples (Evident Technologies Inc.) diluted in toluene were analyzed (Fig. 8). The SPAD detector was mounted to the output port of a microscope (Nikon, TE2000$\mathrm{U})$ and the sample excited by a $470 \mathrm{~nm}$ pico-second pulsed laser diode (PicoQuant, $\mathrm{GmbH}$ ). It was found that the SPAD detector and in-pixel time-gated counters were capable of performing time-resolved analysis of these samples down to a concentration of $10 \mathrm{nM}$. From these decay curves, lifetimes of $13.66,13.65,13.51,13.82,13.10$ and $13.35 \mathrm{~ns}( \pm 408 \mathrm{ps}$ estimated measurement error, based on the timing generator circuit resolution) were extracted for sample concentrations of $50,25,10,1,0.1$ and $0.01 \mu \mathrm{M}$, respectively.

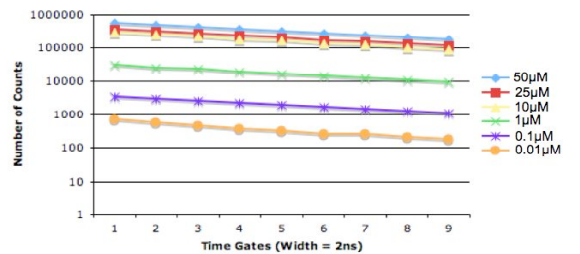

Figure 8: Fluorescence decay curves of quantum dot samples at different concentrations, captured using SPAD detector and in-pixel time-gated concentrations, captured using SPAD detec
counters

Measurements of fluorescence decay curves using the twochip micro-system and external TCSPC hardware were obtained using quantum dots in a toluene solution $($ concentration $=57 \mu \mathrm{M})$ and Rhodamine $6 \mathrm{G}$ (concentration $=$ $250 \mu \mathrm{M}$ ) and Rhodamine B (concentration $=100 \mu \mathrm{M}$ ) in water (Fig. 9). Analysis of these decay curves yields lifetime estimations of $13.81 \mathrm{~ns}, 4.36 \mathrm{~ns}$ and $1.34 \mathrm{~ns}$ for the quantum dot sample, Rhodamine $6 \mathrm{G}$ and Rhodamine B samples, respectively $( \pm 80 \mathrm{ps}$ estimated measurement error, based on SPAD RMS jitter). These results were performed with an LED excitation pulse width of 910ps (FWHM), using a sample volume of $45 \mu \mathrm{L}$, and are consistent with lifetimes reported in the literature [10], [11]. Furthermore, quantum dot lifetimes are consistent with those measured using a conventional microscope system, confirming the ability of the micro-system to accurately resolve fluorescence lifetime data.

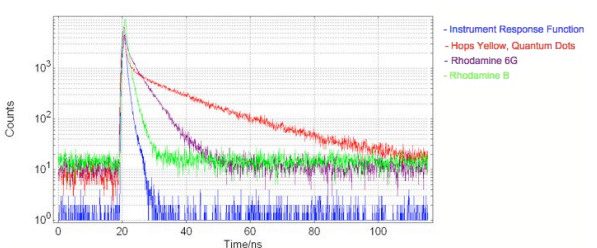

Figure 9: Fluorescence decay curves of quantum dot, Rhodamine $6 \mathrm{G}$ and Rhodamine B samples. An IRF of 910 ps FWHM is also included.

\section{CONCLUSION}

A compact fluorescence lifetime analysis system has been demonstrated which incorporates sample excitation via an $8 \times 8$ CMOS-driven micro-LED array and emission detection using an array of SPAD detectors located on a second CMOS chip. Using an excitation pulse of $910 \mathrm{ps}$ at $450 \mathrm{~nm}$, common fluorophore lifetimes, with a sample volume of $45 \mu \mathrm{L}$ have been sensed. We have demonstrated detection sensitivities down to concentrations of $10 \mathrm{nM}$ using in-pixel signal processing circuitry. This work opens up the possibility for a low-cost and robust micro-system for time-resolved fluorescence analysis, requiring reduced reagent volumes and no optical alignment. On-going work includes incorporation of microfluidics as a sample delivery method.

\section{ACKNOWLEDGMENTS}

We are grateful to Optocap Ltd., U.K. for carrying out the bump-bonding process and Professor Charbon at TU Delft for access to the SPAD devices. We acknowledge the support from the Scottish Funding Council for the Scottish Consortium on Integrated Microphotonic Systems (SCIMPS) and for the Joint Research Institute with Heriot-Watt University, which is a part of the Edinburgh Research Partnership in Engineering and Mathematics (ERPem).

\section{REFERENCES}

[1] A. Rochas, M. Gani, B. Furrer, P.A. Besse and R.S. Popovic, "Single photon detector fabricated in a complementary metal-oxide-semiconductor high-voltage technology," Rev. Sci. Instrum., vol. 74, 3263, 2003.

[2] D.E. Schwartz, E. Charbon and K.L. Shepard, "A single-photon avalanche diode array for fluorescence lifetime imaging microscopy," IEEE Journal of Solid-State Circuits, vol. 43, No. 11, 2008.

[3] D. Mosconi, D. Stoppa, L. Pancheri, L. Gonzo, A. Simoni, "CMOS single-photon avalanche diode array for time-resolved fluorescence 564-7, 2006.

[4] H J. Yoon, S. Itoh and S. Kawahito "A CMOS Image Sensor With In [4] H... Yoon, S. Hoh and S. Kawahito, "A CMOS Image Sensor With InTransactions on Electron Devices, vol. 56, No. 2, 2009.

[5] C. Griffin, E. Gu, H. Choi, C. Jeon, O. Rolinski, D. Birch, J. Girkin, and $\mathrm{M}$. Dawson, "Fluorescence excitation and lifetime measurements using GaN/InGaN micro-led arrays," The 17th Annual Meeting of the IEEE Lasers and Electro-Optics Society, vol. 2, pp. 896-897, November 2004. [6] J.A. Chediak, Z. Luo, J. Seo, N. Cheung, L.P. Lee and T.D. Sands, "Heterogeneous integration of CdS filters with GaN LEDs for fluorescence detection Microsystems," Sensors and Actuators A, vol. 111, pp. 1-7, 2004. [7] C. Jeon, H. Choi, E. Gu, and M. Dawson, "High-density matrixaddressable AlInGaN-based368-nm microarray light-emitting diodes," IEEE Photonics Technology Letters, vol. 16, pp. 2421-2423, 2004

[8] B.R. Rae, C. Griffin, K.R. Muir, J.M. Girkin, E. Gu, D. Renshaw, E. Charbon, M.D. Dawson and R.K. Henderson, "A Microsystem for TimeDiodes and Micro-LEDs", International Solid-State Circuits Conference, $166-7,2008$.

[9] C. Niclass, M. Sergio, and E. Charbon, "A single photon avalanche diode array fabricated in deep-submicron CMOS technology," in Proc. Design, Automation and Test in Europe DATE '06, vol. 1, 6-10, pp. 1-6, 2006.

[10] Evident Technologies, EviDot Specifications,

hitp://www. evidenttech.com/products/evidots/evidot-specifications.html http://www.iss.com/resources/fluorophores.html 


\title{
FPGA Implementation of a Video-rate Fluorescence Lifetime Imaging System with a $32 \times 32$ CMOS Single-Photon Avalanche Diode Array
}

\author{
Day-Uei Li ${ }^{1}$, Richard Walker ${ }^{1,2}$, Justin Richardson ${ }^{1,2}$, Bruce Rae ${ }^{1}$, Alex Buts ${ }^{1}$, David Renshaw ${ }^{1}$, and Robert Henderson ${ }^{1}$ \\ ${ }^{1}$ Institute for Integrated Micro and Nano Systems, Joint Research Institute for Signal \& Image Processing/Integrated \\ Systems/Energy/Civil and Environmental Engineering, School of Engineering, The University of Edinburgh, Edinburgh, UK \\ ${ }^{2}$ Imaging Division, STMicroelectronics, Edinburgh, UK \\ David.Li@ed.ac.uk
}

Abstract-A new integration based fluorescence lifetime imaging microscopy (FLIM) called IEM has been proposed to implement lifetime calculations [1]. A real-time hardware implementation of this IEM FLIM algorithm suitable for a single photon avalanche diode (SPAD) array in $0.13 \mu \mathrm{m}$ CMOS technology is now implemented on FPGA. A widefield microscope was now implennented on FPGA. A widefield microscope was adapted to accommodate the array and test it on biologica applications. Video-rate fluorescence lifetime imaging has been achieved, by perfor realizing the first, compact, and low-cost FLIM camera.

\section{INTRODUCTION}

Time-resolved fluorescence lifetime imaging (FLIM) systems have been widely used in cell-biology research, medical diagnosis, and pharmacological development [1-9]. The independence of fluorescence lifetimes on probe concentration make FLIM more favoured than its counterpart - fluorescence intensity imaging. As shown in Fig. 1(a), a laboratory FLIM experiment usually contains a Ti-Sapphire laser, a photomultiplier tube (PMT), a TCSPC photon counting card, fluorescence lifetime analysis software and a PC graphical user interface (GUI). Available FLIM systems provide excellent time resolution and light sensitivity, but they are quite expensive and cumbersome [3-5]. Commercial applications increasingly demand compact and portable system-on-chip (SOC) FLIM solutions. Thanks to the progress of semiconductor technology, high accuracy time resolution, low cost, and compactness can be achieved by exploiting CMOS SPAD arrays to replace the PMTs [10] and by bumpbonding AlInGaN UV micro-pixellated light-emitting diodes (micro-LEDs) to replace lasers [6] in the general direction of lab-on-chip. A remaining challenge is that the excessive computational demands of available lifetime analysis software such as the iterative least-square method (LSM) or maximumlikelihood-estimation (MLE) [9] renders real-time imaging impossible. In many applications it is desirable to monitor the instantaneous bio-chemical interactions to provide quick

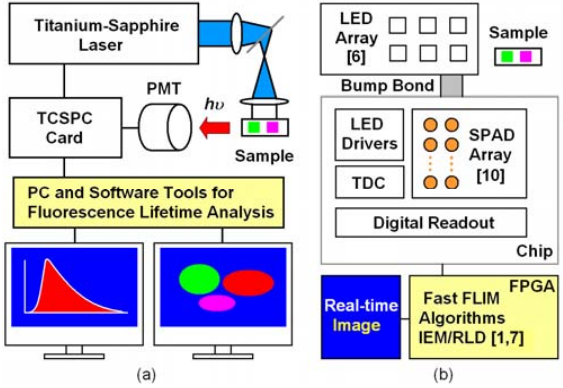

Fig. 1 (a) Laboratory FLIM setup and (b) FLIM system-on-chip.

feedback to corresponding manipulations. The slow speed of software analysis tools becomes the bottleneck and has driven the recent development of non-iterative, fast real-time FLIM algorithms $[1,7-8]$. In the past, rapid lifetime determination methods (RLD) were thought to be the simplest algorithms [8], and they were used in some previously reported video-rate FLIMs [4-5]. However, to implement such algorithms on FPGA for further compactness requires look up tables (LUTs) [7] or natural logarithic functions. It is proven in [1] that IEM has better resolvability and is simpler to implement on FPGA. Moreover, a major drawback of RLD is the requirement to choose a proper time delay between two time gates [4-8], which is quite challenging when specimens with a wide range of lifetimes coexist, such as in micro-fluidic mixing. Figure 1(b) shows a system-on-chip FLIM that will replace that of Fig. 1(a). The IEM introduced for the first time in [1] was performed on the compure and for single pixel only. In this paper, we first introduce simpler IEM algorithm for parallel processing/hardware implementation. The detail of the FPGA implementation for IEM will be given. A real-time lifetime image of quantum dots (Q-dots) will also be shown. 
II. IEM

We have presented in [1] that when the ratio of the FWHM of the instrumental response function (IRF) over the lifetime is much less than 1 , we can assume the fluorescence decay function $f(t)=A \exp (-t / \tau)$ with $\tau$ being the lifetime. For the usual measurement setup in a lab, the FWHM of the IRF is of the order of hundreds of picoseconds so it is reasonable to target lifetimes greater than 500ps. Fig. 2 shows a fluorescence histogram to illustrate the concept of IEM. With $M$ time bins (bin width of $h$ ) generated by the time-to-digital converters (TDCs) in the photon counting module and with the assumption of single-exponential decay, the lifetime $\tau$ is related to the decay function as

$$
\tau\left(f_{0}-f_{M-1}\right)=\int_{t_{0}}^{t_{M-1}} f(t) d t \cong h \sum_{j=0}^{M-1} C_{j} f_{j},
$$

where $f_{j}=f\left(t_{j}\right), j=0, \ldots, M-1$, and Romberg's integration coefficients $\mathbf{C}_{j}=[1 / 2,1, \ldots, 1,1 / 2]$ is used. Multiply (1) on both sides by the factor $\left(1-\mathrm{e}^{-h / \tau}\right)$ to obtain

$$
\frac{\tau_{I E M}}{h} \cong \frac{\sum_{j=0}^{M-1} C_{j} N_{j}}{N_{0}-N_{M-1}}=\frac{N_{c}-\left(N_{0}+N_{M-1}\right) / 2}{N_{0}-N_{M-1}},
$$

where $N_{j}$ is the number of counts in the $j$ th time bin and $N_{c}$ is the total effective signal count. To implement (2) on FPGA, for each pixel, we only need two counters (one for $N_{c}$ and the other for $N_{0}+N_{M-1}$ ) and 1 subtracter for the numerator and 1 up-down counter for the denominator. The division is easily implemented on FPGA by simply choosing

$$
N_{0}-N_{M-1}=2^{L}, L \text { is an integer. }
$$

The hardware implementation of (2) and (3) is much easier than that of the RLD algorithms [4-8] because only addition and subtraction are needed.

\section{FPGA IMPLEMENTATION OF IEM}

Before employing IEM to the SPAD array, we first built a detection model of a SPAD pixel in order to verify the efficiency of the algorithm on the FPGA. Fig. 3 shows the diagram for the IEM implementation for a SPAD pixel. The 8 bits signal coming from a TDC in a SPAD pixel cell can be modeled by a 31-bit pseudo-random bit sequence (PRBS) generator and a look-up table used for generating a photon emission probability function. (Using 8-bit TDC instead of 10bit one is for getting faster simulation results.) The SPAD detection model then feeds the output data into a serializer and detection model then feeds the output data into a serializer and array. The polynomial for generating the 31-bit PRBS is $g(x)$ $=1+x^{28}+x^{31}[11]$. The maximum length of the bit sequence is $2^{31}-1=2.15 \times 10^{9}$, which is much larger than the total photon count of usual TCSPC measurements for a single pixel. For comparison with RLD algorithms, we built a look-up table of digital division inside the IEM implementation block; although in practice there is no need to do so according to (3). Taking a single decay function $f(t)=A \exp [-t /(11 h)]$ as an example, a look-up table for such decay including the overall jitter of SPAD and laser (about $2 h$ in order) and the laser excitation delay between the electrical excitation signal and laser pulse is built right after the 31-bit PRBS generator. If $h$ is of $100 \mathrm{ps}$ (the full range of the TDC $256 h=25.6 \mathrm{~ns}$, which is equivalent to a laser repetition rate (LPR) $=40 \mathrm{MHz}$ ). The lifetime $\tau=11 h=1.1 \mathrm{~ns}$, and if our target is to resolve $\tau$ values greater than $1 \mathrm{~ns}$, a typical jitter of $2 h=200 \mathrm{ps}$ can be tolerated even though only tail-fitting is applied to extract the lifetime without digital de-convolution [1]. Unlike the perfect

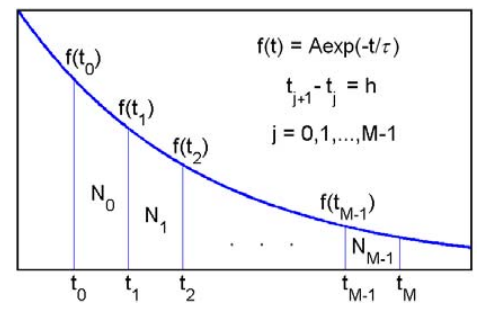

Fig. 2 Concept of IEM algorithm.

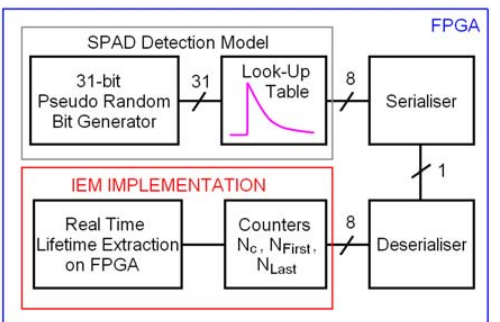

Fig. 3 IEM implementation on FPGA for a single SPAD.

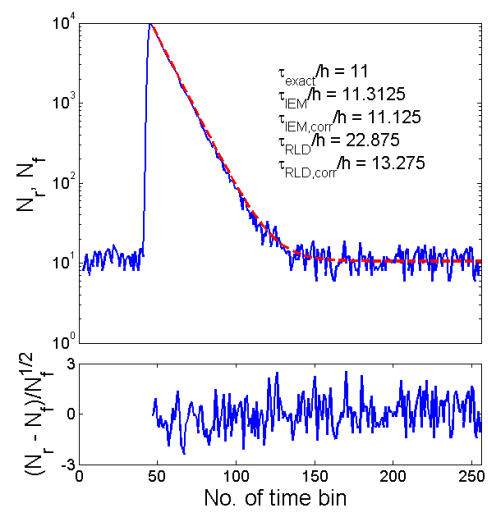

Fig. 4 Decay and fitted curve obtained by the model and IEM. 
exponential histogram shown in Fig. 2 (FIRST $=0, L A S T=$ $M-1$ ), the first and last channel numbers FIRST and LAST are 46 and 255 , respectively. Figure 4(a) shows the decay histogram $N_{r}$ obtained by the model and the fitted curve $N_{f}$ by IEM with background correction. The calculated lifetimes with 4 extra bits for decimal accuracy obtained by IEM and RLD without background correction are also listed and they are 11.3125 and $22.875 \mathrm{~ns}$, respectively. Compared with the exact lifetime, the error is $3 \%$ and $108 \%$ respectively. With background correction, IEM and RLD give $\tau_{\text {IEM,corr }} / h=11.125$ (error $=1 \%)$ and $\tau_{\mathrm{RLD}, \mathrm{cor}} / h=13.275($ error $=21 \%$ ), respectively. For RLD, its optimal window is $1 \tau \leq 2 w_{g} \leq 6 \tau$ [8], where $w_{g}$ is the gate width, and it is a challenging task to resolve lifetimes much less than the effective measurement window ( $\tau \ll 210 h$ in this case). However, for IEM, it has superior resolvability for wider lifetime range [1] and much more insensitive to background noise [12]. Figure 4(b) shows the normalized residual count of $\left(N_{r}-N_{f}\right) / N_{f}^{1 / 2}$ which is well distributed, implying that the model is Poisson distributed as in real cases.

IV. INTEGRATION OF LifETIME EXTRACTION ALGORIthM WITH CMOS SPAD ARRAY

Now we integrate the lifetime extraction block with a $32 \times 32$ SPAD pixel array (split into two $32 \times 16$ SPAD arrays $32 \times 32$ SPAD pixel array (split into two $32 \times 16$ SPAD arrays
with different resolutions, and the measurement is performed on one $32 \times 16$ SPAD array with $h=0.16 \mathrm{~ns}$.). Each SPAD pixel contains a 10-bit TDC, and for each column there is a serializer to minimize the pad number. The details of the SPAD chip will be reported in future publications. The array is capable of generating data at a frame rate of $500 \mathrm{kHz}$. Fig. 5 illustrates how the lifetime extraction algorithm works with the existing SPAD array on FPGA. First the serial data is the existing SPAD array on FPGA. First the serial data is
deserialized and the output data (10-bit for each column) is deserialized and the output data (10-bit for each column) is
connected to a housekeeper to be normalized to a format connected to a housekeeper to be normalized to a format
required by the IEM. Along with the data output, the information of row address, signal ready, and data type: row addr in, ready in, and data type in are also sent out from the deserializers and pipelined to next block. The lifetime extraction module has two extra control signals FIRST and $L A S T$ sent from the GUI via USB connection. These two numbers can be generated automatically by locating the peak of the histogram during the measurement setup phase. Afte

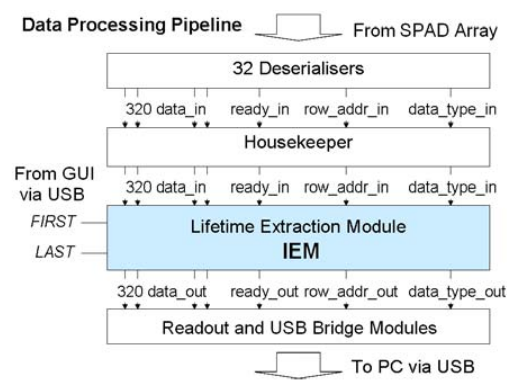

Fig. 5 IEM implementation on FPGA for SPAD array and data path. $\mathrm{r}$ they have been acquired, the system starts lifetime calculations. The extracted lifetime, the counts $N_{c},\left(N_{F i r s t}+\right.$ $\left.N_{\text {Last }}\right)$, and $\left(N_{\text {First }}-N_{\text {Last }}\right)$ are stored in on-FPGA memory. The IEM block only used $11 \%$ of 4 -input LUTs and 1,248 16×1 RAMs (= 20k bits) of Virtex-II Pro FPGA.

$$
\text { V. Measurement Results }
$$

The block diagram and photo of the measurement setup are shown as Fig. 6 and Fig. 7, respectively. It comprises a PicoQuant pulsed diode laser with wavelength of $470 \mathrm{~nm}$, a Nikon TE2000U microscope, a 32×16 SPAD array fabricated in $0.13 \mu \mathrm{m}$ CMOS imaging process and mounted on a daughter board which mounted on a mother board LASP (from EPFL) containing two Xilinx Virtex-II Pro FPGA chips. A sample of Catskill Green quantum dots (Q-dots) (with an emission wavelength of $548 \mathrm{~nm}$, from Evident Technologies) mounted on a micro-cavity slide is measured. The Q-dots are held in a solution of Toluene and the lifetime measurements where Q Q-dots is CdSe/ZnS. The laser repetition rate (LPR) is of $8 \mathrm{MHz}$, and the average output power of laser is $0.12 \mathrm{~mW}$. The extracted lifetimes are then passed from the IEM module to the GUI via the USB link. Assume that a pixel can collect a photon for each frame rate with $L$ being set as 10 in (3), the time (update time) required for the IEM to extract the lifetime of Q-dots is 0.05 second (= $20 \mathrm{frames} / \mathrm{s})$. If the same accuracy

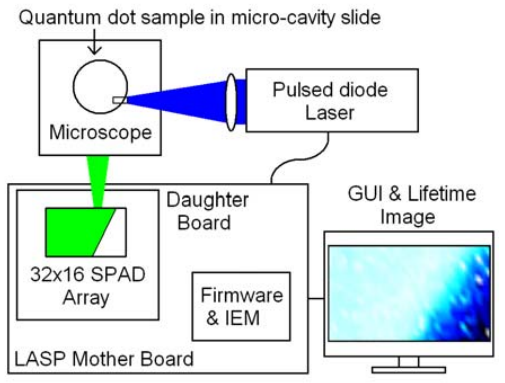

Fig. 6 Block diagram of fluorescence image measurement setup.

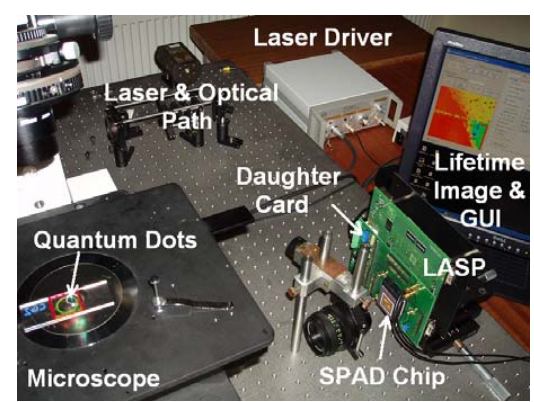

Fig. 7 Fluorescence lifetime measurement setup. 


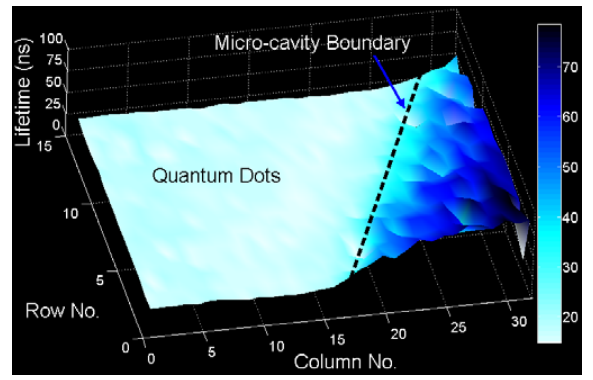

Fig. 8 Fluorescence lifetime image of quantum dots.

of about $25 \mathrm{~dB}$ as [5] is required, the frame rate is better than $30 \mathrm{frames} / \mathrm{s}$. Moreover, the update time will be much smaller if samples with smaller lifetime are used [1]. For example, if a sample with a lifetime of $1 \mathrm{~ns}$, the frame rate is higher than 100 frames/s. Figure 8 shows the fluorescence lifetime image of Q-dots. To the best of our knowledge, this is the first videorate fluorescence lifetime image generated by FPGA. The lifetime range of the Q-dots is usually from 15 to $20 \mathrm{~ns}$, and the extracted lifetime is from $14.6 \mathrm{~ns}$ to $20.3 \mathrm{~ns}$, and the average extracted lifetime is from $14.6 \mathrm{~ns}$ to $20.3 \mathrm{~ns}$, and the average
value within the bright emission area is $16.3 \mathrm{~ns}$. This is in good value within the bright emission area is $16.3 \mathrm{~ns}$. This is in good agreement with the lifetimes quoted by the manufacturer (15The calculation error is mainly from the high dark count noise of the SPAD (around 100kHz). For better resolvability, a new SPAD structure with a lower dark count rate will be implemented on the next chip, or a noise calibration technique can be implemented with the IEM [12]

\section{CONCLUSIONS}

A newly proposed integration based algorithm IEM for real-time FLIM systems has been successfully implemented on FPGA. A photon detection model for single SPAD is proposed to verify the accuracy and efficiency of the algorithm. With a $0.13 \mu \mathrm{m}$ CMOS $32 \times 16$ SPAD array, IEM can work in parallel for each pixel and the calculated lifetime is updated at a certain condition dictated by (3). A widefield microscope was adapted to accommodate the array and test it on biological applications. Without resorting to slow software analysis tools, the real-time image allows instantaneous monitoring of bio-chemical interactions to provide quick feedback for corresponding manipulations. The FLIM prototype successfully updates the lifetime image within tens of milliseconds. The error is mainly due to the high dark count rate of SPADs (around $100 \mathrm{kHz}$ ). To reduce this error, a new SPAD structure with much lower dark count rate will be implemented in future chips or a new noise calibration technique can be applied. To the best of our knowledge, this is the first SOC FLIM system that can generate video-rate fluorescence lifetime images. The usage of hardware resource for implementation is small thanks to the simplicity of (2) and (3). With this merit, IEM can be even implemented in-pixel for further image compression and reduced I/O bandwidth. Along with integrated excitation sources such as low cost, high speed LED arrays [6], this prototype system promises low-cost, miniaturized fluorescence lifetime imaging and sensing for portable instrumentation.

\section{ACKNOWLEDGMENT}

This work has been supported by the European Community within the Sixth Framework Programme of the Information Science Technologies, Future and Emerging Technologies Open MEGAFRAME project (contract 0292172, www.megaframe.eu). We acknowledge the support from the Scottish Funding Council for the Joint Research Institute with the Heriot-Watt University which is a part of the Edinburgh Research Partnership in Engineering and Edinburgh Research Partnership in Engineering and
Mathematics (ERPem). The system has been built with help from Lucio Carrara, Theo Kluter, and Prof. Edoardo Charbon, from Lucio Carrara, Theo Kluter, and Prof. Edoardo Charbon,
EPFL. The measurements have been performed using the EPFL. The measurements have been performed using the
COSMIC laboratory facilities with help from Dr. Jochen Arlt, Andy Garrie, Trevor Whittley, and Dr. David Dryden. The authors would like to express gratitude to them.

Disclaimer: This publication reflects only the authors' views. The European Community is not liable for any use that may be made of the information contained herein.

\section{REFERENCES}

[1] D.-U. Li, E. Bonnist, D. Renshaw, and R. Henderson, "On-chip timecorrelated fluorescence lifetime extraction algorithms and error analysis," J. Opt. Soc. Am. A, vol. 25, pp. 1190-1 198, May 2008.

[2] P. I. H. Bastiaens and A. Squire, "Fluorescence lifetime imaging
microscopy: spatial resolution of biochemical process in the cell," microscopy: spatial resolution of biochemical process in the cell,"
trends in Cell Biology, vol. 9, pp. 48-52, 1999 .

[3] M. A. O'Leary, D. A. Boas, X. D. Li, B. Chance, and A. G. Yodh, "Fluorescence lifetime imaging in turbid media," Opt. Lett., vol. 21, pp. 158-160, 1996.

[4] A. V. Agronskaia, L. Tertoolen, and H. C. Gerritsen, "High frame rate fluorescence lifetime imaging," J. Phys. D: Appl. Phys., pp. 1655-1662, 2003.

[5] D. S. Elson et. al, "Real-time time-domain fluorescence lifetime maging including single-shot acquisistion with a segmented optical image intensifier," New Journal of Phyisss, vol. 6, 2004.

6] B. Rae, C. Griffin, K. Muir, J. Girkin, E. Gu, D. Renshaw, E. Charbon, M. Dawson, and R. Henderson, "A microsystem for time-resolved fluorescence analysis using CMOS single-phost micro-LEDs," ISSCC Dig. Tech. Papers, pp. 166-167, 2008.

[7] D.-U. Li, B. Rae, E. Bonnist, D. Renshaw, and R. Henderson, "On-chip fluorescence lifetime extraction using synchronous gating schemeTheoretical error analysis and practical implementation," in the Proceedings of International Conference on Bio-inspired Systems and Signal Processing (Biosignals 2008), pp. 171-176, 2008.

[8] R. M. Ballew and J. N. Demas, "An error analysis of the rapid lifetime determination method for the evaluation of single exponential decays," Ana. Chem., vol. 61, pp. 30-33, 1998

[9] V. V. Apanasovich and E. G. Novikov, "Methods of analysis of fluorescence decay curves in pulsed fluorometry (Review)," J. Appl. Spectrosc., vol. 56, pp. 317-327, 1992.

10] C. Niclass, M. Gersbach, R. Henderson, L. Grant, and E. Charbon, "A single photon avalanche diode implemented in 130-nm CMOS echnology," IEEE J. Sel. Top. Quantum Electron. vol. 13, pp. 863-869, 2007.

11] P. Alfke, Efficient Shift Registers, LFSR Counters, and Long PseudoRandom Sequence Generators, XAPP052, Application Note, Xlinx, Inc. 1996.

[12] D.-U. Li, R. Walker, J. Richardson, B. Rae, A. Buts, D. Renshaw, and R. Henderson, "Hardware implementation and calibration of background noise for a new integration-based fluorescence lifetime sensing algorithm," accepted for publication on J. Opt. Soc. Am. A.
http://www.opticsinfobase.org/josaa/upcoming.cfm. 


\section{Individually-Addressable AlInGaN Micro-LED Arrays with CMOS Control and Sub- Nanosecond Output Pulses}

Jonathan McKendry, Bruce R. Rae, Zheng Gong, Keith R. Muir, Benoit Guilhabert, David Massoubre, Erdan Gu, David Renshaw, Martin D. Dawson, Fellow, IEEE, and Robert K. Henderson

Abstract-We report the fabrication and characterization of an ultraviolet (UV, $370 \mathrm{~nm}$ ) emitting AlInGaN-based micro lightemitting diode (micro-LED) array integrated with complementary metal oxide silicon (CMOS) control electronics. This configuration allows an $8 \times 8$ array of micro- electronics. This cons, CDD pix $\mu$ can be driver in direct curre pulsed operation, with linear feedback shift registers (LFSR) allowing the output of the micro-LED pixels to mimic that of an optical data transmitter. We present the optical output power versus drive current characteristics of an individual pixel, which show micro-LED output power of up to $570 \mu \mathrm{W}$ in DC operation. Representative optical pulse trains demonstrating the microLEDs driven in square wave and LFSR modes, and controlled optical pulse widths from $300 \mathrm{ps}$ to $40 \mathrm{~ns}$ are also presented.

Index Terms-GaN, microdisplays, micro light-emitting diodes (micro-LEDs), CMOS.

I. INTRODUCTION

$G$ ALLIUM NITRIDE based micro-pixel light emitting $\bigcup_{\text {diode ('micro-LED') arrays have attracted much interest }}$ in recent years as ultraviolet-blue-green spectrally and spatiotemporally selective photo-excitation and photo-stimulation sources [1], [2]. Using computer control to pattern-program the output from these devices, fixed or high frame-rate optical images can be projected into a wide variety of samples and materials, for applications including mask-free photolithography [3], optical lab-on-a-chip, optical sectioning microscopy [4], patterned photo-excitation of biological samples [5], and hybrid organic/inorganic devices [6].

Although micro-LED's can be matrix-addressed to give array control and scalability in a line-scanned format, it has been recognized that, especially in flip-chip format, the devices are advantageously compatible with complementary

Manuscript received (January 5, 2009.) The project was supported by EPSRC under the 'HYPIX' program.

J. McKendry, D. Massoubre, Z. Gong, B. Guilhabert, E. Gu, and M. D. Dawson are with the Institute of Photonics, University of Strathclyde, Glasgow, G4 0NW, U.K. (e-mail: jonathan.mckendry@strath.ac.uk).

B. Rae, K. Muir, D. Renshaw and R. K. Henderson are with the Institute for Integrated Micro and Nano Systons, Joint Research hnstitute for Integrated Kin's Buldings, Mayfield Roul, Ediburh, EH9 3J, UK. (e-Til: Bruce.Rae@ed.ac.uk). metal oxide silicon (CMOS) control electronics for individua and flexible pixel control. In a recent report [7], we demonstrated preliminary results wherein an AlInGaN ultraviolet (UV) micro-LED array was integrated with CMOS device that was designed for electro-wetting [8] rather than being custom-designed for micro-LED compatibility. Under that configuration, multi-nanosecond optical pulses and a limited degree of control was demonstrated. In this paper, we report considerably improved results from a representative $370 \mathrm{~nm}$ micro-LED device driven by a custom-designed CMOS driver. Pulses into the sub-nanosecond regime, high optical output power per pixel and versatile control of the emission characteristics are demonstrated. This device is anticipated to have applications in areas including computercontrolled UV 'direct write' photo-patterning, time-resolved on-chip microfluorimetry, and photo-pumping of organic semiconductor lasers and optical amplifiers.

\section{DEVICE DESIGN AND FABRICATION}

The micro-LED devices, designed for flip-chip bonding, were fabricated from multi-quantum well UV $(370 \mathrm{~nm})$ LED wafers grown on c-plane sapphire substrates, with a similar multilayer structure to those used in earlier reports [9]. The devices contain a $16 \times 16$ array of individually addressable micro-disk pixels, each of $72 \mu \mathrm{m}$ diameter on a $100 \mu \mathrm{m}$ center-to-center pitch. The mesa structure of the pixels was defined using photolithographic patterning and inductively coupled plasma (ICP) etching. $15 \mathrm{~nm}$ of Ni-Au and $200 \mathrm{~nm}$ of $\mathrm{Ti}-\mathrm{Au}$ were then evaporated onto the p-contact area to act as a combined electrical contact and mirror. Ti-Au was then deposited in the spaces between the pixels to act as a common $\mathrm{n}$-contact, and finally a $\mathrm{Si}-\mathrm{O}_{2}$ isolation layer deposited and patterned by plasma enhanced chemical vapor deposition and reactive ion etching. The $300 \mu \mathrm{m}$-thick sapphire substrate was mechanically polished and the patterned wafers diced into individual device chips of area approximately $3 \mathrm{~mm}^{2}$. More detailed descriptions of the micro-LED characteristics and fabrication process can be found elsewhere [10].

The custom CMOS device, implemented in a standard 0.35 um CMOS technology from Austria Microsystems, consists of 64 driver pixels in an $8 \times 8$ array. Each pixel measures $200 \mu \mathrm{m}$ x $200 \mu \mathrm{m}$, which contains a $100 \mu \mathrm{m}$ x $100 \mu \mathrm{m}$ full metal bond 
stack, and dedicated driver circuitry. Fig. 1(a) illustrates a microscope image of a section of the full CMOS array (showing 4 individual CMOS driver pixels) and (b) a representative image showing a simple output pattern.

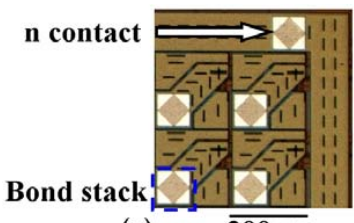

(a) $\overline{200 \mu \mathrm{m}}$

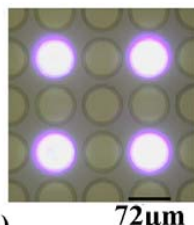

(b)

$7 \overline{2 \mu \mathrm{m}}$

Fig. 1. (a) microscope image of a section of a CMOS device and (b) microscope image of 4 micro-LED/CMOS pixels in operation.

A bump-bonding process was used to electrically and physically contact the micro-LED and CMOS devices. Each CMOS bonding area is connected to a micro-LED pixel by a Au bump-bond, effectively providing an $8 \times 8$ array of CMOS-controlled micro-LED pixels with a $200 \mu \mathrm{m}$ pitch.

The maximum current and voltage supplied by each CMOS pixel was specified to be $100 \mathrm{~mA}$ and $5 \mathrm{~V}$, respectively. Fig. 2 shows a circuit schematic of a section of an individual CMOS driver element. All the CMOS driver input signals were based on 3.3 V logic before the micro-LED bias, labeled LED VDD, is level-shifted to a higher user-definable voltage, which can be set up to the maximum value of $5 \mathrm{~V}$.

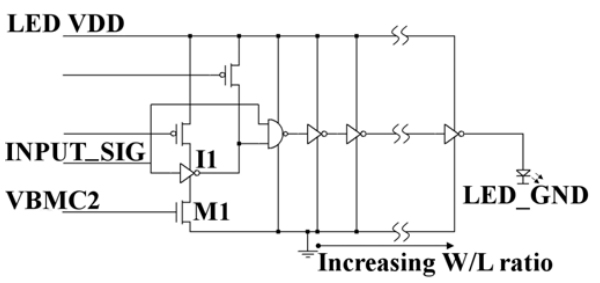

Fig. 2. section of a CMOS driver element, illustrating the output buffer and short pulse generation circuitry.

A key feature of the driver array is that the micro-LED array ground terminal, labeled LED GND, is separated from the ground terminal of the driver circuit. This technique allows the micro-LEDs to be biased above or below ground prior to an excitation signal being applied by the driver. By pulling LED_GND below ground, a voltage greater than $5 \mathrm{~V}$ (limit of the CMOS process) can be placed across each microLED, and hence greater optical output powers can be achieved. The CMOS drivers were also designed to be able to pulse the micro-LEDs to a minimum pulse duration (full width at half maximum, FWHM) of $300 \mathrm{ps}$. To generate these short pulses, a square-wave input signal, labeled INPUT_SIG, is passed through an inverter (I1), and the time delay through the inverter defined the pulse width. The inverter delay can be adjusted via the gate voltage, arbitrarily labeled $\mathrm{V}$ bias mode control 2 (VBMC2), of a current-starving transistor (M1). To minimize the load capacitance seen by the input signal, while maximizing the drive strength of the circuit, an output buffer comprising of a chain of inverters of increasing transistor width/length ratios was implemented. The current that could be delivered by the driver is set by the drive current versus voltage (IV) curve of the micro-LED device and the user definable output voltage (LED VDD).

\section{RESULTS AND DISCUSSION}

Fig. 3(a) shows the current-voltage characteristic and (b) the corresponding optical output power versus drive current (LI) characteristic from an individual micro-LED pixel, driven by CMOS under direct current (DC) bias conditions at room temperature. The output power measurements were performed using a power meter and calibrated Si photodetector $\left(1.13 \mathrm{~cm}^{2}\right.$ active area), placed in close proximity above the device to ensure most of the forward emission from the micro-LED was collected.
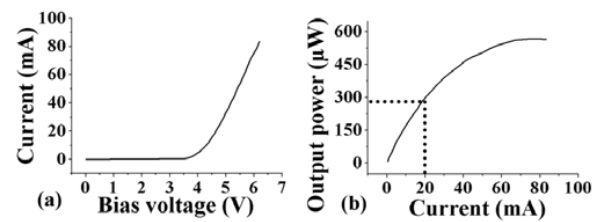

$$
\text { Fig. 3. (a) IV and (b) LI characteristics of a single pixel }
$$

The turn-on voltage of the pixel is approximately $4 \mathrm{~V}$. It can be seen that the output power from a single pixel driven in DC conditions can reach up to $570 \mu \mathrm{W}$ at $83.4 \mathrm{~mA}(290 \mu \mathrm{W}$ at $20 \mathrm{~mA}$ ) of injected current. It should be noted that the maximum input current in this case is limited by the microLED performance, not by the CMOS driver. The CMOS device has an on-chip voltage-controlled oscillator (VCO) that is capable of generating an internal clock ranging from $6 \mathrm{MHz}$ to $800 \mathrm{MHz}$. There is also the functionality to drive the microLEDs from an external clock signal if required. In addition, the CMOS device contains linear feedback shift registers (LFSRs) which allow each column of CMOS elements to output a pseudo-random binary sequence. Optical pulse trains, as measured by a photomultiplier tube (PMT, Hamamatsu H5783P), of a micro-LED pixel being driven by the on-chip VCO and LFSR circuitry can be seen in Fig. 4. The overshoot and oscillation seen on the optical pulses is tentatively attributed to the internal resistance and capacitance of the micro-LEDs exhibiting a low-frequency damped oscillatory behavior when excited by an impulse input.

The LFSR capability is intended to allow the output of the CMOS-controlled micro-LED to mimic the data-modulated output of an optical data transmitter. An integrated micro-LED and CMOS device could potentially be used for optical data transmission applications via either fiber or free-space, similar to those based on resonant-cavity light emitting diodes [11], [12] and, with specifically UV output, could be used for 
modulated white light communications systems or to datamodulate photo-pumped organic semiconductor devices.

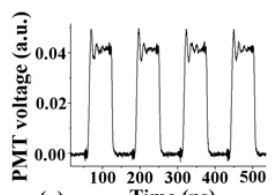

(a)

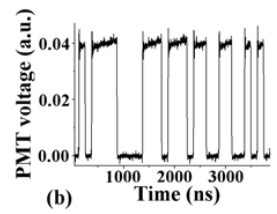

Fig. 4. optical output signal from an individual micro-LED/CMOS pixel driven by a) $8.015 \mathrm{MHz}$ square-wave and b) LFSR signal.

The CMOS device further allows sub-nanosecond optical pulses to be generated from the micro-LEDs. The duration of these pulses is defined by the user, with possible pulse durations set from 300 ps to 40 ns FWHM. We note that the former figure corresponds closely to the minimum pulse duration reported thus far from GaN micro-LEDs [13]. Fig. 5 illustrates the shortest and longest duration optical pulses obtained from a single pixel.
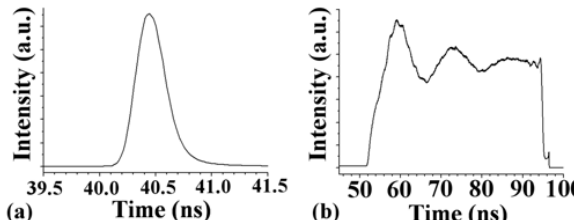

Fig. 5. TCSPC histograms illustrating a) $300 \mathrm{ps}$ and b) $40 \mathrm{~ns}$ optical pulse from a micro-LED pixel.

The energy per pulse is approximately $2.7 \mathrm{fJ}$ and $17.2 \mathrm{pJ}$ for the shortest and longest duration optical pulse, respectively. These values correspond to peak output power densities of 2.21 and $10.56 \mathrm{~W} / \mathrm{cm}^{2}$. These pulses were measured by the time-correlated single photon counting (TCSPC) method, utilizing a PMT and a TCSPC timing module (Becker and Hickl, SPC-730). We previously reported time-resolved lifetime measurements of colloidal quantum dot samples using $\sim 10$ nanosecond timescale pulses from CMOS-controlled micro-LED devices [7]. With sub-nanosecond optical pulses, this opens up the possibility of performing similar timeresolved measurements with more conventional fluorescent samples, with shorter lifetimes in the few nanosecond or subnanosecond regimes.

\section{CONCLUSION}

A UV-emitting AlInGaN $8 \times 8$ micro-LED array interfaced to a dedicated and multi-functional CMOS control circuit has been demonstrated. DC output powers of up to $570 \mu \mathrm{W}$ at 370 $\mathrm{nm}$ were obtained from individual $72 \mu \mathrm{m}$ diameter pixels. Onchip modulation of the micro-LED optical output has been demonstrated with square-wave and pseudo-random electrical signals, and optical pulses of as short as 300 ps FWHM have also been achieved. The performance of this integrated optoelectronic device demonstrates its potential in applications including time-resolved measurements of nanosecond and sub-nanosecond fluorescent samples, and as a transmitter for optical data communications. Such devices can be implemented at any center wavelength over at least the $370-$ $540 \mathrm{~nm}$ range. Future plans include a more detailed characterization of this device, and demonstrations of the practical applications. Nitride-based micro-LEDs at other peak emission wavelengths will also be integrated with this CMOS control device.

\section{REFERENCES}

[1] H. X. Jiang, S. X. Jin, J. Li, J. Shakya, and J. Y. Lin, "III-nitride blue microdisplays," Applied Physics Letters, vol. 78, pp. 1303-1305, 2001

[2] M. D. Dawson and M. A. Neil, "Cluster issue on Micro-pixellated LED' for science and instrumentation," Journal of Physics D: Applied Physics, vol. 41, 2008

[3] C. W. Jeon, E. Gu, and M. D. Dawson, "Mask-free photolithographic exposure using a matrix-addressable micropixellated AlInGaN ultraviole light-emitting diode," Applied Physics Letters, vol. 86, pp. 221105, 2005.

[4] V. Poher, H. X. Zhang, G. T. Kennedy, C. Griffin, S. Oddos, E. Gu, D. S. Elson, J.M. Girkin, P. M. W. French, M. D. Dawson, and M. A. Neil, "Optical sectioning microscopes with no moving parts using a micro-

5] H. Xu, K. M. Davitt, W. Dong, Y.-K. Song, W. R. Patterson, C. D.

H. Xu, K. M. Davitt, W. Dong, Y.-K. Song, W. R. Patterson, C. D.
Aizenman, and A. V. Nurmikko, "Combining Multicore Imaging Fibe Aizenman, and A. V. Nurmikko, "Combining Multicore Imaging Fiber
With Matrix Addressable Blue/Green LED Arrays for Spatiotemporal Photonic Excitation at Cellular Level," Selected Topics in Quantum Electronics, IEEE Journal of, vol. 14, pp. 167-170, 2008.
Elition

[6] C. R. Belton, G. Itskos, G. Heliotis, P. N. Stavrinou, P. G. Lagoudakis, J. Lupton, S. Pereira, E. Gu, C. Griffin, B. Guilhabert, I. M. Watson, A. R. Mackintosh, R. A. Pethrick, J. Feldmann, R. Murray, M. D. Dawson, and D. D. C. Bradley, "New light from hybrid inorganic-organic emitters," D. D. C. Bradley, "New light from hybrid inorganic-organic emitter
Journal of Physics D: Applied Physics, vol. 41, pp. 094006, 2008 .

[7] B. R. Rae, C. Griffin, J. McKendry, J. M. Girkin, H. X. Zhang, E. Gu, D. Renshaw, E. Charbon, M. D. Dawson, and R. K. Henderson, "CMOS driven micro-pixel LEDs integrated with single photon avalanche diodes for time resolved fluorescence measurements," Journal of Physics D: Applied Physics, vol. 41, pp. 094011, 2008.

8] Y. Li, W. Parkes, L. I. Haworth, A. A. Stokes, K. R. Muir, P. Li, A. J. Collin, N. G. Hutcheon, R. Henderson, B. Rae, and A. J. Walton, "Anodic Ta2O5 for CMOS compatible low voltage electrowetting-ondielectric device fabrication," Solid-State Electronics, vol. 52, pp. 1382$1387,2008$.

[9] C. W. Jeon, H.-W. Choi, E. Gu, and M. D. Dawson, "High-density matrix-addressable AlInGaN-based 368-nm microarray light-emitting diodes," Photonics Technology Letters, IEEE, vol. 16, pp. 2421-2423, 2004.

[10] H. X. Zhang, D. Massoubre, J. McKendry, Z. Gong, B. Guilhabert, C. Griffin, E. Gu, P. E. Jessop, J. M. Girkin, and M. D. Dawson, "Individually-addressable flip-chip AlInGaN micropixelated light emitting diode arrays with high continuous and nanosecond outpu power," Opt. Express, vol. 16, pp. 9918-9926, 2008.

[11] P. Maaskant, M. Akhter, B. Roycroft, E. O'Carroll, and B. Corbett, "Fabrication of GaN-Based resonant cavity LEDs," Physica Status Solid a-Applied Research,

D. C. O'Brien, G. E. Faulkner, E. B. Zyambo, K. Jim, D. J. Edwards, P. Stavrinou, G. Parry, J. Bellon, M. J. Sibley, V. A. Lalithambika, V. M. oyner, R. J. Samsudin, D. M. Holburn, and R. J. Mears, "Integrated Ouantum Electronics, IEEE Journal of, vol. 11, pp. 173-183, 2005.

[13] J. Shakya, J. Y. Lin, and H. X. Jiang, "Time-resolved electroluminescence studies of III-nitride ultraviolet photonic-crystal light-emitting diodes," Applied Physics Letters, vol. 85, pp. 2104-2106, 2004. 


\section{Hardware implementation and calibration of background noise for an integration-based fluorescence lifetime sensing algorithm}

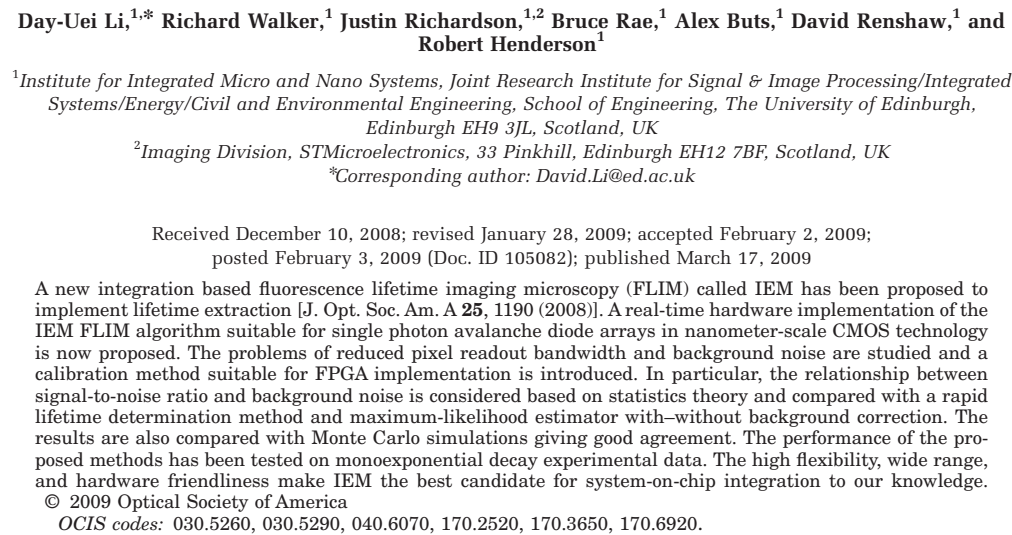

\section{INTRODUCTION}

Fluorescence lifetime imaging (FLIM) is widely used in biology, chemistry, medicine, medical research, and medical diagnosis [1-5]. As shown in Fig. 1(a), a laboratory two-photon microscopy (TPM) FLIM experiment usually contains a titanium-sapphire femtosecond laser system as an excitation source, a photomultiplier tube (PMT) as a fluorescence emission detector, a card for photon counting and as a control system, a PC as a user interface, an fluorescence lifetime analysis software. These existin systems are mainly aimed at research applications and provide excellent time resolution down to tens of picoseconds and excellent light sensitivity [1], although they are lenge is that the available computational methods for generation of lifetime map images such as the iterativ least-square method (LSM) or maximum-likelihood estimation (MLE) [6,7] are very time-consuming, makin real-time imaging impossible. A new FLIM algorithm considering the instrument response based on the Laguerre expansion technique [8] speeds up lifetime calculation but the computation time increases with imager size. However, in many applications such as microfluidic mix ing [3,4], exploratory biological experiments, and clinical diagnosis with endoscopy, it is desirable to monitor the in stantaneous biochemical reactions to provide quick feedback to corresponding manipulations. The slow speed of
LSM-based software analysis tools becomes the bottleneck and has driven the recent development of noniterative, compact, and fast real-time time-domain FLIM systems [9-18] and real-time frequency-domain FLIM algorithms and systems [19-22]. An interesting analog circuit was proposed [17] to calculate lifetimes for singlemolecule microscopy, however, it did not reveal how to remove background noise. In such applications with low fluorescence emission, background-to-signal ratio will be relatively significant.

Commercial applications increasingly demand compact, low-cost, and even portable system-on-chip (SOC) FLIM solutions. Fortunately, high accuracy time resolution can be achieved by exploiting single photon avalanche diode (SPAD) detectors. The feasibility, features, and excellent performance of SPADs in standard complementary metal-oxide-semiconductor (CMOS) technology accompanied by integrated digital readout circuitry [23-28] promise on-chip lifetime extraction and processing. As excitation sources, AlInGaN UV micropixelated light-emitting diodes (micro-LEDs) can be bump-bonded to the digital counters and LED drivers [12] in the general direction of lab-on-chip. In the past, rapid lifetime determination (RLD) methods were thought to be the sim plest algorithms [10], and suitable for real-time applications. A video-rate FLIM was proposed using optomechanical delay control for RLD [14], however, its 


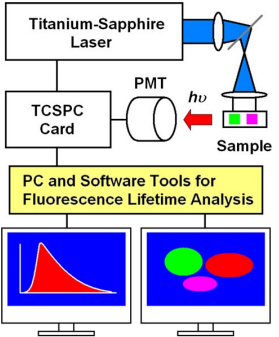

(a)

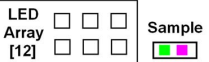

Bump Bond

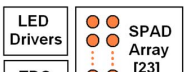

TDC OO ${ }^{[23]}$

Digital Readout

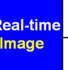

Fast FLIM FPGA

Fig. 1. (Color online) (a) Laboratory FLIM. (b) FLIM system-on-chip.

cumbersome optical setup makes it difficult to image a wide range of fluorophore lifetimes and an electronically controllable delay would be preferable [15]. For this purpose, we evaluated the possibility of applying RLD eithe on-chip or on-FPGA and concluded that RLD can be implemented on FPGA with lookup tables (LUTs) of natural logarithmic or other functions if overlap gating is used [11,13]. However, to build a LUT on-FPGA covering a wide range of lifetimes is inefficient and it is necessary to develop more hardware- or FPGA-friendly algorithms. Moreover, in realistic applications, although video-rat lifetime imagers can provide quick feedback, the raw data must still be processed with software for detailed scientific research. Also, large fluorescence lifetime difference exist in Föster resonance energy transfer (FRET) experimeasure coexisting samples with large lifetime differences. The lifetime range of the imager is therefore from hundreds of picoseconds to tens of nanoseconds. To accommodate these needs, the measurement window is set a several times of the largest lifetime of the samples [with a laser pulse repetition (LPR) rate of several megahertz or a measurement window of hundreds of nanoseconds] [1] It is a challenging task for the standard RLD to meet such requirements, especially when the lifetime is much less than the measurement window. Therefore, to achieve hardware friendliness and wide range of resolvability, a new integration based algorithm called integration for ex traction method (IEM) has been proposed [9] and its performance was successfully verified on single-exponential and multiexponential experimental data obtained by conents. The IEM-based system allows minimum software calculation requirements on both a scanning and a wide-field system. The best way is to make the system selectable and controllable by the end users. The main role of real-time IEM algorithms is for exploratory biological experiments by adapting a wide-field microscope to accommodate the (o scanning mentioned earlier, the fully integrated FLIM system is comparable to a TPM-TCSPC (time-correlated singlephoton counting) system since raw data for precise analysis is also available and light sources and detectors with comparable FWHM are employed. Figure 1(b) shows the

SOC solution suited to lab-on-chip applications, which is intended to replace the system of Fig. 1(a).

The first objective of this paper is to introduce an efficient hardware implementation of IEM [9]. By minimizing the digital circuitry we aim to provide in-pixel or on-FPGA (field programmable gate array) lifetime computation compatible with the increasing numbers of pixels in SPAD arrays (beyond 10,000 in the future).

The second objective is to address the problems of inaccurate lifetime extraction caused by the background noise of the SPADs. The dark count rate (DCR) of the PMT used in the measurement setup of [9] is about $2 \mathrm{kHz}$ at a temperature of $25^{\circ} \mathrm{C}$, which does not have a serious impact on the accuracy of lifetime calculation algorithms since the photon count rate (PCR) at such a single sensor system is much higher than the DCR. Moreover, without using background correction, IEM still provides accurate lifetime calculations. Its insensitivity to the background noise will be explained in Subsection 2.A. Recently pronoise will be explained in Subsection 2 .A. Recently pro-
posed CMOS SPAD structures have reduced the DCR down to hundreds of hertz to several kilohertz at room temperature [24-28] comparable to some state-of-the-art PMTs. However, to achieve parallelism for wide-field microscopy, large arrays of miniaturized SPADs are necessary. It might be argued that increasing light intensity or increasing the LPR rate could improve the ratio of the total photon count causc tive signal count $N_{c}$ ) over that caused by dark count noise (background noise count $N_{b}$ ). This technique is applicable to single-SPAD detection systems with the dead time of a SPAD of tens of nanoseconds, where a DCR of hundreds of kilohertz is perhaps still acceptable. However, as Fig. 2 shows, for modern applications requiring large SPAD arrays, the system becomes readout-bandwidth or framerate limited. Moreover, in FLIM applications, it is necessary to avoid the pileup effect [1], which usually keeps the sary to avoid the pileup effect [1], which usually keeps the
PCR of SPADs much smaller than the readout bandwidth PCR of SPADs much smaller than the readout bandwidth
and this imposes more serious constraints than $3 \mathrm{D}$ ranging applications [23]. On the other hand, there are an increasing number of noisy pixels as the SPAD arrays get larger due to silicon defectivity mechanisms, and which cannot simply be solved by applying pixel interpolationextrapolation method to improve the images due to the requirements of FLIM. In Fig. 2, the frequency margin $\Delta f$ between the DCR of the noisy pixels and the PCR is becoming smaller as SPAD arrays get larger, which makes the ratio of $N_{c}$ over $N_{b}$ decrease. Therefore, in order to in tegrate IEM with such system requirements, the algorithm should include the effect of high background noise.

The importance of developing this theory before th system integration is that we can predict and optimize

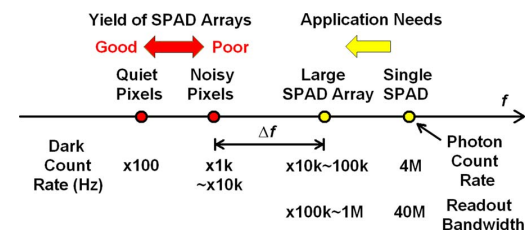

Fig. 2. (Color online) Relationship between DCR and signal readout bandwidth. 
the parameters [29-31], such as the number and width of channels, the depth of memory, and hardware usage according to the required accuracy. In this paper, we start cording to the required accuracy. In this paper, we start
by considering single-exponential decay for simplicity. the single-exponential assumption allows a proper comThe single-exponential assumption allows a proper com-
parison of various fitting algorithms. We first derive the error equations for IEM using Romberg's integration rul to illustrate the impact of background noise. And we also propose a hardware noise calibration technique based on the assumption that be he excitation ligh source. The performance of the proposed methods has been tested on monoexponential experimental data obtained by CMOS SPAD pixels. Because the measurements show that the possibility of after pulsing is extremely low for our CMOS SPADs, the after-pulsing effect is not included in the analysis. Although this paper is mainly motivated by the reduced ratio of the PCR over DCR for larger SPAD arrays, the algorithms can be generalized to include uncorrelated noise caused by ambient light sources.

\section{THEORY}

A. Simpler IEM Formulations for Hardware Implementation

When the FWHM of the instrumental response function (IRF) over the lifetime is much less than 1 , we can assume the fluorescence decay function $f(t)=A \exp (-t / \tau)$ with $\tau$ being the lifetime. For the usual measurement setup in a laboratory, the FWHM is of the order of hundreds of picoseconds, it is reasonable if we target a lifetime larger than 500 ps. Figure 3(a) shows $M$ time bin (bin width of $h$ ) generated by the time-to-digital converters (TDCs) in the photon counting circuitry and also the

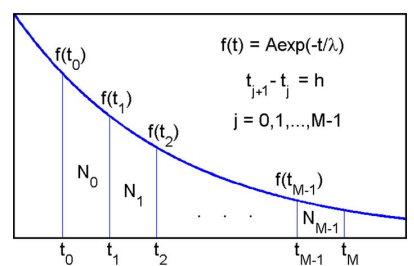

(a)

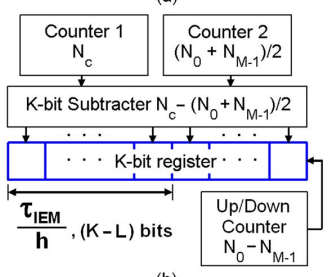

(b)

Fig. 3. (Color online) (a) Single-exponential decay and concept of IEM and (b) hardware implementation of IEM. fluorescence histogram. With the assumption of singleexponential decay, the lifetime $\tau$ is related to the decay function as

$$
\tau\left(f_{0}-f_{M-1}\right)=\int_{t_{0}}^{t_{M-1}} f(t) \mathrm{d} t \cong h \sum_{j=0}^{M-1} C_{j} f_{j},
$$

where $f_{j}=f\left(t_{j}\right), j=0, \ldots, M-1$, and Romberg's integration coefficient $\mathbf{C}_{j}=[1 / 2,1, \ldots, 1,1 / 2]$ is used. Multiply Eq. (1) on both sides by the factor $\left(1-e^{-h / \tau}\right)$ to obtain

$$
\begin{aligned}
\frac{\tau_{\text {IEM }}}{h}= & \frac{\sum_{j=0}^{M-1} C_{j} N_{j}}{N_{0}-N_{M-1}}=\frac{\sum_{j=0}^{M-1} N_{j}-\left(N_{0}+N_{M-1}\right) / 2}{N_{0}-N_{M-1}} \\
= & \frac{N_{c}-\left(N_{0}+N_{M-1}\right) / 2}{N_{0}-N_{M-1}},
\end{aligned}
$$

where $N_{j}$ is the number of counts in the $j$ th time bin and $N_{c}$ is the total effective signal count. To implement Eq. (2) with hardware, we only need two counters (one for $N_{c}$ and the other for $N_{0}+N_{M-1}$ ) and one subtracter for the numerator and one up-down counter for the denominator. The hardware implementation is shown as Fig. 3(b). In Fig. 3(b), a $K$-bit register is used to latch the results from the subtracter when the up-down counter reaches a value of

$$
N_{0}-N_{M-1}=2^{L},
$$

where $L$ is an integer. By this arrangement, we do not even need digital division by only taking the first $(K-L)$ most significant bit (MSB) bits of the register or more than $(K-L)$ MSB bits for decimal accuracy. For low counts, the histogram is almost white and $N_{0} \sim N_{M-1}$, and this condition is easily avoided in hardware implementation. For example, the user can set a photon count rate threshold $\mathrm{PCR}_{\text {th }}$ and when PCR is less than $\mathrm{PCR}_{\mathrm{th}}$, the system will display a black pixel on the screen. Compared with Eq. (6) in [9], which we rewrite as

$$
\frac{\tau_{\text {IEM }}}{h}=\frac{\left[\left(N_{0}+N_{M-1}\right)+4 \sum_{i=1}^{(M-1) / 2} N_{2 i-1}+2 \sum_{i=1}^{(M-3) / 2} N_{2 i}\right]}{3\left(N_{0}-N_{M-1}\right)},
$$

where $M$ is odd, Eq. (2) is much easier to implement in pixel or on-FPGA, because fewer counters are needed and we do not need to use so many logic gates by locating photon counts in the second and third terms of Eq. (4) and doing the division-by-3. Therefore the hardware and chip area can be greatly reduced. The hardware implementation shown in Fig. 3(b) is much simpler than previously proposed video-rat ing data can be compressed in the counters by Eq. (2), the input/output (IO) data rate is more relaxed. Even if divisions in Eq. (2) are done on software rather than on hardware with Eq. (3), IEM is much easier than available realtime algorithms [18-22] 
B. Impact of Background Noise on IEM

In applying large advanced CMOS SPAD arrays, the smaller ratio of the PCR over DCR increases the bias of the lifetime estimators if correction measures are not taken. According to the central limit theory, the spread can be reduced as the number of photons increases, but the bias can never be reduced in the same way. Figure 4 verifies this conclusion and shows how the dark count influences the precision $\sigma \tau / \tau$ and accuracy $\Delta \tau / \tau$ of the MLE $[29,30]$ by the theory with a solid curve and Monte Carlo simulation with open wircs and cross. The total effective signal counts and total background noise counts are $N_{c}=2^{17}$ and $N_{b}=10^{4}$, respectively. If $N_{b}$ is zero, $\Delta \tau / \tau=0$ for $N_{c}=2^{17}$ and $N_{b}=10^{4}$, respectively. If $N_{b}$ is zero, $\Delta \tau / \tau=0$ for
MLE. As $N_{b}=10^{4}, 7 \%$ of total count, $\Delta \tau / \tau$ degrades significantly to less than $22 \mathrm{~dB}$, and $\sigma \tau / \tau$ degrades as well. Now we increase the memory space and store more photon counts $N_{c}=8 \times 2^{17}$ and $N_{b}=8 \times 10^{4}$; the precision is indeed improved by $9 \mathrm{~dB}$ with the accuracy curve still fixed.

Using IEM we assume that the timing jitter of the TDCs with phase-locked loops is negligible [9]. With white dark count noise (assumed as an independent Poisson process from the time-correlated signal), the fluorescence decay should be modified as $f(t)=A \exp (-t / \tau)+N_{b} /(M h)$, $0 \leqslant t \leqslant M h$, and $A=N_{c} /\left[\tau\left(1-e^{-M h / \tau}\right)\right]$. Now the second term of $f(t)$ becomes parasitic such that we cannot separate it from the pure exponential function. The recorded variables $N_{j}$ are independently Poisson distributed with a respective mean value $E N_{j}=\int_{j h}^{(j+1) h} f(t) \mathrm{d} t$ and standard deviation $\sigma N_{j}=\left(E N_{j}\right)^{1 / 2}$, and we thus have

$$
E N_{j}=N_{c} x^{j}(1-x)\left(1-x^{M}\right)^{-1}+N_{b} M^{-1}=\sigma N_{j}^{2},
$$

where $x=\exp (-h / \tau)$. From Eq. (2), we have

$$
\begin{aligned}
\frac{\tau_{\text {IEM }}}{h}= & \frac{\sum_{j=0}^{M-1} C_{j} E N_{j}+\sum_{j=0}^{M-1} C_{j} \sigma N_{j}}{E N_{0}-E N_{M-1}+\sigma N_{0}-\sigma N_{M-1}}=\frac{\bar{U}+\sigma u}{V+\sigma v} \\
= & \frac{\bar{U}(1+\sigma u / \bar{U})}{V(1+\sigma v / V)} \cong \frac{\bar{U}(1+\sigma u / \bar{U}-\sigma v / V)}{V},
\end{aligned}
$$

where

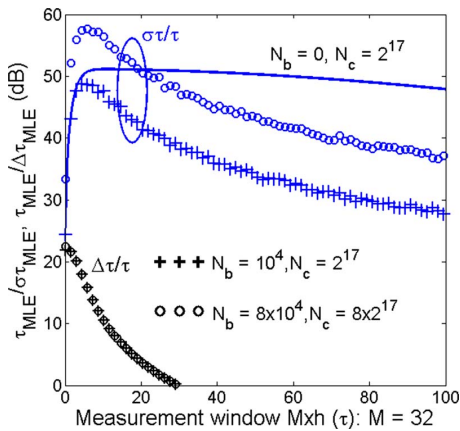

Fig. 4. (Color online) Precision and accuracy curves for the MLE with $\left(N_{c}=2^{17}, \quad N_{b}=0\right),\left(N_{c}=2^{17}, \quad N_{b}=10^{4}\right)$, and $\left(N_{c}=8 \times 2^{17}\right.$ $\left.N_{b}=8 \times 10^{4}\right)$, respectively.
$\bar{U}=\sum_{j=0}^{M-1} C_{j} E N_{j}=\frac{N_{c}}{2} \frac{(1+x)\left(1-x^{M-1}\right)}{1-x^{M}}+\frac{N_{b}(M-1)}{M}=U+B$,

$\sigma u=\sum_{j=0}^{M-1} C_{j} \sigma N_{j}$,

$V=E N_{0}-E N_{M-1}=\frac{N_{c}(1-x)\left(1-x^{M-1}\right)}{1-x^{M}}$,

$\sigma v=\sigma N_{0}-\sigma N_{M-1}$,

and $\quad U=N_{c}(1+x)\left(1-x^{M-1}\right) /\left[2\left(1-x^{M}\right)\right], \quad B=N_{b}(M-1) / M$. Equation (6) can therefore be rewritten as

$$
\begin{aligned}
\tau_{\text {IEM }}= & \frac{h(1+x)}{2(1-x)}\left(1+\frac{B}{U}\right)\left(1+\frac{\sigma u}{\bar{U}}-\frac{\sigma v}{V}\right) \\
= & \tau\left[1+\frac{1}{12} \alpha^{2}+O\left(\alpha^{4}\right)\right]\left(1+\frac{B}{U}\right)\left(1+\frac{\sigma u}{\bar{U}}-\frac{\sigma v}{V}\right) \\
\cong & \tau\left[1+\frac{1}{12} \alpha^{2}+\frac{B}{U}\left(1+\frac{1}{12} \alpha^{2}\right)+\left(1+\frac{1}{12} \alpha^{2}\right)\right. \\
& \left.\times\left(1+\frac{B}{U}\right)\left(\frac{\sigma u}{\bar{U}}-\frac{\sigma v}{V}\right)\right]=\tau\left(1+\frac{\Delta \tau}{\tau}+\frac{\sigma \tau}{\tau}\right),
\end{aligned}
$$

where $\alpha=h / \tau$ and the Taylor's series expansion is used on $h(1+x) /[2(1-x)]$. The accuracy and precision of the IEM are defined as

$$
\begin{aligned}
\frac{\Delta \tau_{\text {IEM }}}{\tau_{\text {IEM }}}= & \frac{1}{12} \alpha^{2}+\frac{B}{U}\left(1+\frac{1}{12} \alpha^{2}\right) \\
= & \frac{1}{12}\left(\frac{h}{\tau}\right)^{2}+\frac{N_{b}}{N_{c}} \frac{2(M-1)\left(1-x^{M}\right)}{M(1+x)\left(1-x^{M-1}\right)} \\
& \times\left[1+\frac{1}{12}\left(\frac{h}{\tau}\right)^{2}\right], \\
\frac{\sigma \tau_{\text {IEM }}}{\tau_{\text {IEM }}}= & \left(1+\frac{1}{12} \alpha^{2}\right)\left(1+\frac{B}{U}\right)\left(\frac{\sigma u}{\bar{U}}-\frac{\sigma v}{V}\right) \\
= & {\left[1+\frac{1}{12}\left(\frac{h}{\tau}\right)^{2}\right] } \\
& \times\left[1+\frac{N_{b}}{N_{c}} \frac{2(M-1)\left(1-x^{M}\right)}{M(1+x)\left(1-x^{M-1}\right)}\right] \sigma a,
\end{aligned}
$$

respectively, and 


$$
\begin{aligned}
\sigma a & =\frac{\sigma u}{\bar{U}}-\frac{\sigma v}{V}=\sqrt{\left(\frac{1}{2 \bar{U}}-\frac{1}{V}\right)^{2} \sigma N_{0}^{2}+\left(\frac{1}{\bar{U}}\right)^{2 M-2} \sum_{j=1}^{2 M} \sigma N_{j}^{2}+\left(\frac{1}{2 \bar{U}}+\frac{1}{V}\right)^{2} \sigma N_{M-1}^{2}}, \\
\sum_{j=1}^{M-2} \sigma N_{j}^{2} & =\frac{N_{c}(1-x)}{1-x^{M}}\left(x+x^{2}+\cdots+x^{M-2}\right)+\frac{M-2}{M} N_{b}=\frac{N_{c}\left(x-x^{M-1}\right)}{1-x^{M}}+\frac{N_{b}(M-2)}{M} .
\end{aligned}
$$

The precision of the IEM can easily be obtained by combining Eqs. (5), (7), and (11). It is clear that the accuracy of Eq. (9) is a function of the ratio $N_{b} / N_{c}$, therefore it cannot be improved by simply increasing the laser intensity or measurement time. And the precision of Eq. (10) is no longer simply proportional to the square root of $N_{c}^{-1}$. It is a function of $N_{c}$ and $N_{b} / N_{c}$, and it deteriorates as $N_{b}$ gets larger. If the ratio $N_{b} / N_{c}$ is fixed, then the precision larger. If the races and precision curves versus measurement window $(M h)$ in for the 128-bin IEM and two-gate RLD (with a gate width $\left.w_{g}=64 h\right)$ at $\left(N_{c}=2^{17}, N_{b}=10^{2}\right)$ and $\left(N_{c}=2^{17}, N_{b}=10^{4}\right)$, respectively. The theoretical results marked as solid curve are compared with Monte Carlo simulations marked with open circles (IEM) and triangles (RLD-2), giving good agreement and proving the correctness of Eqs. (9) and (10). It is clear that the accuracy curves are more sensitive to the background noise. Here we define a new precision value for SNR plots as

$$
\text { Precision } \equiv \frac{\tau}{\sqrt{\sigma \tau^{2}+\Delta \tau^{2}}} .
$$

It is convenient to determine the optimal parameters with this definition of precision. The optimal points for the 128bin IEM and two-gate RLD precision curves are located at $h=0.2 \tau$ and $w_{g}=2.5 \tau$, respectively, at $N_{b} \sim 0$. The signalto-noise ratio (SNR) for RLD is better than IEM in the range of $M h / \tau<10$, while IEM dominates in the range of $M h / \tau>10$. Considering the limitation of pileup effects to the system, in which we have at most one photon count per pixel for ten frames with frame rate $(F R=1 \mathrm{MHz})$, or $\mathrm{PCR}=100 \mathrm{kHz}$, and if a conservative $\mathrm{DCR}=20 \mathrm{kHz}$ is taken, $N_{c}$ can be obtained as

$$
\begin{aligned}
N_{c} & =(P C R-D C R) T_{M} \\
& =T_{M} P C R-N_{b} \Rightarrow \frac{N_{b}}{N_{c}}=\frac{D C R}{P C R-D C R}=0.25,
\end{aligned}
$$

where $T_{M}$ is the measurement time, which can be easily obtained by implementing a counter in the photon counting module, $T_{M}=N_{F} / F R$, and $N_{F}$ is the number of frame this counter accumulates. For larger arrays with much more than 16 pixels per column and the same DCR, $N_{b} / N_{c}$ becomes even larger. With $N_{b} / N_{c}$ larger than $25 \%$, the accuracy is much smaller than $22 \mathrm{~dB}$. If we take $N_{b} / N_{c}=0.25$, and the number of bits of the register used to store the total count of $\left(N_{c}+N_{b}\right)$ is $17 \mathrm{bits}$, the precision and accuracy curves for the 128-bin IEM and 128-bin MLE with $N_{c}=0.8 \times 2^{17}$ and $N_{b}=0.2 \times 2^{17}$ are shown in Figs. 6(a) and 6(b), respectively. In this case, the accuracy is down to less than $12 \mathrm{~dB}$. Although the MLE has the best resolvability for a wide range of lifetimes in cases of $N_{b}=0$, its Monte Carlo simulations show more sensitivity than the IEM to the background noise. Precision and accuracy curves of IEM and MLE for $N_{c}=0.99 \times 2^{17}$ and $N_{b}=0.01 \times 2^{17}$ are also shown in Fig. 6(b). For practical cases with nonzero $N_{b}$, the integration based IEM shows

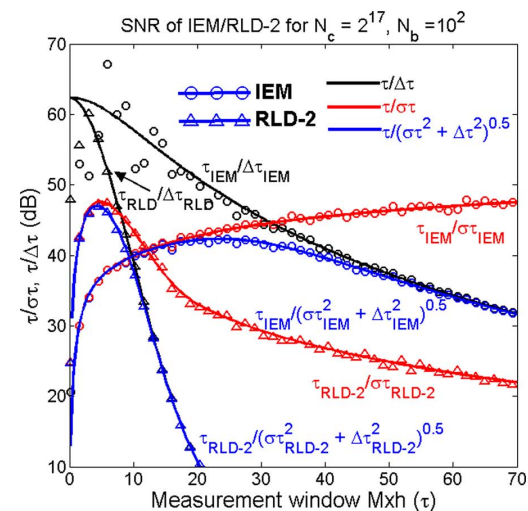

(a)

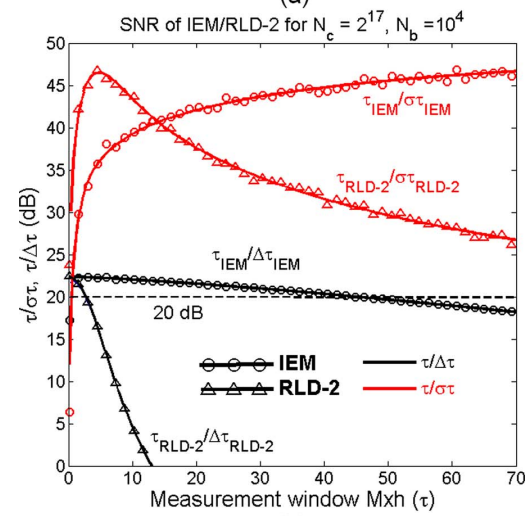

(b)

Fig. 5. (Color online) Precision and accuracy curves for the 128 bin IEM and two-gate RLD with (a) $N_{c}=2^{17}, N_{b}=10^{2}$, and (b) $N_{c}=2^{17}, N_{b}=10^{4}$. 


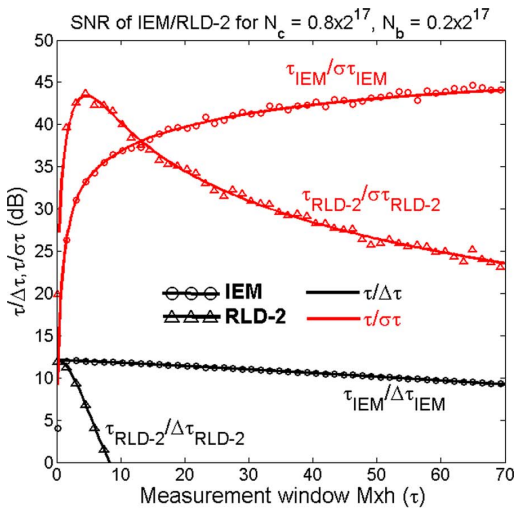

(a)

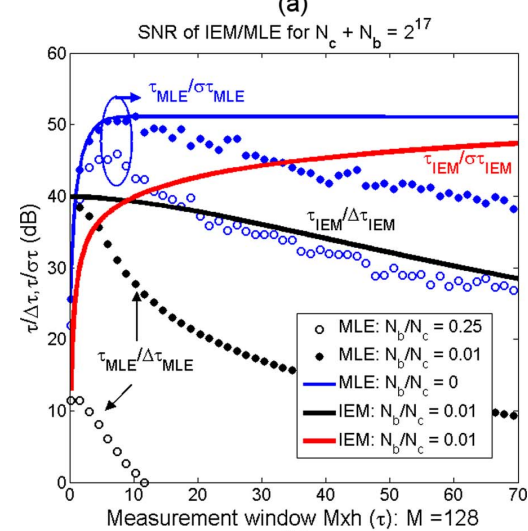

(b)

Fig. 6. (Color online) Precision and accuracy curves for the (a) 128-bin IEM and two-gate RLD and (b) 128-bin IEM and 128-bin MLE with different $N_{b} / N_{c}$ ratios under $N_{c}+N_{b}=2^{17}$.

its superiority over RLD and MLE in terms of accuracy. The other advantages of IEM are: it does not need iterative root calculations of $M$-order polynomials as MLEs tive root calculations of $M$-order polynomials as MLEs
and its hardware implementation in-pixel or on-FPGA is much easier than RLD.

\section{Hardware Calibration}

In most practical lifetime analysis methods background is treated as another fitting parameter or taken into account by subtraction of a DC background value $C_{0}\left(=N_{b} / M\right)$ from the data, and these are easy to do by software. However, for faster real-time imaging, it is desirable that a hardware calibration technique can be integrated into the system. It makes more sense to subtract the background by generating the required $C_{0}$ through available counts than to treat background as a fitting parameter. From the discussion above, the accuracy of algorithms is the dominating factor in cases with low $N_{c}$ to $N_{b}$ ratio. And since the accuracy depends on the mean value instead of the fluctuations of photon counts, we can make use of a backfluctuations of photon counts, we can make use of a back-
ground count collector to remove the unwanted term $N_{b}(M-1) / M$ in the numerator of Eq. (7a). This is equivalent to subtracting $C_{0}$ from the recorded count on each bin. Assume that the temperature of the system is controlled such that the dark count rate is at a fixed level. Similarly to the calibration phase of analog-to-digital conSict verters (ADCs) before the ing, and the result $N_{b 0}(M-1) / M$ is stored in the $K$-bit register, where $N_{b 0}$ is the total noise count during background noise measurement. As shown in Fig. 7, we use a new counter to calculate the measurement time $T_{M 0}$, and the measured information $N_{b 0}$ and $T_{M 0}$ are stored in a memory table. In the lifetime calculation phase, when the up-down counter storing $N_{0}-N_{M-1}$ reaches a value of $2^{L}$ it sends a trigger signal to stop the elapsed time measurement counter storing $N_{F}$. The output of this counter is then connected to the memory table to output a noise count for calibration. A $K$-bit subtracter is used to smooth the noise count, and as before the first $(K-L)$ bits are the calculated lifetime. Without knowing the exact DCR, the mean value term $\bar{U}$ of Eq. (7a) can still be calibrated as

$$
\begin{aligned}
N_{b 0}= & T_{M 0} D C R=N_{F 0} D C R / F R \\
\Rightarrow & N_{b}=N_{b 0} T_{M} / T_{M 0}=N_{b 0} N_{F} / N_{F 0} \cong N_{b 0}, \\
\bar{U}_{\text {cal }}= & \frac{N_{c}(1+x)\left(1-x^{M-1}\right)}{2}+\frac{N_{b}(M-1)}{M} \\
& -\frac{N_{b 0} N_{F}(M-1)}{M N_{F 0}} \cong U,
\end{aligned}
$$

where $N_{F 0}$ is the number of frames for the background measurement time and $F R$ is the frame rate introduced
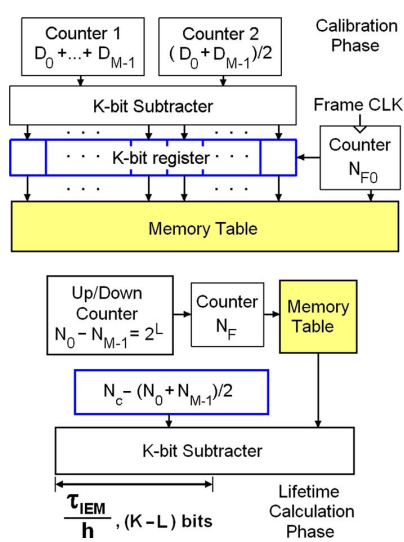

Fig. 7. (Color online) Hardware implementation of background noise calibration. 
earlier. The measured time in normal operation mode $T_{M}$ (or $N_{F}$ ) is compared with $N_{F 0}$ on the memory table and the closest one is chosen. With this arrangement, the accuracy is equivalent to cases without $N_{b}$.

D. Error Analysis of IEM-RLD-MLE with Background Noise Calibration

With only white background noise, the recorded variables $D_{j}$ are still independently Poisson distributed with respective mean value $E D_{j}=\int_{j h}^{(j+1) h} N_{b 0} /(M h) \mathrm{d} t$ and standard deviation $\sigma D_{j}=\left(E D_{j}\right)^{1 / 2}$, and we thus have

$$
E D_{j}=N_{b 0} M^{-1}=\sigma D_{j}^{2}=N_{b} M^{-1} .
$$

By calibrating the mean value term $\bar{U}$ of Eq. (6) and from Eq. (14), we have

$\frac{\tau_{\text {IEM,cal }}}{h}$

$$
=\frac{\sum_{j=0}^{M-1} C_{j} E N_{j}+\sum_{j=0}^{M-1} C_{j} \sigma N_{j}-\left(\sum_{j=0}^{M-1} C_{j} E D_{j}+\sum_{j=0}^{M-1} C_{j} \sigma D_{j}\right)}{E N_{0}-E N_{M-1}+\sigma N_{0}-\sigma N_{M-1}}
$$

$=\frac{U(1+\sigma \bar{u} / U)}{V(1+\sigma v / V)}$,

$$
\sigma \bar{u}=\sum_{j=0}^{M-1} C_{j} \sigma N_{j}-\sum_{j=0}^{M-1} C_{j} \sigma D_{j} .
$$

Comparing Eq. (16) with Eq. (6), and also Eq. (17) with Eq. (7b), it is obvious that we are trading some precision for accuracy since $N_{j}$ and $D_{j}$ are independently Poisson distributed, and

$$
\begin{aligned}
\tau_{\text {IEM,cal }} & =\frac{h(1+x)}{2(1-x)}\left(1+\frac{\sigma \bar{u}}{U}-\frac{\sigma v}{V}\right) \\
& \cong \tau\left[1+\frac{1}{12}\left(\frac{h}{\tau}\right)^{2}\right]\left(1+\frac{\sigma \bar{u}}{U}-\frac{\sigma v}{V}\right) \\
& =\tau\left(1+\frac{\Delta \tau}{\tau}+\frac{\sigma \tau}{\tau}\right) .
\end{aligned}
$$

Therefore, we have

$$
\begin{aligned}
\frac{\Delta \tau_{\text {IEM,cal }}}{\tau_{\text {IEM,cal }}} & =\frac{1}{12}\left(\frac{h}{\tau}\right)^{2}=\left.\frac{\Delta \tau_{\text {IEM }}}{\tau_{\text {IEM }}}\right|_{N_{b}=0}, \\
\frac{\sigma \tau_{\text {IEM,cal }}}{\tau_{\text {IEM,cal }}} & =\left(1+\frac{1}{12} \alpha^{2}\right)\left(\frac{\sigma \bar{u}}{U}-\frac{\sigma v}{V}\right) \\
& =\left[1+\frac{1}{12}\left(\frac{h}{\tau}\right)^{2}\right] \sigma \bar{a},
\end{aligned}
$$

In Eq. (21), if $Q(x)$ dominates, the precision is proportional to $1 / \sqrt{N_{c}}$, whereas if $Z(x)$ dominates, the precision is proportional to $\sqrt{N_{b}} / N_{c}=\sqrt{N_{b} / N_{c}} / \sqrt{N_{c}}=\kappa / \sqrt{N_{c}}$. We can conclude here that under a certain light intensity $(\kappa$ is a fixed value), the precision can be improved $3 \mathrm{~dB}$ by doubling $N_{c}$ as long as the system has enough memory space to store the doubled total count $\left(N_{c}+N_{b}\right)$.

The same hardware background calibration mechanism can also be applied to RLD if it is implemented onFPGA. Neglecting the details of derivation for simplicity, the precision equation is as

$$
\frac{\sigma \tau_{\mathrm{RLD}, \mathrm{cal}}}{\tau_{\mathrm{RLD}, \mathrm{cal}}}=\frac{\tau}{w_{g} \sqrt{N_{c}}} \sqrt{2+y+y^{-1}+\frac{N_{b}}{N_{c}}\left[(1+y)^{2}+\left(1+y^{-1}\right)^{2}\right]},
$$

where $y=\exp \left(-w_{g} / \tau\right)$, and $w_{g}$ is the gate width.

For MLE, also neglecting the details of derivation, the precision equation with the same background calibration can be obtained as

$$
\begin{aligned}
& \frac{\sigma \tau_{\text {MLE,cal }}}{\tau_{\text {MLE,cal }}} \\
& =\frac{\tau}{h \sqrt{N_{c}}} \sqrt{(1-x)^{2}\left(1-x^{M}\right)^{2}\left\{\frac{1}{x G(x)}+\frac{N_{b}}{N_{c} x^{2}[G(x)]^{2}}\right\}}, \\
& \quad x=\exp (-t / \tau), \\
& \quad G(x)=1-M^{2} x^{M-1}+\left(2 M^{2}-2\right) x^{M}-M^{2} x^{M+1}+x^{2 M}, \\
& P(x)=\left[1-(M+1) x^{M}+M x^{M+1}\right]\left[-M+(M+1) x-x^{M+1}\right] \\
& \quad+\frac{(1-x)^{2}\left(1-x^{M}\right)^{2}}{6(M+1)^{-1}(2 M+1)^{-1}} . \\
& \text { From Eqs. }(20)-(23), \text { it can be derived that the SNR of } \\
& \text { IEM is less sensitive to the background noise. From Eq. } \\
& \text { (13), it is possible that } N_{b} / N_{c} \gg 1 \text { for low fluorescence }
\end{aligned}
$$




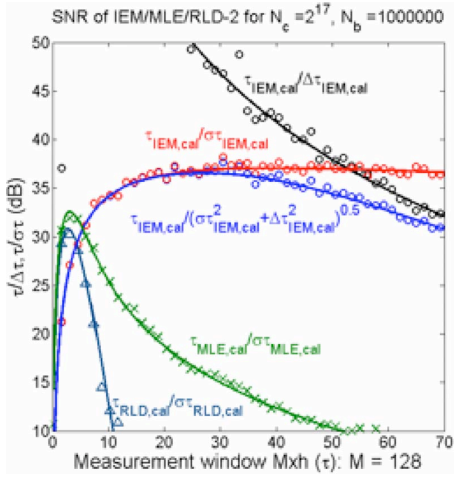

Fig. 8. (Color online) Precision and accuracy curves with background correction for the 128-bin IEM,
gate RLD with $N_{b} / N_{c}=7.63$ and $N_{c}=2^{17}$

emission cases $(P C R \ll 100,000)$. Figure 8 shows the precision and accuracy curves with background calibratio for 128-bin IEM, 128-bin MLE, and two-gate RLD with $N_{c}=2^{17}, N_{b}=10^{6}$. The theoretical results marked as solid curves are compared with Monte Carlo simulations marked with open circles, crosses, and triangles, giving good agreement and proving the correctness of Eqs. (19)-(23). It is clear the accuracy is kept the same with (19)-(23). It is clear the accuracy is kept the same with
cases without background noise. Unlike the cases with cases without background noise. Unlike the cases with
$N_{b} \sim 0$, the peak SNRs of RLD and MLE (with their optimal SNR ranges shrinking) now less than that of IEM indicates that IEM is less sensitive to background noise. For MLE, increasing $M$ does not improve the range of resolvability significantly as in cases of low background noise. It seems this calibration technique can be utilized without limit for IEM, however, to get the same SNR the memory usage and measurement time increase and require much higher laser intensity making the measurement inefficient. A reasonable DCR should be much lowe than the PCR. Without calibration, none of the above algorithms can deal with cases for $N_{b} / N_{c} \gg 1$. For cases with limited hardware resources, we can also use Eqs. (19), and (20) and a simple calibration algorithm introduced in [9] to do the image postprocessing.

\section{EXPERIMENTAL RESULTS}

Measurements of the decay of Catskill Green quantum $\operatorname{dots}(Q$ dots) (with an emission wavelength of $548 \mathrm{~nm}$, from Evident Technologies) mounted on a microcavity slide have been made to test the proposed IEM noise calibration algorithm. The CdSe/ZnS $Q$ dots are held in a solution of toluene with a concentration of $61.3 \mathrm{nmol} / \mathrm{ml}$. The laser pulse rate (LPR) (PicoQuant pulsed diode laser The laser pulse rate (LPR) (PicoQuant pulsed diode laser
with wavelength of $470 \mathrm{~nm}$ ) is $2.5 \mathrm{MHz}$, and the average output power is $0.12 \mathrm{~mW}$. Florescence decay curves were recorded on a time scale of $400 \mathrm{~ns}$, resolved into 4096 channels. With a LPR of $2.5 \mathrm{MHz}$, there is no bleedthrough observed on measured histograms. The fluores- cence emission is captured by a SPAD array fabricated in $0.13 \mu \mathrm{m}$ CMOS imaging process mounted on a daughter board. Figure 9(a) and 9(b) shows two measured histograms detected by two pixels. The one in Fig. 9(a) is close to the average noise level, while that in Fig. 9(b) is obtained by a much noisier pixel. The noisy pixel is deliberately chosen to evaluate how much noise IEM can tolerate. The dark count noise of the SPAD mainly contributes to the noise floor. To calculate the lifetime, the histogram should be corrected by subtracting the background noise. On the FPGA, this is ently done by the method intrOn the rocA, this is eabily done by the method introduced above or simply subtracting an average count $N F$ of $K$ bins on the noise floor (the flat part of the histogram in Fig. 9) since the delay from the first bin to the bin con taining the peak is kept the same through the whole SPAD array. According to the statistics theory, when the number of bins $K$ used to calculate $N F$ is equal to $M$, these two methods are equivalent. When $K$ is much smaller than $M$, the SNR of the calibrated lifetime deteriorates. The worst case is $K=1$, only using the count on a single bin as a reference. Taking the noisier pixel as an example, Figs. 10(a)-10(c) show the calibrated lifetime versus $N F$ for different algorithms with measurement window $M W=17 \mathrm{~ns}(\sim 1 \tau), M W=85 \mathrm{~ns}(\sim 5 \tau)$, and $M W$ $=156 \mathrm{~ns}(\sim 9 \tau)$, respectively. The mean value of $N F$ is 3400 counts, and a range of 20 times the standard deviation is chosen. peak) are chosen to be where the SNR of RLD is higher peak) are chosen to be where the SNR of RLD is higher than that of IEM as shown in Fig. 5(a) to demonstrate
that the higher flexibility and wider range of IEM is indeed much better for system integration. In Fig. 10(a) the measurement window for all algorithms is taken at on lifetime. Without background correction, the calculated lifetimes for IEM, MLE, and RLD are $\tau_{\mathrm{IEM}, 0}=18.0 \mathrm{~ns}$, $\tau_{\mathrm{M}, 0,0}=17.7 \mathrm{~ns}$, and $\tau_{\mathrm{n}}$ $\tau_{\mathrm{MLE}, 0}=17.7 \mathrm{~ns}$, and $\tau_{\mathrm{RLD}, 0}=17.8 \mathrm{~ns}$, respectively. At thi es all algorithm are $0.4 \mathrm{ps}$ per count. At $M W=5 \tau$ (optimal condition for RLD) as in Fig. 10(b), without background calibration, the calculated lifetimes for IEM, MLE, and RLD are $\tau_{\text {IEM, },}$
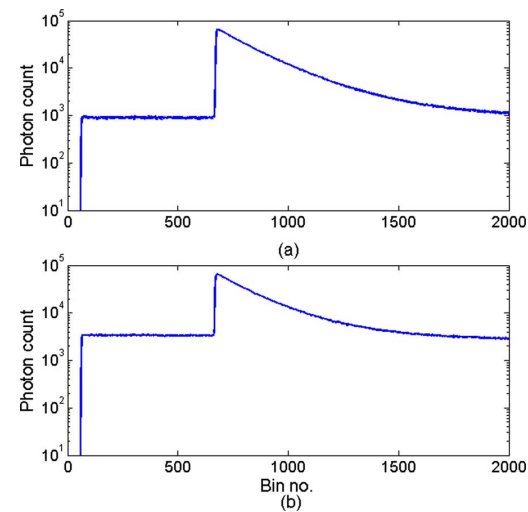

Fig. 9. (Color online) Fluorescence histograms detected by two Fig. 9. (Color
CMOS SPADs. 


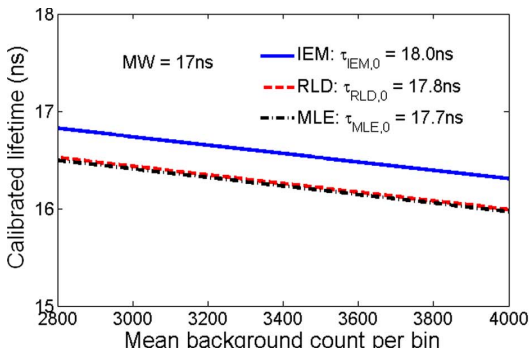

(a)

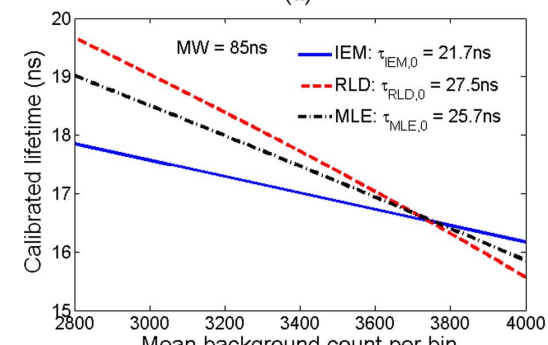

(b)

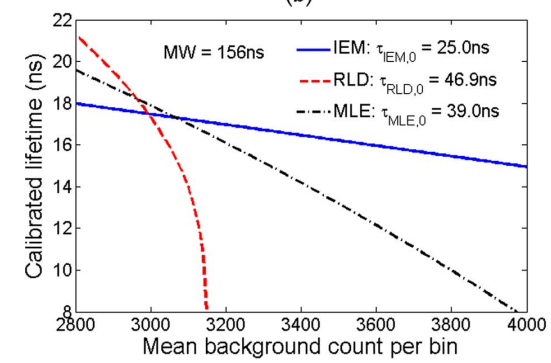

(c)

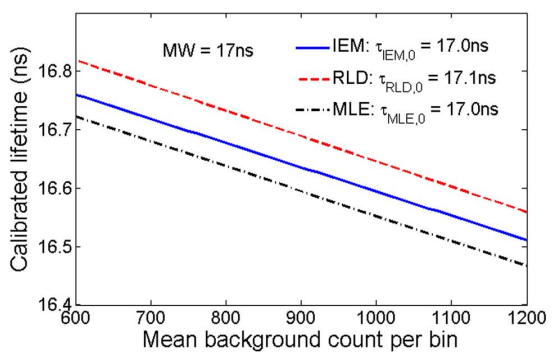

(d)

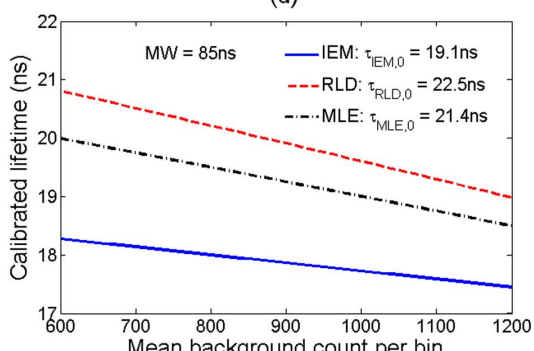

(e)

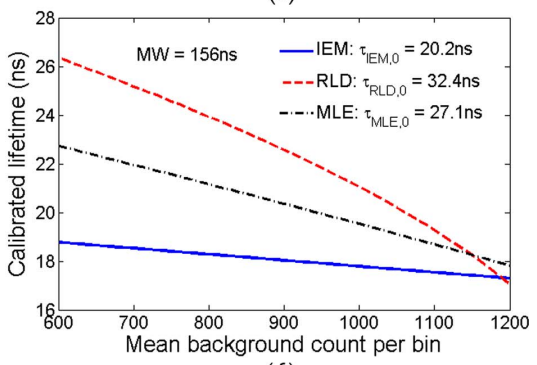

(f)

Fig. 10. (Color online) Calculated lifetimes with background correction versus NF for different algorithms with a mean NF of 3400 and $\mathrm{MW}=$ (a) 17 , (b) 85 , and (c) $156 \mathrm{~ns}$. Calculated lifetimes with background correction versus NF for different algorithms with a mean NF of 900 and $M W=$ (d) 17 , (e) 85 , and (f) $156 \mathrm{~ns}$.

$=21.7 \mathrm{~ns}, \quad \tau_{\mathrm{MLE}, 0}=25.7 \mathrm{~ns}$, and $\tau_{\mathrm{RLD}, 0}=27.5 \mathrm{~ns}$, respec tively. The sensitivities are $1.4,2.6$, and 3.4 ps per count for IEM, MLE, and RLD, respectively. In an exaggerated range of $N F$ (about 20 times the standard deviation around the mean count), the variation of the calibrated lifetime is $8 \%, 15 \%$, and $20 \%$, respectively. This mean that even for a higher-lower $N F$, IEM gets more accurate results, and we can therefore use a smaller er $K$ or a smalle calibrated lifetime of IEM is about $17 \mathrm{~ns}$, which is in a good agreement with the data provided by the manufacturer. At $M W=9 \tau$ as in Fig. 10(c), however, without background correction, the calculated lifetimes are $\tau_{\text {IEM }} 0$ $=25.0 \mathrm{~ns}, \tau_{\mathrm{MLE}, 0}=39.0 \mathrm{~ns}$, and $\tau_{\mathrm{RLD}, 0}=46.9 \mathrm{~ns}$, respec -

tively. The sensitivities are $2.5,10$, and more than $40 \mathrm{ps}$ per count for IEM, MLE, and RLD, respectively. However, in this case, even when a very accurate $N F$ is provided for background correction (around 3400 counts), the calibrated lifetime of RLD is far from the reasonable range. A big error is caused. Figures 10(d)-10(f) show the calibrated lifetimes of the quieter pixel versus $N F$ for different algorithms with measurement window $M W=17 \mathrm{~ns}$ tively. The mean value of $N F$ is 900 counts, and a range of tively. The mean value of $N F$ is 900 counts, and a range of
20 times the standard deviation is chosen. The calculated 20 times the standard deviation is chosen. The calculated
lifetimes without background correction for IEM, MLE, lifetimes without background correction for IEM, MLE,
and RLD are also listed. The calculated lifetime using IEM is also around $17 \mathrm{~ns}$, giving good consistency with 
that obtained by the noisier pixel. Figures 10(a)-10(f) clearly show that with or without background correction for all algorithms, IEM gets much more accurate result as Eqs. (9) and (21)-(23) predict, in good agreement with as Eqs. (9) and (21)-(23) predict, in good agreement with
Figs. 5, 6, and 8, and its range of resolvability is the most insensitive to background noise. More uniform lifetime images can therefore be generated showing the suitability of IEM for widefield imaging.

\section{CONCLUSIONS}

We have modified our previously proposed FLIM algorithm called IEM as Eqs. (2) and (3) for hardware implementation. With the modifications, the hardware resources can be greatly reduced without sacrificing too much precision. Without iteration, IEM offers direct calculation of lifetime and makes real-time imaging feasible. An interesting result of our study is that optimum performance considering both accuracy and precision at low background noise can be obtained at $h=0.2 \tau$. When integrated with large CMOS SPAD arrays with an increasing number of noisy pixels, IEM shows its superior accuracy number of noisy pixels, IEM shows its superior accuracy
over MLE and RLD. Low PCR to DCR ratio renders MLE over MLE and RLD. Low PCR to DCR ratio renders MLE
and RLD inaccurate except in a very limited range of lifetimes. We derived the error equation for IEM with existing background noise and the theoretical results are compared with Monte Carlo simulations, giving good agreement. We also proposed a hardware background noise calibration to correct the accuracy. Its accuracy and noise calibration to correct the accuracy. Its accuracy and
precision equations are also derived and compared with precision equations are also derived and compared with
Monte Carlo simulations. The error equations for RLD and MLE with background correction are also derived fo comparison. The proposed calibration is verified on measured histograms of $Q$ dots using $0.13 \mu \mathrm{m}$ CMOS SPAD arrays and the calculated lifetime is in a good agreement with the data provided by the manufacturer. With background correction for all three algorithms, IEM still ground correction for all three algorithms, IEM still
shows its superior performance over MLE and RLD. The higher flexibility, wider range of resolvability, and hardware friendliness make IEM the best candidate for realtime FLIM system integration so far.

Disclaimer: This publication reflects only the authors' views. The European Community is not liable for any use that may be made of the information contained herein.

\section{ACKNOWLEDGMENTS}

This work has been supported by the European Community within the Sixth Framework Programme of the Information Science Technoogies, Future and Emerging Technologies Open MEGAFRAME project (contract 029217-2, www.megaframe.eu). We acknowledge the support from the Scottish Funding Council for the Joint Research Institute with the Heriot-Watt University, which is a part of the Edinburgh Research Partnership in Engineering and Mathematics (ERPem). The measurements have been performed using the COSMIC laboratory facilities with help from Jochen Arlt, Andy Garrie, Trevor Whittley, an David Dryden. The authors would like to express gratitude to them.

\section{REFERENCES}

1. W. Becker, Advanced Time-Correlated Single Photon Counting Techniques (Springer, 2005

2. P. I. H. Bastiaens and A. Squire, "Fluorescence lifetime imaging microscopy: spatial resolution of biochemica

3. A. D. Elder, S. M. Matthews, J. Swartling, K. Yunus, J.

A. D. Elder, S. M. Matthews, J. Swartling, K. Yunus, J. H
Frank, C. M. Brennan, A. C. Fisher, and C. F. Kaminski, Frank, C. M. Brennan, A. C. Fisher, and C. F. Kaminski,
"Application of frequency-domain fluorescence lifetime imaging microscopy as a quantitative analytical tool for maging microscopy as a quantitative analytical tool for
microfluidic devices," Opt. Express 14, 5456-5467 (2006).

4. D.-A. Mendels, E. M. Graham, S. W. Magennis, A. C. Jones and F. Mendels, "Quantitative comparison of thermal an solutal transport in a T-mixer by FLIM and CFD, Microfluid. Nanofluid. 5, 603-617 (2008)

5. R. K. Neely, D. Daujotyte, S. Grazulis, S. W. Magennis, D T. F. Dryden, S. Klimasauskas, and A. C. Jones, "Timeresolved fluorescence of 2-aminopurine as a probe of base 6953-6960 (2005)

6. A. A. Istratov and O. F. Vyvenko, "Exponential analysis in physical phenomena" Rev, Sci. Instrum. 70, 1233-1257 (1999).

7. S. Pelet, M. J. R. Previte, L. H. Laiho, and P. T. C. So, "A fast global fitting algorithm for fluorescence lifetime imaging microscopy based on image segmentation, Biophys. J. 87, 2807-2817 (2004)

8. J. A. Jo, Q. Fang, and L. Marcu, "Ultrafast method for the analysis of fluorescence lifetime imaging microscopy dat based on the Laguerre expansion technique," IEEE J. Sel. Top. Quantum Electron. 11, 835-845 (2005).

9. D.-U. Li, E. Bonnist, D. Renshaw, and R. Henderson, "On-chip time-correlated fluorescence lifetime extraction 1190-1198 (2008).

10. R. M. Ballew and J. N. Demas, "An error analysis of the rapid lifetime determination method for the evaluation of single exponential decays" Anal. Chem. 61, 30-33 (1989).

11. D.-U. Li, B. Rae, E. Bonnist, D. Renshaw, and R Henderson, "On-chip fluorescence lifetime extraction using synchronous gating scheme-theoretical error analysis and practical implementation," in Proceedings of the International Conference on Bioinspired Systems and
Signal Processing (2008), pp. 171-176.

B. Rae C. Griffin $\mathrm{K}$. M. pr, J. Gistin.

B. Rae, C. Griffin, K. Muir, J. Girkin, E. Gu, D. Renshaw, E. Charbon, M. Dawson, and R. Henderson, "A microsystem for time-resolved fluorescence analysis using Proceedings of the IEEE International Conference on Solid State Circuits (IEEE, 2008), pp. 166-167.

13. C. Moore, S. P. Chan, J. N. Demas, and B. A. Degraff, Comparison of methods for rapid evaluation of lifetime (2004).

14. A. V. Agronskaia, L. Tertoolen, and H. C. Gerritsen, "High frame rate fluorescence lifetime imaging," J. Phys. D $\mathbf{3 6}$

15. D. S. Elson, I. Munro, J. Requejo-Isidro, J. McGinty, C. Dunsby, N. Galletly, G. W. Stamp, M. A. A. Neil, M. J. M. W. French "Real-time time-domain fluorescence lifetime M. W. French, "Real-time time-domain fluorescence lifetim imaging including single-shot acquisition with a segmented
optical image intensifier," New J. Phys. 6, 1-13 (2004).

16. J. Requejo-Isidro, J. McGinty, I. Munro, D. S. Elson, N. P. Galletly, M. J. Lever, M. A. A. Neil, G. W. H. Stamp, P. M. W. French, P. A. Kellett, J. D. Hares, and A. K. L. DymokeBradshaw, "High-speed wide-field time-gated endoscopic fuorescence-lifetime imaging," Opt. Lett. 29, 2249-225

17. W. Trabesinger, C. G. Hübner, B. Hecht, and U. P. Wild, Continuous real-time measurement of fluo
lifetimes," Rev. Sci. Instrum. 73, 3122-3124 (2002).

18. D. Halmer, G. von Basum, P. Hering, and M. Mürtz, "Fast exponential fitting algorithm for real-time instrumental use," Rev. Sci. Instrum. 75, 2187-2191 (2004).

19. H. P. Good, A. J. Kallir, and U. P. Wild, "Comparison of 
fluorescence lifetime fitting techniques," J. Phys. Chem. 88 $5435-5441$ (1984).

and R. M. Clegg, "Rapid acquisition, analysis, and display of fluorescence lifetime-resolve images for real-time applications," Rev. Sci. Instrum. 68, 4107-4119 (1997).

21. J. Mizeret, T. Stepinac, M. Hansroul, A. Studzinski, H. van den Bergh, and G. Wagnières, "Instrumentation for realtime fluorescence lifetime imaging in endoscopy," Rev. Sci.

.

R. A. Colyer, C. Lee, and E. Gratton, "A novel fluorescence Microsc. Res. Tech. 71, 201-213 (2008)

23. C. Niclass, A. Rochas, P. A. Besse, and E. Charbon, "Towar IEEE J. Sel. Top. Quantum Electron. 10, 796-802 (2004).

24. L. Pancheri and D. Stoppa, "Low-noise CMOS singlephoton avalanche diodes with $32 \mathrm{~ns}$ dead time," in Proceedings of the 37th European Solid-State Device Research Confer

25. C. Niclass, M. Gersbach, R. Henderson, L. Grant, and E. Charbon. "A single photon avalanche diode implemented in 130-nm CMOS technology," IEEE J. Sel. Top. Quantum Electron. 13, 863-869 (2007).

26. M. Ghioni, A. Gulinatti, I. Rech, F. Zappa, and S. Cova, "Progress in silicon single-photon avalanche diodes," IEE J. Sel. Top. Quantum Electron. 13, 852-862 (2007).

27. M. Gersbach, C. Niclass, J. Richardson, R. Henderson, L Grant, and E. Charbon, "A single photon detecto implemented in a $130 \mathrm{~nm}$ CMOS imaging process," in
Proceedings of the 38 th European Solid-State Device Research Conference (2008), pp. 270-273.

28. M. A. Marwick and A. G. Anreou, "Single photon avalanche photodetector with integrated " quenching fabricted in TSMC 0.18 m 1.8 V CMOS process," Electron. Lett. 44, 643-644 (2008).

29. M. Köllner and J. Wolfrum, "How many photons are necessary for fluorescence-lifetime measurements?" Chem. Phys. Lett. 200, 199-204 (1992).

30. P. Hall and B. Selinger, "Better estimates of exponentia

decay parameters, J. Phys. Chem. 85, 2941-2946 (1981).

31. J. Philips and K. Carlsson, "Theoretical investigation of th signal-to-noise ratio in fluorescence lifetime imaging," J.
Opt. Soc. Am. A 20, 368-379 (2003). 


\title{
(\$)WILEY Early View publication on www.interscience.wiley.com InterScience ${ }^{\circledR} \quad$ (issue and page numbers not yet assigned; citable using Digital Object Identifier - DOI) \\ Phys. Status Solidi C, 1-4 (2009) / DOI 10.1002/pssc.200880847 \\ Flip-chip, micro-pixellated InGaN

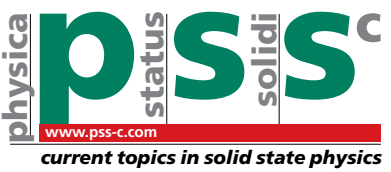 \\ light-emitting diode arrays: attractive sources for micro-displays, colour conversion, and fluorescence detection
}

\author{
Z. Gong ${ }^{*}{ }^{1}$, D. Massoubre', J. McKendry', H. X. Zhang', C. Griffin', B. Guilhabert', E. Gu', J. M. Girkin' \\ M. D. Dawson', B. R. Rael', and R. K. Henderson ${ }^{2}$ \\ 1 Institute of Photonics, University of Strathclyde, Glasgow G4 0NW, UK \\ ${ }_{2}^{2}$ Institute for Integrated Micro and Nano Systems, The School of Electrical and Electronic Engineering, \\ The University of Edinburgh, The King's Buildings, Mayfield Road, Edinburgh, EH9 3JL, UK \\ Received 10 September 2008, revised 16 October 2008, accepted 21 October 2008 \\ Published online 19 January 2009 \\ PACS 81.16.Rf, 85.60.Jb, 85.60.Pg
}

" Corresponding author: e-mail zheng.gong@strath.ac.uk, Phone: +44 1415483447

Flip-chip micro-pixellated InGaN light-emitting diode (micro-LED) arrays fabricated in both matrix-addressable and individually-addressable formats were presented. Despite different addressing schemes, all these LED arrays exhibited superior device operation performance in terms of power output, emission uniformity, and current handling capability, in contrast to the top-emitting counterparts. With the aid of custom-designed driver circuitry, a wide range of promising applications have been demonstrated by using these micro-light sources including micro-display, colour conversion, and fluorescence detection.

2009 WILEY-VCH Verlag GmbH \& CO. KGaA Weinheim

1 Introduction Thanks to their high degree of spatial spectral and temporal control, micro-pixellated GaN-based light-emitting diodes ('micro-LED's) at visible and ultraviolet wavelengths are emerging as promising light sources for various applications including micro-display $[1,2]$ neuron stimulation [3], and fluorescence detection [4], and mask-free lithography [5]. For most of these applications, it is highly desirable that the micro-LEDs can deliver high output power, uniform emission, and are immune from the effect of any tissue, dye, and organic/inorganic structures, which generally need to be brought into proximity with the micro-LEDs. Conventional top-emission micro-LEDs that we have already developed comprise performance by the optical losses in the p-type spreading layer. Flip-chip InGaN LEDs however, where the transparent sapphire substrate is facing up and is inert for most of these substances, should be well suited for the above applications. Here, we report the fabrication and performance of flip-chip micropixellated InGaN LEDs which can be either matrixaddressable or individually-addressable. We also demon-

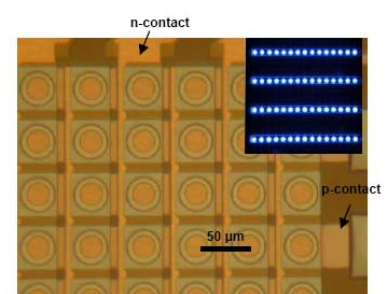

Figure 1 Optical image of the matrix devices taken through the polished sapphire substrate. Inset shows the emission characteristics of several blue LED rows.

strate some promising applications such as micro-displays, colour conversion, and fluorescence detection by using these LEDs as programmable light sources. 


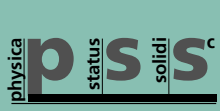

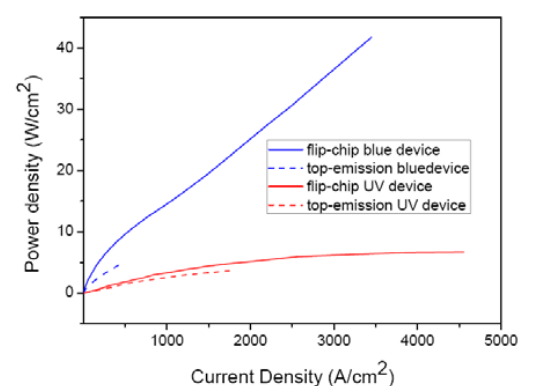

Figure 2 Power output characteristics of one representative pixel in the flip-chip matrix devices emitting at blue and UV wavelengths.

2 Device fabrication and characterization

2.1 Matrix devices The matrix-addressable InGaN LED arrays have 64 x 64 pixels, each with a $20 \mu \mathrm{m}$ emission aperture on a $50 \mu \mathrm{m}$ pitch (Fig. 1). For this device configuration, all pixels in the same row share a common p-contact while all pixels in the same column share a common n-contact. Thus, only 256 electrodes are required for the 64 x 64 LED array, substantially simplifying the fabrication of such a large LED array. The basic fabrication steps include mesa definition, pixel patterning, $\mathrm{SiO}_{2}$ passivation, and $\mathrm{n} / \mathrm{p}$ contact metallization. More fabrication details of the matrix-addressed micro-LED arrays have been reported elsewhere [2]. The devices are then flip-chip bonded onto custom-designed silicon backplanes.

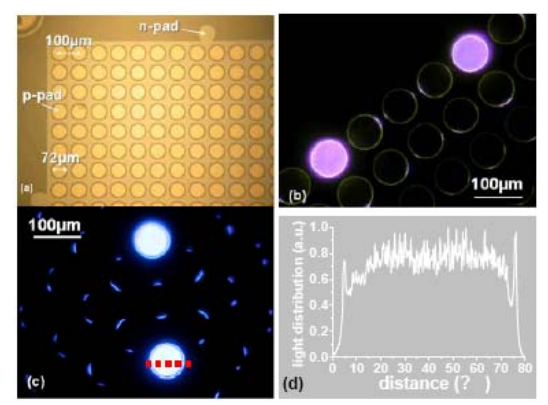

Figure 3 (a) Microscope image of a typical type A device taken through the polished sapphire substrate and showing a portion of the $16 \times 16$ micro-pixel array with two n-pads (on the left and top sides of the image), (b) light emitted by two close UV pixels from a type A device, (c) two operating blue pixel from a type A device, and (d) the light distribution along the dashed line in (c).
The optical power output characteristics of the flipchip matrix device are shown in Fig. 2. Clearly, under the same injection current density, the flip-chip devices can provide a much larger light output than the top-emission ones. For instance, at a current density of $500 \mathrm{~A} / \mathrm{cm}^{2}$, a power density of $10 \mathrm{~W} / \mathrm{cm}^{2}\left(2 \mathrm{~W} / \mathrm{cm}^{2}\right)$ can be achieved from the flip-chip blue (UV) device, 2 (1.5) times as large as that from the top-emission one. It should be noted that all these flip-chip matrix devices can sustain an extremely high injection current density and thus provide a surprisingly large power output, as shown in Fig. 2. For example, the power density of the flip-chip blue (UV) device increases continuously with current densities up to 4000 $\mathrm{A} / \mathrm{cm}^{2}$ before breakdown (power saturation), and reaches a maximum power density of $43 \mathrm{~W} / \mathrm{cm}^{2}\left(6.5 \mathrm{~W} / \mathrm{cm}^{2}\right)$. By contrast, the maximum power density of each pixel in the top-emission LED blue (UV) array is only $5 \mathrm{~W} / \mathrm{cm}^{2}(3.5$ $\mathrm{W} / \mathrm{cm}^{2}$ ), a consequence of a lower maximum current density the device can sustain. We attribute these excellent device performances to the improved thermal dissipation capability after the silicon mount is introduced, and we expect, using an AlN sub-mount instead of the silicon mount, that a further improvement of the power output and current-handling capability can be achieved [9].

2.2 Individually-addressable devices The individually-addressable InGaN micro-disk LED arrays, on the other hand, consist of an array of either $16 \times 16$ pixels (type A) or $32 \times 32$ pixels (type B). The former devices have a pixel diameter (D) of $72 \mu \mathrm{m}$ on a $100 \mu \mathrm{m}$ pitch have a pixel diameter (D) of $72 \mu \mathrm{m}$ on a $100 \mu \mathrm{m}$ pitch
while the latter have a pixel diameter of $30 \mu \mathrm{m}$ on a $60 \mu \mathrm{m}$ pitch. For these devices, all pixels have a common n-
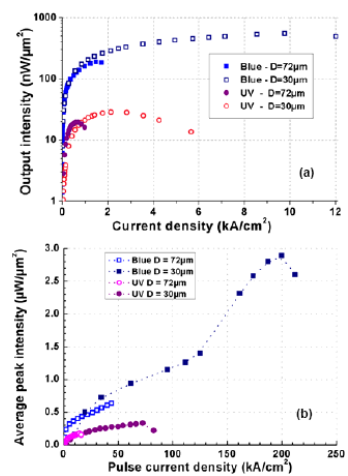

Figure 4 Typical intensities versus continuous injection current densities for blue and UV flip-chip micro-LEDs in type $\mathrm{A}$ and B device formats under (a) DC operation and (b) pulse operation. The repetition rate was fixed at $10.5 \mathrm{kHz}$ with an electrical pulse width of $18 \mathrm{~ns}$. 


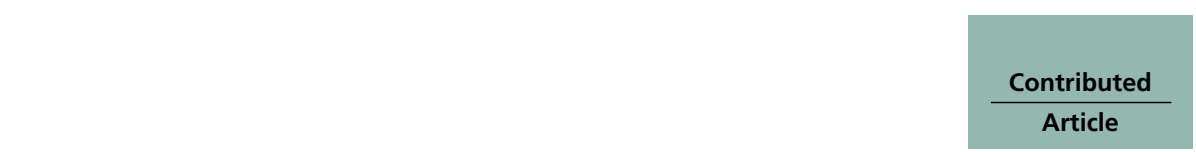

contact whereas each pixel has its own p-contact (Fig. 3(a)). Thus the fabrication of these devices is simpler since the complicated n-bus and p-bus interconnect metal-lines (required for matrix format devices) are not required any more. More detailed fabrication procedures for this format of devices can be found in [6].

Figure 3(a) shows a microphotograph of a portion of a $16 \times 16$ flip-chip micropixellated LED array (type A) with the image taken through the polished sapphire substrate. Two UV and blue operating pixels (type A) are shown on the images of Figs. 3(b) and (c), respectively. All these pictures are from representative devices. From these images, it can be seen that the light emission is uniform, not only within each pixel, but also from pixel to pixel. We note that the pixel sidewalls are angled, to reflect light upwards; light reflected at the pixel sidewalls is evident in the figures [Fig. 3(d)].

Shown in Fig. 4 are the power output characteristics of one representative pixel from the individually-addressable devices, respectively. We note that, whatever the emission wavelength and despite their slightly higher turn-on voltages, the micro-LEDs with reduced size are able to sustain a much higher injection current density before roll-over (power saturation) appears. These results are quite similar to those of the flip-chip matrix device, despite the different addressing scheme. We also noticed that, the power intensity under the pulsed operation is much higher than the one measured in continuous mode due to the use of a low duty cycle $(\sim 0.02 \%)$ and the suppression of thermal effect. Thus, a peak intensity up to $2.9 \mu \mathrm{W} / \mu \mathrm{m}^{2}$ (corresponding to a density of energy of $6.4 \mu \mathrm{J} / \mathrm{cm}^{2}$ ) was obtained for the blue

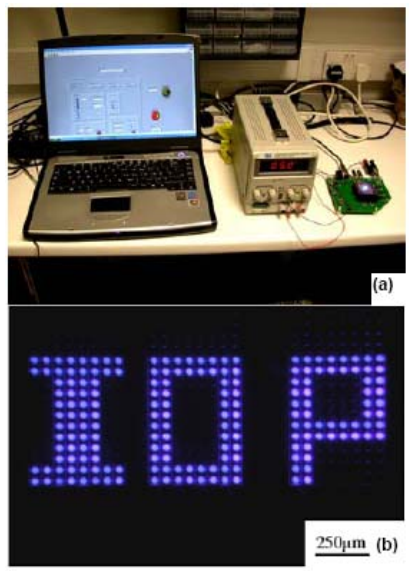

Figure 5 (a) Driver circuit for the matrix LED array, and (b) representative micro-display image generated onto the LED array.

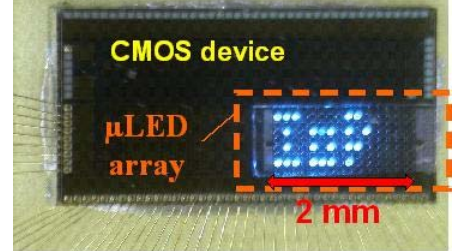

Figure 6 Micro-display image generated onto the individually addressable LED array by CMOS driver.

device B (5-fold higher than in continuous mode) and up to $330 \mathrm{nW} / \mu^{2}$ (corresponding to a density of energy of 570 $\mathrm{nJ} / \mathrm{cm}^{2}$ ) for the UV device B (11-fold higher than in continuous mode). We would also like to emphasize that the type B device has shown the capability to endure extremely high pulsed current densities up to $200 \mathrm{kA} / \mathrm{cm}^{2}$ (blue) and $72 \mathrm{kA} / \mathrm{cm}^{2}$ (UV) before breakdown. Such pulses are powerful enough to be used for 'indirect electrical' pumping of integrated organic semiconductor lasers [7].

\section{Device applications}

3.1 Micro-displays One of the most straightforward applications related to these micro-LEDs is micro-display. For this purpose, we have designed suitable driver circuits for both types of micro-LEDs. The matrix LED arrays are driven in a passive mode (raster scanning) while the individually addressable LED arrays are driven in an active mode using a CMOS circuit. Representative micro-display images generated onto these LEDs by the drivers are shown in Fig. 5, and Fig. 6, respectively. Given the excellent device performance and the pattern-controllable capability of these LED arrays, high-quality micro-displays
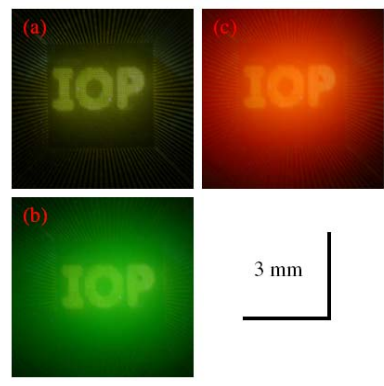

$3 \mathrm{~mm}$

Figure 7 Controllable color-conversion patterns generated by using UV LED arrays combined with a suitable driver to excite the CdSe quantum dots: (a) original UV emission pattern, (b) green conversion pattern, and (c) red conversion pattern. 


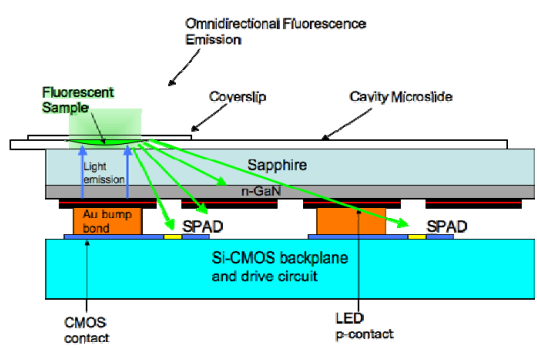

Figure 8 Schematic overview of experimental setup. The transparent sapphire substrate allows the fluorescence emission to pass and reach the single photon avalanche diodes (SPAD) below.

have been obtained from these devices. These controllable emission patterns can be used for a range of other applications, as will be shown later.

3.2 Colour conversion Colour conversion using InGaN LEDs to excite phosphors is another hot topic which has been attracted intensive investigations. These investigations are mainly motivated for white light illumination. Commonly used colour-conversion phosphors however, are not well suited for the micro-LEDs discussed here because of the low conversion efficiency and their large grain size comparable to micro-LEDs. CdSe coreshell quantum dots with a typical size of a few nanometers, on the other hand, should be feasible for this purpose because of their high quantum efficiency and controllable emission wavelength. To illustrate this possibility, here we use our matrix UV LEDs to excite the CdSe quantum dots coated onto the sapphire side of the micro-LED arrays. Noting that our UV LEDs are pattern-controllable (by the driver), more flexible colour conversion patterns have been

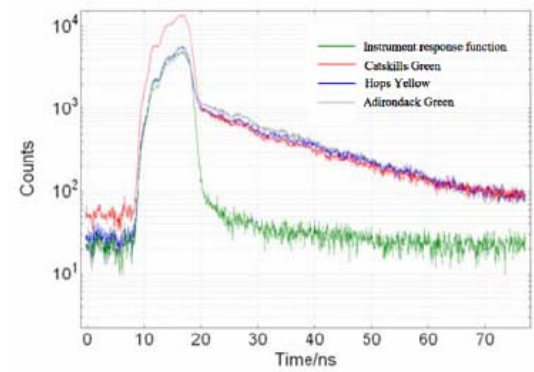

Figure 9 Instrument response function and fluorescent decay curves for quantum dots at three different emission wavelengths. achieved, as demonstrated in Fig. 7. By blending quantum dots with different emission wavelengths together, we are developing full-colour micro-displays.

3.3 On-chip fluorescence detection Still another promising application that we will show here is on-chip time-resolved fluorescence measurement. This is achieved by bonding our individually addressable LED arrays onto a CMOS backplane with integrated single photon avalanche diode (SPAD) arrays [8]. As an illustration, here we use our fluorescence detection system to measure the fluorescence life-time of colloidal CdSe quantum dots. A schematical construction of our fluorescence measurement setup is shown in Fig. 8. A cavity micro-slide containing CdSe quantum dot solution is placed on top of the sapphire side of the LED array. The quantum dots are excited by a pulse train from a single micro-LED. The resulting fluorescence is then collected by the SPAD detector array adjacent to each micro-LED.

Shown in Fig. 9 are the measured fluorescence decay curves of quantum dots at three different emission wavelengths, as well as the instrument response function. We notice that these decay curves are clearly distinguished from the instrument response function (IRF). Discrete deconvolution of the IRF and decay curves in Fig. 7 (by using FAST TM lifetime extraction software by Edinburgh Instruments) yields lifetimes of $17.75 \mathrm{~ns}, 17.23 \mathrm{~ns}$ and 19.09 ns for Adirondack Green, Catskills Green and Hops Yellow quantum dots, respectively. This is in good agreement with the lifetimes quoted by the manufacturer (15-20 ns) and measured independently using a photo-multiplier tube as a detector, indicating the feasibility of our compact fluorescence detection system.

In summary, we have demonstrated the fabrication of high-efficiency micro-pixellated LED arrays in flip-chip formats, which can be matrix-addressable or individuallyaddressable. These devices are then used for a range of applications including micro-display, colour conversion, and fluorescence measurement.

Acknowledgments This work was supported under a RCUK Basic Technology Research Programme and an EPSRC Science and InnovationAward on 'Molecular Nanometrology'.

\section{References}

[1] Z. Gong et al., IEEE Trans. Electron Dev. 54, 2650 (2007)

[2] Z. Gong et al., J. Phys. D: Appl. Phys. 41, 094002 (2008).

[3] V. Poher et al., J. Phys. D: Appl. Phys. 41, 094014 (2008).

[4] B. Guilhabert et al., J. Phys. D: Appl. Phys. 094008 (2008).

[5] C. Jeon et al., Appl. Phys. Lett. 86, 221105 (2005).

[6] H. X. Zhang et al., Opt. Express 16, 9920 (2008).

[7] Y. Yang et al., Appl. Phys. Lett. 92, 163306 (2008).

[8] B. R. Rael et al., J. Phys. D: Appl. Phys. 41, 094011 (2008)

[9] S. Wu et al., Jpn. J. Appl. Phys. 45(12), L352 (2006). 


\title{
ON-CHIP FLUORESCENCE LIFETIME EXTRACTION USING SYNCHRONOUS GATING SCHEME \\ Theoretical Error Analysis and Practical Implementation
}

\author{
Day-Uei Li, Bruce Rae, David Renshaw, Robert Henderson \\ Institute for Integrated Micro and Nano Systems, The University of Edinburgh, The King's Buildings \\ Mayfield Rd. Edinburgh EH9 3JL, UK \\ David.Li@ed.ac.uk \\ Eleanor Bonnist \\ School of Chemistry and the Collaborative Optical SpectroscopyMicromanipulation and Imaging Centre (COSMIC) \\ University of Edinburgh, West Mains Rd., Edinburgh EH9 3JJ, UK \\ e.y.m.bonnist@sms.ed.ac.uk
}

\begin{abstract}
Keywords: Fluorescence lifetime imaging microscopy (FLIM). Error analysis. On-chip implementation. Rapid lifetime determination (RLD). Single photon avalanche diode (SPAD). Time-correlated single-photon counting (TCSPC). Data fitting. Pipe-lined RLD. Instrumental response function (IRF).

Abstract: A synchronous gating technique was proposed for fluorescent photon collecting. The two- and multi-gate rapid lifetime determination (RLD) technique was applied to implement on-chip fluorescence lifetime extraction. Compared with all available iterative least square method (LSM) or maximum likelihood estimation (MLE) based general purpose FLIM analysis software, we offer a method for the direct calculation of lifetime based on the photon counts stored in on-chip memory and deliver faster analysis to enable real-time applications. Theoretical error analysis of the two-gate RLD technique was derived for enable real-time applications. Theoretical error analysis of the two-gate RLD technique was derived for
comparison. The performance of the algorithms were tested on a single-exponential histogram obtained from a CMOS SPAD detector chip using a $468 \mathrm{~nm}$ laser diode light source with optimized gate width. Moreover, a multi-exponential pipelined RLD FLIM technique was also proposed and tested on a fourexponential decay DNA sample containing a single adenine analogue 2-aminopurine.
\end{abstract}

\section{INTRODUCTION}

Fluorescence lifetime measurements have been used widely to study various scientific and practical applications on optics, chemistry, biology, medicine, medical diagnosis. A large number of different techniques including time-domain and frequencydomain methods have been well developed for measuring fluorescence lifetime (Apanasovich and Novikov, 1992). In time-domain methods, the fluorescence intensity decay is measured through a time-correlated single photon-counting (TCSPC) card after excitation with a short pulse of laser light (Cubeddu et al, 2002), whereas in frequency-domain methods, the fluorescent sample is illuminated with a periodic light source to obtain a measured phase difference between the light source and the fluorescent emission. Irrespective of the method used (Jo et al, 2004), the lifetime extraction is done using computer software. For general purpose timedomain analysis tools for scientific research demanding high accuracy down to the picosecond timescale or for practical medical/clinical diagnostic applications demanding fast results, a wide range of faint multi-exponential fluorophores must be computed with a lifetime resolution better than $50 \mathrm{ps}$ (Becker, 2005). Due to the incapability of the LSM or MLE to resolve a small lifetime with a coarse channel width, the number of bits of resolution of TDCs on photon counting cards is therefore expected to be larger than 11-bit (Becker, 2005). To use LSM or MLE properly, the measurement window is usually set as large as possible otherwise the software would treat the measured data as having a DC offset part and therefore the laser pulse repetition rate is kept low, which further lowers the photon collection speed. Data therefore can be gathered in several days. Moreover, because 
fluorescence lifetimes in imaging are determined on a pixel-by-pixel basis, iterative methods can be quite time consuming and make real-time image processing almost impossible. Although one can drop the requirement for short laser pulses by using frequency-domain methods, lifetime extraction still relies on software analysis, which also makes realtime image processing difficult to achieve. As process technology advances, integration of high speed laser drivers and laser diodes on chip is becoming feasible.

\section{THEORETICAL ERROR}

\section{ANALYSIS}

The recorded fluorescence intensity $f(t)$ is related to the true decay function $\mathrm{I}(\mathrm{t})$ through the integral

$$
f(t)=\int_{0}^{t} I(t-\tau) \operatorname{IRF}(\tau) d \tau
$$

where $\operatorname{IRF}(t)$ is the instrumental response function, or the convolution of transition spread of the detector and the pulse function of the laser source. The true response $I(t)$ could be obtained through an on-chip digital de-convolution calculation. However, we need to evaluate whether the enhanced precision can justify the cost of the extra chip area for digital de-convolution. Here we assume $I(t)=A \exp (-t / \tau)$ and the ratio of the full width half maximum (FWHM) of $I R F(t)$ over the lifetime is denoted as $r$. The recorded response $f(t)$ is obtained from (1). As $r$ is larger than 1 , it is difficult to obtain a clea response because of the effects of noise and it is inefficient to accumulate enough photon counts for a certain SNR criteria. The smaller the ratio $r$, the more efficiently and accurately the lifetime can be extracted. Considering the $10 \mathrm{ps}$ jitter in the light source, the $80 \mathrm{ps}$ transition spread of our SPAD structure, and the $30 \mathrm{ps}$ jitter of gate transitions, the overall FWHM is about 100ps. Thus, without onchip de-convolution function, the smallest lifetime that can be obtained is of the order of 200ps. For first time implementation, we simplify by using longer-lifetime samples as test cases. The assumption of $f(t)$ as a single exponential is quite reasonable. In this paper, we applied the RLD method for simplicity.

\subsection{Theory}

The simplest way of calculating fluorescence lifetime is to use the RLD technique with two consecutive gates (Ballew and Demas, 1989) called

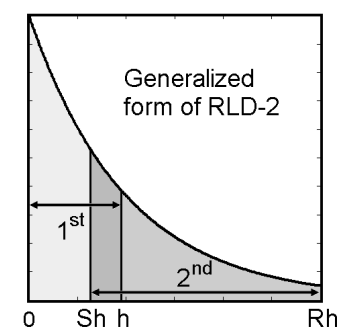

Figure 1: Generalized form of two-gate RLD.

standard RLD. Unlike the LSM or MLE based methods, it is a direct calculation method. The disadvantage of standard RLD is its high sensitivity to the gate width selection. This can be explained by reasoning that when dealing with a short lifetime, the photons are mostly located in the first gate, and the relatively low counts in the second gate becomes the major source of error. To overcome this problem, a gate overlap approach was introduced to the standard RLD (Sharman and Periasamy, 1999) trying to offer greater insensitivity to the Possion noise in the second gate. This method did offer better resolvability for a range of short lifetimes, but it sacrificed precision for the longer lifetimes. Another approach called SWRLD is proposed (Chan et al, 200) using a square wave driven LED as a light source. SWRLD offers uniform high precision in a much wider range of gate width. However, this method does not easily extract lifetimes shorter than $1 \mathrm{~ns}$ because the $1 \mathrm{~ns}$ edge speed of the fastest available LED dictates the minimum lifetime extraction limit, Thus an example of a long lifetime of $2 \mathrm{~ms}$ has been chosen as an illustration. The second challenge is that SWRLD needs many filters to separate fluorophore emission from scattered laser emission (the IRF). Beyond these limits, SWRLD is indeed a precise method for long lifetime extraction ( $>$ 10ns). A better approach to achieve better precision for long lifetimes is make the second gate wider than the first and therefore tolerate much higher counts (Moore et al, 2004). This method, however, needs an iteration method to do lifetime extraction. Plus for on-chip implementation, asymmetric gates require the generation of two synchronized clocks with different pulse widths and thus increase the circuit complexity. The best theoretical solution is not necessarily the right one in terms of cost and feasibility. All the amended algorithms mentioned require Monte Carlo to do error analysis. We derive a generalized formula here 
for calculating the standard deviation of lifetimes much more conveniently and therefore facilitate location of the optimized lifetime region or measurement window. Figure 1 shows the generalized form of two-gate RLD. The counts $N_{1}$ and $N_{2}$ in the two gates are related as

$$
g(x)=N_{2}(1-x)-N_{1}\left(x^{S}-x^{R}\right)=0,
$$

where $x=\exp (-h / \tau)$ and

$$
\begin{aligned}
& N_{1}=N_{c}(1-x) /\left(1-x^{R}\right), \sigma N_{1}=\sqrt{N_{1}}, \\
& N_{2}=N_{c}\left(x^{S}-x^{R}\right) /\left(1-x^{R}\right), \sigma N_{2}=\sqrt{N_{2}} .
\end{aligned}
$$

with $\sigma N_{1}$ and $\sigma N_{2}$ being the standard deviations in $N_{1}$ and $N_{2}$, respectively for Poisson noise and $N_{c}$ the total count number. Together with (2), we have

$$
\begin{gathered}
\sigma g(x)=\sigma x \cdot\left|g^{\prime}(x)\right|, \\
\sigma g=\sqrt{\sigma N_{2}^{2}(1-x)^{2}+\sigma N_{1}^{2}\left(x^{S}-x^{R}\right)^{2}}, \\
\left|g^{\prime}(x)\right|=N_{2}+N_{1}\left(S x^{S-1}-R x^{R-1}\right), \\
\text { and } \sigma x / x=h \sigma \tau / \tau^{2} .
\end{gathered}
$$

From (2) to (7), we could obtain

$$
\begin{aligned}
& \frac{\sigma \tau}{\tau}=\frac{\tau h^{-1} \sqrt{\left(1-x^{R}\right) k(x)} / \sqrt{N_{c}}}{\left[(S-1) x^{S+1}-(R-1) x^{R+1}-S x^{S}+R x^{R}\right]} \\
& k(x)=(1-x)\left(x^{S}-x^{R}\right)^{2}+(1-x)^{2}\left(x^{S}-x^{R}\right) .
\end{aligned}
$$

\subsection{Comparison of RLD-2s and RLD- $N$}

To demonstrate the ability of different RLD schemes, we fix the measurement window (MW). First we compute the standard deviation over the lifetime $\mathrm{SNR}=\sigma \tau / \tau$ in $\mathrm{dB}$. Figure 2 shows the SNR in the range of $\tau / \mathrm{MW}$ within 0.05 and 1 , and gate number $N$ within 2 and 128 under total counts of $2^{17}$. It shows the SNR plot converges as $N>8$ and RLD-2 shows the best resolvability for small lifetime region, but both RLD-2 and RLD- $N$ could not resolve those less than 0.1 . It means that with a laser source of repetition rate of $100 \mathrm{MHz}$, they could not resolve those less than 1ns. Moreover, the complexity of implementing $N$-gate RLD on chip is too large. In terms of implementation, the RLD-2 is much easier than RLD- $N$. Figure 3 shows a comparison plot of lifetime SNR versus lifetime normalized by measurement window (MW) for theoretical equation (8), equation for the multi-gate scheme not shown here, Monte-Carlo RLD methods and the maximum likelihood estimator (MLE) (Kollner and Wolfrum, 1992).

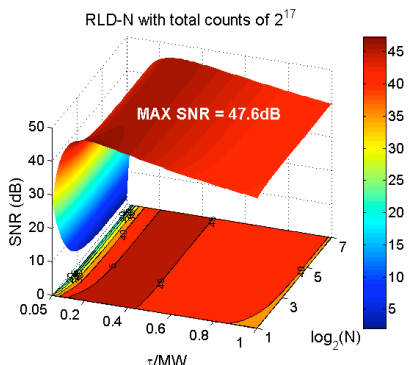

Figure 2: SNR plot for RLD- $N$ under total counts of $2^{17}$.

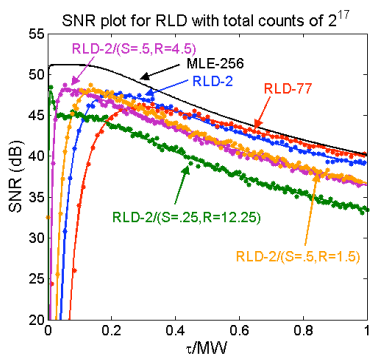

Figure 3: SNR plot for RLD- $N$ with total counts of $2^{17}$

This plot shows the range of lifetime resolvable by each extraction method for a certain laser repetition rate. The results obtained by theoretical equations are marked as solid lines whereas those by MonteCarlo methods are indicated by dots. They match well. We also compare the MLE results and find that the peak value of the RLD-2 occurs at $\tau / \mathrm{MW}=\tau /(2 h)$ $\sim 0.2$ which is coincident with previously reported literature. The plot shows the RLD-77 has best precision and closest to that of MLE only in high lifetime region, while it has the worst resolvability for low lifetime region. Un-equal gate scheme ( $\mathrm{S}=$ $0.5, \mathrm{R}=4.5$ ) offers better resolvability than the equal gate-width one $(\mathrm{S}=0.5, \mathrm{R}=1.5)$. The one with $\mathrm{S}=0.25$ and $\mathrm{R}=12.25$ suggested by (Moore et al, 2004) offers the best resolvability for small lifetime region but sacrifices some precision in the high lifetime region. In terms of implementation complexity, feasibility, and limitations on chip, the equal gate width scheme is the simplest. Of course, if we insist on implementing an un-equal one given 
that we have conquered the problem of transition spread of the IRF, we could build an on-chip lookup table to simplify lifetime extraction. For first time on-chip implementation, we simplify by using the equal gate and non-overlap scheme. The overlap scheme will be implemented in the future. Table 1 lists the summary of RLD schemes. Except the RLD- $N$, the others are all possible candidates.

Table 1: Comparison and summary of RLD schemes.

\begin{tabular}{|c|c|c|c|}
\hline & $\begin{array}{c}\text { Closed } \\
\text { Form }\end{array}$ & $\begin{array}{c}\tau / \text { MW }<0.1 \\
\text { Resolvability }\end{array}$ & $\begin{array}{c}\text { On-chip } \\
\text { Feasibility }\end{array}$ \\
\hline $\begin{array}{c}\text { Standard } \\
\text { RLD-2 }\end{array}$ & Yes & No & $\begin{array}{c}\text { Yes/Look- } \\
\text { up Table }\end{array}$ \\
\hline $\begin{array}{c}\text { Standard } \\
\text { RLD- } N \\
(\mathrm{~N}>2)\end{array}$ & Yes & No & No \\
\hline $\begin{array}{c}\text { Overlap } \\
\text { RLD-2 } \\
(\mathrm{R}=1+\mathrm{S})\end{array}$ & Yes & Yes & $\begin{array}{c}\text { Yes/Look- } \\
\text { up Table }\end{array}$ \\
\hline $\begin{array}{c}\text { Overlap } \\
\text { RLD-2 } \\
(\mathrm{R} \neq 1+\mathrm{S})\end{array}$ & No & Yes & $\begin{array}{c}\text { Yes/Look- } \\
\text { up Table }\end{array}$ \\
\hline
\end{tabular}

\subsection{Synchronous Gating Scheme}

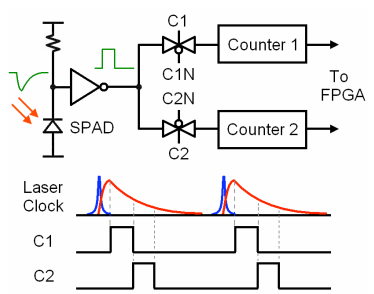

Figure 4: Timing diagram for synchronous gating technique.

Figure 4 shows the block diagram for the photon counting process. The fluorescence emission is detected by a SPAD detector, and the detected signal is converted into a digital one by a comparator and then sent into two synchronous counters controlled by clocks $\mathrm{C} 1$ and $\mathrm{C} 2$, respectively. And the photon counts on counters 1 and 2 are sent to a FPGA for post processing.

\subsection{Pipelined RLD-2 for Multi-Decays}

The above analysis is based on the assumption that the fluorescence emission follows a single-lifetime function. When trying to resolve multi-lifetime fluorescence decay, we need a simple algorithm Figure 5 shows an algorithm for lifetime extraction in a two-lifetime fluorescence histogram similar to the concept of pipelined analog-to-digital converters, called pipelined RLD-2 (PL-RLD-2). The lifetime extraction procedure uses RLD-2 to extract the larger lifetime and intensity with the first memory, and subtraction of the extracted extrapolation function from the photon counts stored in the second memory to obtain the second lifetime and intensity. Pipelined algorithms for higher $(>2)$ decays can follow this procedure until the last lifetime is finally calculated.

\section{EXPERIMENTAL RESULTS}

\subsection{Single-decay}

The chip including a $4 \times 16$ SPAD array and digital readout circuits was implemented on $0.35 \mu \mathrm{m}$ high voltage CMOS process. The die had the polymide passivation removed providing around 3-5x increase in photon detection probability in the $500 \mathrm{~nm}$ range Each pixel contains a single $15 \mu \mathrm{m}$-diameter CMOS SPAD (Niclass, 2006).

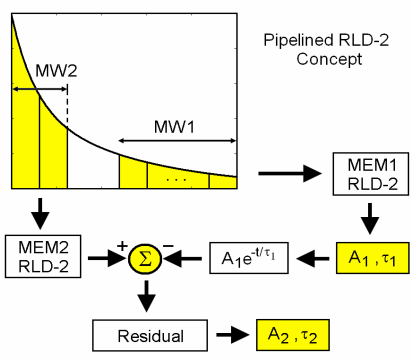

Figure 5: Block diagram of pipelined RLD-2.

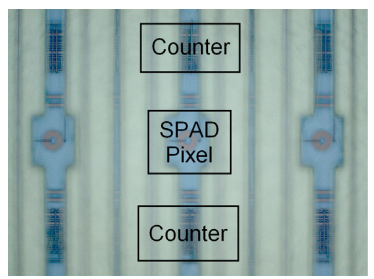

Figure 6: SPAD pixel and two ripple counters. 


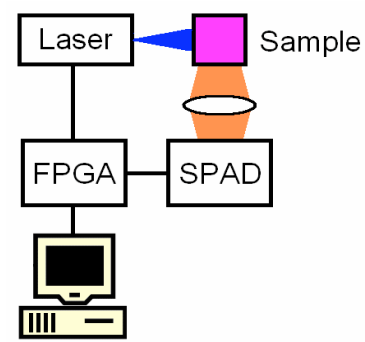

Figure 7: SPAD pixel and two ripple counters.

Figure 6 shows a SPAD pixel with two ripple counters up and down. The gating width could be adjusted over a $48 \mathrm{~ns}$ range with a $408 \mathrm{ps}$ resolution. The imager is controlled by a FPGA and photon count histograms are captured and displayed on a PC. The measurement setup is shown in Figure 7. It consists of a laser diode emitting 88ps pulses at $468 \mathrm{~nm}, 5 \mathrm{~mW}$ average power, synchronized to the system clock. Without using any photon counting card, the photon emitted is converted into a digital signal and processed by on-chip ripple counters in Figure 4. The fluorophore sample is 1 micro-molar Rhodamine B. Table 2 shows the extracted lifetime using the RLD-2 and the LSM based software. The difference between them is about $7 \%$. Jitter performance of the synchronous gate might contribute some error, because a phase-locked loop PLL has not been integrated to minimize the jitter.

Table 2: Comparison of lifetimes extracted by RLD-2 and software.

\begin{tabular}{|c|c|c|}
\hline Sample & RLD-2 & Software \\
\hline Rhodamine B & $2.33 \mathrm{~ns}$ & $2.175 \mathrm{~ns}$ \\
\hline
\end{tabular}

\subsection{Multiple-decays}

The second example is used to test the proposed pipelined RLD-2 algorithm. This data set comes from the fluorophore 2-aminopurine (2AP) inside a singly-labelled 14 base-pair DNA duplex and was measured in an Edinburgh Instruments spectrometer equipped with TCC 900 photon counting electronics (Neely et. al, 2005). The excitation source was a TiSapphire femtosecond laser system producing pulses of $\sim 200 \mathrm{fs}$ at $76 \mathrm{MHz}$ repetition rate. The output of the laser was passed through a pulse picker to reduce the repetition rate to $4.75 \mathrm{MHz}$ and then frequency tripled to give an output at $320 \mathrm{~nm}$. The emission from the sample was collected orthogonal to the excitation direction through a polarizer. The fluorescence was passed through a monochromator, and detected by a Hamamatsu PMT (R3809U-50). The instrument response was 50ps FWHM Florescence decay curves were recorded at emission wavelength of $390 \mathrm{~nm}$ on a timescale of $50 \mathrm{~ns}$, resolved into 4096 channels, to a total 10,000 counts in the peak channel. Decay curves were analyzed using the proposed PL-RLD-2 and using the F900 software with standard iterative reconvolution method, assuming a multi-exponential decay function in the following equation.

$$
I(t)=\sum_{i=1}^{4} A_{i} \exp \left(-\frac{t}{\tau_{i}}\right)
$$
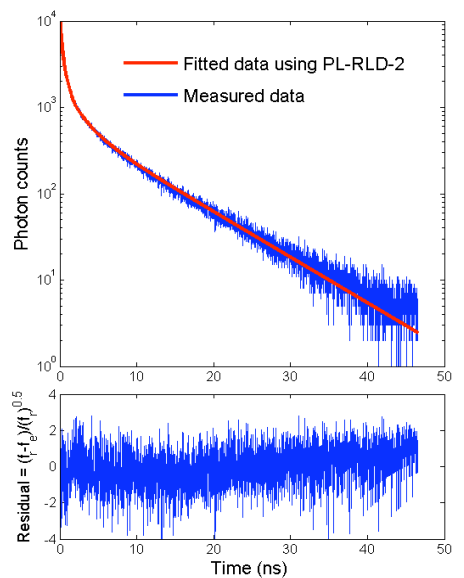

Figure 8: Fitted data and residual using PL-RLD-2.

Table 3: Comparison of lifetimes (ns) and fractional amplitudes (\%) extracted by PL-RLD-2 and F900 software.

\begin{tabular}{|c|c|c|}
\hline$\tau_{i}(\mathrm{~ns}) / A_{i}(\%)$ & PL-RLD-2 & F900 Software \\
\hline$\tau_{1} / A_{1}$ & $0.136 / 27$ & $0.14 / 47$ \\
\hline$\tau_{2} / A_{2}$ & $0.481 / 55$ & $0.47 / 39$ \\
\hline$\tau_{3} / A_{3}$ & $2.179 / 11$ & $2.19 / 9$ \\
\hline$\tau_{4} / A_{4}$ & $8.225 / 7$ & $8.15 / 5$ \\
\hline
\end{tabular}

where $A_{i}$ is the fractional amplitude and $\tau_{i}$ is the fluorescence lifetime of the $i$-th decay component. Figure 8 shows the logarithmic plot for the measured photon counts starting from the channel with peak counts 10,000 and the fitted data using the proposed PL-RLD-2. The residual plot reveals that the proposed method fits well with the experimental data. The extracted lifetimes and fractional 
amplitudes using the PL-RLD-2 and the F900 software are listed in Table 3. The Table shows the extracted lifetimes differ within $4 \%$ whereas the amplitudes differ in a significant range. That is why recent literature (Philip, 2003) suggests that fluorescence lifetime measurements offer better precision. These results highlight the potential of RL-RLD-2 for on-chip multiple exponential lifetime extraction, if adaptive gating width technique could also be introduced on-chip.

\section{CONCLUSIONS}

On-chip fluorescence lifetime extraction including a SPAD array and digital readout circuitry is for the first time implemented on $0.35 \mu \mathrm{m}$ CMOS process using the two-gate RLD. Theoretical error equation for several RLD-2/RLD- $N$ schemes were derived and compared to determine a possible implementation strategy. To implement RLD-2, non-overlap synchronous gating is applied for photon counting. The first on-chip attempt is mainly focused on dealing with single-exponential fluorescence emission, and the extracted result matches with the true value well within $10 \%$ including possible contribution from gating jitter. For possible future on-chip implementation for multi-exponential fluorescence lifetime extraction, we proposed a pipelined RLD-2 (PL-RLD-2) and we test this method on a four-exponential experimental data, and the extracted lifetimes match well with those obtained by iteration based software within $4 \%$.

\section{ACKNOWLEDGEMENTS}

This work has been supported by the European Community within the Sixth Framework Programme IST FET Open MEGAFRAME project (contract No. 029217-2, www.megaframe.eu). The measurements have been performed using the COSMIC laboratory facilities at the University of Edinburgh.

\section{DISCLAIMER}

This publication reflects only the authors' views. The European Community is not liable for any use that may be made of the information contained herein.

\section{REFERENCES}

Apanasovich, V. V. and Novikov, E. G. (1992) 'Methods of analysis of fluorescence decay curves in pulsed fluorometry (Review)', Journal of Applied Spectroscopy, Vol. 56, No. 4, pp. 317-327.

Cubeddu, R., Comelli, D., D'Andrea, C., Taroni, P. and Valentini, G. (2002) 'Time-resolved fluorescence
(T)eddu, R., Comell, D., D'Andrea, C., Taroni, P. and Valentini, G. (2002) 'Time-resolved fluorescence
imaging in biology and medicine', J. Phys. D: Applied Physics, Vol. 35, pp. R61-R76.

Jo, J. A., Fang, Q., Papaioannou, T. and Marcu, L. (2004) "Novel ultra-fast deconvolution method for fluorescence lifetime imaging microscopy based on the Laguerre expansion technique', Conference Proceedings, $26^{\text {th }}$ Annual International Conference of the IEEE EMBS, pp. 1271-1274.

Becker, W. (2005), Advanced Time-Correlated Single Photon Counting Techniques, Wolfgang, Springer.

Ballew, R. M. and Demas, J. N. (1989), 'An error analysis of the rapid lifetime determination method for the evaluation of single exponential decays', Ana. Chem. Vol. 61, pp. 30-33

Sharman, K. K. and Periasamy, A. (1999), 'Error analysis of the rapid lifetime determination method for doubleexponential decays and new windowing schemes', Ana. Chem., Vol. 71, pp. 947-952.

Chan, S. P., Fuller, Z. J., Demas, J. N., Ding, F. and DeGraff, B. A. (2001), 'New method of rapid luminescence lifetime determination using squarewave excitation', Applied Spectroscopy, Vol. 55, No. wave excitation, $1245-1250$

Moore, C., Chan S. P., Demas, J. N. and DeGraff, B. A. (2004), 'Comparison of methods for rapid evaluation of lifetime of exponential decays', Applied Spectroscopy, Vol. 58, No. 5, pp. 603-607.

Kollner, M. and Wolfrum, J. (1992), 'How many photons are necessary for fluorescence-lifetime measurements?', Chemical Physics Letters, Vol. 200, No. 1,2, pp. 199-204.

Niclass, C. L. et al, (2006), 'A single photon avalanche diode array fabricated in deep submicron technology', Conference Proceedings, Design, Automation, and Test Europe Conference, pp. 81-86.

Neely, R. K., Daujotyte, D., Grazulis, S., Magennis, S. W. Dryden, D. T. F., Klimasauskas, S. and Jones, A. C., (2005), 'Time-resolved fluorescence of 2-aminopurine as a probe of base flipping in M.Hhal-DNA complexes', Nucleic Acids Research, Vol. 33, No. 22, pp. 6953-6960. 
Solid-State Electronics 52 (2008) 1382-1387

Anodic $\mathrm{Ta}_{2} \mathrm{O}_{5}$ for CMOS compatible low voltage electrowetting-on-dielectric device fabrication

Y. Li *, W. Parkes, L.I. Haworth, A.A. Stokes, K.R. Muir, P. Li, A.J. Collin, N.G. Hutcheon, R. Henderson, B. Rae, A.J. Walton

Scottish Microelectronic Centre (SMC), School of Engineering and Electronics, Institute for Integrated Micro and Nanosystems,

The University of Edinburgh, King's Buildings, Edinburgh, EH9 3JF, UK

A R T I C L E I N F O

Article history:

Available online 2 July 2008

The review of this paper was arranged by Jurriaan Schmitz

\section{Keyworts:}

MEMS

Microfluidic

EWOD

CMOS

Tantalum pentoxide

High-K dielectric
Post-process

\begin{abstract}
A B S T R A C T
This paper reports a CMOS compatible fabrication procedure that enables electrowetting-on-dielectric (EWOD) technology to be post-processed on foundry CMOS technology. With driving voltages less than $15 \mathrm{~V}$ it is believed to be the lowest reported driving voltage for any material system compatible with post-processing on completed integrated circuits wafers. The process architecture uses anodically grown tantalum pentoxide as a pinhole free high dielectric constant insulator with an overlying $16 \mathrm{~nm}$ layer of Teflon- $\mathrm{AF}^{\oplus}$, which provides the hydrophobic surface for droplets manipulation. This stack provides a very robust dielectric, which maintains a sufficiently high capacitance per unit area for effective operation at a reduced voltage $(15 \mathrm{~V})$ which is more compatible with standard CMOS technology. The paper demonstrates that the sputtered tantalum layer used for the electrodes and the formation of the insulating dielectric can readily be integrated with both aluminium and copper interconnect used in foundry CMOS. ๑) 2008 Published by Elsevier Ltd.
\end{abstract}

\section{Introduction}

In recent years lab-on-a-chip and bio-MEMS systems, which can manipulate and analyse biological fluidic samples in micro- and nano-litre scales, have emerged as a solution for automating repetitive laboratory tasks [1,2]. Digital microfluidic devices based on technologies such as dielectrophoresis (DEP), electrowetting-ondielectrics (EWOD) and surface acoustic waves (SAW) provide a potentially reconfigurable method of obtaining a bio-MEMS system [2,3], enabling different manipulations and transport routes to be programmed using the same device. Of these, EWOD technoto be programmed using the same device. Of these, EWOD technology is an attractive option that has a low power consumption making it well suited for the design and manufacture of microfluidic
systems [2]. EWOD uses surface tension as a driving force, which can be controlled by applying a suitable voltage to an array of electrodes covered by a two layer dielectric.

A key parameter in EWOD technology is the driving voltage $V_{D}$. Earlier work on electrowetting arrays required driving voltages in the range $80-100 \mathrm{~V}[4]$. More recently with a more judicious choice of materials, processes and dielectric thickness, the voltage required to manipulate droplets has been reduced below $15 \mathrm{~V}$ [4].

* Corresponding author. Tel.: +44 1316505609.

E-mail address: Y.Li@ed.ac.uk (Y. Li).

However, the temperatures required for the deposition of one of these dielectric layers is well in excess of $450^{\circ} \mathrm{C}$ [5], making the process incompatible with CMOS post-processing. Being able to construct EWOD structures on top of CMOS technology is attractive because it facilitates large EWOD electrode array with on-chip control and sensing. This paper reports a process architecture that matches the driving voltage of [4] while requiring process temperatures considerably less than $450^{\circ} \mathrm{C}$.

\section{Background}

2.1. Electrowetting-on-dielectrics

The technology of the electrocapillary phenomenon that is used has been extensively described elsewhere [1], and will only be discussed briefly. For an EWOD system, the Young-Lippmann equacussed briefly. For an EWOD system, the Young-Lippmann equa-
tion predicts the contact angle (defining the surface wettability as described in [1]). The change from $\theta(0)$ to $\theta(V)$ for a droplet in terms of the applied voltage $V$, the relative dielectric constant $\varepsilon_{0}$ the liquid-gas surface tension $\gamma_{\mathrm{gg}}$ and the thickness $t$ of the dielectric is given by

$\cos \theta(V)+\frac{\varepsilon_{\mathrm{r}} \varepsilon_{0}}{2 \gamma_{\mathrm{gg}} t} V^{2}=\cos \theta(0)$

0038-1101/\$ - see front matter $\odot 2008$ Published by Elsevier Ltd. doi:10.1016/j.sse.2008.04.030 
Eq. (1) identifies the important role played by the dielectric covering the electrodes in determining the driving voltage $V_{D}$ required to modify the contact angle by the $40^{\circ}$ for droplet manipulation. On a modify the contact angle by the $40^{\circ}$ for droplet manipulation. On a
Teflon-AF $\mathrm{F}^{\circledast}$ surface (commonly used EWOD hydrophobic coating), Teflon-AF
this contact angle change required is from $120^{\circ}$ to $80^{\circ}$, where the Eq. (1) can then be re-written as

$$
V_{\mathrm{D}}^{2}=\frac{0.67 \times 2 \gamma_{\mathrm{gg}} t}{\varepsilon_{\mathrm{r}} \varepsilon_{0}}
$$

From Eq. (2), it is clear that in order to reduce the droplet driving voltage $V_{\mathrm{D}}$, a dielectric with a high permittivity is required. This is in addition to the requirement for this layer to be totally impervious to the liquid that forms the droplet being manipulated. Failure to meet this latter criterion leads to electrolytic action at the electrode the device to cease functioning. Hence, a robust pinhole free dielectric with a sufficiently high breakdown voltage that also acts as barrier to the liquid is essential for any EWOD device. Finally the dielectric also has to display a hydrophobic surface, which is not typically available with materials meeting the above specifications. As a result EWOD dielectric's typically consist of two layers; the insulating dielectric discussed above which is covered by a thin hydrophobic surface layer such as Teflon- $\mathrm{AF}{ }^{\circledR}$.

Table 1 compares the characteristics of some of the dielectrics which have been proposed for EWOD technology.

\subsection{Large EWOD arrays and multi-level metallisation}

In recent years, there has been an increased number of demonstrations of bioassays executed concurrently on a digital microfluidics-based biochip [2,6]. Furthermore, it is clear that system integration and application complexity are expected to increase steadily.

One of the advantages of a digital microfluidic system based on EWOD technology is the ability to reconfigure the system. This means that the different manipulations required can be achieved on the same electrode array by simply modifying the control software. Examples of reconfigurable digital microfluidic systems based on EWOD technology and DEP technology have been reported for sample analysis that use reagent mixing [2,7-10].

Large electrode arrays have the potential to greatly increase the reconfiguration possibilities, including:

- Greater system flexibility: increases the defect tolerance of the system, allowing increased flexibility in route selection $[2,6]$. More functional units, such as droplet mixers, consisting of different numbers of electrodes $[2,6]$.

Table 1

Comparison of EWOD dielectric properties $[4,13]$

\begin{tabular}{|c|c|c|c|}
\hline & $\begin{array}{l}\text { Dielectric } \\
\text { constant }\end{array}$ & $\begin{array}{l}\text { Dielectric } \\
\text { strength }(\mathrm{MV} / \mathrm{cm})\end{array}$ & $\begin{array}{l}\text { Process } \\
\text { temperature }\left({ }^{\circ} \mathrm{C}\right) \\
\end{array}$ \\
\hline BST & $>30^{\mathrm{a}}$ & 10 & 800 \\
\hline $\begin{array}{l}\mathrm{LPCVD} \\
\mathrm{SiO}_{2}\end{array}$ & 3.8 & 10 & 900 \\
\hline $\begin{array}{l}\text { LPCVD } \\
\mathrm{Si}_{3} \mathrm{~N}_{4}\end{array}$ & $6-8$ & 10 & 900 \\
\hline $\begin{array}{l}\mathrm{PECVD} \\
\mathrm{PiO}_{2}\end{array}$ & 3.8 & 8 & 300 \\
\hline $\begin{array}{l}\text { PECVD } \\
\operatorname{SiN}_{x}\end{array}$ & 6-9 & 6 & 300 \\
\hline $\begin{array}{l}\text { Parylene-C } \\
\text { Anodic }\end{array}$ & $\begin{array}{l}3.1 \\
8-25^{\mathrm{b}}\end{array}$ & $\begin{array}{l}2.7 \\
6\end{array}$ & $\begin{array}{l}25 \\
25\end{array}$ \\
\hline
\end{tabular}

- Higher sample processing throughput: more droplet samples can be processed simultaneously.

Finer control of droplet volume: enables the system to have a higher resolution of droplet volumes.

Several examples showing defect tolerant design and spontaneous multiple droplet manipulations on electrode arrays can be found in the literature $[2,6]$.

Obviously in a passive EWOD system, there is no internal control circuitry available, and each driving electrode in the device must be individually addressed from a contact pad via interconnect. While the interconnect for single and double rows of eletrodes can be simply implemed using a single level of , the same is nevel of metallisatin, the same is mith electrode inner electrodes in the $M \times N$ array to the exterior control circuit must run between the electrode gaps. This approach is demonstrated in Fig. 1 which shows a single-level-metallisation micro-heater array using a passive single-level metal addressing mechanism.

In EWOD devices the interconnects are, in effect, just small electrodes and can also suffer from unwanted wetting of non-electrode areas (Fig. 2). This wetting phenomenon will potentially affect areas (Fig. 2). in routed between electrodes as can be observed in Fig. 1. Even when the interconnect is held at ground potential, the electrode gaps may be unacceptably wide when several tracks pass between electrodes. Hence, for EWOD arrays equal to or larger than $3 \times 3$ electrodes, multi-level metallisation is required to avoid parasitic effect of interconnects by burying them beneath the EWOD functional electrodes.

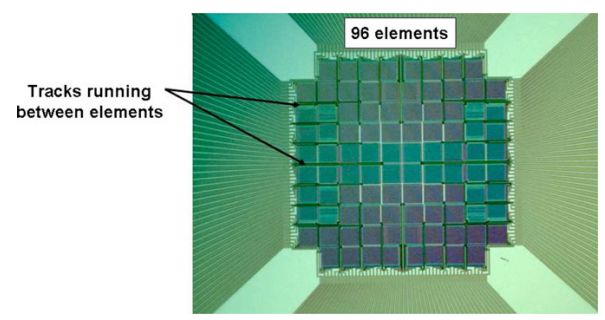

Fig. 1. Ninety six element micro-heater array, an example of a single-level-metallisation large array having interconnect tracks running between elements electrodes.
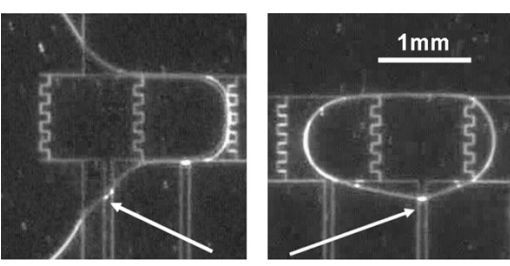

Unwanted wetting of the interconnect track causes droplet distortion Fig. 2. Wetting phenomenon on interconnects in a single metallisation EWOD
device. 
2.3. Large EWOD arrays on a CMOS backplane

For arrays of EWOD electrodes, the realistic number of electrodes for passive systems is also limited by packaging considerations. For example a $30 \times 30$ array would require a 900 pin package which is not particulary practical.

Gong et al. partly address the packaging problem by using printed circuit board (PCB) technology together with land grid array (LGA) sockets [7]. The advantage of this solution is its low-cost and system flexibility (i.e., scalable). However, this does not solve the practical aspect of the interconnect problem entirely since a $32 \times 32$ digital microfluidic array used in [10] requires over 1000 pins. This is at the practical pins. This is at the practical limit of a passive electrode drive system and any size bigger really requires an active controlling ackplane.

CMOS technology has been widely used for row-column addressing of large numbers elements, of which the largest application is related to memory devices. Others examples include CMOS imaging chips [11] and micro displays [12]. Addressing arrays using this approach is obviously ideally suited to the realisation of EWOD arrays with a large matrix of electrodes. In addition the underlying CMOS also makes it possible to provide the electrodes with additional capabilities such as sensing (e.g., $\mathrm{pH}$, temperature, light, voltage, etc) and actuation (e.g., tempera$\mathrm{pH}$, temperat

Obviously a clear advantage of using on-chip addressing for large two-dimensional arrays is the significant reduction in the number of bond pads which also simplifies the packaging. A dielectrophoresis (DEP) system with a $32 \times 32$ array of individually addressable electrodes using a CMOS solution has already been demonstrated [10]. Manipulating droplets having more than a 100 fold volume range, it shows a scalable architecture of digital microfluidic systems based on CMOS technology [10]

In another commercialised prototype system, more than 600,000 electrodes have been embedded and separately controlled to create more than 100,000 DEP cages for droplet manipulation ${ }^{1}$ Similar success can be achieved with active EWOD electrode arrays (i.e. integrated CMOS backplane electrode control), if appropriate post-processing technology is available.

As part of this work an EWOD/CMOS chip has been fabricated and tested [13] (Fig. 3). This used conventional foundry processes and materials, with the EWOD post-processing involving the deposition of appropriate dielectric and surface treatment layers.

\subsection{Low voltage EWOD processes}

Previously reported low voltage EWOD fabrication processes have all used dielectrics deposited at high temperature (e.g. $700^{\circ} \mathrm{C}$ MOCVD for barium strontium titanate [4], thermal oxidation [14], $700{ }^{\circ} \mathrm{C}$ annealed $\mathrm{BZN}\left(\mathrm{Bi}_{2} \mathrm{O}_{3}-\mathrm{ZnO}-\mathrm{Nb}_{2} \mathrm{O}_{5}\right)$ [15]). None of these are compatible with $\mathrm{CMOS}$ technology incorporating aluminium (or copper) interconnect as deposition temperatures in excess of $450^{\circ} \mathrm{C}$ are required.

In this work the CMOS foundry process that was used as the backplane of the EWOD device employed aluminium as its interconnect. Hence, the electrode metallisation and the passivation layer were determined by the foundry. For typical foundry processes the passivation is a relatively thick dielectric layer $(0.5$ $1.0 \mu \mathrm{m}$ ) of silicon dioxide or nitride (or oxynitride), neither of which possesses a very high dielectric constant.

For an EWOD implementation using foundry passivation as the insulating dielectric a comparatively high operating voltage in the region of $70 \mathrm{~V}$ is required to drive droplets. This is as a direct con-

\footnotetext{
${ }^{1}<$ http://www/siliconbiosystems.com/DEPArray.page>.
}

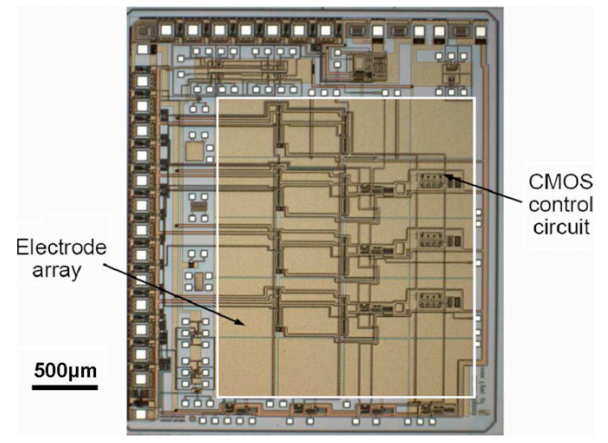

Fig. 3. EWOD electrode array (boxed area) controlled by backplane CMOS circuit [13].

sequence of the low dielectric constant of the passivation materials. The resulting high drive voltage requirement drove the selection of a $100 \mathrm{~V}$ CMOS foundry process for the demonstration EWOD backplane.

Having fabricated and post-processed a high voltage CMOS EWOD device the challenge was to identify and demonstrate an improved material system that was fully compatible with EWOD, while at the same time being suitable for integration with a lowe voltage CMOS technology. The material system selected is based upon a tantalum pentoxide insulating layer with a high $\varepsilon_{\mathrm{r}}$ dielectric constant (in our case around 19 at a thickness of $95 \mathrm{~nm}$ ), which can be grown pinhole-free. This is covered by a uniform and thin (apthe required $m$, which involves no high temperature process (except for sputtering tantalum), simply consists of $\mathrm{Ta} / \mathrm{Ta}_{2} \mathrm{O}_{5} /$ teflon layers, which is compatible with standard foundry CMOS IC technology with aluminium interconnect.

\section{Multi-level metallisation EWOD array}

Multi-level metallisation EWOD arrays using chromium and chromium/platinum on glass and silicon substrates have previously been fabricated for reconfigurable multi-functional microfluidic systems $[16,6]$.

Aluminium multilayer interconnect structures have been widely employed in standard CMOS circuitry fabrication, while a single layer of aluminium has been demonstrated as a passive EWOD electrode material in [13]. Hence, a two-level aluminium metallisation process for $M \times N$ EWOD electrode array fabrication is clearly feasible. Similar multi-level aluminium metallisation structures are commonly employed in standard CMOS integrated circuits devices.

The process flow for fabricating a two-level metallisation EWOD electrode array is detailed below and illustrated in Fig. 4:

(a) The bottom aluminium layer is sputtered on an $\mathrm{SiO}_{2}$ insulated silicon substrate.

(b) It is then patterned to form the bond pads and the interconnects, thicknesses ranges between 0.1 and $1 \mu \mathrm{m}$.

(c) After patterning, $1 \mu \mathrm{m}$ of PECVD $\mathrm{SiO}_{2}$ is deposited as the inter-metal dielectric. Vias are then opened by patterning the PECVD $\mathrm{SiO}_{2}$ and the second aluminium layer is then sputtered to form the electrodes an dielectrical connection to the bottom aluminium layer. 

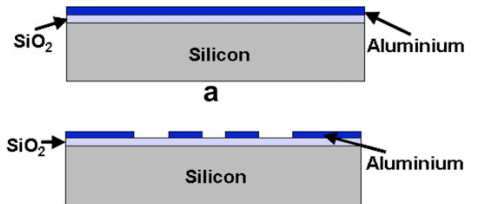

b

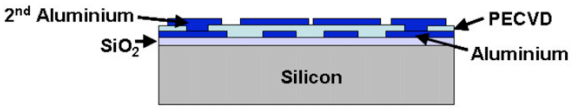

c

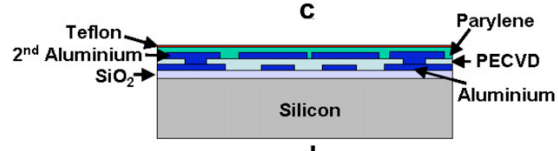

d

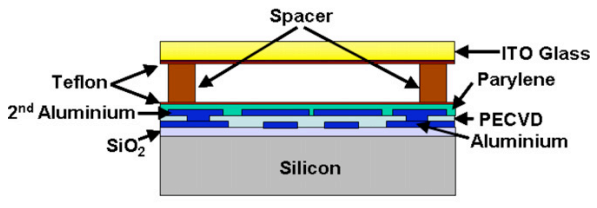

e

Fig. 4. Process flow for a two-level metal EWOD electrode array with aluminium a the interconnect and electrode material.

(d) After the patterning the second aluminium layer, the insulating dielectric $500 \mathrm{~nm}$ parylene-C (room temperature vapour deposition), and $50 \mathrm{~nm}$ Teflon- $\mathrm{AF}^{\circledast}$ hydrophobic layers (spin-coating) were finally deposited.

(e) After dicing the microscope slide sized chip can then be probed or packaged in the same manner as for single metal systems [13].

Using the same bond pad layout and chip size discussed in [13] a $5 \times 8$ EWOD electrode array was designed. The bottom aluminium interconnect lines were $10 \mu \mathrm{m}$ wide. The top metal electrodes were $1 \mathrm{~mm} \times 1 \mathrm{~mm}$ interdigited square electrodes with $100 \mu \mathrm{m}$ wide gaps in between. The electrodes were covered with a

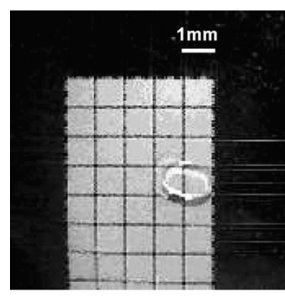

a

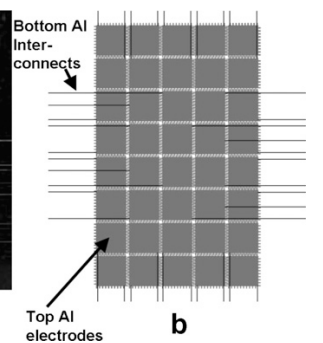

electrodes

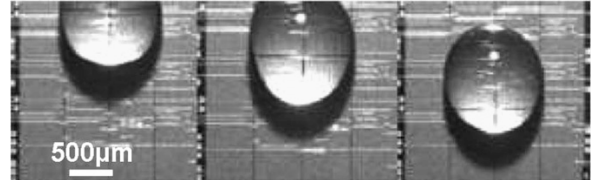

Fig. 6. Droplet movement (three frames, left to right) on a post-processed EWOD electrode array controlled by a CMOS backplane.

$500 \mathrm{~nm}$ layer of parylene-C covered by $50 \mathrm{~nm}$ of Teflon- $\mathrm{AF}^{\circledast}$. This required a driving voltage of $60 \mathrm{~V}$ and Fig. 5 shows droplet manipulation on a $5 \times 8$ EWOD electrode array.

By using the same post-process steps on a custom designed By using the same post-process steps on a custom designed
CMOS backplane chip, droplet movement was achieved with a drive voltage of $60 \mathrm{~V}$ as shown in Fig. 6.

\section{Tantalum-aluminium structures for low voltage EWOD-} CMOS systems

As has been previously mentioned a low voltage EWOD electrode array based upon a high-K tantalum pentoxide insulating layer has been developed. This involves no high temperature process and simply consists of $\mathrm{Ta} / \mathrm{Ta}_{2} \mathrm{O}_{5} / \mathrm{Teflon}^{-\mathrm{AF}^{\circledast}}$ or $\mathrm{CYTOP}^{\circledR}$ layers. cess and simply consists of $\mathrm{Ta} / \mathrm{Ta}_{2} \mathrm{O}_{5} / \mathrm{Teflon}^{-\mathrm{AF}^{\circledast}}$ or CYTOP ${ }^{\star}$ layers.
This is compatible with standard foundry CMOS IC technology with This is compatible with standard foundry CMOS IC technology with
conventional aluminium interconnect. This section will focus on the fabrication of EWOD systems based on tantalum-aluminium structures.

\subsection{Structure design and fabrication}

To demonstrate the $\mathrm{Ta}_{2} \mathrm{O}_{5}-\mathrm{Teflon}^{-} \mathrm{AF}^{\circledast}$ dielectric system, the top aluminium layer in Fig. 4 was replaced by sputtered tantalum which was patterned using the same mask.

There are a number of options for etching tantalum. It can be etched in fluorine-containing plasmas such as $\mathrm{CF}_{4}, \mathrm{SF}_{6}$, and $\mathrm{CF}_{3} \mathrm{Cl}$ with $\mathrm{CH}_{3} \mathrm{~F}$, sometimes mixed with $\mathrm{O}_{2}[17,18]$. The drawback is that these processes will potentially attack any underlying PECVD $\mathrm{SiO}_{2}$ layer, which may be problematic if the tantalum etching is not uniform. By using $\mathrm{SiCl}_{4}$ mixed with $\mathrm{NF}_{3}$ plasma, Shimada et al obtained an etch selectivity greater than $80: 1$ between tantalum and $\mathrm{SiO}_{2}\left(10: 1\right.$ in absence of $\left.\mathrm{NF}_{3}\right)$ [19].

An alternative is $\mathrm{XeF}_{2}$ dry etching. This is commonly used for silicon etch release in MEMS fabrication, especially post-CMOS silicon etch release in MEMS fabrication, especially post-CMOS
etch release due to its high selectivity with other materials (greater etch release due to its high selectivity with other materials (greater
than 1000:1 for silicon to $\mathrm{SiO}_{2}$ and aluminium) [20]. $\mathrm{XeF}_{2}$ rapidly etches tantalum and no aluminium or $\mathrm{SiO}_{2}$ attack was observed when using a Memsstar ${ }^{\circledR}$ tool. The only potential issue is the degree of undercut, as shown in Fig. 7 with an average value of

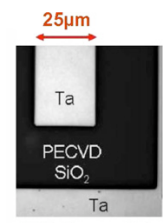

$\mathrm{SiCl}_{4}$ Plasma Etch

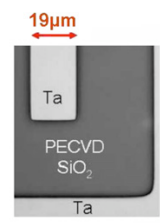

$\mathrm{XeF}_{2}$ Etch
Fig. 5. (a) Photograph of a moving droplet on a two-level metallisation (aluminium) $5 \times 8$ EWOD electrode array with a driving voltage of $60 \mathrm{~V}$. This is a two-plate EWOD device with a $440 \mu \mathrm{m}$ droplet height. (b) Layout of the $5 \times 8$ EWOD electrode array.
Fig. 7. Microscope photos showing a higher etching selectivity of tantalum to $\mathrm{SiO}_{2}$ in $\mathrm{XeF}_{2}$ gas (right) than in $\mathrm{SiCl}_{4}$ plasma (left). Isotropically etched tantalum patterns

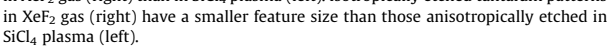


$3.0 \mu \mathrm{m}$ on each side being measured when etching $0.45 \mu \mathrm{m}$ thick tantalum. As the gap between EWOD electrodes in this case is $30 \mu \mathrm{m}$, this undercut rate is acceptable and if need be, could be accounted for by a bias in the mask.

After patterning, the tantalum electrodes are anodised with gel form citric acid solution to form the tantalum pentoxide insulating layer [21]. In this work the applied voltage was $50 \mathrm{~V}$ and for the electrode array shown in Fig. 5 the current compliance was $2 \mathrm{~mA}$. A thin Teflon- $\mathrm{AF}^{\circledast}$ layer is then deposited using a standard spin coater on the oxidised tantalum electrodes. The surface roughness of the anodic $\mathrm{Ta}_{2} \mathrm{O}_{5}$ has been measured using AFM to have a mean roughness $R_{\text {a }}$ between 0.4 and $0.6 \mathrm{~nm}$ (peak-to-valley value down to fer value down to few nanometers) with the Te

The resulting two-level metal EWOD array has aluminium as the bottom metal with tantalum as the top metal electrode. The $50 \mathrm{~V}$ anodisation voltage used in this process resulted in $95 \mathrm{~nm}$ of $\mathrm{Ta}_{2} \mathrm{O}_{5}$. This was followed by a $0.3 \%$ Teflon- $-\mathrm{F}^{\circledast}$ solution (diluted in Fluorinert solvent FC-75) being spin coated at $2000 \mathrm{rpm}$ for $50 \mathrm{~s}$, giving $16 \mathrm{~nm}$ of Teflon- $\mathrm{AF}^{\circledR}$.

\subsection{Experiment and results}

4.2.1. Low voltage EWOD manipulation on large arrays

A common two-plate configuration EWOD chip [22] has been used in the experiments to evaluate the $\mathrm{Ta}_{2} \mathrm{O}_{5} / \mathrm{Teflon}^{-\mathrm{AF}^{\circledast}}$ system. A conductive indium tin oxide (ITO) covered glass plate coated with $20 \mathrm{~nm}$ Teflon- $\mathrm{AF}^{\circledR}$ was placed above the $5 \times 8$ electrode array previously presented. Spacers were used to set the distance between the plates and hence the height of droplets. In this case the spacers were 258 microns. The above combination of dielectric materials on the EWOD device ( $95 \mathrm{~nm} \mathrm{nt}_{2} \mathrm{O}_{5}$ and $16 \mathrm{~nm}$ of Teflon. of $14 \mathrm{~V}$ (Fig. 8).

4.2.2. Droplet size manipulation using low voltage

Fig. 9 shows a digital droplet dispense system which uses a glass fibre capillary attached through a plastic ferrule as a liquid in-

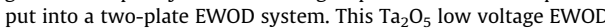

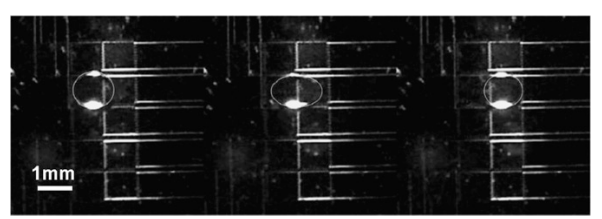

Fig. 8. Three frames (left to right) showing a moving droplet on a two-level metal EWOD chip coated with $95 \mathrm{~nm} \mathrm{Ta} \mathrm{O}_{5}$ and $16 \mathrm{~nm}$ Teflon-AF${ }^{\circledast 2}$ (the outlines of the droplet have been enhanced for clarity).

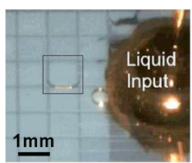

a

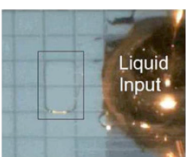

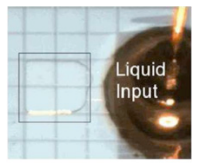

Fig. 9. Moving droplets (black boxed) with different volumes (a) $80 \mathrm{~nL}$, (b) $160 \mathrm{~nL}$ and (c) $320 \mathrm{~nL}$, dispensed from a liquid input capillary fibre (on the right of each figures system is based on a tantalum electrode array with aluminium interconnects and uses a $15 \mathrm{~V}$ DC drive voltage. The liquid input is pressurised through the fibre capillary from a syringe which fills up the reservoir attached to the EWOD electrodes. The liquid is then extruded and cut into droplets of by manipulating the electrodes in the EWOD array in a similar manner to that described in $[22,13]$.

Fig. 9 shows the creation of droplets with volumes of $80 \mathrm{~nL}$ $160 \mathrm{~nL}$ and $320 \mathrm{~nL}$ by switching on 1, 2 or 4 electrodes simultaneously. The volume is defined by the size of a single electrode and the gap between the two EWOD plates. In this case, each unit electrode was $1 \times 1 \mathrm{~mm}$ in size and the gap $80 \mu \mathrm{m}$. More volume the volume choices are obviously available as the electrode size reduces and

\section{Conclusion and future work}

This paper has described what is believed to be the first fully CMOS compatible EWOD system that can drive liquid droplets using voltages less than $20 \mathrm{~V}$. The method of producing a thin uniform film of high permittivity dielectric by the anodisation of tantalum, together with a reliable method of spinning thin uniform teflon films, are the key to achieving the required low operating potential. The resulting system, with its robust and pinhole free anodised $\mathrm{Ta}_{2} \mathrm{O}_{5}$ provides a high dielectric constant and an impervious barrier to the liquids being transported, which is not always the case with deposited dielectrics.

The integration of CMOS and EWOD technologies helps solve the issues relate to increasing the EWOD electrode array scales. The benefits have already been shown in other CMOS integrated system [1] and othe advantages such as possible on-chip sensing can also be obtained by integrating sensing circuits with the EWOD systems.

In addition an EWOD post-process foundry CMOS chip has been processed and droplet movement demonstrated. The nex step is to significantly increase the number of electrodes so that it becomes possible to implement a programmable electrode array size and start integrating further functionality into the electrodes. Fig. 10 shows part of a prototype design that provides an example of both of these elements. It consists of an EWOD a ex with $200 \mu 100 \mu$ electrodes inted with SPAD single photon avalanche diodes) for light detection and this gives one example of the direction digital microfluidics will be moving in the future.

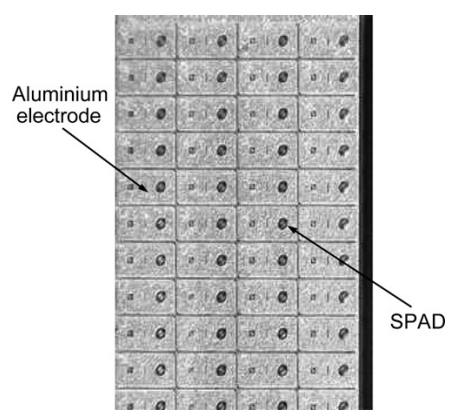

Fig. 10. A prototype design of an EWOD array with electrodes integrated with $S P A D$ (single photon avalanche diodes) [23]. 


\section{Acknowledgments}

The authors would like to acknowledge the financial support of EPSRC, the IIS (part of the Edinburgh Research Partnership in Engineering and Mathmatics (ERPEM)), the RASOR project (EPSRC and BBSRC) and GOLEM project (EC).

The authors would also like to acknowledge Dr. Edoardo Charbon from EPFL for providing the underlying SPAD design integrated into the EWOD device shown in Fig. 10 and Memsstar ${ }^{\otimes}$ technology (Tony O'Hara and Graeme Pringle) for support with the $\mathrm{XeF}_{2}$ etching process.

\section{References}

[1] Mugele F, Baret JC. Electrowetting: from basics to applications.J Phys, Condens

2] Chakrabarty K, Su F. Digital microfluidic biochips: synthesis, testing, and 3] Wixforth A. Acousticaques. CRC; 2006, ISBN 0849390095. chemical applications. JALA 2006:399-405.

[4] Moon H, Cho SK, Garrell RL, Kim CJ. Low voltage electrowetting-on-dielectric.] Appl Phys 2002;92(7):4080-7.

. Integration of IC technology with MEMS: silicon+ technology for the future. Ite 6] Su F, Ozev S, Chakrabarty K. Concurrent testing of digital microfluidics-base biochips. ACM Trans Des Autom Electron Syst 2006;11:442-64

7] Gong J, Fan SK, Kim CJ. Portable digital microfluidics platform with active but disposable lab-on-chip. In: Micro electro mechanical systems conference

[8] SK, Hashi C, Kim C]. Manipulation of multiple droplets on $N \times M$ grid by crossreference EWOD driving scheme and pressure-contact packaging. Micro electro-mechanical systems, MEMS, Kyoto, 2003. p. 694-7.
[9] Gong J, Kim CJ. Two-dimensional digital microfluidic system by multi-layer printed circuit board. MEMS. In: 18 th IEEE conference, 2005. p. 726-9. [10] Gascoyne PRC, Vykoukal JV, Schwartz JA, Anderson TJ, Vykoukal DM, Current
KW, et al. Dielectrophoresis-based programmable fluidic processors. Lab Chip 2004:4:299-309. Wang $G$ Renshaw

100-3. 27-31 May.

12] Underwood I, Gourlay J. Light-emitting polymer on CMOS: a new photonic technology. Proc SPIE 2003;5181:110-9. Li Y, Li P, Kazantzis A, Haworth LI, Muir K, Ross AWS, et al. Building EWOD actuators. The IET seminar Lon

(hin 5] Kim, Hong SM, Park SS, Hong YP. The movement of micro droplet with the effects of dielectric layer and hydrophobic surface treatment with atmir $\mathrm{RB}$. Digital mich flidics: is a true lab-on-a-chip possible? Microfluid Nanofluid 2007:3:245-81.

17] Hsiao R, Miller D. Etching of tantalum in fluorine-containing high density plasmas. J Electrochem Soc 1996;143:3266-70

18] Kuo Y. Reactive ion etching of sputter deposited tantalum with $\mathrm{CF}_{4}, \mathrm{CF}_{3} \mathrm{Cl}$, and

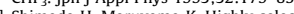

thin gate dielectrics using $\mathrm{SiCl}_{4}-\mathrm{NF}_{3}$ gas mixture of tantalum electrode to

[20] Chang FI, Yeh R, Lin G, Chu PB, Hoffman E, Kruglick EJJ, et al. Gas-phase silico micromachining with xenon difluoride. Proc SPIE - Int Soc Opt Eng 1995

21] Li Y, Developing CMOS compatible electrowetting-on-dielectric (EWOD) 22] Pollack MG, Fair RB. Electrowesting-based actuation of liquid droplets fo microfluidic applications. Appl Phys Lett 2000;77:1725-6.

23] Niclass $C$, Sergio M, Charbon E. A single photon avalanche diode array fabricated in deep submicron technology. In: Design automation and tes 


\title{
CMOS driven micro-pixel LEDs integrated with single photon avalanche diodes for time resolved fluorescence measurements
}

\author{
B R Rae ${ }^{1,4}$, C Griffin ${ }^{2}$, J McKendry ${ }^{2}$, J M Girkin ${ }^{2}$, H X Zhang ${ }^{2}$, E Gu ${ }^{2}$, \\ D Renshaw ${ }^{1}$, E Charbon ${ }^{3}$, M D Dawson ${ }^{2}$ and R K Henderson \\ ${ }^{1}$ Institute for Integrated Micro and Nano Systems, The School of Electrical and Electronic Engineering, \\ The University of Edinburgh, The King's Buildings, Mayfield Road, Edinburgh, EH9 3JL, UK \\ ${ }^{2}$ Institute of Photonics, SUPA, University of Strathclyde, 106 Rottenrow, Glasgow G4 0NW, UK \\ ${ }^{3}$ Ecole Polytechnique Fédérale de Lausanne (EPFL), CH-1015, Lausanne, Switzerland \\ E-mail: Bruce.Rae@ed.ac.uk \\ Received 17 September 2007, in final form 6 December 2007 \\ Published 4 April 2008 \\ Online at stacks.iop.org/JPhysD/41/094011 \\ Abstract \\ We describe a single chip approach to time resolved fluorescence measurements based on time \\ correlated single photon counting. Using a single complementary metal oxide silicon (CMOS) \\ chip, bump bonded to a $4 \times 16$ array of AlInGaN UV micro-pixellated light-emitting diodes, a \\ prototype integrated microsystem has been built that demonstrates fluorescence excitation and \\ detection on a nanosecond time scale. Demonstrator on-chip measurements of lifetimes of \\ fluorescence colloidal quantum dot samples are presented.
}

(Some figures in this article are in colour only in the electronic version)

\section{Introduction}

The use of fluorescence-based methodologies is at the core of many modern instrumentation technologies, especially in the life sciences. Originally, the interest was in the specific labelling of biological samples for imaging applications, but more recently this has expanded rapidly with the growth of DNA sequencing and micro-array applications. In principle, the equipment needed for such spectroscopic instrumentation has not changed over several decades, in that a narrow wavelength source is required to excite the fluorophore of interest and the resulting fluorescence must pass through an optical device to separate the excitation light from the fluorescence emission, before being detected by a light sensitive instrument and the level of fluorescence determined. However, using modern fabrication and manufacturing techniques, such instrumentation can now be made in complete integrated systems with the potential for volume production. The range of methods for interpreting the fluorescence data has

${ }^{4}$ Author to whom any correspondence should be addressed. also expanded with the growth of fast electronics, originally destined for the telecommunications and computer markets, now being applied to fluorescence instrumentation.

Most commonly used fluorophores are designed to be excited in the near ultraviolet (UV) and blue regions of the optical spectrum. The advent of GaN and other blue emitting semiconductor sources, around 15 years ago [1], has helped stimulate interest in the use of such sources in place of lasers or mercury discharge lamps for excitation in fluorescence-based instrumentation. The advantages of these sources include low cost, reliability, compactness and good wavelength matching to many standard fluorophores. In addition, their ability to produce short $(\approx 1 \mathrm{~ns})$ pulse lengths offers the opportunity for low cost fluorescence lifetime measurements. It has been appreciated for a long time [2] that the fluorescence decay from a molecule provides a significant level of information about the sample and the surrounding conditions of the fluorophore, from the local viscosity in cell membranes [3] to $\mathrm{pH}$ [4]. Most recently interest has been in the rapid growth of Föster resonance energy transfer (FRET) methods, where the spatial separation of two fluorescent molecules significantly affects 
the fluorescence lifetime of the shorter wavelength 'donor' molecule. Through advances in molecular chemistry, FRET based assays and experiments utilizing lifetime measurement as the monitor of the FRET process are emerging as the method of choice for many applications in the life sciences [5].

Several methods of lifetime measurement are now in common use in lifetime instrumentation, but all require the common features of a short pulsed, or high frequency modulated, light source, fast detector and timing electronics. Time correlated single photon counting (TCSPC) is potentially the most accurate method of determining complex decays (multiple lifetimes) and was therefore the method selected to demonstrate the performance of our system. The phase method, in which the phase change in the fluorescence emission relative to the excitation pulse is measured [6], is less sensitive compared with TCSPC and applies more light to the sample which can lead to faster sample decomposition. A slight variation on TCSPC is the use of time gated detectors which provide a slightly less accurate lifetime measurement, but are excellent at providing a method of detecting changes in lifetime [7]. More recently, a variation on the phase method has been developed, whereby the change in output intensity is measured [8]. This method uses a slow detector and thus was not suitable in a system that makes use of rapid detectors. Therefore, we chose the TCSPC method as we believed that this would demonstrate the capability of our novel excitation source and detector. Furthermore, if the system worked in this configuration it should also work in the slightly less demanding phase and gated configurations, though these might be the preferred options in a final instrument configuration.

In 1995, Araki and Misawa [9] and in 2005, Davitt et al [10] both reported the use of light-emitting diodes (LEDs) for lifetime measurements; however, their system used a conventional photomultiplier. Although this work demonstrated the capability of LEDs for lifetime measurements with the use of a photomultiplier as the detector, the system was not capable of low cost miniaturization. In this paper, we describe a single chip embodiment for such measurements based on time correlated single photon counting. In our approach, a $16 \times 4$ array of micropixel AlInGaN LEDs emitting at $370 \mathrm{~nm}$ [11] has been bonded to an equivalent array of aluminium electrodes made from the top metal of a $0.35 \mu \mathrm{m}$ high-voltage CMOS chip. Each electrode has an associated driver circuit capable of switching up to $50 \mathrm{~V}$. Incorporated into each electrode is a single photon avalanche diode (SPAD). The output of the SPAD detectors is processed in real time by a TCSPC module that generates a histogram of the fluorescence decay [12]. Other pertinent features of the CMOS chip include on-chip control of the micro-LED pulse width $(1.5-48 \mathrm{~ns}$ optical pulse width) and the ability to separately address any micro-LED or SPAD in the array. With this device, we have successfully performed demonstration measurements in a colloidal suspension of quantum dots, achieving lifetimes consistent with manufacturer's specifications and our own reference measurements taken using a photomultiplier tube detector. This microsystem solution will enable the development of low cost, portable fluorescence lifetime readers

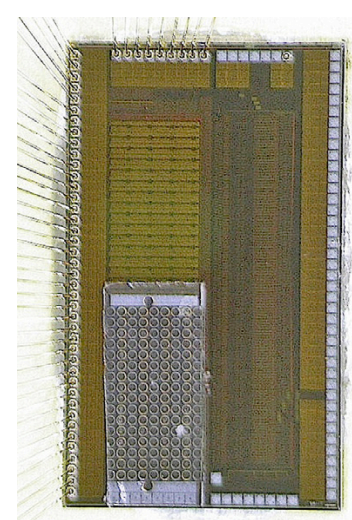

Figure 1. Micrograph image of CMOS chip with bump-bonded micro-LED array.

for many optical lab-on-a-chip applications such as point-ofcare diagnostics equipment and the synthesis and/or read out of DNA micro-arrays. With each element being separately addressable, there is the potential to excite many fluorescen samples in parallel [11]. By integrating the excitation source with a photodetector and on-chip driving electronics, our devices will contribute to the development of lab-on-a-chip (LoC) systems [13]. This includes work by Chodavarapu et al [14], which aims to carry out fluorescence detection using a CMOS-based system which incorporates a detector with signal processing circuitry. Their system does not, however, include an integrated excitation source and uses a discrete LED. Cleary et al [15] demonstrated TCSPC on a microscale using integrated optics and microfluidics; however, their system relies on a pulsed diode laser as an excitation source and a discrete SPAD detector. The system presented here offers a greater level of integration, placing the excitation source in the same micro-scale system as the sample and detector.

\section{Experimental setup}

A micrograph image of the CMOS chip with bump-bonded micro-LED array can be seen in figure 1 .

The micro-LEDs consist of a $16 \times 4$ array of individually addressable pixels, each pixel having a diameter of $72 \mu \mathrm{m}$ on a $100 \mu \mathrm{m}$ pitch. The devices were fabricated from 'standard' UV LED wafers grown on $c$-plane sapphire substrates by metal organic chemical vapour deposition and have a peak emission at $370 \mathrm{~nm}$ [16]. The array of electrodes was depassivated by pad opening at the foundry to reveal the aluminium topplate electrodes for bump bonding. A post-processing step of oxygen plasma etching removed the polyimide layer of the chip which has been shown to improve the photon detection probability (PDP) of the underlying SPADs by a factor of $2-5$ [17]. An example of the top metal driver plates can be seen in figure 2. An electrical connection between the micro-LED 
array and the driving CMOS chip was made using a bumpbonding process with each element of the micro-LED array being bonded to a dedicated driver site. A four metal layer, high voltage, $0.35 \mu \mathrm{m}$ CMOS technology was used to fabricate the driving CMOS chip. The first two layers of metal were used for signal routing, the third layer formed a shield to protect the underlying electronics from the high-voltage signal being driven onto the array and the fourth layer was used to pattern an array of driver plates onto which the micro-LED array was bonded. Driving circuitry for each electrode and SPAD is contained within a $200 \times 100 \mu \mathrm{m}^{2}$ cell, with $19090 \mu \mathrm{m}^{2}$ of top metal exposed as the electrode surface. Electrode selection is controlled using row and column addressing.

The SPAD used in this test chip is essentially an avalanche photodiode operating in Geiger mode. This mode of operation enables detection of single photons, when the diode is equipped with the means to detect and regenerate the avalanche currents associated with photon detection. A schematic cross section of a SPAD is shown in figure 3. The high voltage, twin-tub CMOS technology allows the construction of a $\mathrm{p}+$ /deep $n$-tub avalanche multiplication region. Premature lateral breakdown of the junction is avoided by a guard ring of lightly doped p-type diffusion. A detailed description of the SPAD structure

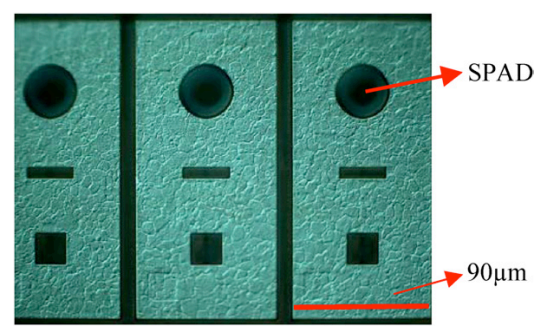

Figure 2. Scanning electron microscope (SEM) image of the individual top layer metal plates upon which each micro-LED in the array is bump bonded. The SPAD detectors are situated in the circular gap in the metal. and measured performance can be found in [18]. The detection of a photon and the subsequent avalanche breakdown of the SPAD generates a voltage pulse, the duration of which is approximately $30 \mathrm{~ns}$, which is quenched passively on chip. Single incident photons generate digital pulses, with around $100 \mathrm{ps}$ of timing jitter, which are readily processed by on-chip electronics.

Measurement of fluorescence decay curves have been demonstrated using CdSe/ZnS quantum dots (Evident Technologies Inc., USA) in a toluene solution [19] at concentrations as they were received from the supplier. These particles have an excitation spectrum that peaks at wavelengths less than $400 \mathrm{~nm}$ and emissions at $526 \mathrm{~nm}$ (Adirondack Green), $543 \mathrm{~nm}$ (Catskill Green) and $555 \mathrm{~nm}$ (Hops Yellow), respectively. Table 1 contains a summary of the quantum dot samples used.

The samples are held in a cavity microslide (Agar Scientific Ltd, UK) and sealed by a $24 \mathrm{~mm} \times 24 \mathrm{~mm}$ cover slip to avoid evaporation. The slide is placed on top of the chip and excited by a pulse train from a single micro-LED. The LED is biased with $4 \mathrm{~mA}$ of bias current and the electrode driven to $13 \mathrm{~V}$, producing short pulses with fast rise and fall times ( $2.15 \mathrm{~ns}$ and $3.31 \mathrm{~ns}$ respectively, for a pulse width of $8 \mathrm{~ns}$ ). Each micro-LED driver site also contains an addressable SPAD detector, as described above. The sapphire substrate of the micro-LED array is optically transparent and therefore any returning fluorescence light passes through the micro-LED array and reaches the SPAD array. Figure 4 shows a schematic of the optical stack.

The number and position of the LED pixels that are active is user definable and any combination from a single LED up to all 64 elements of the array can be active at any one time. During analysis all 64 SPADs in the array are active. However, the address chosen by the user defines which SPADs are present on the output bus; thus, the address of the detecting SPAD does not have to correspond to the address of the exciting micro-LED. To avoid direct coupling of excitation light from the micro-LED, the SPAD chosen to detect is situated eight rows away $(800 \mu \mathrm{m})$ from the emitting LED pixel. It should be noted that this does not completely

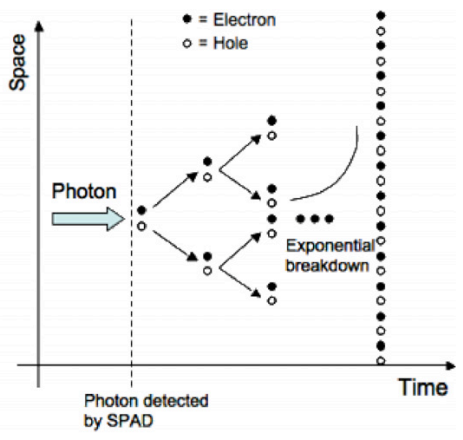

Figure 3. Schematic cross section of CMOS SPAD and illustration of avalanche breakdown. 
J. Phys. D: Appl. Phys. 41 (2008) 094011

Table 1. Summary of quantum dot samples used to evaluate system.

\begin{tabular}{lllll}
\hline Sample & $\begin{array}{l}\text { Peak } \\
\text { absorption } \\
(\mathrm{nm})\end{array}$ & $\begin{array}{l}\text { First } \\
\text { absorption } \\
\text { peak }(\mathrm{nm})\end{array}$ & $\begin{array}{l}\text { Emission } \\
\text { peak }(\mathrm{nm})\end{array}$ & $\begin{array}{l}\text { Concentration } \\
\left(\mathrm{nmol} \mathrm{ml}^{-1}\right)\end{array}$ \\
\hline $\begin{array}{c}\text { Adirondack } \\
\text { Green }\end{array}$ & $<400$ & 510 & 526 & 77.03 \\
$\begin{array}{c}\text { Catskill } \\
\text { Green }\end{array}$ & $<400$ & 525 & 543 & 68.77 \\
$\begin{array}{c}\text { Hops } \\
\text { Yellow }\end{array}$ & $<400$ & 531 & 555 & 57.17 \\
\hline
\end{tabular}

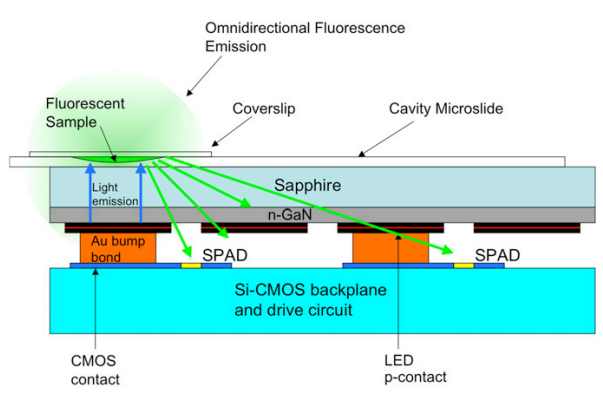

Figure 4. Schematic overview of experimental setup. The transparent sapphire substrate allows the fluorescence emission to pass and reach the SPAD below.

eliminate photon counts due to direct LED excitation of the SPAD. Photon counts due to directly coupled light from the LEDs have been further minimized by choosing a $370 \mathrm{~nm}$ UV excitation source. At $370 \mathrm{~nm}$, the PDP of the SPADs is $7 \%$. However, this wavelength is ideal for the excitation of the selected quantum dots. At $526 \mathrm{~nm}$ (the emission wavelength of the Adirondack Green quantum dots) the PDP of the SPADs increases to 25\% (after depassivation). This insensitivity to shorter wavelengths results in filtering of the excitation as summarized in figure 5 , which shows the spectral characteristics of the LED, Adirondack Green sample and SPAD. The LED and Adirondack Green emission spectra were measured using a Triax 550 spectrometer, with the Adirondack Green excited by a Power Technology $15 \mathrm{~mW}$, $375 \mathrm{~nm}$ UV laser. For the absorption, $200 \mu \mathrm{l}$ of Adirondack Green was mixed with $1800 \mu \mathrm{l}$ of toluene in a quartz cuvette and its absorption spectrum measured with a Lambda 2 spectrophotometer.

Each micro-LED pulse is synchronized to the rising edge of the system clock. This clock is therefore used as the synchronization pulse for the TCSPC card (Becker and Hickl, SPC-730). The TCSPC card is operated in reverse mode and the pulse train from the SPADs are passed via a pulse inverter to the constant fraction discriminator input of the card. The micro-LED pulse frequency is set by the system clock and is user definable, for these experiments a pulse frequency of $3.6864 \mathrm{MHz}$ was chosen. The micro-LED electrical drive pulse width is also user definable via software control of the chip. The user can select pulse widths from $400 \mathrm{ps}$ up to $48 \mathrm{~ns}$ in steps of $400 \mathrm{ps}$. This allows the user to
B R Rae et al select an excitation pulse that best suits the sample of interest. For the quantum dots used in this study, an excitation pulse width of $8 \mathrm{~ns}$ was chosen. A photograph of the micro-LED array exciting a sample of Adirondack Green quantum dots mounted on a micro-cavity slide is shown in figure 6.

\section{Results}

The signal generated by the on-chip SPADs is passed to the TCSPC card which creates a histogram based on photon arrival times, relative to the excitation pulse. As mentioned above, due to the absence of any optical spectral filtering in the system, the excitation pulse is also detected by the SPADs and can be observed in the resulting histogram as the large broad peak on the left. The ratio of detected excitation light to detected fluorescence emission is dependent on several factors. One such factor is the intensity of excitation light from the LED. This can be controlled by the user by adjusting the peak voltage, the bias current of the driver and the width of the excitation pulse. The intensity of fluorescence emission from the sample is also an important factor. Finally, the relative distance from the excitation micro-LED and the relevant detection SPAD affects the level of directly coupled light from the LED reaching the detector. This affects the ratio of the number of counts due to the LED pulse and the fluorescence emission, as demonstrated in figure 7.

As can be seen from the results in figure 7, the fluorescence decay curves can be clearly distinguished from the instrument response function (IRF). The data gathered are exported and analysed using FAST $^{\mathrm{TM}}$ lifetime extraction software by Edinburgh Instruments. Discrete deconvolution of the IRF and decay curves presented in figure 7 yields lifetimes of $17.8 \mathrm{~ns}$, $17.2 \mathrm{~ns}$ and $19.1 \mathrm{~ns}( \pm 104 \mathrm{ps}$ estimated measurement error, based on $100 \mathrm{ps}$ jitter from the SPAD and $4 \mathrm{ps}$ resolution of the TCSPC hardware) for Adirondack Green, Catskill Green and Hops Yellow, respectively. This is in good agreement with the lifetimes quoted by the manufacturer (15-20 ns) and measured independently using a photomultiplier tube as a detector. The noise floor of the results presented in figure 7 is also in good agreement with the $50 \mathrm{~Hz}$ dark count rate (DCR) of the SPADs quoted in the literature, which is strongly temperature dependent [17].

\section{Conclusions and discussion}

A compact fluorescence lifetime analysis system has been demonstrated, which incorporates sample excitation via a CMOS driven UV $16 \times 4$ micro-LED array and emission detection using an array of SPAD detectors located on the same CMOS chip. The AlInGaN micro-LED array was bump bonded to the SPAD and driver array which has been fabricated in a high-voltage $0.35 \mu \mathrm{m}$ CMOS technology and allows the user to define the excitation pulse and intensity.

The CMOS micro-LED driver was designed to deliver electrical pulses of variable width, from $400 \mathrm{ps}$ to $48 \mathrm{~ns}$. As a result of a larger than expected load capacitance, due to the bump-bonded micro-LED elements an optical pulse of $400 \mathrm{ps}$ was not achieved. Figure 8 demonstrates the longest 


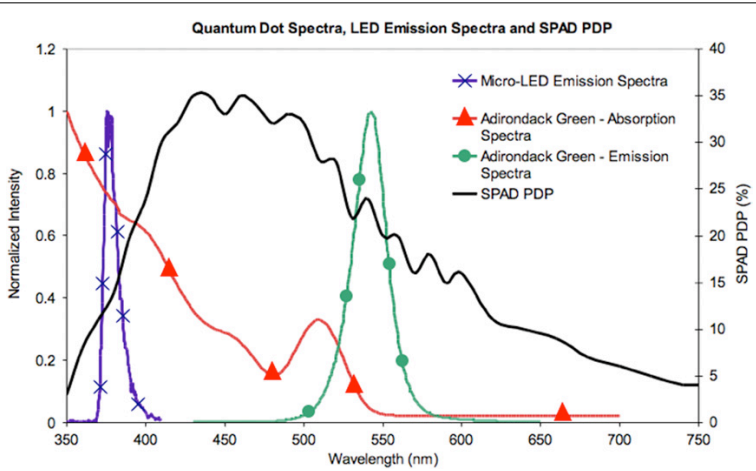

Figure 5. Graph of micro-LED emission spectra, SPAD PDP and quantum dot absorption (Adirondack Green) and emission spectra versus wavelength. This clearly highlights the spectral separation of the excitation wavelength and the detector sensitivity range.

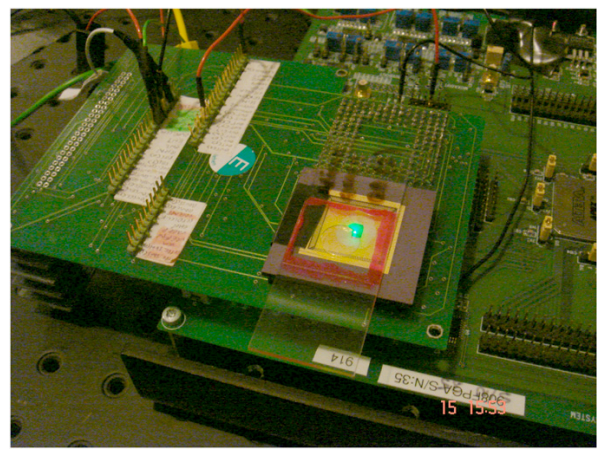

Figure 6. Photograph of micro-LED array exciting a sample of Adirondack Green quantum dots on a micro-cavity slide.

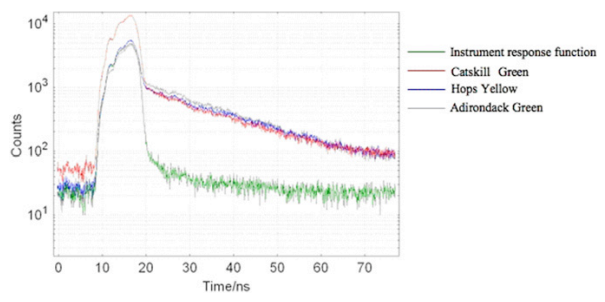

Figure 7. IRF and fluorescent decay curves for quantum dots at three different emission wavelengths.

(47.87 ns full width half maximum, FWHM) and shortest $(1.12 \mathrm{~ns}, \mathrm{FWHM})$ optical pulses achieved. While it has been shown that a pulse of $1.12 \mathrm{~ns}$ (FWHM) is possible, a pulse of $8 \mathrm{~ns}$ was chosen to conduct the lifetime experiments. As the pulse width is decreased, the intensity of the micro-LED emission is reduced, which in turn reduces the fluorescence

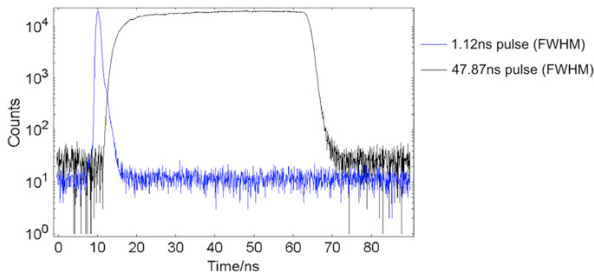

Figure 8. The longest ( $47.87 \mathrm{~ns}$, FWHM) and shortest (1.12 ns, FWHM) optical pulses achieved. Greater than expected capacitive load on the driver circuit output prevented shorter pulses being achieved.

emission. This leads to a lower signal-to-noise ratio and thus a less accurate lifetime measurement. To allow an $8 \mathrm{~ns}$ excitation pulse to be used, fluorophores with a relatively long lifetime were used. In addition to their compatible wavelength properties, as described previously, the 15-20 ns lifetimes of the quantum dot samples were ideally matched for excitation with an $8 \mathrm{~ns}$ excitation pulse.

Results have been presented which demonstrate the system's ability, in conjunction with external TCSPC hardware, to accurately measure fluorescence lifetime data of commercially available quantum dot fluorophore samples. The system removes the need for complicated optical alignment, with the sample being introduced into the system on a simple micro-cavity slide. The SPADs natural insensitivity to UV wavelengths has also been used to remove the need for optical filters. This system is, to the best of our knowledge, the first demonstration of a complete microsystem for TCSPC to incorporate both an array of excitation sources and an array of detectors with single photon sensitivity. While results are not yet comparable to more established experimental setups using commercially available equipment, this prototype system demonstrates the benefits and potential of an integrated system. The ability of such a microsystem, based on a standard CMOS process, to conduct lifetime analysis without 
J. Phys. D: Appl. Phys. 41 (2008) 094011

the need for expensive optics and filters should open the way to the development of a compact (potentially portable), robust and inexpensive solution to time resolved fluorescence measurements.

\section{Acknowledgments}

This work was funded by the UK Engineering and Physical Sciences Research Council, in part under the Basic Technology programme and under a Scottish Funding Council SRDG Award. We are grateful to Optocap Ltd, Livingston, UK for carrying out the bump-bonding process. We further thank Alec Ruthven at the Scottish Microelectronic Centre, Edinburgh, for chip bonding, the COSMIC laboratory facility at the University of Edinburgh for providing access to test equipment and laboratory space, Dr Paul R Edwards and Professor Robert W Martin at the Department of Physics, University of Strathclyde, for providing the quantum dot samples and Dr Olaf J Rolinski and Professor David J S Birch for allowing access to the spectrophotometer for absorption measurements.

\section{References}

[1] Nakamura S and Fasol G 1997 The Blue Laser Diode: GaN Based Light Emitters and Lasers (New York: Springer)

[2] Lakowicz J R 1999 Principles of Fluorescence Spectroscopy 2nd edn (Dordrecht/New York: Kluwer/Plenum)

[3] Kung C E and Reed J K 1986 Microviscosity measurements in phospholipids bilaters using fluorescent dyes that undergo

[4] Sipior J, Carterm G M, Lakowicz J R and Rao G 1997 Blue light emitting diode demonstration as an ultra-violet excitation source for nanosecond phase-modulation fluorescence lifetime measurements Rev. Sci. Instrum. $682666-70$

[5] Jares-Erijman E A and Jovin T M 2003 FRET Imaging Nature Biotechnology 21 1387-95

[6] Gratton E and Limkeman M 1983 A continuosly variable frequency cross-correlation phase fluorometer with picosecond resolution Biophys. J. 44 215-324

[7] Buurman E P, Sanders R, Draaijer A, Gerritsen H C Van Veen J J, Houp P M and Levine Y K 1992 Fluorescence lifetime imaging using a confocal laser scanning microscope Scanning 14 155-59

[8] Matthews D R, Summers H D, Njoh K, Errington R J, Smith P J, Barber P, Ameer-Beg S and Vojnovic B 2006 Technique for measurement of fluorescence lifetime by use of stroboscopic excitation and continuous-wave detection Appl. Opt. $452115-23$

[9] Araki T and Misawa H 1995 Light emitting diode-based nanosecond ultraviolet light source for fluorescence lifetime measurements Rev. Sci. Instrum. 66 5469-72

[10] Davitt K, Song Y-K, Patterson W, Nurmikko A, Gherasimova M, Han J, Pan Y-L and Chang R 2005290 and $340 \mathrm{~nm}$ UV LED arrays for fluorescence detection from single airborne particles Opt. Express 13 9548-55

[11] Griffin C, Gu E, Choi H, Jeon C, Rolinski O, Birch D, Girkin J and Dawson M 2004 Fluorescence excitation and lifetime measurements using Ga/InGaN micro-LED arrays The 17t Annual Meeting of the IEEE Lasers and Electro-Optics Society (Western Rio Mar, Puerto Rico) vol 2 pp 896-97

[12] Becker W 2005 Advanced Time-correlated Single Photon Counting Techniques (Springer Series in Chemical Physics) (Berlin: Springer)

[13] Voh-Dinh T and Askari M 2001 Micro-arrays and biochips: applications and potential in genomics and proteomics J. Curr. Genomics 2 399-415

[14] Chodavarapu V P, Shubin D O, Barkowski R M, Titus A H, Cartwright A N and Bright F V 2007 CMOS-based phase fluorometric oxygen sensor system IEEE Trans. Circuits Syst. 54 111-18

[15] Cleary A, Glidle A, Laybourn P J R, Garcia-Blanco S, Pellegrini S, Helfter C, Buller G S, Aitchison J S and Cooper J M 2007 Integrating optics and microfluidics for time-correlated single-photon counting in lab-on-a-chip devices Appl. Phys. Lett. 91071123

[16] Gong Z, Zhang H X, Gu E, Griffin C, Dawson M D, Poher V, Kennedy G, French P M W and Neil M A A 2007 Matrix-addressable micropixellated InGaN light-emitting diodes with uniform emission and increased light output IEEE Trans. Electron. Devices $\mathbf{5 4} 2650$

[17] Niclass C, Sergio M and Charbon E 2006 A single photon avalanche diode array fabricated in deep-submicron CMOS technology Proc. Conf. on Design, Automation and Test in Europe (Munich, Germany) pp 81-6

[18] Niclass C, Rochas A, Besse P-A, Popovic R S and Charbon E 2005 CMOS imager based on single photon avalanche diodes IEEE Transducers 1 1030-4

[19] Evident Technologies EviDot Specifications http://www. evidenttech.com/products/evidots/evidot-specifications.html 
ISSCC 2008 / SESSION 8 / MEDICAL \& DISPLAYS / 8.3

8.3 A Microsystem for Time-Resolved Fluorescence Analysis using CMOS Single-Photon Avalanche Diodes and Micro-LEDs

Bruce R. Rae', Chris Griffin ${ }^{2}$, Keith R. Muir', John M. Girkin ${ }^{2}$ Erdan $\mathrm{Gu}^{2}$, David R. Renshaw', Edoardo Charbon', Martin D. Dawson', Robert K. Henderson

'The University of Edinburgh, Edinburgh, United Kingdom 2University of Strathclyde, Glasgow, United Kingdom
${ }^{3}$ Ecole Polytechnique Fédérale, Lausanne, Switzerland

Although microfluidics and microarray technologies are revolutionizing the throughput, sensitivity and cost in many areas of biodiag nostics [1], they are still reliant on bulky and expensive fluorescence analysis instrumentation. Conventional fluorescence intensity measurements are prone to misinterpretation due to illumination and fluorophore concentration non-uniformities. Thus, there is a growing interest in time-resolved fluorescence detection, whereby the characteristic fluorescence decay time-constant (or lifetime) in response to

We describe the first complete micro-scale time-resolved fluorescence analyzer to include excitation, detection and time-gated histogram generation (Fig. 8.3.1). It comprises a CMOS chip in 0.35um highvoltage technology incorporating a $16 \times 4$ array of SPADs [2], digital counters and LED drivers bump-bonded to an AlInGaN UV micropixellated light-emitting diode (micro-LEDs) array [3]. This system replaces instrumentation based on lasers, photomultiplier tubes, bulk optics and discrete electronics with a PC-based micro-system.

The micro-LEDs in the $16 \times 4$ array are individually addressable, have a $100 \mu \mathrm{m}$ pitch and each have a diameter of $72 \mu \mathrm{m}$. They were fabricated from 'standard UV LED wafers grown on c-plane sapphire substrates by metal-organic chemical vapour deposition (peak ar array and the driving CMOS chip was made using a bump-bonding pres river each eloring circuitry for within $200 \times 100 m^{2}$ ell, with $19090 \mathrm{~m}^{2}$ of inctro tectrode surface. The array of electrodes had their passivation selectively ro bum thodes for bump-bonding. A post-processing step of oxygen plasma shown to improve the photon detection probability of the SPADs a factor of 2 to 5 [3].

The SPAD allows single photon detection through the action of avalanche breakdown in a $\mathrm{p}+$ /deep $\mathrm{n}$-tub photodiode, reverse biase above its breakdown voltage (Geiger mode). The detection of a photon and the subsequent avalanche breakdown of the SPAD generate $40 \mathrm{~ns}$ digital pulses with $114 \mathrm{ps}$ full width at half-maximum jitter, which are processed by time-gated on-chip ripple counters [4]. These counters are situated in a second array (Fig. 8.3.1) pitch-matched to the micro-LEDs, allowing histogram and lifetime analysis withou the need for external photon counting hardware and significantly reducing the amount of data to be broadcast off-chip. Direct observation of SPAD output pulses is also possible from an array of addressable SPADs situated directly within the micro-LEDs for confirmatio of the integrated lifetime analysis techniques. Figure 8.3.2 illustrates the optical paths of excitation light and the returning fluores cence emission to these SPADs from a sample held in a micro-cavit slide.

The counter array circuitry consists of X- and Y-address decoders, timing generation circuitry and the pixel array (Fig. 8.3.3). Each SPAD has two associated 9b ripple counters (Fig. 8.3.4) as well as time gate and address decode logic. The two counters allow photons from two different time periods to be collected simultaneously. Thi algorithm proposed in [5];

$$
\tau=\Delta T / \ln \left(\frac{T G 1}{T G 2}\right)
$$

where $\tau$ is the lifetime of the fluorophore, $\Delta T$ is the width of both the time gates and TG1 and TG2 are the number of counts gathered in the first and second time gates, respectively.
SPAD pulses provide the asynchronous clock to the first T-type flipflop $(\mathrm{FF})$ in the counter. A ripple counter was chosen to minimize the clock loading, since no synchronous count behaviour is required. Time-gated operation is accomplished by providing the toggle input of the first T-type FF in the counter with short pulses, which are genof the fist T-type Fin the counter with short pulses, which are generated within the pixel from delayed versions of the $3.68 \mathrm{MHz}$ system the loading on the clock waveforms and the required bandwidth of the bus drivers are minimized.

The timing generator consists of a 120-element tapped delay line composed of current limited buffers. The buffer unit delay is $408 \mathrm{ps}$ with $44 \mathrm{ps}$ RMS jitter at $3.3 \mathrm{~V}$ at room temperature. Three delayed versions of the $3.68 \mathrm{MHz}$ system clock are generated, each delayed output can be selected independently under the control of a latched shift register. Time-gate widths can be selected from $408 \mathrm{ps}$ to $48 \mathrm{~ns}$ with a resolution of $408 \mathrm{ps}$.

LEDs are addressed using the same X- and Y-decoders and electrical pulses are defined using the on-chip timing generator (Fig. 8.3.3). pulses are defined using the on-chip timing generator (Fig. 8.3.3). Fig. 8.3.5. Short electrical pulses are applied to the micro- sED, with Fig. 8.3.5. Short electrical pulses are applied to the micro-LED, with a width equal to the delay between SQIN and SQIND. The turn-on
voltage of the UV micro-LEDs is around 4.5V, so high voltage D-MOS voltage of the UV micro-LEDs is around $4.5 \mathrm{~V}$, so high voltage D-MOS transistors have been employed in the driver circuit. Current pulstem clock.

Photon counts due to directly coupled light from the LEDs have been minimized by choosing a $370 \mathrm{~nm}$ UV excitation source where the photon detection probability (PDP) of the SPADs is $7 \%$. This wavelength is also ideal for the excitation of quantum dot fluorophores. At $526 \mathrm{~nm}$, the emission wavelength of the Adirondack Green quantum dots, the PDP of the SPADs increases to $25 \%$. Measurements were obtained using CdSe/ZnS quantum dots [6]. The quoted lifetime of these samples is 15 to $20 \mathrm{~ns}$.

Using the on-chip 2-gate RLD method with 10.2ns gate widths, lifetimes of $16.576 \mathrm{~ns}, 17.349 \mathrm{~ns}$ and $19.174 \mathrm{~ns}$ for Adirondack Green (concentration: $77.03 \mathrm{nmol} / \mathrm{ml}$ ), Catskills Green $(68.77 \mathrm{nmol} / \mathrm{ml})$ and tively. These are in good agreement with the lifetimes quoted by the manufacturer and those obtained by conventional quosurement. manufacturer and those obtained by conventional measurement.
Using SPADs situated within the micro-LED array, the decay of Adirondack Green and the instrument response function (IRF) were Adirondack Green and the instrument response function (IRF) were
captured and the data processed by external Becker and Hickl SPCcaptured and the data processed by external Becker and Hickl SPCgated counters, an equivalent fluorescence decay histogram of the Catskills Green quantum dot sample was captured in 26 time gates, each 800ps wide (Fig. 8.3.7).

Acknowledgements:

This work was funded by the UK EPSRC, in part under the Basic Technology programme and under a Scottish Funding Council SRDG Award. We are grate-
ful to Optocap Ltd, Livingston Microelectronic Centre, the COSMIC laboratory facility at the University of Edinburgh and Dr. Paul Edwards, Prof. Robert Martin, Dr. Olaf J. Rolinski and References:

[1] T. Vo-Dinh and M. Askari, "Micro Arrays and Biochips: Applications and

No. 2, pp. 399-415, 2001 .
[2] C. Niclass, M. Sergio and E. Charbon, "A Single Photon Avalanche Diode
Array Fabricated in Deep-Submicron CMOS Technology," IEEE Proc. of Design, Automation and Test in Europe, vol. 1, pp 81-86, March 2006. [3] C. W. Jeon, H. W. Choi, E. Gu and M. D. Dawson, "High-density matrix addressable AlInGaN-Based 368nm microarray light-emitting diodes," IEEE

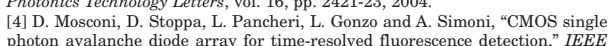
photon avalanche diode array for time-resolved fluorescence detection," IEEE Proc. of ESSCIRC, pp. 564-567, September 2006.

[5] H. C. Gerritsen, M. Asselbergs, A. V. Agronskaia and W. Van Sark, "Fluorescence lifetime imaging in scanning microscopes: acquisition speed, pho-
ton economy and lifetime resolution", Journal of Microscopy, Vol. 206, pt 3, pp. 218-224, June 2002

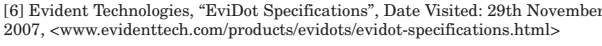


ISSCC 2008 / February 5, 2008 / 9:30 AM

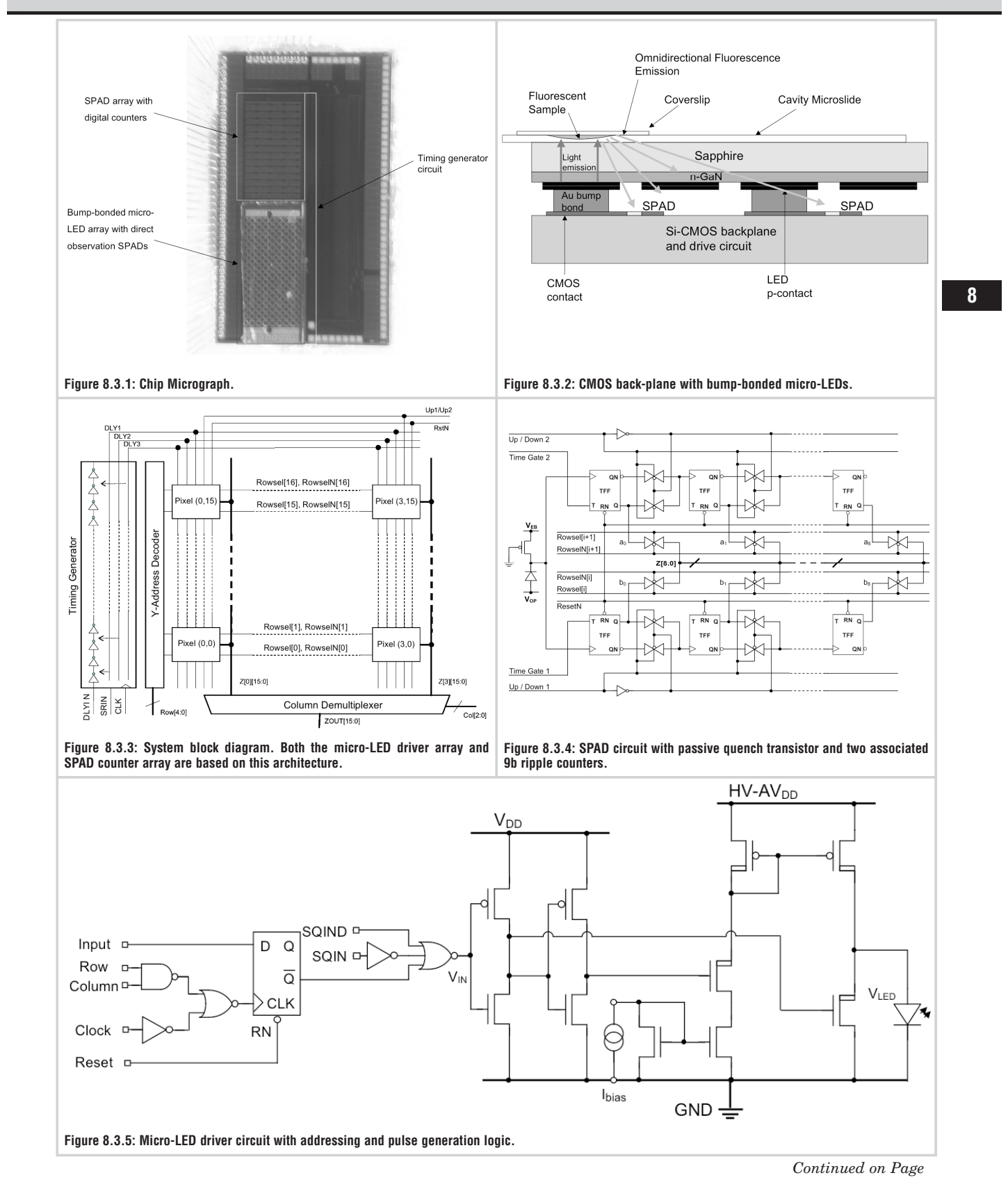


ISSCC 2008 PAPER CONTINUATIONS

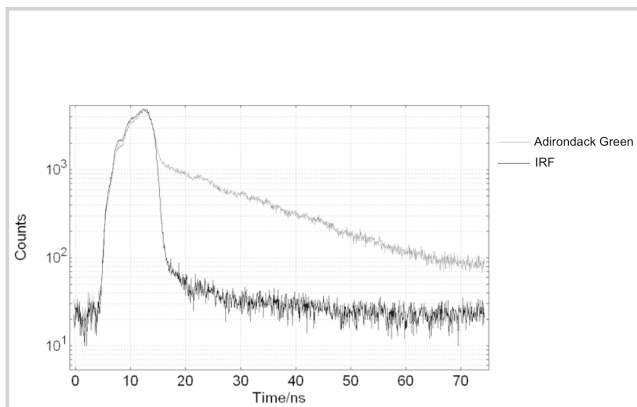

Fluorescence Lifetime Decay Curve of Catskills Green

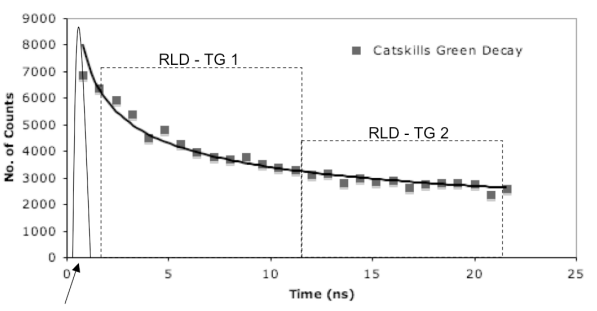

Micro-LED excitation

pulse

Figure 8.3.7: Fluorescence decay of Catskills Green quantum dot sample capFigure 8.3.6: Fluorescence decay of Adirondack Green quantum dot sample tured using the on-chip circuitry, in 26 time gates, each 800ps wide. An illusprocessed off chip using TCSPC hardware. vided. 


\title{
CMOS-integrated flip-chip, micro-pixel InGaN LED arrays for on-chip microfluorimetry
}

\author{
C. Griffin ${ }^{1}$, J. McKendry ${ }^{1}$, H. X. Zhang ${ }^{1}$, E. Gu ${ }^{1}$, B. R. Rae ${ }^{2}$, R. Henderson ${ }^{2}$, D. Renshaw ${ }^{2}$, J. M. Girkin ${ }^{1}$, and \\ Martin D. Dawson ${ }^{1}$ \\ ${ }^{1}$ Institute of Photonics, University of Strathclyde, Wolfson Centre, 106 Rottenrow, Glasgow G4 0NW, Scotland, UK \\ ${ }^{2}$ Institute for Integrated Micro and Nano Systems, Faraday Building, School of Engineering and Electronics, The University of \\ Edinburgh, The King's Buildings, Mayfield Rd, Edinburgh EH9 3JL, Scotland, UK \\ Abstract- $4 x 16$ arrays of micro-pixellated InGaN LEDs, each of diameter $72 \mu \mathrm{m}$, have been flip-chipped onto CMOS driver \\ backplanes which also contain single-photon avalanche photodiodes. Pattern-programmable control is demonstrated in \\ backplanes which also contain single-photon avalanche photodiodes. Pattern-programmable control is demonstrated in
continuous and nanosecond modes. Such devices show promise as miniaturized excitation and detection systems for \\ continuous and nanosec
microfluorimetry studies.
}

\section{INTRODUCTION}

Micro-pixellated InGaN light-emitting diodes ('micro-LEDs'), consisting of high-density 1-D or 2-D arrays of separatelyaddressable micro-sized elements that may each be pulsed in the nanosecond regime [1], are very promising sources for applications such as highly-parallel microfluorimetry and fluorescence lifetime studies $[2,3]$. There is also potential for these devices to form part of a monolithically integrated fluorescence detection systems with filters and microchannel sample delivery [4]. Initially investigated in 'epitaxy-up' configurations, where parallelism was accomplished primarily via matrix-addressing schemes, these devices have recently begun to be developed in flip-chip format. Flip-chipping offers device performance improvements and, importantly, facilitates direct bonding to a silicon backplane, allowing local access to the emitter pixels for true individual-element addressing. Furthermore, this format presents an optically-flat and chemically-resistant protective upper sapphire window (the epitaxial substrate, polished afterwards on its upper surface) to (i) keep the samples under analysis separate from the LED's, (ii) allow integration e.g. of microfluidics, and (iii) allow access to photodetectors which may be co-located on the silicon backplane. Here, we bring these considerations together by integrating a custom flip-chip micro-LED array with a the silicon backplane. Here, we bring these considerations together by integrating a custom flip-chip micro-LED array with a
dedicated Complimentary Metal-Oxide Semiconductor (CMOS) driver backplane which also contains silicon single-photon avalanche photodiodes (SPADs.)

\section{LED ARRAY / CMOS BONDING}

The micro-LEDs used were 16x16 arrays of individually addressable pixels, each pixel having a diameter of $72 \mu \mathrm{m}$ on a $100 \mu \mathrm{m}$ pitch. The devices were fabricated from 'standard' GaN LED wafers for blue $(470 \mathrm{~nm})$ and UV $(370 \mathrm{~nm})$ wavelength grown on cplane sapphire substrates by metal-organic chemical vapour deposition. Details of the respective wafer structures are given in references [5] and [6].

The CMOS chips consisted of a $4 \times 16$ array of $180 \mu \mathrm{m}$ wide $\mathrm{Al}$ pads on a $200 \mu \mathrm{m}$ pitch, plus extra chip area for control electronics and wirebonding. A high voltage chip fabrication process [7] was used to fabricate MOSFETs capable of switching up to $50 \mathrm{~V}$, while a low power level shifter transistor architecture [8] provided the interface between $5 \mathrm{~V}$ control signals and the high voltage output via a current mirror. Each CMOS pad was capable of providing $5 \mathrm{~mA}$ to an LED, and pulsed operation was possible clocked in the range of $1 \mathrm{kHz}$ to several $\mathrm{MHz}$ through the use of an external oscillator. The electrical pulse width could be varied down to a minimum of $400 \mathrm{ps}$ through varying the number of delay gates.

In addition, each CMOS plate comprised a Single-Photon Avalanche Diode (SPAD) of diameter 25 $\mu \mathrm{m}$, intended to detect the fluorescence signals of a sample placed on top of and excited by one of the LEDs. These were connected in turn to a Becker \& fluorescence signals of a sample placed on top of and excited by one of the LEDs. These were connected in turn to a Becker \&
Hickl SPC-730 photon counting card operated in reverse mode, in order to detect the LED pulses and fluorescence lifetimes of

samples.

The LED array and CMOS devices were bonded together using a thermosonic flip-chip process, whereby Au bumps were bonded onto the CMOS pads and the LED array was inverted then bonded on top. A fluorescent or scattering sample could be placed on top of the array (Fig. 1) and the LED temporal response or fluorescent signal could be detected by the SPAD.

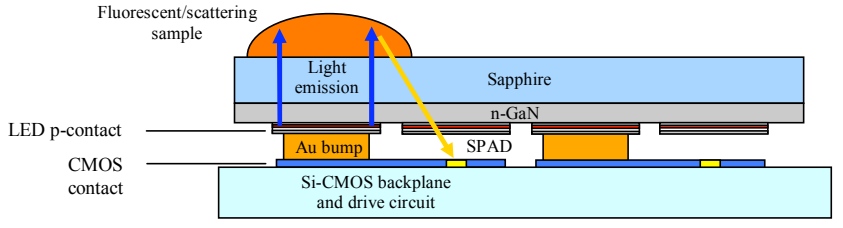

Fig. 1 Schematic of flip-chip bonded LED array/CMOS device 
The bonding process was successful as the LED elements were fully operational, and when being driven at the limits of the CMOS gave an optical output of $163 \mu \mathrm{W}$ with $25 \mathrm{~V}$ on the pad at $5 \mathrm{~mA}$ current [Note: this high voltage is not due to the LED I-V requirments, but the current limited CMOS driver]. Fig. 2 shows, by way of example, a programmable pattern on the combined CMOS/LED $470 \mathrm{~nm}$ array device being driven at $2.5 \mathrm{~mA}$ and $3.5 \mathrm{~V}$. This illustrates the highly visible light output from the device, its apparent uniformity, and its programmability.

The SPADs were measured to have an impulse response of 380ps FWHM when detecting pulsed output from a Pico-Quant 88ps, 480nm wavelength laser diode (Fig. 3). Using a Hamamatsu H5783 PMT, the minimum LED pulse width measured was 5ns FWHM (Fig. 4). This is considerably larger than the theoretical minimum of 400ps provided by the CMOS driver, but is attributed to the LED device having a larger capacitance than the CMOS was designed to drive. The LED capacitance was subsequently measured to be $3.5 \mathrm{pF}$. In addition the PMT had a rise/fall time of $780 \mathrm{ps}$ which would have contributed to the pulse width measurement.

Measurements with scattering and fluorescent samples placed on top of the LED array are presently underway in order to Measurements with scattering and fluorescent samples placed on top of the LED array are presently underway in order to
determine the LED pulse characteristics and time resolved fluorescence measurement capabilities with the SPADs. This points determine the LED pulse characteristics and time resolved fluorescence measurement capabilities with the SPADs. This points
the way towards a miniaturized time-resolved fluorescence system requiring a small sample volume, and capable of on-chip the way towards a min
single photon counting.
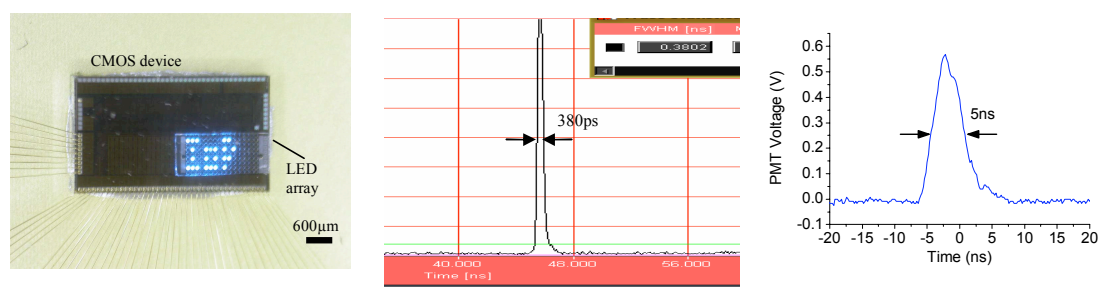

Fig. 2 Programmable operation of the LED/CMOS Fig. 3 Minimum time response of SPAD (380ps)

Fig. 4 Minimum pulse width (5ns) of micro-LED device showing 'IOP' pattern

IV. CONCLUSIONS

In summary, we have demonstrated a fully operational flip-chip micro-LED array driven by a CMOS backplane. This device is capable of showing a user programmed pattern and individual pixels have been driven in pulsed operation to a minimum width of $5 \mathrm{~ns}$. UV devices are currently under test and developments are under way in developing a full 16x16 CMOS driver for driving the full LED array. This driver will be capable of driving $100 \mathrm{~mA}$ to each LED element and will be impedance matched to the LEDs in order to produce sub-ns pulses. Results on this and the UV device will be presented, as will progress towards a fully minitarized microfluorimetry sytem for fluorescence lifetime detection.

ACKNOWLEDGEMENTS

We would like to thank Optocap of Livingston, UK for flip chip bonding.

REFERENCES

[1] S. X. Jin, J. Shakya, J. Y. Lin, and H. X. Jiang, "Size dependence of III- nitride microdisk light- emitting characteristics," Appl. Phys. Lett, vol. 78, pp. 3532-3534, 2001 .

H. Peng, E. Makarona, Y. He, Y. K. Song, A. V. Nurmikko, J. Su, Z. Ren, M. Gherasimova, S. R. Jeon, G. Cui, and J. Han, "Ultraviolet light-emitting diodes operating in the $340 \mathrm{~nm}$ wavelength range and application to time-resolved fluorescence spectroscopy," Appl. Phys. Lett., vol. 85, pp. 1436-

[3] C. Griffin, E. Gu, H. W. Choi, C. W. Jeon, O. J. Rolinski, D. J. S. Birch, J. M. Girkin, and M. D. Dawson, "Fluorescence excitation and lifetime measurements using GaN/InGaN micro-LED arrays," presented at 2004 IEEE LEOS Annual Meeting Conference Proceedings, Rio Grande, Puerto

E. Thrush, O. Levi, W. Ha, G. Carey, L. J. Cook, J. Deich, S. J. Smith, W. E. Moerner, and J. S. Harris, "Integrated Semiconductor Vertical-Cavity Surface-Emitting Lasers and PIN Photodetectors for Biomedical Fluorescence Sensing," IEEE J. Quant. Elec., vol. 40, pp. 491-498, 2004. C. W. Jeon, H. W. Choi, and M. D. Dawson, "Fabrication of Matrix-Addressable InGaN-Based Microdisplays of High Array Density," IEEE Phot. Tech. Lett., vol. 15, pp. 1516-1518, 2003.

C. W. Jeon, H. W. Choi, E. Gu, and M. D. Dawson, "High-Density Matrix-Addressable AlInGaN-Based 368-nm Microarray Light-Emitting Diodes,"

Industrial Technology, Maribor, Slovenia 2003 .

J. Doutreloigne, H. De Smeet, J. Van den Steen, and G. Van Doorselaer, "Low-power high-voltage CMOS level-shifters for liquid crystal display drivers," presented at The Eleventh International Conference on Microelectronics, Kuwait, 1999 
$2^{\text {nd }}$ EOS Topical Meeting on Optical Microsystems, $30^{\text {th }}$ Sept. $-3^{\text {rd }}$ Oct. 2007, Capri, Italy

TOPIC: New active and passive devices in optoelectronics

\section{MICRO-PIXELLATED FLIP-CHIP InGaN LIGHT EMITTING DIODES INTEGRATED WITH CMOS.}

J. McKendry ${ }^{1}$, C. Griffin ${ }^{1}$, H. X. Zhang ${ }^{1}$, Z. Gong ${ }^{1}$, B. Guilhabert ${ }^{1}$, D. Massoubre ${ }^{1}$, E. $\mathrm{Gu}^{1}$, M. D. Dawson 1 , B. R. Rae ${ }^{2}$, D. Renshaw ${ }^{2}$, and R. Henderson ${ }^{2}$.

${ }^{1}$ Institute of Photonics, University of Strathclyde, Glasgow, UK.

${ }^{2}$ School of Engineering and Electronics, The University of Edinburgh, Edinburgh, UK.

We report developments in micro-pixellated light-emitting diode arrays ('micro-LEDs') with elements that can be individually addressed via a silicon backplane. These microLEDs can be designed to emit spectrally-selective output at any wavelength in the UV to blue and green range.

These versatile micro-structured devices have potential in many areas of optical lab-ona-chip, including sectioning optical microscopy, time resolved fluorescence imaging or assays and the synthesis or read-out of DNA microarrays.

The devices to be reported are 'flip-chipped', resulting in improved current-spreading, heat dissipation and, hence, output power. This scheme utilises the transparent sapphire substrate as the top surface, thereby protecting the LED elements and offering a stable surface on which to locate e.g. microfluidics and tissue, dye or polymer samples. This geometry allows the integration of microlenses onto the sapphire window, enabling projection of the light from each element.

The current flip-chip devices consist of a $16 \times 16$ array of elements, each with a $72 \mu \mathrm{m}$ diameter on a $100 \mu \mathrm{m}$ pitch, which can be individually addressed. These have been controlled from a CMOS drive circuit chip, which also contains an array of SinglePhoton Avalanche Diode (SPAD) photodetectors.

We have shown this configuration to be capable of addressing individual LEDs in constant drive or nanosecond pulsed regimes, with output pulses as short as 5ns. Given our results we believe this is a promising route to highly-parallel, on-chip single-photon microfluorimetry. 


\title{
Anodic $\mathrm{Ta}_{2} \mathrm{O}_{5}$ for CMOS Compatible Low Voltage Electrowetting-On-Dielectric Device Fabrication
}

\author{
Y. Li, W. Parkes, L.I. Haworth, A.A. Stokes, K.R. Muir, P. Li, A.J. Collin, N.G. Hutcheon, \\ R. Henderson, B. Rae and A.J. Walton \\ Scottish Microelectronic Centre (SMC), School of Engineering and Electronics, \\ The University of Edinburgh, King's Buildings \\ Edinburgh, EH9 3JF, UK +44 1316505609 \\ Email: Y.Li@ed.ac.uk
}

\begin{abstract}
This paper reports a CMOS compatible fabrication procedure that enables ElecroWetting On Dielectric (EWOD) technology to be post-processed on foundry technology. With driving voltages less than $15 \mathrm{~V}$ it is believed to be the lowest reported driving voltage for any material system compatible with post-processing on integrated circuits. The process architecture uses anodically grown tantalum pentoxide as the pinhole free high dielectric constant insulator with the overlying $16 \mathrm{~nm}$ layer of Teflon- $A F^{\circledR}$, which provides the hydrophobic surface upon which droplets can be molpulat. This stack provides a very which droplets can be manipulated. This stack provides a very robust dielectric, which maintains a sufficiently high capacitance per unit area for effective operation at the lower voltage favoured by more standard CMOS technology. The paper demonstrates that the sputtered tantalum layer can be integrated with the aluminium (or copper) interconnect of foundry CMOS processes by standard microfabrication techniques.
\end{abstract}

\section{INTRODUCTION}

In recent years lab-on-a-chip and bio-MEMS systems, which can manipulate and analyse biological fluidic samples in micro- and nano-litre scales, have emerged as a solution for automating repetitive laboratory tasks [1], [2]. Digital microfluidic devices based on technologies such as dielectrophoresis (DEP), electrowetting on dielectrics (EWOD) and surface acoustic waves (SAW) provide a potentially reconfigurable method of obtaining a bio-MEMS system [2], [3]. Of these, EWOD technology is an attractive option that has a low power consumption making it well suited for the design and manufacture of microfluidic systems [2]. EWOD uses surface tension as a driving force, which can be controlled by applying a suitable voltage to an array of electrodes covered by a two layer dielectric.

A key parameter in EWOD technology is the driving voltage $V$. The initial work on electrowetting arrays required driving voltages in the range $80-100 \mathrm{~V}$ [4]. More recently with a more judicious choice of materials, processes and dielectric thickness, the voltage required to manipulate droplets has been reduced below 15V [4]. However, the temperatures required for the deposition of one of these dielectric layers is well in excess of $450^{\circ} \mathrm{C}$ [5] making the process non-compatible with CMOS post-processing. This paper reports a process architecture that matches the driving voltage of [4] while not involving processing temperatures anywhere near $450^{\circ} \mathrm{C}$.

\section{A. Background}

The technology of the electrocapillary phenomenon has been extensively described elsewhere [1], and will only be discussed briefly. For an EWOD system, the Young-Lippmann equation describes the wetting angle change for a droplet in terms of the applied voltage $V$, the relative dielectric constant $\varepsilon_{r}$, the liquid-gas surface tension $\gamma_{l g}$ and the thickness $t$ of the dielectric:

$$
\cos \theta(V)-\cos \theta(0)=\frac{\varepsilon_{r} \varepsilon_{0}}{2 \gamma_{l g} t} V^{2}
$$

Equation (1) identifies the important role played by the dielectric covering the electrodes in determining the driving voltage $V$ required to modify the contact angle $\theta\left(40^{\circ}\right.$ typically required for droplet movement). From the Young-Lippmann equation, it is clear that in order to reduce the droplet driving voltage, a dielectric with a higher permittivity is required. This is in addition to the requirement for this layer to be totally impervious to the liquid that forms the droplet being manipulated. Failure to meet this latter criterion leads to electrolytic action at the electrode causing the device to cease functioning. Hence, a robust pinhole free dielectric with a sufficiently high breakdown voltage that also acts as a barrier to the liquid is essential for any EWOD device. Finally the dielectric also has to display a hydrophobic surface, which is not normally available with materials meeting the above specifications. As a result, EWOD dielectrics typically consist of two layers; there is the insulating dielectric discussed above and the thin hydrophobic surface layer (e.g. Teflon- $\mathrm{AF}^{\circledR}$ ) that covers it.

B. Large EWOD arrays on a CMOS backplane

Practical, flexible and programmable EWOD systems designed to move, split and merge droplets with predetermined volume, require a large number of electrodes for complex operations. Examples are given in references [2], [6], [7] for sample analysis that use reagent mixing. Obviously each driving electrode in an EWOD device must be individually addressed from a contact pad via interconnect. While the interconnect for single and double rows of electrodes can be simply implemented on a single level of metallisation, this is not the case for arrays with electrode counts of $3 \times 3$ or greater. For 


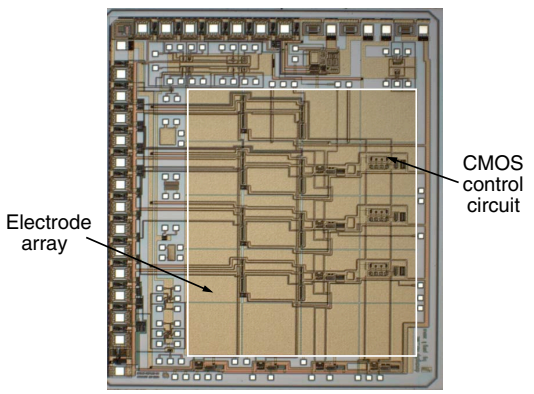

Fig. 1. EWOD electrode array (boxed area) controlled by backplane CMOS circuit [11].

arrays of EWOD electrodes multilevel metallisation is required with the realistic number of electrodes for passive systems being limited by packaging considerations. For example a $30 \times 30$ array would require a 900 pin package which is not practical.

Gong et al. partly address the packaging problem by using printed circuit board (PCB) technology together with land grid array (LGA) sockets [7]. The advantage of this solution is its low-cost and system flexibility (i.e., scalable). However, this does not solve the practical aspect of the interconnect problem entirely since a $32 \times 32$ digital microfluidic array used in [8] requires over 1000 pins. This is at the limit of a passive electrode drive system and any size bigger really requires an active controlling backplane.

CMOS technology has been widely used for row-column addressing of large numbers of elements, of which the largest application is related to memory devices. Others examples include CMOS imaging chips [9] and micro displays [10]. This approach to addressing arrays obviously lends itself to the realisation of EWOD arrays and, with a large matrix of electrodes, the use of CMOS for backplane row-column addressing enables the utilisation of existing technology. In addition it also makes it possible to provide the electrodes with additional capabilities such as sensing (e.g. pH, temperature, light, voltage etc) and actuation (e.g. temperature control).

Obviously clear advantages exist using on-chip addressing for large two-dimensional arrays. These include a significant reduction in the number of bond pads as well as the simplification of packaging. A dielectrophoresis (DEP) system using a CMOS solution has already been demonstrated [8]

As part of this work an EWOD/CMOS chip has been fabricated and tested [11] (figure 1) using conventional foundry processes and materials. Preparing it for EWOD activation requires post-processing involving the deposition of appropriate dielectric and surface treatment layers.

Previously reported low-voltage EWOD fabrications have used dielectrics deposited at high temperature (e.g. $700^{\circ} \mathrm{C}$ MOCVD for Barium Strontium Titanate [4], thermal oxida- tion [12], $700^{\circ} \mathrm{C}$ annealed $\mathrm{BZN}\left(\mathrm{Bi}_{2} \mathrm{O}_{3}-\mathrm{ZnO}-\mathrm{Nb}_{2} \mathrm{O}_{5}\right)$ [13]), none of which are compatible with CMOS technology with aluminium interconnect (deposition temperatures in excess of $450^{\circ} \mathrm{C}$ are required).

In this work the CMOS foundry process that was used to manufacture the EWOD device employed aluminium at its interconnect. Hence, this determined the electrode metallisation and the passivation layer. For typical foundry processes the passivation is a relatively thick dielectric layer $(0.5-1.0 \mu \mathrm{m})$ of silicon dioxide or nitride (or oxynitride), neither of which possesses a very high dielectric constant.

For an effective EWOD implementation using this dielectric technology, a comparatively high operating voltage in the region of $70 \mathrm{~V}$ is required to drive droplets. This is a direct consequence of the low dielectric constant associated with the passivation layer. Hence the high drive voltage requirement determined the selection of $100 \mathrm{~V}$ CMOS foundry process for the EWOD backplane. The post-processing and the demonstration of the completed EWOD device is presented in the next section.

Having fabricated and post-processed a high voltage CMOS EWOD device the challenge was to select an improved material system that was fully compatible with EWOD, while at the same time being suitable for integration with a lower voltage CMOS technology. The material system selected is based upon a tantalum pentoxide insulating layer (a high $\varepsilon_{r}$ dielectric which can be grown pinhole free), covered by a uniform and thin $\left(<20 \mathrm{~nm}\right.$ thick) overlying layer of Teflon- $\mathrm{AF}^{\circledR}$ to provide the required hydrophobic surface. This material system, which involves no high temperature process, simply consists of $\mathrm{Ta} / \mathrm{Ta}_{2} \mathrm{O}_{5} / \mathrm{Teflon}-\mathrm{AF}^{\circledR}$ layers. This is compatible with standard foundry CMOS IC technology for which the metallisation scheme is conventional aluminium interconnect.

\section{ANOdic $\mathrm{Ta}_{2} \mathrm{O}_{5}$ IN Low voltage EWOD For CMOS INTEGRATION}

A. Multilevel metallisation structure

In order to mimic the technology of a standard CMOS chip with aluminium interconnect (figure 2(a)), a two-level aluminium metallisation process was designed and an electrode array fabricated.

Initially, aluminium was used for both metal levels, as in the foundry CMOS chip discussed above. After the top-level metal was patterned it was covered with post-process dielectric layers of vapour deposited Parylene-C ${ }^{\circledR}$ and spin coated Teflon- $\mathrm{AF}^{\circledR}$ (figure 2(b)), both processes being performed at room temperature.

This completes the processing and droplet manipulation for this structure has been achieved using a drive voltage of $80 \mathrm{~V}$ (figure 3). By applying the same post-processes on the CMOS backplane chip in figure 1, droplet movement was achieved with a drive voltage of $60 \mathrm{~V}$ as shown in figure 4 .

B. Anodic $\mathrm{Ta}_{2} \mathrm{O}_{5}$ process

Anodically grown tantalum pentoxide is widely used in electrolytic capacitors to provide large area pinhole free di- 


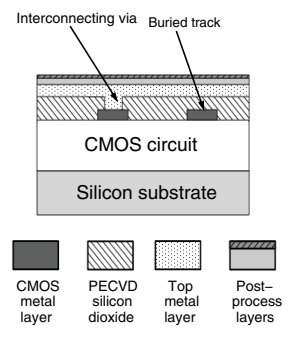

(a)

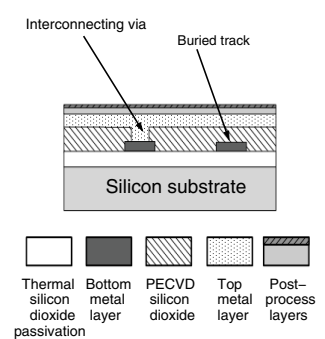

(b)
Fig. 2. (a) Cross-section of a CMOS backplane chip and the post-processed
layers, (b) cross-section of a two-level metallisation chip representing the CMOS chip and the post-processed layers.

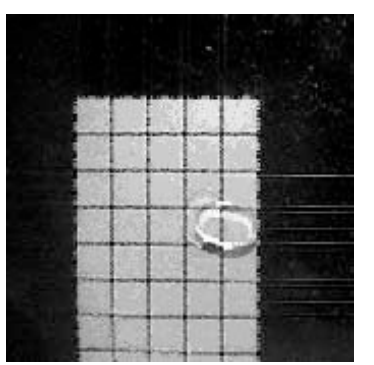

Fig. 3. A moving droplet on a two-level metallisation (aluminium) EWOD chip with a high driving voltage of $80 \mathrm{~V}$.

electrics. $\mathrm{Ta}_{2} \mathrm{O}_{5}$ has a higher $\varepsilon_{r}(\sim 20)$, than $\mathrm{SiO}_{2}$ or $\mathrm{Si}_{3} \mathrm{~N}_{4}$, and in combination with a thin Teflon- $\mathrm{AF}^{\circledR}(<20 \mathrm{~nm})$ layer results in a high capacitance, which reduces the driving voltage to less than $15 \mathrm{~V}$. The low temperature process associated with the EWOD dielectric sandwich allows it to be post-processed on top of standard CMOS foundry technology.

To demonstrate the $\mathrm{Ta}_{2} \mathrm{O}_{5}$-Teflon- $\mathrm{AF}^{\circledR}$ dielectric system the top aluminium layer in figure 2(b) was replaced by sputtered tantalum and patterned using the same mask. Tantalum can be etched in fluorine-containing plasmas such as $\mathrm{CF}_{4}, \mathrm{SF}_{6}$, $\mathrm{CF}_{3} \mathrm{Cl}$ with $\mathrm{CH}_{3} \mathrm{~F}$, sometimes mixed with $\mathrm{O}_{2}$ [14], [15]. The drawback is that these processes will potentially attack any

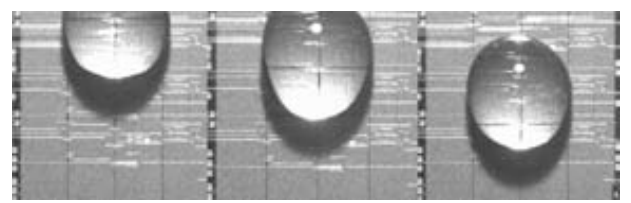

Fig. 4. A moving droplet (3 frames, left to right) on post-processed EWOD electrode array controlled by CMOS backplane. underlying PECVD $\mathrm{SiO}_{2}$ layer, which may be problematic if the tantalum etching is not uniform.

By using $\mathrm{SiCl}_{4}$ mixed with $\mathrm{NF}_{3}$ plasma, Shimada et al obtained an etch selectivity greater than 80:1 between tantalum and $\mathrm{SiO}_{2}\left(10: 1\right.$ in absence of $\left.\mathrm{NF}_{3}\right)$ [16].

An alternative is $\mathrm{XeF}_{2}$ dry etching, which is commonly used for silicon etch release in MEMS fabrication, especially postCMOS etch release due to its high selectivity to other materials (greater than 1000:1 for silicon to $\mathrm{SiO}_{2}$ and aluminium) [17]. It (greater than 1000:1 for silicon to $\mathrm{SiO}_{2}$ and aluminium) [17]. It
has also been observed to rapidly etch tantalum as well. The etch process was evaluated using a Memsstar ${ }^{\circledR}$ tool and no aluminium or $\mathrm{SiO}_{2}$ attack was observed. The only potential issue is the degree of undercut with an average value of $3.0 \mu \mathrm{m}$ on each side being measured when etching $0.45 \mu \mathrm{m}$ thick tantalum. As the gap between EWOD electrodes in this case is $30 \mu \mathrm{m}$, this undercut rate is acceptable and if need be, could be accounted for by a bias in the mask.

After patterning, the tantalum electrodes are anodised with a gel form citric acid solution. The anodising voltage is applied to every electrode on the chip through the EWOD control circuit in same manner as required for droplet manipulation.

The electrode control system consists of a PC digital I/O card controlled by a C program. The $5 \mathrm{~V}$ TTL digital outputs are optically isolated from the drive transistors which switch the drive voltage to any combination of electrodes under program control. This drive voltage can be set to any value between 0 and $100 \mathrm{~V}$.

After anodisation a thin Teflon- $\mathrm{AF}^{\circledR}$ layer is deposited using a standard spin coater on the oxidised tantalum electrodes. The measured surface roughness of the anodic $\mathrm{Ta}_{2} \mathrm{O}_{5}$ has been measured to have a mean roughness $R_{a}$ between 0.4 to $0.6 \mathrm{~nm}$ with the Teflon- $\mathrm{AF}^{\circledR}$ layer thickness uniformity across the wafer within $10 \%$.

The resulting EWOD array is a two-level metallisation structure which has aluminium as the bottom metal with tantalum as the second (top) metal electrode. A $50 \mathrm{~V}$ anodisation voltage was used resulting in $95 \mathrm{~nm}$ of $\mathrm{Ta}_{2} \mathrm{O}_{5}$. This was followed by a $0.3 \%$ Teflon- $\mathrm{AF}^{\circledR}$ (diluted in Fluorinert solvent FC-75) being spin coated at $2000 \mathrm{rpm}$ for $50 \mathrm{sec}$, giving $16 \mathrm{~nm}$ of Teflon-AF ${ }^{\circledR}$.

\section{EXPERIMENT AND RESUlts}

A common two-plate configuration EWOD chip [18] with asymmetric electrode arrangement has been used in the experiments to evaluate the $\mathrm{Ta}_{2} \mathrm{O}_{5} /$ Teflon- $\mathrm{AF}^{\circledR}$ system. A conductive indium tin oxide (ITO) covered glass plate coated with $20 \mathrm{~nm}$ Teflon-AF ${ }^{\circledR}$ was placed above the EWOD electrode array. Spacers were used to define the distance between the plates and hence the height of droplets. In this case the spacers were 258 microns.

This combination of dielectric materials on the EWOD device $\left(95 \mathrm{~nm} \mathrm{Ta}_{2} \mathrm{O}_{5}\right.$ and $16 \mathrm{~nm}$ of Teflon- $\left.\mathrm{AF}^{\circledR}\right)$ enabled deionized water to move at $14 \mathrm{~V}$, with a speed of $2 \mathrm{~mm} \cdot \mathrm{s}^{-1}$ (shown in figure 5). 


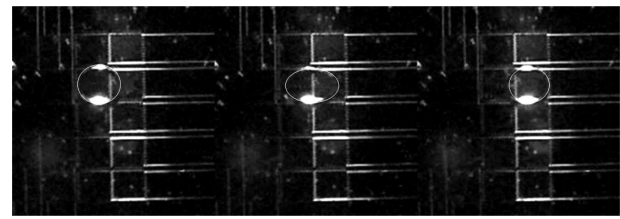

Fig. 5. Three frames (left to right) showing a moving droplet on a two-level
metallisation EWOD chip coated with $95 \mathrm{~nm} \mathrm{Ta}_{2} \mathrm{O}_{5}$ and $16 \mathrm{~nm}$ Teflon- $\mathrm{AF}^{\circledR}$ metallisation EWOD chip coated with $95 \mathrm{~nm} \mathrm{Ta}_{2} \mathrm{O}_{5}$ and $16 \mathrm{n}$ (the outlines of the droplet have been enhanced for clarity).

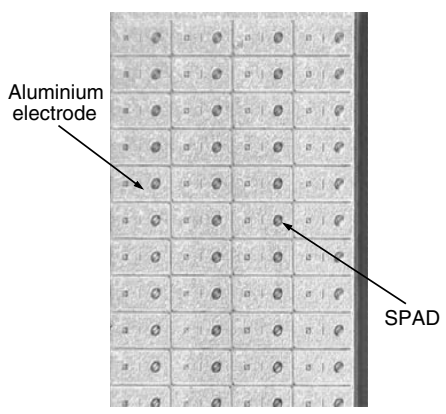

Fig. 6. A prototype design of an EWOD array with electrodes integrated with SPAD (Single Photon Avalanche Diodes) [19].

\section{Conclusion}

This paper has described what is believed to be the first fully CMOS compatible EWOD system that can drive liquid droplets using voltages less than $15 \mathrm{~V}$. The method of producing a thin uniform film of high permittivity dielectric by the anodisation of tantalum, together with a reliable method of spinning thin uniform Teflon- $\mathrm{AF}^{\circledR}$ films, are the keys to achieving the required low operating potential. The resulting system, with its robust and pinhole free anodised $\mathrm{Ta}_{2} \mathrm{O}_{5}$, provides a high dielectric constant and an impervious barrier to the liquids being transported, which is not always the case with deposited dielectrics.

In addition an EWOD post-process foundry CMOS chip has been processed and droplet movement demonstrated. The next step is to significantly increase the number of electrodes so that it becomes possible to implement a programmable electrode array size and start integrating further functionality into the electrodes. Figure 6 shows part of a prototype design that provides an example of both of these elements. It consists of an EWOD array with $200 \mu \mathrm{m} \times 100 \mu \mathrm{m}$ electrodes integrated with SPAD (Single Photon Avalanche Diodes) for light detection and this gives one example of the direction digital microfluidics will be moving in the future.

\section{AcKNowLEDGMEN}

The authors would like to acknowledge the financial support of EPSRC and the Edinburgh Research Partnership,
Dr. Edoardo Charbon from EPFL for providing the SPAD design integrated into the EWOD device shown in Figure 6 and $\mathrm{Memsstar}^{\circledR}$ technology for providing the $\mathrm{XeF}_{2}$ etching process.

\section{REFERENCES}

[1] F. Mugele and J.C. Baret "Electrowetting: from basics to applications", Journal of Physics, Condens. Matter, vol. 17, pages 705-774, 2005. . Oct 2006.

[3] A. Wixforth, "Acoustically Driven Programmable Microfluidics for Biological and Chemical Applications", JALA, pages 399-405, December 2006.

[4] H. Moon, S.K. Cho, R.L. Garrell and C.J. Kim, "Low voltage electrowetting-on-dielectric", Journal of Applied Physics, vol. 92, 7, pages 4080-4087, 2002.

[5] A.J. Walton, J.T.M. Stevenson, I. Underwood, J.G. Terry, S. Smith, W. Parkes, C. Dunare, H. Lin, Y. Li, R. Henderson, D. Renshaw, K. Muir, M. Desmulliez, D. Flynn, M.J. MacIntosh, W.S. Holland, A. F. Murray, T.B. Tang, A. Bunting, A.M. Gundlach, "Integration of IC technology with MEMS: Silicon+ technology for the future", IET Micro ElectroMechanical Systems (MEMS) Technology, To be presented, 25 April 2007,

[6] H. Moon, A.R. Wheeler, R.L. Garrell, J.A. Loo and C.J. Kim, "An integrated digital microfluidic chip for multiplexed proteomic sample preparation and analysis by

7] J. Gong and C.J. Kim, "Two-dimensional digital microfluidic system by multi-layer printed circuit board", MEMS2005, 18th IEEE conference,

8] P.R.C. Gascoyne, J.

Vykoukal, K.W. Current, C. McConaghy, F.F. Becker and C Andrews, "Dielectrophoresis-based programmable fluidic processors", Lab on a Chip, vol. 4, pages 299-309, 2004

[9] Wang, G.; Renshaw, D.; Denyer, P.B., "CMOS video cameras", Euro ASIC '91, pages 100-103, 27-31 May 1991.

10] I. Underwood, J. Gourlay, "Light-emitting polymer on CMOS: a new photonic technology", Proceedings of the SPIE, vol. 5181, pages 110-119,

11] Y. Li, P. Li, "Building EWOD Microfluidic Array Technology on Top of Foundry CMOS", MEMS Sensors and Actuators, The IET Seminar,

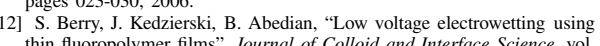
303, pages 517-524, 2006.

[13] N.Y. Kim, S.M. Hong, S.S. Park and Y.P. Hong, "The Movement of Micro Droplet with the effects of Dielectric Layer and Hydrophobic Micro Droplet with the effects of Dielectric Layer and Hydrophobic Surface Treatment with R.F. Atmospheric Plasma in EWOD Structure",
Journal of Physics, Conference Series, vol. 34, pages 650-655, 2006. [14] R. Hsiao and D. Miller, "Etching of Tantalum in Fluorine-Containing
High Density Plasmas", Journal of Electrochemical Society, vol. 143, pages 3266-3270, 1996.

[15] Y. Kuo, "Reactive Ion Etching of Sputter Deposited Tantalum with $\mathrm{CF}_{4}$ $\mathrm{CF}_{3} \mathrm{Cl}$, and $\mathrm{CHF}_{3}$ ", Japanese Journal of Applied Physics, vol. 32, pages

179-185, 1993.
[16] H. Shimada, K. Maruyama, "Highly selective Etching of Tantalum Electrode to Thin Gate Dielectrics Using $\mathrm{SiCl}_{4}-\mathrm{NF}_{3}$ Gas Mixture Plasma", Japanese Journal of Applied Physics, vol. 43, pages 1768-1772, 2004. 17] F.I. Chang, R. Yeh, G. Lin, P.B. Chu, E. Hoffman, E.J.J. Kruglick, and K.S.J. Pister, "Gas-phase silicon micromachining with xenon difluoride", vol. 2614, pages 117-128, 1995 .
vitonal Society for Optical Engineering,

18] M.G. Pollack and R.B. Fair, "Electrowetting-based actuation of liquid droplets for microluidic applications", Applied Physics Letters, vol. 77,

19] C. Niclass, M. Sergio

array fabricated in deep and E. Charbon, "A single photon avalanche diode Test Europ Conference, pages 81-86, 2006. 


\section{A 2-D Time Resolved Photon Counting Imager in 0.35 $\mu \mathrm{m}$ Technology}

\section{Bruce R. Rae ${ }^{1}$, David Renshaw ${ }^{1}$, Edoardo Charbon ${ }^{2}$ and Robert Henderson ${ }^{1}$}

${ }^{1}$ The University of Edinburgh, Institute for Integrated Micro and Nano Systems,

Edinburgh, U.K.

${ }^{2}$ Ecole Polytechnique Fédérale de Lausanne, Lausanne, Switzerland email: Bruce.Rae@ed.ac.uk

\section{Summary}

We describe a $16 \times 4$ pixel imaging system, realised in $0.35 \mu \mathrm{m}$ high voltage CMOS technology. Each $100 \mu \mathrm{m}$ pixel contains a single photon avalanche diode (SPAD) and two 9-bit counters capable of time-gated counting with 408ps resolution. Time-resolved data is displayed as $64 \times 120$ point histograms at a maximum rate of 3.05 frame/s.

\section{Introduction}

Here we introduce a 2-D CMOS time-resolved imaging system consisting of a fully addressable array of $16 \times 4$ 100um SPADs integrated with on-chip signal processing and timing circuits. The pixels incorporate two 9-bit ripple up-down counters with novel time-gating mechanism and address-decode logic, allowing fully programmable scanning of time-resolved events over a 48ns range with a 408ps resolution. The imager is controlled by a FPGA and photon count histograms are captured and displayed on a $\mathrm{PC}$ at a maximum refresh rate of $3.05 \mathrm{frame} / \mathrm{s}$. Histograms can be displayed real-time by a $P C$ video image frame-grabber allowing continuous monitoring of the profile and characteristics of fast time domain events such as would be encountered in time-of flight imaging [1] and fluorescence lifetime imaging [2-3]. These developments open the way to low-cost compact time-resolved, photon-counting imaging systems.

\section{Operating Modes}

Time-gated photon-counting is achieved by employing the SPAD pulses as the clock of the two 9-bit up-down ripple counters. Variable time windows are applied to the toggle input of the first counter. A ripple counter has been chosen for minimum area and fast operation speed as no synchronous count behaviour is required. Up-down capability is included in the counters for rejection of ambient illumination. Time gates are generated internally within the pixel from delayed versions of the system clock waveform broadcast to

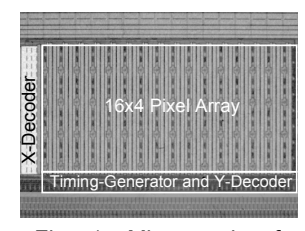

Fig. 1: Micrograph of Pixel Array the array. In this way, loading on the clock waveforms and

bandwidth requirements of the bus drivers are minimised. The entire pixel array has been implemented with standard library logic cells with no requirement for $A / D$ conversion as in conventional imagers.

The timing generator consists of a 120-element tapped delay line composed of current-limited buffers. The buffer unit delay is $408 \mathrm{ps}$, with $44 \mathrm{ps}$ RMS jitter at $3.3 \mathrm{~V}$ at room temperature. Three delayed versions of the system clock are generated and can be selected independently. Two time gating schemes have been implemented based on 
ideas from fluorescent lifetime imaging [4]. The first scheme sweeps the start and end times of two time windows across the two 9-bit counters in 408ps steps over a maximum range of $48 \mathrm{~ns}$. Thus, all photons in the $48 \mathrm{~ns}$ time interval are detected by either counter. The second scheme is a novel time gating method using a fixed time gate width (minimum 408ps). The start and end times of the time gate are swept across the range acting as a photon-counting oscilloscope. Data from each time bin is output as a count value in real time and can be displayed as a histogram image.

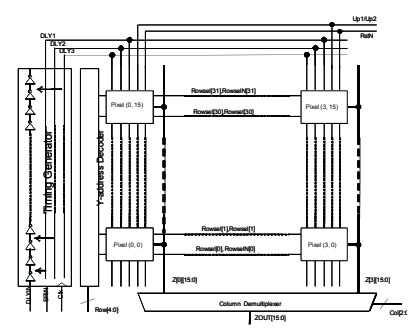

Fig. 2: Chip Block Diagram

\section{Results}

The die (Fig. 1) has had the polyimide passivation removed by oxygen plasma, providing around $3-5 x$ increase in photon detection probability in the $500 \mathrm{~nm}$ range. The system diagram (Fig. 2) consists of $X$ and $Y$ address decoders, timing-generation circuitry and the $16 \times 4$ pixel array. Each pixel contains a single $15 \mu \mathrm{m}$-diameter CMOS SPAD with performance reported in [5].

The laboratory evaluation setup consists of a laser diode emitting $88 \mathrm{ps}$ pulses at $468 \mathrm{~nm}, 5 \mathrm{~mW}$ average power, synchronized to the system clock. Fig. 3 demonstrates the two time gating schemes implemented. Sharp laser pulses impinging directly on the sensor are detected in specific time bins. The dramatic change in count value signifies where the laser pulse falls out with the time gate period.

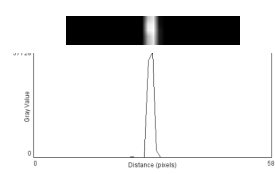

(a)

Fig. 3: Real time images from chip captured with both (a) scanning time gate and (b) decreasing time gate width. Each column represents a different time gate window. A profile of each pixel value in the $x$ plane is included.

\section{Conclusions}

We have demonstrated a CMOS time resolved single photon counting imager that can be operated in a number of user-definable time-gating schemes and can output real-time data which can be displayed in an easily interpretable image. On-chip signal processing reduces the need for any additional data analysis hardware while integrated timing generation results in low-jitter, accurate sub-nanosecond timed events.

\section{References}

[1] C. L. Niclass, Rochas, A., P. A. Besse and E. Charbon, "A CMOS single photon avalanche diode array for 3-D imaging", IEEE Solid-State Circuits Conference, Vol. 1, pp. 120-517, 2004

[2] G. Patounakis, K. L. Shepard and R. Levicky, "Active CMOS Biochip for Time-Resolved Fluorescence Detection", VLSI Circuits Symposium, pp. 68-71, 2005

[3] D. Mosconi, D. Stoppa, L. Pancheri, L. Gonzo and A. Simoni, "CMOS Single-Photon Avalanche Diode Array for Time-Resolved Fluorescence Detection", IEEE European Solid State Circuits Conference, Montreux, 2006

[4] H. C. Gerritsen, M. Asselbergs, A. V. Agronskaia and W. Van Sark, "Fluorescence lifetime imaging in scanning microscopes: acquisition speed, photon economy and lifetime resolution", Journal of Microscopy, Vol. 206, pt. 3, pp. 218-224, 2002

[5] C. L. Niclass, M. Sergio and E. Charbon, "A single photon avalanche diode array fabricated in deep submicron technology", Design, Automation and Test Europe Conference, pp. 81-86, 2006 


\section{A Buried Triple-Junction Self-Reset Pixel in a $0.35 \mu \mathrm{m}$ High Voltage CMOS Process}

Thomas D. Ross, Robert K. Henderson, Bruce Rae, David Renshaw

Institute of Micro and Nano Systems, University of Edinburgh, Edinburgh EH9 $3 \mathrm{JL}$

Tel +44 131650 5568, Fax: +44 131650 6554,E-mail: Robert.Henderson@ed.ac.uk

ABSTRACT - Light to frequency converters are used to sense the photocurrents of a buried triplejunction pixel achieving high dynamic range and low dark current colour sensing without the use of colour filters. The pixel is realised in a high voltage $0.35 u \mathrm{~m}$ CMOS enabling sample manipulation by electrowetting and spectral sensing for a FRET biosensor.

\section{INTRODUCTION}

Colour sensing without the need for surface optical filters has been achieved by using buried p-n junction structures in various CMOS processes [1$3]$. In addition to reducing process costs, this increases overall photon collection efficiency and eliminates the colour aliasing introduced by conventional Bayer pattern sub-sampling [4-5]. BiCMOS processes provide several $\mathrm{p}-\mathrm{n}$ junctions at different depths which can be employed to realise stacked triple-junction photodiodes [6]. In this paper, we present the use of a $0.35 \mu \mathrm{m}$ high voltage CMOS process for the realisation of such buried triple-junction (BTJ) photodiode structures (Fig. 1). High voltage handling and optical filterless colour sensing are required for electrowetting and spectral sensing for FRETbased biosensors [7-8]. The pixel itself employs light-to-frequency (L2F) techniques, and is presented in section II.

\section{CIRCUIT OPERATION}

BTJ sensors generally employ integrating mode photodiodes (Fig. 2) whereby the three junctions are reset to various potentials to keep the photodiodes reverse-biased. The diodes then integrate at different rates dependent on the spectral content of the light and their respective responsivities. Because the junctions are coupled, they will become forward biased if the signal in one spectral band is particularly high relative to the others, resulting in crosstalk between colour channels. Moreover, the stack of voltage levels uses up voltage headroom and requires careful management.

Self-resetting L2F converters are a popular means of realising very high dynamic range image sensors [9-11]. In the sensor proposed here, L2F converters are employed to sense the currents through each of the reverse-biased $\mathrm{p}-\mathrm{n}$ junctions of the BTJ structure (Fig. 3). The virtual ground of the amplifiers allows the junction potentials to be held constant at arbitrary levels during operation. In this case, the charge amplifier bias voltage, $\mathrm{V}_{\mathrm{CM}}$, is chosen to be $1.6 \mathrm{~V}$; approximately half of the $3.3 \mathrm{~V}$ supply. Thus, the blue and green junctions have zero potential across them, resulting in minimal dark current [12]. A $1 \mathrm{~V}$ swing was chosen for the integrators, resulting in $\mathrm{V}_{\mathrm{CP}}=2.6 \mathrm{~V}$ (Fig. 4). The charge amplifier of the middle junction integrates in the opposite sense, and so the comparator has been inverted, and its voltage threshold set to $\mathrm{V}_{\mathrm{CN}}=0.6 \mathrm{~V}$. The output frequencies of the top and bottom charge amplifiers are given by (1) and (3), while that at the output of the middle charge amplifier is given by (2), shown below:

$$
\begin{gathered}
f_{b}=\frac{I_{\text {blue }}}{2 C_{f}\left(V_{C P}-V_{C M}\right)} \\
f_{b g}=\frac{I_{\text {blue }}+I_{\text {green }}}{2 C_{f}\left(V_{C M}-V_{C N}\right)} \\
f_{\text {gr }}=\frac{I_{\text {green }}+I_{\text {red }}}{2 C_{f}\left(V_{C P}-V_{C M}\right)}
\end{gathered}
$$

Note that the $\mathrm{L} 2 \mathrm{~F}$ output frequencies represent the $I_{\text {blue }}, I_{\text {blue }}+I_{\text {green }}$, and $I_{\text {green }}+I_{\text {red }}$, respectively because of the summations of currents into the amplifier virtual earths. The frequencies are directly proportional to the photocurrents. $100 \mathrm{fF}$ poly-poly capacitors have been chosen as integrating 
capacitors setting the sensitivity of the sensor. The high linearity of these capacitors assures a linear relationship between photocurrent and frequency. The denominator terms in (1), (2) and (3) are designed to be identical by choosing matched integrating capacitance and $\left(\mathrm{V}_{\mathrm{CP}^{-}} \mathrm{V}_{\mathrm{CM}}\right)=\left(\mathrm{V}_{\mathrm{CM}^{-}}\right.$ $\mathrm{V}_{\mathrm{CN}}$ ). This allows the contributions of the individual $\mathrm{I}_{\text {blue }}, \mathrm{I}_{\text {green }}$ and $\mathrm{I}_{\text {red }}$ photocurrents to be dissociated by simple subtraction of the frequencies. A convenient hardware implementation is possible using up-down counters clocked by the output transitions of the blue/green or red/green L2F outputs.

An extremely high dynamic range is obtained $(>150 \mathrm{~dB})$. Saturation does not occur regardless of the spectral content of the illumination leading to good colour detection over a very high dynamic range.

In Fig. 5, an L2F structure is proposed with improved full scale-range. A switched-capacitor feedback structure allows independent choice of the photodiode voltage level and the comparator threshold voltage. The full range of the power supply can thus be used (between $\mathrm{V}_{\mathrm{CN}}$ and $\mathrm{V}_{\mathrm{CP}}$ ), maximising SNR and full-well capacity.

\section{MEASURED RESULTS}

The chip micrograph is shown in Fig. 6. The die has had the polyimide passivation removed by oxygen plasma, providing around $3-5 x$ increase in quantum efficiency in the $500 \mathrm{~nm}$ range. Four different BTJ structures were implemented using the various junction depths available. Fig. 7 shows a typical set of outputs of the L2F converters. The spectral response curves of photocurrents corresponding to the n-diffusion, p-well, deep nwell BTJ structures are shown in Fig. 8. The sensor performance is summarized in Table. 1. Dark current of the blue and green diodes with 0V reverse bias is not measurable while the dark current of the red photodiode with $1.6 \mathrm{~V}$ bias is $2 \mathrm{nA} / \mathrm{cm}^{2}$. A dynamic range of $>150 \mathrm{~dB}$ with frequencies ranging from $1 \mathrm{mHz}$ to $5 \mathrm{MHz}$ is obtained.

\section{CONCLUSION}

Self-reset loop structures can be applied to buried triple junction photodiodes to provide highdynamic range, filterless colour sensing. Low dark current with simple digital colour outputs are beneficial for biosensor applications.

\section{REFERENCES}

[1] Nozaki et al., "Color sensor," US patent 4,677,289, 1987.

[2] Gay et al., "Vertically integrated solid-state color imager," US patent 4,581,625, 1986.

[3] B. Chouikha et al., "Photodetector based on buried junctions and a corresponding method of manufacture," US patent 5,883,421, 1999.

[4] R.B. Merrill, "Color separation in an active pixel cell imaging array using a triple-wellstructure," US patent 5,965,875, 1999.

[5] R. Lyon and P. Hubel, "Eyeing the camera: into the next century," $10^{\text {th }}$ Color Imaging Conference: Color Science and Engineering Systems, Technologies, Applications, Scottsdale, Arizona, pp. 349-355, Nov. 2002.

[6] M. Ben Chouikha, G.N. Lu, M. Sedjil, and G. Sou1, "Colour detection using buried triple pn junction structure implemented in BiCMOS process," Electronics Letters, Vol. 34, No. 1, pp. 120-122, 1998.

[7] A R Mount et al., "The stability and characteristics of a DNA Holliday junction switch," J. Biophys Chem., Vol. 124, pp.214-221, Dec. 2006.

[8] K. Current et al., "A high-voltage CMOS VLSI programmable fluidic processor chip," Symposium on VLSI Circuits, pp. 72-75, 2005.

[9] J. Rhee, Y. Joo, "Wide dynamic range CMOS image sensor with pixel level ADC," Electronics Letters, Vol. 39, No. 4, pp. 360-361, 2003.

[10]S. Kavusi, K. Ghosh and A. El Gamal, "Architectures for high dynamic range, high speed image sensor Readout Circuits," IFIP VLSISoC, Nice, Oct. 2006.

[11] G. de Graaf, R.F. Wolffenbuttel, "Optical CMOS sensor system for detection of light sources," Sensors and Actuators A, Vol. 110, pp. 77-81, 2004.

[12] L. Simpson et al., "An integrated CMOS microluminometer for low-level luminescence sensing in the bioluminescent bioreporter integrated circuit," Sensors and Actuators B, Vol. 72, pp. 134-140, 2001. 


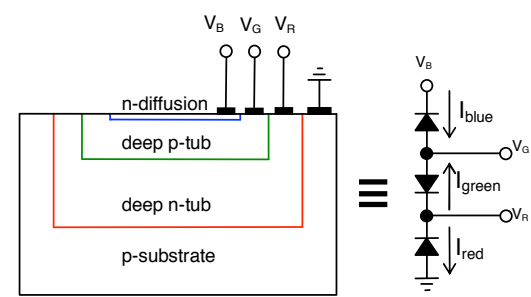

Fig. 1 Diagram of buried triple structure showing junctions and diodes

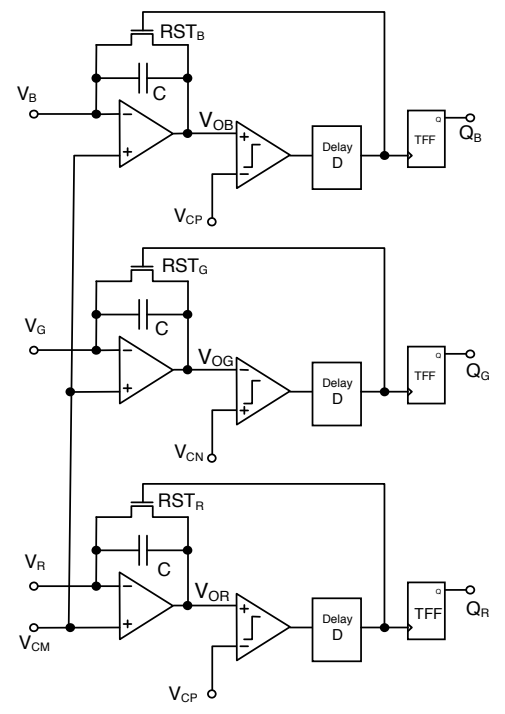

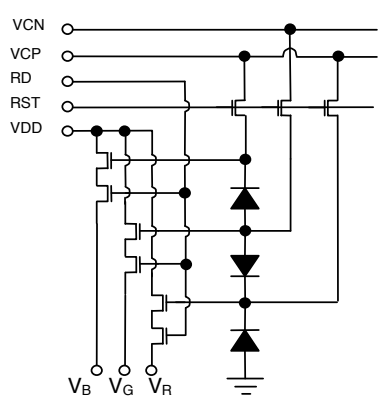

Fig. 2 BTJ source-follower readout
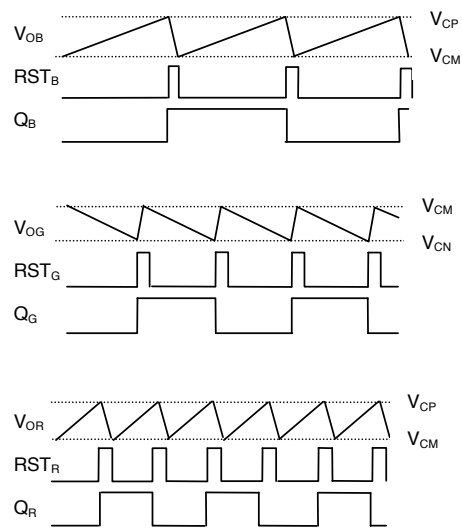

Fig. 4 Timing diagram showing the operation of the $\mathrm{L} 2 \mathrm{~F}$ readout

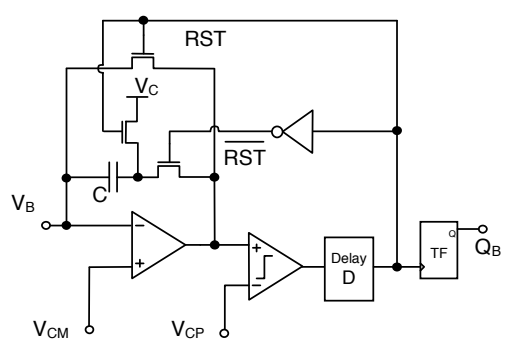

Fig. 5 L2F converter with optimised output swing 


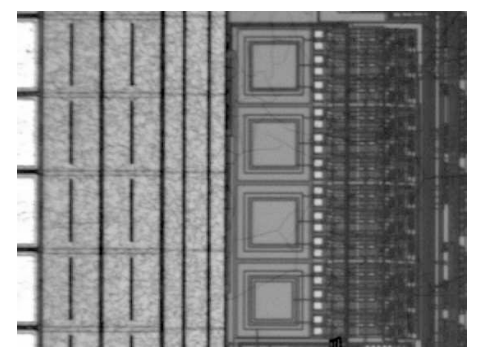

Fig. 6 Chip micrograph showing four $80 \mu \mathrm{m} x$ $80 \mu \mathrm{m}$ BTJ diode structures with L2F readouts

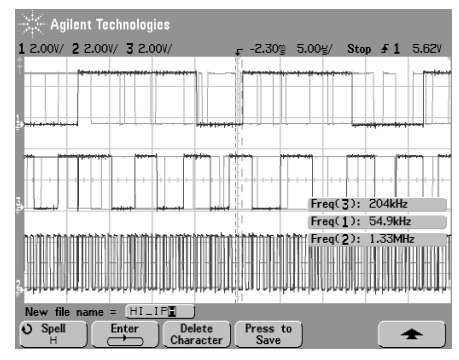

Fig. 7 Oscilloscope trace showing the three L2F outputs

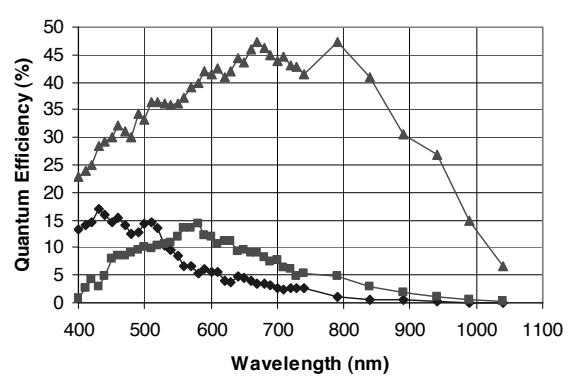

$\rightarrow$ Blue

$\rightarrow-$ Green

$\rightarrow$ Red

Fig. 8 Quantum efficiency versus wavelength for the three junctions

\begin{tabular}{|c|c|c|}
\hline Parameter & Value & units \\
\hline Process & $0.35 \mu \mathrm{m} 50 \mathrm{~V}$ 4LM 2P & $\mathrm{CMOS}$ \\
\hline Dynamic Range & $>150$ & $\mathrm{~dB}$ \\
\hline Dark current (red) & 2 & $\mathrm{nA} / \mathrm{cm}^{2}$ \\
\hline Dark current (blue, green) & Not observable & $\mathrm{nA} / \mathrm{cm}^{2}$ \\
\hline Capacitance & 100 (poly-poly) & $\mathrm{fF}$ \\
\hline Area & 0.016 & $\mathrm{~mm}^{2}$ \\
\hline Current consumption & 500 & $\mu \mathrm{A}$ \\
\hline Supply voltage & 3.3 & $\mathrm{~V}$ \\
\hline Full well & $625 \mathrm{k}$ & $\mathrm{e}$ \\
\hline Maximum frequency & 5 & $\mathrm{MHz}$ \\
\hline
\end{tabular}

Table 1 BTJ Sensor Performance Summary 


\section{Oversampled Time Estimation Techniques for Precision Photonic Detectors}

\author{
Robert Henderson, Bruce Rae, David Renshaw \\ School of Engineering and Electronics \\ University of Edinburgh \\ Edinburgh, Scotland, UK \\ Robert.Henderson@ed.ac.uk
}

Abstract-The use of oversampling to reduce I/O requirements of time-to-digital converters for arrays of high precision photonic detectors is considered. Simulation results show that the high linearity offered by oversampled converters can be applied to time estimation. The averaging and lowpass filtering inherent in these techniques reduce jitter and enhance estimates of mean time delay. Various sigma-delta TDC architectures are studied in the presence of background illumination noise. Applications in time-of-flight Ladar and fluorescence detection are studied.

\section{INTRODUCTION}

Accurate time measurement is commonly required for space science, high energy physics, range finding and fluorescence lifetime sensing. The key component of such systems, integrated Time-to-Digital Converters (TDCs), Time to Analogue Converters (TACs) or gated counters have achieved single-shot resolutions of 10's of picoseconds [1]. Often however, the quantity that must be estimated accurately is an average time delay between a cyclical stimulus and response. The conventional approach is to take many repeated single-shot time measurements and to construct event histograms. The average delay is then extracted from the mean of the photon arrival histogram. Averaging has two favourable effects; to reduce time uncertainty due to jitter and to increase SNR from spuriously generated events due to background noise. Particular examples of this are time-of-flight measurement or time-correlated single-photon counting techniques for fluorescence [2,3]

Detectors with both high time precision and sensitivity include photomultiplier tubes and avalanche photodiodes. Single-photon Avalanche Photodiodes (SPADs) have recently been realised in deep submicron CMOS processes [4]. Such detectors promise massively-parallel, singlephoton detection with extremely high timing accuracy and low dark count. The ability to integrate arrays of SPADs with on-chip TDCs or counters is expected to yield imagers with unprecedented sensitivity and dynamic range.

\author{
Edoardo Charbon \\ Ecole Polytechnique Fédérale de Lausanne (EPFL) \\ CH-1015 Lausanne, Switzerland \\ Edoardo.Charbon@epfl.ch
}

However, the high data bandwidths required to transmit photon arrival times or counts to off-chip memories for histogram construction are likely to have serious implications for power consumption, thermal effects and pin-count.

In this paper, we will apply oversampled techniques to improve the accuracy of average photon arrival time estimation and greatly reduce $\mathrm{i} / \mathrm{o}$ data bandwidth. This is of particular interest for arrays of photonic detectors such as SPADs which can be integrated together with the other readout and processing circuitry in a single chip.

\section{BACKGROUND}

A. Time to Digital Converters

Time to digital conversion is the process of converting time delay linearly into a numeric digital representation. Various architectures have been proposed with time resolutions down to a few picoseconds. However, the linearity of these converters has conventionally been limited to around 10-bits by inverter delay matching []. Jitter and temperature stabilization are other key performance criteria.

Sigma-delta converters have been employed very successfully to achieve very high resolutions and linearity at the cost of reduced bandwidth. A recent paper has proposed the use the advantages of a sigma-delta modulator in the context of estimation of on-chip clock jitter []. The authors construct a cascade of a mixer and lowpass filter with a sigma-delta modulator in order to achieve femtosecond time resolutions.

\section{B. Optoelectronic System}

The optoelectronic system which will be studied in this paper consists of a illumination source (usually a laser or laser diode) producing very short light pulses (femtosecond or picosecond) at $10-100 \mathrm{MHz}$. The illumination is reflected from a target and returns to a detector and TDC system synchronized to the laser by a clock. This system has been used to perform ranging by the time-of-flight method []. 
III. Sigma-DELTA TDC

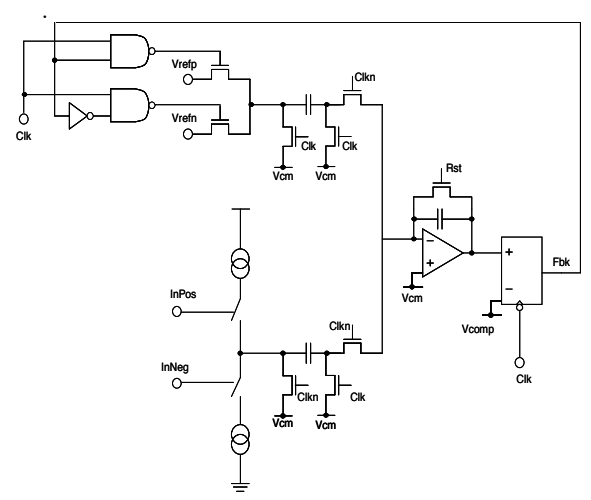

Figure 1. Sigma-delta Time to Digital Converter based on a first order MASH architecture

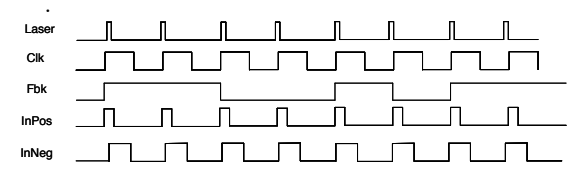

Figure 2. Timing for sigma delta TDC

Fig. 2 shows a circuit diagram of a simple first order MASH sigma-delta modulator with a time to voltage conversion input. There are many other modulator structures with superior performance to which the same concepts may be applied []. The operation of the modulator is controlled by the timing waveforms of Fig. 2 and is appropriate to any system with a repetitive pulsed illumination source

\section{A. Operation}

The two-phase switched-capacitor implementation of a first order MASH modulator operates to produce an output bit-stream fbk which will be passed to a lowpass decimation filter (not shown). The clock clk can operate at 10 's of $\mathrm{MHz}$ synchronized with the pulse repetition rate of the pulsed light source. Fast triggering events from the SPAD or other optical detector generate the waveforms InPos and InNeg. In particular falling edge the first photon after the laser pulse. Thus the time delay or the first photon after the laser pulse. Thus the time delay or
time-of flight is represented by the delay time between the falling edges of InPos and InNeg.
The current source charge the capacitor $\mathrm{C} 1$ to a produce a voltage proportional to the time delay in a similar way to a time-to-analogue converter (TAC) or the charge pump of a PLL. The overlap time between the on-state of the positive and negative current source is based on technique is used in PLL charge pumps to extend the linear range of conversion to very short time delays []. Since the current sources are to very short time delays []. Since the current sources are
connected passively to the capacitor $\mathrm{C} 1$ and common mode voltage $\mathrm{Vcm}$ during clk, fast open-loop settling to the nanosecond time intervals of the photonic detector can be achieved. The settling and current requirements of the integrating OTA are determined during the next phase clkn and have a full half clock period. At the end of the period $\mathrm{clk}$, capacitor $\mathrm{C} 1$ has been charged to a voltage linearly related to the delay time interval from laser pulse to the first photonic detected. A feedback decision has also been made by the comparator fbk to select either of the reference voltage Vrefn or Vrefp.

During the next phase clkn, the selected reference voltage and the voltage on $\mathrm{C} 1$ are integrated on the capacitor Cint. This process is repeated over many repetitions of the laser and clock waveform.

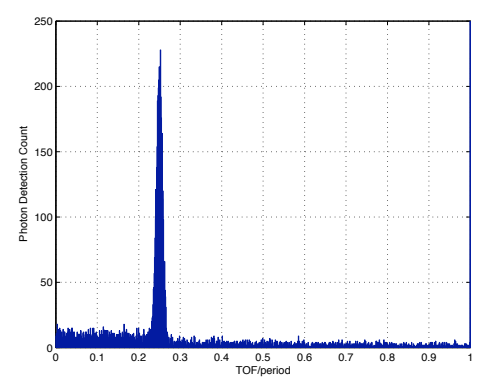

Figure 3. Sample histogram of first detected photon for $25 \mathrm{MHz}$ laser repetition rate, $10 \mathrm{~ns}$ time of flight and $2 \mathrm{Mphotons} / \mathrm{sec}$ background.

\section{B. Modelling}

To investigate the properties of the system a software model of the modulator and signal source has been developed. We take the particular example of a time-of-flight system where the return signal from the emitted femtosecond pulsed light source is consider to be a Gaussian distributed photon detection peak. This represents the aggregate jitter in the detection system [] and may originate from a number of sources. The mean of the Gaussian has an adjustable offset representing the time of flight (TOF) and standard deviation representing the jitter.

We also consider a background signal from ambient light or detector dark signal as a Poisson random process parameterized by a mean arrival rate in photons/sec. The 
output from the detector is considered to be a sequence of delay times of the first photon detected after the repeated laser pulse. This event may either be triggered by the reflected laser pulse or by a background event photon, whichever occurs first. The detector is considered to generate only one event per clock cycle.

Fig. 3 shows a histogram of photon detections for a clock frequency of $25 \mathrm{MHz}$, jitter of $300 \mathrm{ps}$ and background arrival rate of $10 \mathrm{Mphotons} / \mathrm{sec}$. In this example, we consider that photons are only received from the reflected source according to a linearly distributed probability. Thus some detector triggered events are received after the time-of-flight mean from background. Note that the jitter and TOF are normalized to the clock period in the forthcoming treatment.

\section{Simulation Results}

A second order MASH sigma delta modulator with oversampling rate $\mathrm{N}=256$ and $3^{\text {rd }}$ oder comb filter has been simulated. A sweep of TOF normalized to the clock period has been performed and a least mean squares fitting algorithm applied to the decimated modulator output in order to estimate linearity. A number of clock periods (10000) are used before analysis to avoid any transient effects. The noise level at the comb filter output is estimated from the standard deviation of the code over 10000 clock cycles.

Fig. 4 shows the output of the modulator and comb filter with no background noise and a 300ps jitter input with a $25 \mathrm{MHz}$ laser and system clock. As expected, the noise has been reduced by $\operatorname{sqrt}(\mathrm{N})$ or a factor of 8 from $300 \mathrm{ps}$ to $37.5 p s$. The linearity of the modulator is estimated to be around 10bits, limited by the RMS noise of the input. As the oversampling factor is increased both jitter and linearity are improved. Note that the jitter on the input signal also acts as a dither and reduces the build up of tones which are known to reduce modulator resolution [].

Fig. 5 shows the analysis repeated in the presence of 2Mphotons/sec Poisson arrival rate of background illumination. As the TOF is increased there is a greater probability of a background photon triggering the detector rather than the TOF signal. Below $0.1 \mathrm{TOF} /$ period we obtain the same improvement in resolution as in the case without background. Above this level the noise level and hence distance accuracy is steadily degraded. In Fig. 6 the level of background illumination is varied whilst keeping a fixed TOF input. The TOF estimates from the modulator are accurate until a background arrival rate.

The minimum and maximum errors from a least mean squares linearity fit on the data is shown in Fig. 7. Below the $0.1 \mathrm{TOF} /$ Period the data has good linearity and above we see a nonlinear departure and increasing uncertainty.

$$
\text { V. DISCUSSION }
$$

The simulation results indicate that without background light improvements in linearity, jitter and data rate can be obtained over building histograms from single-shot TDCs The effect of background is to cause departure from linearity above a TOF delay.

Suppression of integrated background detections can be obtained using windowed detection. Triggers from the detector are inhibited until the falling edge of the InNeg pulse. This pulse can be scanned by a variable delay to to a position close to the mean TOF. Thus spurious integrations due to background can be minimized.

An effect which has not been considered is the possibility that the detector has not been triggered within a clock period as would be the case in low light environments such as in fluorescent imaging. The absence of a trigger will result in a full-scale input to the sigma delta which will skew the average integrated pulse delay. This problem may be solved by creating an event-triggered sigma-delta modulator whereby an integration clock cycle is only generated when a signal pulse arrives. In the system of Fig. 1 the positive edge of clk must be suppressed if the state of InPos is still high at the falling edge of clkn.

A $1^{\text {st }}$ order sigma-delta TDC with a SPAD detector has recently been designed and sent for manufacture in a 0.35 um CMOS technology.

\section{ACKNOWLEDGMENT}

The assistance of Seyed Danesh in Matlab scripting is gratefully acknowledged. We acknowledge the support of the Scottish Funding Council for the Joint Research Institute with Heriot-Watt University which is a part of the Edinburgh Research Partnership

\section{REFERENCES}

[1] R. Staszewski, S. Vemulapalli, P Vallur, J. Wallberg, and P. T Balsara, "1.3 V 20 ps Time-to-digital converter for frequency synthesis in 90-nm CMOS", IEEE Transactions on Circuits an

[2] J. C. Jackson et al., "Characterization of geiger mode avalanche photodiodes for fluorescence decay measurements", Proc. of SPIE Vol. 4650-07, Photonics West, San Jose, CA, Jan. 2002.

[3] C. Niclass A. Rochas, P.A. Besse, and E. Charbon, "Design and Characterization of a CMOS 3-D image sensor based on single photon avalanche diodes", IEEE Journal of Solid-State Circuits,

[4] Collins, M.; Al-Hashimi, B.M.; Wilson, P.R., "On-chip timing measurement architecture with femtosecond resolution", Electronic Letters, Volume 42, Issue 9, 27, pp. 39-40, April 2006.

[5] C. Niclass, M. Sergio and E. Charbon, "A single photon avalanche diode array fabricated in deep submicron technology", Design

[6] S. Pellegrini, G. S. Buller, J. M. Smith, A. M. Wallace and S. Cova, "Laser-based distance measurement using picosecond resolution time-correlated single-photon counting", Meas. Sci. technology, 11 pp. 712-716, 2000 .

[7] W. Becker, "Advanced Time-Correlated Single Photon Counting Techniques", Springer-Verlag, Berlin 2005.

[8] Gokturk, S.B. Yalcin, H. Bamji, C., "A Time-Of-Flight Depth Sensor - System Description", Issues and Solutions", Computer Vision and Pattern Recognition Workshop, pp. 35-39, June 2004. 


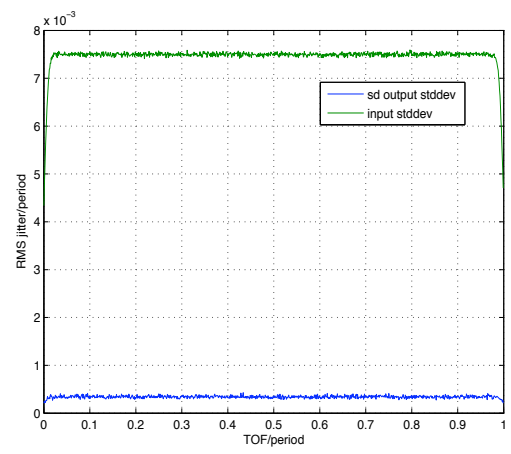

Figure 4. relative jitter of a $2^{\text {nd }}$ order modulator/comb filter versus TOF DC level for overampling ratio 256 , period $40 \mathrm{~ns}$, input jitter $300 \mathrm{~ns}$. An $8 \mathrm{x}$ reduction in jitter at the output has been

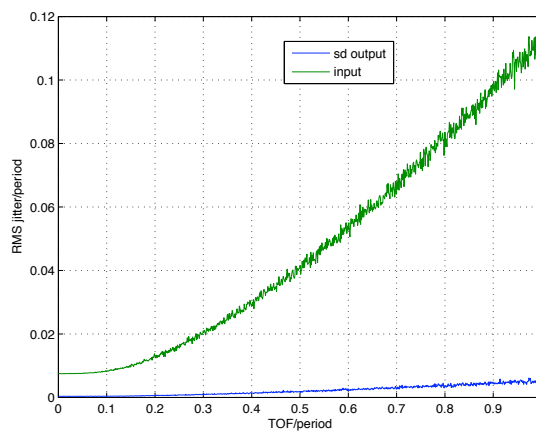

Figure 5. relative jitter of a $2^{\text {nd }}$ order modulator/comb filter versus TOF DC level for overampling ratio 256 , period $40 \mathrm{~ns}$, input jitter $300 \mathrm{~ns}$ in the presence of $2 \mathrm{Mphotons} / \mathrm{sec}$ background illumination.

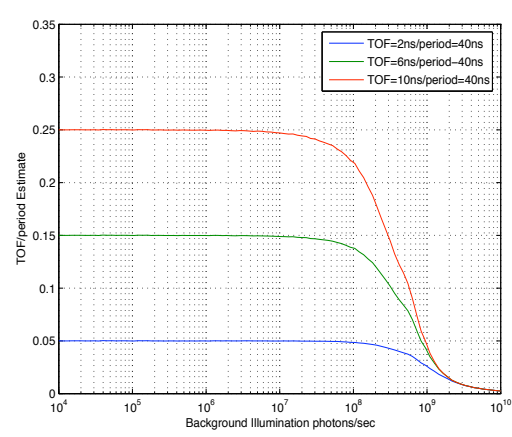

Figure 6. modulator ouptut estimate of TOF versus background illumination level for three different TOFs. Shorter TOFs are more resistant to background illumination level.

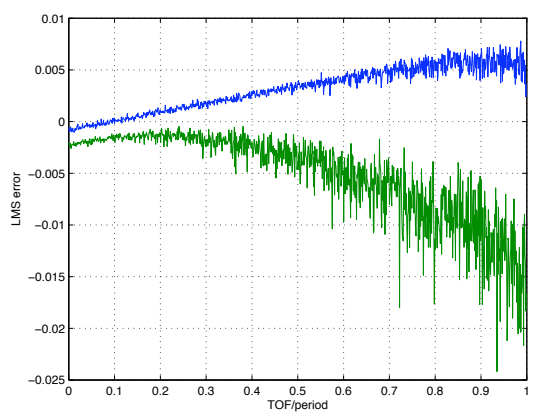

Figure 7. Minumum and maximum linearity error of a $2^{\text {nd }}$ order sigma-delta modulator versus TOF level for oversampling ratio 256 , period $40 \mathrm{~ns}$, input jitter $300 \mathrm{~ns}$ in the presence of
2 Mphotons/sec background illumination level. 


\section{References}

[1] X. Michalet, A. Kapanidis, T. Laurence, F. Pinaud, S. Doose, M. Pflughoefft, and S. Weiss, "The power and prospects of fluorescence microscopies and spectroscopies," Annual Review of Biophysics and Biomolecular Structure, vol. 32, pp. 161-82, 2003.

[2] R. Cubeddu, D. Comelli, C. D'Andrea, P. Taroni, and G. Valentini, “Time-resolved fluorescence imaging in biology and medicine," Journal of Physics D: Applied Physics, vol. 35, pp. 61-76, April 2002.

[3] PicoQuant-GmbH, “www.picoquant.com,” 2009.

[4] Edinburgh-Instruments, “www.edinst.com,” 2009.

[5] M. Davenport, "Detection and processing strategies for integrated xerogel-based sensor systems," Ph.D. dissertation, State University of New York at Buffalo, 2004.

[6] R. K. Henderson and C. Campbell, "A cmos microfluorometer for time-resolved fluorescence detection," RCUK Grant Proposal, 2005.

[7] J. Lakowicz, Principles of Fluorescence Spectroscopy, 2nd ed. Kluwer Academic / Plenum Publishers, 1999, no. ISBN 0306460939.

[8] Y. Ardeshirpour, M. Jamaldeen, and S. Shirani, "Two-dimensional cmos-based image sensor system for fluorescent detection," Canadian Journal of Electrical and Computing Engineering, vol. 29, no. 4, pp. 231-235, October 2004.

[9] G. Patounakis, K. Shepard, and R. Levicky, "Active cmos biochip for time-resolved fluorescence detection," 2005 Symposium on VLSI Circuits, vol. 2005 Digest of Technical Papers, pp. 68-71, June 16-18, 20052005.

[10] S. Turconi, R. P. Bingham, U. Haupts, and A. J. Pope, "Developments in fluorescence lifetime-based analysis for ultra-hts," Drug Discovery Today, vol. 6, pp. 27-39, 2001.

[11] M. Christenson and S. Sternberg, "Fluorescence lifetime imaging microscopy (flim) lets biologists study cellular processes," SPIE's oemagazine, pp. 28-30, January 2004. 
[12] R. Pepperkok, A. Squire, S. Geley, and P. I. H. Bastiaens, "Simultaneous detection of multiple green fluorescent proteins in live cells by fluorescence lifetime imaging microscopy," Current Biology, vol. 9, pp. 269-274, 1999.

[13] P. N. Prasad, Introduction to Biophotonics. Wiley-Interscience, 2003.

[14] V. Chodavarapu, S. Khanol, E. Tehan, A. Titus, A. Cartwright, and F. Bright, "Cmos integrated optical sensor using phase detection," Proceedings of IEEE Sensors, vol. 2, pp. 1266-1270, October 2003.

[15] A. Squire, P. J. Verveer, and P. I. H. Bastiaens, "Multiple frequency fluorescence lifetime imaging microscopy," Journal of Microscopy, vol. 197, pp. 136-149, 2000.

[16] W. Becker, Advanced Time-Correlated Single Photon Counting Techniques, 1st ed. Springer, 2005, no. ISBN-10 3-540-26047-1.

[17] H. Gerritsen, M. Asselbergs, A. Agronskaia, and W. van Sark, "Fluorescence lifetime imaging in scanning microscopes: Acquisition speed, photon economy and lifetime resolution," Journal of Microscopy, vol. 206, no. 3, pp. 218-224, June 2002.

[18] R. Krishnan, H. Saitoh, H. Terada, V. Centonze, and B. Herman, "Development of a multiphoton fluorescence lifetime imaging microscopy (flim) system using a streak camera," Review of Scientific Instruments, vol. 74, no. 5, pp. 2714-2721, May 2003.

[19] M. Straub and S. Hell, "Fluorescence lifetime three-dimensional microscopy with picosecond precision using a multifocal multiphoton microscope," Applied Physics Letters, vol. 73, pp. 1769-1771, 1998.

[20] D. Elson, S. Webb, J. Siegel, K. Suhling, D. Davis, J. Lever, D. Phillips, A. Wallace, and P. French, "Biomedical applications of fluorescence lifetime imaging," Optics and Photonics News, pp. 26-32, November 2002.

[21] S. Kleinfelder, K. Kwiatkowski, and A. Shah, "A solid state streak camera," Proceedings of SPIE, vol. 5580, pp. 235-243, 2005.

[22] D. Matthews, H. Summers, K. Njoh, R. Errington, P. Smith, S. Ameer-Beg, and B. Vojnovic, "Time-resolved fluorescence measurements using stroboscopic excitation," Proceedings of SPIE-OSA Biomedical Optics, vol. 5864, 2005. 
[23] D. Matthews, H. Summers, K. Njoh, R. Errington, P. Smith, P. Barber, S. Ameer-Beg, and B. Vojnovic, "Technique for measurement of fluorescence lifetime by use of stroboscopic excitation and continuous-wave detection," Applied Optics, vol. 45, pp. 2115-2123, 2006.

[24] D. Matthews, H. Summers, K. Njoh, R. Errington, P. Smith, S. Ameer-Beg, and B. Vojnovic, "Time-resolved fluorescence measurements using self-pulsing 650-nm laser diodes," Proceedings of SPIE, vol. 5705, pp. 23-31, 2005.

[25] P. Porta and H. Summers, "Vertical-cavity semiconductor devices for fluoresence spectroscopy and microfluidic platforms," Journal of Biomedical Optics, vol. 10, no. 3, May/June 2005.

[26] E. Thrush, O. Levi, W. Ha, G. Carey, L. Cook, J. Deich, S. Smith, W. Moerner, and H. J. J.S., "Integrated semiconductor vertical-cavity surface-emitting lasers and pin photodetectors for biomedical fluorescence sensing," IEEE Journal of Quantum Electronics, vol. 40, no. 5, pp. 491-498, May 2004.

[27] K. Davitt, Y.-K. Song, W. Patterson, A. Nurmikko, M. Gherasimova, J. Hans, Y.-L. Pan, and R. Chang, "290 and 340nm uv led arrays for fluorescence detection from single airborne particles," Optics Express, vol. 13, pp. 9548-9555, 2005.

[28] T. Araki and H. Misawa, "Light emitting diode-based nanosecond ultraviolet light source for fluorescence lifetime measurements," Review of Scientific Instruments, vol. 66, no. 12, pp. 5469-5472, December 1995.

[29] C. Griffin, E. Gu, H. Choi, C. Jeon, O. Rolinski, D. Birch, J. Girkin, and M. Dawson, "Fluorescence excitation and lifetime measurements using gan/ingan micro-led arrays," The 17th Annual Meeting of the IEEE Lasers and Electro-Optics Society, vol. 2, pp. 896-897, November 2004.

[30] R. Buss, M. Gross, T. Alder, W. Brockherde, and D. Jager, "8 x 8 gaasp/gap led arrays fully integrated with 64 channel si-driver circuits," Applications of Photonic Technology, 1995.

[31] R. Buss, F. Pramassing, D. Puttjer, N. Stawski, and D. Jager, "Photonic technologies for visual implants," International Conference on Application of Photonic Technology, 2002. 
[32] G. Patounakis, K. Shepard, and R. Levicky, "Active cmos array sensor for time-resolved fluorescence detection," IEEE Journal of Solid-State Circuits, vol. 41, no. 11, pp. 2521-2530, November 2006.

[33] H. Yoon, S. Itoh, and S. Kawahito, "A cmos image sensor with in-pixel two-stage charge transfer for fluorescence lifetime imaging," IEEE Transactions on Electron Devices, vol. 56, no. 2, 2009.

[34] I. ISS, "Lifetime data of selected fluorophores," www.iss.com, 2008.

[35] A. Rochas, M. Gani, B. Furrer, P.-A. Besse, and R. Popovic, "Single photon detector fabricated in a complementary metal-oxide-semiconductor high-voltage technology," Review of Scientific Instruments, vol. 74, p. 3263, 2003.

[36] D. E. Schwartz, E. Charbon, and K. L. Shepard, "A single-photon avalanche diode array for fluorescence lifetime imaging microscopy," IEEE Journal of Solid-State Circuits, vol. 43, no. 11, pp. 2546-2557, Nov. 2008.

[37] D. Mosconi, D. Stoppa, L. Pancheri, L. Gonzo, and A. Simoni, "Cmos single-photon avalanche diode array for time-resolved fluorescence detection," in Proc. 32nd European Solid-State Circuits Conference ESSCIRC 2006, Sept. 2006, pp. 564-567.

[38] M. Gersbach, D. Boiko, M. Sergio, C. Niclass, C. Petersen, and E. Charbon, "Time-correlated two-photon fluorescence imaging with arrays of solid-state single photon detectors," in Proc. and the International Quantum Electronics Conference Lasers and Electro-Optics CLEOE-IQEC 2007. European Conference on, 17-22 June 2007, pp. 1-1.

[39] E. Charbon, "Towards large scale cmos single-photon detector arrays for lab-on-chip applications," Journal of Physics D: Applie Physics, vol. 41, 2008.

[40] C. Niclass, M. Sergio, and E. Charbon, "A single photon avalanche diode array fabricated in $0.35 \mathrm{um}$ cmos and based on an event-driven readout for tcspc experiments," Proceedings of SPIE: Advanced Photon Counting Techniques, vol. 6372, 2006.

[41] D. Stoppa, L. Pancheri, M. Scandiuzzo, L. Gonzo, G.-F. Betta, and A. Simoni, "A cmos 3-d imager based on single photon avalanche diode," IEEE Transactions on Circuits and Systems, vol. 54, no. 1, pp. 4-12, Jan. 2007. 
[42] F. Zappa, A. Gulinatti, P. Maccagnani, S. Tisa, and S. Cova, "Spada: Single photon avalanche diode arrays," IEEE Photonics Technology Letters, vol. 17, no. 3, pp. 657-659, March 2005.

[43] F. Zappa, A. Giudice, A. Ghioni, and S. Cova, "Fully-integrated active-quench circuit for single-photon detection," Proceedings of the European Solid-State Circuits Conference, pp. 355-358, 2002.

[44] S. Cova, M. Ghioni, A. Loitito, I. Rech, and F. Zappa, "Evolution and prospects for single-photon avalanche diodes and quenching circuits," Journal of Modern Optics, vol. 51, no. 9-10, pp. 1267-1288, 2004.

[45] S. Pellegrini, R. E. Warburton, L. J. J. Tan, J. S. Ng, A. B. Krysa, K. Groom, J. P. R. David, S. Cova, M. J. Robertson, and G. Buller, "Design and performance of an ingaas-inp single photon avalanche diode detector," IEEE Journal of Quantum Electronics, vol. 42, no. 4, pp. 397-403, 2006.

[46] J. Song, M. Culha, P. Kasili, G. Griffin, and T. Voh-Dinh, "A compact cmos biochip immunosensor towards the detection of a single bacteria," Biosensors and Bioelectronics, vol. 20, pp. 2203-2209, May 2005.

[47] T. Vo-Dinh, G. D. Griffin, A. Wintenberg, D. L. Stokes, J. Mobley, M. Askari, and R. Maples, "Advanced biochip: Principle and applications in medical diagnostics and pathogen detection," Proceedings of the International Symposium on Circuits and Systems, vol. 3, pp. 622-625, 2003.

[48] J. Kling, "Moving diagnostics from the bench to the bedside," Nature Biotechnology, vol. 24, pp. 891-893, 2006.

[49] J. A. Chediak, Z. Luo, J. Seo, N. Cheung, L. P. Lee, and T. D. Sands, "Heterogeneous integration of cds filters with gan leds for fluorescence detection microsystems," Sensors and Actuators A: Physical Review, vol. 111, pp. 1-7, 2004.

[50] E. Thrush, O. Levi, W. Ha, J. Harris Jr., S. Smith, A. Kurtz, J. Hwang, and E. Moerner, "Integrated semiconductor fluorescence sensor for portable bio-medical diagnostics," CLEO, 2003.

[51] R. P. Hertzberg and A. J. Pope, "High-throughput screening: New technology for the 21 st century," Current Opinion in Chemical Biology, vol. 4, pp. 445-451, 2000. 
[52] S. Bellis, J. C. Jackson, and A. Mathewson, "Towards a disposable in vivo miniature implantable fluorescence detector," Proceedings of SPIE: Optical Fibers and Sensors for Medical Diagnostics and Treatment Applications, vol. 6083, 2006.

[53] M. Davenport, A. Titus, E. Tehan, Z. Tao, Y. Tang, R. M. Bukowski, and F. Bright, "Chemical sensing systems using xerogel-based sensor elements and cmos photodetectors," IEEE Sensors Journal, vol. 4, no. 2, pp. 108-188, April 2004.

[54] R. Shinar, B. Choudhury, Z. Zhou, H. S. Wu, L. B. Tabatabai, and J. Shinar, "Structurally integrated organic light-emitting device-based sensors for oxygen, glucose, hydrazine and anthrax," Analytica Chimica Acta, vol. 568, pp. 190-199, 2006.

[55] V. Namasivayam, R. Lin, B. Johnson, S. Brahmassandra, Z. Razzacki, D. Burke, and M. Burns, "Advances in on-chip photodetection for applications in miniaturized gentic analysis systems," Journal of Micromechanics and Microengineering, vol. 14, pp. 81-90, 2004.

[56] E. Thrush, O. Levi, W. Ha, K. Wang, S. J. Smith, and J. Harris Jr., "Integrated bio-fluorescence sensor," Journal of Chromatography A, vol. 1013, pp. 103-110, 2003.

[57] S. Sonkusale, M. Cheng, L. Kish, A. Agarwal, Y. Kim, L. Liu, and S. Seo, “A cmos sensor array ic of nanowell devices for molecular sensing," Proceedings of IEEE Sensors, vol. 2, pp. 963-966, October 2004.

[58] P. Porta and H. Summers, "Vertical-cavity semiconductor devices for generation and detection of fluorescence emission on a single chip," Applied Physics Letters, vol. 85, no. 11, pp. 1889-1891, 2004.

[59] H. Summers and P. A. Porta, "Resonant-cavity optoelectronic devices for fluorimetry," IEEE Journal of Selected Topics in Quantum Electronics, vol. 11, pp. 854-857, 2005.

[60] B. Jang, P. Cao, A. Chevalier, A. Ellington, and A. Hassibi, "A cmos fluorescent-based microarray," Digest of IEEE International Solid-State Circuits Conference, 2009.

[61] R. Aebersold and M. Mann, "Mass spectrometry-based proteomics," Nature, vol. 422, pp. 198-207, 2003.

[62] J. Wang, A.-N. Kawde, A. Erdem, and M. Salazar, "Magnetic bead-based label-free electrochemical detection of dna hybridization," The Analyst, vol. 126, pp. 2020-2024, October 2001. 
[63] I. Lauks, "Microfabricated biosensors and microanalytical systems for blood analysis," Accounts of Chemical Research, vol. 31, no. 5, pp. 317-324, 1998.

[64] J. R. Baena and B. Lendl, "Raman spectroscopy in chemical bioanalysis," Current Opinion in Chemical Biology, vol. 8, pp. 534-539, 2004.

[65] K. Swinney and D. Bornhop, "A chip-scale universal detector for electrophoresis based on backscattering interferometry," The Analyst, vol. 125, pp. 1713-1717, September 2000 .

[66] C. Wong, K. Lei, W. Chow, H. Li, W. Kong, and K. Chan, "Chemical and biological detection using microfluidic platform and surface plasmon resonance imaging sensor," IEEE Sensors, 2005, pp. 187-190, October 2005.

[67] A. Moe, S. Marx, I. Bhinderwala, and D. Wilson, "A miniaturized lock-in amplfier design suitable for impedance measurements in cells," IEEE Proceedings of Sensors, vol. 1, pp. 215-218, October 2004.

[68] R. Thewes, "T4: Introduction to cmos bio sensor arrays: Electrical specifications, cmos processing, circuit and system design," ISSCC 2006 Tutorial, February 2006.

[69] P. J. Tadrous, J. Siegel, P. M. W. French, S. Shousha, E.-N. Lalani, and G. W. H. Stamp, "Fluorescence lifetime imaging of unstained tissues: Early results in human breast cancer," Journal of Pathology, vol. 199, pp. 309-317, 2003.

[70] R. Haitz, "Studies on optical coupling between silicon p-n junctions," Solid-State Electronics, vol. 8, pp. 417-425, 1965.

[71] H. Finkelstein, M. J. Hsu, and S. C. Esener, "Sti-bounded single-photon avalanche diode in a deep-submicrometer cmos technology," IEEE Electron Device Letters, vol. 27, pp. 887-889, 2006.

[72] M. Gersbach, C. Niclass, E. Charbon, J. Richardson, R. Henderson, and L. Grant, "A single photon detector implemented in a 130nm cmos imaging process," in Proc. 38th European Solid-State Device Research Conference ESSDERC 2008, 15-19 Sept. 2008, pp. 270-273.

[73] A. Rochas, A. Pauchard, P.-A. Besse, D. Pantic, Z. Prijic, and R. Popovic, "Low-noise silicon avalanche photodiodes fabricated in conventional cmos technologies," IEEE Transactions on Electron Devices, vol. 49, pp. 387-394, 2002. 
[74] C. Niclass, M. Sergio, and E. Charbon, "A single photon avalanche diode array fabricated in deep-submicron cmos technology," in Proc. Design, Automation and Test in Europe DATE '06, vol. 1, 6-10 March 2006, pp. 1-6.

[75] S. M. Sze, Physics of Semiconductor Devices. Wiley-Interscience, 1981.

[76] S. Cova, A. Lacaita, and G. Ripamonti, "Trapping phenomena in avalanche photodiodes on nanosecond scale," IEEE Electron Device Letters, vol. 12, pp. 685-687, 1991.

[77] J. C. Jackson, D. Phelan, A. P. Morrison, R. M. Redfern, and A. Mathewson, "Toward integrated single-photon-counting microarrays," Optical Engineering, vol. 42, pp. $112-118,2002$.

[78] A. Rochas, "Single photon avalanche diodes in cmos technology," Ph.D. dissertation, Ecole Polytechnique Federale deLausanne, 2003.

[79] A. Spinelli, A. Ghioni, S. D. Cova, and L. M. Davis, "Avalanche detector with ultraclean response for time-resolved photon counting," IEEE Journal of Quantum Electronics, vol. 34, pp. 817-821, 1998.

[80] C. Niclass, A. Rochas, P. Besse, and E. Charbon, "A cmos single photon avalanche diode array for 3d imaging," in Proc. Digest of Technical Papers Solid-State Circuits Conference ISSCC. 2004 IEEE International, 15-19 Feb. 2004, pp. 120-517.

[81] A. Gallivanoni, I. Rech, D. Resnati, M. Ghioni, and S. Cova, "Monolithic active quenching and picosecond timing circuit suitable for large-area single photon avalanche diodes," Optics Express, vol. 14, pp. 5021-5030, 2006.

[82] N. Faramarzpour, J. M. Deen, S. Shirani, and Q. Fang, "Fully integrated single photon avalanche diode detector in standard cmos 0.18-um technology," IEEE Transactions on Electron Devices, vol. 55, pp. 760-767, 2008.

[83] C.-W. Jeon, H. Choi, and M. Dawson, "A novel fabrication method for a $64 \times 64$ matrix-addressable gan-based micro-led array," Physica Status Solidi (a), vol. 200, no. 1, pp. 79-82, 2003.

[84] C. Jeon, H. Choi, E. Gu, and M. Dawson, "High-density matrix-addressable alingan-based 368-nm microarray light-emitting diodes," IEEE Photonics Technology Letters, vol. 16, pp. 2421-2423, 2004. 
[85] C. Jeon, H. Choi, and M. Dawson, "Fabrication of matrix-addressable ingan-based microdisplays of high array density," IEEE Photonics Technology Letters, vol. 15, pp. 1516-1518, 2003.

[86] E. F. Schubert, Light-Emitting Diodes, 2nd ed. Cambridge University Press, 2006.

[87] E. T. Ltd., www.evidenttech.com, 2008.

[88] K. J. Liu, Y. Zhang, and T. H. Wang, "Detect the dots," IEEE Nanotechnology Magazine, vol. 2, pp. 15-16, 2008.

[89] G. E. Moore, "Cramming more components onto integrated circuits," Proceedings of the IEEE, vol. 86, pp. 82-85, 1998, reprint of original paper published in Electronics, 1965.

[90] S. Spiesshoefer and L. Schaper, "Ic stacking technology using fine pitch, nanoscale through silicon vias," Proceedings of the 53rd Electronic Components and Technology Conference, pp. 632-633, 2003.

[91] idQuantique, “www.idquantique.com,” 2009.

[92] MicroPhotonDevices, “www.microphotondevices," 2009.

[93] S. Donati, G. Martini, and M. Norgia, "Microconcentrators to recover fill-factor in image photodetectors with pixel on-board processing circuits," Optics Express, vol. 15, no. 26, pp. 18 066-18 075, December 2007.

[94] N. Scheidegger, R. Krpoun, H. Shea, C. Niclass, and E. Charbon, "A new concept for a low-cost earth sensor: Imaging oxygen airglow with arrays of single photon detectors," American Astronautical Society, 30th Annual Guidance and Control Conference, 2007.

[95] S. Tudisco, F. Musumeci, L. Lanzano, A. Scordino, S. Privitera, A. Campisi, L. Cosentino, G. Condorelli, P. Finocchiaro, G. Fallica, S. Lombardo, M. Mazzillo, D. Sanfilippo, and E. Sciacca, "A new generation of spad-single-photon avalanche diodes," vol. 8, no. 7, pp. 1324-1329, 2008.

[96] J. A. Jo, Q. Fang, T. Papaionnou, and L. Marcu, "Novel ultra-fast deconvolution method for fluorescence lifetime imaging microscopy based on the laguerre expansion technique," Proceedings of the 26th Annual International Conference of the IEEE EMBS, pp. 1271-1274, 2004. 
[97] A. Agronskaia, L. Tertoolen, and H. Gerritsen, "High frame rate fluorescence lifetime imaging," Journal of Physics D: Applied Physics, vol. 36, pp. 1655-1662, July 2003.

[98] R. M. Ballew and J. Demas, "An error analysis of the rapid lifetime determination method for the evaluation of single exponential decays," Analytical Chemistry, vol. 61, pp. 30-33, 1989.

[99] D.-U. Li, E. Bonnist, D. Renshaw, and R. Henderson, "On-chip, time-correlated, fluorescence lifetime extraction algorithms and error analysis," Journal of Optical Society of America A, vol. 25, pp. 1190-1198, 2008.

[100] Z. D. Popovic, R. A. Sprague, and G. A. Neville Connell, "Technique for monolithic fabrication of microlens arrays," Applied Optics, vol. 27, pp. 1281-1284, 1988.

[101] F. Borghetti, D. Mosconi, L. Pancheri, and D. Stoppa, "A cmos single-photon avalanche diode sensor for fluorescence lifetime imaging," IEEE International Image Sensors Workshop, 2007.

[102] D. Stoppa, D. Mosconi, L. Pancheri, and L. Gonzo, "Single-photon avalanche diode cmos sensor for time-resolved fluorescence measurements," IEEE Sensors, p. Awaiting Publication, Submitted for Publication Oct. 2008.

[103] J. Richardson, L. Grant, and R. Henderson, "Low dark-count single-photon avalanche diode structure compatible with standard nanometer scale cmos technology," IEEE Photonics Technology Letters, vol. 21, pp. 1020-1022, 2009.

[104] R. Brederlow, S. Zauner, A. Scholtz, K. Aufinger, W. Simburger, C. Paulus, A. Martin, M. Fritz, H.-J. Timme, H. Heiss, S. Marksteiner, L. Elbrecht, R. Aigner, and R. Thewes, "Biochemical sensors based on bulk acoustic wave resonators," in Proc. IEDM '03 Technical Digest Electron Devices Meeting IEEE International, 8-10 Dec. 2003, pp. 32.7.1-32.7.3.

[105] S. Nakamura and G. Fasol, The Blue Laser Diode: GaN Based Light Emitters and Lasers. Springer: New York, 1997.

[106] C. Jeon, H. Choi, P. Edwards, A. Bryce, and M. Dawson, "64x64 matrix-addressable arrays of gan-based microleds," in The 15th Annual Meeting of the IEEE Lasers adn Electro-Optics Society, 2002. 
[107] H. Zhang, D. Massoubre, J. McKendry, Z. Gong, B. Guilhabert, C. Griffin, E. Gu, P. Jessop, J. Girkin, and M. Dawson, "Individually-addressable flip-chip alingan micropixelated light emitting diode arrays with high continuous and nanosecond output power," Optics Express, vol. 16, pp. 9918-9926, 2008.

[108] K.-Y. Chou, C. M.-J, and C.-W. Liu, "Active devices under cmos i/o pads," IEEE Transactions on Electron Devices, vol. 49, no. 12, pp. 2279-2287, 2002.

[109] V. Poher, H. Zhang, G. Kennedy, C. Griffin, S. Oddos, E. Gu, D. Elson, J. Girkin, P. French, M. Dawson, and M. Neil, "Optical sectioning microscopes with no moving parts using a micro-stripe array light emitting diode," Optics Express, vol. 15, pp. 11 196-11 206, 2007.

[110] E. Instruments, "Fast - advanced analysis of fluorescence kinetics," Operating Manual, September 2007.

[111] A. El Gamal and H. Eltoukhy, "Cmos image sensors," IEEE Circuits and Devices Magazine, vol. 21, pp. 6-20, 2005.

[112] B. E. Bayer, “Colour imaging array,” US Patent, March 1975, patent No. 3,971,065.

[113] D. Magde, R. Wong, and P. G. Seybold, "Fluorescence quantum yields and their relation to lifetimes of rhodamine $6 \mathrm{~g}$ and fluorescein in nine solvents: Improved absolute standards for quantum yields," Photochemistry and Photobiology, vol. 75, pp. 327-334, 2002.

[114] D.-U. Li, R. Walker, J. Richardson, B. Rae, A. Buts, D. Renshaw, and R. K. Henderson, "Hardware implementation and calibration of background noise for integration-based fluorescence lifetime sensing algorithms," 2009, accepted for publication by Journal of the Optical Society of America A.

[115] T. Vo-Dinh and M. Askari, "Micro-arrays and biochips: Applications and potential in genomics and proteomics," Journal of Current Genomics, vol. 2, no. 4, pp. 399-415, December 2001.

[116] A. Cleary, A. Glidle, P. Laybourn, S. Garcia-Blanco, S. Pellegrini, C. Helfter, G. Buller, J. Aitchison, and J. Cooper, "Integrating optics and microfluidics for time-correlated single-photon counting in lab-on-a-chip devices," Applied Physics Letters, vol. 91, p. 071123, 2007. 
[117] H. X. Jiang and J. Y. Lin, "Advances in iii-nitride micro-size light emitters," III-Vs Review, vol. 14, p. 32, 2001.

[118] Z. Gong, H. Zhang, E. Gu, C. Griffin, M. Dawson, V. Poher, G. Kennedy, P. M. W. French, and M. Neil, "Matrix-addressable micropixellated ingan light-emitting diodes with uniform emission and increased light output," IEEE Transactions on Electron Devices, vol. 54, pp. 2650-2658, 2007.

[119] K. H. Kim, Z. Y. Fan, M. Khizar, M. L. Nakami, J. Y. Yin, and H. X. Jiang, “Algan-based ultraviolet light-emitting diodes grown on ain epilayers," Applied Physics Letters, vol. 85, pp. 4777-4779, 2004.

[120] H. Xu, J. Zhang, K. M. Davitt, Y.-K. Song, and A. V. Nurmikko, "Application of blue-green and ultraviolet micro-leds to biological imaging and detection," Journal of Physics D: Applied Physics, vol. 41, 2008.

[121] Y. Yang, G. A. Turnbull, and I. D. W. Samuel, "Hybrid optoelectronics: A polymer laser pumped by a nitride light-emitting diode," Applied Physics Letters, vol. 92, p. 163306, 2008.

[122] I. D. W. Samuel, “Laser physics: Fantastic plastic,” Nature, vol. 429, pp. 709-711, 2004.

[123] L. Li, H. Hu, H. Lin, and D. tian Ye, "Electrowetting of the blood droplet on the hydrophobic film of the ewod chips," in Proc. 27th Annual International Conference of the Engineering in Medicine and Biology Society IEEE-EMBS 2005, 2005, pp. 1941-1944.

[124] Y. Li, P. Li, A. Kazantzis, L. Haworth, K. Muir, A. Ross, J. Terry, J. Stevenson, A. Gundlach, A. Bunting, and A. Walton, "Building ewod microfluidic array technology on top of foundry cmos," in Proc. Institution of Engineering and Technology Seminar on MEMS Sensors and Actuators, 28-28 April 2006, pp. 23-30.

[125] Y. Li, M. Yoshio, L. Haworth, W. Parkes, M. Kubota, and A. Walton, “Test structure for characterising low voltage coplanar ewod system," in Proc. IEEE International Conference on Microelectronic Test Structures ICMTS 2008, 24-27 March 2008, pp. $80-85$. 
[126] N. Manaresi, A. Romani, G. Medoro, L. Altomare, A. Leonardi, M. Tartagni, and R. Guerrieri, "A cmos chip for individual cell manipulation and detetection," IEEE Journal of Solid-State Circuits, vol. 38, no. 12, pp. 2297-2305, December 2003. 